\title{
Vegetation analysis of the subalpine beech forest on the upper forest line in the Julian Alps (NW Slovenia and NW Italy) and in the northern Dinaric Alps
}

\author{
Igor Dakskobler ${ }^{1}$ \& Andrej Rozman² (1)
}

Key words: phytosociology, synsystematics, hierarchical classification, UPGMA, beech communities, upper forest line, Julian Alps, Trnovo Forest Plateau, Triglav National Park, Natura 2000, Slovenia, Italy.

Ključne besede: fitocenologija, sinsistematika, hierarhična klasifikacija, UPGMA, bukove zružbe, zgornja gozdna meja, Julijske Alpe, Trnovski gozd, Triglavski narodni park, Natura 2000, Slovenija, Italija.
Received: 26. 9. 2020

Revision received: 10. 2. 2021

Accepted: 12. 2. 2021

\begin{abstract}
Using hierarchical clustering with unweighted pair-group method with arithmetic mean (UPGMA) we arranged 603 phytosociological relevés of beech forests on the present upper forest line, mainly from the Julian Alps and the Trnovo Forest Plateau (we also included the relevés from the Karawanks and the Kamnik Alps), into 32 clusters. Based on their analysis and comparison with previously described similar (alti)montane-subalpine beech communities we classified most of the relevés into the association Polysticho lonchitis-Fagetum and its new subassociations ericetosum carneae, cardaminetosum trifoliae, luzuletosum niveae, luzuletosum luzuloidis, calamagrostietosum variae, allietosum victorialis, adoxetosum moschatellinae, stellarietosum nemorum and several new variants. The altitude of the studied stands is predominantly 1400 to $1550 \mathrm{~m}$ (the upper line is at $1660 \mathrm{~m}$ ); they occur at all aspects, frequently on steep and very steep slopes, mainly on limestone and dolomite limestone, the predominant soil type is rendzina. These stands are species rich (on average 61 species per relevé, altogether more than 500 vascular plants) and have many species in common with the stands of associations Rhododendro hirsuti-Fagetum and Rhodothamno-Laricetum.
\end{abstract}

\section{Izvleček}

S hierarhično klasifikacijo z metodo kopičenja na podlagi povezovanja (netehtanih) srednjih razdalj (UPGMA) smo 603 fitocenoloških popisov bukovih gozdov na zdajšnji gozdni meji, večinoma iz Julijskih Alp in Trnovskega gozda (vključili smo tudi popise iz Karavank in Kamniških Alp), razdelili v 32 skupin. Na podlagi njihove analize in primerjave $\mathrm{z}$ do zdaj opisanimi podobnimi (alti) montansko-subalpinskimi bukovimi združbami smo večino popisov uvrstili v asociacijo Polysticho lonchitis-Fagetum in v naslednje njene nove subasociacije: ericetosum carnea, cardaminetosum trifoliae, luzuletosum niveae, luzuletosum luzuloidis, calamagrostietosum variae, allietosum victorialis, adoxetosum moschatellinae, stellarietosum nemorum in več novih variant. Nadmorska višina preučenih sestojev je najbolj pogosto med $1400 \mathrm{~m}$ in $1550 \mathrm{~m}$ (zgornja meja je $1660 \mathrm{~m}$ ), uspevajo v vseh legah, pogosto na strmih in zelo strmih pobočjih, predvsm na apnencu in dolomitnem apnencu, talni tip je večinoma rendzina. So vrstno bogati (povprečno 61 vrst na popis, skupno več kot 500 praprotnic in semenk) in imajo veliko skupnih vrst s sestoji asociacij Rhododendro hirsutiFagetum in Rhodothamno-Laricetum.

\footnotetext{
1 Scientific Research Centre of the Slovenian Academy of Sciences and Arts, Institute of Biology, Regional Unit Tolmin, Brunov drevored 13, SI-5220 Tolmin, Slovenia. E-mail: igor.dakskobler@zrc-sazu.si

2 University of Ljubljana, Biotechnical Faculty, Department of Forestry and Renewable Forest Resources, Večna pot 83, 1000 Ljubljana, Slovenia. E-mail: andrej.rozman@bf.uni-lj.si
} 


\section{Introduction}

The characteristics of the upper forest line in Slovenia have been studied by numerous authors and from various aspects, from ecological and silvicultural to geographical. Wraber (1970) investigated the ecological aspect of the upper forest line. Forest research at the upper forest line has been incorporated in many graduation and master theses. Some of the more notable ones have been Robič (1998), Pogačnik \& Prosen (1998), Kadunc \& Rugani (1999), Ferreira et al. (2000) and Firm et al. (2006). The geographical aspect of the upper forest line has been studied by Gams (1976, 1977, 2002), Vrtačnik Merčun (1999) and in particular by Lovrenčak $(1977,1987,1989$, 2007), along with other authors. Properties of beech at the upper forest line were discussed by Torelli (2001). A number of older sources, e.g. some of those focusing on the Julian Alps and their foothills (Stur 1857, Marek 1910, Tuma 1925), give evidence of the human impact on its current course today.

Investigations into the vegetation of beech forests at the upper forest line have a long history. Fanta (1981) contributed an overview of the wider Alpine region. The fundamental source for the Illyrian floral province (the Southeastern Alps and the northern part of the Dinaric Alps) was Horvat's work (1938), which included the description of the association Fagetum sylvaticae croaticum subalpinum. An association with a similar name Fagetum subalpinum Horvat was reported in Slovenia by Tregubov (1957), who published three relevés from the Snežnik Mountains (the Dinaric Alps). He reported the following characteristic species: Adenostyles glabra, Ranunculus platanifolius, Luzula sylvatica, Veratrum album, Polystichum lonchitis and Cicerbita alpina. A more extensive phytosociological table with 10 relevés made at the forest line under Mt. Ratitovec and Mt. Blegoš (the foothills of the Julian Alps) titled Fagetum subalpinum prealpinum was published by Marinček (1980), who listed following diagnostic species of this association: Fagus sylvatica, Polystichum lonchitis, Viola biflora, Carex ferruginea, Rhododendrum hirsutum, Centaurea montana, Salix waldsteiniana, S. glabra, Ribes alpinum and Geranium sylvaticum. Marinček (1987) also published a vegetation outline (without vegetation tables) and a silvicultural description of the beech forest at the upper forest line in the Alps and the northern part of the Dinaric Alps, namely Fagetum subalpinum prealpinum and Fagetum subalpinum dinaricum. Subsequently, Zukrigl (1989) described altimontane-subalpine beech forests in the immediate vicinity of Slovenia, on the northern slopes of the Karawanks and a part of the Carnic Alps, as the association
Saxifrago rotundifoliae-Fagetum, and presented it with a table comprising 73 relevés, which he classified into several subassociations and variants. This relevé material, with stands recorded in the belt spanning 1000 to 1500 $\mathrm{m}$, comprises several stands in the vicinity of the upper forest line (Saxifrago rotundifoliae-Fagetum calamagrostietosum variae, five relevés, Saxifrago-Fagetum rhododendretosum hirsuti, one relevé). Poldini \& Nardini (1993) published a phytosociological table with five relevés of the subalpine beech forest from the Carnic Alps in Friuli (at 1390 to $1650 \mathrm{~m}$ a.s.l.) and classified them into the syntaxon Polysticho lonchitis-Fagetum Marinček in Poldini et Nardini 1993 var. geogr. Anemone trifolia. They refer to Polystichum lonchitis, Luzula sylvatica, Homogyne alpina, Rhododendron hirsutum and Rhododendron ferrugineum as diagnostic species of the association. The spatial distribution and ecological description of the stands of this association in the Italian part of the Julian Alps was published by Del Favero et al. (1998: 90-91).

Marinček et al. (1993) selected Horvat's relevé (1938) from the table of the association Fagetum sylvaticae croaticum subalpinum as the nomenclatural type (lectotype) of the association Polysticho lonchitis-Fagetum. The association Saxifrago rotundifoliae-Fagetum p.p. is listed in the synonymy of two associations, Polysticho lonchitis-Fagetum and Ranunculo platanifolii-Fagetum. In terms of the altitude and floristic composition the holotype of the association Saxifrago rotundifolli-Fagetum as selected in Willner (2002: 403), but see also Zukrigl (1989, Table I), cannot be classified into the association Polysticho lonchitis-Fagetum in the sense of Marinček et al. (1993). Marinček (1996) published a description of a new geographical variant Polysticho lonchitis-Fagetum var. geogr. Allium victorialis with three subassociations (polystichetosum, adenostyletosum alliariae and hacquetietosum) based on the relevés from the Dinaric Alps, namely the Snežnik Mountains and the Trnovo Forest Plateau. He listed Polystichum lonchitis, Carex ferruginea, Pinus mugo, Clematis alpina, Rhododendron hirsutum, Ribes alpinum, Salix appendiculata, Sorbus chamaemespilus, Ribes petraeum and Lonicera caerulae as diagnostic species. Willner (2002, 2007a, 2007b) opted for Saxifrago rotundifoliae-Fagetum Zukrigl 1989 as the valid name for altimontane and subalpine beech forests on calcareous bedrock in the Southeastern Alps and the northern Dinaric Alps, and included the stands of associations Polysticho lonchitis-Fagetum and Ranunculo platanifolii-Fagetum into this association. In Zukrigl's publication (1989), however, the nomenclatural type of the association Saxifrago rotundifolii-Fagetum is missing, and was only published in Willner (2002: 403): "Nomenklatorischer Typus: Zukrigl l. c., Tab. I, Laufende Nr. 151 (Holotypus)”. Willner (2007b) also published a 
synoptic table of this association based on the relevés from Austria (primarily Zukrigl's relevés from 1989) and divided it into several subassociations, among which subassociations calamagrostietosum variae and typicum with their species composition have the most similarities with the subalpine beech forest in the Julian Alps. According to Willner the differential species of the subassociation Saxifrago rotundifoliae-Fagetum calamagrostietosum variae are Calamagrostis varia, Sesleria caerulea, Erica carnea, Rhododendron hirsutum, Vaccinium vitis-idaea, Carex alba, Polygala chamaebuxus, Sorbus aria, S. chamaemespilus and Pinus mugo, whereas Carex ferruginea, Aster bellidiastrum, Polystichum lonchitis, Rubus saxatilis, Ranunculus montanus, Gymnocarpium robertianum, Betonica alopecuros and Cirsium erisithales are differential species of the subassociation typicum. Surina \& Rakaj (2007) described a new subassociation Polysticho lonchitis-Fagetum rhodendretosum hirsuti in the Snežnik Mountains. They listed Polystichum lonchitis, Salix appendiculata, Carex ferruginea, Lonicera caerulea in Ribes alpinum as the diagnostic species of the association, and Rhododendron hirsutum, Rubus saxatilis, Clematis alpina and Rosa pendulina as the differential species of the new subassociation. By comparing previously published material on altimontane-subalpine beech forests with Rhododendron birsutum (Horvat 1938, Marinček 1980, 1996, Poldini \& Nardini 1993, Dakskobler 2003, 2004, Dakskobler et al. 2000, Willner 2007b) they observed that Willner's (Zukrigl's) subassociations Saxifrago rotundifoliae-Fagetum typicum and calamagrostietosum variae group with relevés of the association Polysticho lonchitis-Fagetum, whereas other subassociations of the association Saxifrago rotundifoliae-Fagetum group separately, which indicates that this association comprises very diverse communities.

Marinček \& Čarni (2010) ignored Willner's findings in their synthetic study of altimontane beech forests of Slovenia and briefly described three races (geographical variants) of the association Polysticho lonchitis-Fagetum: var. geogr. Salix waldsteniana (relevés from Blegoš and Ratitovec), var. geogr. Anemone trifolia (SE-Alps) and var. geogr. Allium victorialis (northern Dinaric Alps). In the same year, the authors of this article (Dakskobler \& Rozman 2010) published a description of the new subassociation Polysticho lonchitis-Fagetum betuletosum pubescens based on four relevés from the cirque $\mathrm{Za}$ Akom. As diagnostic species we identified also Juniperus sibirica and Rhodothamnus chamaecistus. Two years later we mutated the name of the new subassociation in keeping with taxonomic findings (namely that the taxon Betula pubescens subsp. carpatica occurs in some of the cirques in the eastern Julian Alps) to Polysticho lonchitis-Fagetum betuletosum carpaticae (Dakskobler et al. 2012). Subsequently, in the framework of our research of forest and shrub communities with Alnus viridis, we published a table with 27 relevés, of which 25 were classified into the association Polysticho lonchitis-Fagetum (Dakskobler et al. 2013). We identified Aster bellidiastrum, Laserpitium peucedanoides, Festuca calva, Senecio cacaliaster, Astrantia bavarica, Clematis alpina, Alnus viridis and Aconitum lycoctonum subsp. ranunculifolium as diagnostic (or differential) species of this association. We also expressed our disagreement with Willner's (2007a) conclusions on a single association of altimontane and subalpine beech forests on carbonate bedrock, which had been based on elaboration of previously collected relevé material with more than 400 relevés of beech forests at the upper forest line. In his extensive comparison of altimontane beech and maple-beech forests Zupančič (2012) argued that, based on the material published therein, it was not necessary to differentiate between associations Ranunculo platanifolii-Fagetum and Polysticho lonchitis-Fagetum and proposed for the stands of the races that had until then been described in Slovenia (var. geogr. Salix waldsteiniana and var. geogr. Allium victorialis) to be incorporated into the association Ranunculo platanifolii-Fagetum as altitudinal variants (var. alt.). He identified Luzula sylvatica, Ranunculus platanifolius and Polystichum lonchitis as its characteristic species.

It was therefore necessary to collect and edit all the collected material (excluding several ten relevés made during field seasons of 2018 and 2019) in the same way we had analysed larch forests several years previous (Dakskobler et al. 2018). Our goal was to determine:

- Into how many associations can we classify beech forests at the upper forest line in the Julian Alps?

- Does our relevé material support the existing division into lower syntaxonomic units, races (geographical variants), subassociations, variants?

- Which ecological factors are the most critical for the species diversity of these forests?

- What are the similarities between beech forests on extreme sites at their upper distribution limit and subalpine larch forests with which they occasionally come into contact?

- Are subalpine beech forests on the southern edge of the Alps that directly transition to dwarf pine, rocks or Alpine swards floristically distinctly different from subalpine beech forests in the interior of the Alps, where they are succeeded by the larch belt?

- What is the percentage and role of sycamore and conifers such as spruce, fir and larch in subalpine beech forests? 
- Does our vegetation analysis reflect the effects of threat factors and anthropogenic impact, in particular pasture, in these forests?

- Does their species composition reflect the spread of more thermophytic or even invasive alien species, and can we observe the influence of the climate change in recent decades in their species composition?

\section{Methods}

\section{Study area}

Our research was limited to the study of beech forest vegetation at the current upper distribution limit. Most of the relevés were made between 1986 and 2017 across the Julian Alps, partly also in the western Karawanks, the Kamnik Alps and the Trnovo Forest Plateau.

Our stands were therefore recorded in the highest belt that still allows for the growth of beech. We distinguished at least three groups of beech stands:

A: stands on the climatic upper forest line (the highest forest belt) where beech forest transitions through dwarf pine to alpine swards (the Tolmin-Bohinj Mts., the Krn Mts., Porezen, Matajur, the Stol range, Muzci / Cime del Monte Musi, Krnica / Fossa di Carnizza under Krniška Glavica / Jôf do Somdogna, the Kanin Mts., partly the Bala valley, the Loška Koritnica valley, the Lepena and Upper Soča valleys);

B: stands on the orographic forest line, where rocks and rock faces prevent the forest to extend higher (cirques Pod Špikom and Za Akom, Bukovlje in the Vrata valley, partly the Bala valley, the Loška Koritnica valley);

C: the highest belt of beech stands in the areas, where open canopy spruce or larch forests occur in the upper forest line (Komna with the Lopučnica valley, valleys of Krma, Kot, Vrata, Beli potok, Planica-Tamar).

In a large part of the study area the current course of the upper forest line has been shaped by climatic factors and orographic conditions as well as by man, either through deforestation and pasture or military activity after World War I. Human impact is the most obvious in parts of the Krn and Kanin Mountains, on Mts. Matajur, Porezen and Črna prst, but is less significant elsewhere, and the course of the upper forest line has been determined by natural factors (also because of the significant protective function of beech forests). Such localities are high-karst plateau Lopata at Vogel, sunny slopes of the TolminBohinj Mts. under Hohkovbl / Matajurski Vrh, Rodica, Vogel and Žabijski Kuk; Grušnica, Kožljak and the Slemenske Peči range above the Tolminka valley, shady slopes of the Stol ridge, Muzci / Cime del Monte Musi range above Rezija / Val Resia, Trbiška Krnica / Carnizza di Rio Freddo above the valley of Mrzla voda /Valle di Rio Freddo and Krnica / Fossa di Carnizza under Krniška Glavica / Jôf do Somdogna above the Zajzera Valley / Val Saisera in the western Julian Alps, promontories in the rock walls of Mt. Rombon above the Možnica valley, the Bala valley, promontories and ledges on the northern slopes of Loška Stena rock wall from Krnica under Mt. V Gradu to Planinica and Ruševa Glava, the slopes under Jerebica, Planja, Nemške Glave / Cime del Mughi and Predelske Glave / Cima Predil, Mangart and Jalovec, all above the Loška Koritnica valley, cirques Pod Špikom and $\mathrm{Za}$ Akom in the upper Sava Valley, ledges above the valleys of Tamar, Vrata, Kot and Krma.

\section{Vegetation data processing}

A total of 603 of our own relevés of subalpine beech stands are stored in the FloVegSi database (Seliškar et al. 2003). All relevés were initially arranged in one table, in which we merged the stand layers recorded on site (the upper tree layer, lower tree layer, upper shrub layer, lower shrub layer, herb and moss layer) into four main layers: the tree layer (E3), the shrub layer (E2), the herb layer (E1) and the moss layer (E0).

We transformed Braun-Blanquet's scale $(r,+, 1,2,3,4,5)$ - Braun-Blanquet (1964) - into cover percentages $(0-100 \%)$ and calculated, for different layers (two shrub layers and two tree layers), the total coverage of the main layers using the below equation (Jennings et al. 2009, Maarel van der \& Franklin 2013),

$C_{i}=\left[1-\prod_{j=1}^{n}\left(1-\frac{\% \operatorname{cov} j}{100}\right)\right] \times 100$

where $\operatorname{cov} j$ is species cover in layer $j$. In the phytosociological table we converted the calculated total covers back to the original Braun-Blanquet scale.

The relevés were compared by means of hierarchical classification using the unweighted average linkage clustering method (UPGMA) and nonmetric multidimensional scaling (nMDS), where only the first two axes were taken into account. In both cases, Wishart's similarity ratio coefficient was used as the dissimilarity measure. Percentage covers (0-100\%) were modified by square root $(\sqrt[2]{\mathrm{cov}})$. Based on the results, we arranged the relevés into partial tables.

In identifying the indicator species of the syntaxa we used the Indicator Value Index (Legendre \& Anderson 1999, De Caceres \& Legendre 2009) and $\phi$ (phi) value (Chytrý et al. 2002). The permutation test was used to eliminate the species with a non-significant occurrence 
optimum in a particular cluster. Species with frequency $\geq 15 \%$, a phi coefficient $\geq 0.25$ and a difference in frequencies among clusters $\geq 10 \%$ were considered to be good candidates for differential species (Slezak et al. 2016).

Numerical comparisons were made with the software package SYN-TAX (Podani 2001) and R (R Core Team 2017), using the package vegan (Oksanen et al. 2017) and indicspecies (De Caceres \& Legendre 2009).

In describing new subassociations and variants we used the concept of relative differential species. It refers to a species that is usually abundant in the stands of the researched communities, but has an obviously higher frequency or medium coverage in a certain group of relevés and thus distinctly characterises them. Some of the syntaxa could only be named after such species, because we could not identify differential species that do not occur in stands of other similar syntaxa.

Geoelemental, ecological and phytosociological designations of plant species follow the Flora alpina (Aeschimann et al. 2004a, 2004b) but for the diagnostic species of the syntaxa Vaccinio-Piceetea, Erico-Pinetea, Quercetalia pubescenti-petraeae, Fagetalia sylvaticae, Querco-Fagetea, Elyno-Seslerietea, Festuco-Brometea and Asplenietea trichomanis we rely also on our own experience and the opinion of our experienced colleague Mitja Zupančič. There are several species that can be assigned to more than one syntaxonomical unit. In such cases we also relied on our own experience with sites and plant communities in Slovenia.

The nomenclatural source for the names of vascular plants is the Mala flora Slovenije (MFS) (Martinčič et al. 2007). The nomenclature of Flora alpina - Sesleria caerulea (Aeschimann et al. 2004b) was used for the taxon Sesleria caerulea subsp. calcaria (MFS). We also used the names Molinia arundinacea Schrank (instead of Molinia caerulea subsp. arundinacea), Alnus viridis (Chaix) DC in Lam \& DC (instead of Alnus alnobetula (Ehrh.) Hartig) and Heracleum pollinianum Bertol. (instead of Heracleum sphondylium subsp. pollinianum (Bertol.) Neum.). According to Rottensteiner (personal communication), taxon Aconitum lycoctonum subsp. ranunculifolium, which is reported in MFS, is in fact Aconitum lupicida. Martinčič $(2003,2011)$ is the nomenclatural source for the names of mosses and Suppan et al. (2000) is the nomenclatural source for the names of lichenized fungi. The determination of some less frequent mosses and lichenized fungi is not always reliable. The nomenclatural sources for the names of syntaxa are Theurillat (2004) and Šilc \& Čarni (2012). Buser (2009) is the source of data on the geological bedrock, and the source for the nomenclature of soil types is Urbančič et al. (2005). Climate data (precipi- tation volume, mean temperature, mean moisture and snow cover duration) were obtained from high resolution raster maps provided by the Environmental Agency of the Republic of Slovenia, Ministry of the Environment and Spatial Planning (http://www.arso.gov.si/).

\section{Results and discussion}

\section{Ecological conditions in the studied subalpine beech stands}

The average annual daily temperature in the study area is between 3 and $5^{\circ} \mathrm{C}$ and the annual precipitation level ranges between (1800) 2000 and $3500 \mathrm{~mm}$. The snow cover persists for (80) 120 to 150 (180) days. The vertical range of the localities of the relevés ranges between 1200 to $1660 \mathrm{~m}$ a.s.l., the highest density of relevés is at the elevations 1400 do $1550 \mathrm{~m}$ (Figure 1, source http://www. arso.gov.si/).

The ratio between shady (N, NE, E, NW) and sunny aspects (S, SE, SW) is 48: 52 . More than $70 \%$ of the relevés were recorded on slopes of 25 degrees or steeper (Figure 2). Limestone ( $46 \%$ of all plots) and limestonedolomite bedrock (35\% of all plots) prevail. Dolomite is the geological bedrock in $6 \%$ of all relevés, and the same percentage of relevés (about 6\%) have mixed bedrock (limestone, rarely dolomite, with admixture of marlstone, chert or claystone). In 5\% of the plots the parent material is gravel (debris), moraine (till), breccia, talus or rockslide.

The soil type of more than $97 \%$ of the relevés is rendzina, on $2 \%$ of the plots calcareous brown soil (brown soil on limestone) and eutric brown soil on $1 \%$ of the plots. The relevés were made at the peak of the vegetation period, which lasts from July to September, in the period between 1986 and 2017.

Subalpine beech stands are generally slightly open, the tree layer cover is mostly 65 to $90 \%$, the shrub layer covers between 5 and 35\%, the species-rich herb layer covers 50 to $80 \%$ and the moss ground cover is about $10 \%$ (5 to $25 \%$ ).

Most of the plots, measuring mainly $400 \mathrm{~m}^{2}$ (some also $200 \mathrm{~m}^{2}$ ), comprised between 45 and 75 plant taxa (on average 61 taxa); the highest number of taxa per plot was 103. The average Shannon diversity index is between 2.8 and 3.5 per plot (Figure 3).

In most of the stands, the maximum diameter at breast height is 25 to $55 \mathrm{~cm}$ and the upper tree height less than $20 \mathrm{~m}$ (mostly 8 to $18 \mathrm{~m}$ ), with some trees (admixed larch or spruce) substantially larger (Figure 3 ). 

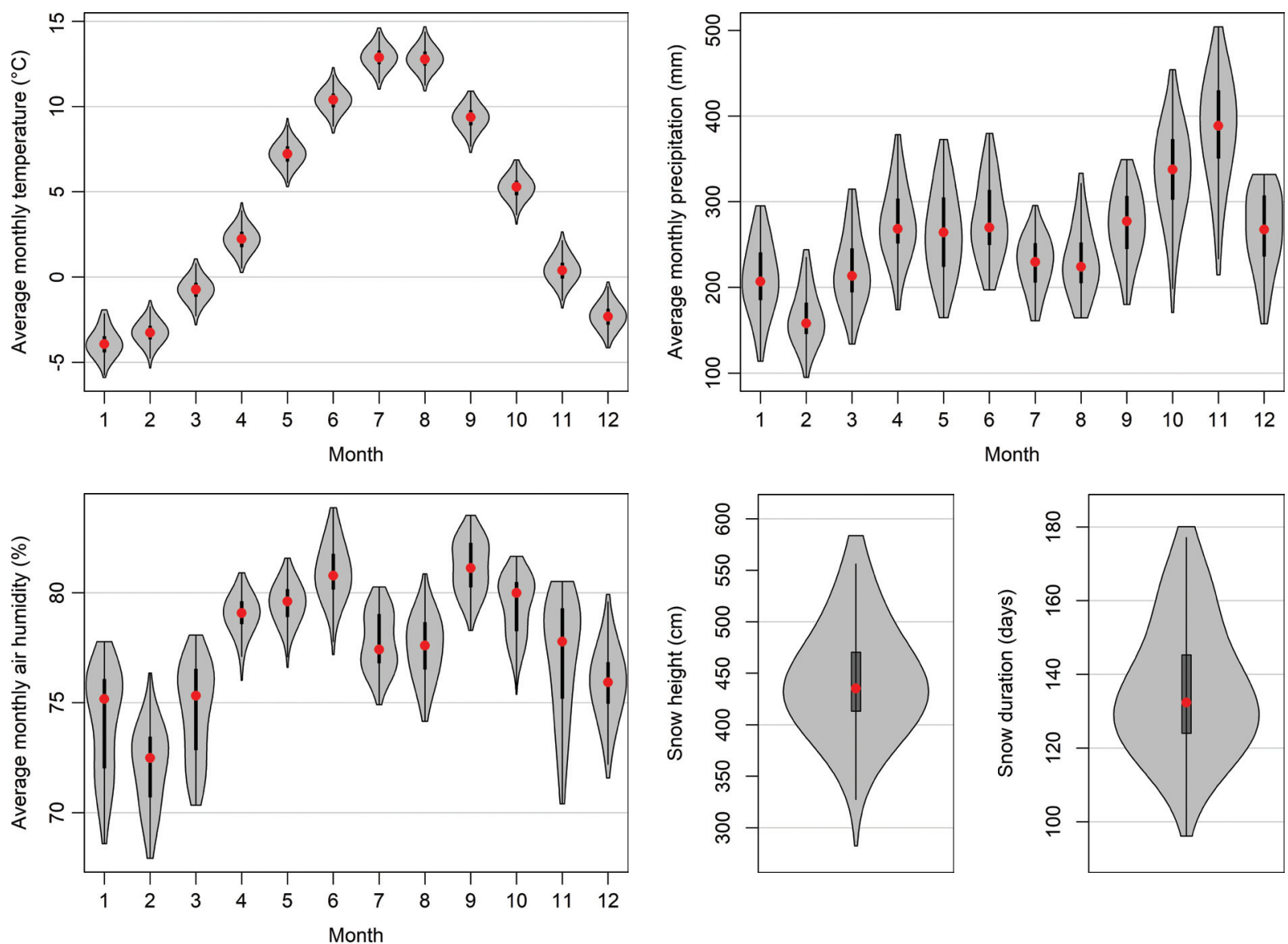

Figure 1: Density plots of average monthly temperature, precipitation and air humidity, the total height of new snow and the duration of the snow cover in subalpine beech forests. Red dots represent medians.

Slika 1: Gostote porazdelitve povprečne mesečne temperature, padavin in zračne vlažnosti ter skupne višina novega snega in trajanja snežne odeje v subalpinskem bukovju. Rdeče točke so mediane.

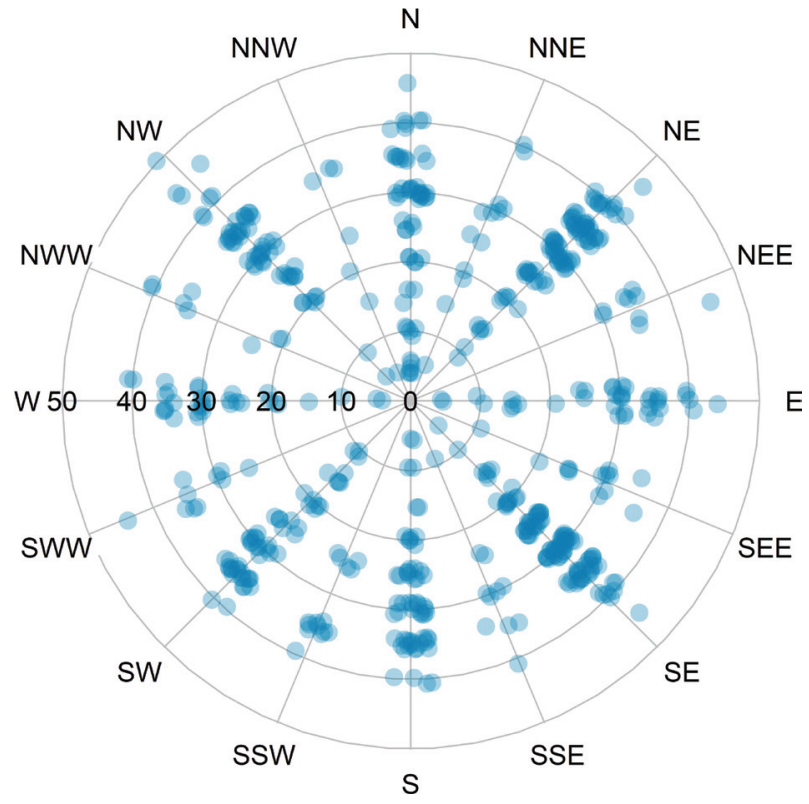

Figure 2: Radial diagram of aspects and slopes $\left(^{\circ}\right)$ in subalpine beech stands.

Slika 2: Radialni diagram nebesnih leg in nagibov terena $\left(^{\circ}\right)$ v sestojih subalpinskega bukovja. 

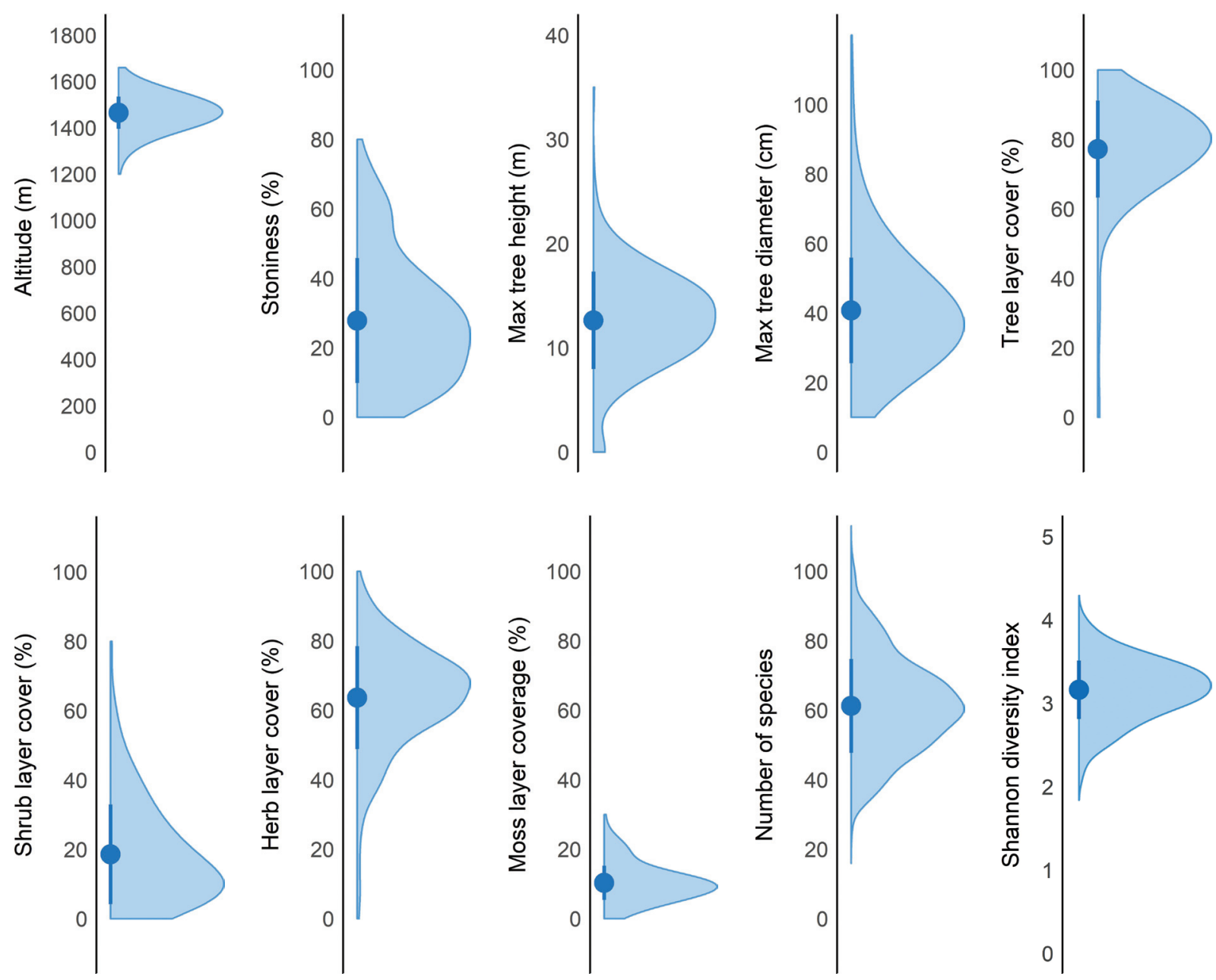

Figure 3: Density plots of certain ecological variables and stand parameters in subalpine beech forests.

Slika 3: Gostote porazdelitve nekaterih ekoloških spremenljivk in sestojnih parametrov v subalpinskem bukovju.

\section{Intercomparison of the studied} stands and their comparison with similar montane-altimontane beech communities

In hierarchical classification, 602 relevés from the Southeastern Alps and northeastern Dinaric Alps (Figure 4) formed 32 groups (clusters) - Table S17, with several relevés distinctly different from others. Similarity between 32 groups that can be classified into a specific syntaxonomic rank was determined with their hierarchical classification (Figure 5) and according the results arranged into 23 Tables: Tables 1-8, which are printed, and Tables S2-S16, which are available only in electronic Appendix). Relevés which were distinctly different from others were arranged in Table S1 (also only in electronic Appendix).
32 groups of relevés from Table S17 (synoptic table in electronic Appendix), arranged according to their similarities into Tables $1-8$ and into Tables S1-S16 and classified into 38 different syntaxa were compared with different forms (subassociations, races) of similar associations Polysticho lonchitis-Fagetum, Ranunculo platanifolii-Fagetum, Saxifrago rotundifoliae-Fagetum, Anemono trifoliae-Fagetum, Rhododendro hirsuti-Fagetum, Stellario montanae-Fagetum, Aconito paniculati-Fagetum, MyrrhidoFagetum, Cardamino waldsteinii-Fagetum (see Table S18 in electronic Appendix). The syntaxa from this table were compared using hierarchical classification (Figure 6) and ordination (Figure 7).

Based on the relevés from publications listed in Table S18 we made a synoptic table with associations Aconito paniculati-Fagetum, Anemono trifoliae-Fagetum, Cardamino waldsteinii-Fagetum var. Abies alba, Myrrhido-Fagetum, Polysticho lonchitis-Fagetum, Ranunculo platanifolii-Fage- 
- Hacquetia 20/2 •2021,373-564

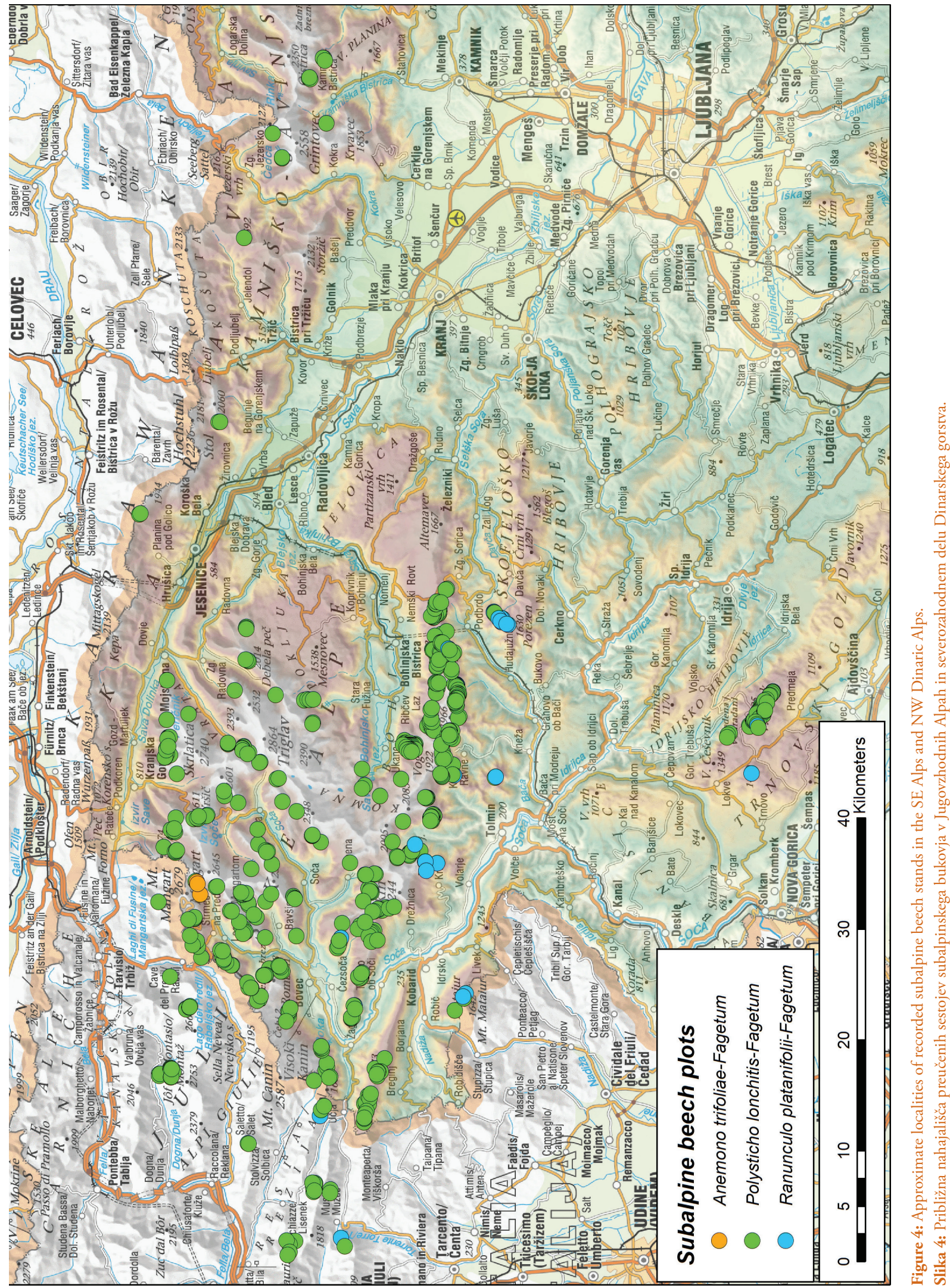




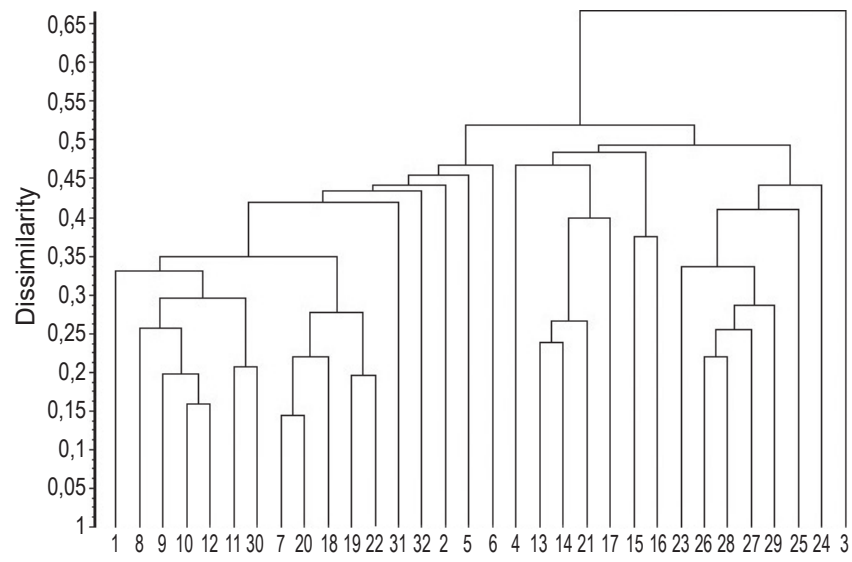

Figure 5: Hierarchical classification of 32 clusters of subalpine beech forests (Table S17, explanation in text, see also Tables 1-8, Tables S2-S16 and Table S18) in SE Alps and NW Dinaric Alps (UPGMA, similarity ratio).

Slika 5: Hierarhična klasifikacija 32 skupin subalpinskih bukovih gozdov (Tabela S17, razlaga v besedilu, glej tudi Tabele 1-8, S2-S16 in S18) v Jugovzhodnih Alpah in severozahodnem delu Dinarskega gorstva (UPGMA, similarity ratio).

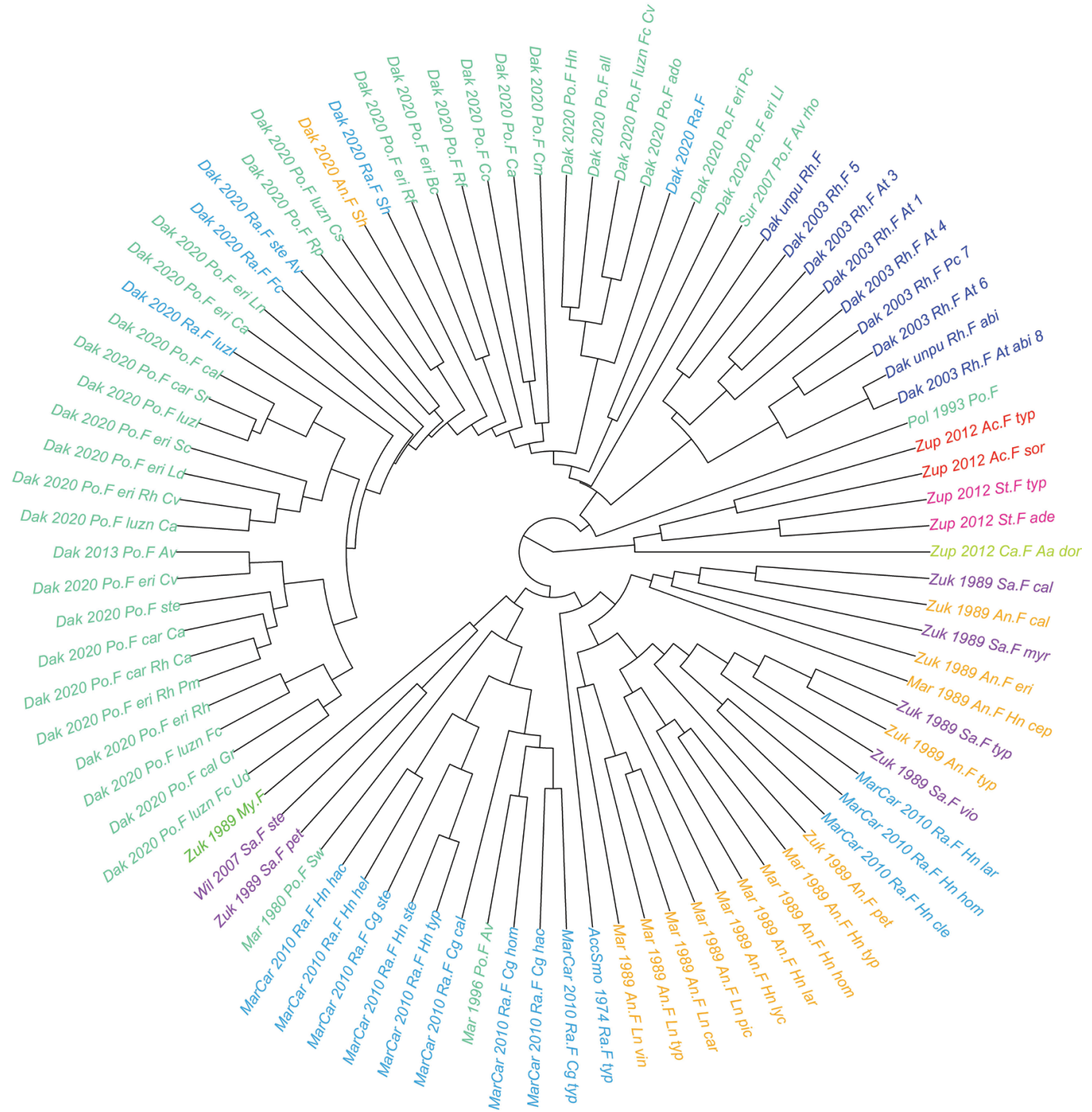

Figure 6: Hierarchical classification of 90 syntaxa of (alti)montane and subalpine beech forests in the SE Calcareous Alps (Table S18).

Slika 6: Hierarhična klasifikacija 90-tih sintaksonov (alti)montanskih in subalpinskih bukovih gozdov iz Jugovzhodnih apneniških Alp (Tabela S18). 

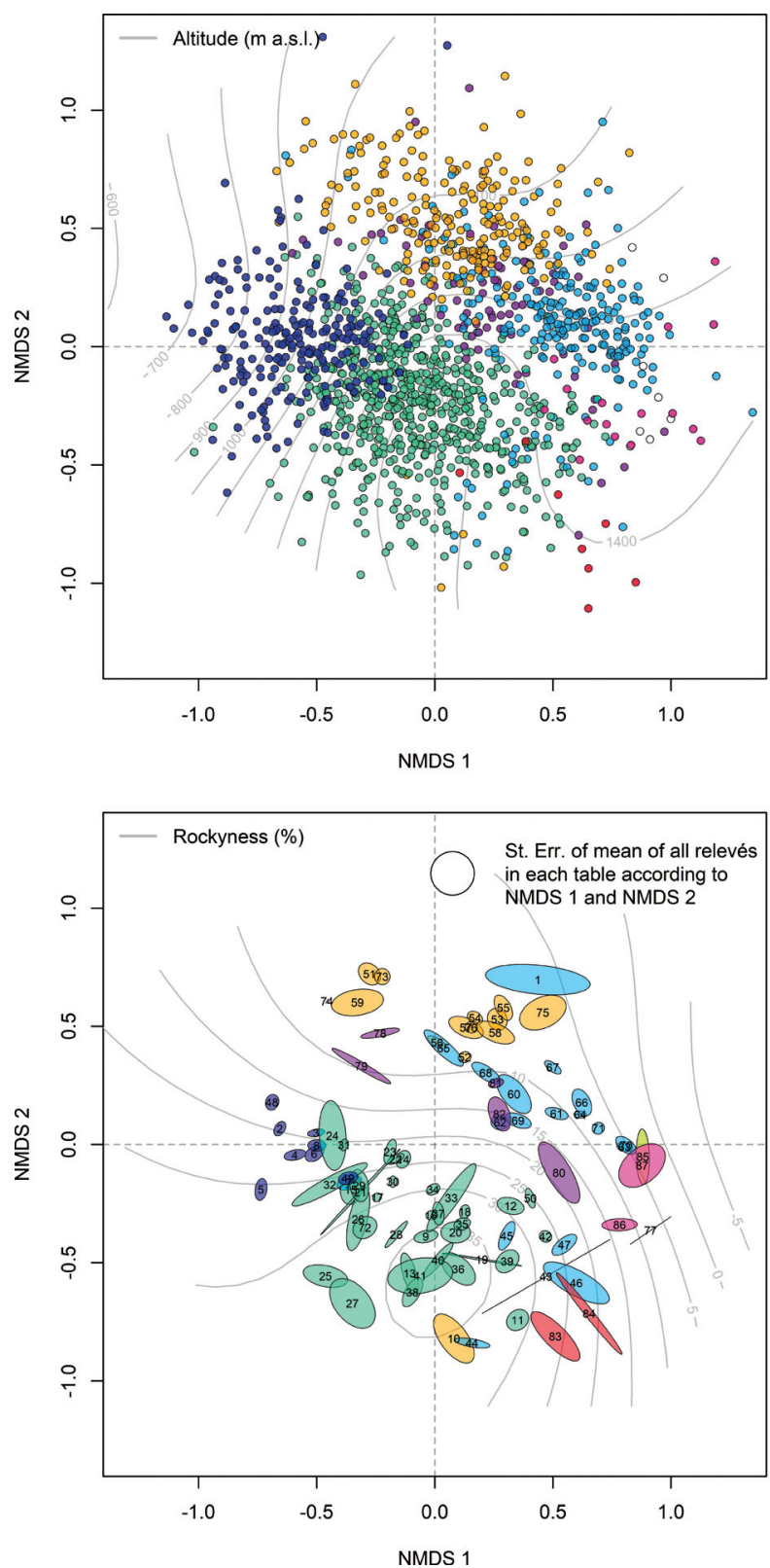

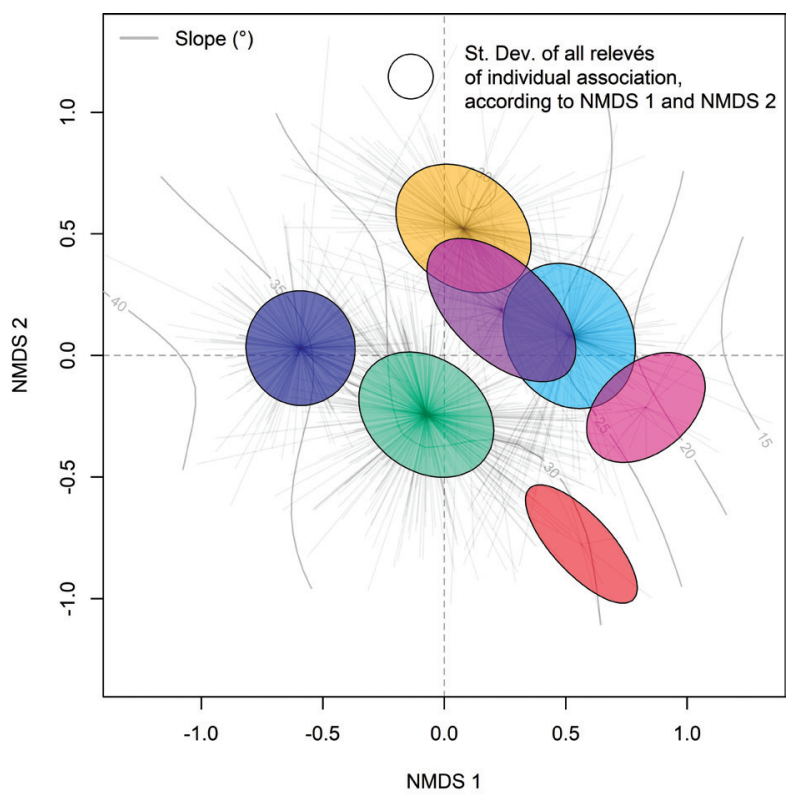

Legend

\section{Aconito-Fagetum \\ Anemono trifoliae-Fagetum \\ Cardamino-Fagetum \\ Myrrhido-Fagetum \\ Polysticho lonchitis-Fagetum \\ Ranunculo platanifolii-Fagetum \\ Rhododendro hirsuti-Fagetum \\ Saxifrago rotundifoliae-Fagetum \\ Stellario-Fagetum}

Figure 7: nMDS ordination plot of 1419 relevés of (alti)montane and subalpine beech communities in SE Calcareous Alps. The syntaxa were analysed at the level of: (a) relevés, (b) associations, where each ellipse represents an area of one standard deviation related to all relevés of an individual association on the nMDS ordination plot, and (c) compared syntaxa, where each ellipse represents the standard error of the mean related to all relevés of an individual syntaxon table (Table S20) on the nMDS ordination plot. Altitude, rockiness and slope were added onto ordination plots as isolines.

Slika 7: Slika ordinacije po metodi nMDS na podlagi 1419 popisov (alti)montanskih in subalpinskih bukovih združb iz JV apneniških Alp. Sintaksoni so prikazani na treh nivojih: (a) prikaz posameznih popisov fitocenoz, (b) prikaz asociacij, kjer vsaka elipsa prikazuje površino enega standardnega odklona vseh popisov posamezne asociacije na sliki ordinacije nMDS in (c) prikaz primerjanih sintaksonov (Tabela S20), kjer vsaka elipsa prikazuje območje standardne napake aritmetične sredine popisov posamezne fitocenološke tabele na sliki ordinacije nMDS. Nadmorska višina, skalnatost in naklon so na sliki ordinacije prikazani z izočrtami.

tum, Rhododendro hirsuti-Fagetum, Saxifrago rotundifoliaeFagetum and Stellario montanae-Fagetum (Table 9), and compared them using hierarchical classification (Figure 8).

The results of these comparisons (Figures 6,7 and 8, Table 9, which comprises the calculated $\phi$ (phi) values) indicate that the syntaxa described in this paper are well differentiated from the stands of the previously listed associations. Our relevés (all columns) grouped separately from the columns comprising the relevés of associations Saxifrago rotundifoliae-Fagetum, Ranunculo platanifoliiFagetum and Anemono trifoliae-Fagetum, which means that they, for the most part, cannot be included into the listed associations. In terms of floristics, our relevés have the most in common with the stands of the association Rhododendro hirsuti-Fagetum. This association comprises those stands on extreme sites (steep, shady rocky slopes) 


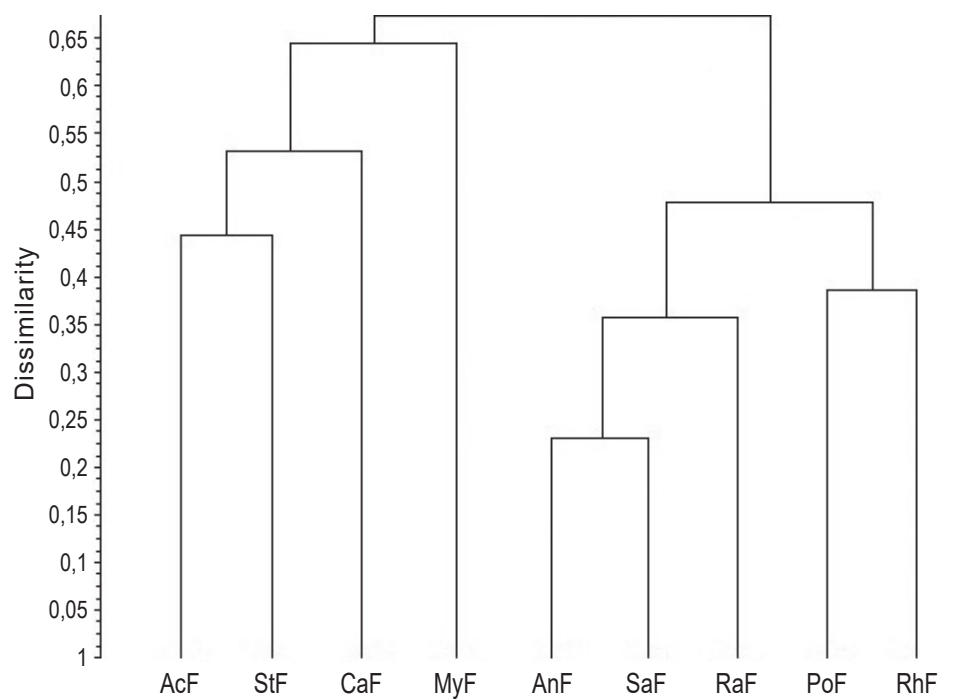

\section{Legend / Legenda}

AcF Aconito paniculati-Fagetum

StF Stellario montanae-Fagetum

$\mathrm{CaF}$ Cardamino waldsteinii-Fagetum (Cardamino savensi-Fagetum var. Abies alba)

MyF Myrrhido-Fagetum

AnF Anemono trifoliae-Fagetum

$\mathrm{SaF}$ Saxifrago rotundifolii-Fagetum

$\mathrm{RaF}$ Ranunculo platanifolii-Fagetum

PoF Polysticho lonchitis-Fagetum

RhF Rhododendro hirsuti-Fagetum

Figure 8: Hierarchical classification of the (alti)montane and subalpine beech communities from the suballiance Saxifrago-Fagenion (UPGMA, similarity ratio). Slika 8: Hierarhična klasifikacija (alti)motanskih in subalpinskih bukovih gozdov iz podzveze SaxifragoFagenion (UPGMA, similarity ratio). in the submontane $(300 \mathrm{~m})$ to the upper montane belt (1200, even $1280 \mathrm{~m})$ that are similar in appearance to the subalpine beech stands, and is a vicariant of the studied community (Dakskobler 2003, Accetto 2015).

Stands of the association Rhododendro hirsuti-Fagetum are clearly differentiated from subalpine beech stands by the presence of thermophytic species such as Fraxinus ornus, Ostrya carpinifolia, Euonymus verrucosa, Viburnum lantana, Amelanchier ovalis, Campanula rapunculoides, higher constancy of Laburnum alpinum, Sorbus aria, Melittis melissophyllum, Convallaria majalis, Carex alba, Vincetoxicum hirundinaria, Cirsium erisithale, Bupthalmum salicifolium, Salvia glutinosa, Neottia nidus-avis, Platanthera bifolia, Primula vulgaris, presence of species that only rarely or not at all occur in the subalpine belt (Phyteuma scheuchzeri subsp. columnae, Epipactis helleborine, Tilia cordata, Acer platanoides, Veratrum nigrum) and certain acidophilic species (Pteridium aquilinum), and very low presence or even absence of subalpine-alpine species such as Festuca calva, Astrantia bavarica, Cerastium subtriflorum, Poa alpina and species of subalpine shrub communities and tall herbs (Sorbus chamaemespilus, Ribes alpinum, Juniperus sibirica, Alnus viridis, Geum rivale, Adenostyles alliariae, Geranium sylvaticum, Saxifraga rotundifolia, Myrrhis odorata, Chaerophyllum hirsutum, C. villarsii). The most floristically similar to the subalpine beech stands are the stands of the subassociation Rhododendro hirsuti-Fagetum abietetosum from the northern fringe of the Trnovo Forest Plateau (Bukov Vrh, Zeleni Rob), which are also the most frequently situated at the contact with these stands, but are differentiated from them by the high constancy and mean cover of the differential species Abies alba (Dakskobler 2003). Fir is also the differential species between the subalpine beech stands described herein and slightly similar montane and altimontane fir-beech stands of subassociations Omphalodo-Fagetum rhododendretosum hirsuti and Homogyno sylvestris-Fagetum rhododendretosum hirsuti (Dakskobler et al. 2000, Dakskobler 2002a, 2002b, 2004, Surina \& Dakskobler 2013).

\section{Diagnostic species of the association Polysticho lonchitis-Fagetum}

Based on hierarchical classification of the relevés that we synthetically presented in Table S17, and hierarchical classification in Figure 5, we arranged the relevés in 24 analytical tables. In addition to the above comparisons we based their classification into the syntaxonomic system also on the presence of the species that are diagnostic (differential) for subalpine beech stands, taking into account previous findings described in the introduction, the synthetic table of all compared associations (Table 9) and the constancy of all recorded species, which was established based on 603 relevés.

The diagnostic species of subalpine beech stands are in particular those that clearly indicate their position at or just below the forest line, i.e. on the fringes of or at the contact with other subalpine communities, larch, dwarf pine, subalpine grasslands, screes and rocks.

Based on these criteria we identified the following diagnostic species of the association Polysticho lonchitis-Fagetum: Polystichum lonchitis (total constancy 76\%; it has similarly high constancy also in the stands of the association Aconito paniculati-Fagetum, which is an altimontane-subalpine maple-beech community documented with only 8 relevés), Clematis alpina (66\%, with $40 \%$ constancy in the stands of the association Rhododendro hirsuti-Fagetum), Luzula sylvatica (60\%, with higher con- 
stancy of $86 \%$ in the stands of the association Cardamino waldsteinii-Fagetum from Pohorje), Rhododendron hirsutum $(51 \%$, with higher constancy of $91 \%$ in the stands of the association Rhododendro hirsuti-Fagetum), Paraleucobryum sauteri (50\%), Sorbus chamaemespilus (48\%, with higher constancy of $62 \%$ in the stands of the association Aconito paniculati-Fagetum), Aconitum lycoctonum subsp. ranunculifolium or A. lupicida (48\%, with $38 \%$ in the stands of the association Aconito paniculati-Fagetum), Laserpitium peucedanoides (33\%, with constancy of $26 \%$ in the stands of association Rhododendro hirsuti-Fagetum), Pinus mugo (33\%, with constancy of $11 \%$ in the stands of the association Rhododendro hirsuti-Fagetum), Salix appendiculata (29\%, with higher constancy of $75 \%$ in the stands of the association Aconito paniculati-Fagetum and similar constancy of $37 \%$ in the stands of the association Rhododendro hirsuti-Fagetum), Paederota lutea (27\%, with higher constancy of $53 \%$ in the stands of the association Rhododendro hirsuti-Fagetum), Aster bellidiastrum (26\%, with similar constancy of $30 \%$ in the stands of the association Rhododendro-Fagetum), Homogyne alpina (22\%, 7\% in the stands of associations Anemono trifoliae-Fagetum and Saxifrago rotundifoliae-Fagetum), Carex ferruginea (21\%, 33\% in stands of the association Rhododendro-Fagetum, and 36\% in the stands of the association Saxifrago-Fagetum), Festuca calva (21\%, 2\% in the stands of the association Ranunculo platanifolii-Fagetum), Lonicera caerulae (20\%) and Rhodothamnus chamaecistus (16\%, $8 \%$ in the stands of the association RhododendroFagetum).

Most of the listed species are therefore present also in certain forms of altimontane beech communities from associations Saxifrago rotundifoliae-Fagetum, Dentario pentaphylli-Fagetum, Ranunculo platanifoli-Fagetum, Anemono trifoliae-Fagetum, Homogyno sylvestris-Fagetum, Cardamino waldsteinii-Fagetum and Aconito paniculati-Fagetum. Some of them (Rhododendron hirsutum, Rhodothamnus chamaecistus, Clematis alpina, Salix appendiculata and Carex ferruginea) are characteristic also for the submontanemontane association Rhododendro hirsuti-Fagetum.

Based on the results of hierarchical classification in Figures 2, 3 and 4, and the presence of the above-listed species we classified the researched stands into one of the associations that have already been described in this area and elevation belt (Polysticho lonchitis-Fagetum, Saxifrago rotundifoliae-Fagetum, Ranunculo platanifolii-Fagetum, Anemono trifoliae-Fagetum). We identified the differential species of lower units by taking into account also fidelity calculated with the Indicator Value Index and $\phi$ (phi) value. Analysis of the percentages of diagnostic species (Table 10) helped us in the division into lower syntaxonomic units.

\section{Overview of established syntaxa and their nomenclatural types}

The established groups are syntaxonomically evaluated as follows:

Anemono trifoliae-Fagetum var. Saxifraga hostii (minor part of cluster 23) - Table S11, Columns 1-4.

Ranunculo platanifolii-Fagetum var. Saxifraga petraea, Table S1, Columns 1-3.

Ranunculo platanifolii-Fagetum var. Sedum hispanicum (cluster 3) - Table S2, Columns 1-3.

Ranuculo platanifolii-Fagetum luzuletosum luzuloidis (minor part of cluster 19), the nomenclatural type, holotypus, is releve 45 in Table 3-Table 3, Columns 35-49.

Ranunculo platanifolii-Fagetum stellarietosum nemorum Marinček et Čarni 2010 var. Alnus viridis (cluster 14, minor part) - Table 8, Columns 28-36.

Polysticho lonchitis-Fagetum var. Rosa pendulina prov. Table S1, Columns 4-7.

Polysticho lonchitis-Fagetum betuletosum carpaticae Dakskobler et Rozman 2010 (cluster 6) - Table S2, Columns 4-7.

Polysticho lonchitis-Fagetum ericetosum carneae subass. nov., the nomenclatural type, holotypus, is relevé 38 in Table 1. var. Rhamnus fallax (cluster 5) - Table S2, Columns 8-10. var. Phegopteris connectilis (cluster 2) - Table S2, Columns 11-13.

var. Sorbus chamaemespilus (cluster 31) - Table S2, Columns 14-17.

var. Laserpitium latifolium (cluster 32) - Table S2, Columns 18-22.

var. Larix decidua (cluster 1) - Table S3.

var. Calamagrostis arundinacea (cluster 8) - Table S4. var. Rhododendron hirsutum (cluster 9) - Table S5.

subvar. Calamagrostis villosa (cluster 10) - Table S6.

subvar. Calamagrostis varia (cluster 12) - Table 1.

var. Luzula nivea (cluster 11) - Table S7.

var. Calamagrostis varia (cluster 30) - Table S8.

Polysticho lonchitis-Fagetum cardaminetosum trifoliae subass. nov., the nomenclatural type, holotypus, is relevé 6 in Table 2. var. Rhododendron hirsutum subvar. Calamagrostis arundinacea (cluster 7) - Table S9.

var. Calamagrostis arundinacea (cluster 20) - Table 2. var. Saxifraga rotundifolia (cluster 18) - Table S10.

Polysticho lonchitis-Fagetum luzuletosum luzuloidis subass. nov., the nomenclatural type, holotypus, is relevé 12 in Table 3 (major part of cluster 19) - Table 3, Columns 1-34.

Polysticho lonchitis-Fagetum calamagrostietosum variae subass. nov., the nomenclatural type, holotypus, is relevé 24 in Table 4 (cluster 22) - Table 4.

var. Genista radiata - Table 4, Columns 32-48.

Polysticho lonchitis-Fagetum luzuletosum niveae subass. nov., the nomenclatural type, holotypus, is relevé 29 in Table 5 (cluster 28). 

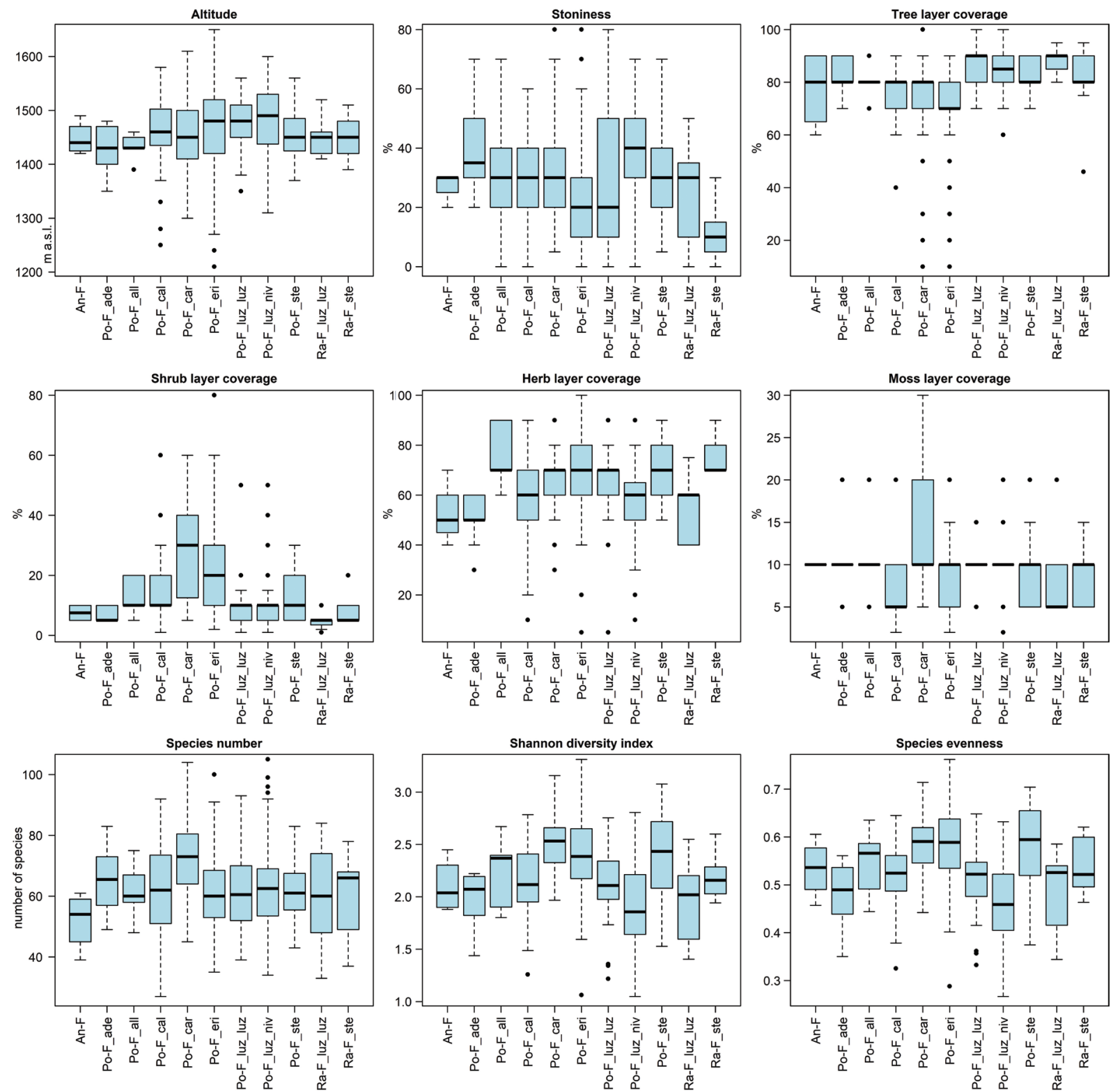

Figure 9: Boxplots showing some ecological data for identified syntaxa of subalpine beech communities.

Slika 9: Okvirji z ročaji za nekatere ekološke podatke pri ugotovljenih sintaksonih subalpinskih bukovih združb.

\section{Legend / Legenda}

An-F Anemono trifoliae-Fagetum

Po-F_ade Polysticho lonchitis-Fagetum var. Adenostyles alliariae prov.

Po-F_all Polysticho lonchitis-Fagetum allietosum victorialis

Po-F_cal Polysticho lonchitis-Fagetum calamagrostietosum variae

Po-F_car Polysticho lonchitis-Fagetum cardaminetosum trifoliae

Po-F_er Polysticho lonchitis-Fagetum ericetosum carneae (incl. betuletosum carpaticae)

Po-F_luz-luz Polysticho lonchitis-Fagetum luzuletosum luzuloidis

Po-F_luz_niv Polysticho lonchitis-Fagetum luzuletosum niveae

Po-F_ste Polysticho lonchitis-Fagetum stellarietosum nemorum

Ra-F_luz_luz Ranunculo platanifolii-Fagetum luzuletosum luzuloidis

Ra-F_ste Ranunculo platanifolii-Fagetum stellarietosum nemorum 
var. Cerastium subtriflorum (cluster 23, major part) -

Table S11, Columns 5-8.

var. Calamagrostis arundinaceae (cluster 26) - Table S12.

var. Festuca calva (cluster 28) - Table 5.

subvar. Urtica dioica (cluster 27) - Table S13, Col.

$1-16$.

subvar. Calamagrostis varia (cluster 29) - Table S14,

Columns 2-13.

Polysticho lonchitis-Fagetum adoxetosum moschatellinae, the nomenclatural type, holotypus, is relevé 4 in Table 6 (cluster 24) - Table 6, Columns 1-10.

Polysticho lonchitis-Fagetum var. Helleborus niger prov. (cluster 25) - Table 6, Columns 11-15.

Polysticho lonchitis-Fagetum allietosum victorialis subass. nov., the nomenclatural type, holotypus, is relevé 5 in Table 7 (cluster 13) - Table 7.
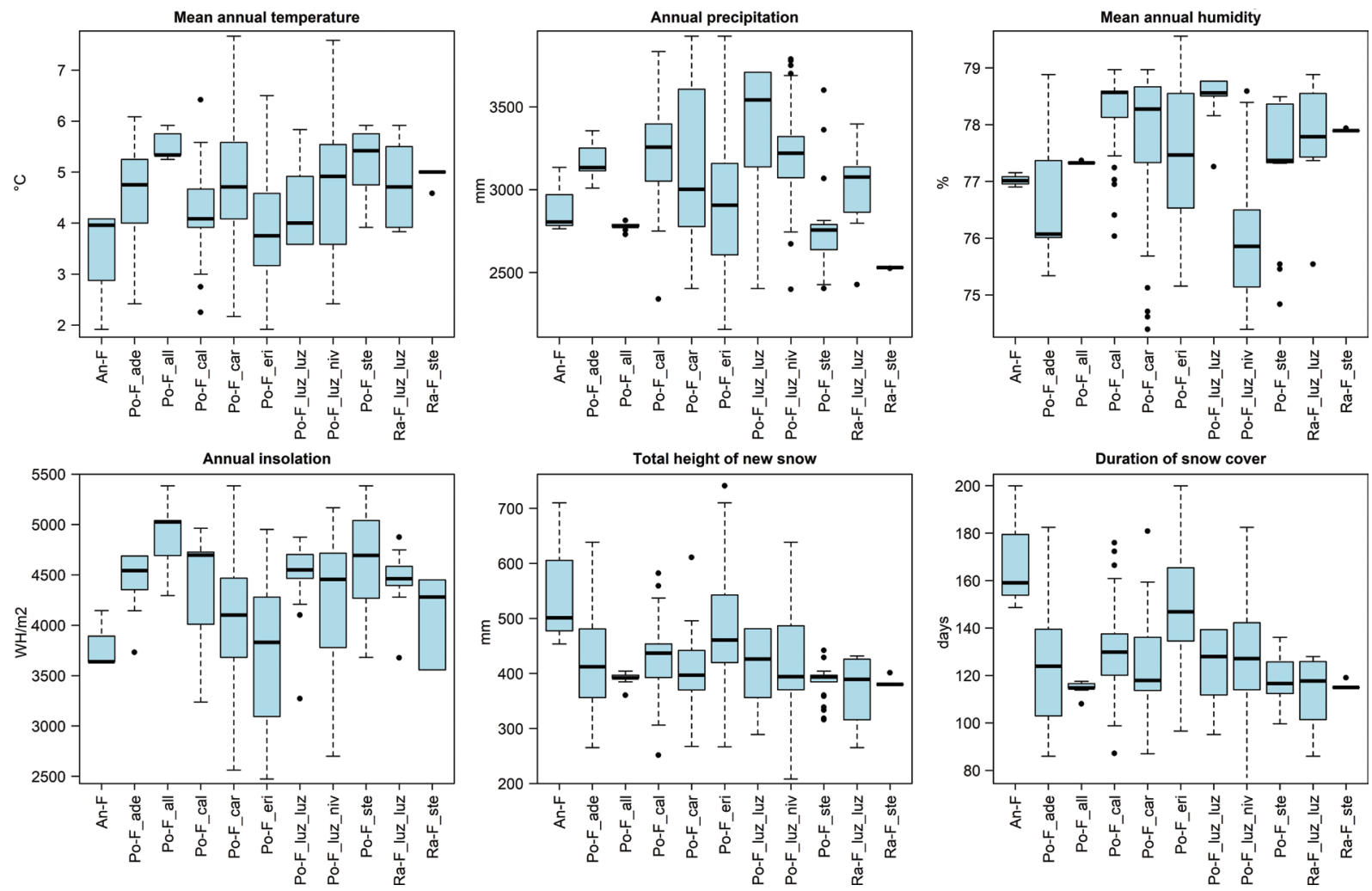

var. Adoxa moschatellina, Columns 1-4.

Polysticho lonchitis-Fagetum stellarietosum nemorum subass. nov., the nomenclatural type, holotypus, is relevé 21 in Table 8 (cluster 14, major part) - Table 8, Columns $1-27$.

var. Chaerophyllum villarsii, Columns 23-27.

Polysticho lonchitis-Fagetum var. Adenostyles alliariae prov. (cluster 21) - Table S15, Columns 1-5.

Polysticho lonchitis-Fagetum var. Centaurea montana prov. (cluster 17) - Table S15, Columns 6-9.

Polysticho lonchitis-Fagetum var. Cirsium carniolicum prov. (cluster 4) - Table S16, Columns 1-3.

Polysticho lonchitis-Fagetum var. Conocephalum conicum prov. (cluster 15) - Table S16, Columns 4-8.

Polysticho lonchitis-Fagetum var. Digitalis grandiflora prov. (cluster 16) - Table S16, Columns 9-12.

Figure 10: Boxplots showing climatic data for identified syntaxa of subalpine beech communities.

Slika 10: Okvirji z ročaji za nekatere podnebne podatke pri ugotovljenih sintaksonih subalpinskih bukovih združb.

\section{Legend / Legenda}

\section{An-F Anemono trifoliae-Fagetum}

Po-F_ade Polysticho lonchitis-Fagetum var. Adenostyles alliariae prov. Po-F_all Polysticho lonchitis-Fagetum allietosum victorialis

Po-F_cal Polysticho lonchitis-Fagetum calamagrostietosum variae Po-F_car Polysticho lonchitis-Fagetum cardaminetosum trifoliae Po-F_er Polysticho lonchitis-Fagetum ericetosum carneae

(incl. betuletosum carpaticae) 

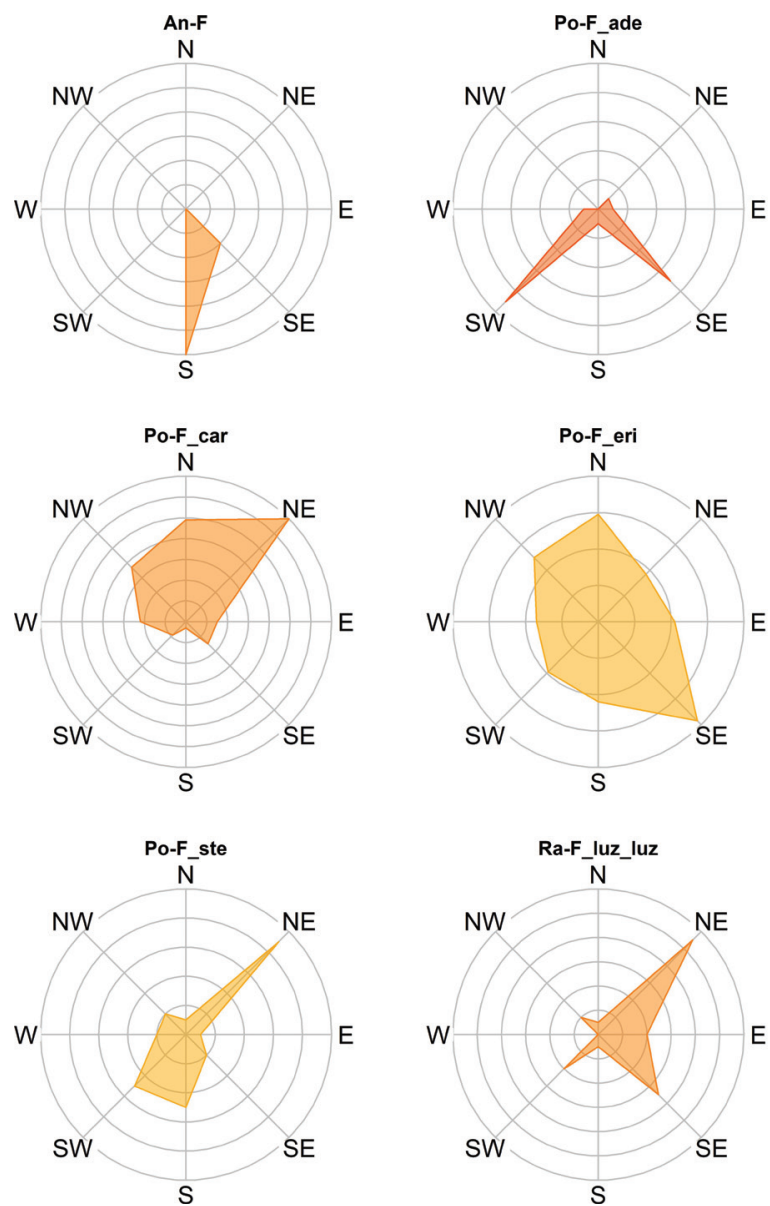

Figure 11: Rose diagrams of aspects with mean slope by syntaxa.

\section{Legend / Legenda}

An-F Anemono trifoliae-Fagetum

Po-F_ade Polysticho lonchitis-Fagetum var. Adenostyles alliariae prov. Po-F_all Polysticho lonchitis-Fagetum allietosum victorialis

Po-F_cal Polysticho lonchitis-Fagetum calamagrostietosum variae Po-F_car Polysticho lonchitis-Fagetum cardaminetosum trifoliae Po-F_er Polysticho lonchitis-Fagetum ericetosum carneae

(incl. betuletosum carpaticae)

\section{Description of syntaxa -}

commentary to Tables 1-8 and Tables S1-S16, see also Figures 5, 6, 9, 10 and 11

\section{Polysticho lonchitis-Fagetum var. Rosa pendulina prov.}

Table $S 1$ comprises the relevés that are not included in any of 32 identified clusters and have clearly grouped separately. Based on the above criteria we classified the first three relevés in this table (from the ridge of Polovnik, above the Tolminka valley and under Mt. Matajur) into
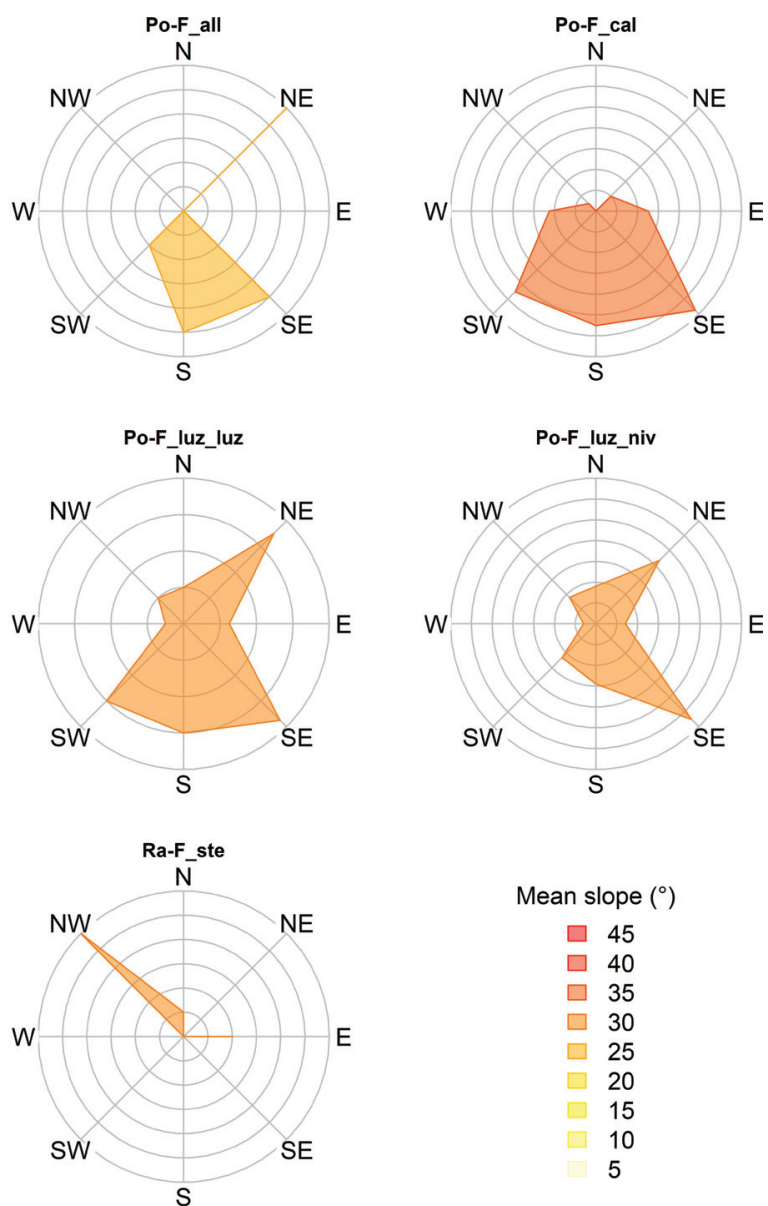

Mean slope $\left({ }^{\circ}\right)$

$\square 45$

$\square \quad 40$

$\square \quad 35$

$\square \quad 30$

$\square 25$

$\square 20$

15

$\square \quad 10$

5

Slika 11: Rože nebesnih leg s prikazom povprečne strmine po sintaksonih.

Po-F_luz-luz Polysticho lonchitis-Fagetum luzuletosum luzuloidis Po-F_luz_niv Polysticho lonchitis-Fagetum luzuletosum niveae Po-F_ste Polysticho lonchitis-Fagetum stellarietosum nemorum Ra-F_luz_luz Ranunculo platanifolii-Fagetum luzuletosum luzuloidis

Ra-F_ste Ranunculo platanifolii-Fagetum stellarietosum nemorum

the association Ranunculo platanifolii-Fagetum as a special variant with Saxifraga petraea (its differential species are also Festuca calva and Campanula carnica) - these are stands of an extreme form of this association on very steep and stony sunny slopes. Relevés 4-7 in this table (from the slopes under Loška Stena, the Stol ridge, Črna Gora at Črna Prst and the ridge of Muzci / Cime del Monte Musi) undoubtedly belong to the association Polysticho lonchitis-Fagetum and are provisionally classified into the variant with Rosa pendulina. They are indicative of subalpine forests on steep and stony shady slopes. 


\section{Polysticho lonchitis-Fagetum ericetosum carneae}

Relevés from several clusters in the synthetic table, which usually comprise only a few relevés (clusters 3, 6, 5, 2, 32 and 31), are presented in Table S2. The first three relevés in this table (cluster 3, which stands out from all others) were made on the Trnovo Forest Plateau (Mrzovec, Mali Golak, Javorški Vrh). They comprise very few diagnostic species of the association Polysticho lonchitis-Fagetum and are therefore classified into the association Ranunculo platanifolii-Fagetum, the variant with Sedum hispanicum (differential species include Millium effusum, Crepis aurea and Cardamine bulbifera). They are distinguished from other relevés in this table by a substantially higher percentage of mesophytic species from the alliance TilioAcerion and order Fagetalia sylvaticae.

Relevés in cluster 6 belong to the subassociation betuletosum carpaticae Dakskobler et Rozman 2010, whose differential species are also Gymnocarpium dryopteris and Juniperus sibirica (see also Dakskobler \& Rozman 2010). They are floristically close to the stands of the subassociation ericetosum carneae and therefore in our analyses treated together with them.

In the dendrogram in Figure 2, other clusters in Table S2 indicate a certain similarity with the bigger group of relevés (clusters 1, 8, 9, 10,12, 11, 30 on the left side of the dendrogram), whose common characteristic is the predominating proportion of species of classes VaccinioPiceetea and Erico-Pinetea. These are the highest-situated beech stands from the central parts of the Julian Alps, which are frequently in contact with subalpine larch, occasionally also spruce forests, whereas the predominant beech community in the montane and altimontane belt is the association Anemono trifoliae-Fagetum. The soil is rendzina with raw or moder humus, decomposition of organic matter is slow. In the tree layer, beech is frequently admixed with individual mountain ash (Sorbus aucuparia), larch (Larix decidua) and spruce (Picea abies) trees, rarely with fir (Abies alba). These stands grouped into clusters mainly based on higher cover of certain species from said two classes (Picea abies, Larix decidua, Vaccinium myrtillus, V. vitis-idaeae, Calamagrostis arundinaceae, C. villosa, C, varia, Rhododendron hirsutum, Pinus mugo), but have no distinctive species that could serve as name-giving species of subassociations. The bigger group of relevés in clusters on the left side of the dendrogram in Figure 2 is therefore classified into the same subassociation. We identified Erica carnea as its name-giving species, as it characterises and differentiates these sites against the sites of other groups of relevés. The differential species of the subassociation Polysticho lonchitis-Fagetum ericetosum carneae are also Larix decidua, Calamagrostis villosa and Homogyne alpina, as they are clearly more frequent in the stands of this group than in other groups. In the phytogeographical division these stands belong to the race (geographical variant) Polysticho lonchitis-Fagetum var. geogr. Anemone trifolia.

This subassociation comprises also other relevés in Table S2. Relevés of cluster 5 are classified into the variant with Rhamnus fallax and were made in the Tolmin-Bohinj range; its differential species include Pinus mugo and Lonicera caerulea. Relevés in cluster 2 are classified into the variant with Phegopteris connectilis (one of the differential species is Astrantia bavarica); two relevés are from the Karawanks, one also from the Lopata plateau (Voglova Jelovica). Relevés in cluster 31 were made on Lopata, the valley of Lopučnica and slopes above the Voje valley, and are classified into the variant with Sorbus chamaemespilus (its differential species include Gentiana asclepiadea and Geranium sylvaticum); relevés in cluster 32 are classified into the variant with Laserpitium latifolium, whose differential species include Calamagrostis varia, Betonica alopecuros, Cirsium erisithales, Carex alba, Mercurialis perennis and Neottia nidus-avis, and characterise rather open forests on extreme sites on sunny aspects (above the Vrata Valley under Stenar, in the Lopučnica valley, at mountain pasture Trstje in Čiprje).

Stands in Table S3 (cluster 1) are classified into the syntaxon Polysticho lonchitis-Fagetum ericetosum carneae var. Larix decidua. Its differential species are Larix decidua (with high mean cover in the tree layer) and Euphorbia amygdaloides. The diagnostic species of the association are well represented. This variant comprises subalpine stands at the upper distribution limit of beech at the contact with subalpine larch forests (Rhodothamno-Laricetum). The stands demonstrate some similarity with the stands of the subassociation Ranunculo platanifolii-Fagetum laricetosum, but they grouped separately in the extended synthetic table. The stands of this variant were found in the Trenta Valley, under Srebrnjak, at Pokljuka (mountain pasture Klek), under Visoka Ponca in Tamar and in two areas in Kamniška Bistrica - Repov Kot and Kalce.

Stands in Table S4 (cluster 8) are classified into the variant Polysticho lonchitis-Fagetum ericetosum carneae var. Calamagrostis arundinacea. Its differential species are acidophytic Calamagrostis arundinacea and Vaccinium myrtillus with high mean cover. All relevés of this variant were made in Bohinj, mainly in the virgin forest remnant Lopata at Vogel (Budkovič et al. 1996) and under Orlove Glave nearby, one relevé also on the mountain pasture Suha and one in the Triglav range (Čiprje). Many of the stands are on a more level, karstified limestone tablelands. Decomposition of organic matter is very slow and due to an accumulating layer of raw humus the acidophytic species of spruce forests dominate. 
Stands in Table S5 (cluster 9) are classified into the variant Polysticho lonchitis-Fagetum ericetosum carneae var. Rhododendron hirsutum. Stands of this variant typically occur on steep shady slopes, more often on dolomite than on limestone bedrock. Rhododendron hirsutum is differential due to its high constancy and mean cover. Pinus mugo also has high constancy in these stands, whereas spruce, larch and fir are very rare. Most of the relevés of this variant were made in the western Julian Alps, in the Bovec region (Korita above the Možnica valley), in Rezija / Val Resia (under Mala Baba / Baba Piccola) under the ridge of Muzci / Cime del Monte Musi above Dol po Meji / Valle di Musi, above the Zajzera Valley / Val Saisera in Krnica / Fossa di Carnizza under Krniška Glavica / Jôf di Somdogna, and in the westernmost part of the Julian Alps under Lopič / Monte Plauris, two relevés also in the Tolmin-Bohinj mountains (Lisec at Črna Prst and Za Robom under Konjski Vrh), mainly on sites that are very extreme for forest vegetation.

In terms of their full species composition the stands in Tables S6 and Table 1 are very similar to the stands in Table S5 and are therefore classified into the same variant Polysticho lonchitis-Fagetum ericetosum carneae var. Rhododendron hirsutum. Within the race (geographical variant) Anemone trifolia and subassociation ericetosum carneae these stands are the most typical stands of subalpine beech forests within the Julian Alps, and the least affected by man. Stands in Table S6 are classified into the subvariant with Calamagrostis villosa (the differential species of the subvariant is also Gymnocarpium dryopteris). The parent material is limestone, dolomite limestone, moraine (till) or alluivial fan, the soil is initial rendzina with raw humus. Some of their localities are in the Italian part of the Julian Alps: the ridge of Muzci (Cime del Monte Musi, Sella Carnizza) above Rezija / Val Resia, in Zajzera / Val Saisera: Krnica / Fossa di Carnizza under Krniška Glavica / Jôf di Somdogna; above the Dunja Valley / Val Dogna under Krniška Glavica / Jôf di Somdogna; in the Koritnica valley in the Bovec region: under northern rock walls of Loška Stena (Planinica, Veliki Ozebnik), Mangartski Potok-Gorenji Stan, Možnica-Čez Brežič, Rombon-Kanja; in the Upper Soča Valley with Trenta: Pod Lemežem-V Sleču, Plaski Kuk-Krbulnik, Trenta-Zadnjiški Dol, and in the Upper Sava Valley: Vrata-Bukovlje, Vrata-Macesence-Stenar, Pod Špikom, Za Akom.

Stands in Table 1 are classified into the subvariant Polysticho lonchitis-Fagetum ericetosum carneae var. Rhododendron hirsutum subvar. Calamagrostis varia. Calamagrostis varia and Vaccinium vitis-idaea are differential mainly because of high mean cover. Also frequent are Pinus mugo and Larix decidua, so some of the stands of this subvariant, in which larch has higher mean cover in the tree layer, are similar to the stands of the variant Polysticho lonchitis-Fagetum ericetosum carneae var. Larix decidua (Table S3, cluster 1). The predominant parent material is dolomite and dolomite limestone, the soil is moder rendzina. The localities of the stands of this subvariant are partly in the western Julian Alps: above the Mrzla Voda Valley / Valle di Rio Freddo: in Trbiška Krnica / Carnizza di Rio Freddo and under Prednje Lastovice / Cime delli Rondini, in Rezija / Val Resia under Kadin / M. Cadin, under Kopa / Picco di Mezzodi above Bela Peč Lakes / Laghi di Fusine, under Loška Stena, Predelske Glave, Rombon and above Mangartski Potok in the valley of the Koritnica, under Mt. Svinjak near Bovec, in the Kanin Mts. above the mountain pasture Krnica, under Plaski Kuk-Krbulnik, in Trenta (Mlinarica, Zadnjiški Dol, Korita under Luknja, Na Grivah), in the Tolmin-Bohinj range (Grušnica, Prodi-Ovčja Suha, Planina Poljana, Planina Suha), in the Upper Sava Valley (in Krma under Debela Peč and in Prodi, in Kot under Macesnovec, in Vrata in Bukovlje under Sovatna, Pod Špikom and Pod Srcem - Srednji Kotel, in the Beli Potok valley under Macesnje, in Mala Pišnica, Tamar under Srednja Ponca). This subvariant comprises also several relevés from the Kamnik Alps (Presedlaj above Kamniška Bela, Ravenska Kočna and Makekova Kočna).

Stands in Tables S7 and S8 occur mainly on steep sunny slopes. Stands in Table S7 are classified into the variant Polysticho lonchitis-Fagetum ericetosum carneae var. Luzula nivea. Luzula nivea is differential because of high mean cover, which is characteristic also of Calamagrostis villosa and Vaccinium myrtillus in this variant. Luzula sylvatica and Campanula scheuchzeri also suggest certain affiliation with this variant. Most of the localities are in the Bovec region: in Trenta above the mountain pasture Zapotok and under Staro Utro (Berebica), in the Kanin Mts. at the mountain pasture Globoko, under Jerebica above the Možnica valley and in Izgora under Loška Stena.

Stands in Table S8 are classified into the variant Polysticho lonchitis-Fagetum ericetosum carneae var. Calamagrostis varia. This species is differential on account of high mean cover. The localities are in Trenta (Strmarica, Na Česmu under Srebrnjak, Velika Glava above the mountain pasture Trebiščina, Ravni Dol under Plazjanski Vršac), under Jerebica and Rombon above the Možnica valley, above Mangartski Potok and under the peaks of the ridge in the vicinity of Predel, in the Kanin Mts., also above the mountain pasture Krnica, in Rezija / Val Resia under Sart / M. Sart, at Rabelj / Cave del Predil (Mala Kraljevska Špica / M. Re), one relevé from the Upper Sava Valley (under Kukova Špica-Čorlovec) and one from Makekova Kočna in the Kamnik Alps. 


\section{Polysticho lonchitis-Fagetum cardaminetosum trifoliae}

The floristic composition of the relevés that grouped in clusters 7,20,18,19 and 22 is very similar to the stands of the subassociation ericetosum carneae, but their localities are more frequently situated in areas where beech forests is the highest form of forest vegetation and is not succeeded by larch, but dwarf pine. The decomposition of humus is slightly more complete (moder rendzina) and consequently there more species that indicate mesophytic sites (with a slightly higher percentage of diagnostic species of the alliance Tilio-Acerion, order Fagetalia sylvatice and class Mulgedio-Aconitetea). These stands are therefore classified into the new subassociation Polysticho lonchitis-Fagetum cardaminetosum trifoliae. The species that differentiate it against the subassociation ericetosum carneae and indicate more distinctly mesophytic sites include Anemone nemorosa, Actaea spicata, Dryopteris filixmas and Phyteuma ovatum as well as certain diagnostic species of the alliance Tilio-Acerion (Acer pseudoplatanus, Aruncus dioicus, Polystichum aculeatum), which usually have substantially higher constancy in these stands than in the stands of the previous subassociation. In terms of phytogeography the stands of this subassociation belong to two races (geographical variants), namely the Alpine, var. geogr. Anemone trifolia, and the northern-Dinaric, var. geogr. Allium victorialis, but the distribution area of Allium victorialis is wider and this species occurs also in relevés from the Alps.

Stands in Table S9 are classified into the subvariant Polysticho lonchitis-Fagetum cardaminetosum trifoliae var. Rhododendron hirsutum subvar. Calamagrostis arundinacea. They are distributed across shady, steep to very steep dolomite, rarely limestone, slopes. The soil is slightly acidic due to slow decomposition of organic matter. These sites are also considered extreme for forest vegetation. Rhododendron hirsutum is the differential species of the variant due to $100 \%$ constancy and high mean cover. The differential species of the subvariant are Calamagrostis arundinaceae, Phegopteris connectilis and Huperzia selago. The localities of the stands of this variant are on northern slopes of the Stol ridge above the Učja valley, in the forest reserve Grušnica and under Kuntar and Vrh Planje above the Tolminka valley, in the Tolmin-Bohinj Mts. also under Orlova Glava, Konjski Vrh, Slatnik, Lisec, at mountain pastures Poljana and Suha, and in the Bovec region at V Sleču under Lemež and under Debeljak in the Polovnik ridge. Only one relevé from the Upper Sava Valley (Vrata, Bukovlje) was classified into this subvariant, along with several relevés from the forest reserves of Govci and Golaki (i.e. beech stands on peak ridges of the Trnovo Forest Plateau). Most localities of these stands are in areas where the upper forest line consists of beech forests; the admixture of larch is slightly higher only in a few relevés under Grušnica.

Stands in Table 2 are classified into the variant Polysticho lonchitis-Fagetum cardaminetosum trifoliae var. Calamagrostis arundinacea. Calamagrostis arundinacea is differential mainly on account of high mean cover. These stands occur on steep, shady limestone, rarely dolomite slopes with moder rendzina and slightly moist soils. This is indicated by Acer pseudoplatanus and Aruncus dioicus, which have higher constancy here compared to the stands of the previously described subvariant. The localities are mainly in the southern part of the Julian Alps the Stol ridge (including its westernmost part - Lanež / Cuel di Lanis in Italy), the Krn Mountains (Krasji Vrh in the Polovnik ridge, Kuntar above the Tolminka valley), Tolmin-Bohinj Mountains (under Žabijski Kuk above the mountain pasture Razor, above the mountain pasture Suha, Orlove Glave at Vogel, vicinity of Mt. Črna Prst - Lisec, Črna Gora, Šoštar, Babji Zob), Golaki in the Trnovo Forest Plateau.

Stands in Table S10 are classified into the variant Polysticho lonchitis-Fagetum cardaminetosum trifoliae var. Saxifraga rotundifolia. Its differential species include Acer pseudoplatanus, Polystichum aculeatum, Stellaria nemorum and Ranunculus auricomus agg. (most likely R. braunblanquetii). Steep, shady limestone slopes prevail, more rarely also tablelands in the central (Lopata at Vogel, mountain pasture Razor-Žabijski Kuk, Konjski Vrh) and eastern part of the Tolmin-Bohinj range (Slatnik, Šavnik, Dravh, Možic, Šoštar, Črna Gora, Lisec), only one relevé is from the central part of the eastern Julian Alps (Kačji Graben under Mt. Špik).

\section{Polysticho lonchitis-Fagetum Iuzuletosum Iuzuloidis and Ranunculo platanifolii-Fagetum luzuletosum Iuzuloidis}

Stands in Table 3, relevés in columns 1-34, are classified into the subassociation Polysticho lonchitis-Fagetum luzuletosum luzuloidis, whereas relevés in columns 35-49 are classified into the subassociation Ranuculo platanifoliiFagetum luzuletosum luzuloidis. Localities of these stands are mainly in the Tolmin-Bohinj range (Prodi above the Kneža valley, Grantarski, Rutarski and Stržiškarski Gozd, Črna Gora, Dravh, Kobla, Grušnica and Smrečje above the Tolminka valley) on steep, both sunny and shady slopes, on limestone, dolomite limestone and talus, occasionally with a slight admixture of chert. The differential species of the subassociation Polysticho lonchitis-Fagetum luzuletosum luzuloidis are Luzula luzuloides, Calamagrostis arundinacea (with high mean cover), Cirsium erisithales and Primula vulgaris. Relevés 35-49 in Table 3 were made more or less 
in the same area (the southern edge of the Julian Alps: Slemenske Peči above the Tolminka valley, Stržiškarski Gozd, Kobilja Glava, Šoštar, Matajur, Porezen, Lanež / Cuel di Lanis in the Stol ridge), but in slightly different site conditions - limestone is frequently admixed with claystone and marlstone, the soils include calcareous brown soil (brown soil on limestone) and eutric brown soils. Different site conditions are reflected also in poor presence of diagnostic species of the association Polysticho lonchitis-Fagetum and based on this criterion these relevés can no longer be classified into this association - instead, they are classified into the association Ranunculo platanifolii-Fagetum (syn. Saxifrago rotundifolii-Fagetum Zukrigl 1989 sensu Willner) and into the new subassociation luzuletosum luzuloidis. The differential species of the subassociation are Luzula luzuloides and Calamagrostis arundinacea.

\section{Polysticho lonchitis-Fagetum calamagrostietosum variae}

Relevés in Table 4 are classified into the subassociation Polysticho lonchitis-Fagetum calamagrostietosum variae. The differential species of the subassociation are Calamagrostis varia, Cyclamen purpurascens and Rhamnus fallax. Their site characteristics are predominantly sunny, very steep limestone and dolomite slopes, including scree slopes, both in the southern part of the Julian Alps (where beech forest forms the upper forest line) and in the central part of the Julian Alps, where the beech belt is occasionally succeeded by larch. With their entire species composition these stands are slightly similar also to the stands of the subassociation ericetosum carneae, but occur in different relief and soil conditions, not on raw humus, but on shallow soils with more decomposed organic matter (litter). Their localities are predominantly in the Tolmin-Bohinj range (slopes above the Tolminka valley, Prodi above the Kneža valley, mountain pasture Suha, mountain pasture Poljana, Konjski Vrh, Črna Gora, Grantarski Gozd, Rutarski Gozd), several relevés are also from other parts of the Julian Alps (Vršič, Tamar, Vrata, Trenta, Bavšica, Loška Koritnica) and from the slopes above the valleys of Rezija / Val Resia and Dunja / Val Dogna. Most of the relevés from steep sunny scree slopes between Hohkovbl / Matajurski Vrh and Rodica, where subalpine beech stands transition into dwarf pine, are classified into a special thermophytic variant with Genista radiata. Melittis melissophyllum also has higher constancy in these stands.

\section{Polysticho lonchitis-Fagetum Iuzuletosum niveae}

Table S11 comprises eight relevés from cluster 23. All of them have high mean cover of Luzula nivea in the herb layer, but they differ considerably in terms of the presence of diagnostic species of the association Polysticho lonchitis-
Fagetum and site conditions. Based on these considerations we classify relevés $1-4$ into a special variant of the association Anemono trifoliae-Fagetum var. Saxifraga hostii (extreme form of this association on steep, stony, sunny slopes near the present upper forest line, its localities are in the gable end of the Loška Koritnica valley), and relevés 5-8 into the subassociation Polysticho lonchitis-Fagetum luzuletosum niveae var. Cerastium subtriflorum (predominantly steep, shady limestone slopes above the valleys of Bala and Bavšica as well as in Rezija / Val Resia and in the westernmost part of the Julian Alps under Lopič / Mt. Plauris).

Relevés in Table $S 12$ are classified into the syntaxon Polysticho lonchitis-Fagetum luzuletosum niveae var. Calamagrostis arundinaceae. Cluster 26 and Table S12 comprise the relevés from steep, shady limestone slopes of the Stol and Polovnik ridges, the ridge of Muzci above Rezija / Cime del Monte Musi, and the slopes of Javoršečk and Lemež. In terms of the ecology of their sites and species composition these relevés strongly resemble the relevés of the subassociation Polysticho-Fagetum cardaminetosum trifoliae var. Calamagrostis arundinacea from Table 2. They are differentiated from them primarily by Luzula nivea, Adoxa moschatellina, Poa nemoralis and Festuca calva (differential species of the subassociation), as well as by high constancy of Luzula sylvatica. Luzula nivea is a phytogeographical rather than ecological differential species, as it is the most densely distributed in the northwestern part of the Slovenian Alps, and characterises the stands in the Bovec region also with its high mean cover. It is frequent also in several variants of the subassociation ericetosum carneae, but there it occurs on different sites.

Relevés in Table 5 are classified into the syntaxon Polysticho lonchitis-Fagetum luzuletosum niveae var. Festuca calva. This group comprises relevés on steep sunny, rarely shady limestone slopes from the Krn and Kanin Mountains, several relevés are also from the slopes of Tolminski Migovec, the ridge of Bavški Grintavec and Loška Stena above the Bala valley, one relevé is from the Upper Sava Valley (under Špik). These are mainly beech stands on the upper forest line that continue into stony subalpine grasslands or open dwarf pine stands, occasionally into rock walls. In the past, these beech forests were probably shaped also by small cattle grazing.

The impact of former small cattle grazing practice is probably even more evident in the stands of the syntaxon Polysticho lonchitis-Fagetum luzuletosum niveae var. Festuca calva subvar. Urtica dioica - Table S13, which comprises relevés from the same area (the Krn and Kanin Mountains, the Bala valley). The differential species of the subvariant is Urtica dioica. Relevé 17 in this Table is classified into the association Ranunculo platanifolii-Fagetum, be- 
cause it has very few diagnostic species of the association Polysticho lonchitis-Fagetum.

Most of the relevés in Table S14, whose localities are mainly in the upper part of the Bala valley, virgin forest stands on sunny rocky slopes of Loška Stena, a few relevés are also from the Krn and Kanin Mountains, are classified into the syntaxon Polysticho lonchitis-Fagetum luzuletosum niveae var. Festuca calva subvar. Calamagrostis varia. This is a natural subalpine forest that continues into subalpine grasslands and rocks, and probably has not been exposed to human disturbance in at least 70 years. The differential species of the subvariant are Calamagrostis varia, Cerastium subtriflorum, Geranium macrorrhizum, Primula auricula and Campanula carnica. In terms of species composition, relevé 1 in this table should be classified into the association Ranunculo platanifolii-Fagetum, the variant with Festuca calva.

\section{Polysticho lonchitis-Fagetum adoxetosum moschatellinae}

Phytosociological table 6 comprises relevés of two separate clusters -24 and 25 . Relevés 1-10 (cluster 24) demonstrate certain similarity with the syntaxon Polysticho lonchitis-Fagetum luzuletosum niveae var. Festuca calva, but their entire species composition speaks against their classification into this syntaxon. Diagnostic species of the association Polysticho lonchitis-Fagetum are rare in these relevés, and based on this criterion they could be classified into the association Ranunculo platanifolii-Fagetum (Saxifrago rotundifoliae-Fagetum Zukrigl sensu Willner), despite the absence of Ranunculus platanifolius. Due to the presence of several other species of tall herbs and shrubs that are mainly limited to the subalpine belt (Senecio cacaliaster, Aconitum angustifolium, Heracleum pollinianum, Rumex arifolius, Ribes uva-crispa) these beech stands on steep, sunny limestone and scree slopes in the Krn ridge to the west of Krničica (localities include Matajur, Kobilja Glava and the Bala valley) are still classified into the association Polysticho lonchitis-Fagetum, subassociation adoxetosum moschatellinae. Its differential species are Adoxa moschatellina, Festuca calva, Geranium robertianum, Sedum hispanicum, Aegopodium podagraria, Lamium orvala, Moehringia trinervia, Stellaria holostea, S. montana, Corydalis solida and Verbascum lanatum, which indicate slightly nitrophytic and moist sites at the contact with subalpine grasslands from the association Avenastro parlatorei-Festucetum calvae. In the past, the development of these stands was shaped by pasture (in part it still is), in the Krn Mountains also by the immediate vicinity of the combat zone, or the front line.

Stands in relevés 11-15 (cluster 25) are provisionally classified into the syntaxon Polysticho lonchitis-Fagetum var. Helleborus niger prov. (the differential species of the variant are also Hepatica nobilis and Saxifraga cuneifolia). Its relevés were made above and under the Vršič Pass, under Pirhovec in the Polovnik ridge and under Debela Peč above the Krma valley.

\section{Polysticho lonchitis-Fagetum allietosum victorialis}

Relevés of cluster 13 in Table 7 are classified into the new subassociation Polysticho lonchitis-Fagetum allietosum victorialis. Its differential species are Allium victorialis (mainly on account of high mean cover), Acer pseudoplatanus, Calamagrostis arundinaceae, Lonicera nigra and Rubus idaeus. With high constancy of Cardamine trifolia they show certain similarity also with the stands of the syntaxon Polysticho-Fagetum cardaminetosum trifoliae var. Calamagrostis arundinacea. Its sites are steep, shady and sunny limestone slopes under the ridge of Golaki on the Trnovo Forest Plateau. Marinček (1996) classified slightly similar stands into the geographical race and subassociation Polysticho lonchitis-Fagetum var. geogr. Allium victorialis polystichetosum aculeati. The altimontane association Allio victorialis-Fagetum Smettan ex Karner et Mucina 1993 or its homonym, Allio victorialis-Fagetum Accetto 2002 (Dakskobler 2008) is known in the Austrian Alps and the Dinaric region of Slovenia (Krokar virgin forest, Snežnik Mts.). According to Willner (2007a) it belongs to the association Saxifrago rotundifoliae-Fagetum Zukrigl 1989. In Slovenia, however, it is documented with only one published relevé (Accetto 2002), so there is no basis for a proper comparison, but it is definitely very different from the Austrian community with the same name. Allium victorialis occasionally occurs also in subalpine beech stands in the Julian Alps, which includes the stands of var. geogr. Anemone trifolia. The stands of the new subassociation characterise specific sites, slightly concave slopes with persistent snow cover. Diagnostic species of the association Polysticho lonchitis-Fagetum are relatively rare in these stands, which on the other hand comprise several species mainly associated with the subalpine belt, such as Cicerbita alpina, Pleurospermum austriacum, Senecio cacaliaster, Poa hybrida and Myrrhis odorata, which justifies their classification into this association. In addition to the typical variant we also distinguish the variant with Adoxa moschatellina (the differential species is also Adenostyles glabra).

\section{Polysticho lonchitis-Fagetum stellarietosum nemorum and Ranunculo platanifolii-Fagetum stellarietosum nemorum var. Alnus viridis}

The relevés that grouped in cluster 14 in Table 8 indicate a contact of two associations, Polysticho lonchitis-Fagetum and Ranunculo platanifollii-Fagetum. In terms of Zukrigl (1989) and Willner's (2007a) syntaxonomic classification 
they belong to the association Saxifrago rotundifoliaeFagetum. In terms of differentiation between altimontane and subalpine beech stands (Marinček 1996, Marinček \& Čarni 2010) and based on the results of hierarchical classification and ordination in Figures 3 and 4, as well as the presence of diagnostic species of the association Polysticho lonchitis-Fagetum (to which we can attribute also Ribes alpinum in this table) relevés $1-27$ in Table 8 can still be classified into this association as a new subassociation Polysticho lonchitis-Fagetum stellarietosum nemorum. Its differential species are Stellaria nemorum, Corydalis cava, Adenostyles alliariae and Myrrhis odorata. The stands of this subassociation were found on shady, rarely on sunny limestone slopes under Golaki on the Trnovo Forest Plateau, under Šavnik (there var. Chaerophyllum villarsii) and Možic, under Hoč at Porezen, under Matajur and Breginjski Stol. Relevés 28-36 in Table 8 were made on shady slopes of Porezen and are provisionally classified into the new variant of the subassociation Ranunculo platanifoliiFagetum stellarietosum nemorum Marinček et Čarni 2010 var. Alnus viridis. The differential species of this variant is also Chaerophyllum villarsii.

Stands in Table S15 (clusters 21 and 17) are classified into the association Polysticho lonchitis-Fagetum. Relevés $1-5$ could provisionally be classified into the variant Polysticho lonchitis-Fagetum var. Adenostyles alliariae prov. Its localities are Breginjski Stol, Kobla, Črna Gora at Črna Prst and Golaki on the Trnovo Forest Plateau. Floristically are quite different from the stands of the subassociation Polysticho lonchitis-Fagetum var. geogr. Allium victorialis adenostyletosum alliariae (Marinček 1996). Relevés 6-9 could provisionally be classified into the variant Polysticho lonchitis-Fagetum var. Centaurea montana prov. (its differential species is also Cirsium erisithales, localities are Golaki, mountain pasture Vrtaška Planina and Stržiškarski Gozd).

Stands in relevés 1-3 in Table S16 (cluster 4) are from the Karawanks (Kočna saddle, Begunjščica) and are classified into the variant Polysticho lonchitis-Fagetum var. Cirsium carniolicum (differential species include Primula elatior and Chaerophyllum hirsutum). Due to high mean cover relevés 4-8 in the same table are provisionally classified into the variant Polysticho lonchitis-Fagetum var. Conocephalum conicum (differential species include Vicia sylvatica, Pulmonaria officinalis, and Neckera crispa) - localities are Kobla, Črna Prst and Kuntar. Relevés 9-12 are classified into the variant Polysticho lonchitis-Fagetum var. Digitalis grandiflora (localities Komna, Kobla, Črna Gora and Lisec), differential species include Rhamnus fallax and Prenanthes purpurea. For the time being, these stands cannot be classified into any of previously described subassociations.

\section{The characteristic combination of constant species}

With 603 relevés we determined 504 taxa of vascular plants, 64 mosses, 16 lichens and 2 tree funghi. The average number of species per relevé is 61 (in the range of 26 to 103 species). As many as 53 species have constancy of $40 \%$ or more ( 48 vascular plants and 5 mosses) - Table 11. The highest constancy (70\% and more) have beech as well as Polygonatum verticillatum, Picea abies, Cardamine enneaphyllos, Polystichum lonchitis, Asplenium viride, Valeriana tripteris, Lonicera alpigena, Adenostyles glabra, Aposeris foetida, Veratrum album and Athyrium filix-femina (Table 11). Stands of the association Rhodothamno-Laricetum comprise the constant combination of 56 species, five of which are mosses (Dakskobler et al. 2018).

We compared constant combinations of three forest communities with extreme sites that are documented with a large number of relevés (Rhodothamno-Laricetum, Polysticho lonchitis-Fagetum and Rhododendro hirsuti-Fagetum - Table 12) and found that the stands of all three syntaxa share 17 species of the constant combination: $P i$ cea abies, Sorbus aucuparia, Asplenium viride, Rhododendron hirsutum, Adenostyles glabra, Clematis alpina, Rubus saxatilis, Anemone trifolia, Daphne mezereum, Calamagrostis varia, Solidago virgaurea, Erica carnea, Rosa pendulina, Valeriana tripteris, Melica nutans, Ctenidium molluscum and Tortella tortuosa, which can be considered character species of southeast-Alpine and northernDinaric montane forests on extreme sites. Associations Rhodothamno-Laricetum and Polysticho lonchitis-Fagetum share 27 species of the characteristic combination (i.e. a half of all species), namely the above listed species as well as Luzula sylvatica, Polystichum lonchitis, Sesleria caerulea, Sorbus chamaemespilus, Oxalis acetosella, Veratrum album, Athyrium filix-femina, Gymnocarpium dryopteris, Polygonatum verticillatum and Saxifraga rotundifolia. In addition to the above-listed 17 species the associations Polysticho lonchitis-Fagetum and Rhododendro hirsuti-Fagetum comprise another 10 species of the characteristic combination: Fagus sylvatica, Cardamine enneaphyllos, Cyclamen purpurascens, Mercurialis perennis, Lonicera alpigena, Galeobdolon flavidum, Prenanthes purpurea, Veronica urticifolia, Gentiana asclepiadea and Acer pseudoplatanus, which means that they share 27 species of the characteristic combination. All species in the constant combination of the association Rhodothamno-Laricetum occur with high or low constancy also in the stands of the association Polysticho lonchitis-Fagetum, whereas some of the species of the constant combination of the 
associations Rhodothamno-Laricetum and (or) Polysticho lonchitis-Fagetum do not occur or are very rare in the stands of the association Rhododendro hirsuti-Fagetum: Sorbus chamaemespilus, Homogyne alpine, Geranium sylvaticum, Heliosperma alpestre, Astrantia bavarica, Festuca nitida, Campanula scheuchzeri and Saxifraga rotundifolia. There are also several species from the characteristic (constant) combination of the association Rhododendro hirsuti-Fagetum, which are very rare or absent from the stands of the other two compared associations: Ostrya carpinifolia, Fraxinus ornus, Phyteuma scheuchzeri subsp. columnae.

\section{Species composition of subalpine beech stands in the Julian Alps and NW Dinaric Alps by groups of diagnostic species}

Table 10 (Column 33) demonstrates that species of spruce forests (class Vaccinio-Piceetea) have the largest proportion in the researched subalpine beech communities $(21 \%$, in the stands of the association Rhodothamno-Laricetum 24\%), whereas the character species of beech forests (Fagetalia sylvaticae) constitute around $18 \%$ (only about 7\% in Rhodothamno-Laricetum). The third dominant group are diagnostic species of the class Mulgedio-Aconitetea (11\%, in RhodothamnoLaricetum 9\%). Also important are proportions of diagnostic species of SE Alpine-NW Dinaric neutrobasophilous beech forests from the alliance AremonioFagion (about 5\%, in Rhodothamno-Laricetum about $3 \%$ ), diagnostic species of basophilous pine forests (Erico-Pinetea) - 5\% (in Rhodothamno-Laricetum 8\%), diagnostic species of the subalpine-alpine basophilous grasslands and swards (Elyno-Seslerietea) - about 4\% (in Rhodothamno-Laricetum about 12\%), diagnostic species of scree communities (Thlaspietea rotundifolii) - about 3\% (in Rhodothamno-Laricetum about 6\%) and diagnostic species of moist chasmophytic communities (alliance Cystoperidion fragilis) - about 4\% (in RhodothamnoLaricetum 1\%). Stands of the association Rhododendro hirsuti-Fagetum (Dakskobler 2003, Table 1) have a higher proportion of diagnostic species of the alliance AremonioFagion (5-8\%), diagnostic species of thermophytic oak forests from the order Quercetalia pubescenti-petraeae (around $7 \%$ ) and diagnostic species of the class EricoPinetea (around 11\%) and class Asplenieta trichomanis (around 7\%), whereas the proportion of diagnostic species of classes Vaccinio-Piceetea (around 17\%) and Mulgedio-Aconitetea (about 3\%) is much smaller.

\section{Nature conservation function of subalpine beech forests in the Julian Alps and the northern part of the Dinaric Alps}

The majority of the researched subalpine beech forests are protection forests and therefore excluded from regular forest management. They are a constituent part of two national parks, the Triglav National Park (Slovenia) and the Julian Prealps Nature Park (NE Italy), and of several forest reserves: Govci, Smrekova Draga-Golaki, Golaki, Grušnica, Savica-Ukanc, Trstje-Tosc, Mala Pišnica, Vrš̌ičZa Akom, Kalce and Bela-Dol-Sedelšček (Mlinšek et al. 1980, Marenče 2003). They belong to the natural habitat type of community interest 91K0 Illyrian Fagus sylvatica forests. Some of the areas with these forests bear little evidence of human activity and some of their stands are therefore considered virgin forests: Gajzd under Lipnik above the Tolminka valley, Krnica on the southern slopes of the peak V Gradu, on the southwestern edge of Loška Stena, promontories under Loška Stena and Jalovec above Loška Koritnica, the upper part of the Bala valley, Lopata at Vogel, Čiprje at the mountain pasture Trstje and Golaki on the Trnovo Forest Plateau.

They are a habitat of the wood grouse (Tetrao urogallus) and the black grouse (Tetrao tetrix). In the researched stands are some localities of four species of European conservation concern (Čušin et al. 2004) - Cypripedium calceolus, Aquilegia iulia, Campanula zoysii and Primula carniolica. Subalpine beech forest species comprise other protected species as well (Anon. 2004): Cephalanthera rubra, Coeloglossum viride, Convallaria majalis, Corallorhiza trifida, Cyclamen purpurascens, Dactylorhiza fuchsii, D. sambucina, Epipactis helleborine, E, atrorubens, Galanthus nivalis, Gentiana clusii, G. lutea subsp. symphyandra, G. pannonica, Goodyera repens, Gymnadenia conopsea, Helleborus niger, H. odorus, Hemerocallis lilioasphodelus, Huperzia selago, Iris graminea, Leontopodium alpinum, Lilium martagon, L. carniolicum, Listera ovata, Lycopodium annotinum, Neottia nidus-avis, Pinguicula alpina, Platanthera bifolia, Primula auricula, Primula auricula var. tolminensis, Pulsatilla alpina subsp. austroalpina, Pseudorchis albida and Sedum maximum, a protected fungus Laricifomes officinalis as well as several Red List species (Anon. 2002): Arabis pauciflora, Betula pubescens subsp. carpatica, Hypericum richeri subsp. grisebachii, Ranunculus auricomus agg. (R. braun-blanquetii), Rhododendron $x$ intermedium, Spiraea decumbens, Streptopus amplexifolius, Veratrum nigrum and Viola pyrenaica. Collectively, these forests therefore serve as a habitat of 48 vascular plants and one fungus of conservation concern. 


\section{Verification of the hypotheses stated in the introduction}

By analysing a large number of relevés and comparing them with similar communities we determined that most of the studied stands can be classified into the association Polysticho lonchitis-Fagetum. Nevertheless, stands whose floristic composition allows us to classify them also into associations Anemono trifoliae-Fagetum and Ranunculo platanifolii-Fagetum also occur on or slightly below the present upper forest line. In nature, it is not always straightforward to differentiate between the stands of these three associations and we faced the same problem when processing our material. In several cases (Tables S1, S2, 3, S13 S14, 8), the assessment of diagnostic species suggested that one, a few or more relevés could be classified into a different association than the rest of the relevés. Nevertheless, in most cases the stands of these three associations are well differentiated from one another also in nature. The concern or assumption that the stands of the subassociation ericetosum carneae, for example, could be classified into an altitudinal variant (subassociation) of the association Anemono trifoliae-Fagetum, and the stands of subassociations adoxetosum moschatellinae, allietosum victorialis and stellarietosum nemorum into altitudinal forms of the association Ranunculo platanifolii-Fagetum (Saxifrago rotundifoliae-Fagetum), is not sufficiently justified if taking into account the entire spectrum (diversity) of the stands of the association Polysticho lonchitis-Fagetum.

Our analysis showed that the same cluster (group) could comprise relevés from areas of two races (var. geogr. Anemone trifolia and var. geogr. Allium victorialis). Some relevés from the Trnovo Forest Plateau that belong to the latter race otherwise grouped together as well, most obviously in the case of the subassociation allietosum victorialis.

The same as our analysis of larch forests this analysis also confirmed our finding that the division into lower units, i.e. subassociations, is possible, but there are no available name-giving species that are associated exlusively with the sites and stands of one subassociation and could serve as absolute differential species. It is the entire species combination that is differential for a specific subunit, but species with high mean cover are usually defining.

The species diversity of subalpine beech forests is the result of their geographical position (we established certain differences between the interior of the Alps and their southern and western fringes), aspect (sunny/shady), parent material (admixture of marlstone or chert), soil conditions (the level of decomposition of organic matter, litter), in part also slope and stoniness of the sites.
With their entire species composition subalpine beech forests are very similar to (in places) contact subalpine larch forests and share with them many diagnostic species and species with the highest constancy. Larch forests comprise substantially more species of subalpine-alpine non-forest sites (screes, grasslands) and substantially fewer species exclusive to beech forests.

The differences between subalpine beech forests in the interior of the Julian Alps, where larch stands usually constitute the highest forest belt, and subalpine beech forests on the fringes of this mountain range, where beech forests form the highest belt of forest vegetation and give way to dwarf pine, alpine swards and rocks, are obvious, although certain relevés from both areas also grouped in the same cluster. At the level of lower syntaxonomic units the stands of the subassociation ericetosum carneae prevail in the first type of subalpine beech stands, and the stands of subassociations cardaminetosum trifoliae, luzuletosum niveae and calamagrostietosum variae in the second type. Stands of subassociations luzuletosum luzuloidis, adoxetosum moschatellinae, stellarietosum nemorum and allietosum victorialis were found exclusively in the second type of subalpine beech stands.

The most frequent among other tree species in subalpine beech stands are mountain ash (Sorbus aucuparia) and spruce (Picea abies), but often with only a few specimens and exclusively in the shrub layer. The proportion of spruce and larch is higher in certain forms of subalpine beech stands in the interior of the Julian Alps (larch is differential for the subassociation ericetrosum carnae), on shady aspects also in their southern fringe area (Grušnica). Fir (Abies alba) also occurs in subalpine beech forests, but never with a high proportion or a large number of individuals. The proportion of sycamore (Acer pseudoplatanus) is higher in stands of certain subassociations (stellarietosum nemorum, adoxetosum moschatellinae, allietosum victorialis), although individual trees occur across the entire distribution area of this community, at least in the shrub and herb layers.

In the course of our research (1986-2019) we did not come across signs of significant human impact in these forests. This can partly be attributed to their remoteness and difficult access. Some of the stands, however, are located near ski slopes and forest roads - e.g. virgin forest Lopata and stands under Orlova Glava on Vogel as well as stands in Stržiškarski Gozd, Rutarski Gozd and Grantarski Gozd. A few years ago, a new forest road was cut through Stržiškarski Gozd just below the forest line. A new ski slope, which is planned for the area between Slatnik, Šavnik and Kobla, would also involve clear-cutting of subalpine beech stands. In some places (Porezen, mountain pasture Suha, mountain pasture Klek), these 
stands are part of or situated at the contact with grazing areas. Mountain trails lead through some of the stands and increasing number of visitors to the mountains and random movement (including bicycles and even motorbikes) have caused erosion gullies and left other marks on the landscape. Major natural hazards include avalanches (e.g. in the Kanin Mts., the Bala valley), fires in contact dwarf pine (e.g. on the slopes of Hohkovbl / Matajurski Vrh above Rut), rarely sleet (Golaki on the Trnovo Forest Plateau), strong wind (windstorms) and rockfall (e.g. Slemenske Peči under Rdeči Rob).

We identified several species in beech stands at or just below the forest line that are otherwise considered character species of submontane beech and beech-oak, even hornbeam forests, communities that thrive in very different climatic conditions or other species that are usually (at least in Slovenia) distributed only up to the (upper) montane belt. Some of these species and their constancy in 603 relevés are listed below:

Primula vulgaris 48 relevés, constancy $8 \%$

Helleborus odorus 39 relevés, constancy 6\%

Primula veris subsp. columnae 20 relevés, constancy 3\%

Lathyrus vernus subsp. flaccidus 18 relevés, constancy 3\%

Carex flacca 17 relevés, constancy 3\%

Corylus avellana, 12 relevés, constancy $2 \%$

Saxifraga petraea 12 relevés, constancy $2 \%$

Iris graminea 10 relevés, constancy $2 \%$

Vincetoxicum hirundinaria 8 relevés, constancy 1\%

Arabis turrita 7 relevés, constancy $1 \%$

Carex pilosa 6 relevés, constancy $1 \%$

Lonicera xylosteum 6 relevés, constancy 1\%

Ornithogalum pyrenaicum 5 relevés, constancy 1\%

Galanthus nivalis 4 relevés, constancy 1\%

Crocus napolitanus (C. vernus subsp. vernus) 2 relevés, constancy $0.3 \%$

Hemerocallis lilioasphodelus, 2 relevés, constancy $0.3 \%$

Veratrum nigrum 2 relevés, constancy $0.3 \%$

Phyllitis scolopendrium, 2 relevés, constancy $0.3 \%$

Platanthera bifolia, 2 relevés, constancy $0.3 \%$

Asarum europaeum subsp. caucasicum 2 relevés, constancy $0.3 \%$

Ostrya carpinifolia 1 relevé, constancy $0.1 \%$

Anthericum ramosum 1 relevé, constancy $0.1 \%$

Cephalanthera rubra 1 relevé, constancy $0.1 \%$

Ceterach javorkeanum 1 relevé, constancy $0.1 \%$

Alliaria petiolata 1 relevé, constancy $0.1 \%$

The listed species were mainly found in subalpine beech stands on sunny slopes and may also indicate more favourable thermal conditions in the last decades. Our analysis, however, could not yet identify significant changes in vegetation that would reflect the climate change in recent decades.

No alien invasive species were detected in any of our 603 relevés and only a few relevés comprised ruderal species (Stellaria media, Geum urbanum, Alliaria petiolata, Cirsium eriophorum, Taraxacum sect. Ruderalia). Generally speaking, the researched forests are some of the best preserved in Slovenia and the least affected by man.

\section{Conclusions}

In terms of their entire species composition the beech forests at the present forest line in the Julian Alps and the northern Dinaric Alps (these findings partly apply also to the Karawanks and Kamnik Alps) are definitely a unique plant community. In most cases, our analysis of more than 600 phytosociological relevés did not provide sufficient basis to classify it into the associations of beech forests of the altimontane belt in this area (Anemono trifoliae-Fagetum, Saxifrago rotundifoliae-Fagetum, Ranunculo platanifolii-Fagetum, Aconito paniculati-Fagetum) as proposed by Zukrigl (1989), Willner (2002, 2007a, 2007b) and Zupančič (2012), and we therefore confirm its classification into the association Polysticho lonchitisFagetum (the older synonym is Fagetum subalpinum) as justified or described by Marinček (1980), Poldini \& Nardini (1993), Marinček et al. 1993, Marinček (1996) and Marinček \& Čarni (2010), Dakskobler (2008, 2015) and Dakskobler et al. (2013). In terms of species composition and structure their submontane-montane vicariant consists of the stands of the association Rhododendro hirsuti-Fagetum that overgrow extreme, steep, rocky, mainly shady slopes in the same area and elsewhere (Alpine, preAlpine, Dinaric and pre-Dinaric part of Slovenia), and are differentiated from subalpine beech stands by certain more thermophytic species. The studied subalpine beech stands share many species with the eastern-Alpine larch community (Rhodothamno-Laricetum), but larch stands are an even more extreme and open community with canopy gaps and comprise more subalpine-alpine species of screes and grasslands.

Illyrian (southeastern-Alpine-northern-Dinaric) beech communities on carbonate and limestone-marl/flysch bedrock comprise relatively many diagnostic species, none of which is exclusive to only one of these communities. These associations, including Polysticho lonchitisFagetum, have no absolute character species. We found that the studied community is characterised by the characteristic structure by groups of diagnostic species, where the percentage of species of spruce forests is similar to the percentage of species of beech forests, and tall herbs as well as species of basophytic pine forests and moist 
rock crevices are well represented; another characteristic is frequent co-occurrence of at least a third of the following species: Polystichum lonchitis, Clematis alpina, Luzula sylvatica, Rhododendron hirsutum, Paraleucobryum sauteri, Sorbus chamaemespilus, Aconitum lycoctonum subsp. ranunculifolium (A. lupicida), Laserpitium peucedanoides, Pinus mugo, Salix appendiculata, Paederota lutea, Aster bellidiastrum, Homogyne alpina, Carex ferruginea, Festuca calva, Lonicera caerulae, Rhodothamnus chamaecistus.

In some parts of the Southeastern Alps (mainly in the areas with the Submediterranean influence) the studied subalpine beech communities are treated as zonal vegetation on the climatic upper forest line (in the highest forest belt) where beech forest transitions through dwarf pine to alpine swards. In other parts of these Alps with a more continental climate, where the zonal vegetation on the climatic forest line consists of spruce and larch forests, they are connected with specific geological, geomorphological and local climatic conditions. Human impact (deforestation and pasture, military activity during and after World War I) does not have a decisive role in described vertical zonation, although the upper forest line has evidently lowered (in some places) due to anthropogenic factors.

Hierarchical classification of a large number of very similar relevés (communities) of beech forests at the present upper forest line did not produce clusters that would be reflect the presence or absence of one or more differential species. We did, however, identify groups of relevés that are sufficiently clearly differentiated by some of the following factors: geological bedrock (limestone/dolomite/marlstone), soil conditions (the rate of decomposition of organic matter, raw humus, moder/soil moisture), geographical position (central/fringe areas of the mountain range), aspect (sunny/shady) and presence of other tree species (spruce, larch, sycamore). With high constancy and/or high mean cover these identified groups characterise individual species or groups of species that can otherwise be present in most of the other clusters of relevés and are therefore only relative differential species. The newly described subassociations ericetosum carneae, cardaminetosum trifoliae, luzuletosum niveae, adoxetosum moschatellinae, calamagrostietosum variae are therefore not justified by the exclusive occurrence of name-giving species in their stands, but by their entire species composition. Only subassociations luzuletosum luzuloidis, stellarietosum nemorum and allietosum victorialis are slightly more (but not entirely) justified based on the concept of differential species as understood by Willner (2002, 2007a).

Subalpine beech forests in the Julian Alps and the northern Dinaric Alps are some of the most natural, best preserved and least affected by man in this part of Europe; some are assumed to be virgin forests and are species rich, comprising many organisms of conservation concern. They are endangered by extensive and aggressive silvicultural practice that disregards their nature conservation function, increased tourism activity (planned ski slopes) and potentially also climate change, even though this is not yet reflected in their species composition and structure.

\section{Povzetek}

Vegetacijska analiza bukovega gozda na zgornji gozdni meji v Julijskih Alpah (severozahodna Slovenija in severovzhodna Italija) in severnem delu Dinarskega gorstva

Bukovi gozdovi na zdajšnji gozdni meji v Julijskih Alpah in severnem delu Dinarskega gorstva (deloma te ugotovitve veljajo tudi za Karavanke in Kamniške Alpe) so po svoji celotni vrstni sestavi zagotovo svojska/posebna/edinstvena rastlinska združba, ki jo na podlagi analize več kot 600 fitocenoloških popisov v večini primerov nismo mogli uvrstiti $\mathrm{v}$ asociacije bukovih gozdov altimontanskega pasu v tem območju (Anemono trifoliae-Fagetum, Saxifrago rotundifoliae-Fagetum, Ranunculo platanifoliiFagetum, Aconito paniculati-Fagetum) - kot predpostavljajo Zukrigl (1989), Willner (2002, 2007a,b) in Zupančič (2012), zato potrjujemo njeno uvrstitev v asociacijo Polysticho lonchitis-Fagetum (starejši sinonim Fagetum subalpinum), v smislu, kot so to asociacijo utemeljili oz. opisali Marinček (1980), Poldini \& Nardini (1993), Marinček et al. 1993, Marinček (1996) in Marinček \& Čarni (2010), Dakskobler $(2008,2015)$ in Dakskobler et al. (2013). Po vrstni sestavi in zgradbi so njihova podgorsko-gorska vikarianta sestoji asociacije Rhododendro hirsuti-Fagetum, ki poraščajo $\mathrm{v}$ istem območju in tudi drugod (alpski, predalpski, dinarski in preddinarski del Slovenije) skrajna, strma, skalnata, večinoma osojna pobočja in jih od subalpinskega bukovja razlikujejo neketere bolj toploljubne vrste. Veliko skupnih vrst ima preučeno subalpinsko bukovje z vzhodoalpsko združbo macesna (RhodothamnoLaricetum), s to razliko, da je macesnovje še bolj skrajna, odprta in vrzelasta združba, $\mathrm{z}$ več subalpinsko-alpinskimi vrstami melišč in traviščc.

Ilirske (jugovzhodnoalpsko-severnodinarske) bukove združbe na karbonatni in apnenčasto-lapornati/flišni podlagi vsebujejo razmeroma veliko diagnostičnih vrst, od katerih pa nobena ni navezana samo na eno od teh združb. Absolutnih značilnic te asociacije nimajo in to velja tudi za asociacijo Polysticho lonchitis-Fagetum. Po 
naši presoji preučeno združbo ob značilni strukturi po skupinah diagnostičnih vrst, kjer so vrste smrekovih gozdov po deležu blizu vrstam bukovih gozdov, dobro pa so zastopane tudi vrste visokih steblik, bazofilnih borovih gozdov in vlažnih skalnih razpok, označuje pogosto skupno uspevanje vsaj tretjine od naslednjih vrst: Polystichum lonchitis, Clematis alpina, Luzula sylvatica, Rhododendron hirsutum, Paraleucobryum sauteri, Sorbus chamaemespilus, Aconitum lycoctonum subsp. ranunculifolium (A. lupicida), Laserpitium peucedanoides, Pinus mugo, Salix appendiculata, Paederota lutea, Aster bellidiastrum, Homogyne alpina, Carex ferruginea, Festuca calva, Lonicera caerulae, Rhodothamnus chamaecistus.

$S$ hierarhično klasifikacijo velikega števila med seboj precej podobnih popisov (združb) bukovega gozda na zdajšnji zgornji gozdni meji nismo mogli dobiti skupin, ki bi bile utemeljene na prisotnosti/odsotnosti ene ali več razlikovalnih vrst. Prepoznali pa smo skupine popisov, ki se med seboj dovolj očitno razlikujejo po nekaterih od naštetih dejavnikov: po geološki podlagi (apnenec/dolo$\mathrm{mit} /$ laporovec), talnih razmerah (stopnja razkrojenosti organske snovi, surov humus, prhnina/vlažnost tal), geografski legi (osrednji deli gorovja/ robni deli), nebesni legi (prisojna/osojna), prisotnost drugih drevesnih vrst (smreke, macesna, gorskega javorja). Te prepoznane skupine $\mathrm{z}$ veliko stalnostjo in/ali velikim srednjim zastiranjem označujejo posamezne vrste ali skupine vrst, ki so sicer lahko prisotne tudi v večini drugih skupin popisov, torej so samo relativne razlikovalnice. Novo opisane subasociacije ericetosum carneae, cardaminetosum trifoliae, luzuletosum niveae, adoxetosum moschatellinae, calamagrostietosum variae torej nimajo podlage $\mathrm{v}$ izključnem pojavljanju po njih imenovanih vrst v njihovih sestojih, pač pa v njihovi celokupni vrstni sestavi. Le subasociacije luzletosum luzuloidis, stellarietosum nemorum in allietosum victorialis so nekoliko bolj (a ne povsem) utemeljene na konceptu razlikovalnih vrst v smislu Willnerja (2002, 2007a).

Subalpinski bukovi gozdovi v Julijskih Alpah in severnem delu Dinarskega gorstva so med najbolj naravni$\mathrm{mi}$, ohranjenimi in od človeka najmanj vplivanimi bukovimi gozdovi v tem delu Evrope, nekateri so domnevno pragozdni in so vrstno bogati, vsebujejo veliko naravovarstveno pomembnih organizmov. Ogroža jih ekstenzivna in agresivna gozdarska dejavnost, ki ne spoštuje njihove varovalnosti, povečanje turistične dejavnosti (načrtovana smučišča) in morda tudi podnebne spremembe, čeprav se za zdaj vse to v njihovi vrstni sestavi in zgradbi očitno še ne kaže.

\section{Acknowledgements}

The authors acknowledge the financial support from the Slovenian Research Agency (research core funding No. P1-0236) and the Recharge.green project funded by the European Regional Development Fund. Sincere thanks to Mag. Andrej Seliškar, Branko Zupan, Dr. Aleš Poljanec, Dr. Branko Vreš, late Iztok Mlekuž, late Vid Dakskobler, Ivan Veber, Brane Anderle, Iztok Sajko and Kostja Jerovšek for their help in the field work. Two anonymous reviewers and Prof. Dr. Jean-Paul Theurillat helped us with valuable improvements and corrections. English translation by Andreja Šalamon Verbič and authors.

Andrej Rozman (I) https://orcid.org/0000-0003-0797-5452

\section{Supplementary material on-line}

\section{References}

Accetto, M. 2002: Pragozdno rastlinje rezervata Krokar na Kočevskem. Gozdarski vestnik 60 (10): 419-443.

Accetto, M. 2015: Gozdno in drugo rastje na levem bregu Iškega vintgarja. Acta Silvae et Ligni 106: 1-121. DOI: https://doi. org/10.20315/ASetL.106.1

Aeschimann, D., Lauber, K., Moser, D. M. \& Theurillat, J.-P. 2004a: Flora alpina. Bd. 1: Lycopodiaceae-Apiaceae. Haupt Verlag, Bern, Stuttgart, Wien, 1159 pp.

Aeschimann, D., Lauber, K., Moser, D. M. \& Theurillat, J.-P. 2004b: Flora alpina. Bd. 2: Gentianaceae-Orchidaceae. Haupt Verlag, Bern, Stuttgart, Wien, $1188 \mathrm{pp}$.

Anonymous 2002: Pravilnik o uvrstitvi ogroženih rastlinskih in živalskih vrst v rdeči seznam. Uradni list RS 82/2002.

Anonymous 2004: Uredba o zavarovanih prosto živečih rastlinskih vrstah. Uradni list RS 46/2004.

Braun-Blanquet, J. 1964: Pflanzensoziologie. Grundzüge der Vegetationskunde. 3. Auflage. Springer, Wien - New York, 865 pp.

Budkovič, L., Gašperin, M. \& Veber, I. 1996: Drevesa velikani v Bohinju. Gozdarsko društvo Bled, Bohinjka Bistrica, 48 pp.

Buser, S. 2009: Geološka karta Slovenije 1: 250.000. Geological map of Slovenia 1.250,000. Geološki zavod Slovenije, Ljubljana.

Chytrý, M., Tichý L., Holt J. \& Botta-Dukát Z. 2002:

Determination of diagnostic species with statistical fidelity measures. Journal of Vegetation Science 13 (1): 79-90. DOI: https://doi. org/10.1111/j.1654-1103.2002.tb02025.x

Čušin, B., Babij, V., Bačič, T., Dakskobler, I., Frajman, B., Jogan, N., Kaligarič, M., Praprotnik, N., Seliškar, A, Skoberne, P., Surina, B., Škornik, S., Vreš, B. 2004: Natura 2000 v Sloveniji, Rastline. Založba ZRC, ZRC SAZU, Ljubljana, 172 pp.

Dakskobler, I. 2002a: Jelovo-bukovi gozdovi v dolinah Kneže, Zadlaščice in Tolminke (južne Julijske Alpe, zahodna Slovenija) Razprave 4. razreda SAZU 43-3: 111-165. 
Dakskobler, I. 2002b: Jelovo-bukovi gozdovi na Bovškem (Julijske Alpe, severozahodna Slovenija). Razprave 4. razreda SAZU 43-2: 109-155.

Dakskobler, I. 2003: Asociacija Rhododendro hirsuti-Fagetum Accetto ex Dakskobler 1998 v zahodni Sloveniji. Razprave 4. razreda SAZU 44-2: 5-85.

Dakskobler, I. 2004: Jelovo-bukovi gozdovi v dolini Loške Koritnice v Julijskih Alpah (severozahodna Slovenija). Gozdarski vestnik 62 (7-8): 299-315.

Dakskobler, I. 2008: Pregled bukovih rastǐ̌č v Sloveniji. Zbornik gozdarstva in lesarstva 87: 3-14.

Dakskobler, I. 2015: Gozdna vegetacija Triglavskega narodnega parka. Acta Triglavensia 3: 9-39.

Dakskobler, I., Urbančič, M. \& Wraber, A. 2000: Gozd bukve in jelke $\mathrm{z}$ dlakavim slečem (Omphalodo-Fagetum rhododendretosum hirsuti) v Trnovskem gozdu (zahodna Slovenija). Zbornik gozdarstva in lesarstva 62: $5-52$.

Dakskobler, I. \& Rozman, A. 2010: Novi nahajališči puhaste breze (Betula pubescens Ehrh.) in smrdljivega brina (Juniperus sabina L.) $\mathrm{v}$ Julijskih Alpah. New localities of Betula pubescens Ehrh. and Juniperus sabina L. in the Julian Alps. Gozdarski vestnik 68 (2): 107-122.

Dakskobler, I., Rozman, A. \& Franz, W. R. 2012: Betula pubescens Ehrh. subsp. carpatica (Willd.) Ascherson \& Graebner, a new taxon in the flora of the Julian Alps and Slovenia and its new association Rhododendro hirsuti-Betuletum carpaticae ass. nov. Folia biologica et geologica 53 (1-2): 5-23.

Dakskobler, I., Rozman, A. \& Seliškar, A. 2013: Forest and scrub communities with green alder (Alnus viridis) in Slovenia. Hacquetia 12 (2): 95-185. DOI: https://doi.org/ 10.2478/HACQ-2013-0012

Dakskobler, I., Seliškar, A. \& Rozman, A. 2018: Phytosociological analysis of European larch forests in the Southeastern Alps. Hacquetia 17 (2): 247-519. DOI: https://doi.org/10.2478/hacq-2018-0004

De Caceres M., \& Legendre P. 2009: Associations between species and groups of sites: indices and statistical inference. Ecology 90: 12: 3566-3574. DOI: https://doi.org/10.1890/08-1823.1

Del Favero, R., Poldini, L., Bortoli, P., Dreossi, G., Lasen, C. \& G. Vanone, G. 1998: La vegetazione forestale e la selvicoltura nella regione Friuli - Venezia Giulia. Vol. 2. Regione autonoma Friuli-Venezia Giulia. Direzione Regionale delle Foreste, Servizio delle Selvicoltura, Udine, 303 pp. + Appendix.

Dufrene, M. \& Legendre, P. 1997: Species assemblages and indicator species: the need for a flexible asymmetrical approach. Ecological Monographs 67 (3): 345-366. DOI: https://doi.org/10.1890/00129615(1997)067[0345:SAAIST]2.0.CO;2

Fanta, J. 1981: Fagus sylvatica L. und das Aceri-Fagetum an der alpinen Waldgrenze in mitteleuropäischen Gebirgen. Vegetatio 44: 13-24.

Ferreira, A., Kušar, G. \& Hočevar, M. 2000: Kartiranje zgornje gozdne meje v Triglavskem narodnem parku z uporabo metod digitalne fotogrametrije. In: Hladnik, D. et al. (eds..): GIS v Sloveniji 1999-2000, Ljubljana, pp. 187-199.

Firm, D., Rozman, A. \& Diaci, J. 2006: Zgradba in razvo visokogorske vegetacije v gozdnem rezervatu na Dleskovški planoti. In: Jurc, M. (ed.): Podnebne spremembe - vplivi na gozdarstvo. Biotehniška fakulteta, Oddelek za gozdarstvo in obnovljive gozdne vire, Ljubljana. Strokovna in znanstvena dela 130, pp. 133-151.
Gams, I. 1976: O zgornji gozdni meji na jugovzhodnem Koroškem. Geografski zbornik 16 (2): 155-192.

Gams, I. 1977: Gozd ob gornji meji uspevanja in varstvo narave. Varstvo narave 10: 23-31.

Gams, I. 2002: Koliko visokogorja in hribovja imamo v Sloveniji. Planinski vestnik 102 (3): 24-28.

Horvat, I. 1938: Biljnosociološka istraživanja šuma u Hrvatskoj. Glasnik za šumarske pokuse 6: 127-279.

Jennings, M.D., Faber-Langendoen, D., Loucks, O.L., Peet, R.K. \& Roberts, D. 2009: Standards for associations and alliances of the U.S. National Vegetation Classification. Ecological Monographs 79 (2): 173-199. DOI: https://doi.org/10.1890/07-1804.1

Kadunc, A. \& Rugani, T. 1999: Zgornja gozdna meja v Notranjem Bohinju. Gozdarski vestnik 57 (1): 23-33.

Landolt, E., Bäumler, B. Erhardt, A., Hegg, O., Klötzli, F., Lämmler, W., Nobis, M., Rudmann-Maurer, K., Schweingruber, F. H., Theurillat, J.-P., Urmi, E., Vust, M. \& Wohlgemuth, T. 2010: Flora indicativa. 2. Auflage. Haupt Verlag, Bern-Stuttgart-Wien, 323 pp.

Legendre, P. \& Anderson, M. J. 1999: Distance-based redundancy analysis: testing multispecies responses in multifactorial ecological experiments. Ecological Monographs 69: 1-24. DOI: https://doi. org/10.1890/0012-9615(1999)069[0001:DBRATM]2.0.CO;2

Lovrenčak, F. 1977: Zgornja gozdna meja v Kamniških Alpah v geografski luči (v primerjavi s Snežnikom). Geografski zbornik 16 (1): $1-144$.

Lovrenčak, F. 1987: Zgornja gozdna meja v Julijskih Alpah in na visokih kraških planotah Slovenije. Geografski zbornik 26 (1): 5-62.

Lovrenčak, F. 1989: The upper forest line in the Julian Alps. Biogeographia 13 (1987): 113-118.

Lovrenčak, F. 2007: Zgornja gozdna meja slovenskih Alp, visokih kraških planot in Prokletij. Znanstvenoraziskovalni inštitut Filozofske fakultete Univerze v Ljubljani, Ljubljana, 217 pp.

Marek, R. 1910: Waldgrenzstudien in den österreichischen Alpen. Dr. A. Petermanns Mitteilungen aus Justus Perthes' Geographischer Anstalt. Heft. 168, pp. 1-102 + 4 Graphs and map, Justus Perthes, Gotha.

Maarel, van der E. 1979: Transformation of cover-abundance values in phytosociology and its effects on community similarity. Vegetatio 39 (2): 97-114. DOI: https://doi.org/10.1007/BF00052021

Maarel, van der E. \& Franklin, J. 2013: Vegetation Ecology. 2nd Edition. Wiley-Blackwell. 572 pp.

Marenče, M. 2003: Gozdni rezervati v Triglavskem narodnem parku. Triglavski razgledi 7 (11): 1-24.

Marinček, L. 1980: Subalpsko bukovje Škofjeloškega pogorja. Loški razgledi 27: 182-192.

Marinček, L. 1987: Bukovi gozdovi na Slovenskem. Delavska enotnost, Ljubljana, 153 pp.

Marinček, L., 1996: Subalpine Buchenwälder in den Westlichen Dinariden. Atti del $24^{\circ}$ Simposio della Societa Estalpino-Dinarica di Fitosociologia. - Ann. Mus. Civ. Rovereto. Sez.: Arch., St., Sc. nat. Suppl. II, vol. 11 (1995), pp. 197-208. 
Marinček, L., Mucina, L., Zupančič, M., Poldini, L., Dakskobler, I. \& Accetto, M. 1993: Nomenklatorische Revision der illyrischen Buchenwälder (Verband Aremonio-Fagion). Studia Geobotanica 12 (1992): 121-135.

Marinček, L., Poldini, L. \& Zupančič, M. 1989: Beitrag zur Kenntniss der Gesellschaft Anemono-Fagetum. Razprave 4. razreda SAZU 30 (1): $3-64$.

Marinček, L. \& Čarni, A. 2010: Altimontanski bukovi gozdovi podzveze Saxifrago-Fagenion (Aremonio-Fagion). Scopolia 69: 1-107.

Martinčič, A. 2003: Seznam listnatih mahov (Bryopsida) Slovenije. Hacquetia 2 (1): 91-166.

Martinčič, A. 2011: Annotated Checklist of Slovenian Liverworts (Marchanthiophyta) and Hornworts (Anthocerotophyta). Scopolia 72: 1-38.

Martinčič, A., Wraber, T., Jogan, N., Podobnik, A., Turk, B., Vreš, B., Ravnik, V., Frajman, B., Strgulc Krajšek, S., Trčak, B., Bačič, T., Fischer, M. A., Eler, K. \& Surina, B. 2007: Mala flora Slovenije. Ključ za določanje praprotnic in semenk. Četrta, dopolnjena in spremenjena izdaja. Tehniška založba Slovenije, Ljubljana, 967 pp.

Mlinšek, D., Accetto, M., Anko, B., Piskernik, M. Robič, D., Smolej, I. \& Zupančič, M. 1980: Gozdni rezervati v Sloveniji. Inštitut za gozdno in lesno gospodarstvo pri Biotehniški fakulteti v Ljubljani, Ljubljana, $414 \mathrm{pp}$.

Novosel, J. 1974: Gozdne združbe G.e. Tržič. Biro za gozdarsko načrtovanje Ljubljana, Elaborat.

Oksanen, J., Blanchet, F. G., Friendly, M., Kindt, R., Legendre, P., McGlinn, D., Minchin, P. R., O'Hara, R. B., Simpson, G. L., Solymos, P., Stevens, M. H. H., Szoecs, E. \& Wagner, H. 2017: vegan: Community Ecology Package. R package version 2.4-4. https:// CRAN.R-project.org/package=vegan

Podani, J. 2001: SYN-TAX 2000. Computer Programs for Data Analysis in Ecology and Systematics. User's Manual, Budapest, 53 pp.

Pogačnik, N. \& Prosen M. 1998: Zgradba bukovega gozda ob gozdni meji na Snežniku. Gozdarski vestnik 56 (10): 443-459.

Poldini, L. \& Nardini, S. 1993: Boschi di forra, faggete e abieteti in Friuli. Studia Geobotanica 13: 215-298.

R Core Team 2017: R: A language and environment for statistical computing. R Foundation for Statistical Computing, Vienna, Austria. URL https://www.R-project.org/.

Robič, D. 1998: Gorski gozd v Sloveniji, poizkus opredelitve in nekatere posebnosti ravnanja z njim. In: J. Diaci (ed.): Gorski gozd. Zbornik referatov. 19. gozdarski študijski dnevi, Logarska dolina 26.-27. 3. 1998, pp. 1-16, Ljubljana.

Seliškar, T., Vreš, B. \& Seliškar, A. 2003: FloVegSi 2.0. Fauna, Flora, Vegetation and Paleovegetation of Slovenia. Computer programme for arranging and analysis of biological data. Biološki inštitut ZRC SAZU, Ljubljana.

Slezák M., Hrivnák R., Ujházy K., Ujházyová M., Máliš F., Petrášová, A. 2016: Syntaxonomy and ecology of acidophilous beech forest vegetation in Slovakia. Phytocoenologia 46 (1): 69-87. DOI: https:// doi.org/ 10.1127/phyto/2016/0047

Suppan, U., Prügger, J. \& Mayrhofer, H. 2000: Catalogue of the lichenized and lichenicolous fungi of Slovenia. Bibliotheca Lichenologica 76: 1-215.
Surina, B. \& M. Rakaj 2007: Subalpine beech forests with Hairy Alpenrose (Polysticho lonchitis-Fagetum rhododendretosum hirsuti subass. nova) on Mt. Snežnik (Liburnian Karst, Dinaric Mts). Hacquetia 6 (2): 195-208. DOI: https://doi.org/10.2478/v10028$007-0011-y$

Surina, B. \& Dakskobler, I. 2013: Phytosociology and ecology of the Dinaric fir-beech forests (Omphalodo-Fagetum) at the north-western part of the Illyrian floral province (NW Dinaric Alps). Hacquetia 12 (1): 11-85. DOI: https://doi.org/10.2478/HACQ-2013-0002

Stur, D. 1857: Über den Einfluss des Bodens auf die Vertheilung der Pflanzen. Sitzungsber. d. Akad. d. Wiss. Wien, Mathem.- naturw. Kl. Bd. 25 (1-2): 349-421.

Šilc, U. \& Čarni, A. 2012: Conspectus of vegetation syntaxa in Slovenia. Hacquetia 11 (1): 113-164. DOI: https://doi.org/10.2478/ v10028-012-0006-1

Theurillat, J.-P. 2004: Pflanzensoziologisches System. In: Aeschimann, D., Lauber, K., Moser D. M. \& Theurillat J.-P.: Flora alpina 3: Register. Haupt Verlag, Bern, Stuttgart, Wien, pp. 301-313.

Tregubov, V. 1957: Gozdne rastlinske združbe. In: Tregubov, V. \& Čokl, M. (eds.): Prebiralni gozdovi na Snežniku. Inštitut za gozdno in lesno gospodarstvo, Strokovna in znanstvena dela 4, Ljubljana. pp. $23-65$

Torelli, N. 2001: Odziv drevja na globoke in površinske poškodbe na primeru bukve (Fagus sylvatica L.) s poudarkom na nastanku in ekologiji ranitvenega lesa (»rdeče srce») (pregled). Gozdarski vestnik 59 (2): 85-94.

Tuma, H. 1925: Naše planine. Gospodarska čitanka (ed. J. Rustja), Goriška Mohorjeva družba, Gorica. pp. 36-47.

Urbančič, M., Simončič, P., Prus, T. \& Kutnar, L. 2005: Atlas gozdnih tal. Zveza gozdarskih društev Slovenije, Gozdarski vestnik \& Gozdarski inštitut Slovenije, Ljubljana. 100 pp.

Vrtačnik Merčun, V. 1999: Gozdna meja v porečjih Martuljka in Belega potoka. Geografski obzornik 46 (2): 13-17.

Willner, W. 2002: Syntaxonomische Revision der südmitteleuropäischen Buchenwälder. Phytocoenologia 32 (3): 337-453.

Willner, W. 2007a: Fagion sylvaticae Luquet 1926 1971. In: Willner, W. \& Grabherr, G. (eds.): Die Wälder und Gebüsche Österreichs. Ein Bestimmungswerk mit Tabellen. 1. Textband. Spektrum Akademischer Verlag in Elsevier, Heidelberg, pp. 144-166.

Willner, W. 2007b: Saxifrago rotundifoliae-Fagetum. In: Willner, W. \& Grabherr, G. (eds.): Die Wälder und Gebüsche Österreichs. Ein Bestimmungswerk mit Tabellen. 2. Tebellenband. Spektrum Akademischer Verlag in Elsevier, Heidelberg, pp. 172-176.

Wraber, M. 1970: Die obere Wald- und Baumgrenze in den slowenischen Hochgebirgen in ökologischer Betrachtung. Mittl. Ostalp.-din. Ges. f. Vegetationskunde 11: 235-248.

Zukrigl, K. 1989: Die montanen Buchenwälder der Nordabdachung der Karawanken und Karnischen Alpen. Naturschutz in Kärnten 9. Amt der Kärntner Landesregierung, Klagenfurt, 114 pp.

Zupančič, M. 2012: Syntaxonomic problems of altimontane beech forests of the alliance Aremonio-Fagion in Slovenia. Folia biologica et geologica 53 (1-2): 83-127. 


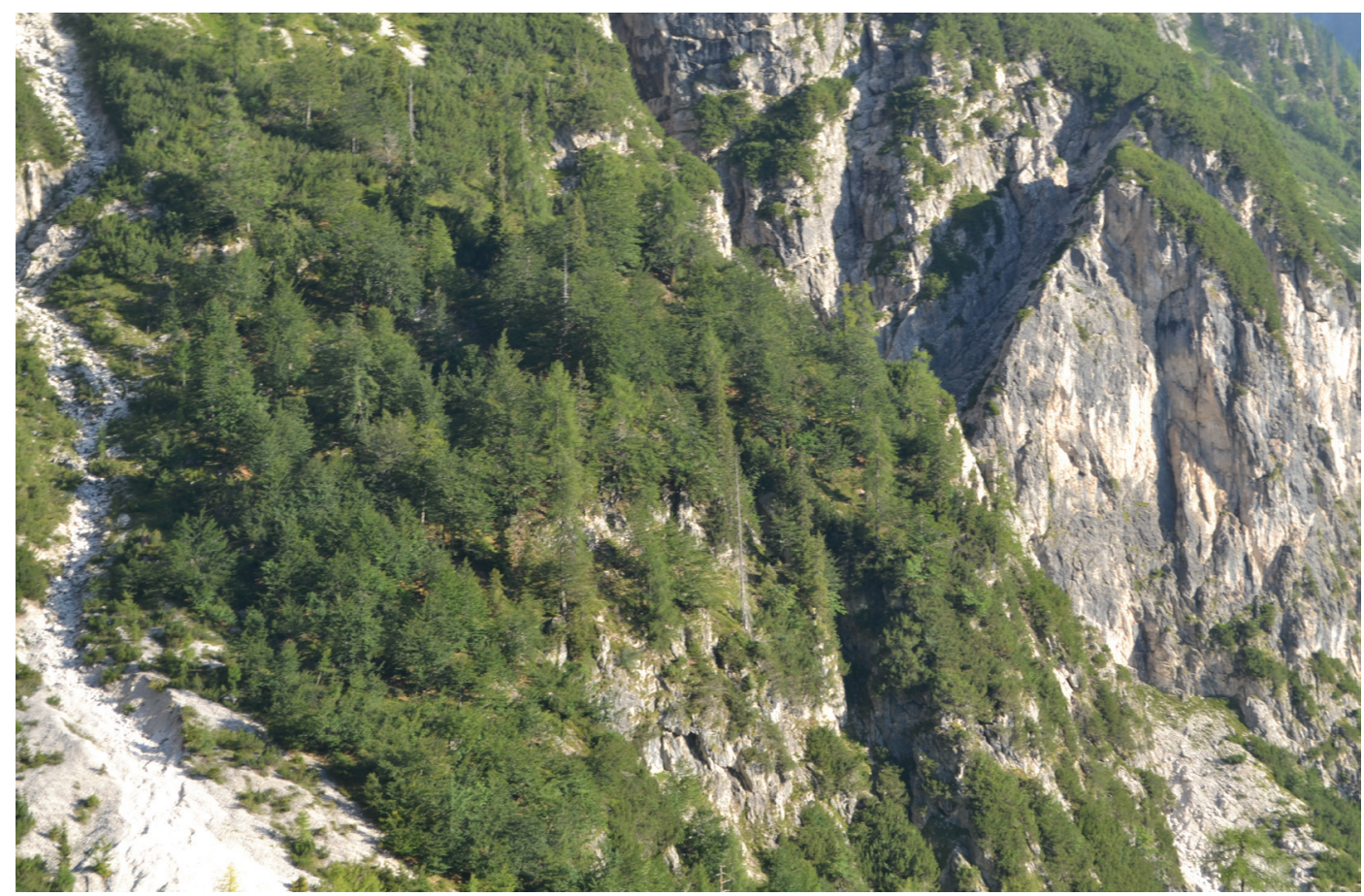

Figure 12: Stand of the subassociation Polysticho lonchitis-Fagetum ericetosum carneae, above the Mrzla Voda Valley / Velle di Rio Freddo, under Prednje Lastovice / Cime delli Rondini in the Italian part of the Julian Alps. Photo: Igor Dakskobler.

Slika 12: Sestoj subasociacije Polysticho lonchitis-Fagetum ericetosum carneae, above the Mrzla Voda Valley / Velle di Rio Freddo, pod Prednjimi Lastovicami / Cime delli Rondini v italijanskem delu Julijskih Alp. Foto: Igor Dakskobler.

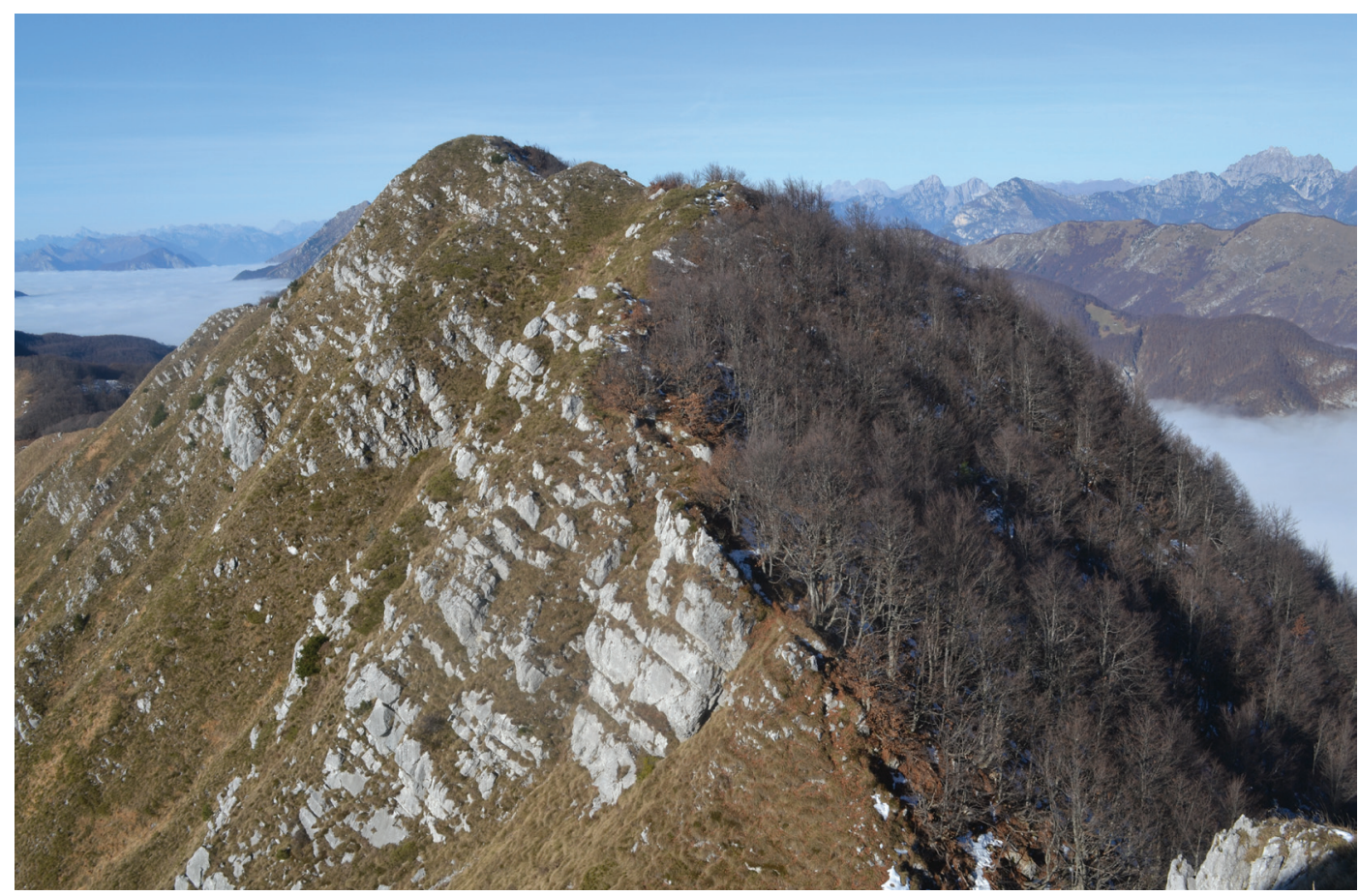

Figure 13: Nothern slopes of the peak Gabrovc (Veliki Muzec) in the Stol Mts. above the Učja valley, stands of the subassociation Polysticho lonchitis-Fagetum cardaminetosum trifoliae. Photo: Igor Dakskobler.

Slika 13: Severna pobočja vzpetine Gabrovc Veliki Muzec) v Stolovem pogorju nad dolino Učje, sestoj subasociacije Polysticho lonchitis-Fagetum cardaminetosum trifoliae. Foto: Igor Dakskobler. 


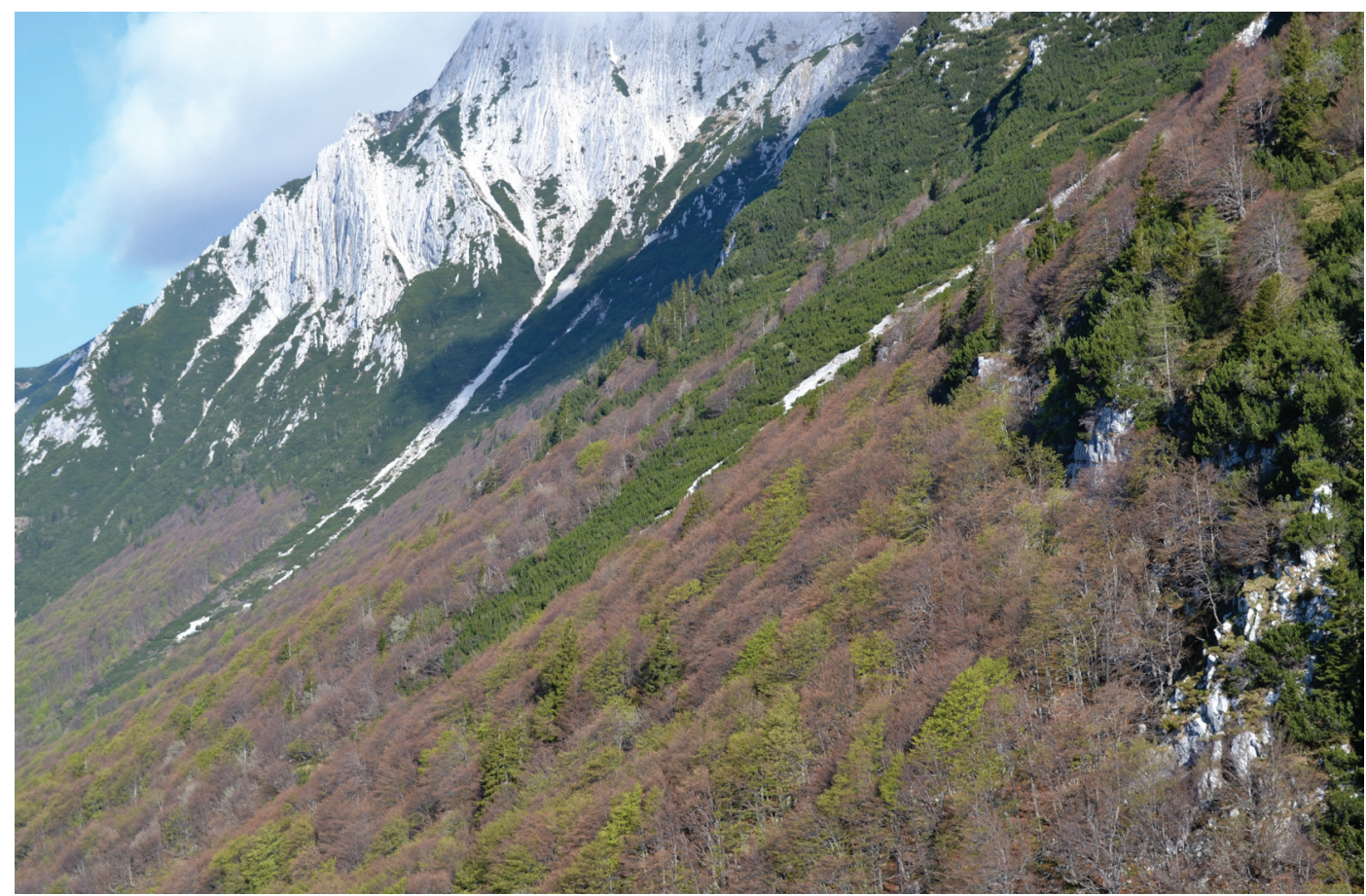

Figure 14: Subalpine beech forests (Polysticho lonchitis-Fagetum calamagrostietosum variae) on the climatic upper forest line in Rutarski Gozd under Hohkovbl / Matajurski Vrh in the Tolmin-Bohinj Mts. Photo: Igor Dakskobler.

Slika 14: Subalpinski bukovi sestoji (Polysticho lonchitis-Fagetum calamagrostietosum variae) na klimatski zgornji meji v Rutarskem gozdu pod Hohkovblom (Matajurskim vrhom) v Tolminsko-Bohinjskem pogorju. Foto: Igor Dakskobler.

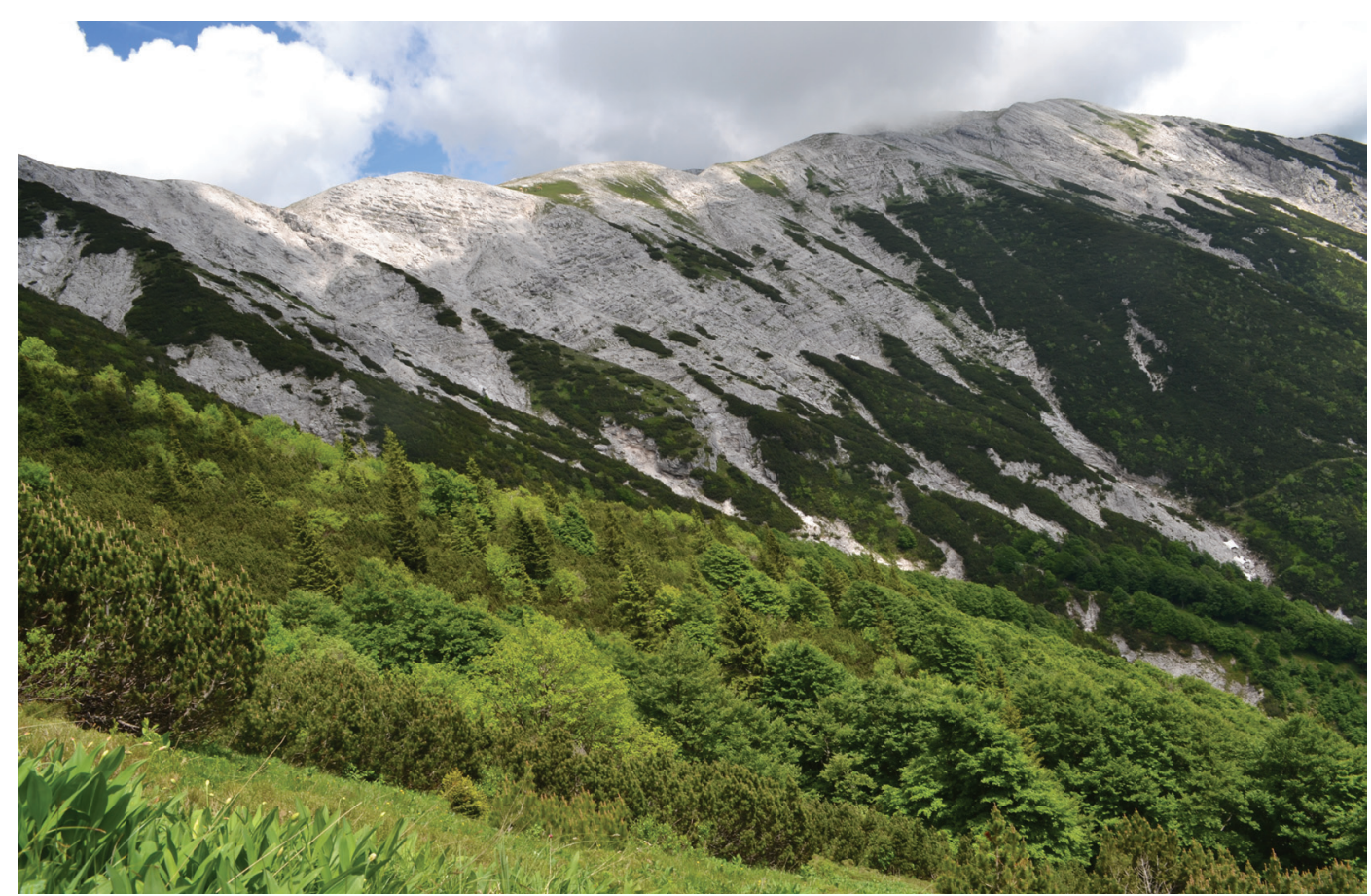

Figure 15: Subalpine beech stands (Polysticho lonchitis-Fagetum luzuletosum luzuloidis) at Ovčja Suha above the Kneža valley in the Tolmin-Bohinj Mts. Photo: Igor Dakskobler.

Slika 15: Subalpinski bukovi sestoji (Polysticho lonchitis-Fagetum luzuletosum luzuloidis), pri Ovčji Suhi nad dolino Kneže v Tolminsko-Bohinjskem pogorju. Foto: Igor Dakskobler. 


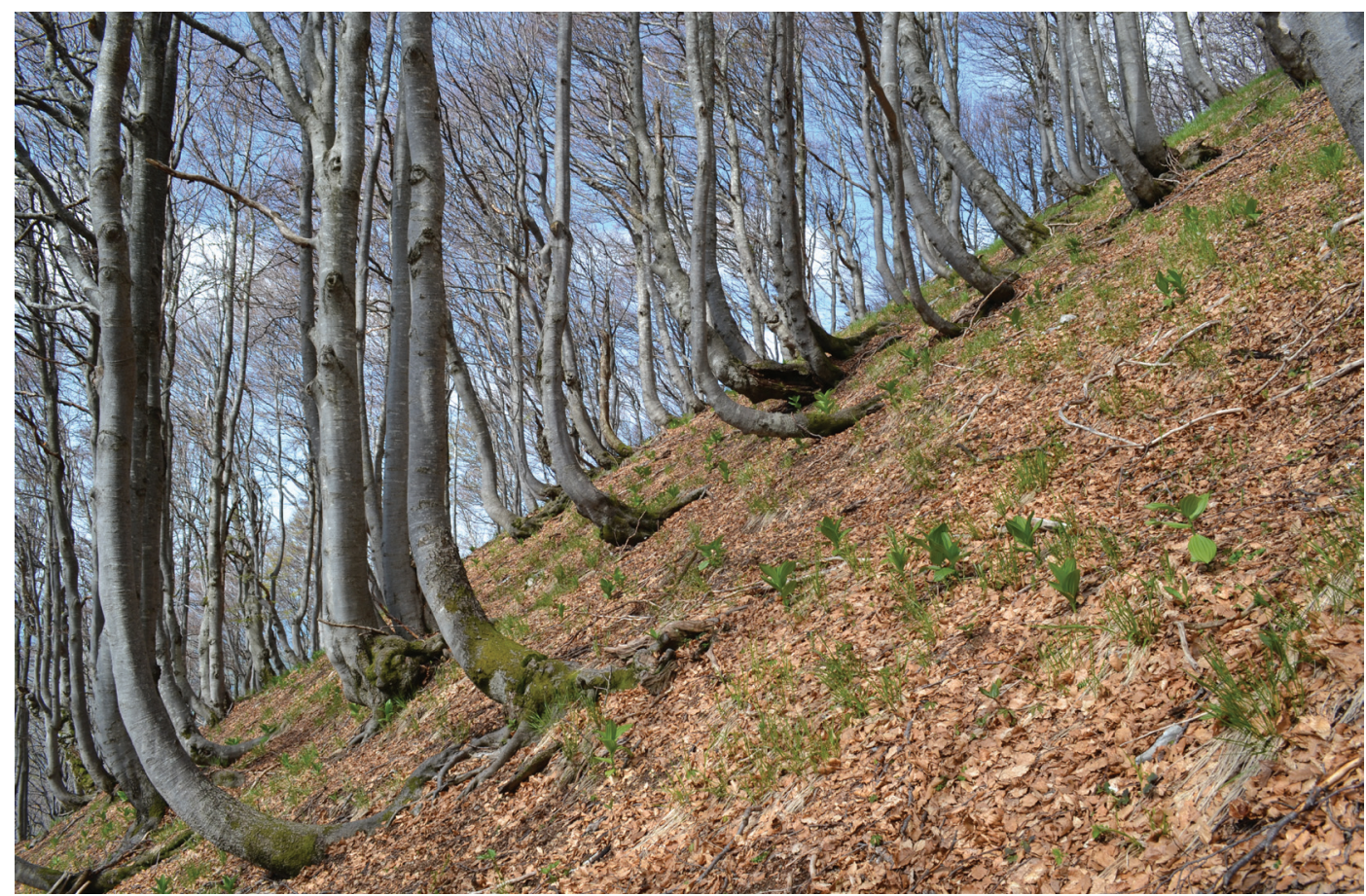

Figure 16: Stand of the subassociation Polysticho lonchitis-Fagetum luzuletosum luzuloidis, Pajlen above Stržišče in the upper Bača Valley (Tolmin-Bohinj Mts.). Photo: Igor Dakskobler.

Slika 16: Sestoj subasociacije Polysticho lonchitis-Fagetum luzuletosum luzuloidis, Pajlen nad Stržiščami v zgornji Baški dolini

(Tolminsko-Bohinjko pogorje). Foto: Igor Dakskobler.

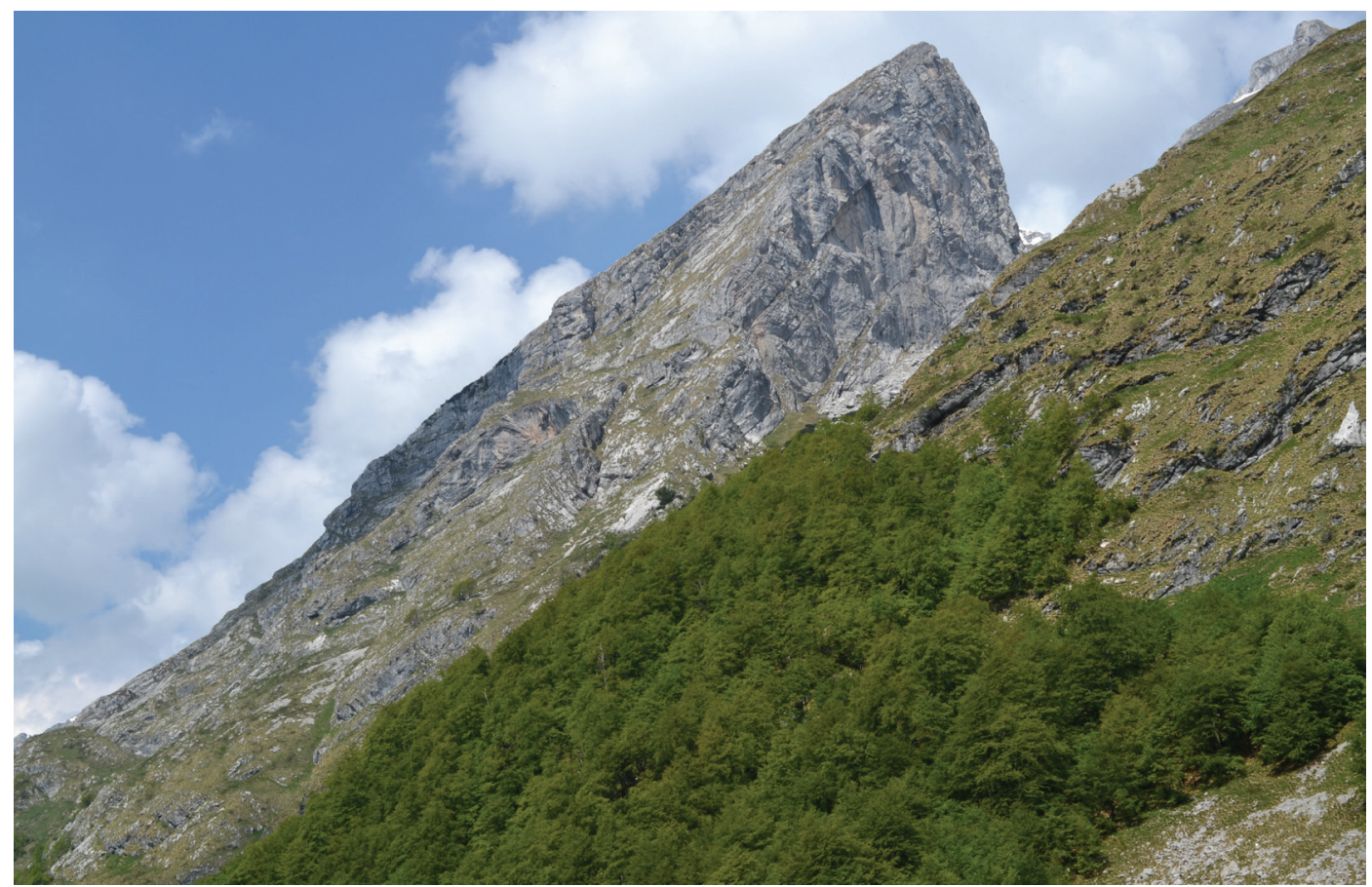

Figure 17: Subalpine beech forests in the Bala valley under Zorcev Rob and Stador, stands of the subassociation Polysticho lonchitis-Fagetum luzuletosum niveae. Photo: Igor Dakskobler.

Slika 17: Subalpinski bukovi gozdovi v dolini Bele, pod Zorcevim robom in Stadorjem, sestoji subasociacije Polysticho lonchitis-Fagetum luzuletosum niveae. Foto: Igor Dakskobler. 
Hacquetia $20 / 2 \cdot 2021,373-564$

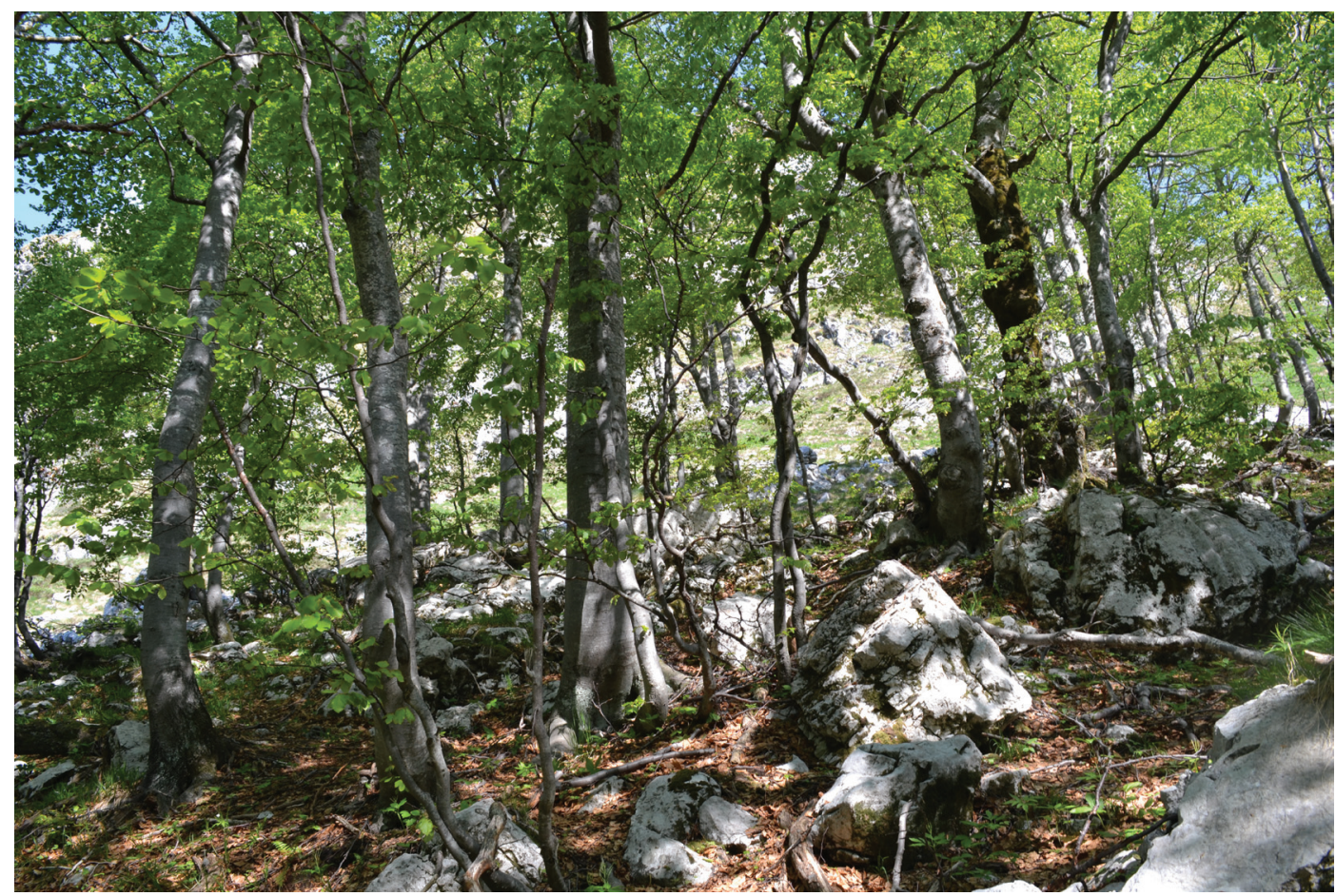

Figure 18: Stand of the subassociation Polysticho lonchitis-Fagetum luzuletosum niveae under Vršrič in the upper Bala valley. Photo: Igor Dakskobler. Slika 18: Sestoj subasociacije Polysticho lonchitis-Fagetum luzuletosum niveae pod Vršičem v zgornjem delu doline Bale. Foto: Igor Dakskobler.

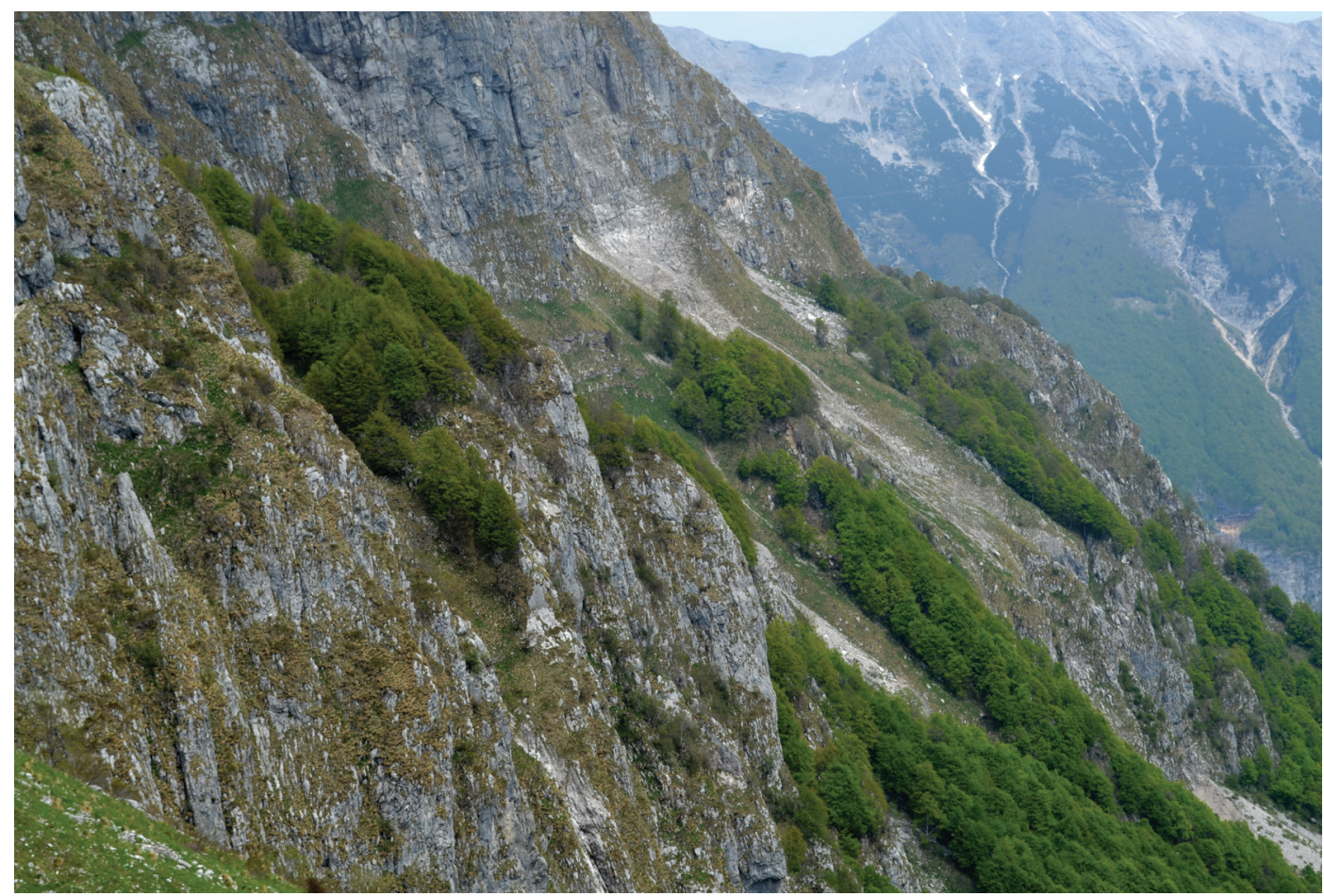

Figure 19: The orographic upper forest line in Slemenske Peči under Rdeči Rob above the Tolminka valley, stands of the subassociation Polysticho lonchitis-Fagetum adoxetosum moschatellinae. Photo: Igor Dakskobler.

Slika 19: Orografska zgornja meja v Slemenskih pečeh pod Rdečim robom, sestoji subasociacije Polysticho lonchitis-Fagetum adoxetosum moschatellinae. Foto: Igor Dakskobler. 


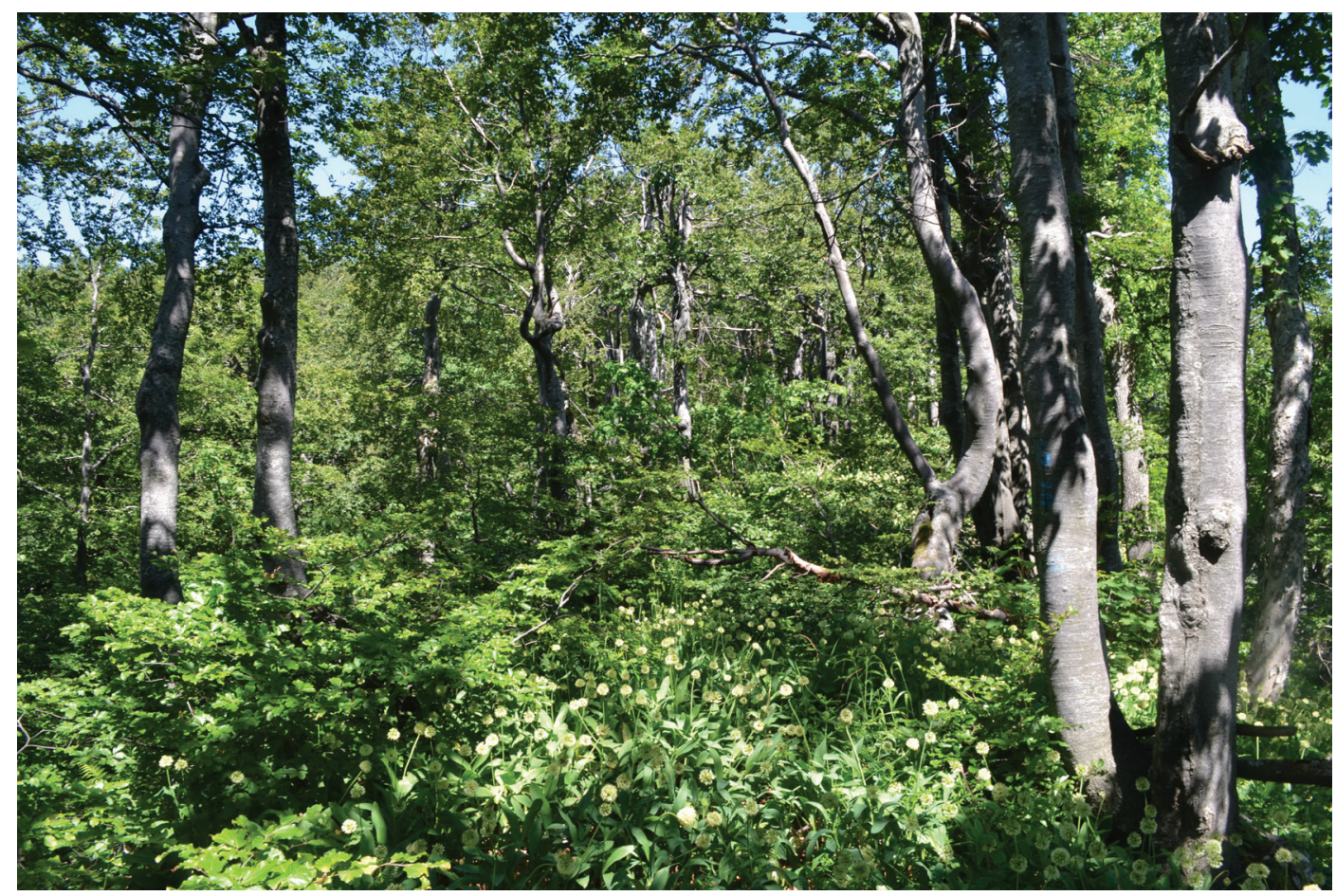

Figure 20: Stand of the subassociation Polysticho lonchitis-Fagetum allietosum victorialis. Golaki in the Trnovo Forest Plateau. Photo: Igor Dakskobler. Slika 20: Sestoj subasociacije Polysticho lonchitis-Fagetum allietosum victorialis. Golaki v Trnovskem gozdu. Foto: Igor Dakskobler.

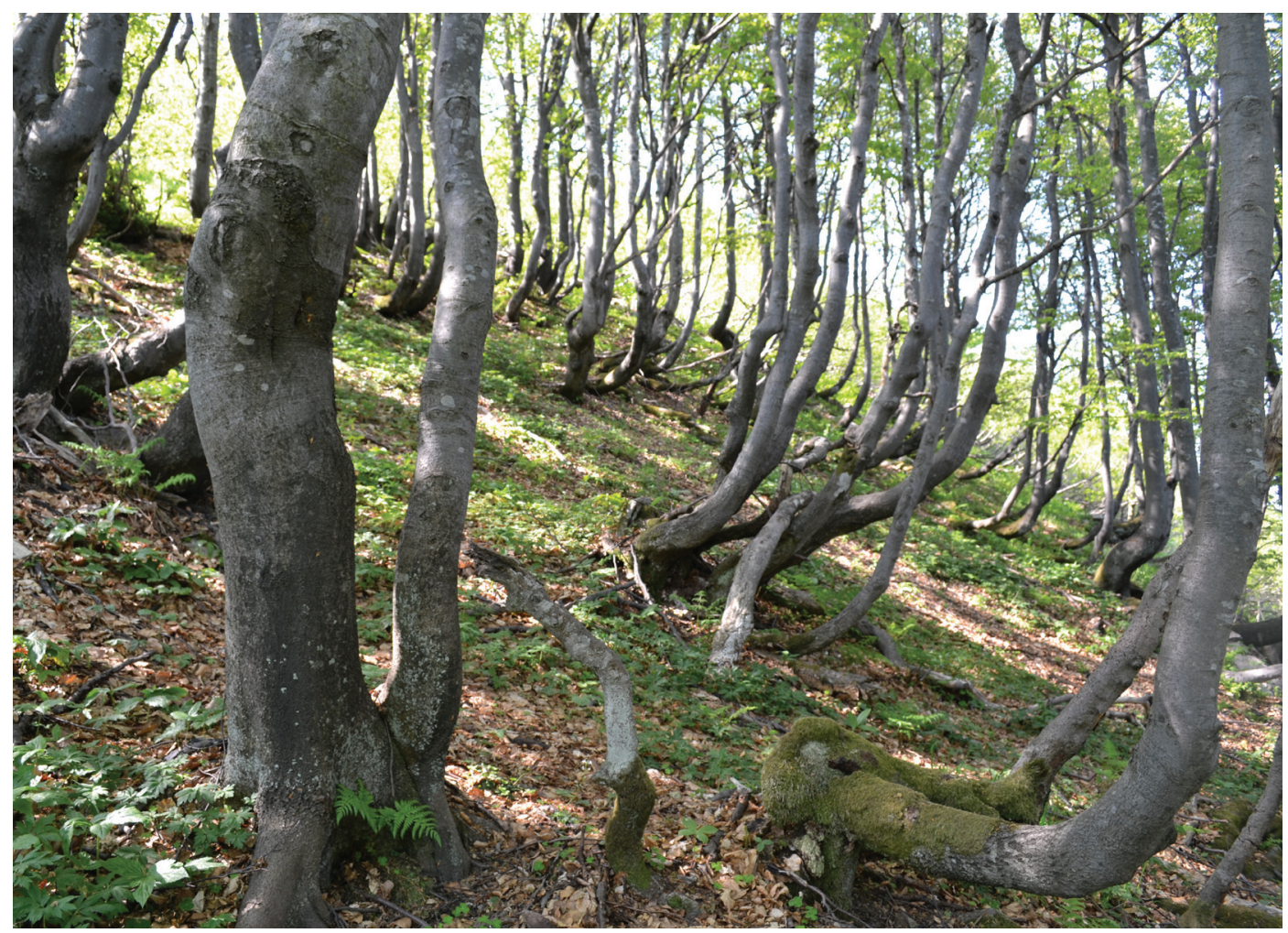

Figure 21: Stand of the subassociation Polysticho lonchitis-Fagetum stellarietosum nemorum, Mt. Porezen. Photo: Igor Dakskobler. Slika 21: Sestoj subasociacije Polysticho lonchitis-Fagetum stellarietosum nemorum, Porezen. Foto: Igor Dakskobler. 
Table 1: Polysticho lonchitis-Fagetum ericetosum carneae var. Rhododendron hirsutum subvar. Calamagrostis varia - cluster 12.

Relevé number 1-29.

\begin{tabular}{|c|c|c|c|c|c|c|c|c|c|c|c|}
\hline Number of relevé (Zaporedna štev. popisa) & & 1 & 2 & 3 & 4 & 5 & 6 & 7 & 8 & 9 & 10 \\
\hline $\begin{array}{l}\text { Database number of relevé } \\
\text { (Delovna številka popisa) }\end{array}$ & & 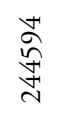 & $\frac{\underset{\sim}{\Xi}}{\underset{\sim}{\Xi}}$ & 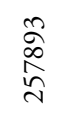 & 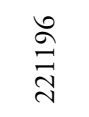 & 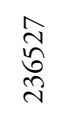 & 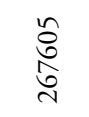 & $\begin{array}{l}\text { in } \\
\hat{\sigma}\end{array}$ & 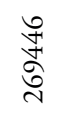 & 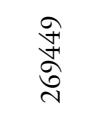 & 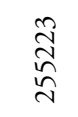 \\
\hline Author of the relevé (Avtor popisa) & & $\theta$ & $\Leftrightarrow$ & $\Theta$ & $\ominus$ & $\ominus$ & 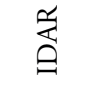 & $\theta$ & $\ominus$ & 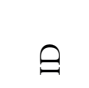 & $\ominus$ \\
\hline Elevation in $\mathrm{m}$ (Nadmorska višina $\mathrm{v} \mathrm{m})$ & & 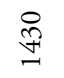 & 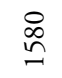 & $\stackrel{\&}{\stackrel{\Xi}{ \pm}}$ & $\tilde{n}$ & $\stackrel{\infty}{\stackrel{\infty}{n}}$ & $\stackrel{n}{\sim}$ & $\stackrel{8}{\circ}$ & 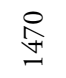 & $\stackrel{8}{\circ}$ & 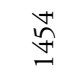 \\
\hline Aspect (Lega) & & SE & SW & $\mathrm{NE}$ & SWW & SW & NNW & NNW & $\mathrm{NE}$ & NNW & NNE \\
\hline Slope in degrees (Nagib v stopinjah) & & 15 & 25 & 35 & 25 & 10 & 35 & 15 & 35 & 25 & 25 \\
\hline Parent material (Matična podlaga) & & A & $\mathrm{DA}$ & $\mathrm{D}$ & Mo & $\mathrm{DA}$ & DA & Gr & $\mathrm{DA}$ & $\mathrm{D}$ & $\mathrm{D}$ \\
\hline Soil (Tla) & & $\operatorname{Re}$ & $\operatorname{Re}$ & $\operatorname{Re}$ & $\operatorname{Re}$ & $\operatorname{Re}$ & $\operatorname{Re}$ & $\operatorname{Re}$ & $\operatorname{Re}$ & $\operatorname{Re}$ & $\operatorname{Re}$ \\
\hline Stoniness in \% (Kamnitost v \%) & & 40 & 5 & 5 & 20 & 10 & 30 & 20 & 15 & 10 & 0 \\
\hline Cover in \% (Zastiranje v \%): & & & & & & & & & & & \\
\hline Upper tree layer (Zgor. drevesna plast) & $\mathrm{E} 3 \mathrm{~b}$ & 70 & 60 & 80 & 80 & 70 & 80 & 50 & 60 & 60 & 40 \\
\hline Lower tree layer (Spod. drevesna plasti) & E3a & . & 10 & 10 & 10 & 20 & 10 & 20 & 20 & 20 & 10 \\
\hline Shrub layer (Grmovna plast) & E2 & 50 & 20 & 5 & 20 & 40 & 20 & 30 & 30 & 40 & 80 \\
\hline Herb layer (Zeliščna plast) & E1 & 60 & 80 & 75 & 70 & 80 & 80 & 80 & 90 & 80 & 95 \\
\hline Moss layer (Mahovna plast) & E0 & 10 & 10 & 5 & 10 & 5 & 10 & 10 & 5 & 10 & 5 \\
\hline $\begin{array}{l}\text { Maximum tree diameter } \\
\text { (Maksimalni premer dreves) }\end{array}$ & $\mathrm{cm}$ & 25 & 50 & 40 & 50 & 80 & 45 & 60 & 70 & 100 & 40 \\
\hline $\begin{array}{l}\text { Maximum tree height } \\
\text { (Maksimalna višina dreves) }\end{array}$ & $\mathrm{m}$ & 9 & 16 & 17 & 20 & 24 & 20 & 28 & 25 & $\begin{array}{l}35 \\
(20)\end{array}$ & 15 \\
\hline Number of species (Število vrst) & & 34 & 44 & 48 & 63 & 50 & 51 & 63 & 70 & 59 & 39 \\
\hline Relevé area (Velikost popisne ploskve) & $\mathrm{m}^{2}$ & 200 & 400 & 400 & 400 & 400 & 400 & 400 & 400 & 400 & 500 \\
\hline Date of taking relevé (Datum popisa) & & 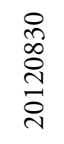 & 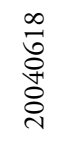 & 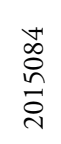 & $\begin{array}{l}0 \\
\infty \\
\infty \\
\infty \\
\stackrel{\lambda}{0}\end{array}$ & 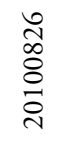 & 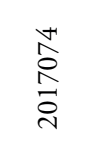 & 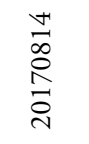 & 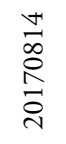 & $\begin{array}{l}\stackrel{+}{1} \\
\infty \\
\stackrel{2}{\curvearrowright} \\
\stackrel{\lambda}{0}\end{array}$ & 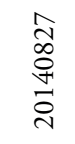 \\
\hline Day (Dan) & & 30 & 18 & 4 & 6 & 26 & 4 & 14 & 14 & 14 & 27 \\
\hline Month (Mesec) & & 8 & 6 & 8 & 8 & 8 & 7 & 8 & 8 & 8 & 0 \\
\hline Year (Leto) & & $\stackrel{\text { ก }}{\stackrel{2}{1}}$ & $\stackrel{\text { : }}{\stackrel{2}{2}}$ & $\underset{\sim}{\stackrel{n}{n}}$ & 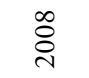 & $\stackrel{\circ}{\stackrel{2}{2}}$ & 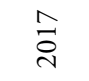 & $\vec{\sim}$ & 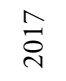 & $\stackrel{ }{\tilde{i}}$ & $\stackrel{\Downarrow}{\stackrel{\sim}{N}}$ \\
\hline
\end{tabular}

Locality (Nahajališče)

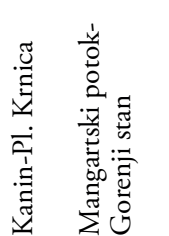

苞

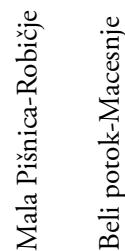

JA JA JA JA JA JA

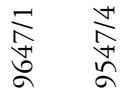

Quadrant (Kvadrant)

Coordinate (Koordinate) GK Y (D-48)

Coordinate (Koordinate) GK X (D-48) $\mathrm{m} \quad \stackrel{r}{\sim}$

$\mathrm{m} \quad \infty$




\begin{tabular}{|c|c|c|c|c|c|c|c|c|c|c|c|c|c|c|c|c|c|c|}
\hline 1 & 12 & 13 & 14 & 15 & 16 & 17 & 18 & 19 & 20 & 21 & 22 & 23 & 24 & 25 & 26 & 27 & 28 & 29 \\
\hline $\begin{array}{l}\stackrel{+}{\mathbb{Z}} \\
\underset{\mathrm{S}}{\mathrm{d}}\end{array}$ & $\begin{array}{l}\underset{N}{J} \\
\underset{\nabla}{v}\end{array}$ & $\begin{array}{l}\stackrel{n}{\tilde{\&}} \\
\underset{\sim}{\sim}\end{array}$ & $\begin{array}{l}\stackrel{n}{\sqrt{n}} \\
\hat{\sim}\end{array}$ & 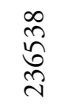 & $\begin{array}{l}0 \\
\underset{\sim}{\tilde{N}} \\
\tilde{\sim}\end{array}$ & $\infty$ & $\overrightarrow{\mathrm{\sigma}}$ & $\overrightarrow{\mathrm{\sigma}}$ & $\overline{\mathrm{g}}$ & 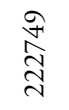 & 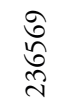 & స్రి & 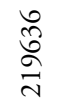 & $\begin{array}{l}\hat{\infty} \\
\sim \\
\infty \\
\\
\sim\end{array}$ & $\begin{array}{l}\infty \\
\infty \\
\sim\end{array}$ & $\stackrel{\infty}{\stackrel{+}{\sim}}$ & $\begin{array}{l}\text { त్ర } \\
\text { तె }\end{array}$ & 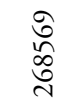 \\
\hline$\theta$ & $\theta$ & $\theta$ & $\ominus$ & $\theta$ & $\Theta$ & $\ominus$ & 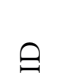 & $\ominus$ & $\ominus$ & $\theta$ & 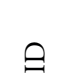 & $\theta$ & $\theta$ & 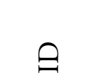 & $\theta$ & $\begin{array}{l}\text { N } \\
\text { ق }\end{array}$ & 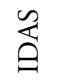 & $\exists$ \\
\hline 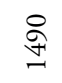 & $\stackrel{ }{\sigma}$ & $\stackrel{8}{\circ}$ & $\underset{\overbrace{}}{\stackrel{\overbrace{}}{\Psi}}$ & $\begin{array}{l}\stackrel{\infty}{\infty} \\
\stackrel{+}{+}\end{array}$ & $\underset{\mathbb{J}}{\mathbb{J}}$ & 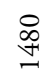 & 离 & $\hat{\sigma}$ & $\underset{\approx}{\approx}$ & 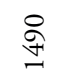 & $\stackrel{ }{\stackrel{\sim}{n}}$ & $\simeq$ & $\begin{array}{l}\stackrel{\infty}{\infty} \\
\stackrel{\infty}{+}\end{array}$ & $\underset{\underbrace{}}{\stackrel{n}{\forall}}$ & 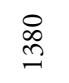 & $\stackrel{R}{n}$ & $\stackrel{\infty}{\stackrel{\infty}{+}}$ & 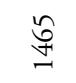 \\
\hline VI & $S$ & SE & SSE & SE & SE & SE & 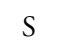 & $\mathrm{SE}$ & SEE & NW & SSW & V & W & NWW & SE & & $\mathrm{N}$ & sWw \\
\hline 30 & 30 & 30 & 35 & 25 & 25 & 30 & 25 & 35 & 30 & 35 & 35 & 3 & 5 & & 4 & J & 40 & 30 \\
\hline & A & DA & DA & DA & DA & D & DA & DA & DA & A & $\mathrm{DL}$ & $\mathrm{DA}$ & $\mathrm{DA}$ & & DA & $D A$ & & D \\
\hline $\mathrm{Re}$ & $\mathrm{Re}$ & $\operatorname{Re}$ & $\mathrm{Re}$ & $\mathrm{Re}$ & $\mathrm{Re}$ & $\operatorname{Re}$ & $\operatorname{Re}$ & $\operatorname{Re}$ & $\mathrm{Re}$ & $\operatorname{Re}$ & $\operatorname{Re}$ & $\mathrm{Re}$ & $\mathrm{Re}$ & $\mathrm{Re}$ & $\operatorname{Re}$ & $\mathrm{Re}$ & Re & $\operatorname{Re}$ \\
\hline 5 & 10 & 30 & 0 & 0 & 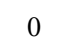 & 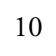 & & 20 & 1 & 20 & & 10 & 20 & & & & 0 & 10 \\
\hline 70 & 60 & 80 & 80 & 80 & 8 & 7 & & & & 6 & & 80 & 75 & & & & 70 & 90 \\
\hline 20 & 1 & 5 & 3 & 10 & 5 & 10 & & 10 & . & 10 & 10 & & . & & & 10 & & \\
\hline 30 & 10 & 5 & 10 & 10 & 10 & 2 & 30 & 20 & 3 & 40 & 15 & 10 & 60 & 40 & 10 & 60 & 30 & 10 \\
\hline 90 & 60 & 60 & 80 & 80 & 100 & 0 & 80 & 75 & 60 & 60 & 70 & 70 & 70 & 80 & 80 & 30 & 50 & 70 \\
\hline 5 & 10 & 10 & 5 & 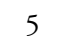 & 5 & 10 & 10 & 10 & 5 & 10 & & 5 & 10 & & U & & & 10 \\
\hline 60 & 25 & 50 & 40 & 50 & 40 & 50 & 60 & 60 & $\begin{array}{c}50 \\
(35)\end{array}$ & 40 & 45 & 50 & 30 & 30 & 50 & 40 & 2 & 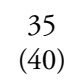 \\
\hline 18 & 15 & 18 & $\begin{array}{c}22 \\
(18)\end{array}$ & 20 & 17 & 17 & 16 & $\begin{array}{c}20 \\
(17)\end{array}$ & $\begin{array}{c}22 \\
(16)\end{array}$ & 10 & 18 & 18 & 12 & 10 & 15 & 12 & 12 & $\begin{array}{l}17 \\
15)\end{array}$ \\
\hline 44 & 48 & 52 & 40 & 49 & 39 & 54 & 66 & 55 & 47 & $0 t$ & 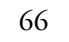 & 52 & 46 & & 4 & 34 & 66 & 56 \\
\hline 00 & 200 & 400 & 500 & 400 & 500 & 400 & 400 & 400 & 400 & 200 & 400 & 400 & 400 & 400 & 400 & 200 & 400 & 400 \\
\hline 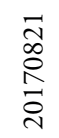 & $\begin{array}{l}\infty \\
\stackrel{\infty}{0} \\
\stackrel{+}{0} \\
\stackrel{\sim}{0}\end{array}$ & 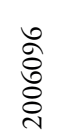 & 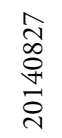 & $\begin{array}{l}0 \\
0 \\
\stackrel{0}{0} \\
\stackrel{\sim}{\circ}\end{array}$ & \begin{tabular}{l}
$\hat{A}$ \\
o \\
0 \\
\multirow{+}{*}{} \\
$\stackrel{i}{*}$
\end{tabular} & 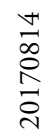 & 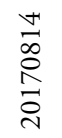 & 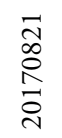 & $\begin{array}{l}\vec{N} \\
\stackrel{0}{0} \\
\stackrel{\sim}{\sigma}\end{array}$ & $\begin{array}{l}\hat{o} \\
\text { } \\
\text { ¿े }\end{array}$ & 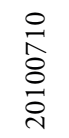 & $\begin{array}{l}\stackrel{ }{A} \\
\stackrel{\circ}{\circ} \\
\stackrel{ }{\circ}\end{array}$ & 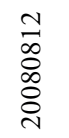 & 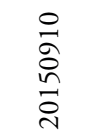 & $\begin{array}{l}0 \\
0 \\
\infty \\
O \\
\stackrel{+}{0} \\
\stackrel{\sim}{1}\end{array}$ & 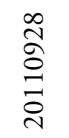 & $\begin{array}{l}\stackrel{N}{\approx} \\
\stackrel{ }{0} \\
\stackrel{ }{0}\end{array}$ & $\stackrel{\infty}{\sim}$ \\
\hline 21 & 18 & 6 & 27 & 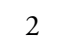 & 27 & 14 & 14 & 21 & 21 & 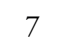 & 10 & 10 & 12 & 10 & 16 & 28 & 12 & 18 \\
\hline 8 & 6 & 9 & 8 & 8 & 8 & 8 & $\sigma$ & 8 & 8 & 9 & 7 & & & & 8 & 7 & 7 & 8 \\
\hline$\triangleq$ & 䓂 & ঃ্ণ & $\underset{\sim}{\stackrel{\sim}{\sim}}$ & $\stackrel{\circ}{\stackrel{\sim}{\sim}}$ & 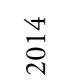 & $\hat{\text { సे }}$ & $\widehat{\sim}$ & $\widehat{\stackrel{\sim}{\circ}}$ & $\hat{\stackrel{i}{\sim}}$ & ஓ্ণ & $\stackrel{\circ}{\stackrel{\sim}{\sim}}$ & $\stackrel{\circ}{\stackrel{\circ}{\sim}}$ & 总 & $\stackrel{\sim}{\stackrel{\sim}{\sim}}$ & 苍 & $\overrightarrow{\vec{N}}$ & $\stackrel{\circ}{\stackrel{\sim}{\sim}}$ & \\
\hline
\end{tabular}

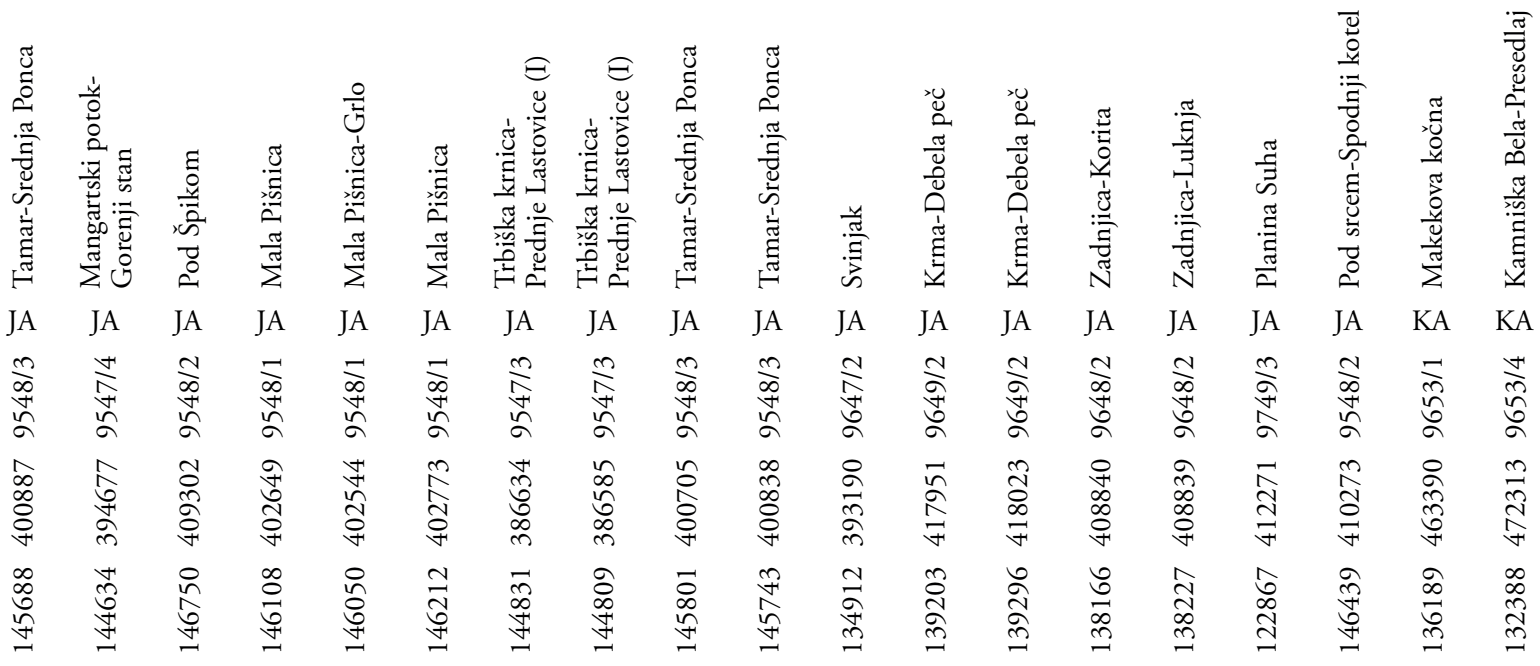




\begin{tabular}{|c|c|c|c|c|c|c|c|c|c|c|c|c|}
\hline & Number of relevé (Zaporedna štev. popisa) & & 1 & 2 & 3 & 4 & 5 & 6 & 7 & 8 & 9 & 10 \\
\hline & \multicolumn{12}{|c|}{ Diagnostic species of the association (Diagnostične vrste asociacije) } \\
\hline EP & Rhododendron hirsutum & E2 & 3 & $\mathrm{r}$ & + & . & 1 & 2 & 1 & 4 & 3 & 3 \\
\hline EP & Pinus mugo & $\mathrm{E} 2$ & + & . & + & + & 2 & + & . & 1 & + & 3 \\
\hline VP & Polystichum lonchitis & E1 & 1 & . & . & 1 & + & . & 1 & 1 & 1 & . \\
\hline $\mathrm{BA}$ & Sorbus chamaemespilus & E2 & . & 1 & $\mathrm{r}$ & + & + & . & 1 & + & 1 & 2 \\
\hline VP & Clematis alpina & E2 & $\mathrm{r}$ & + & . & . & + & 1 & + & + & + & 1 \\
\hline VP & Luzula sylvatica & E1 & 2 & 1 & 2 & . & 2 & 1 & . & 1 & 2 & 1 \\
\hline $\mathrm{CA}$ & Laserpitium peucedanoides & E1 & . & . & . & . & + & . & . & + & . & + \\
\hline ML & Paraleucobryum sauteri & E0 & . & . & . & + & + & . & . & + & . & . \\
\hline EP & Rhodothamnus chamaecistus & E1 & + & . & + & . & . & + & + & + & + & . \\
\hline VP & Homogyne alpina & E1 & + & . & 1 & . & . & . & + & + & + & + \\
\hline ES & Aster bellidiastrum & E1 & . & . & . & . & + & + & . & + & + & . \\
\hline $\mathrm{CF}$ & Carex ferruginea & E1 & . & + & . & . & + & . & . & + & + & . \\
\hline $\mathrm{MuA}$ & Aconitum lycoctonum subsp. ranunculifolium & E1 & . & . & . & . & . & . & + & . & . & . \\
\hline $\mathrm{BA}$ & Salix appendiculata & E2 & . & . & . & . & . & + & . & + & . & . \\
\hline VP & Lonicera caerulea & E2 & . & . & . & . & . & . & + & . & . & . \\
\hline PS & Paederota lutea & E1 & . & . & . & + & . & + & + & . & . & . \\
\hline \multirow[t]{2}{*}{$\mathrm{CA}$} & Festuca calva & E1 & + & . & . & . & . & . & . & . & . & . \\
\hline & \multicolumn{12}{|c|}{ Differential species of lower units (Razlikovalnice nižjih enot) } \\
\hline EP & Erica carnea & E1 & 3 & 2 & 2 & 2 & 2 & 3 & 2 & 2 & 2 & 4 \\
\hline EP & Calamagrostis varia & E1 & . & + & 1 & 2 & 2 & 1 & 3 & 2 & 2 & 2 \\
\hline VP & Vaccinium vitis-idaea & E1 & 1 & 2 & 1 & 1 & 1 & 1 & 1 & + & + & 2 \\
\hline VP & Larix decidua & E3 & . & . & + & + & 2 & 3 & 3 & 3 & 3 & 2 \\
\hline VP & Larix decidua & E2 & . & . & 1 & $\mathrm{r}$ & + & . & + & + & + & + \\
\hline VP & Larix decidua & E1 & . & . & . & . & . & . & . & . & . & . \\
\hline VP & Calamagrostis villosa & E1 & . & 3 & 2 & . & 2 & 2 & 2 & 2 & 2 & 1 \\
\hline \multirow[t]{10}{*}{$\mathrm{AF}$} & Aremonio-Fagion & & & & & & & & & & & \\
\hline & Anemone trifolia & E1 & . & 1 & 1 & . & 1 & 1 & 1 & 1 & 1 & 1 \\
\hline & Cyclamen purpurascens & E1 & . & . & + & 1 & 1 & . & . & . & . & 1 \\
\hline & Cardamine enneaphyllos & E1 & + & 1 & . & . & . & . & + & . & . & + \\
\hline & Knautia drymeia & E1 & . & . & . & . & 1 & + & 1 & + & + & . \\
\hline & Helleborus niger & E1 & . & . & 1 & 2 & . & . & . & . & . & 1 \\
\hline & Rhamnus fallax & E2 & . & . & . & . & . & . & . & . & . & . \\
\hline & Cardamine trifolia & E1 & . & . & . & + & . & . & . & . & . & . \\
\hline & Euphorbia carniolica & E1 & . & . & . & . & . & . & . & . & . & . \\
\hline & Anemone $x$ pittonii & E1 & . & . & . & . & . & . & . & . & . & . \\
\hline \multirow[t]{2}{*}{$\mathrm{EC}$} & Erythronio-Carpinion & & & & & & & & & & & \\
\hline & Primula vulgaris & E1 & . & . & . & . & . & . & . & . & . & . \\
\hline \multirow[t]{6}{*}{ TA } & Tilio-Acerion & & & & & & & & & & & \\
\hline & Acer pseudoplatanus & E3 & . & . & . & . & . & . & . & . & . & . \\
\hline & Acer pseudoplatanus & E2 & . & . & . & . & . & . & . & . & . & . \\
\hline & Acer pseudoplatanus & E1 & . & . & . & + & . & + & + & + & . & . \\
\hline & Polystichum aculeatum & E1 & . & . & . & + & . & . & . & . & . & . \\
\hline & Aruncus dioicus & E1 & . & . & . & . & . & . & . & . & . & . \\
\hline \multirow[t]{7}{*}{ FS } & Fagetalia sylvaticae & & & & & & & & & & & \\
\hline & Fagus sylvatica & E3 & 4 & 4 & 5 & 4 & 3 & 3 & 3 & 3 & 3 & 3 \\
\hline & Fagus sylvatica & E2 & + & 1 & + & 1 & + & 1 & 2 & 2 & 2 & 1 \\
\hline & Fagus sylvatica & E1 & + & 1 & 2 & . & 1 & 1 & . & 1 & 1 & 1 \\
\hline & Daphne mezereum & E2 & . & + & . & + & + & . & 1 & 1 & 1 & + \\
\hline & Melica nutans & E1 & + & + & + & . & 1 & . & + & 1 & + & 1 \\
\hline & Prenanthes purpurea & E1 & . & . & + & + & + & 1 & + & + & 1 & 1 \\
\hline
\end{tabular}




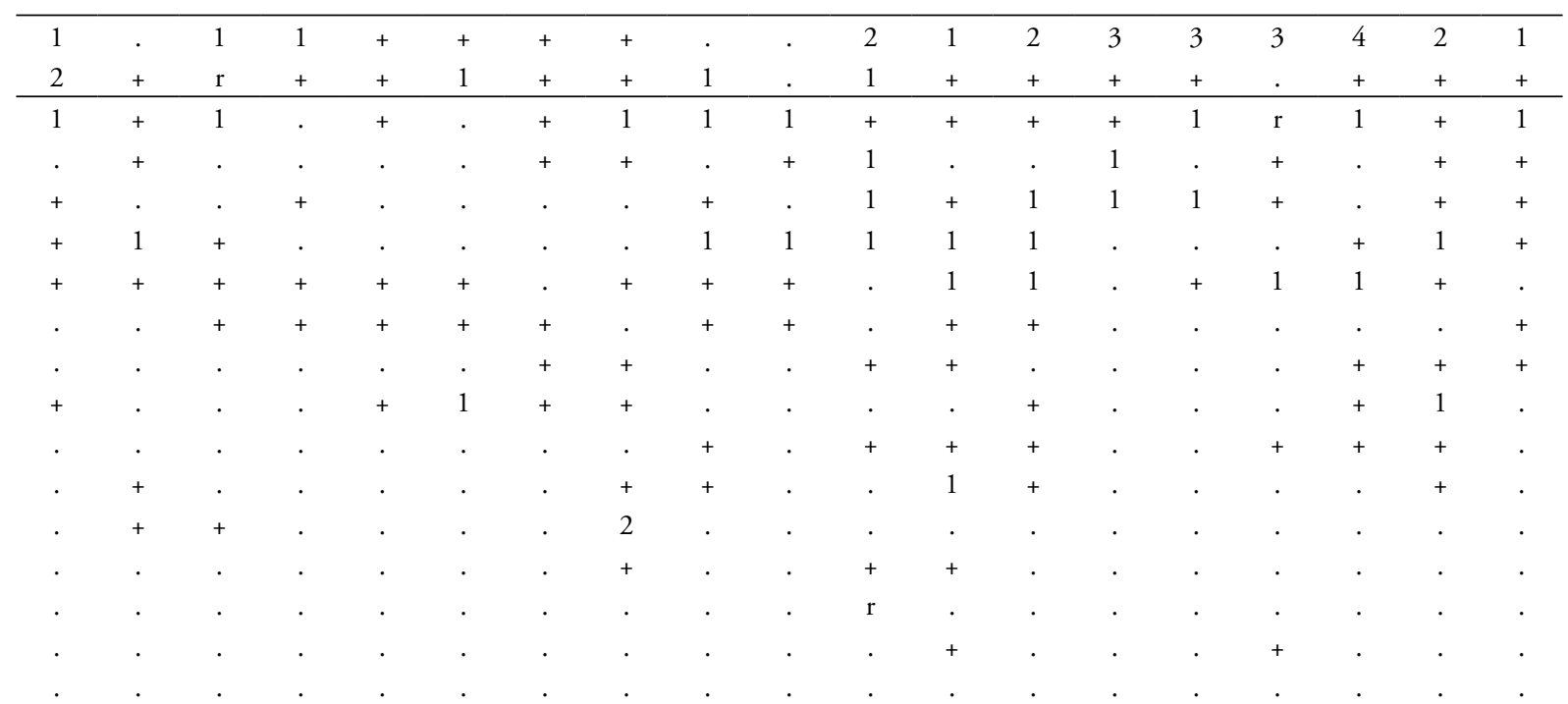

\begin{tabular}{|c|c|c|c|c|c|c|c|c|c|c|c|c|c|c|c|c|c|}
\hline 2 & 2 & 2 & 4 & 3 & 3 & 2 & 2 & 3 & 2 & 2 & 2 & 3 & 3 & 2 & 3 & 3 & 3 \\
\hline 2 & 1 & 1 & 4 & 3 & 3 & 3 & 3 & 3 & 3 & 1 & 1 & 1 & 1 & 3 & 3 & 3 & 3 \\
\hline 4 & 2 & 2 & 1 & 1 & 2 & + & 1 & 2 & 2 & $\mathrm{r}$ & + & 1 & . & + & . & 1 & 1 \\
\hline 3 & 1 & 1 & + & 3 & 2 & + & $\mathrm{r}$ & 1 & 1 & 2 & + & + & $\mathrm{r}$ & + & . & + & 1 \\
\hline . & . & . & . & . & . & + & + & + & . & 1 & . & . & . & . & . & 1 & . \\
\hline . & . & . & . & . & . & . & + & . & . & . & . & + & . & . & . & . & + \\
\hline 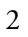 & + & . & + & 1 & 3 & 1 & + & + & + & . & + & + & . & + & . & . & + \\
\hline
\end{tabular}

\begin{tabular}{|c|c|c|c|c|c|c|c|c|c|c|c|c|c|c|c|c|c|}
\hline 3 & 3 & 4 & 4 & 3 & 4 & 4 & 4 & 4 & 5 & 3 & 4 & 4 & 4 & 4 & 5 & 4 & 3 \\
\hline 1 & 1 & + & 1 & 1 & + & 1 & 2 & 1 & . & + & + & + & + & 1 & . & + & + \\
\hline 1 & + & 1 & 1 & 1 & 1 & + & 1 & + & 1 & . & . & . & . & + & + & + & + \\
\hline+ & + & + & + & + & + & 1 & 1 & 1 & + & 1 & + & + & 1 & 1 & + & 1 & + \\
\hline . & + & + & + & + & + & + & . & + & 1 & + & + & + & . & 1 & + & . & + \\
\hline+ & . & + & + & + & + & 1 & . & . & + & 1 & + & 1 & 1 & 1 & + & . & 1 \\
\hline
\end{tabular}




\section{$4 \quad 5 \quad 6$}

$\begin{array}{llll}7 & 8 & 9 & 10\end{array}$

Lonicera alpigena

Mercurialis perennis

E2

Lilium martagon

Galium laevigatum

Paris quadrifolia

Laburnum alpinum

Laburnum alpinum

Laburnum alpinum

Luzula nivea

Galeobdolon flavidum

Euphorbia amygdaloides

Dryopteris filix-mas

Phyteuma spicatum

Neottia nidus-avis

Mycelis muralis

Epipactis helleborine

Symphytum tuberosum

Ranunculus lanuginosus

Lathyrus vernus

Festuca altissima

QP

Quercetalia pubescenti-petraeae

Sorbus aria (Aria edulis)

Sorbus aria (Aria edulis)

Convallaria majalis

Carex flacca

Melittis melissophyllum

Ostrya carpinifolia

QF Querco-Fagetea

Hepatica nobilis

Carex digitata

Viola riviniana

Anemone nemorosa

Poa nemoralis

Melampyrum pratense

Dactylorhiza fuchsii

Pteridium aquilinum

VP

\section{Vaccinio-Piceetea}

Vaccinium myrtillus

Rosa pendulina

Aposeris foetida

Gymnocarpium dryopteris

Picea abies

Picea abies

Picea abies

Maianthemum bifolium

Veronica urticifolia

Gentiana asclepiadea

Hieracium murorum

Lonicera nigra

Huperzia selago

Abies alba

E1

E2

E1

E1

E1

E1

E1

E1

E1

E1

E1

E1

E1

\begin{tabular}{|c|c|c|c|c|c|c|c|c|c|}
\hline E1 & 3 & 3 & 3 & 2 & 1 & 1 & 3 & 2 & 3 \\
\hline E2 & . & + & . & + & . & + & + & + & + \\
\hline E1 & . & 1 & + & + & 1 & . & + & 1 & . \\
\hline E1 & . & . & 1 & + & $\cdot$ & 1 & + & 1 & + \\
\hline E3 & + & + & + & + & + & . & + & + & + \\
\hline E2 & . & . & 1 & + & + & 1 & + & + & + \\
\hline E1 & . & + & . & . & . & 1 & . & + & . \\
\hline E1 & . & . & + & 1 & + & 1 & + & + & + \\
\hline E1 & . & . & + & + & $\cdot$ & 1 & $\cdot$ & 1 & . \\
\hline E1 & . & . & $\cdot$ & . & + & . & . & + & + \\
\hline E1 & . & . & . & . & + & . & + & . & + \\
\hline E2 & . & . & . & + & . & + & + & . & . \\
\hline E1 & . & . & 1 & . & . & + & . & . & . \\
\hline E3 & . & . & . & + & + & $\mathrm{r}$ & + & $\mathrm{r}$ & + \\
\hline
\end{tabular}




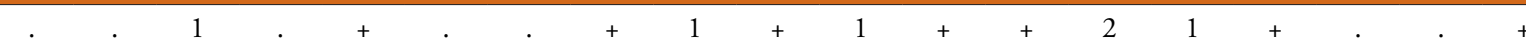


Abies alba

Abies alba

Lycopodium annotinum

Oxalis acetosella

Phegopteris connectilis

Solidago virgaurea

Melampyrum sylvaticum

Homogyne sylvestris

Saxifraga cuneifolia

Calamagrostis arundinacea

Orthilia secunda

Pyrola minor

Pyrola rotundifolia

Thelypteris limbosperma

Dryopteris dilatata

Luzula luzuloides

Ajuga pyramidalis

Dryopteris expansa

Luzula pilosa

Pyrola chlorantha

\section{Erico-Pinetea}

Rubus saxatilis

Carex alba

Polygala chamaebuxus

Aquilegia nigricans

Molinia arundinacea

Genista radiata

Cotoneaster tomentosus

Amelanchier ovalis

Carex ornithopoda

Peucedanum austriacum subsp. rablense

Chamaecytisus hirsutus

SSC Sambuco-Salicion capreae, Rhamno-Prunetea

Sorbus aucuparia

Sorbus aucuparia

E1

Sorbus aucuparia

E1

Sambucus racemosa

$\begin{array}{llllllllll}1 & 2 & 3 & 4 & 5 & 6 & 7 & 8 & 9 & 10\end{array}$

\section{Betulo-Alnetea}

Juniperus sibirica

Salix glabra

Sorbus austriaca

Ribes alpinum

Salix waldsteiniana

Alnus viridis

MuA Mulgedio-Aconitetea

Polygonatum verticillatum

E1

Geranium sylvaticum

Athyrium filix-femina

Veratrum album

Viola biflora

Phyteuma ovatum 


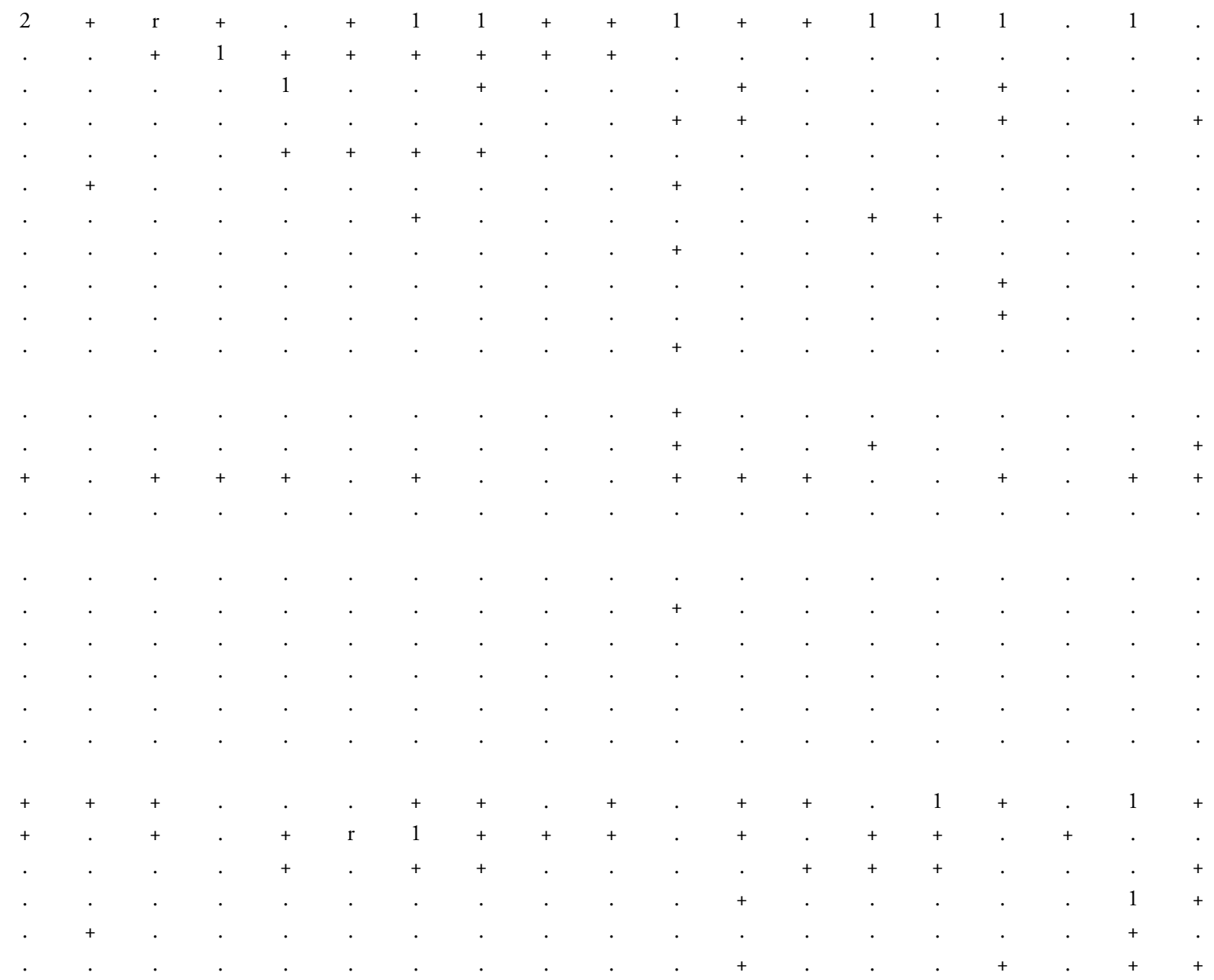




\section{E1}

Hypericum maculatum

Chaerophyllum villarsii

Primula elatior

Ranunculus platanifolius

Aconitum degenii subsp. paniculatum

Tanacetum corymbosum subsp. clusii

Cicerbita alpina

Geum rivale

Allium victorialis

Thalictrum aquilegiifolium

Adenostyles alliariae

CA Caricion austroalpinae

Pulsatilla alpina subsp. austroalpina

CF Caricion ferrugineae

Hypericum richeri subsp. grisebachii

Cfir Caricion firmae

Carex firma

Sesleria sphaerocephala

ES Elyno-Seslerietea

Betonica alopecuros

Sesleria caerulea

Senecio abrotanifolius

Campanula witasekiana

Carduus crassifolius

Phyteuma orbiculare

Carex sempervirens

Ranunculus montanus

Thymus praecox subsp. polytrichus

Lotus alpinus

Scabiosa lucida subsp. lucida

Bartsia alpina

Selaginella selaginoides

Daphne striata

Hieracium pilosum

Scabiosa lucida subsp. stricta

E1

E1

E1

E1

E1

E1

E1

E1

E1

E1

Ranunculus carinthiacus

E1

Carex mucronata

E1

Globularia cordifolia

E1

Helictotrichon parlatorei

E1

Astrantia bavarica

E1

Carduus defloratus

E1

Hieracium villosum

E1

Juncus monanthos

E1

E1

Campanula scheuchzeri

E1

Potentilla erecta

E1

Tofieldia calyculata

E1 
Number of relevé (Zaporedna štev. popisa)

\section{4}

$5 \quad 6$

FB Festuco-Brometea
Cirsium erisithales

Buphthalmum salicifolium

E1

Carex humilis

Carlina acaulis

Linum catharticum

TG Trifolio-Geranietea

Laserpitium latifolium

Verbascum lanatum

E1

E1

E1

E1

E1

E1

PaT Poo alpinae-Trisetetalia

Ranunculus nemorosus

Poa alpina

Crocus albiflorus

Trollius europaeus

MA Molinio-Arrbenatheretea

Crepis paludosa

Festuca rubra agg.

EA Epilobietea angustifolii, Galio-Urticetea

Fragaria vesca

Rubus idaeus

GU Urtica dioica

AC Arabidetalia caeruleae

Soldanella alpina

Soldanella minima

E1

E1

E1

E1

E1

E1

E1

E2

E1

E1

E1

TR Thlaspietea rotundifolii

Adenostyles glabra

Gymnocarpium robertianum

Astrantia carniolica

Heliosperma alpestre

Valeriana montana

Trisetum argenteum

Hieracium bifidum

Biscutella laevigata

Cystopteris montana

Aquilegia einseleana

E1

E1

E1

E1

E1

E1

E1

E1

E1

E1

Petasites paradoxus

E1

Molopospermum peloponnesiacum subsp. bauhinii

Heracleum pollinianum

E1

E1

Saxifraga caesia

E1

Cy Cystopteridion fragilis

Valeriana tripteris

E1

Asplenium viride

Carex brachystachys

Moehringia muscosa

Cystopteris fragilis

Cystopteris regia

Physoplexido comosae-Saxifragion petraeae

Campanula cespitosa

Paederota bonarota

Saxifraga crustata

Potentilla nitida

E1

E1

E1

E1

E1

E1

E1

E1

E1

1

1

1

(1)

1

$+$

$\begin{array}{rllllllllll} & + & + & \cdot & + & \cdot & \cdot & \cdot & \cdot & \cdot & \cdot \\ 2 & \cdot & \cdot & \cdot & \cdot & \cdot & + & \cdot & \cdot & \cdot & \cdot\end{array}$

1

$\cdot+\cdot$




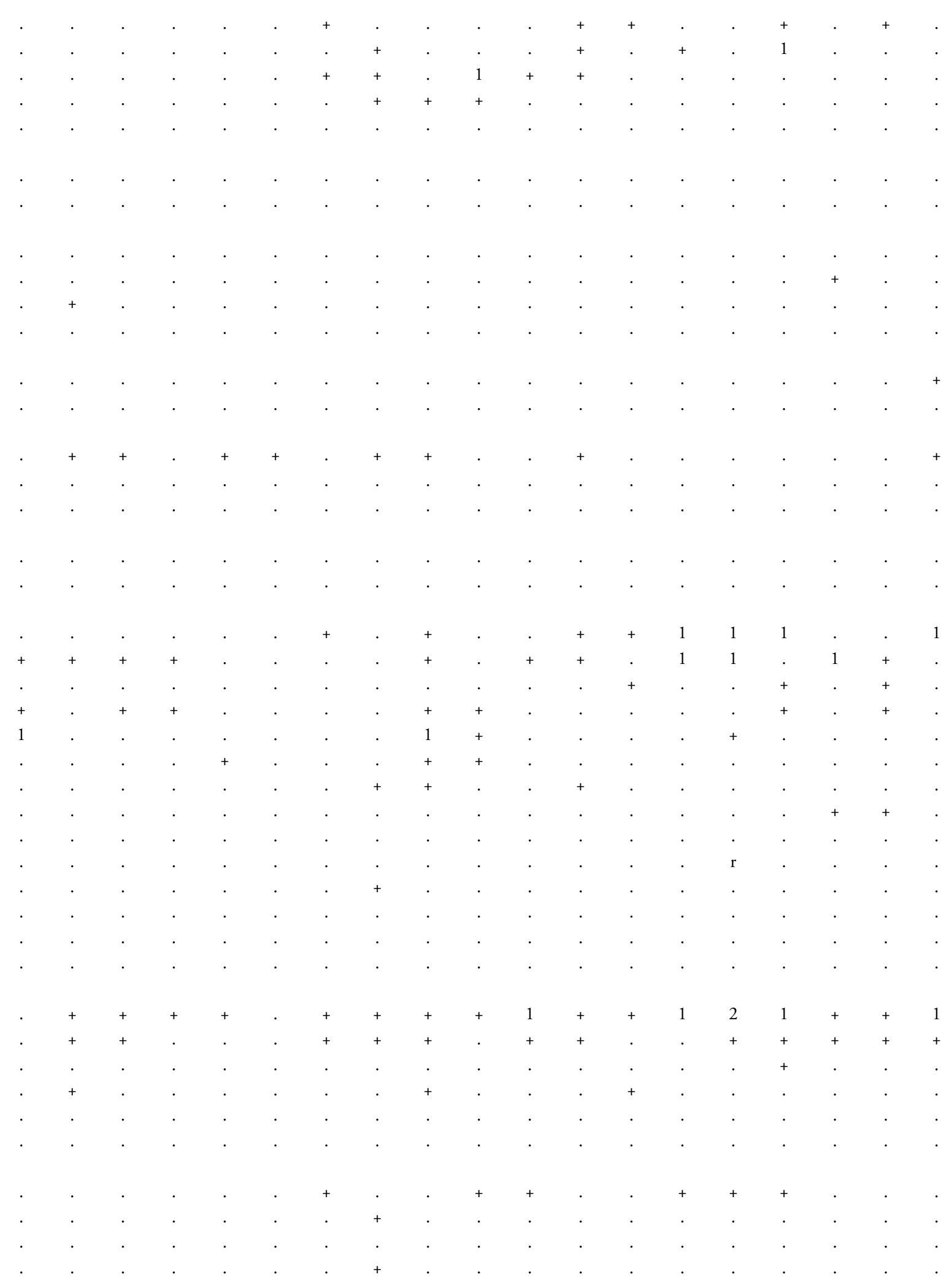


E1

E1

PC

Spiraea decumbens

E1

Campanula cochleariifolia

Valeriana saxatilis

Potentilla caulescens

E1

E1

AT Asplenietea trichomanis

Asplenium ruta-muraria

Kernera saxatilis

Asplenium trichomanes

Polypodium vulgare

ML Mosses and lichens (Mahovi in lišaji)

Tortella tortuosa

Ctenidium molluscum

Fissidens dubius

Dicranum scoparium

Schistidium apocarpum

Polytrichum formosum

Marchantia polymorpha

Peltigera canina

Isothecium alopecuroides

Rhytidiadelphus triquetrus

Cladonia pyxidata

Plagiochila porelloides

E1

Peltigera leucophlebia

Hookeria lucens

Pseudoleskeella catenulata

Orthothecium rufescens

Cladonia sp.

Bartramia pomiformis

Neckera crispa

Rhizomnium punctatum

Cladonia rangiferina

Hylocomium splendens

Lobaria pulmonaria

Conocephalum conicum

Metzgeria furcata

Plagiothecium denticulatum

E0

E0

E0

E0

E0

E0

E0

E0

E0

E0

E0

E0

E0

E0

E0

E0

E0

E0

E0

E0

E0

E0

E0

E0

E0

E0

Solorina saccata

E0

$\mathrm{Fu}$ Fungi (Glive)

Laetiporus sulphureus agg. (L. horoniensis)

E3

Laricifomes officinalis

\section{$\begin{array}{llllllllll}1 & 2 & 3 & 4 & 5 & 6 & 7 & 8 & 9 & 10\end{array}$}

(1)

$\begin{array}{ll}1 & \cdot \\ 1 & \cdot \\ 1 & \\ 1 & \\ 1 & \cdot \\ 1 & .\end{array}$

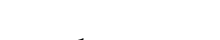

2

(1)




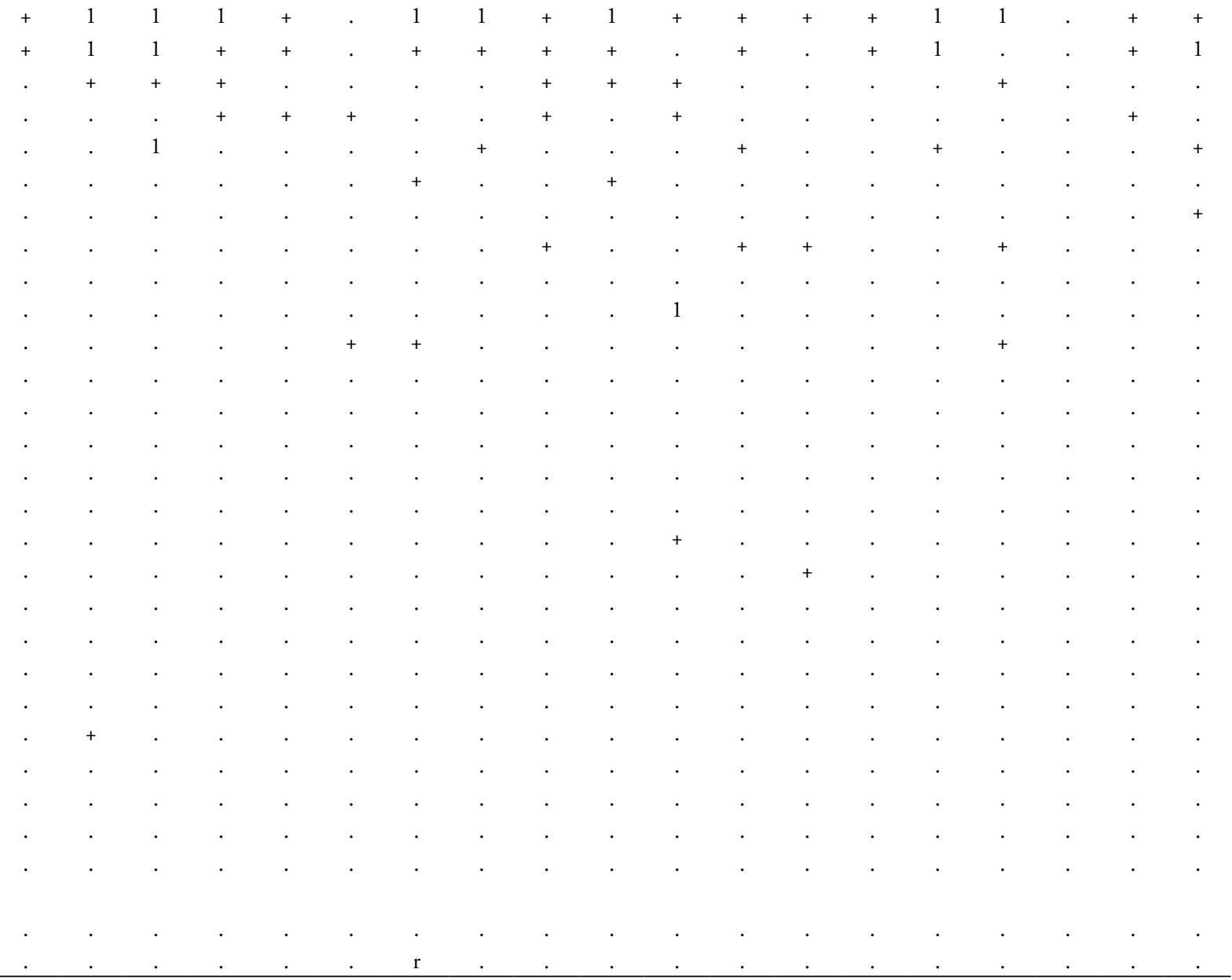


Table 1: Polysticho lonchitis-Fagetum ericetosum carneae var. Rhododendron hirsutum subvar. Calamagrostis varia - cluster 12. Relevé numbers 30-57.

\begin{tabular}{|c|c|c|c|c|c|c|c|c|c|c|c|}
\hline Number of relevé (Zaporedna štev. popisa) & & 30 & 31 & 32 & 33 & 34 & 35 & 36 & 37 & 38 & 39 \\
\hline $\begin{array}{l}\text { Database number of relevé } \\
\text { (Delovna številka popisa) }\end{array}$ & & $\begin{array}{l}\stackrel{R}{N} \\
\text { م) } \\
\text { d }\end{array}$ & 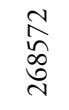 & $\begin{array}{l}\overrightarrow{\tilde{\sigma}} \\
\vec{\sim}\end{array}$ & $\begin{array}{l}\vec{\widetilde{ }} \\
\text { } \\
\text { }\end{array}$ & $\begin{array}{l}\text { 站 } \\
\text { ปี }\end{array}$ & 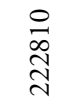 & $\begin{array}{l}\text { oे } \\
\text { ते } \\
\text { त }\end{array}$ & $\begin{array}{l}= \\
\text { ज्ञ } \\
\text { ה }\end{array}$ & $\begin{array}{l}\overline{\widehat{ૂ}} \\
\text { तิ }\end{array}$ & 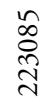 \\
\hline Author of the relevé (Avtor popisa) & & $\Theta$ & $\Theta$ & $\Leftrightarrow$ & 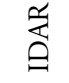 & $\Leftrightarrow$ & $\theta$ & $\theta$ & $\theta$ & 至 & $\ominus$ \\
\hline Elevation in $\mathrm{m}$ (Nadmorska višina $\mathrm{v} \mathrm{m}$ ) & & 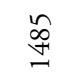 & $\stackrel{R}{2}$ & $\stackrel{尺}{\stackrel{尺}{\sharp}}$ & $\underset{\substack{+n}}{\stackrel{8}{n}}$ & $\underset{\&}{\&}$ & 孛 & $\stackrel{Ð}{Ð}$ & 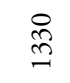 & $\stackrel{尺}{n}$ & $\begin{array}{l}\infty \\
\infty \\
\stackrel{\infty}{2}\end{array}$ \\
\hline Aspect (Lega) & & SSW & NW & $\mathrm{NE}$ & W & $\mathrm{E}$ & SW & NW & $\mathrm{NW}$ & $\mathrm{NE}$ & $\mathrm{N}$ \\
\hline Slope in degrees (Nagib v stopinjah) & & 35 & 40 & 30 & 15 & 40 & 35 & 35 & 35 & 40 & 35 \\
\hline Parent material (Matična podlaga) & & $\mathrm{D}$ & $\mathrm{D}$ & DA & $\mathrm{DA}$ & A & DA & DA & $\mathrm{DA}$ & $\mathrm{DA}$ & DA \\
\hline Soil (Tla) & & $\operatorname{Re}$ & $\mathrm{Re}$ & $\mathrm{Re}$ & $\operatorname{Re}$ & $\operatorname{Re}$ & $\operatorname{Re}$ & $\operatorname{Re}$ & $\operatorname{Re}$ & $\operatorname{Re}$ & $\operatorname{Re}$ \\
\hline Stoniness in \% (Kamnitost v \%) & & 5 & 5 & 5 & 1 & 40 & 20 & 30 & 40 & 10 & 5 \\
\hline Cover in \% (Zastiranje v \%): & & & & & & & & & & & \\
\hline Upper tree layer (Zgor. drevesna plast) & $\mathrm{E} 3 \mathrm{~b}$ & 80 & 80 & 60 & 70 & 80 & 70 & 60 & 80 & 70 & 70 \\
\hline Lower tree layer (Spod. drevesna plasti) & E3a & . & . & . & 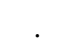 & . & . & 20 & . & . & \\
\hline Shrub layer (Grmovna plast) & $\mathrm{E} 2$ & 10 & 20 & 30 & 80 & 20 & 30 & 40 & 30 & 30 & 40 \\
\hline Herb layer (Zeliš̌na plast) & E1 & 70 & 90 & 90 & 90 & 60 & 60 & 60 & 50 & 60 & 70 \\
\hline Moss layer (Mahovna plast) & E0 & 5 & 5 & 5 & 5 & 10 & 10 & 10 & 20 & 10 & 5 \\
\hline $\begin{array}{l}\text { Maximum tree diameter } \\
\text { (Maksimalni premer dreves) }\end{array}$ & $\mathrm{cm}$ & 35 & 30 & 30 & 45 & 25 & 30 & 40 & 30 & 30 & 20 \\
\hline $\begin{array}{l}\text { Maximum tree height } \\
\text { (Maksimalna višina dreves) }\end{array}$ & $\mathrm{m}$ & 16 & 14 & 10 & $\begin{array}{c}10 \\
(14)\end{array}$ & 10 & 10 & 12 & 14 & 10 & 8 \\
\hline Number of species (Število vrst) & & 49 & 51 & 55 & 46 & 58 & 65 & 66 & 61 & 64 & 54 \\
\hline Relevé area (Velikost popisne ploskve) & $\mathrm{m}^{2}$ & 400 & 400 & 200 & 200 & 200 & 400 & 400 & 400 & 400 & 200 \\
\hline Date of taking relevé (Datum popisa) & & $\begin{array}{l}\infty \\
\infty \\
\stackrel{\infty}{0} \\
\stackrel{\sim}{*}\end{array}$ & $\begin{array}{l}\infty \\
\infty \\
\infty \\
\stackrel{0}{0} \\
\stackrel{\sim}{\sim}\end{array}$ & 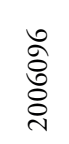 & 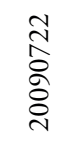 & $\begin{array}{l}\vec{\sigma} \\
\stackrel{\sigma}{\sigma} \\
\sigma\end{array}$ & 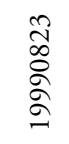 & $\begin{array}{l}\exists \\
\sigma \\
\sigma \\
\sigma\end{array}$ & 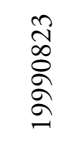 & $\begin{array}{l}\vec{\infty} \\
\hat{0} \\
\stackrel{0}{8} \\
\text { ํ. }\end{array}$ & $\begin{array}{l}\stackrel{0}{0} \\
\stackrel{0}{0} \\
\stackrel{2}{\sigma}\end{array}$ \\
\hline Day (Dan) & & 18 & 18 & 6 & 22 & 11 & 23 & 11 & 23 & 31 & 20 \\
\hline Month (Mesec) & & 8 & 8 & 9 & 7 & 10 & 8 & 10 & 8 & 7 & 8 \\
\hline Year (Leto) & & $\hat{\text { సे }}$ & $\hat{\check{\lambda}}$ & ठ্ণ & ठे & $\stackrel{n}{\sigma}$ & Дે & $\stackrel{\partial}{\sigma}$ & $\hat{\sigma}$ & $\overrightarrow{\grave{d}}$ & $\bar{\sigma}$ \\
\hline Locality (Nahajališče) & & 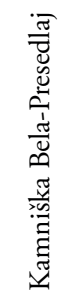 & 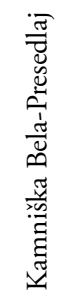 & 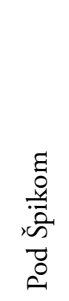 & 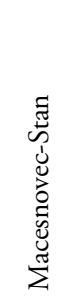 & 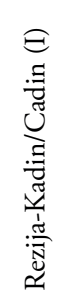 & 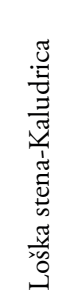 & 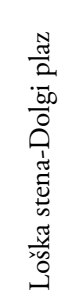 & 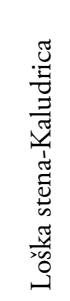 & 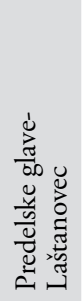 & 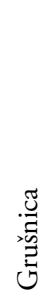 \\
\hline Mountain range (Pogorje) & & KA & KA & $\mathrm{JA}$ & JA & $\mathrm{JA}$ & JA & JA & JA & JA & $\mathrm{JA}$ \\
\hline Quadrant (Kvadrant) & & $\begin{array}{l}\frac{1}{\infty} \\
\grave{n} \\
\curvearrowright\end{array}$ & 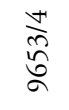 & \begin{tabular}{l}
$\frac{1}{\infty}$ \\
$\vdots$ \\
\multirow{\sigma}{\alpha}{} \\
$\sigma$
\end{tabular} & $\begin{array}{l}\stackrel{m}{a} \\
\stackrel{\hbar}{\alpha}\end{array}$ & 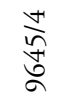 & 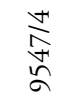 & 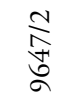 & 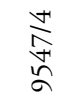 & \begin{tabular}{l}
$\underset{⿱}{*}$ \\
\multirow{n}{\kappa}{}
\end{tabular} & $\begin{array}{l}\stackrel{+}{\infty} \\
\stackrel{+}{+} \\
\hat{\sigma}\end{array}$ \\
\hline Coordinate (Koordinate) GK Y (D-48) & $\mathrm{m}$ & 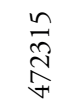 & 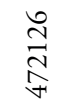 & $\begin{array}{l}\text { â } \\
\text { oे } \\
\text { के }\end{array}$ & 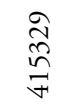 & $\begin{array}{l}\overrightarrow{\hat{O}} \\
\stackrel{0}{0} \\
\text { no }\end{array}$ & $\begin{array}{l}\vec{\infty} \\
\stackrel{\leftrightarrow}{*} \\
\tilde{m}\end{array}$ & 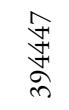 & 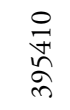 & $\begin{array}{l}\hat{n} \\
\approx \\
\approx\end{array}$ & $\begin{array}{l}\vec{F} \\
\hat{\tilde{\hat{\sigma}}} \\
\stackrel{+}{+}\end{array}$ \\
\hline Coordinate (Koordinate) GK X (D-48) & $\mathrm{m}$ & 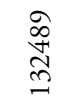 & $\begin{array}{l}\stackrel{m}{\approx} \\
\ddot{\sim}\end{array}$ & 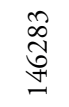 & $\begin{array}{l}\text { SU } \\
\stackrel{+}{+} \\
\stackrel{+}{+}\end{array}$ & $\begin{array}{l}\infty \\
\stackrel{\infty}{0} \\
\stackrel{n}{2}\end{array}$ & $\begin{array}{l}\stackrel{P}{1} \\
\infty \\
\stackrel{+}{-}\end{array}$ & 茫 & $\begin{array}{l}\underset{\mathbb{N}}{N} \\
\stackrel{+}{+}\end{array}$ & 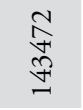 & 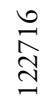 \\
\hline
\end{tabular}


Tabela 1: Polysticho lonchitis-Fagetum ericetosum carneae var. Rhododendron hirsutum subvar. Calamagrostis varia - skupina 12.

Zaporedne številke popisov 30-57.

\begin{tabular}{|c|c|c|c|c|c|c|c|c|c|c|c|c|c|c|c|c|c|}
\hline 40 & 41 & 42 & 43 & 44 & 45 & 46 & 47 & 48 & 49 & 50 & 51 & 52 & 53 & 54 & 55 & 56 & 57 \\
\hline 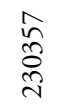 & 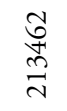 & 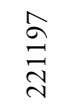 & 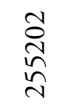 & 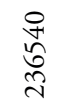 & $\begin{array}{l}\infty \\
\stackrel{+}{1} \\
\infty \\
\stackrel{\sim}{\sim}\end{array}$ & $\begin{array}{l}\stackrel{\Im}{+} \\
\infty \\
\stackrel{\sim}{N}\end{array}$ & $\begin{array}{l}\curvearrowright \\
n \\
n \\
\sim\end{array}$ & 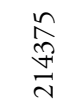 & $\underset{\stackrel{N}{\sim}}{\stackrel{N}{N}}$ & 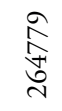 & $\begin{array}{l}\vec{n} \\
\stackrel{\approx}{\approx}\end{array}$ & $\begin{array}{l}\tilde{n} \\
\approx \\
\approx \\
\approx\end{array}$ & 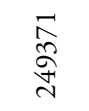 & 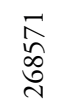 & $\begin{array}{l}m \\
\tilde{n} \\
\stackrel{n}{n} \\
\stackrel{n}{n}\end{array}$ & $\begin{array}{l}\stackrel{\circlearrowright}{二} \\
\underset{\nabla}{\sim}\end{array}$ & $\begin{array}{l}n \\
\infty \\
\stackrel{\infty}{*}\end{array}$ \\
\hline ○ & $\ominus$ & $\ominus$ & $\ominus$ & $\theta$ & $\ominus$ & $\ominus$ & $\ominus$ & 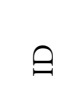 & $\ominus$ & $\theta$ & $\Theta$ & $\theta$ & $\ominus$ & $\Leftrightarrow$ & $\stackrel{\text { 秃 }}{\Theta}$ & $\ominus$ & $\Theta$ \\
\hline 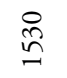 & 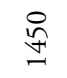 & $\stackrel{\stackrel{\triangleright}{\&}}{\stackrel{\infty}{+}}$ & $\underset{I}{\stackrel{I}{\sharp}}$ & $\stackrel{\circ}{\stackrel{n}{n}}$ & $\begin{array}{l}\stackrel{\infty}{\infty} \\
\stackrel{+}{ \pm}\end{array}$ & $\stackrel{\$}{\nsubseteq}$ & $\stackrel{8}{8}$ & $\begin{array}{l}\stackrel{\infty}{\infty} \\
\stackrel{n}{n}\end{array}$ & 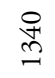 & $\begin{array}{l}\stackrel{D}{\infty} \\
\stackrel{\infty}{-}\end{array}$ & $\underset{\Im}{\stackrel{I}{I}}$ & $\begin{array}{l}\stackrel{\infty}{\infty} \\
\stackrel{(}{=}\end{array}$ & 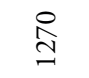 & $\begin{array}{l}n \\
\stackrel{2}{n} \\
n\end{array}$ & 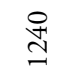 & $\stackrel{\text { }}{\sim}$ & $\stackrel{\$}{ \pm}$ \\
\hline$S$ & NW & NW & $\mathrm{NE}$ & $\mathrm{NE}$ & SWW & W & $\mathrm{NE}$ & NNE & $\mathrm{N}$ & $\mathrm{N}$ & SW & $\mathrm{N}$ & NNW & W & NW & $S$ & SW \\
\hline 30 & 35 & 25 & 10 & 35 & 35 & 30 & 25 & 40 & 20 & 5 & 25 & 25 & 20 & 20 & 25 & 30 & 20 \\
\hline A & DA & DA & $\mathrm{Gr}$ & DA & DA & DA & DA & $\mathrm{D}$ & $\mathrm{D}$ & DA & $\mathrm{DA}$ & $\mathrm{Gr}$ & $\mathrm{Gr}$ & $\mathrm{D}$ & DA & DA & DA \\
\hline $\mathrm{Re}$ & $\operatorname{Re}$ & $\operatorname{Re}$ & $\mathrm{Re}$ & $\operatorname{Re}$ & $\operatorname{Re}$ & $\operatorname{Re}$ & $\operatorname{Re}$ & $\operatorname{Re}$ & $\mathrm{Re}$ & $\operatorname{Re}$ & $\operatorname{Re}$ & $\operatorname{Re}$ & $\operatorname{Re}$ & $\operatorname{Re}$ & $\mathrm{Re}$ & $\operatorname{Re}$ & $\operatorname{Re}$ \\
\hline NA & 10 & 0 & 5 & 5 & 10 & 10 & 10 & 5 & 10 & 20 & 5 & 20 & 20 & 5 & 5 & 10 & 0 \\
\hline 70 & 60 & 80 & 70 & 80 & 70 & 80 & 60 & 80 & 60 & 70 & 60 & 70 & 80 & 70 & 90 & 70 & 70 \\
\hline 10 & . & . & . & . & . & . & 20 & . & 20 & 10 & . & . & . & 10 & . & . & . \\
\hline 40 & 50 & 40 & 20 & 40 & 50 & 30 & 40 & 10 & 40 & 20 & 40 & 60 & 60 & 30 & 20 & 40 & 40 \\
\hline 60 & 80 & 70 & 80 & 80 & 80 & 70 & 80 & 80 & 80 & 70 & 70 & 70 & 60 & 70 & 80 & 60 & 60 \\
\hline 10 & 10 & 10 & 5 & 5 & 10 & 5 & 10 & 10 & 10 & 10 & 5 & 20 & 5 & 10 & 5 & 10 & 10 \\
\hline 90 & 20 & 15 & 30 & 30 & 60 & 35 & 40 & 30 & 40 & 60 & 20 & 20 & 25 & 30 & 35 & 20 & 50 \\
\hline $\begin{array}{c}18 \\
(10)\end{array}$ & 6 & 5 & 12 & 12 & 15 & 9 & 16 & 15 & 14 & 17 & 7 & 10 & 8 & 15 & 14 & 8 & 11 \\
\hline 67 & 59 & 47 & 51 & 56 & 58 & 51 & 54 & 56 & 61 & 52 & 61 & 66 & 65 & 52 & 45 & 53 & 48 \\
\hline 400 & 200 & 400 & 500 & 200 & 400 & 400 & 200 & 200 & 400 & 400 & 400 & 400 & 400 & 400 & 400 & 200 & 400 \\
\hline 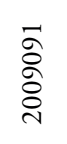 & 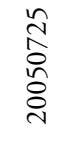 & 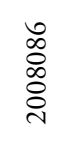 & 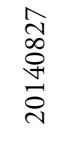 & $\begin{array}{l}\text { N } \\
\infty \\
O \\
0 \\
0 \\
0\end{array}$ & 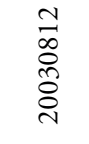 & 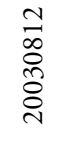 & 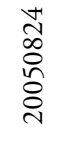 & 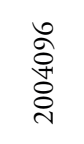 & 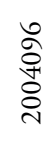 & $\begin{array}{l}\overrightarrow{\hat{o}} \\
0 \\
\stackrel{0}{0}\end{array}$ & 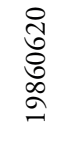 & 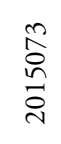 & $\begin{array}{l}0 \\
\infty \\
0 \\
\\
\stackrel{\sim}{0}\end{array}$ & $\begin{array}{l}\infty \\
\infty \\
\stackrel{\infty}{\curvearrowright} \\
\stackrel{\sim}{\circ}\end{array}$ & 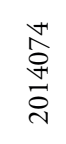 & \begin{tabular}{l}
$\hat{a}$ \\
oे \\
\multirow{+}{8}{} \\
ㄱ.
\end{tabular} & 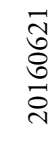 \\
\hline 1 & 25 & 6 & 27 & 2 & 12 & 12 & 24 & 6 & 6 & 1 & 20 & 3 & 6 & 18 & 4 & 7 & 21 \\
\hline 9 & 7 & 8 & 8 & 8 & 8 & 8 & 8 & 9 & 9 & 9 & 6 & 7 & 8 & 8 & 7 & 9 & 6 \\
\hline 융 & 苂 & $\stackrel{\infty}{\stackrel{\circ}{\circ}}$ & $\stackrel{+}{\stackrel{*}{*}}$ & $\stackrel{\circ}{\stackrel{\sim}{\circ}}$ & ஜ̊ & ڤి & 苂 & 苍 & ষ্ণ & 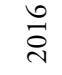 & $\stackrel{\circ}{2}$ & $\frac{n}{\stackrel{i}{d}}$ & $\stackrel{n}{\stackrel{2}{\sim}}$ & $\stackrel{ }{\stackrel{i}{~}}$ & $\stackrel{\stackrel{\sim}{\circ}}{\stackrel{\sim}{*}}$ & ஓ્ટ & $\stackrel{0}{\circ}$ \\
\hline
\end{tabular}

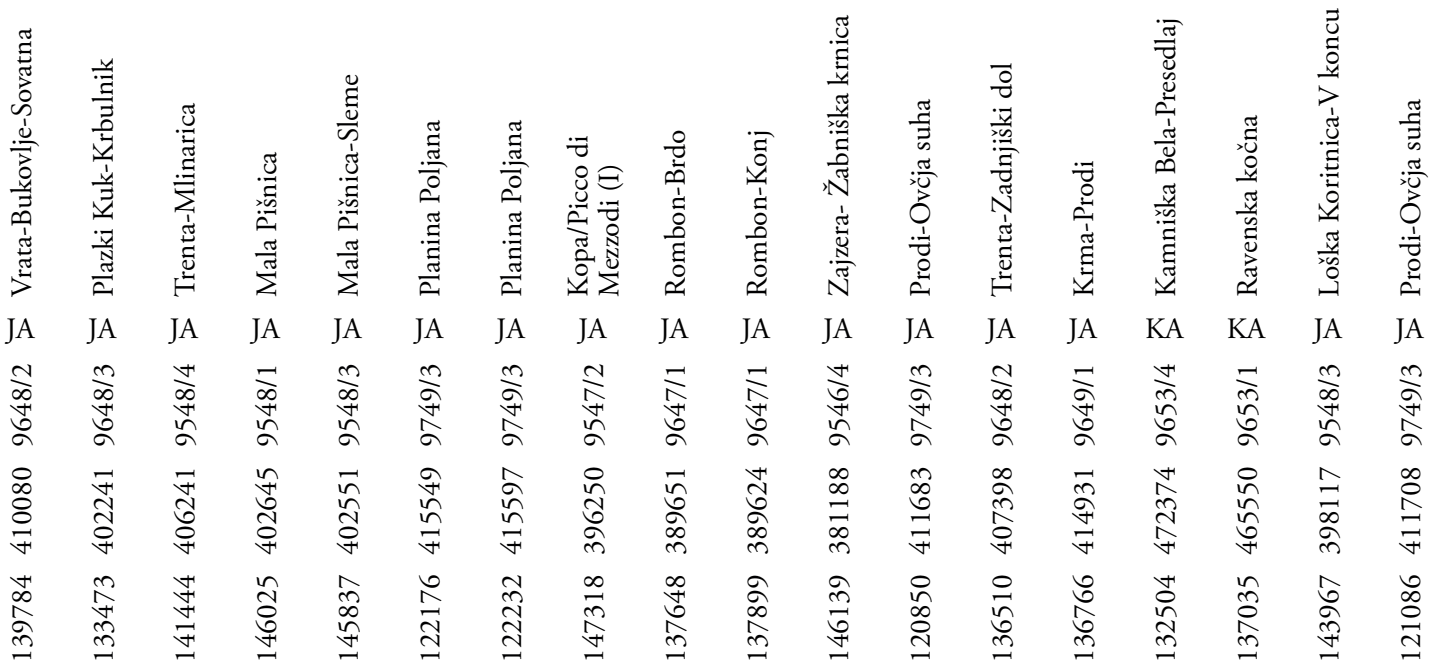




\begin{tabular}{|c|c|c|c|c|c|c|c|c|c|c|c|c|}
\hline & Number of relevé (Zaporedna štev. popisa) & & 30 & 31 & 32 & 33 & 34 & 35 & 36 & 37 & 38 & 39 \\
\hline & \multicolumn{12}{|c|}{ Diagnostic species of the association (Diagnostične vrste asociacije) } \\
\hline EP & Rhododendron hirsutum & E2 & + & 2 & 4 & 4 & 1 & 2 & 3 & 2 & 2 & 3 \\
\hline $\mathrm{EP}$ & Pinus mugo & $\mathrm{E} 2$ & + & + & . & + & . & $\mathrm{r}$ & + & + & + & + \\
\hline VP & Polystichum lonchitis & E1 & . & + & + & + & 1 & + & 1 & 1 & + & + \\
\hline BA & Sorbus chamaemespilus & $\mathrm{E} 2$ & + & + & 1 & 1 & + & 1 & + & $\mathrm{r}$ & + & + \\
\hline VP & Clematis alpina & E2 & + & + & + & 1 & + & + & + & . & 1 & 1 \\
\hline VP & Luzula sylvatica & E1 & 1 & + & 1 & 1 & . & . & 1 & . & + & 1 \\
\hline CA & Laserpitium peucedanoides & E1 & . & $\cdot$ & + & 1 & + & + & $\mathrm{r}$ & . & + & + \\
\hline ML & Paraleucobryum sauteri & E0 & . & . & + & + & + & + & + & . & + & . \\
\hline EP & Rhodothamnus chamaecistus & E1 & . & . & . & + & . & 1 & 1 & + & + & . \\
\hline VP & Homogyne alpina & E1 & . & . & 1 & + & . & . & 1 & . & . & + \\
\hline ES & Aster bellidiastrum & E1 & . & $\cdot$ & + & . & . & $\mathrm{r}$ & + & + & . & . \\
\hline $\mathrm{CF}$ & Carex ferruginea & E1 & $\cdot$ & $\cdot$ & $\cdot$ & . & . & . & . & $\cdot$ & + & + \\
\hline $\mathrm{MuA}$ & Aconitum lycoctonum subsp. ranunculifolium & E1 & . & $\cdot$ & . & . & + & + & + & 1 & + & . \\
\hline BA & Salix appendiculata & E2 & . & + & . & . & + & . & + & . & + & . \\
\hline VP & Lonicera caerulea & E2 & . & . & $\mathrm{r}$ & + & + & . & . & . & + & . \\
\hline PS & Paederota lutea & E1 & $\cdot$ & $\cdot$ & $\cdot$ & . & . & + & + & + & . & . \\
\hline \multirow[t]{2}{*}{ CA } & Festuca calva & E1 & $\cdot$ & $\cdot$ & $\cdot$ & . & . & . & . & $\cdot$ & . & $\cdot$ \\
\hline & \multicolumn{12}{|c|}{ Differential species of lower units (Razlikovalnice nižjih enot) } \\
\hline $\mathrm{EP}$ & Erica carnea & E1 & 3 & 2 & 2 & 3 & + & 1 & 1 & 1 & 1 & + \\
\hline EP & Calamagrostis varia & E1 & 3 & 3 & + & 1 & 2 & 1 & 1 & 1 & 2 & 2 \\
\hline VP & Vaccinium vitis-idaea & E1 & + & + & + & 1 & . & 1 & 1 & + & + & . \\
\hline VP & Larix decidua & E3 & + & + & $\mathrm{r}$ & + & . & . & . & . & . & . \\
\hline VP & Larix decidua & E2 & . & . & + & + & . & . & . & . & . & . \\
\hline VP & Larix decidua & E1 & . & $\cdot$ & . & . & . & . & . & . & . & . \\
\hline VP & Calamagrostis villosa & E1 & . & . & 3 & 2 & . & . & + & . & 1 & . \\
\hline \multirow[t]{10}{*}{ AF } & Aremonio-Fagion & & & & & & & & & & & \\
\hline & Anemone trifolia & E1 & . & $\cdot$ & 1 & 1 & . & 1 & + & + & 1 & 1 \\
\hline & Cyclamen purpurascens & E1 & 1 & 1 & 1 & + & 1 & 1 & 1 & 1 & 1 & 1 \\
\hline & Cardamine enneaphyllos & E1 & $\cdot$ & $\cdot$ & $\cdot$ & . & · & . & · & $\cdot$ & 1 & 1 \\
\hline & Knautia drymeia & E1 & 1 & $\cdot$ & + & . & . & $\cdot$ & . & $\cdot$ & $\mathrm{r}$ & . \\
\hline & Helleborus niger & E1 & 1 & $\cdot$ & $\cdot$ & . & . & . & . & $\cdot$ & . & . \\
\hline & Rhamnus fallax & E2 & . & . & $\cdot$ & . & . & . & · & $\cdot$ & . & $\cdot$ \\
\hline & Cardamine trifolia & E1 & . & $\cdot$ & $\cdot$ & . & . & . & . & . & . & . \\
\hline & Euphorbia carniolica & E1 & $\cdot$ & + & $\cdot$ & . & . & . & . & $\cdot$ & . & . \\
\hline & Anemone $x$ pittonii & E1 & $\cdot$ & $\cdot$ & $\cdot$ & . & . & . & . & $\cdot$ & . & . \\
\hline \multirow[t]{2}{*}{$\mathrm{EC}$} & Erythronio-Carpinion & & & & & & & & & & & \\
\hline & Primula vulgaris & E1 & $\cdot$ & $\cdot$ & $\cdot$ & . & . & . & . & $\cdot$ & . & . \\
\hline \multirow[t]{6}{*}{ TA } & Tilio-Acerion & & & & & & & & & & & \\
\hline & Acer pseudoplatanus & E3 & . & + & . & $r$ & . & . & $\mathrm{r}$ & $\cdot$ & . & . \\
\hline & Acer pseudoplatanus & E2 & . & + & . & . & + & . & . & . & + & . \\
\hline & Acer pseudoplatanus & E1 & 1 & 2 & $\cdot$ & + & + & $\cdot$ & $\mathrm{r}$ & $\cdot$ & + & . \\
\hline & Polystichum aculeatum & E1 & . & $\cdot$ & . & + & + & + & . & $\cdot$ & . & . \\
\hline & Aruncus dioicus & E1 & . & . & . & $\cdot$ & $\cdot$ & $\cdot$ & . & $\cdot$ & + & . \\
\hline \multirow[t]{7}{*}{ FS } & Fagetalia sylvaticae & & & & & & & & & & & \\
\hline & Fagus sylvatica & E3 & 5 & 4 & 4 & 4 & 5 & 4 & 4 & 4 & 4 & 5 \\
\hline & Fagus sylvatica & E2 & + & + & 1 & 1 & 1 & + & 1 & 1 & 1 & 1 \\
\hline & Fagus sylvatica & E1 & + & + & 1 & + & . & + & 1 & + & . & . \\
\hline & Daphne mezereum & E2 & + & $\cdot$ & 1 & 1 & . & 1 & 1 & 1 & 1 & . \\
\hline & Melica nutans & E1 & . & + & . & + & + & + & . & + & + & . \\
\hline & Prenanthes purpurea & E1 & $\cdot$ & + & $\cdot$ & 1 & $\cdot$ & + & + & $\cdot$ & + & . \\
\hline
\end{tabular}




\begin{tabular}{|c|c|c|c|c|c|c|c|c|c|c|c|c|c|c|c|c|c|c|c|}
\hline 3 & 3 & 3 & 2 & 3 & 4 & 2 & 2 & 2 & 3 & 3 & + & 1 & 2 & 1 & 1 & 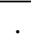 & 1 & 52 & 91 \\
\hline+ & 1 & 1 & . & + & 1 & 1 & + & . & + & + & 2 & 2 & 1 & 2 & 1 & 2 & 2 & 49 & 86 \\
\hline 2 & + & 1 & + & 1 & . & + & 1 & 1 & 1 & 1 & + & + & 1 & 1 & . & 1 & . & 47 & 82 \\
\hline+ & 1 & 1 & 1 & 1 & 2 & 2 & 1 & + & 1 & + & 1 & 1 & 2 & 1 & 2 & + & 1 & 45 & 79 \\
\hline 1 & 1 & . & + & + & 1 & . & + & + & + & + & + & + & 1 & 1 & . & 1 & . & 42 & 74 \\
\hline . & . & 1 & 1 & + & . & . & + & + & + & + & + & 1 & + & 1 & + & . & + & 39 & 68 \\
\hline . & . & . & . & 1 & 1 & + & . & + & . & . & . & + & + & . & + & + & + & 34 & 60 \\
\hline+ & + & . & . & . & + & + & + & . & + & . & 1 & + & . & + & + & $\cdot$ & . & 29 & 51 \\
\hline . & + & . & . & . & + & . & + & + & + & . & . & 1 & + & . & 1 & $\cdot$ & . & 26 & 46 \\
\hline+ & . & . & . & + & . & . & + & . & 1 & 1 & . & . & . & . & . & . & . & 23 & 40 \\
\hline . & . & . & . & . & . & . & . & + & + & + & . & + & . & . & . & . & . & 19 & 33 \\
\hline . & . & . & . & + & 1 & . & . & . & . & + & + & . & . & . & . & . & . & 16 & 28 \\
\hline . & . & + & + & + & . & . & . & . & . & . & + & . & + & . & . & . & + & 15 & 26 \\
\hline . & + & . & . & . & + & . & . & . & . & . & + & . & + & + & . & . & . & 14 & 25 \\
\hline+ & + & + & . & . & . & . & + & . & . & . & . & . & + & . & . & . & + & 12 & 21 \\
\hline+ & . & . & . & . & . & . & . & . & $r$ & . & . & + & . & . & . & . & . & 11 & 19 \\
\hline . & . & . & . & . & . & . & . & . & . & . & . & . & . & . & . & $\mathrm{r}$ & . & 2 & 4 \\
\hline
\end{tabular}

$\begin{array}{ccccccccccccccccccccc}1 & + & 2 & . & 1 & 2 & 1 & 2 & + & 3 & 2 & + & + & 1 & 1 & 3 & 2 & 2 & 56 & 98 \\ 1 & 1 & 1 & 3 & 2 & 2 & 2 & 1 & 2 & 2 & 1 & 2 & 1 & 2 & 3 & 2 & 2 & + & 56 & 98 \\ + & 1 & 1 & 1 & 1 & + & 1 & 2 & 1 & 1 & 1 & + & + & + & 1 & 1 & + & . & 51 & 89 \\ 1 & . & . & + & + & . & \mathrm{r} & + & + & . & + & \mathrm{r} & + & \mathrm{r} & \mathrm{r} & \mathrm{r} & . & . & 4274 \\ + & . & . & + & + & . & . & + & \mathrm{r} & . & . & . & . & . & . & . & . & . & 19 & 33 \\ . & . & . & . & . & . & . & . & . & . & . & . & . & \mathrm{r} & . & . & . & . & 4 & 7 \\ . & 1 & 1 & + & . & . & + & 3 & 1 & 1 & 2 & . & . & . & . & . & . & + & 34 & 60\end{array}$

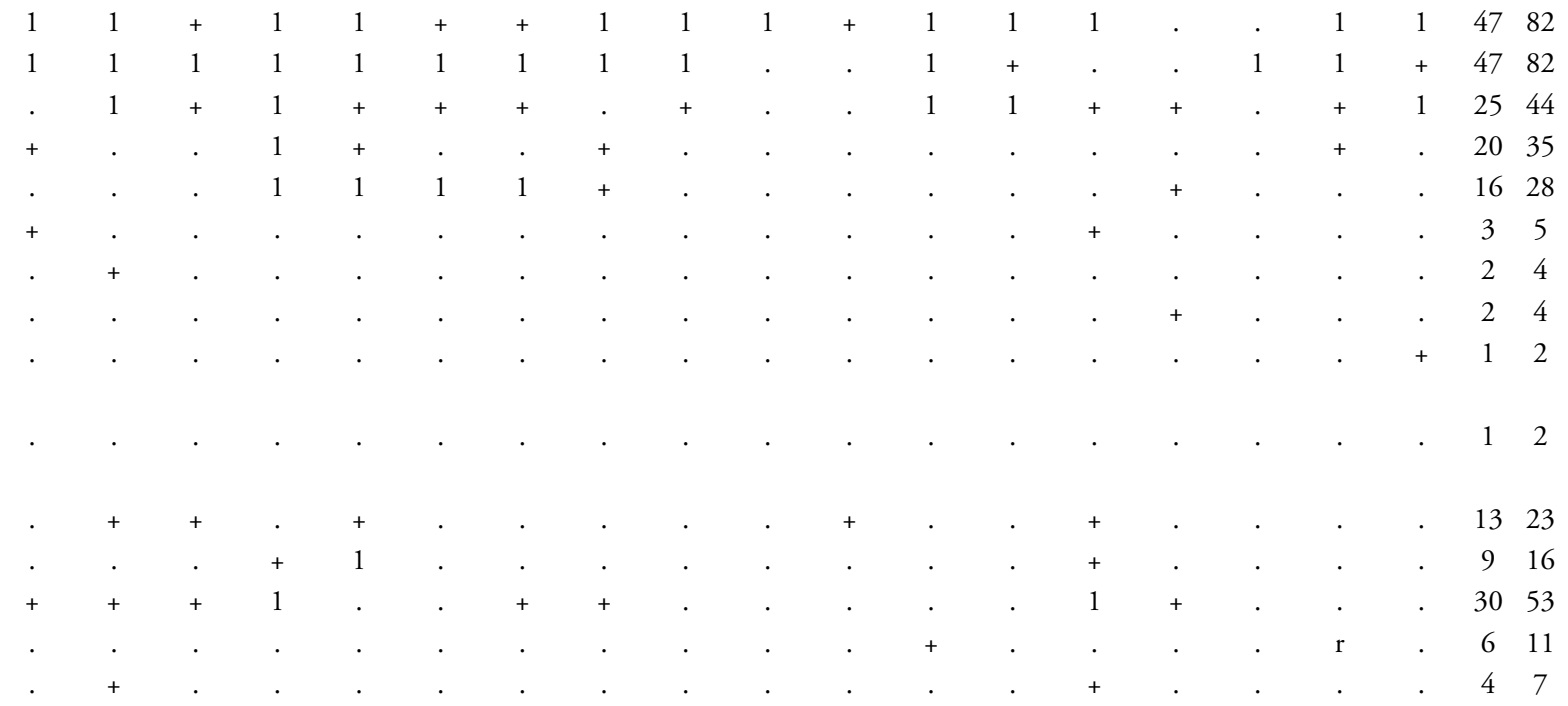

$$
\begin{array}{lllllllllllllllllllll}
4 & 4 & 4 & 4 & 4 & 4 & 5 & 4 & 5 & 4 & 4 & 4 & 4 & 5 & 4 & 5 & 4 & 4 & 57 & 100 \\
1 & 2 & . & 2 & 1 & 2 & 1 & 2 & 1 & 2 & 1 & 1 & . & . & . & 1 & 1 & . & 50 & 88 \\
+ & . & . & 1 & . & + & . & + & 1 & 1 & 1 & . & . & + & + & . & . & . & 38 & 67 \\
1 & 1 & 1 & + & + & + & + & + & + & 1 & . & 1 & + & + & + & + & 1 & + & 50 & 88 \\
+ & 1 & . & + & + & . & + & . & 1 & 1 & + & + & + & 1 & + & 1 & 1 & 1 & 4375 \\
+ & . & . & . & + & + & 1 & 1 & 1 & 1 & 1 & + & + & . & + & 1 & + & . & 41 & 72
\end{array}
$$




\begin{tabular}{|c|c|c|c|c|c|c|c|c|c|c|c|c|}
\hline & Number of relevé (Zaporedna štev. popisa) & & 30 & 31 & 32 & 33 & 34 & 35 & 36 & 37 & 38 & 39 \\
\hline & Lonicera alpigena & E2 & + & + & . & + & + & + & + & + & 1 & + \\
\hline & Mercurialis perennis & E1 & . & + & . & . & 1 & 1 & + & + & 1 & . \\
\hline & Lilium martagon & E1 & + & + & 1 & + & . & . & . & . & $\mathrm{r}$ & . \\
\hline & Galium laevigatum & E1 & . & + & . & . & 1 & . & . & . & . & + \\
\hline & Paris quadrifolia & E1 & . & + & . & . & . & . & . & . & . & . \\
\hline & Laburnum alpinum & E1 & . & . & . & . & . & $\mathrm{r}$ & . & . & . & . \\
\hline & Laburnum alpinum & E2 & . & + & . & $\mathrm{r}$ & + & . & $\mathrm{r}$ & . & . & . \\
\hline & Laburnum alpinum & E3 & . & $\cdot$ & . & . & . & + & $\mathrm{r}$ & . & . & . \\
\hline & Luzula nivea & E1 & . & . & . & . & . & . & . & + & . & . \\
\hline & Galeobdolon flavidum & E1 & + & + & . & . & + & . & . & . & . & . \\
\hline & Euphorbia amygdaloides & E1 & + & + & . & . & . & . & . & + & . & . \\
\hline & Dryopteris filix-mas & E1 & . & . & $\mathrm{r}$ & . & + & . & + & . & . & . \\
\hline & Phyteuma spicatum & E1 & . & . & . & . & . & . & . & . & . & . \\
\hline & Neottia nidus-avis & E1 & + & . & . & . & . & . & . & . & . & . \\
\hline & Mycelis muralis & E1 & . & . & + & . & . & . & . & . & . & . \\
\hline & Epipactis helleborine & E1 & . & . & . & . & . & . & . & . & . & . \\
\hline & Symphytum tuberosum & E1 & . & + & $\cdot$ & $\cdot$ & $\cdot$ & $\cdot$ & $\cdot$ & $\cdot$ & $\mathrm{r}$ & . \\
\hline & Ranunculus lanuginosus & E1 & . & . & + & . & . & . & . & . & . & . \\
\hline & Lathyrus vernus & E1 & . & . & . & . & . & . & . & . & . & + \\
\hline & Festuca altissima & E1 & . & . & . & . & . & . & . & . & . & . \\
\hline \multirow[t]{7}{*}{ QP } & Quercetalia pubescenti-petraeae & & & & & & & & & & & \\
\hline & Sorbus aria (Aria edulis) & E2 & . & + & . & . & . & $\mathrm{r}$ & . & . & . & + \\
\hline & Sorbus aria (Aria edulis) & E1 & . & . & . & . & . & . & . & . & . & . \\
\hline & Convallaria majalis & E1 & . & + & . & . & . & . & . & . & + & . \\
\hline & Carex flacca & E1 & + & . & . & . & . & . & . & . & . & . \\
\hline & Melittis melissophyllum & E1 & . & . & . & . & + & $\cdot$ & $\cdot$ & $\cdot$ & $\cdot$ & . \\
\hline & Ostrya carpinifolia & E2 & . & . & . & . & . & $\cdot$ & . & . & . & . \\
\hline \multirow[t]{9}{*}{ QF } & Querco-Fagetea & & & & & & & & & & & \\
\hline & Hepatica nobilis & E1 & + & 1 & . & . & + & + & . & . & . & . \\
\hline & Carex digitata & E1 & . & + & . & + & . & + & . & + & . & . \\
\hline & Viola riviniana & E1 & . & . & . & . & . & + & . & . & . & . \\
\hline & Anemone nemorosa & E1 & . & . & . & . & . & . & . & . & . & + \\
\hline & Poa nemoralis & E1 & + & . & . & . & . & . & . & . & . & . \\
\hline & Melampyrum pratense & E1 & . & . & . & . & . & . & . & . & . & . \\
\hline & Dactylorhiza fuchsii & E1 & . & . & . & . & . & $\cdot$ & . & $\cdot$ & $\cdot$ & . \\
\hline & Pteridium aquilinum & E1 & . & . & . & . & . & . & . & . & . & . \\
\hline \multirow[t]{15}{*}{ VP } & Vaccinio-Piceetea & & & & & & & & & & & \\
\hline & Vaccinium myrtillus & E1 & 1 & + & 2 & + & 1 & 2 & 2 & 1 & 1 & 1 \\
\hline & Rosa pendulina & E2 & + & + & + & + & + & . & + & . & + & + \\
\hline & Aposeris foetida & E1 & 1 & . & 1 & 1 & . & 1 & 1 & 1 & + & + \\
\hline & Gymnocarpium dryopteris & E1 & . & 1 & + & . & . & 1 & + & 1 & + & + \\
\hline & Picea abies & E3 & $\mathrm{r}$ & . & . & . & . & + & . & . & . & + \\
\hline & Picea abies & $\mathrm{E} 2$ & + & + & $\mathrm{r}$ & + & . & $\mathrm{r}$ & . & + & $\mathrm{r}$ & . \\
\hline & Picea abies & E1 & . & . & . & . & . & . & + & + & . & . \\
\hline & Maianthemum bifolium & E1 & . & + & + & + & . & . & . & + & . & . \\
\hline & Veronica urticifolia & E1 & . & + & $\mathrm{r}$ & . & . & . & . & + & . & . \\
\hline & Gentiana asclepiadea & E1 & . & . & . & . & + & + & $\mathrm{r}$ & . & + & + \\
\hline & Hieracium murorum & E1 & . & . & + & . & + & + & . & + & . & + \\
\hline & Lonicera nigra & E2 & . & . & . & . & $\mathrm{r}$ & . & + & . & + & . \\
\hline & Huperzia selago & E1 & . & . & + & . & + & . & + & + & . & + \\
\hline & Abies alba & E3 & . & . & . & . & . & . & . & . & . & . \\
\hline
\end{tabular}




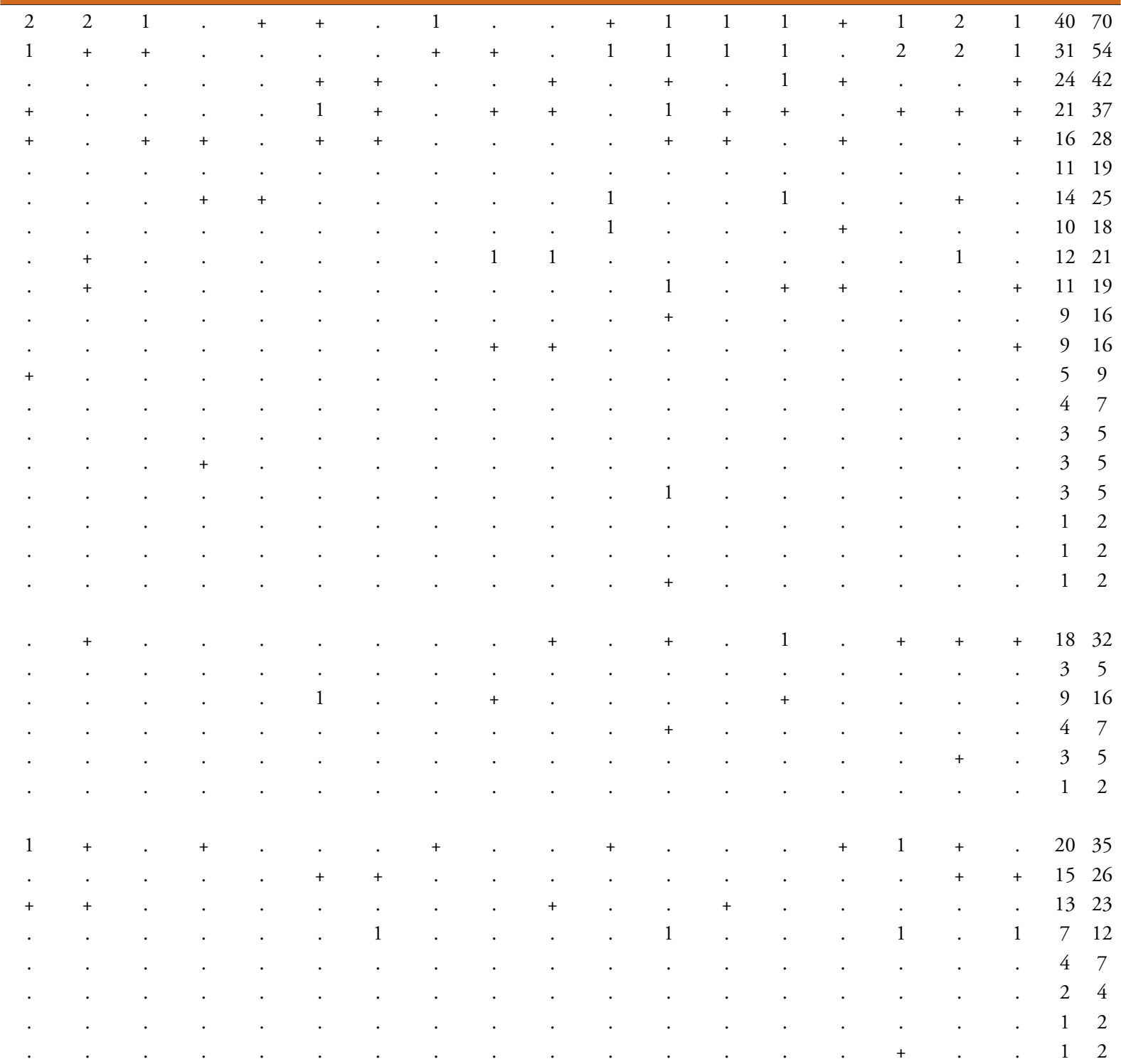

$$
\begin{array}{llllllllllllllllllllll}
3 & 2 & 2 & 3 & 3 & 3 & 3 & 3 & 3 & 3 & 3 & 1 & 2 & 2 & 2 & 3 & 2 & 2 & 53 & 93 \\
2 & 2 & 1 & . & + & + & + & 1 & + & + & + & + & + & . & + & . & 1 & 1 & 48 & 84 \\
. & + & . & 1 & 1 & 1 & 1 & 1 & + & + & 1 & . & 1 & + & + & . & + & . & 41 & 72 \\
1 & 2 & 1 & 1 & + & . & + & 1 & 1 & 1 & 1 & . & 1 & 1 & + & . & . & . & 37 & 65 \\
. & . & + & + & . & . & . & + & . & . & + & + & . & . & . & . & . & + & 26 & 46 \\
. & . & . & + & . & + & . & . & . & . & + & + & . & + & . & + & + & . & 33 & 58 \\
. & . & . & + & . & + & + & + & . & . & . & . & . & . & . & + & . & . & 15 & 26 \\
+ & . & + & + & + & + & 1 & . & . & 1 & 1 & . & . & + & 1 & + & + & . & 31 & 54 \\
+ & + & + & . & + & . & . & 1 & + & + & . & 1 & . & 1 & . & 1 & . & + & 30 & 53 \\
1 & + & . & . & + & 1 & + & + & + & + & + & + & + & 1 & . & . & . & + & 28 & 49 \\
+ & . & . & . & . & + & + & . & + & + & + & + & + & . & . & . & . & . & 28 & 49 \\
. & + & . & + & . & + & + & + & + & + & . & . & + & + & + & + & + & . & 23 & 40
\end{array}
$$


Abies alba

Abies alba

Lycopodium annotinum

Oxalis acetosella

Phegopteris connectilis

Solidago virgaurea

Melampyrum sylvaticum

Homogyne sylvestris

Saxifraga cuneifolia

Calamagrostis arundinacea

Orthilia secunda

Pyrola minor

Pyrola rotundifolia

Thelypteris limbosperma

Dryopteris dilatata

Luzula luzuloides

Ajuga pyramidalis

Dryopteris expansa

Luzula pilosa

Pyrola chlorantha

E2

E1

E1

E1

E1

E1

E1

E1

E1

E1

E1

E1

E1

EP Erico-Pinetea

Rubus saxatilis

Carex alba

Polygala chamaebuxus

Aquilegia nigricans

Molinia arundinacea

Genista radiata

Cotoneaster tomentosus

Amelanchier ovalis

Carex ornithopoda

Peucedanum austriacum subsp. rablense

Chamaecytisus hirsutus

SSC Sambuco-Salicion capreae, Rhamno-Prunetea

Sorbus aucuparia

Sorbus aucuparia

Sorbus aucuparia

Sambucus racemosa

E1

E1

E1

E1

E1

E1

E3

E2

E1

E2

E2

E2

E2

E2

E2

E2

Alnus viridis

MuA Mulgedio-Aconitetea

Polygonatum verticillatum

Geranium sylvaticum

Athyrium filix-femina

Veratrum album

Viola biflora

Phyteuma ovatum \\ $\begin{array}{llllllllll}30 & 31 & 32 & 33 & 34 & 35 & 36 & 37 & 38 & 39\end{array}$}

1
1
1
1
1
1
1
1
1
1
1
1
1
1

1
1
1
2
2
2
1
1

3
2
2



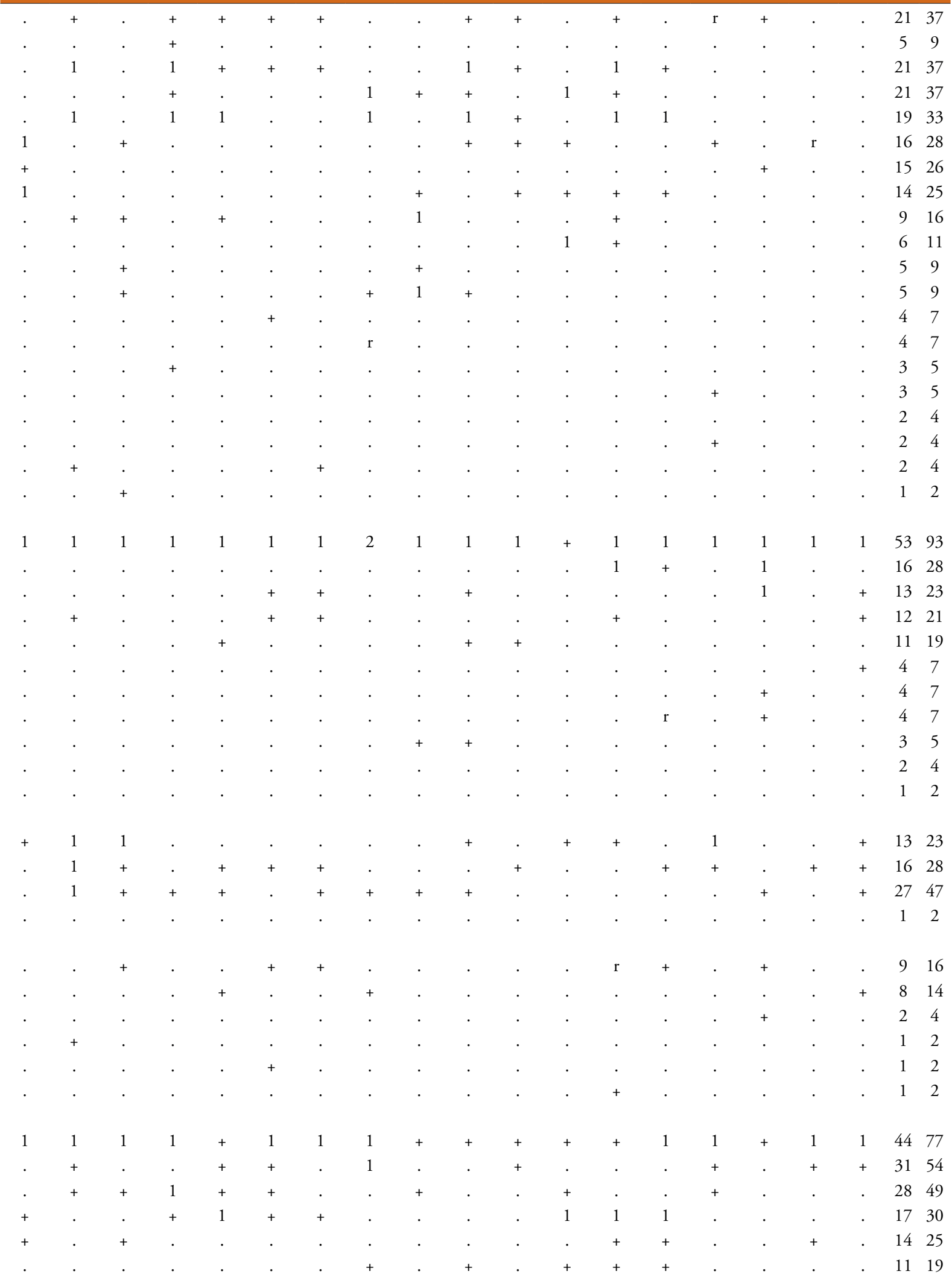
Senecio ovatus

Hypericum maculatum

Chaerophyllum villarsii

Primula elatior

Ranunculus platanifolius

Aconitum degenii subsp. paniculatum

Tanacetum corymbosum subsp. clusii

Cicerbita alpina

Geum rivale

Allium victorialis

Thalictrum aquilegiifolium

Adenostyles alliariae

CA Caricion austroalpinae

Pulsatilla alpina subsp. austroalpina

CF Caricion ferrugineae

Hypericum richeri subsp. grisebachii

Cfir Caricion firmae

Carex firma

Sesleria sphaerocephala

ES Elyno-Seslerietea

Betonica alopecuros

Sesleria caerulea

Senecio abrotanifolius

Campanula witasekiana

Carduus crassifolius

Phyteuma orbiculare

Carex sempervirens

Ranunculus montanus

Thymus praecox subsp. polytrichus

Lotus alpinus

Scabiosa lucida subsp. lucida

Bartsia alpina

Selaginella selaginoides

Daphne striata

Hieracium pilosum

Scabiosa lucida subsp. stricta

Ranunculus carinthiacus

Carex mucronata

Globularia cordifolia

Helictotrichon parlatorei

E1

Astrantia bavarica

Carduus defloratus

Hieracium villosum

NS Nardion strictae, Juncetea trifidi

CD Caricetalia davallianae

Tofieldia calyculata 
$\begin{array}{lllll} & . & 4 & 7\end{array}$ 35

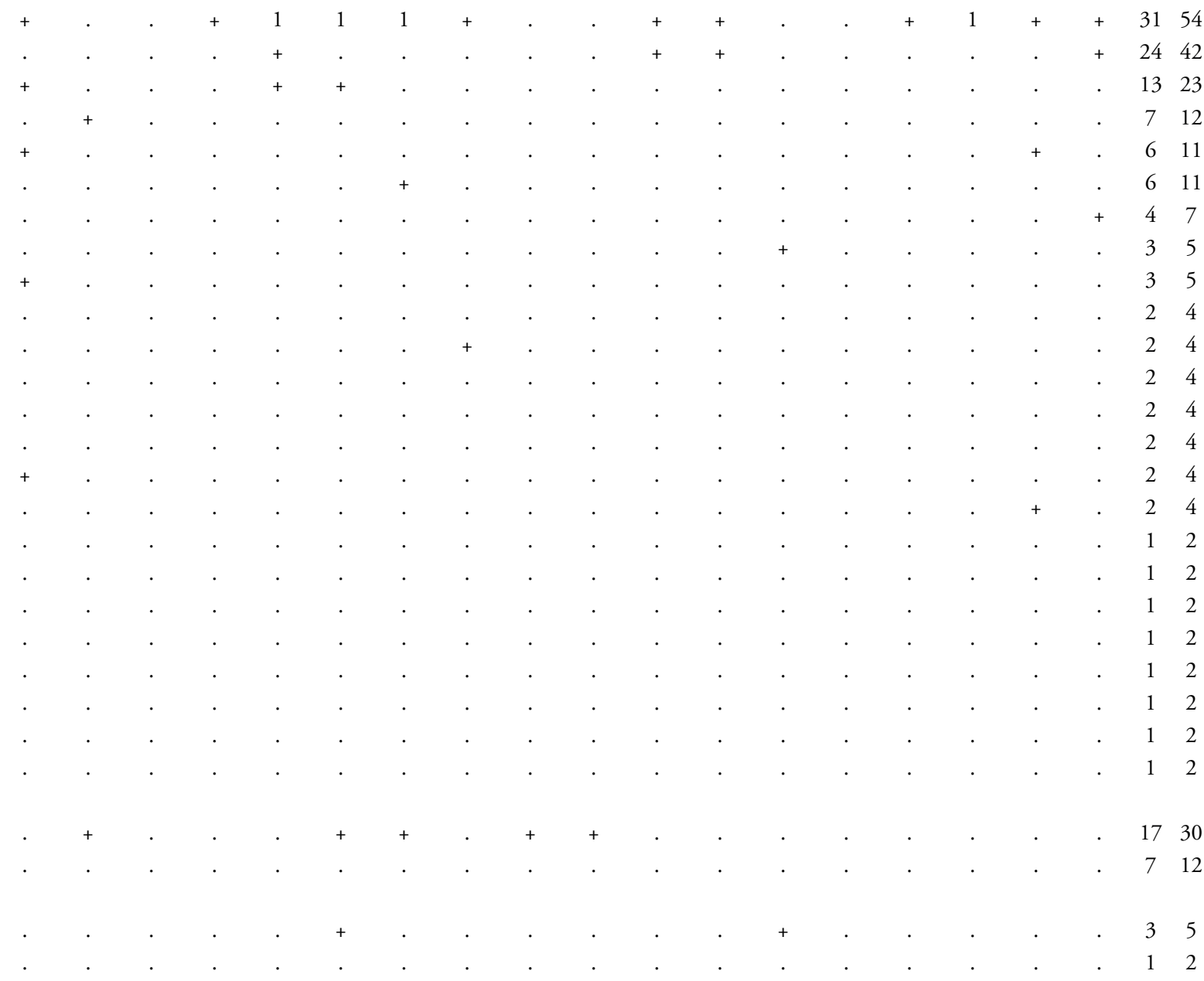




\section{Number of relevé (Zaporedna štev. popisa)

$\begin{array}{llllllllll}30 & 31 & 32 & 33 & 34 & 35 & 36 & 37 & 38 & 39\end{array}$

FB Festuco-Brometea

Cirsium erisithales

Buphthalmum salicifolium

Carex humilis

Carlina acaulis

Linum catharticum

E1

E1

E1

E1

E1

TG Trifolio-Geranietea

Laserpitium latifolium

Verbascum lanatum

E1

PaT Poo alpinae-Trisetetalia

Ranunculus nemorosus

Poa alpina

Crocus albiflorus

Trollius europaeus

MA Molinio-Arrhenatheretea

Crepis paludosa

E1

Festuca rubra agg.

E1

EA Epilobietea angustifolii, Galio-Urticetea

Fragaria vesca

E1

Rubus idaeus

E2

GU Urtica dioica

E1

AC Arabidetalia caeruleae

Soldanella alpina

Soldanella minima

E1

E1

TR Thlaspietea rotundifolii

Adenostyles glabra

Gymnocarpium robertianum

Astrantia carniolica

Heliosperma alpestre

Valeriana montana

Trisetum argenteum

Hieracium bifidum

Biscutella laevigata

Cystopteris montana

Aquilegia einseleana

Petasites paradoxus

Molopospermum peloponnesiacum subsp. bauhinii

Heracleum pollinianum

Saxifraga caesia

E1

E1

E1

E1

E1

Cy Cystopteridion fragilis

Valeriana tripteris

Asplenium viride

Carex brachystachys

Moehringia muscosa

Cystopteris fragilis

Cystopteris regia

E
E
E
E
E
E
E
E
E

E1

E1

E1

E1

E

E1

Physoplexido comosae-Saxifragion petraeae

Campanula cespitosa

Paederota bonarota

Saxifraga crustata

Potentilla nitida 


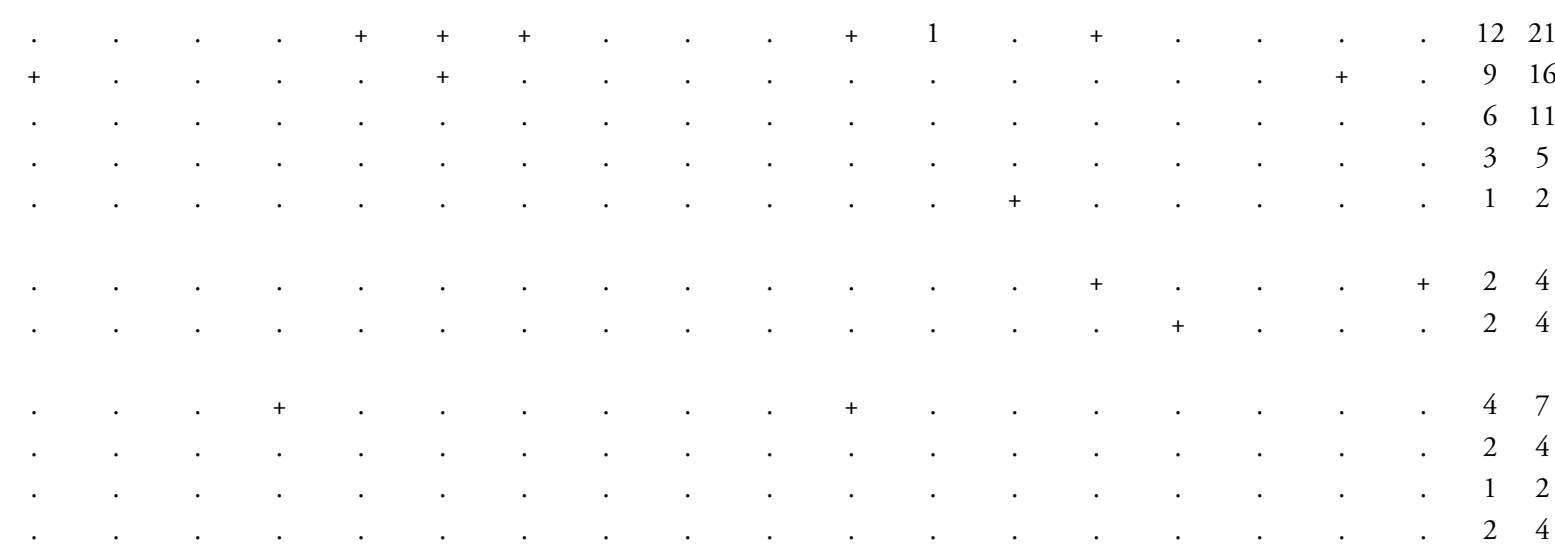

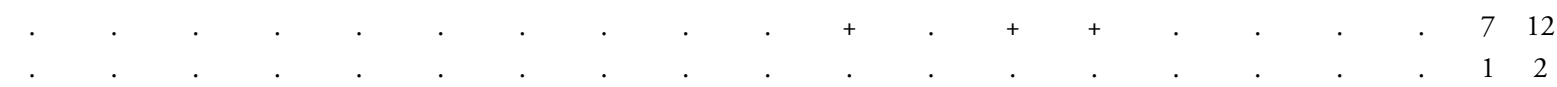

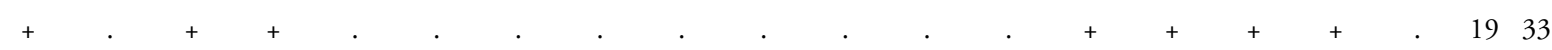

$$
\begin{aligned}
& +35 \\
& 12 \\
& 35 \\
& 12
\end{aligned}
$$

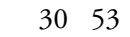

$$
\begin{aligned}
& 2646 \\
& 12 \quad 21 \\
& \begin{array}{ll}
10 & 18
\end{array} \\
& \begin{array}{ll}
6 & 11
\end{array} \\
& 47 \\
& 35 \\
& 35 \\
& 12 \\
& 24 \\
& 12 \\
& 12 \\
& 12 \\
& 12 \\
& \begin{array}{rllllllllllllllllllllll}
1 & . & + & . & 1 & + & + & 2 & 1 & . & + & 1 & 1 & 1 & 1 & 1 & 1 & . & 43 & 75 \\
+ & + & + & + & + & + & . & + & + & \mathrm{r} & . & + & + & + & + & . & + & . & 41 & 72 \\
+ & \cdot & \cdot & . & . & . & . & . & . & . & . & . & 1 & + & . & . & . & . & 8 & 14 \\
. & \cdot & \cdot & . & . & . & . & + & . & . & . & . & . & . & . & . & . & . & 8 & 14 \\
+ & . & . & . & . & . & . & . & . & . & . & . & . & . & . & . & . & . & 2 & 4 \\
. & . & . & . & . & . & . & . & . & . & . & . & . & . & . & . & . & . & 1 & 2
\end{array}
\end{aligned}
$$

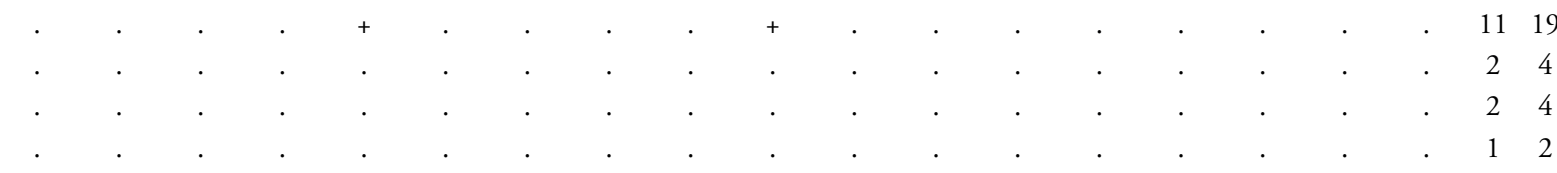


Campanula carnica

E1

Spiraea decumbens

E1

PC Potentilletalia caulescentis

Campanula cochleariifolia

Valeriana saxatilis

Potentilla caulescens

E1

E1

E1

AT Asplenietea trichomanis

Asplenium ruta-muraria

Kernera saxatilis

Asplenium trichomanes

Polypodium vulgare

ML Mosses and lichens (Mahovi in lišaji)

Tortella tortuosa

Ctenidium molluscum

Fissidens dubius

Dicranum scoparium

Schistidium apocarpum

Polytrichum formosum

Marchantia polymorpha

Peltigera canina

Isothecium alopecuroides

Rhytidiadelphus triquetrus

Cladonia pyxidata

Plagiochila porelloides

Peltigera leucophlebia

Hookeria lucens

Pseudoleskeella catenulata

Orthothecium rufescens

Cladonia sp.

Bartramia pomiformis

Neckera crispa

Rhizomnium punctatum

Cladonia rangiferina

Hylocomium splendens

Lobaria pulmonaria

Conocephalum conicum

Metzgeria furcata

Plagiothecium denticulatum

E1

Solorina saccata

Fu Fungi (Glive)

Laetiporus sulphureus agg. (L. horoniensis)

\section{Legend - Legenda}

ID Igor Dakskobler

AS Andrej Seliškar

AR Andrej Rozman

BZ Branko Zupan

A Limestone - apnenec

D Dolomite - dolomit

L Marlstone - laporovec

Re Rendzina - rendzina
Mo Moraine (Till) - morena (til)

Gr Gravel - grušč

JA Julian Alps - Julijske Alpe

KA Kamnik-Savinja Alps - Kamniško-Savinjske Alpe

I Italy (Italia, Italija)

Pr. Presence (number of relevés in which the species is presented) - število popisov, v katerih se pojavlja vrsta

Fr. Frequency in $\%$ - frekvenca $v \%$

Relevé No. 38, holotypus 


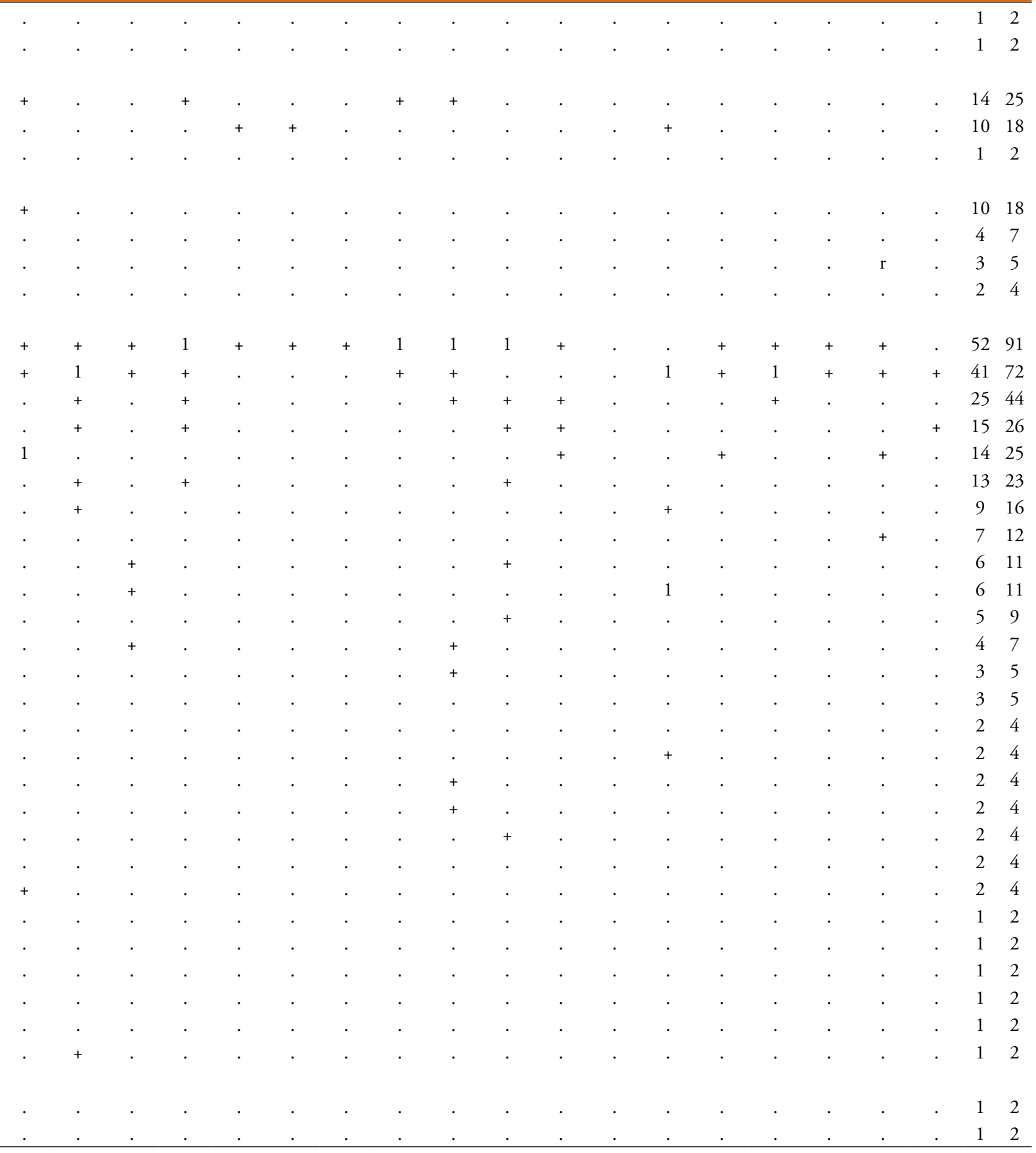


Table 2: Polysticho lonchitis-Fagetum cardaminetosum trifoliae var. Calamagrostis arundinacea - cluster 20.

\begin{tabular}{|c|c|c|c|c|c|c|c|c|c|c|c|c|c|c|c|c|}
\hline Number of relevé (Zaporedna številka & & 1 & 2 & 3 & 4 & 5 & 6 & 7 & 8 & 9 & 10 & 11 & 12 & 13 & 14 & 15 \\
\hline $\begin{array}{l}\text { Database number of relevé } \\
\text { (Delovna številka popisa) }\end{array}$ & & $\begin{array}{l}\text { J్ } \\
\stackrel{\widetilde{N}}{\text { ते }}\end{array}$ & 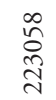 & 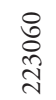 & 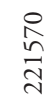 & 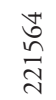 & 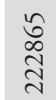 & 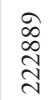 & $\begin{array}{l}\overrightarrow{\mathrm{J}} \\
\stackrel{\sim}{\sim}\end{array}$ & $\begin{array}{l}\text { ¿゙ } \\
\stackrel{\text { సे }}{\text { ते }}\end{array}$ & $\begin{array}{l}\stackrel{n}{\approx} \\
\hat{n} \\
\approx\end{array}$ & $\begin{array}{l}\stackrel{\sim}{\sim} \\
\stackrel{\sim}{\sim}\end{array}$ & 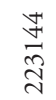 & $\begin{array}{l}\hat{\infty} \\
\infty \\
\infty \\
\hat{\sim}\end{array}$ & 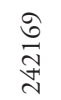 & $\underset{\underset{\sim}{\sim}}{\stackrel{\sim}{\sim}}$ \\
\hline Author of the relevé (Avtor popisa) & & ID & ID & ID & ID & ID & ID & ID & ID & ID & ID & ID & ID & ID & ID & ID \\
\hline Elevation in $\mathrm{m}$ (Nadmorska višina $\mathrm{v} \mathrm{m}$ ) & & $\stackrel{8}{\circ}$ & $\stackrel{\circ}{\underset{\varpi}{\leftrightarrows}}$ & $\stackrel{\stackrel{9}{+}}{\rightarrow}$ & $\stackrel{\widetilde{N}}{\sim}$ & $\begin{array}{l}\infty \\
\stackrel{\infty}{ \pm} \\
-1\end{array}$ & $\stackrel{\text { }}{\sim}$ & 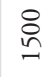 & $\underset{n}{\stackrel{2}{n}}$ & $\stackrel{\circ}{\stackrel{n}{n}}$ & $\stackrel{\infty}{\stackrel{\oplus}{+}}$ & $\underset{\&}{\stackrel{8}{+}}$ & 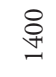 & $\stackrel{\infty}{\stackrel{\infty}{\leftrightarrows}}$ & 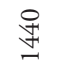 & 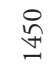 \\
\hline Aspect (Lega) & & $\geqslant$ & $\geqslant$ & 之 & 되 & 空 & 晷 & Z & Z & Z & 㟐 & 岂 & 岂 & 之 & 学 & 㭊 \\
\hline Slope in degrees (Nagib v stopinjah) & & 25 & 25 & 25 & 30 & 20 & 30 & 25 & 30 & 40 & 40 & 40 & 35 & 40 & 25 & 30 \\
\hline Parent material (Matična podlaga) & & A & A & A & A & A & A & A & $\mathrm{DA}$ & A & AG & A & A & A & A & A \\
\hline Soil (Tla) & & $\mathrm{Re}$ & $\operatorname{Re}$ & $\mathrm{Re}$ & $\operatorname{Re}$ & $\operatorname{Re}$ & $\operatorname{Re}$ & $\operatorname{Re}$ & $\mathrm{Re}$ & $\operatorname{Re}$ & $\operatorname{Re}$ & $\operatorname{Re}$ & $\operatorname{Re}$ & $\operatorname{Re}$ & $\operatorname{Re}$ & $\operatorname{Re}$ \\
\hline Stoniness in \% (Kamnitost $\mathrm{v} \%$ ) & & 30 & 30 & 40 & 30 & 40 & 30 & 10 & 10 & 30 & 5 & 50 & 20 & 40 & 30 & 60 \\
\hline \multicolumn{17}{|l|}{ Cover in \% (Zastiranje v \%): } \\
\hline Upper tree layer (Zgornja drevesna plast) & $\mathrm{E} 3 \mathrm{~b}$ & 70 & 80 & 80 & 70 & 90 & 70 & 70 & 80 & 80 & 70 & 80 & 80 & 70 & 70 & 70 \\
\hline Lower tree layer (Spodnja drevesna plasti) & E3a & . & . & . & . & . & . & . & . & . & . & . & 10 & 10 & . & . \\
\hline Shrub layer (Grmovna plast) & E2 & 30 & 30 & 20 & 40 & 5 & 15 & 20 & 30 & 20 & 10 & 10 & 10 & 30 & 50 & 50 \\
\hline Herb layer (Zeliščna plast) & E1 & 30 & 40 & 40 & 70 & 70 & 70 & 80 & 80 & 60 & 80 & 60 & 70 & 70 & 60 & 60 \\
\hline Moss layer (Mahovna plast) & E0 & 10 & 20 & 15 & 10 & 10 & 10 & 10 & 10 & 10 & 5 & 30 & 15 & 20 & 20 & 20 \\
\hline Maximum tree diameter (Maks. premer dreves) & $\mathrm{cm}$ & 15 & 25 & 25 & 30 & 30 & 40 & 15 & 40 & 35 & 35 & 40 & 45 & 45 & 50 & 40 \\
\hline Maximum tree height (Maksimalna višina dreves) & $\mathrm{m}$ & 6 & 10 & 8 & 10 & 7 & 15 & 5 & 10 & 14 & 10 & 12 & 14 & 10 & 16 & 10 \\
\hline Number of species (Število vrst) & & 73 & 66 & 71 & 54 & 72 & 72 & 80 & 61 & 68 & 77 & 86 & 68 & 90 & 71 & 77 \\
\hline Relevé area (Velikost popisne ploskve) & $\mathrm{m}^{2}$ & 200 & 400 & 400 & 200 & 400 & 400 & 400 & 200 & 400 & 400 & 200 & 200 & 200 & 400 & 400 \\
\hline Date of taking relevé (Datum popisa) & & 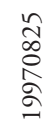 & 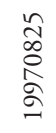 & 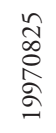 & 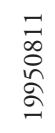 & 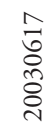 & $\begin{array}{l}\vec{N} \\
\hat{\sigma} \\
\aleph \\
\approx\end{array}$ & $\begin{array}{l}\Xi \\
\Xi \\
\precsim \\
\precsim\end{array}$ & 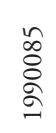 & 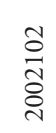 & $\begin{array}{l}\stackrel{n}{\circ} \\
\stackrel{\circ}{\circ} \\
\stackrel{\infty}{\varrho}\end{array}$ & 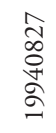 & $\begin{array}{l}\hat{A} \\
\infty \\
\stackrel{+}{+} \\
\stackrel{+}{\sigma}\end{array}$ & 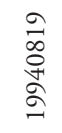 & 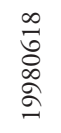 & 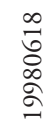 \\
\hline Day (Dan) & & 25 & 25 & 25 & 11 & 17 & 21 & 11 & 5 & 2 & 13 & 27 & 27 & 19 & 18 & 18 \\
\hline Month (Mesec) & & 8 & 8 & 8 & 8 & 6 & 7 & 10 & 8 & 10 & 9 & 8 & 8 & 8 & 6 & 6 \\
\hline Year (Leto) & & $\widehat{\widehat{\partial}}$ & $\widehat{\widehat{ૂ}}$ & $\widehat{\widehat{\Omega}}$ & 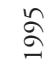 & 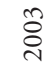 & 气ૂ & ڤ & ڤ & ญิ & $\stackrel{\infty}{\beth}$ & $\stackrel{+2}{2}$ & 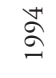 & 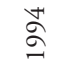 & $\stackrel{\infty}{\check{\beth}}$ & $\stackrel{\infty}{\check{\beth}}$ \\
\hline Locality (Nahajališče) & & 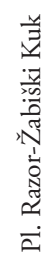 & 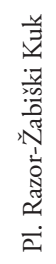 & 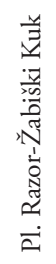 & 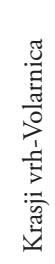 & 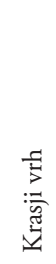 & 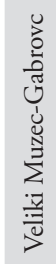 & 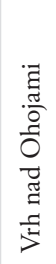 & 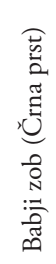 & 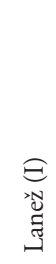 & 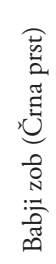 & 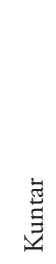 & 泀 & 胥 & 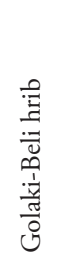 & 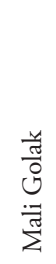 \\
\hline Mountain range (Pogorje) & & JA & JA & JA & JA & JA & JA & JA & JA & JA & JA & JA & JA & JA & TG & TG \\
\hline Quadrant (Kvadrant) & & $\begin{array}{l}\frac{\mathbb{N}}{\infty} \\
\stackrel{+}{\alpha} \\
\hat{\sigma}\end{array}$ & $\begin{array}{l}\frac{+}{\infty} \\
\stackrel{\infty}{+} \\
\stackrel{N}{\sigma}\end{array}$ & $\begin{array}{l}\frac{+}{\infty} \\
\stackrel{\infty}{+} \\
\stackrel{\alpha}{\sigma}\end{array}$ & $\frac{\stackrel{N}{N}}{\stackrel{N}{a}}$ & $\frac{\stackrel{N}{N}}{\stackrel{+}{\alpha}}$ & \begin{tabular}{l}
$\sqrt{5}$ \\
\multirow{+}{*}{} \\
N
\end{tabular} & $\begin{array}{l}\vec{\sigma} \\
\stackrel{+}{\alpha} \\
\hat{\sigma}\end{array}$ & $\frac{\stackrel{+}{a}}{\stackrel{+}{a}}$ & 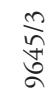 & $\frac{\stackrel{+}{a}}{\stackrel{+}{\alpha}}$ & $\begin{array}{l}\vec{\infty} \\
\stackrel{+}{+} \\
\stackrel{\alpha}{\sigma}\end{array}$ & $\begin{array}{l}\vec{\Phi} \\
\stackrel{\infty}{+} \\
\stackrel{\alpha}{\sigma}\end{array}$ & $\frac{\stackrel{+}{a}}{\stackrel{+}{a}}$ & $\frac{\vec{a}}{\stackrel{+}{8}}$ & वे \\
\hline Coordinate (Koordinate) GK Y (D-48) & $\mathrm{m}$ & $\begin{array}{l}n \\
\stackrel{n}{\infty} \\
\hat{o} \\
\hat{+}\end{array}$ & $\begin{array}{l}\hat{n} \\
\hat{n} \\
\hat{\sigma}\end{array}$ & 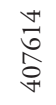 & $\begin{array}{l}\text { ర్ర } \\
\text { స్ } \\
\text { }\end{array}$ & 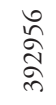 & 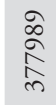 & $\begin{array}{l}\infty \\
\text { రి } \\
\text { مे }\end{array}$ & $\begin{array}{l}\stackrel{\infty}{\cong} \\
\stackrel{\infty}{\approx} \\
\stackrel{F}{*}\end{array}$ & 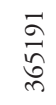 & 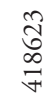 & \begin{tabular}{l}
$\bar{D}$ \\
$\varkappa$ \\
$\&$ \\
\multirow{+}{*}{}
\end{tabular} & 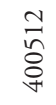 & $\begin{array}{l}\Xi \\
\precsim \\
\precsim\end{array}$ & $\begin{array}{l}\stackrel{n}{\infty} \\
\stackrel{\sim}{\sim} \\
\exists\end{array}$ & 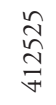 \\
\hline Coordinate (Koordinate) GK X (D-48) & $\mathrm{m}$ & $\begin{array}{l}\stackrel{2}{\infty} \\
\stackrel{\Xi}{\beth}\end{array}$ & $\frac{\infty}{\stackrel{\sim}{\bumpeq}}$ & $\begin{array}{l}\hat{\Omega} \\
\stackrel{\beth}{\beth}\end{array}$ & $\begin{array}{l}\stackrel{m}{\infty} \\
\stackrel{\infty}{N}\end{array}$ & 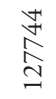 & 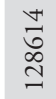 & 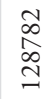 & 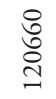 & 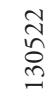 & 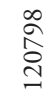 & 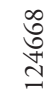 & 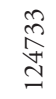 & $\begin{array}{l}\underset{\widetilde{N}}{\infty} \\
\underset{\sim}{\sim}\end{array}$ & $\underset{\widetilde{\sigma}}{\tilde{\sigma}}$ & $\hat{\tilde{n}}$ \\
\hline
\end{tabular}

Diagnostic species of the association (Diagnostične vrste asociacije)

BA Sorbus chamaemespilus

ML Paraleucobryum sauteri

EP Rhododendron hirsutum

$\begin{array}{lllllllllllllllll}\mathrm{E} 1 & + & + & + & + & \mathrm{r} & + & + & + & + & + & 1 & \mathrm{r} & 1 & + & + \\ \mathrm{E} 2 & + & + & + & 1 & 2 & 1 & + & 1 & 1 & . & 1 & + & + & . & 1 \\ \mathrm{E} 2 & + & \cdot & + & + & + & + & + & . & + & . & . & . & + & + & + \\ \mathrm{E} 0 & \cdot & \cdot & \cdot & \cdot & \cdot & + & 1 & + & + & + & 1 & . & 1 & + & 1 \\ \mathrm{E} 2 & + & 1 & + & + & \mathrm{r} & 1 & 1 & + & + & . & + & . & 1 & . & 1\end{array}$


Tabela 2: Polysticho lonchitis-Fagetum cardaminetosum trifoliae var. Calamagrostis arundinacea - skupina 20.

$\begin{array}{llllllllllllllllllllllllllll}16 & 17 & 18 & 19 & 20 & 21 & 22 & 23 & 24 & 25 & 26 & 27 & 28 & 29 & 30 & 31 & 32 & 33 & 34 & 35 & 36 & 37 & 38 & 39 & 40 & 41 & \text { Pr. } & \text { Fr. }\end{array}$

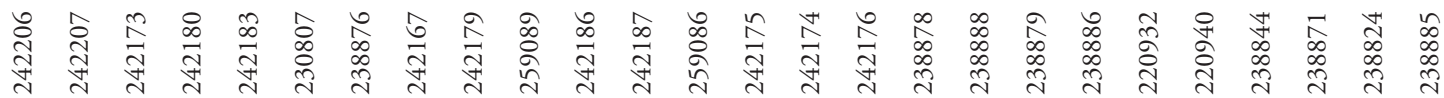
ID ID ID ID ID ID ID ID ID ID ID ID ID ID ID ID ID ID ID ID ID ID ID ID ID ID

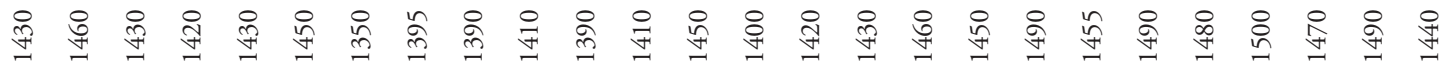

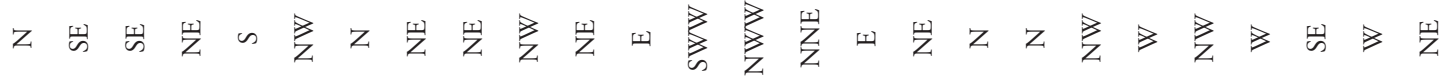
$\begin{array}{llllllllllllllllllllllllll}25 & 25 & 25 & 30 & 30 & 35 & 15 & 35 & 35 & 30 & 35 & 30 & 30 & 35 & 30 & 35 & 30 & 45 & 20 & 35 & 35 & 35 & 25 & 30 & 35 & 30\end{array}$

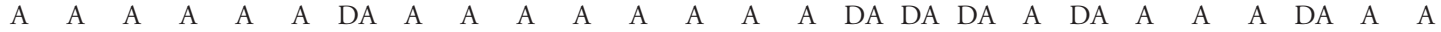
$\operatorname{Re} \operatorname{Re} \operatorname{Re} \operatorname{Re} \operatorname{Re} \operatorname{Re} \operatorname{Re} \operatorname{Re} \operatorname{Re} \operatorname{Re} \operatorname{Re} \operatorname{Re} \operatorname{Re} \operatorname{Re} \operatorname{Re} \operatorname{Re} \operatorname{Re} \operatorname{Re} \operatorname{Re} \operatorname{Re} \operatorname{Re} \operatorname{Re} \operatorname{Re} \operatorname{Re} \operatorname{Re} \operatorname{Re}$ $\begin{array}{llllllllllllllllllllllllll}30 & 20 & 40 & 30 & 60 & 30 & 50 & 40 & 60 & 40 & 60 & 60 & 30 & 70 & 60 & 40 & 30 & 40 & 20 & 30 & 20 & 30 & 50 & 40 & 60 & 40\end{array}$

$\begin{array}{llllllllllllllllllllllllll}80 & 90 & 90 & 90 & 80 & 80 & 80 & 80 & 80 & 80 & 80 & 80 & 80 & 70 & 80 & 80 & 70 & 60 & 80 & 80 & 90 & 70 & 70 & 80 & 80 & 80\end{array}$ $\begin{array}{llllllllllllllllllllllllll}10 & 5 & . & . & 10 & 10 & 20 & 10 & 5 & 10 & 10 & 10 & 20 & 10 & . & 10 & 10 & 10 & 5 & . & . & 10 & 10 & 20 & 10 & .\end{array}$ $\begin{array}{llllllllllllllllllllllllll}30 & 10 & 30 & 20 & 30 & 20 & 10 & 30 & 30 & 10 & 30 & 20 & 10 & 40 & 30 & 30 & 20 & 20 & 10 & 20 & 10 & 30 & 20 & 10 & 10 & 30\end{array}$ $\begin{array}{llllllllllllllllllllllllll}80 & 70 & 80 & 60 & 60 & 70 & 60 & 70 & 50 & 70 & 60 & 70 & 70 & 60 & 70 & 70 & 80 & 70 & 70 & 80 & 70 & 80 & 70 & 70 & 60 & 80\end{array}$ $\begin{array}{llllllllllllllllllllllllll}15 & 10 & 10 & 10 & 20 & 20 & 20 & 20 & 30 & 20 & 20 & 10 & 10 & 20 & 10 & 10 & 20 & 20 & 10 & 15 & 10 & 20 & 10 & 10 & 10 & 20\end{array}$ $\begin{array}{llllllllllllllllllllllllll}35 & 35 & 40 & 50 & 60 & 70 & 70 & 45 & 60 & 40 & 35 & 40 & 45 & 45 & 40 & 40 & 35 & 35 & 50 & 35 & 40 & 60 & 50 & 50 & 45 & 30\end{array}$ $\begin{array}{llllllllllllllllllllllllll}10 & 10 & 12 & 14 & 14 & 20 & 17 & 12 & 16 & 15 & 12 & 10 & 15 & 14 & 10 & 12 & 15 & 10 & 16 & 7 & 15 & 18 & 12 & 12 & 15 & 7\end{array}$ $\begin{array}{llllllllllllllllllllllllll}64 & 59 & 63 & 85 & 71 & 80 & 72 & 82 & 84 & 65 & 77 & 72 & 74 & 75 & 77 & 79 & 70 & 80 & 61 & 75 & 61 & 53 & 99 & 80 & 68 & 81\end{array}$ $400400400400400400400400400400400400400400400400400200400 \quad 200400400400400400 \quad 200$

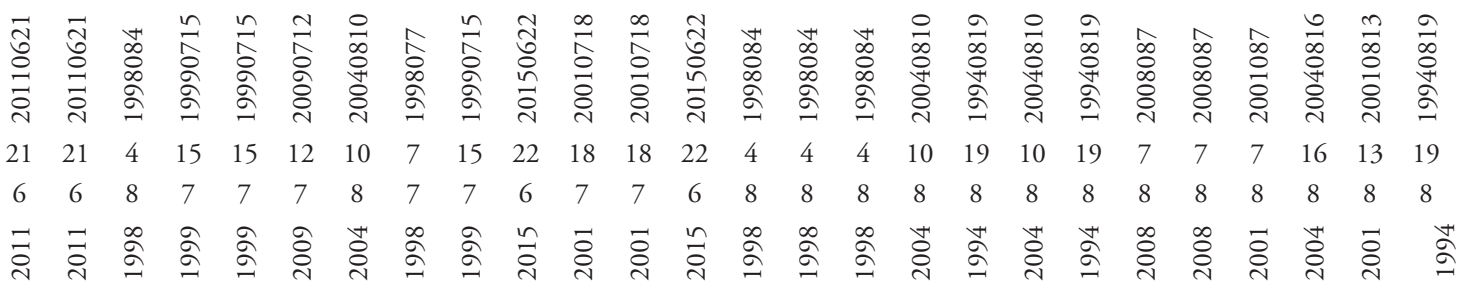

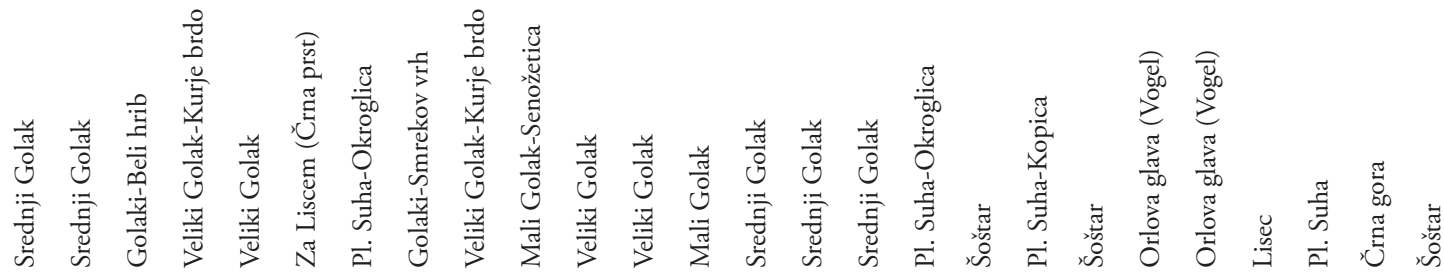
TG TG TG TG TG JA JA TG TG TG TG TG TG TG TG TG JA JA JA JA JA JA JA JA JA JA

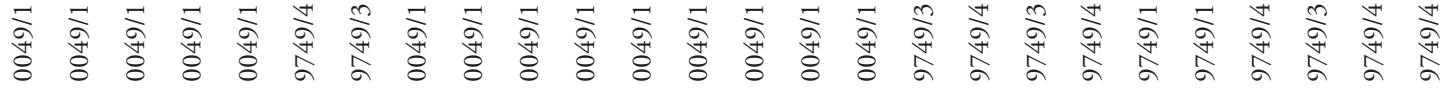

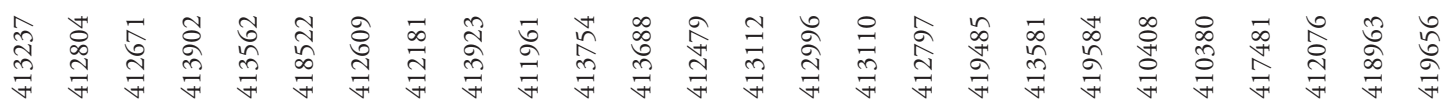

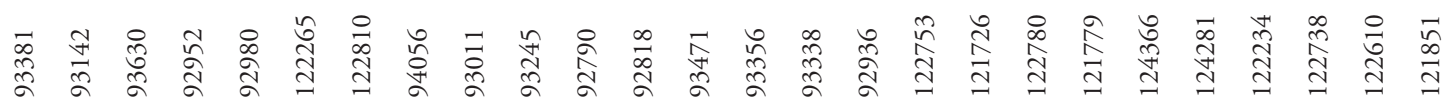

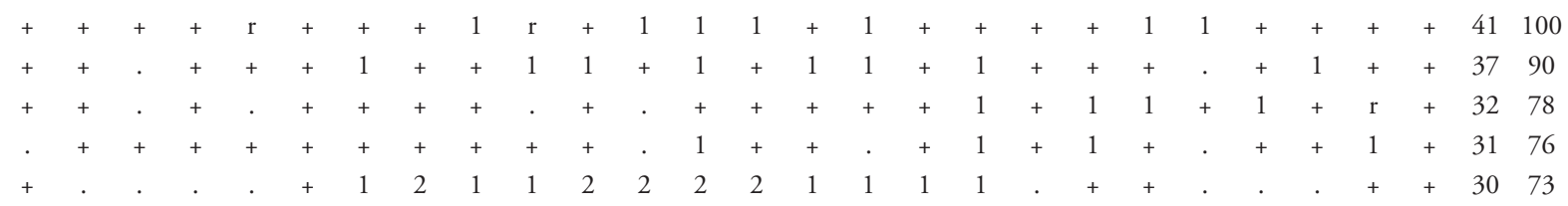


Number of relevé (Zaporedna številka popisa)

BA Salix appendiculata

ES Aster bellidiastrum

PS Paederota lutea

VP Luzula sylvatica

VP Lonicera caerulea

MuA Aconitum lycoctonum subsp. ranunculifolium

CA Laserpitium peucedanoides

EP Pinus mugo

EP Rhodothamnus chamaecistus

VP Homogyne alpina

CF Carex ferruginea

CA Festuca calva

Differential species of lower units (Razlikovalnice nižjih enot)

VP Calamagrostis arundinacea

AF Cardamine trifolia

QF Anemone nemorosa

FS Dryopteris flix-mas

FS Actaea spicata

MuA Phyteuma ovatum

AF Aremonio-Fagion

Cardamine enneaphyllos

Cyclamen purpurascens

Omphalodes verna

Euphorbia carniolica

Rhamnus fallax

Anemone trifolia

Knautia drymeia

Helleborus niger

Lamium orvala

$\begin{array}{lllllllllllllllll}\text { E1 } & 1 & 1 & 1 & 2 & 2 & 1 & 2 & 3 & 2 & 4 & 3 & 3 & 1 & 2 & 2 \\ \text { E1 } & 1 & 1 & 1 & 1 & . & 1 & 1 & 1 & + & 1 & . & . & 1 & 1 & 1 \\ \text { E1 } & + & + & + & . & . & . & . & 1 & . & + & + & 1 & + & 1 & 1 \\ \text { E1 } & . & + & + & + & + & + & + & + & 1 & + & + & + & + & + & + \\ \text { E1 } & + & \text { r } & . & . & . & + & . & . & . & + & + & + & . & + & + \\ \text { E1 } & . & . & . & . & . & . & . & + & + & + & . & + & 1 & . & .\end{array}$

EC Erythronio-Carpinion

Primula vulgaris

TA Tilio-Acerion

Aruncus dioicus

Acer pseudoplatanus

Acer pseudoplatanus

Acer pseudoplatanus

Polystichum aculeatum

Adoxa moschatellina

Polystichum x illyricum

Tilia platyphyllos

Ulmus glabra

Polystichum $x$ luerssenii

FS Fagetalia sylvaticae

Fagus sylvatica

Fagus sylvatica

Fagus sylvatica

Lonicera alpigena

Prenanthes purpurea

Galium laevigatum

Daphne mezereum

Lilium martagon

Galeobdolon flavidum

Mercurialis perennis

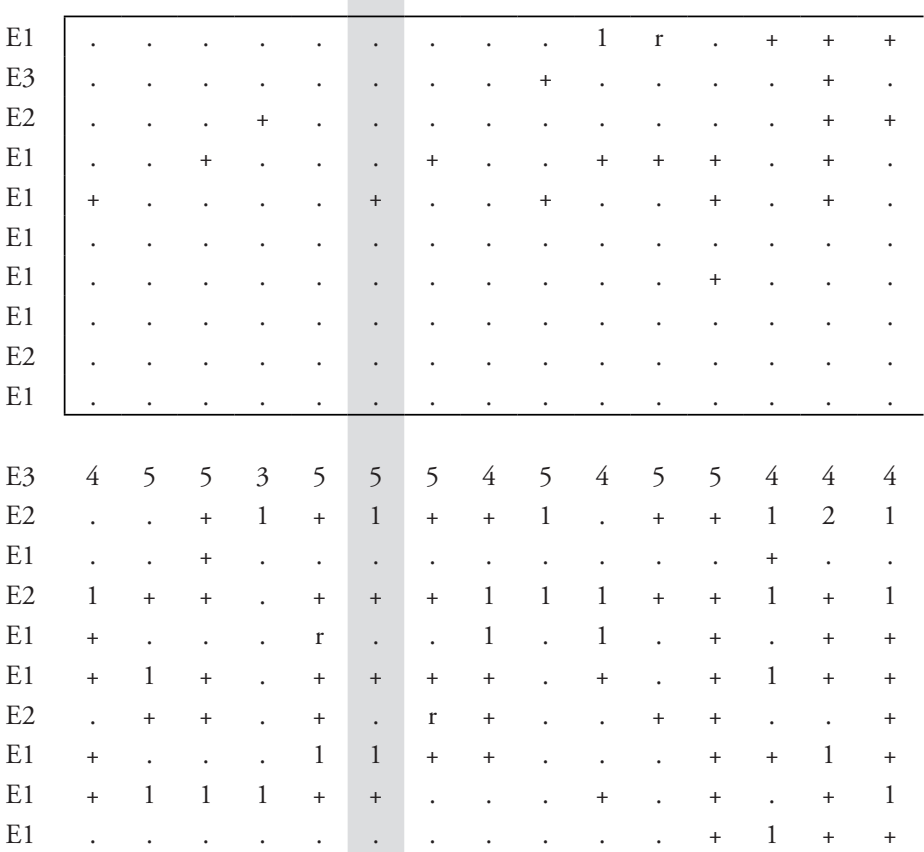




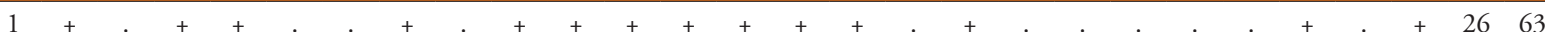

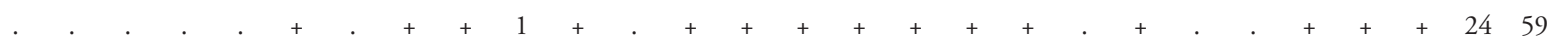
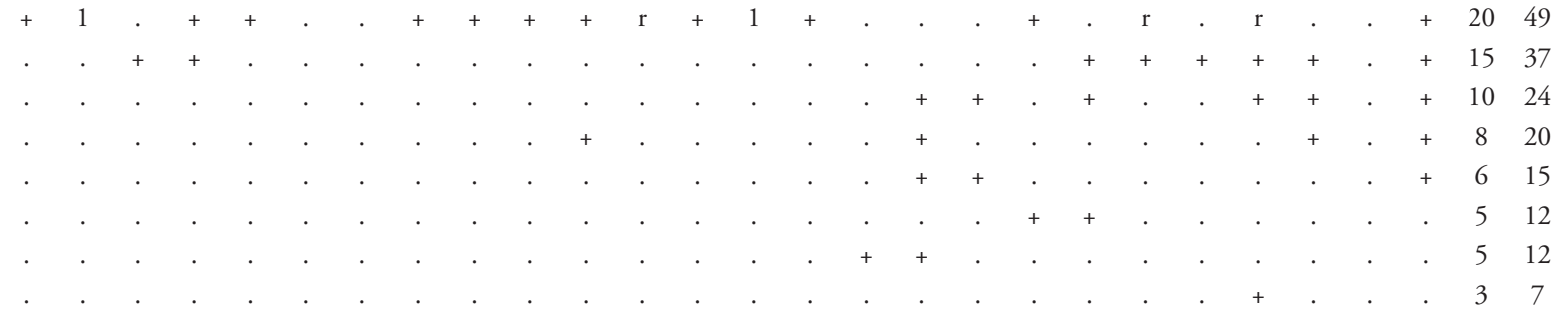

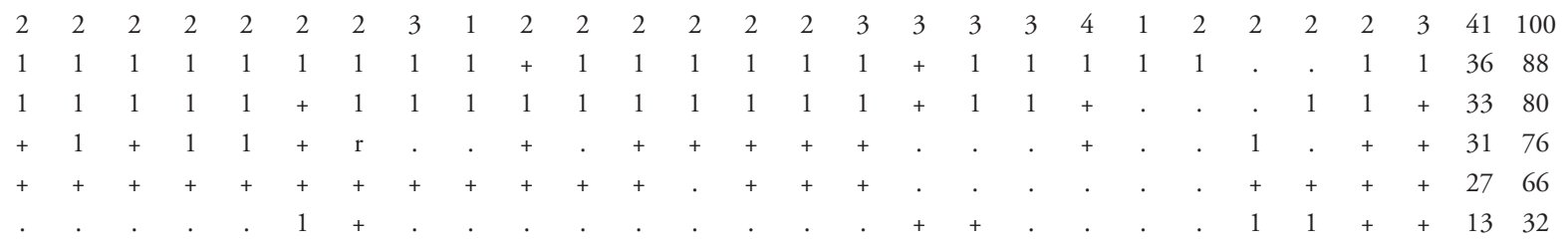
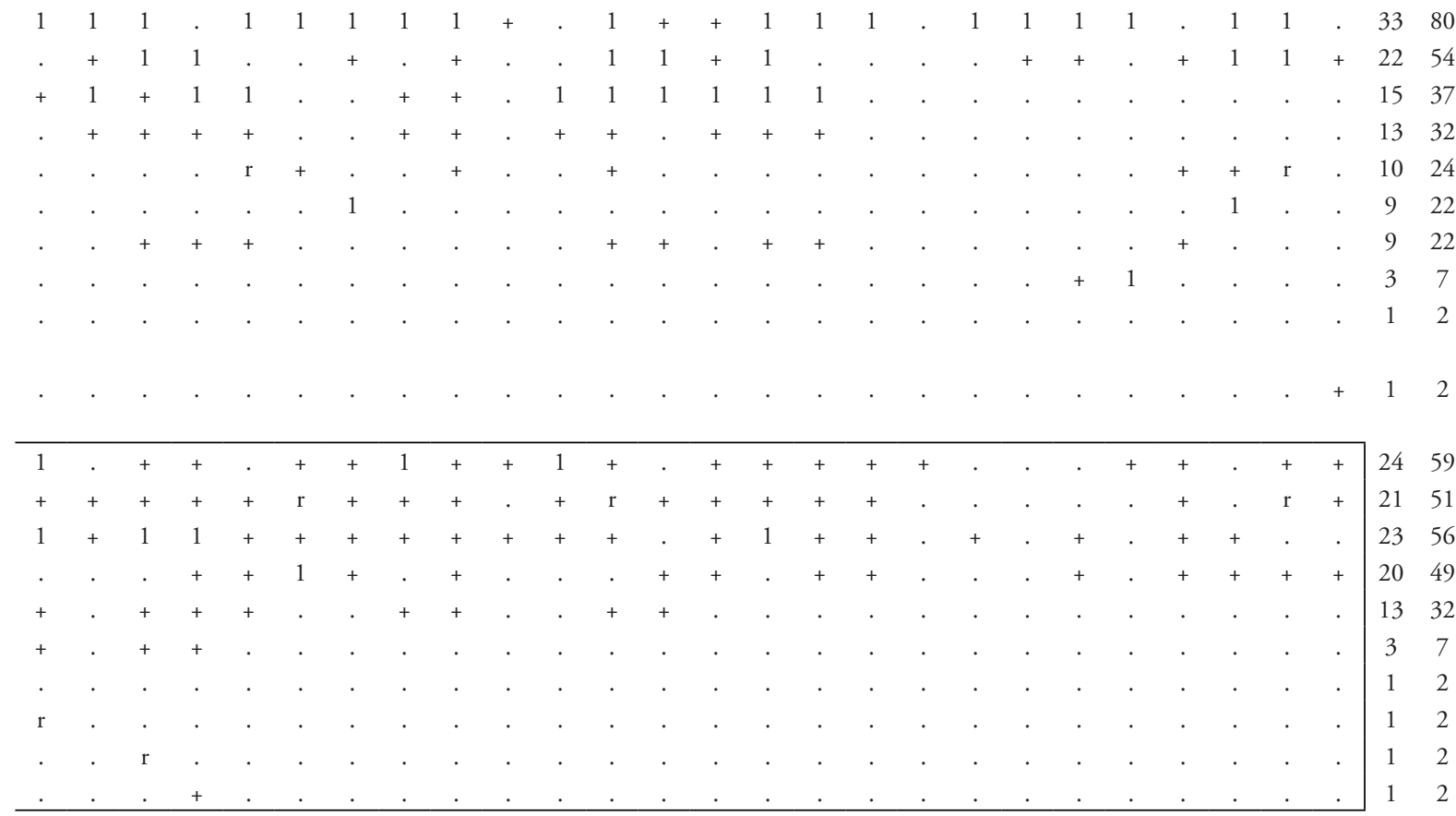

\begin{tabular}{|c|c|c|c|c|c|c|c|c|c|c|c|c|c|c|c|c|c|c|c|c|c|c|c|c|c|c|}
\hline 4 & 5 & 5 & 5 & 5 & 5 & 5 & 5 & 5 & 5 & 5 & 4 & 5 & 5 & 5 & 5 & 4 & 4 & 5 & 5 & 5 & 4 & 4 & 5 & 5 & 5 & 41 \\
\hline 1 & + & 1 & 1 & 1 & 1 & 1 & 1 & 1 & . & 1 & 1 & 1 & 1 & 1 & 1 & 1 & 1 & 1 & + & 1 & 1 & 1 & 1 & 1 & 1 & 37 \\
\hline & + & . & . & . & . & 1 & . & + & + & + & + & + & . & . & + & 1 & . & + & . & 1 & & . & . & & & 14 \\
\hline & 1 & $r$ & 1 & 1 & + & 1 & 1 & 1 & + & + & + & + & + & + & 1 & . & + & + & 1 & 1 & & 1 & + & & & 39 \\
\hline & + & 1 & 1 & + & 1 & + & + & + & 1 & + & + & + & 1 & 1 & 1 & 1 & 1 & 1 & + & 1 & & 1 & 1 & 1 & 1 & 32 \\
\hline & 1 & 1 & + & + & 1 & + & + & . & . & . & 1 & . & . & 1 & 1 & + & + & . & 1 & + & & 1 & 1 & & & 31 \\
\hline & . & 1 & + & + & . & + & + & + & + & + & 1 & 1 & + & 1 & 1 & 1 & + & 1 & + & + & & + & + & & & 30 \\
\hline & + & + & + & + & + & . & . & + & 1 & + & + & + & + & + & + & + & + & & + & + & & + & + & & & 30 \\
\hline & 1 & 1 & 1 & 1 & + & + & + & + & . & + & + & 1 & . & + & + & . & . & & . & 1 & & + & + & & & 28 \\
\hline & 1 & 1 & 1 & 1 & . & 1 & + & 1 & 1 & 1 & 1 & 1 & 1 & 1 & 1 & . & 1 & 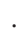 & 1 & 1 & 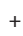 & + & 1 & 1 & 2 & 27 \\
\hline
\end{tabular}


Melica nutans

Symphytum tuberosum

Paris quadrifolia

Epilobium montanum

Phyteuma spicatum subsp. coeruleum

Lathyrus vernus

Mycelis muralis

Heracleum sphondylium

Luzula nivea

Carex sylvatica

Pulmonaria officinalis

Festuca altissima

Petasites albus

Galium odoratum

Laburnum alpinum

Laburnum alpinum

Viola reichenbachiana

Scrophularia nodosa

QP Quercetalia pubescenti-petraeae

Sorbus aria (Aria edulis)

Sorbus aria (Aria edulis)

Convallaria majalis

QF

\section{Querco-Fagetea}

Hepatica nobilis

Carex digitata

Poa nemoralis

Viola riviniana

Dactylorhiza fuchsii

Corylus avellana

VP Vaccinio-Piceetea

Gentiana asclepiadea

Vaccinium myrtillus

Rosa pendulina

Solidago virgaurea

Veronica urticifolia

Aposeris foetida

Maianthemum bifolium

Lonicera nigra

Oxalis acetosella

Gymnocarpium dryopteris

Phegopteris connectilis

Huperzia selago

Dryopteris dilatata

Saxifraga cuneifolia

Homogyne sylvestris

Picea abies

Picea abies

Picea abies

Hieracium murorum

Luzula luzuloides

Abies alba

Abies alba

Abies alba

\section{E1}

E1

E1

E1

E1

E1

E1

E1

E1

E1

E3

E2

E1

E1

E2

E1

E1

E1

E1

E1

E1

E2

E1

E1

E2

E1

E1

E1

E1

E2

E1

E1

E1

E1

E1

E1

E1 

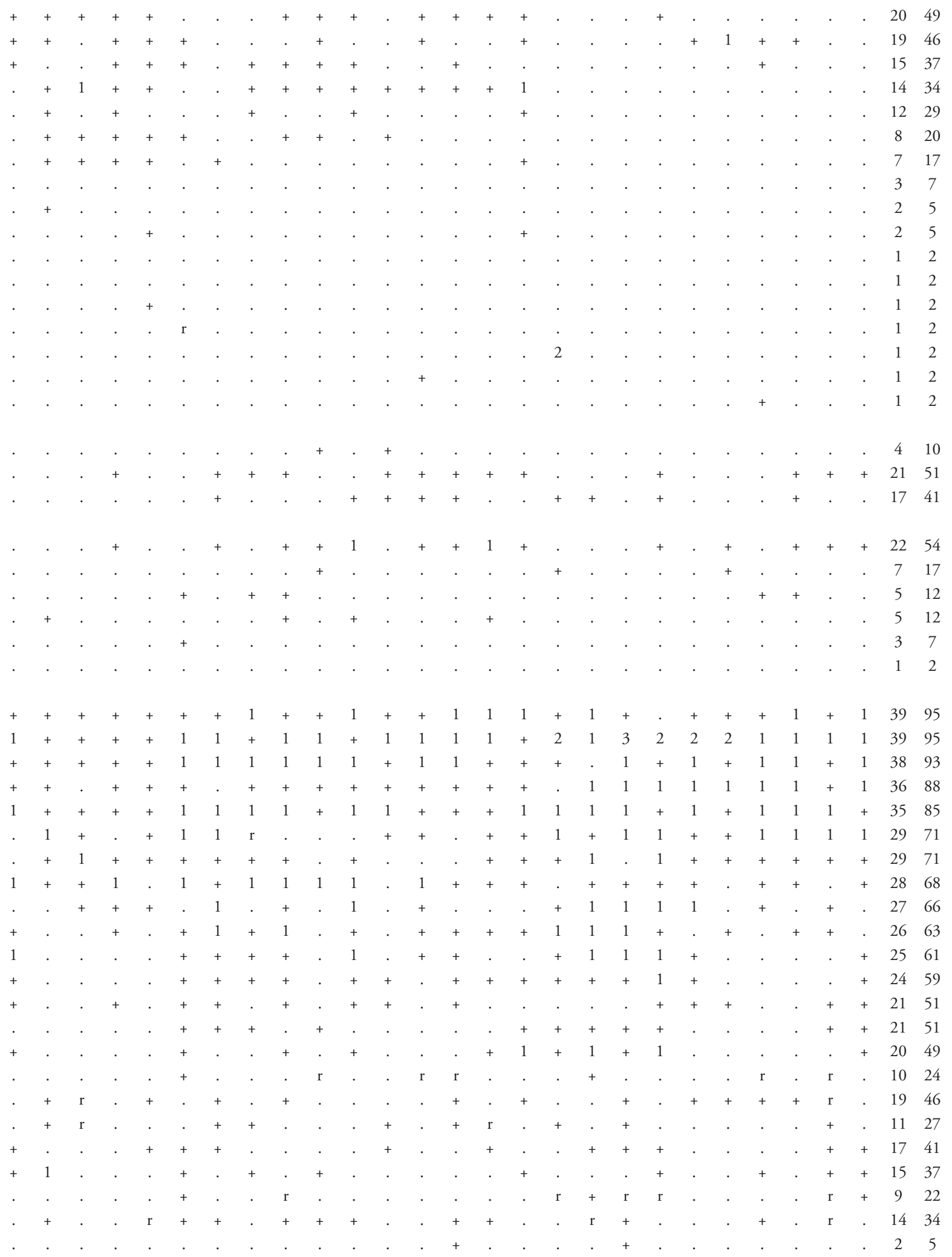
Dryopteris expansa

Lycopodium annotinum

Calamagrostis villosa

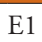

Vaccinium vitis-idaea

Larix decidua

Larix decidua

Melampyrum sylvaticum

Luzula pilosa

Luzula luzulina

Corallorhiza trifida

Goodyera repens

EP Erico-Pinetea

Rubus saxatilis

Aquilegia nigricans

Erica carnea

Calamagrostis varia

Carex ornithopoda

Peucedanum austriacum subsp. rablense

SSC Sambuco-Salicion capreae, Rhamno-Prunetea

Sorbus aucuparia

Sorbus aucuparia

Sorbus aucuparia

Sambucus racemosa

Rosa sp.

BA Betulo-Alnetea

Ribes alpinum

Alnus viridis

Sorbus austriaca

Salix waldsteiniana

Salix glabra

Ribes uva-crispa

Juniperus sibirica

MuA Mulgedio-Aconitetea

Polygonatum verticillatum

Athyrium filix-femina

Veratrum album

Thalictrum aquilegiifolium

Ranunculus platanifolius

Viola biflora

Geranium sylvaticum

Saxifraga rotundifolia

Chaerophyllum villarsii

Doronicum austriacum

Pleurospermum austriacum

Senecio ovatus

Adenostyles alliariae

Geum rivale

Myrrhis odorata

Senecio cacaliaster

Hypericum maculatum

Chaerophyllum hirsutum

Tanacetum corymbosum subsp. clusii

Allium victorialis

\section{E1}

E1

E1

E1

E2

E1

E1

E1

E1

E1

E1

E1

E1

E1

E1

E1

E3

E2

E1

E2

E2

E2

E2

E2

E2

E2

E2

E2

E1

E1

E1

E1

E1

E1

E1

E1

E1

E1

E1

E1

E1

E1

E1

E1

E1

E1

E1

E1 

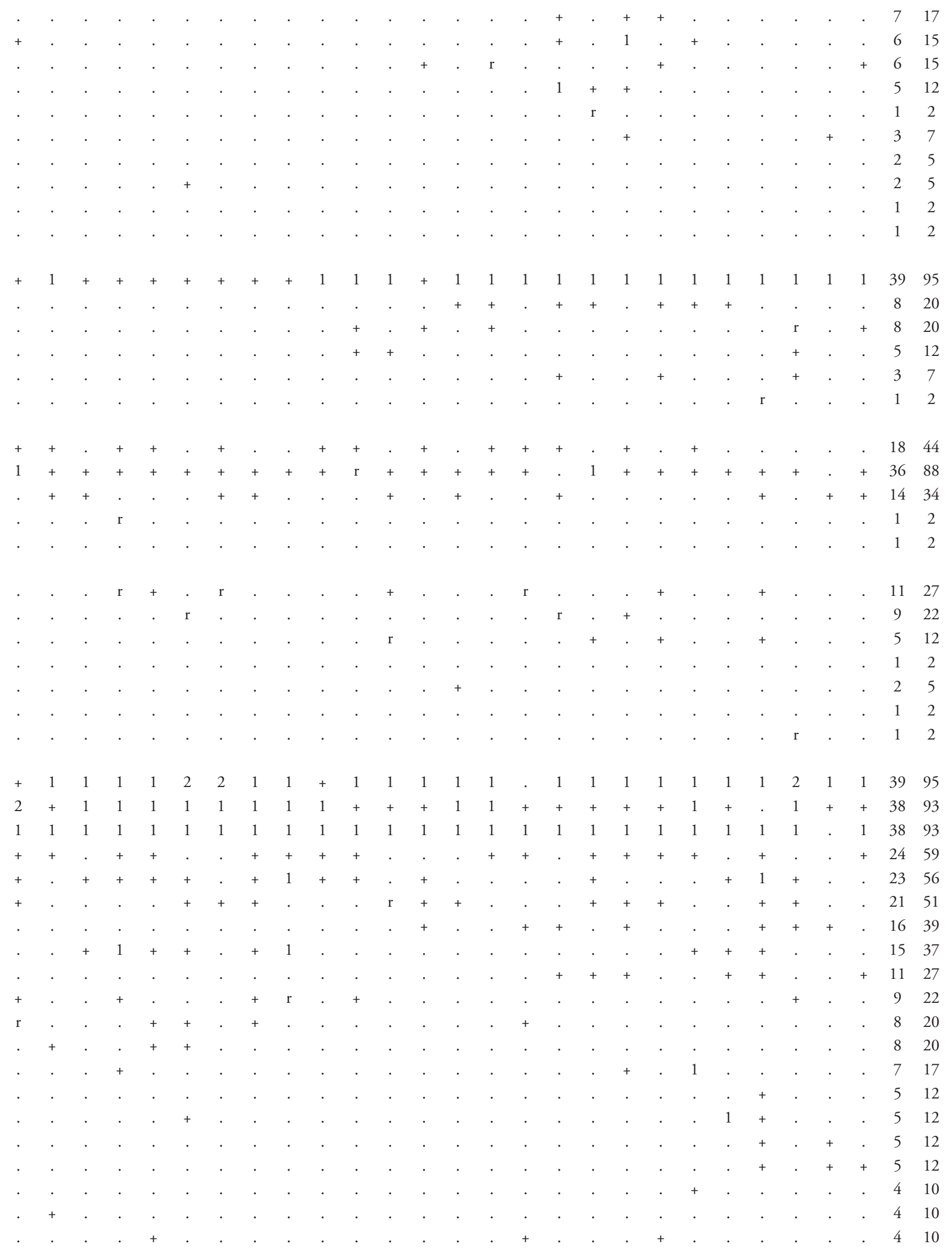
Centaurea montana

\section{E1}

Primula elatior

Aconitum angustifolium

Lathyrus occidentalis var. montanus

Poa hybrida

Aconitum degenii subsp. paniculatum

Cicerbita alpina

Serratula macrocephala

Senecio nemorensis

Crepis pyrenaica

Rumex arifolius

CA Caricion austroalpinae

Pulsatilla alpina subsp. austroalpina

Gentiana lutea subsp. symphyandra

CF Caricion ferrugineae

Knautia longifolia

Cerastium subtriflorum

ES Elyno-Seslerietea

Sesleria caerulea

Betonica alopecuros

Campanula witasekiana

Astrantia bavarica

Phyteuma orbiculare

Helianthemum nummularium subsp. grandiflorum

Senecio abrotanifolius

Pimpinella alpina

Hieracium villosum

Scabiosa lucida subsp. stricta

Ranunculus carinthiacus

Anemone narcissiflora

Phleum hirsutum

NS Nardion strictae, Juncetea trifidi

Campanula scheuchzeri

Potentilla erecta

Selaginella helvetica

CD Caricetalia davallianae

Parnassia palustris

FB Festuco-Brometea

Cirsium erisithales

Koeleria pyramidata

Buphthalmum salicifolium

Carlina acaulis

TG Trifolio-Geranietea

Laserpitium latifolium

Digitalis grandiflora

Grafia golaka

Iris graminea

Vicia sylvatica

Verbascum lanatum

$\mathrm{PaT}$ Poo alpinae-Trisetetalia

Poa alpina

Ranunculus nemorosus

Trollius europaeus 


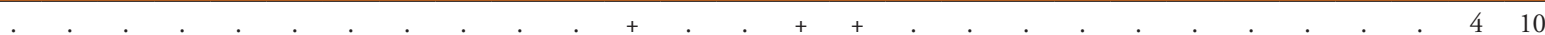

37

37

25

25

25

12

12

$\begin{array}{ll}1 & 2\end{array}$

12

12

$\begin{array}{ll}1 & 2 \\ 1 & 2\end{array}$

$\begin{array}{ll}1 & 2 \\ 1 & 2\end{array}$

2

$14 \quad 34$

$+\quad 922$

$8 \quad 20$

615

$\begin{array}{ll}4 & 10\end{array}$

3 7

$\begin{array}{ll}1 & 2\end{array}$

25

12

$\begin{array}{ll}1 & 2\end{array}$

12

12

12

$12 \quad 29$

12

2 
MA Molinio-Arrhenatheretea

Angelica sylvestris

Crepis paludosa

Festuca rubra agg.

Galium album

Dactylis glomerata

E1

E1

E1

E1

EA Epilobietea angustifolii, Galio-Urticetea

Rubus idaeus

Fragaria vesca

Hypericum hirsutum

Galeopsis speciosa

Cirsium eriophorum

AC Arabidetalia caeruleae

Soldanella alpina

TR Thlaspietea rotundifolii

Adenostyles glabra

Dryopteris villarii

Astrantia carniolica

Heracleum pollinianum

Arabis alpina

Trisetum argenteum

Ligusticum seguieri

Gymnocarpium robertianum

Heliosperma alpestre

Cy Cystopteridion fragilis

Asplenium viride

Valeriana tripteris

Cystopteris fragilis

Moehringia muscosa

Cystopteris regia

Carex brachystachys

Sedum hispanicum

Heliosperma pusillum

PS Physoplexido comosae-Saxifragion petraeae

Phyteuma scheuchzeri subsp. columnae

Saxifraga crustata

Campanula carnica

Primula carniolica

PC Potentilletalia caulescentis

Valeriana saxatilis

Campanula cochleariifolia

Festuca stenantha

AT Asplenietea trichomanis

Asplenium ruta-muraria

Polypodium vulgare

Asplenium trichomanes

O Other species (Druge vrste)

Festuca sp.

Hieracium sp.

ML Mosses and lichens (Mahovi in lišaji)

Ctenidium molluscum

Tortella tortuosa

Fissidens dubius

1

1

E2

E1

E1

E1

E1

E1

E1

E1

E1

E1

E1

E1

E1

E1

E1

E1

E1

E1

E1

E1

E1

E1

E1

E1

E1

E1

E1

E1

E1

E1

E1

E1

E1

E1

E1

$\begin{array}{lllllllllllllllll}\mathrm{E} 0 & + & 1 & 1 & 1 & + & 1 & 1 & + & 1 & + & 1 & 1 & 2 & . & . \\ \mathrm{E} 0 & 1 & 1 & 1 & 1 & + & 1 & 1 & + & 1 & 1 & 1 & 1 & 1 & 1 & 1 \\ \mathrm{E} 0 & 1 & + & 1 & 1 & & + & + & & + & 1 & + & 1 & 1 & 1 & 1\end{array}$



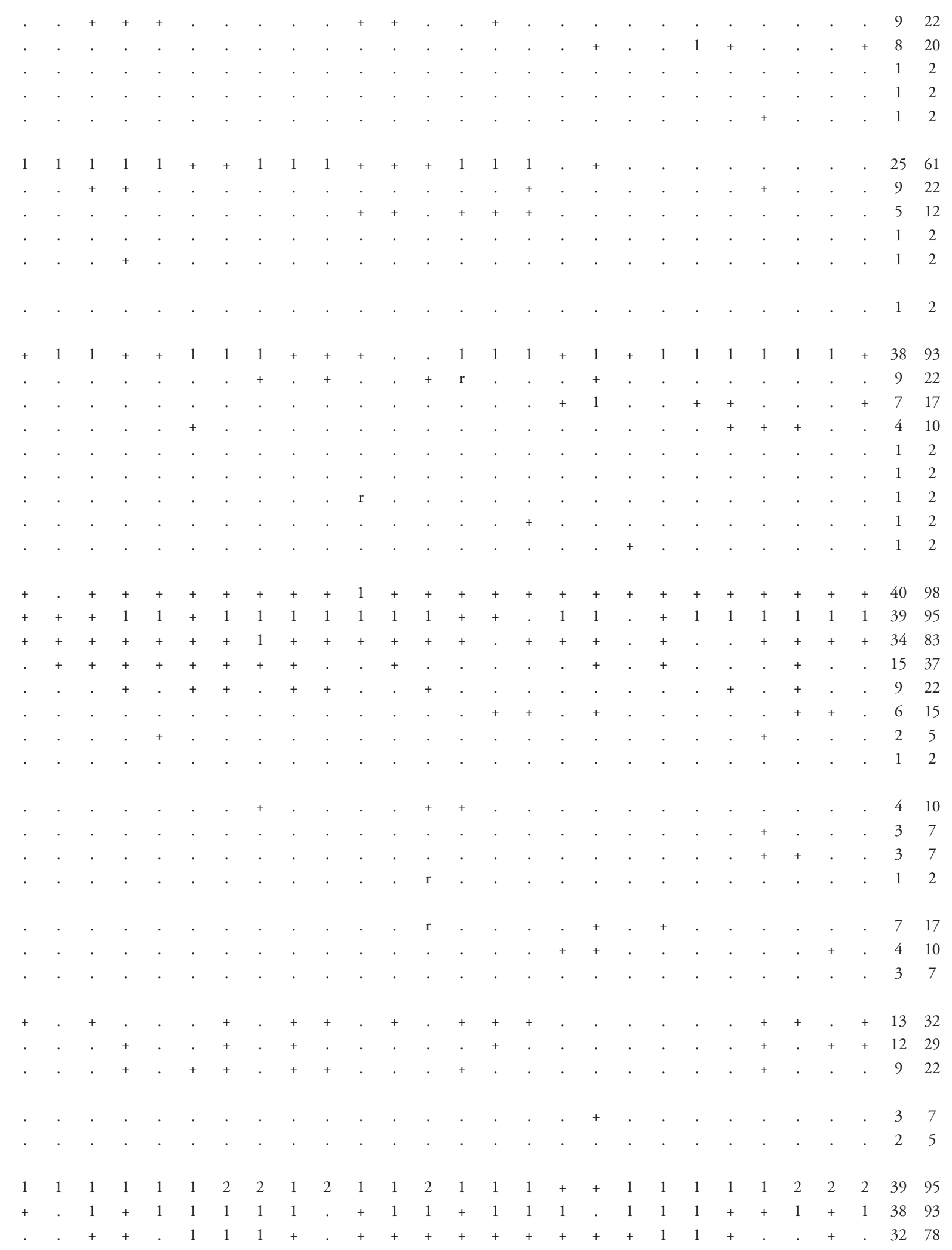
Polytrichum formosum

Isothecium alopecuroides

Schistidium apocarpum

Plagiochila porelloides

Peltigera canina

Conocephalum conicum

Mnium thomsonii

Cladonia sp.

Dicranum scoparium

Rhizomnium punctatum

Bryum capillare

Rhytidiadelphus triquetrus

Pseudoleskeella catenulata

Peltigera leucophlebia

Marchantia polymorpha

Hylocomium splendens

Eurhynchium angustirete

Mnium sp.

Metzgeria furcata

Cladonia pyxidata

Homalothecium philippeanum

Mnium marginatum

Isothecium myosuroides

Plagiothecium denticulatum

Cladonia rangiferina

Collema cristatum

Orthothecium rufescens

Dermatocarpon miniatum

Neckera crispa

Distichium capillaceum

Plagiothecium nemorale

Scapania nemorea

Bartramia pomiformis

Plagiomnium undulatum

Dicranodontium denudatum

Plagiomnium cuspidatum

Brachythecium rutabulum

Atrichum undulatum

Bryum sp.

\begin{tabular}{|c|c|c|c|c|c|c|c|c|c|c|c|c|c|c|c|}
\hline E0 & + & + & + & + & + & + & + & + & + & 1 & 1 & 1 & + & & . \\
\hline E0 & . & . & . & . & . & . & . & + & . & . & . & . & 1 & . & + \\
\hline E0 & $\cdot$ & . & . & . & + & . & . & . & + & . & 1 & . & . & 1 & + \\
\hline E0 & + & + & . & . & . & . & . & . & + & 1 & + & + & + & 1 & . \\
\hline E0 & + & + & . & . & . & . & . & . & . & . & + & + & . & . & + \\
\hline E0 & + & . & . & + & . & + & . & . & + & + & + & . & 1 & + & . \\
\hline E0 & . & + & . & . & . & + & . & . & 1 & + & + & + & + & . & . \\
\hline E0 & . & . & . & . & + & . & . & . & . & . & . & . & . & + & + \\
\hline E0 & . & . & . & + & . & . & + & . & . & . & + & . & . & . & . \\
\hline E0 & . & . & . & $\cdot$ & . & + & + & . & + & + & . & + & . & + & . \\
\hline E0 & + & . & . & . & . & . & . & . & . & . & . & . & . & . & + \\
\hline E0 & . & . & . & . & . & + & 1 & . & 1 & + & . & . & + & . & . \\
\hline E0 & . & . & . & $\cdot$ & . & $\cdot$ & $\cdot$ & . & . & . & . & . & . & + & . \\
\hline E0 & . & . & . & . & . & . & . & . & . & . & . & . & . & . & . \\
\hline E0 & . & . & . & $\cdot$ & + & . & . & . & . & . & . & . & . & . & . \\
\hline E0 & . & . & . & . & . & . & + & . & + & + & . & . & . & . & . \\
\hline E0 & . & . & . & . & . & . & . & + & + & + & . & . & . & $\cdot$ & . \\
\hline E0 & $\cdot$ & . & . & . & . & . & . & . & . & + & + & . & . & . & . \\
\hline E0 & . & . & . & . & . & . & . & . & . & + & . & . & . & . & . \\
\hline E0 & . & . & . & . & . & . & . & . & . & . & . & . & . & . & . \\
\hline E0 & . & . & . & . & + & . & . & . & . & . & . & . & . & . & . \\
\hline E0 & . & . & . & . & . & . & . & + & . & . & + & . & . & . & . \\
\hline E0 & . & . & . & . & . & . & . & . & . & . & + & + & . & $\cdot$ & . \\
\hline E0 & . & . & . & . & . & . & . & . & . & . & 1 & . & . & . & . \\
\hline E0 & . & . & . & $\cdot$ & . & . & $\cdot$ & . & . & . & . & . & . & . & . \\
\hline E0 & . & . & . & . & . & . & . & . & . & . & . & . & . & $\cdot$ & . \\
\hline E0 & . & . & . & . & . & . & . & . & . & . & . & . & . & . & . \\
\hline E0 & . & . & . & $\cdot$ & + & . & . & . & . & . & . & . & . & . & . \\
\hline E0 & . & . & . & . & . & . & . & 1 & . & . & . & . & . & . & . \\
\hline E0 & . & . & . & . & . & . & . & . & + & . & . & . & . & . & . \\
\hline E0 & . & . & . & . & $\cdot$ & . & . & . & . & + & . & . & . & . & . \\
\hline E0 & . & . & . & . & . & . & . & . & . & + & . & . & . & . & . \\
\hline E0 & . & . & . & . & . & . & . & . & . & + & . & . & . & . & . \\
\hline E0 & . & . & . & . & $\cdot$ & . & . & . & . & . & + & . & . & . & . \\
\hline E0 & . & . & . & . & $\cdot$ & . & . & . & . & . & . & . & . & + & . \\
\hline E0 & . & . & . & $\cdot$ & . & . & . & . & . & . & . & . & . & . & . \\
\hline E0 & . & . & . & . & . & . & . & . & . & . & . & . & . & . & . \\
\hline E0 & . & . & . & . & . & . & . & . & . & . & . & . & . & . & . \\
\hline E0 & . & . & . & . & . & . & $\cdot$ & . & . & . & . & . & & &. \\
\hline
\end{tabular}

\section{Legend - Legenda}

ID Igor Dakskobler

A Limestone - apnenec

D Dolomite - dolomit

G Claystone-glinavec

Re Rendzina - rendzina

JA Julian Alps - Julijske Alpe

TG Trnovski Gozd plateau - Trnovski gozd

I Italy (Italia, Italija)

Pr. Presence (number of relevés in which the species is presented) - število popisov, v katerih se pojavlja vrsta

Fr. Frequency in $\%$ - frekvenca $v \%$

Relevé No. 6, holotypus 


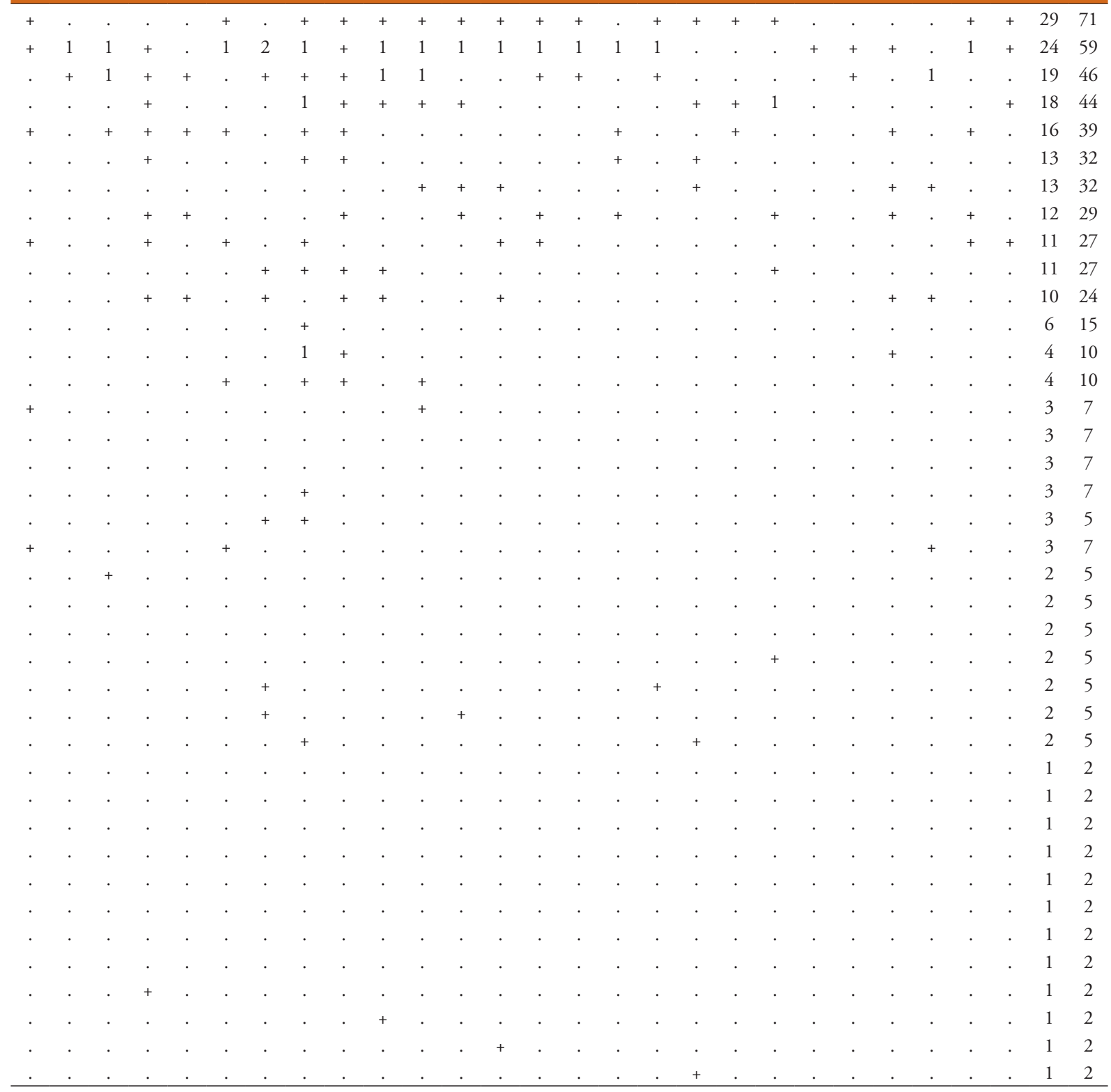


Table 3: Polysticho lonchitis-Fagetum luzuletosum luzuloidis and Ranunculo platanifolii-Fagetum luzuletosum luzuloidis - cluster 19. Relevé numbers 1-37.

\section{Number of relevé (Zaporedna številka popisa)}

Database number of relevé

(Delovna številka popisa)

Author of the relevé (Avtor popisa)

Elevation in $\mathrm{m}$ (Nadmorska višina $\mathrm{v} \mathrm{m})$

Aspect (Lega)

Slope in degrees (Nagib v stopinjah)

Parent material (Matična podlaga)

Soil (Tla)

Stoniness in \% (Kamnitost v \%)

Cover in \% (Zastiranje v \%):

Upper tree layer (Zgornja drevesna plast)
Lower tree layer (Spodnja drevesna plasti)
Shrub layer (Grmovna plast)
Herb layer (Zeliščna plast)
Moss layer (Mahovna plast)

Maximum tree diameter (Maks. premer dreves)

Maximum tree height (Maksimalna višina dreves)

Number of species (Število vrst)

Relevé area (Velikost popisne ploskve)

Date of taking relevé (Datum popisa)

Day (Dan)

Month (Mesec)

Year (Leto)

Locality (Nahajališče)

Mountain range (Pogorje)

Quadrant (Kvadrant)

Coordinate (Koordinate) GK Y (D-48)

Coordinate (Koordinate) GK X (D-48)

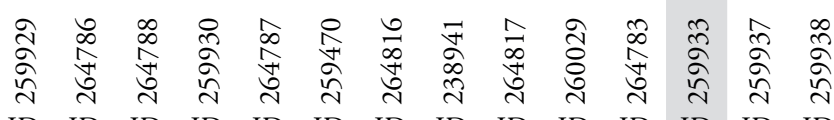

ID ID ID ID ID ID ID ID ID ID ID ID ID ID

กิ

SE $\quad$ SE $\quad$ SE $S$ SW SW SE $S$ SE SW SW NEE S SE

$\begin{array}{llllllllllllll}30 & 39 & 35 & 20 & 20 & 30 & 35 & 30 & 35 & 30 & 30 & 30 & 30 & 35\end{array}$

DA DA DA DA DA DA DA Gr DA DA DA DA DA DA $\operatorname{Re} \operatorname{Re} \operatorname{Re} \operatorname{Re} \operatorname{Re} \operatorname{Re} \operatorname{Re} \operatorname{Re} \operatorname{Re} \operatorname{Re} \operatorname{Re} \operatorname{Re} \operatorname{Re} \operatorname{Re}$ $\begin{array}{llllllllllllll}10 & 10 & 10 & 5 & 2 & 20 & 10 & 50 & 10 & 50 & 0 & 40 & 10 & 15\end{array}$

\begin{tabular}{|c|c|c|c|c|c|c|c|c|c|c|c|c|c|}
\hline $\mathrm{E} 3 \mathrm{~b} \quad 90$ & 80 & 80 & 95 & 85 & 90 & 85 & 90 & 90 & 100 & 80 & 90 & 90 & 80 \\
\hline E3a & & . & . & & . & . & . & . & . & . & 1 & & . \\
\hline E2 & 10 & 5 & 5 & 5 & 15 & 1 & 10 & 5 & 5 & 10 & 5 & 10 & 10 \\
\hline 90 & 70 & 70 & 80 & 70 & 80 & 80 & 60 & 80 & 70 & 70 & 70 & 70 & 70 \\
\hline E0 & 5 & 5 & 10 & 10 & 10 & 10 & 5 & 10 & 10 & 10 & 10 & 5 & 10 \\
\hline 30 & 35 & 35 & 40 & 45 & 30 & 50 & 30 & 30 & 35 & 35 & 40 & 50 & 60 \\
\hline 10 & 15 & 12 & 10 & 12 & 10 & 10 & 10 & 10 & 14 & 12 & 10 & 10 & 12 \\
\hline 58 & 69 & 54 & 58 & 73 & 69 & 50 & 48 & 49 & 61 & 54 & 58 & 46 & 57 \\
\hline 400 & 400 & 400 & 400 & 400 & 400 & 400 & 200 & 400 & 400 & 400 & 400 & 400 & 400 \\
\hline $\begin{array}{l}\text { त్రి } \\
\text { ठ } \\
\stackrel{0}{0} \\
\stackrel{-}{二}\end{array}$ & 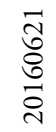 & $\begin{array}{l}\overrightarrow{\widehat{J}} \\
\stackrel{0}{0} \\
\stackrel{\sim}{0}\end{array}$ & 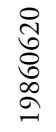 & 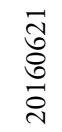 & 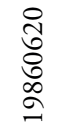 & 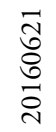 & 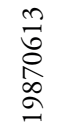 & 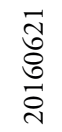 & 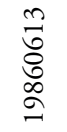 & 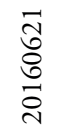 & 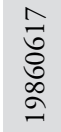 & 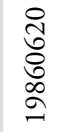 & 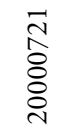 \\
\hline 20 & 21 & 21 & 20 & 21 & 20 & 21 & 13 & 21 & 13 & 21 & 17 & 20 & 21 \\
\hline 6 & 6 & 6 & 6 & 6 & 6 & 6 & 6 & 6 & 6 & 6 & 6 & 6 & 7 \\
\hline 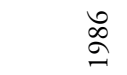 & $\begin{array}{l}\stackrel{\circ}{*} \\
\stackrel{\sim}{*}\end{array}$ & $\begin{array}{l}\stackrel{0}{*} \\
\stackrel{\sim}{\sim}\end{array}$ & 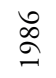 & $\begin{array}{l}\text { 웅 } \\
\stackrel{\sim}{\sim}\end{array}$ & $\begin{array}{l}\stackrel{2}{\infty} \\
\stackrel{2}{\beth}\end{array}$ & $\begin{array}{l}0 \\
\stackrel{\sim}{\sim}\end{array}$ & $\begin{array}{l}\hat{\infty} \\
\stackrel{\Omega}{\sigma}\end{array}$ & $\begin{array}{l}\text { 울 } \\
\stackrel{\sim}{\sim}\end{array}$ & 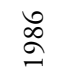 & $\begin{array}{l}\stackrel{0}{*} \\
\stackrel{\sim}{\sim}\end{array}$ & 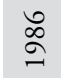 & 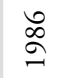 & 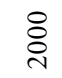 \\
\hline
\end{tabular}

Diagnostic species of the association (Diagnostične vrste asociacije)

MuA Aconitum lycoctonum subsp. ranunculifolium

ML Paraleucobryum sauteri

VP Polystichum lonchitis

VP Clematis alpina

VP Luzula sylvatica

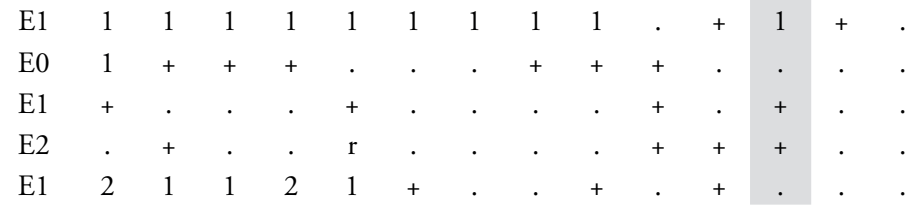




\begin{tabular}{|c|c|c|c|c|c|c|c|c|c|c|c|c|c|c|c|c|c|c|c|c|c|c|}
\hline 15 & 16 & 17 & 18 & 19 & 20 & 21 & 22 & 23 & 24 & 25 & 26 & 27 & 28 & 29 & 30 & 31 & 32 & 33 & 34 & 35 & 36 & \\
\hline $\begin{array}{l}\exists \\
\infty \\
\infty \\
\stackrel{\sim}{\sim}\end{array}$ & $\begin{array}{l}\stackrel{\sim}{\infty} \\
\infty \\
\approx\end{array}$ & $\begin{array}{l}\text { N } \\
\infty \\
\infty \\
\sim\end{array}$ & $\begin{array}{l}\stackrel{ }{\sigma} \\
\stackrel{\sim}{\sim}\end{array}$ & 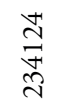 & 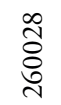 & $\begin{array}{l}\stackrel{\infty}{N} \\
\stackrel{N}{N}\end{array}$ & 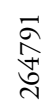 & 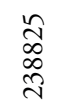 & $\begin{array}{l}\hat{A} \\
\hat{\sigma} \\
\stackrel{\sim}{N}\end{array}$ & $\begin{array}{l}\stackrel{\circ}{\circ} \\
\stackrel{\text { }}{\text { ते }}\end{array}$ & $\begin{array}{l}\text { مे } \\
\text { ते } \\
\stackrel{\sim}{N}\end{array}$ & 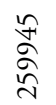 & $\stackrel{\vec{\sigma}}{\stackrel{\sim}{\approx}}$ & 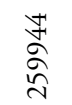 & 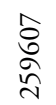 & 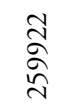 & $\begin{array}{l}\infty \\
\infty \\
\infty \\
\approx\end{array}$ & 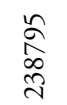 & $\begin{array}{l}\approx \\
\infty \\
\infty \\
\infty \\
\sim\end{array}$ & $\begin{array}{l}\hat{m} \\
\underset{\sim}{\sim} \\
\underset{\sim}{n}\end{array}$ & $\stackrel{\vec{\sim}}{\vec{\sim}}$ & \\
\hline ID & ID & ID & ID & ID & ID & ID & ID & ID & ID & ID & ID & ID & ID & ID & ID & ID & ID & ID & ID & ID & ID & \\
\hline $\begin{array}{l}\stackrel{\infty}{\infty} \\
\stackrel{+}{+}\end{array}$ & 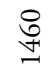 & $\begin{array}{l}8 \\
\stackrel{+}{+}\end{array}$ & $\underset{-}{\stackrel{+}{+}}$ & $\begin{array}{l}\stackrel{\infty}{\infty} \\
\stackrel{m}{\sim}\end{array}$ & $\begin{array}{l}\stackrel{\circ}{n} \\
\stackrel{2}{n}\end{array}$ & $\begin{array}{l}\stackrel{0}{n} \\
\stackrel{n}{n}\end{array}$ & 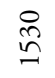 & 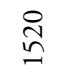 & 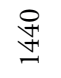 & $\stackrel{\stackrel{R}{\rightleftarrows}}{+}$ & $\begin{array}{l}8 \\
\stackrel{Ð}{ \pm}\end{array}$ & $\underset{\stackrel{P}{+}}{+}$ & $\underset{Ð}{\stackrel{す}{\leftrightarrows}}$ & 왐 & $\underset{\nsubseteq}{\stackrel{+}{\nsubseteq}}$ & $\underset{\underset{J}{ \pm}}{n}$ & $\underset{\nsubseteq}{\stackrel{+}{\sharp}}$ & $\stackrel{\stackrel{R}{ \pm}}{\square}$ & $\begin{array}{l}\mathscr{1} \\
\stackrel{\infty}{ \pm}\end{array}$ & 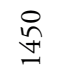 & $\begin{array}{l}\stackrel{̊}{\sharp} \\
\stackrel{\forall}{\sharp}\end{array}$ & \\
\hline $\mathrm{NE}$ & SW & $S$ & E & $\mathrm{N}$ & $S$ & NW & SW & $\mathrm{NE}$ & $\mathrm{N}$ & SE & S & $\mathrm{NE}$ & $\mathrm{NE}$ & NEE & W & NW & $\mathrm{NE}$ & $\mathrm{NE}$ & SEE & $\mathrm{NE}$ & E & \\
\hline 30 & 35 & 40 & 35 & 15 & 20 & 40 & 40 & 30 & 35 & 35 & 30 & 30 & 40 & 35 & 40 & 35 & 30 & 25 & 30 & 35 & 40 & \\
\hline AG & AG & A & A & A & $\mathrm{AB}$ & DA & DA & A & DA & $\mathrm{DA}$ & $\mathrm{Gr}$ & DA & DA & DA & DA & DA & $\mathrm{AL}$ & $\mathrm{DR}$ & A & A & AL & \\
\hline $\mathrm{Re}$ & $\mathrm{Re}$ & $\operatorname{Re}$ & $\mathrm{Re}$ & $\mathrm{Re}$ & $\mathrm{Re}$ & $\operatorname{Re}$ & $\mathrm{Re}$ & $\mathrm{Re}$ & $\mathrm{Re}$ & $\operatorname{Re}$ & $\mathrm{Re}$ & $\mathrm{Re}$ & $\mathrm{Re}$ & $\operatorname{Re}$ & $\mathrm{Re}$ & $\mathrm{Re}$ & $\mathrm{Re}$ & $\operatorname{Re}$ & $\operatorname{Re}$ & $\mathrm{Rj}$ & $\mathrm{Rj}$ & \\
\hline 60 & 80 & 30 & 70 & 60 & 20 & 10 & 10 & 40 & 20 & 50 & 60 & 30 & 60 & 40 & 10 & 10 & 20 & 10 & 50 & 50 & 30 & \\
\hline 80 & NA & 70 & 90 & 90 & 100 & 70 & 80 & 80 & 85 & 80 & 90 & 100 & 100 & 90 & 80 & 80 & 90 & 90 & 80 & 80 & 90 & \\
\hline 10 & $\cdot$ & $\cdot$ & . & . & . & . & $\cdot$ & 10 & . & . & 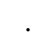 & r. & . & S & . & . & $\cdot$ & 10 & 10 & . & r & \\
\hline 10 & 10 & 20 & 5 & 10 & 2 & 20 & 10 & 20 & 10 & 10 & 10 & 1 & 1 & 5 & م & 20 & 10 & 50 & 5 & 2 & 5 & \\
\hline 60 & 70 & 70 & 70 & 40 & 40 & 70 & 70 & 70 & 50 & 60 & 70 & 60 & 50 & 50 & 70 & 70 & 60 & 5 & 40 & 60 & 40 & \\
\hline 10 & 10 & 10 & 5 & 10 & 10 & 10 & 10 & 10 & 10 & 10 & 10 & 10 & 15 & 10 & 5 & 5 & 10 & NA & 10 & 10 & 5 & \\
\hline 35 & 55 & 35 & 40 & 40 & 30 & 25 & 40 & 35 & 25 & 40 & 35 & 30 & 35 & 70 & 30 & 30 & 20 & 60 & 40 & 25 & 30 & \\
\hline 12 & 12 & 10 & 12 & 18 & 18 & 10 & 12 & 14 & 12 & 12 & 10 & 12 & 10 & 9 & 9 & 10 & 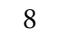 & 14 & 12 & 8 & 0 & \\
\hline 81 & 63 & 92 & 65 & 52 & 40 & 61 & 70 & 82 & 60 & 85 & 58 & 40 & 38 & 45 & 61 & 48 & 62 & 80 & 60 & 65 & 69 & \\
\hline 400 & 400 & 400 & 200 & 200 & 400 & 400 & 400 & 400 & 200 & 400 & 200 & 400 & 400 & 400 & 400 & 400 & 100 & 400 & 400 & 200 & 200 & \\
\hline 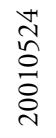 & 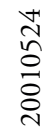 & 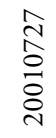 & 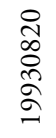 & 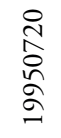 & 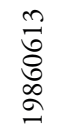 & $\begin{array}{l}\infty \\
\stackrel{\infty}{\circ} \\
\stackrel{\circ}{2} \\
\curvearrowright\end{array}$ & $\begin{array}{l}n \\
\hat{N} \\
0 \\
0 \\
0 \\
0\end{array}$ & $\begin{array}{l}m \\
\infty \\
\infty \\
0 \\
0 \\
\stackrel{\sim}{\sim}\end{array}$ & 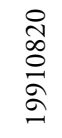 & 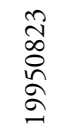 & 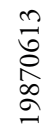 & $\begin{array}{l}\infty \\
\stackrel{\infty}{\circ} \\
\stackrel{2}{\alpha} \\
\check{\sigma}\end{array}$ & 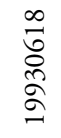 & 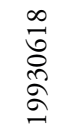 & 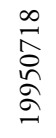 & 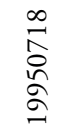 & 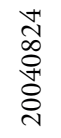 & 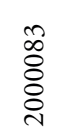 & $\begin{array}{l}\text { m } \\
\infty \\
0 \\
0 \\
\stackrel{0}{0} \\
\stackrel{\sim}{1}\end{array}$ & 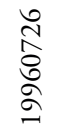 & 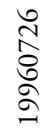 & \\
\hline 24 & 24 & 27 & 20 & 20 & 13 & 18 & 15 & 13 & 20 & 23 & 13 & 18 & 18 & 18 & 18 & 18 & 24 & 3 & 13 & 26 & 26 & \\
\hline 5 & 5 & 7 & 8 & 7 & 6 & 7 & 7 & 8 & 8 & 0 & 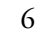 & 6 & 6 & 6 & 7 & 7 & 8 & 8 & 8 & 7 & 7 & \\
\hline ¿্ণ & ¿্ণ & 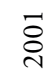 & 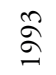 & 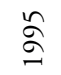 & $\begin{array}{l}\stackrel{2}{\infty} \\
\stackrel{2}{\beth}\end{array}$ & $\tilde{\sigma}$ & $\begin{array}{l}\text { O) } \\
\stackrel{\sim}{\sim}\end{array}$ & ¿্ণ & $\bar{\sigma}$ & $\check{\curvearrowright}$ & $\begin{array}{l}\stackrel{\infty}{\cong} \\
\stackrel{\sim}{二}\end{array}$ & $\tilde{\sigma}$ & 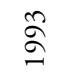 & 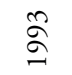 & 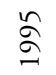 & $\check{\Omega}$ & 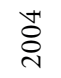 & ¿ & 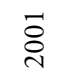 & $\stackrel{\text { }}{\check{\beth}}$ & $\stackrel{\curvearrowright}{\curvearrowright}$ & \\
\hline
\end{tabular}
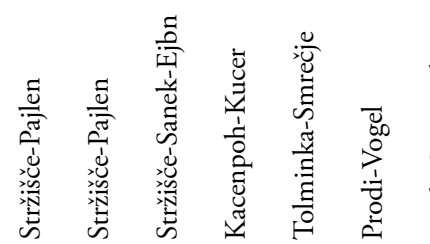

JA JA JA JA

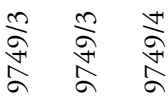

苑 趈

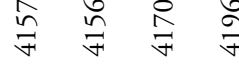

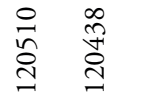

索离
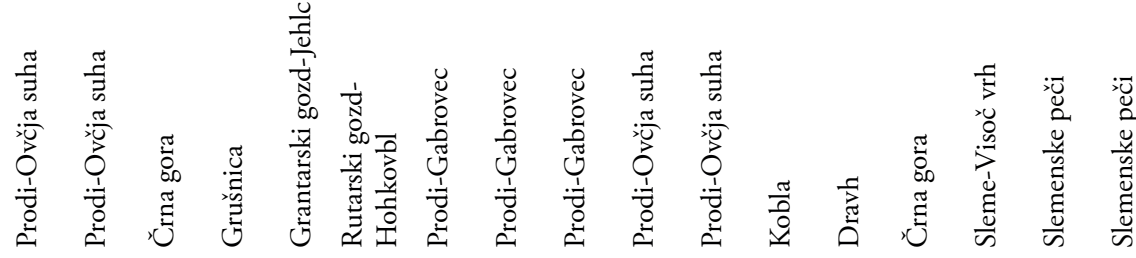

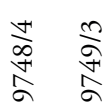

$\stackrel{n}{\frac{n}{a}} \stackrel{n}{a}$

$\stackrel{\frac{a}{a}}{\stackrel{2}{a}}$

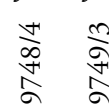

$\stackrel{n}{\stackrel{n}{a} \stackrel{n}{a}}$

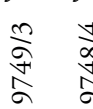

JA JA

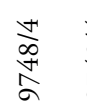

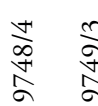

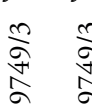

JA JA JA JA JA JA

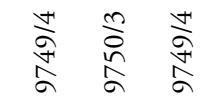

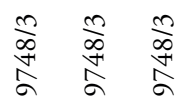

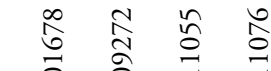

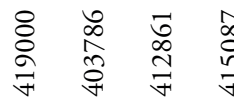

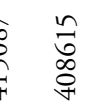

盛

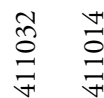

ঙ্ণ

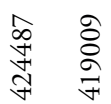

ลิ

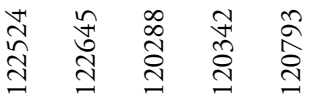

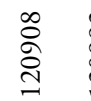




\begin{tabular}{|c|c|c|c|c|c|c|c|c|c|c|c|c|c|c|c|}
\hline & Number of relevé (Zaporedna številka popisa) & & 1 & 2 & 3 & 4 & 5 & 6 & 7 & 8 & 9 & 10 & 11 & 12 & 13 \\
\hline $\mathrm{EP}$ & Pinus mugo & E2 & + & + & + & + & + & . & . & + & . & + & + & + & 1 \\
\hline $\mathrm{BA}$ & Sorbus chamaemespilus & E2 & + & + & + & + & + & + & . & $\cdot$ & . & . & + & + & + \\
\hline CF & Carex ferruginea & E1 & + & + & + & + & + & 2 & 1 & + & 1 & $\cdot$ & $\mathrm{r}$ & . & . \\
\hline CA & Laserpitium peucedanoides & E1 & $\cdot$ & . & + & . & + & 1 & 1 & $\cdot$ & + & + & + & . & + \\
\hline EP & Rhododendron hirsutum & E2 & $\cdot$ & . & . & . & . & . & . & . & $\cdot$ & + & $\mathrm{r}$ & + & 1 \\
\hline PS & Paederota lutea & E1 & $\cdot$ & . & . & . & . & . & $\cdot$ & . & . & 1 & . & + & . \\
\hline BA & Salix appendiculata & E3 & . & . & . & . & . & . & . & . & . & . & . & . & $\cdot$ \\
\hline BA & Salix appendiculata & E2 & $\cdot$ & . & $\cdot$ & . & . & . & . & $\cdot$ & . & $\cdot$ & $\cdot$ & + & . \\
\hline CA & Festuca calva & E1 & . & . & . & . & . & . & . & $\cdot$ & . & $\cdot$ & . & + & . \\
\hline ES & Aster bellidiastrum & E1 & $\cdot$ & . & . & . & . & + & + & $\cdot$ & . & + & . & + & . \\
\hline VP & Lonicera caerulea & E2 & . & . & . & . & + & . & . & . & . & $\cdot$ & . & . & . \\
\hline VP & Homogyne alpina & E1 & . & . & . & + & $\mathrm{r}$ & . & . & . & . & . & . & . & . \\
\hline EP & Rhodothamnus chamaecistus & E1 & . & . & . & . & . & . & . & . & . & . & . & + & $\cdot$ \\
\hline
\end{tabular}

\section{Differential species of the subassociation (Razlikovalnice subasociacije)}

VP Luzula luzuloides

FB Cirsium erisithales

EC Primula vulgaris

AF Aremonio-Fagion

Cardamine enneaphyllos

Cyclamen purpurascens

Cardamine trifolia

Anemone trifolia

Lamium orvala

Rhamnus fallax

Anemone x pittonii

Knautia drymeia

Aremonia agrimonoides

Hacquetia epipactis

Vicia oroboides

Omphalodes verna

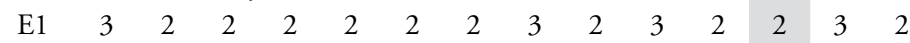

$\mathrm{E} 1$

\section{Erythronio-Carpinion}

Helleborus odorus

Ornithogalum pyrenaicum

TA Tilio-Acerion

Adoxa moschatellina

Acer pseudoplatanus

Acer pseudoplatanus

Acer pseudoplatanus

Polystichum aculeatum

Aruncus dioicus

Geranium robertianum

Lunaria rediviva

Polystichum braunii

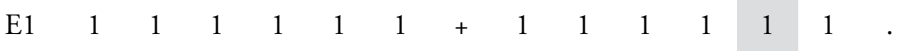

Chrysosplenium alternifolium

Cardamine flexuosa

Polystichum $x$ luerssenii

Stellaria montana

Corydalis solida

FS Fagetalia sylvaticae

Fagus sylvatica

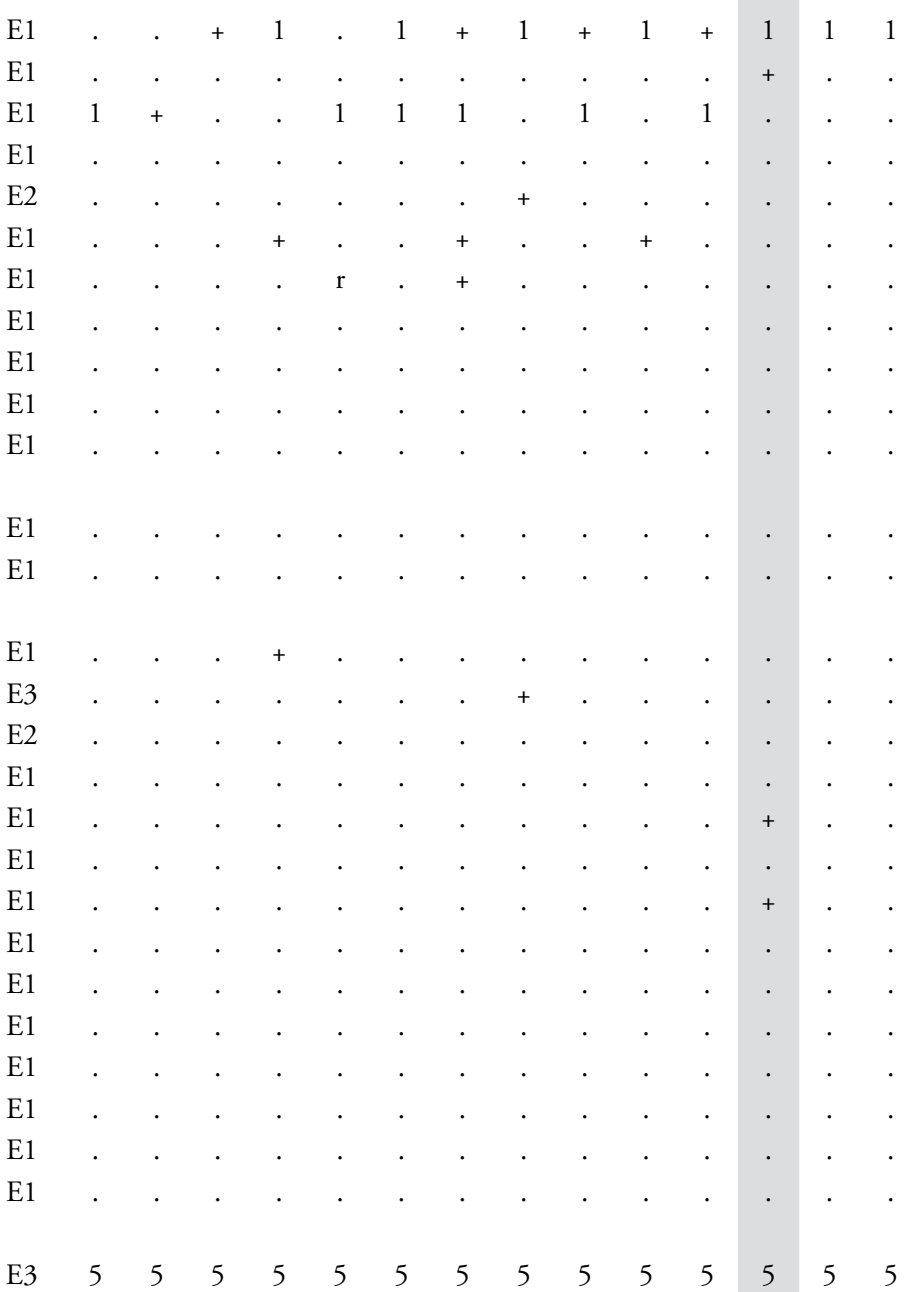



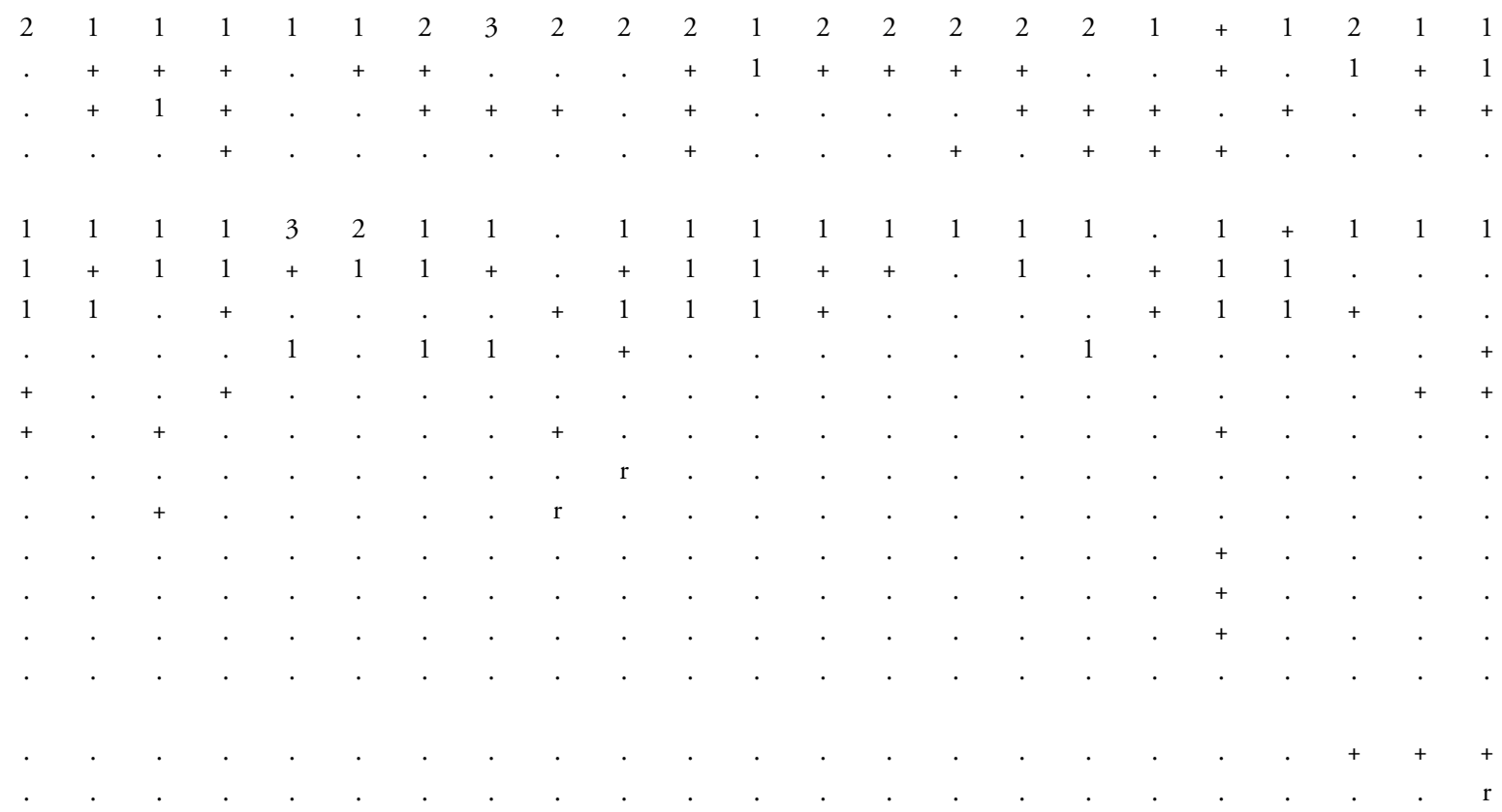
Fagus sylvatica

Fagus sylvatica

Lilium martagon

Galium laevigatum

Lonicera alpigena

Mercurialis perennis

Daphne mezereum

Galeobdolon flavidum

Symphytum tuberosum

Dryopteris filix-mas

Paris quadrifolia

Melica nutans

Actaea spicata

Prenanthes purpurea

Euphorbia amygdaloides

Epilobium montanum

Lathyrus vernus

Scrophularia nodosa

Mycelis muralis

Lathyrus vernus subsp. flaccidus

Luzula nivea

Campanula trachelium

Corydalis cava

Pulmonaria officinalis

Myosotis sylvatica agg.

Festuca altissima

Laburnum alpinum

Laburnum alpinum

Laburnum alpinum

Ranunculus lanuginosus

Galium odoratum

Petasites albus

Viola reichenbachiana

Asarum europaeum subsp. caucasicum

Neottia nidus-avis

Cardamine bulbifera

Salvia glutinosa

Asarum europaeum subsp. europaeum

Polygonatum multiflorum

Carex sylvatica

Cardamine impatiens

Leucojum vernum

E2

E1

E1

E1

E2

E1

E2

E1

E1

E1

E1

E1

E1

E1

E1

E1

E1

E1

E1

E1

E1

E3

E2

E1

E1

E1

E1

E1

E1

E1

E1

E1

E1

E1

E1

E1

E1

QP

Sorbus arias (Aria edulis)

E3

E2

E1

Sorbus arias (Aria edulis)

Convallaria majalis

Carex flacca

QF Querco-Fagetea

Anemone nemorosa

(1)

\section{$\begin{array}{llllllllllllll}1 & 2 & 3 & 4 & 5 & 6 & 7 & 8 & 9 & 10 & 11 & 12 & 13 & 14\end{array}$}

$+++.+++$

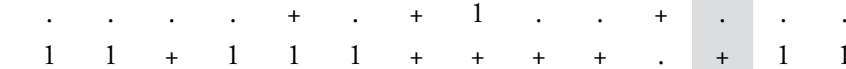

$+++\begin{array}{llllll}+ & 1 & 1 & 1 & + & 1\end{array}$

$1+++11+++++++$

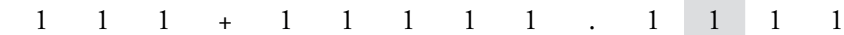

$1++2+2+1+1+++$

$+1+++++++++++$

$+$

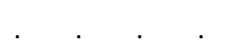

(1)

(1)

(1)

(1)

(1)

El

1

El

1

E1

El

$\mathrm{E} 1$

1




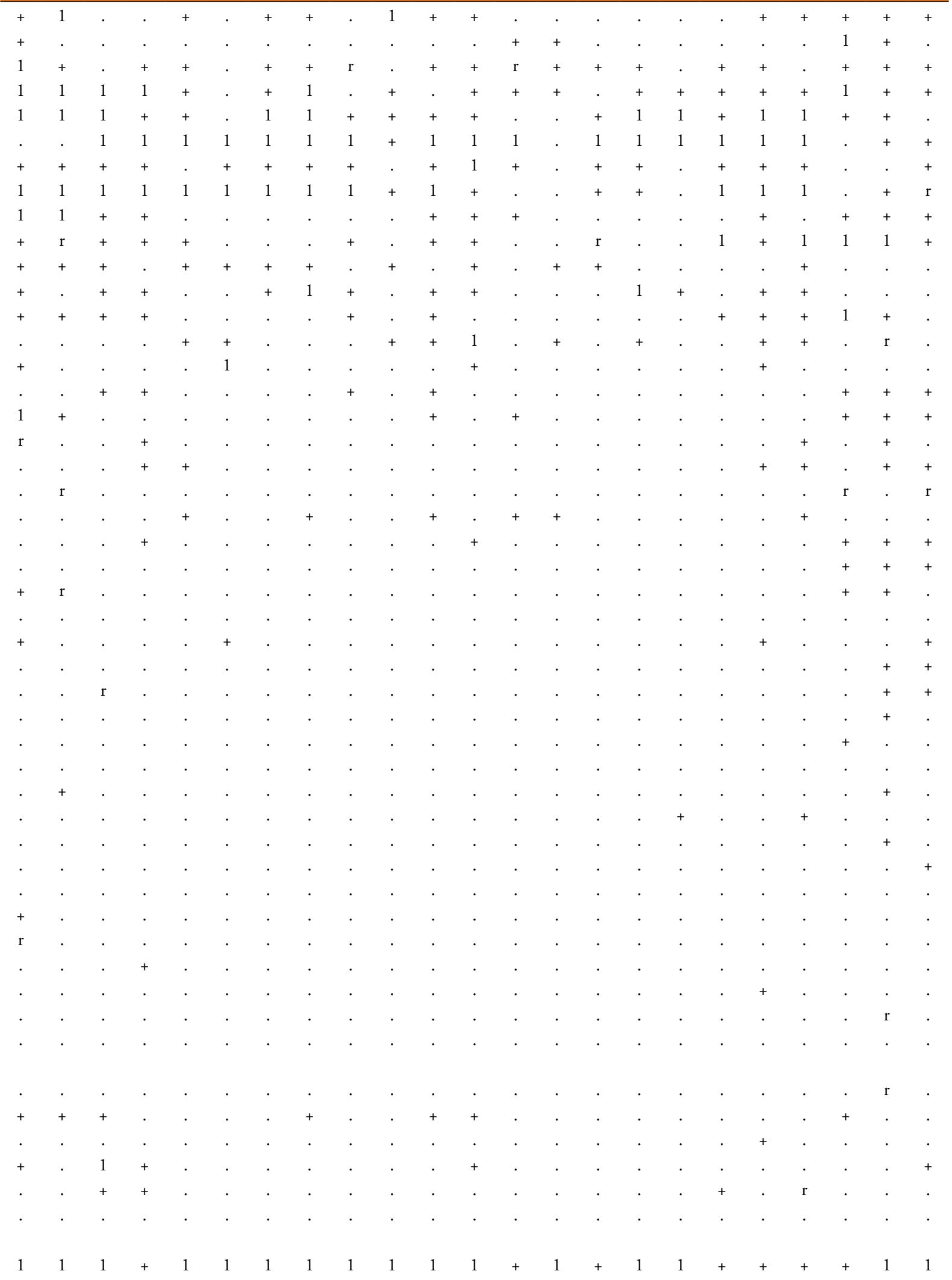


Hepatica nobilis

Poa nemoralis

Carex digitata

Festuca heterophylla

Carex pilosa

Dactylorhiza fuchsii

Stellaria holostea

Ranunculus auricomus agg. (R. braun-blanquetii)

Spiraea chamaedryfolia

Aegopodium podagraria

Scilla bifolia

Dactylis glomerata subsp. lobata (D. polygama)

Lonicera xylosteum

VP Vaccinio-Piceetea

Aposeris foetida

Gentiana asclepiadea

Maianthemum bifolium

Veronica urticifolia

Oxalis acetosella

Picea abies

Picea abies

Picea abies

Rosa pendulina

Solidago virgaurea

Hieracium murorum

Vaccinium myrtillus

Dryopteris dilatata

Huperzia selago

Saxifraga cuneifolia

Homogyne sylvestris

Gymnocarpium dryopteris

Lonicera nigra

Vaccinium vitis-idaea

Phegopteris connectilis

Larix decidua

Dryopteris expansa

Calamagrostis villosa

Lycopodium annotinum

Abies alba

Luzula luzulina

Avenella flexuosa

Monotropa hypopitys

Thelypteris limbosperma

Blechnum spicant

\section{Erico-Pinetea}

Rubus saxatilis

Calamagrostis varia

Aquilegia nigricans

Erica carnea

Carex alba

Carex ornithopoda 
- Hacquetia $20 / 2 \cdot 2021,373-564$

Igor Dakskobler \& Andre Rozman

Vegetation analysis of the subalpine beech forest on the upper forest line in the

Julian Alps (NW Slovenia and NW Italy) and in the northern Dinaric Alps

$\begin{array}{llllll}15 & 16 & 17 & 18 & 19 & 20\end{array}$

21

$22 \quad 23$

$\begin{array}{lllll}23 & 24 & 25 & 26 & 27\end{array}$

$28 \quad 29$

$30 \quad 31$

$31 \quad 32$

33

35

$36 \quad 37$

37
+
+

1

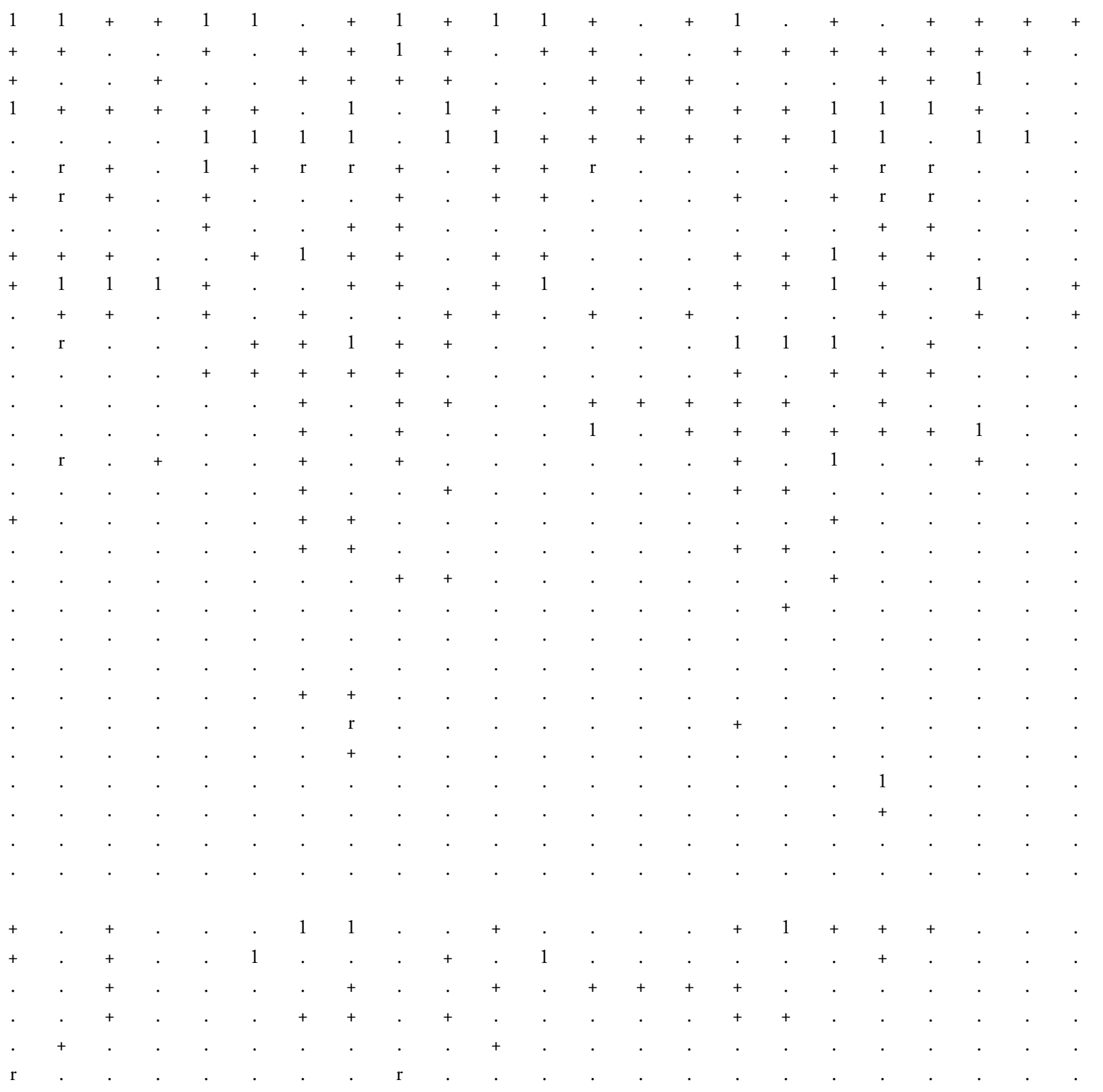

455 
Genista radiata

Peucedanum austriacum subsp. rablense

Chamaecytisus hirsutus

Polygala chamaebuxus

Peucedanum austriacum subsp. austriacum

SSC Sambuco-Salicion capreae, Rhamno-Prunetea

Sorbus aucuparia

Sorbus aucuparia

Sorbus aucuparia

Rosa sp.

Sambucus racemosa

BA Betulo-Alnetea

Alnus viridis

Ribes alpinum

Sorbus austriaca

Salix glabra

Juniperus sibirica

MuA Mulgedio-Aconitetea

Polygonatum verticillatum

Veratrum album

Athyrium filix-femina

Phyteuma ovatum

Senecio ovatus

Saxifraga rotundifolia

Ranunculus platanifolius

Tanacetum corymbosum subsp. clusii

Thalictrum aquilegiifolium

Aconitum degenii subsp. paniculatum

Chaerophyllum villarsii

Allium victorialis

Viola biflora

Geranium sylvaticum

Hypericum maculatum

Centaurea montana

Aconitum angustifolium

Pleurospermum austriacum

Senecio cacaliaster

Doronicum austriacum

Chaerophyllum hirsutum

Primula elatior

Rumex arifolius

Lathyrus occidentalis var. montanus

Geum rivale

Agropyron caninum

Myrrhis odorata

Milium effusum

Stellaria nemorum

Chaerophyllum aureum

Carduus personata

Poa hybrida

Streptopus amplexifolius
E2

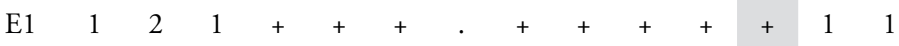

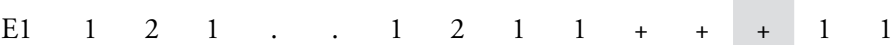




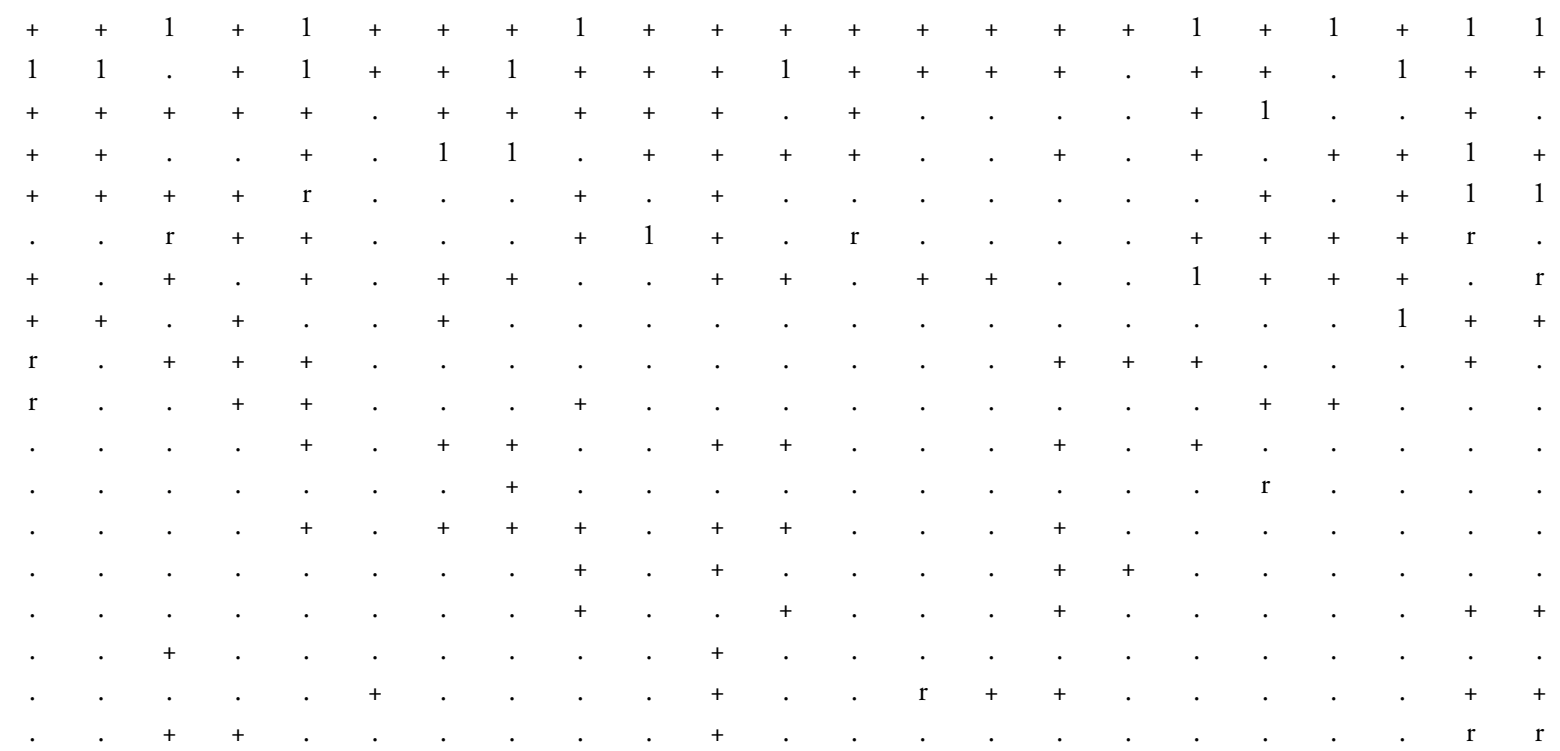




\begin{tabular}{|c|c|c|c|c|c|c|c|c|c|c|c|c|c|c|c|c|}
\hline & Number of relevé (Zaporedna številka popisa) & & 1 & 2 & 3 & 4 & 5 & 6 & 7 & 8 & 9 & 10 & 11 & 12 & 13 & 14 \\
\hline & Silene dioica & E1 & . & $\cdot$ & . & . & . & . & . & . & . & . & . & . & . & . \\
\hline & Adenostyles alliariae & E1 & . & . & . & . & . & . & . & . & . & . & . & . & . & . \\
\hline & Cicerbita alpina & E1 & . & . & . & . & . & . & . & . & . & . & . & . & . & . \\
\hline \multirow[t]{2}{*}{$\mathrm{CF}$} & Caricion ferrugineae & & & & & & & & & & & & & & & \\
\hline & Cerastium subtriflorum & E1 & . & . & . & . & . & . & . & . & . & . & . & . & . & . \\
\hline \multirow[t]{12}{*}{ ES } & Elyno-Seslerietea & & & & & & & & & & & & & & & \\
\hline & Betonica alopecuros & E1 & + & . & 1 & + & + & 1 & 1 & + & . & + & 1 & . & + & 1 \\
\hline & Sesleria caerulea & E1 & . & + & . & + & + & 1 & + & + & + & 2 & + & 1 & 1 & + \\
\hline & Campanula witasekiana & E1 & . & . & . & . & . & + & . & . & . & + & . & + & + & + \\
\hline & Astrantia bavarica & E1 & . & . & . & . & $\mathrm{r}$ & . & . & . & . & $\cdot$ & . & . & $\cdot$ & + \\
\hline & Pimpinella alpina & E1 & . & . & . & . & . & . & . & . & . & . & . & . & $\cdot$ & + \\
\hline & Carex sempervirens & E1 & . & . & . & . & . & . & + & . & + & . & + & . & . & . \\
\hline & Phyteuma orbiculare & E1 & . & . & . & . & . & . & + & . & . & . & . & . & . & . \\
\hline & Ranunculus montanus & E1 & . & . & . & . & + & . & . & . & . & . & . & . & . & . \\
\hline & Achillea clavenae & E1 & . & . & . & . & . & . & . & . & . & . & . & + & . & . \\
\hline & Carduus crassifolius & E1 & . & . & . & . & . & . & . & . & . & . & . & . & . & . \\
\hline & Phleum hirsutum & E1 & . & . & . & . & . & . & . & . & . & . & . & . & . & . \\
\hline \multirow[t]{5}{*}{ NS } & Nardion strictae, Juncetea trifidi & & & & & & & & & & & & & & & \\
\hline & Campanula scheuchzeri & E1 & . & . & . & . & . & . & . & . & . & . & . & . & . & . \\
\hline & Festuca ovina agg. & E1 & . & . & . & . & + & . & . & . & . & · & + & . & $\cdot$ & . \\
\hline & Coeloglossum viride & E1 & . & . & . & . & . & + & . & . & . & . & . & . & . & . \\
\hline & Festuca nigrescens & E1 & . & . & . & . & . & . & . & . & . & . & . & . & $\cdot$ & . \\
\hline \multirow[t]{2}{*}{$\mathrm{CD}$} & Caricetalia davallianae & & & & & & & & & & & & & & & \\
\hline & Parnassia palustris & E1 & . & . & . & . & . & . & . & . & . & . & . & . & . & . \\
\hline \multirow[t]{8}{*}{ FB } & Festuco-Brometea & & & & & & & & & & & & & 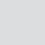 & & \\
\hline & Buphthalmum salicifolium & E1 & . & . & . & . & . & . & + & . & . & . & . & . & . & . \\
\hline & Carex humilis & E1 & . & . & . & . & . & . & + & . & . & . & . & . & . & . \\
\hline & Carlina acaulis & E1 & . & . & . & . & . & + & . & . & . & . & . & . & $\cdot$ & . \\
\hline & Bromopsis transsilvanica & E1 & . & . & . & . & . & . & . & . & . & . & . & . & $\cdot$ & . \\
\hline & Linum viscosum & E1 & . & . & . & . & . & . & . & . & . & . & . & . & . & . \\
\hline & Centaurea triumfettii & E1 & . & . & . & . & . & . & . & . & . & . & . & . & $\cdot$ & . \\
\hline & Koeleria pyramidata & E1 & . & . & . & . & . & . & . & . & . & . & . & . & $\cdot$ & . \\
\hline \multirow[t]{15}{*}{ TG } & Trifolio-Geranietea & & & & & & & & & & & & & & & \\
\hline & Digitalis grandiflora & E1 & . & . & . & . & . & . & . & . & . & . & . & . & . & . \\
\hline & Laserpitium latifolium & E1 & . & . & . & . & . & . & + & . & . & . & . & . & . & . \\
\hline & Arabis turrita & E1 & . & . & . & . & . & . & . & . & . & . & . & . & $\cdot$ & . \\
\hline & Calamintha sylvatica & E1 & + & . & . & . & . & + & . & . & . & . & . & . & $\cdot$ & . \\
\hline & Lilium carniolicum & E1 & . & . & . & . & . & . & + & . & . & . & . & . & . & . \\
\hline & Vicia sylvatica & E1 & . & . & . & . & . & . & . & . & . & . & . & . & . & . \\
\hline & Clinopodium vulgare & E1 & . & . & . & . & . & . & . & . & . & . & . & . & . & . \\
\hline & Iris graminea & E1 & . & . & . & . & . & . & . & . & . & . & . & . & $\cdot$ & . \\
\hline & Arabis pauciflora & E1 & . & . & . & . & . & . & . & . & . & . & . & . & . & . \\
\hline & Laserpitium siler & E1 & . & . & . & . & . & . & . & . & . & . & . & . & . & . \\
\hline & Polygonatum odoratum & E1 & . & . & . & . & . & . & . & . & . & . & . & . & . & . \\
\hline & Thalictrum minus & E1 & . & . & . & . & . & . & . & . & . & . & . & . & $\cdot$ & . \\
\hline & Verbascum lanatum & E1 & . & . & . & . & . & . & . & . & . & . & . & . & $\cdot$ & . \\
\hline & Grafia golaka & E1 & . & . & . & . & . & $\cdot$ & . & . & . & . & . & . & $\cdot$ & . \\
\hline \multirow[t]{4}{*}{$\mathrm{PaT}$} & Poo alpinae-Trisetetalia & & & & & & & & & & & & & & & \\
\hline & Trollius europaeus & E1 & + & $\cdot$ & + & + & . & . & . & . & . & . & . & . & $\cdot$ & . \\
\hline & Astrantia major & E1 & . & . & + & . & . & . & . & . & . & . & . & . & $\cdot$ & . \\
\hline & Ranunculus nemorosus & E1 & . & . & . & . & . & . & . & . & . & . & . & . & $\cdot$ & . \\
\hline
\end{tabular}


Poa alpina

MA Molinio-Arrbenatheretea

Dactylis glomerata

Angelica sylvestris

Galium album

Crepis paludosa

Vicia cracca

Deschampsia cespitosa

Taraxacum sect. Ruderalia

EA Epilobietea angustifolii, Galio-Urticetea

Rubus idaeus

Galeopsis speciosa

GU Urtica dioica

Hypericum hirsutum

Fragaria vesca

GU Alliaria petiolata

Stachys alpina

TR Thlaspietea rotundifolii

Adenostyles glabra

Heracleum pollinianum

Astrantia carniolica

Gymnocarpium robertianum

Arabis alpina

Dryopteris villarii

Hieracium bifidum

Ligusticum seguieri

Geranium macrorrhizum

Trisetum argenteum

Cy Cystopteridion fragilis

Asplenium viride

Valeriana tripteris

Cystopteris fragilis

Moehringia muscosa

Carex brachystachys

Cystopteris regia

Sedum hispanicum

PS Pvysoplexido comosae-Saxifragion petraeae

Campanula carnica

Campanula cespitosa

Primula auricula var. tolminensis

Saxifraga crustata

PC Potentilletalia caulescentis

Campanula cochleariifolia

Festuca stenantha

Primula auricula

AT Asplenietea trichomanis

Asplenium trichomanes

Asplenium ruta-muraria

Polypodium vulgare

$\mathrm{O}$ Other species (Druge vrste)

Hieracium sp.
E1

E1

E1

E1

E1

E1

E1

E2

E1

E1

E1

E1

E1

E1

E1

E1

E1

E1

E1

E1

E1

E1

E1

E1

E1

E1

E1

E1

E1

E1

E1

E1

E1

E1

E1

E1

E1

E

E1 

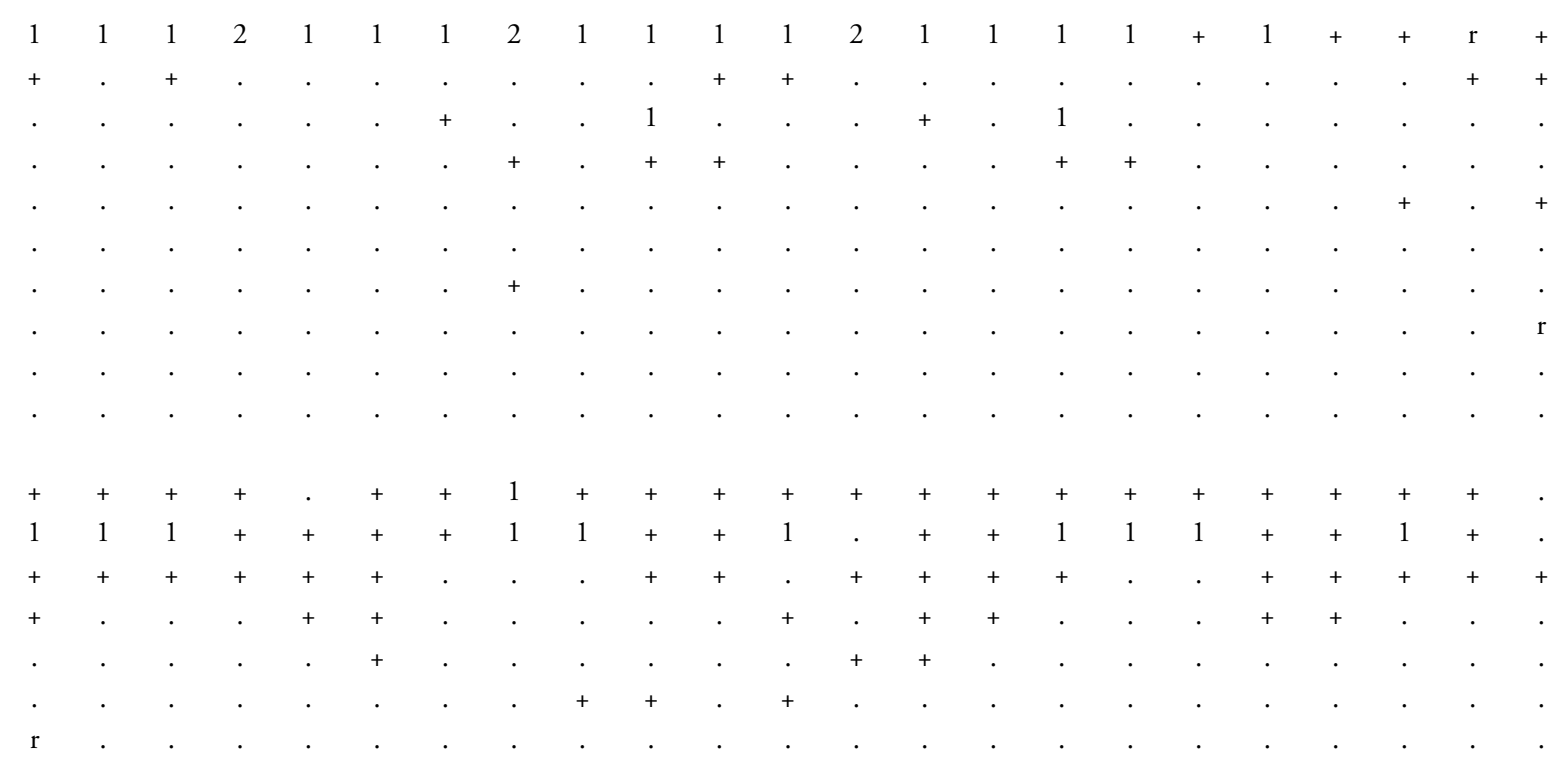
Number of relevé (Zaporedna številka popisa) $\quad \begin{array}{lllllllllllllll}1 & 2 & 3 & 4 & 5 & 6 & 7 & 8 & 9 & 10 & 11 & 12 & 13 & 14\end{array}$

ML Mosses and lichens (Mahovi in lišaji)

Ctenidium molluscum

Tortella tortuosa

Polytrichum formosum

Fissidens dubius

Schistidium apocarpum

Isothecium alopecuroides

Peltigera canina

Pseudoleskeella catenulata

Mnium thomsonii

Plagiochila porelloides

Dicranum scoparium

Bryum capillare

Cladonia sp.

Collema cristatum

Homalothecium lutescens

Conocephalum conicum

Plagiothecium undulatum

Brachythecium sp.

Homalothecium philippeanum

Encalypta streptocarpa

Mnium marginatum

Cladonia pyxidata

Marchantia polymorpha

Thuidium tamariscinum

Hypnum cupressiforme

Neckera crispa

Plagiomnium undulatum

Atrichum undulatum

Dermatocarpon miniatum

Rhizomnium punctatum

Cladonia fimbriata

Mnium sp.

Peltigera leucophlebia

Eurhynchium angustirete

Anomodon longifolius

Homalothecium sericeum

E0

E0

E0

Plagiomnium cuspidatum

E0

Entodon sp.

E0

Rhytidiadelphus triquetrus

E0

Plagiothecium denticulatum

E0

Plagiothecium sp.

E0

Hylocomium splendens 


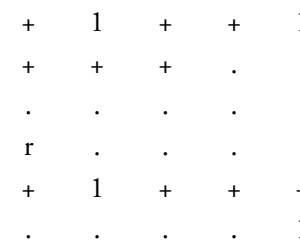


Table 3: Polysticho lonchitis-Fagetum luzuletosum luzuloidis and Ranunculo platanifolii-Fagetum luzuletosum luzuloidis - cluster 19. Relevé numbers 38-49.

Tabela 3: Polysticho lonchitis-Fagetum luzuletosum luzuloidis in Ranunculo platanifolii-Fagetum luzuletosum luzuloidis - skupina 19. Zaporedne številke popisov 38-49.

\section{Number of relevé (Zaporedna številka popisa)}

Database number of relevé

(Delovna številka popisa)

Author of the relevé (Avtor popisa)

\section{$\begin{array}{llllllllllllll}38 & 39 & 40 & 41 & 42 & 43 & 44 & 45 & 46 & 47 & 48 & 49 & \text { Pr. } & \text { Fr. }\end{array}$}

Elevation in $\mathrm{m}$ (Nadmorska višina $\mathrm{v} \mathrm{m}$ )

Aspect (Lega)

Slope in degrees (Nagib v stopinjah)

Parent material (Matična podlaga)

Soil (Tla)

Stoniness in \% (Kamnitost v \%)

Cover in \% (Zastiranje v \%):

Upper tree layer (Zgornja drevesna plast)

Lower tree layer (Spodnja drevesna plasti)

Shrub layer (Grmovna plast)

Herb layer (Zeliščna plast)

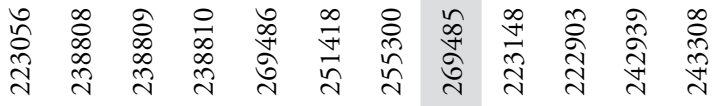

ID ID ID ID ID ID ID ID ID ID ID ID

Moss layer (Mahovna plast)

Maximum tree diameter (Maks. premer dreves)

Maximum tree height (Maksimalna višina dreves)

Number of species (Število vrst)

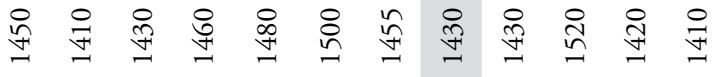

Relevé area (Velikost popisne ploskve)

Date of taking relevé (Datum popisa)

Day (Dan)

Month (Mesec)

Year (Leto)

Locality (Nahajališče)

Mountain range (Pogorje)

Quadrant (Kvadrant)

Coordinate (Koordinate) GK Y (D-48)

Coordinate (Koordinate) GK X (D-48)

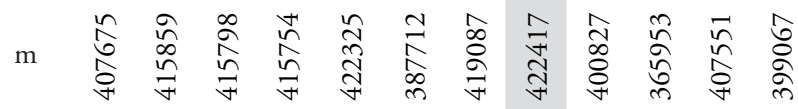

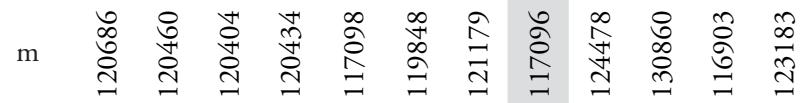

$\begin{array}{llllllllllll}35 & 25 & 30 & 30 & 15 & 30 & 30 & 35 & 35 & 35 & 35 & 35\end{array}$

AR AG AG AG AL AL AG AL A A AR ALR

$\begin{array}{llllllllllll}\mathrm{Re} & \mathrm{Eu} & \mathrm{Eu} & \mathrm{Eu} & \mathrm{Rj} & \mathrm{Eu} & \mathrm{Re} & \mathrm{Rj} & \operatorname{Re} & \operatorname{Re} & \mathrm{Re} & \mathrm{Re}\end{array}$

$\begin{array}{llllllllllll}20 & 10 & 10 & 10 & 0 & 40 & 30 & 10 & 40 & 30 & 30 & 50\end{array}$

$\begin{array}{lllllllllllll}\mathrm{E} 3 \mathrm{~b} & 90 & 90 & 90 & 90 & 95 & 90 & 80 & 90 & 80 & 90 & 80 & 90\end{array}$

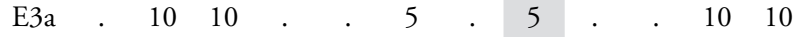

$\begin{array}{lllllllllllll}\mathrm{E} 2 & 2 & 5 & 5 & 5 & 1 & 5 & 5 & 5 & 10 & 10 & 5 & 10\end{array}$

$\begin{array}{lllllllllllll}\mathrm{E} 1 & 50 & 40 & 40 & 60 & 60 & 40 & 70 & 75 & 70 & 60 & 60 & 60\end{array}$

$\begin{array}{lllllllllllll}\text { E0 } & 10 & 5 & 5 & 5 & 5 & 10 & 10 & 5 & 20 & 5 & 10 & 10\end{array}$

$\begin{array}{lllllllllllll}\mathrm{cm} & 35 & 50 & 45 & 40 & 30 & 50 & 35 & 45 & 25 & 25 & 50 & 40\end{array}$

$\begin{array}{lllllllllllll}\mathrm{m} & 12 & 15 & 16 & 12 & 10 & 17 & 10 & 17 & 6 & 8 & 14 & 16\end{array}$

$\begin{array}{llllllllllll}59 & 36 & 35 & 32 & 55 & 49 & 73 & 50 & 80 & 80 & 66 & 43\end{array}$

$\mathrm{m}^{2} \quad 400 \quad 400 \quad 400 \quad 400 \quad 400 \quad 200 \quad 200 \quad 400 \quad 200 \quad 400 \quad 400 \quad 400$

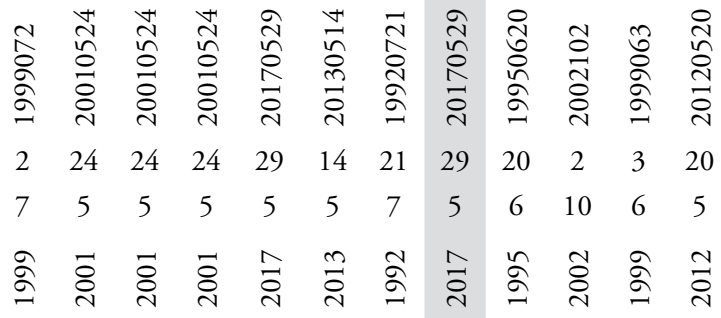

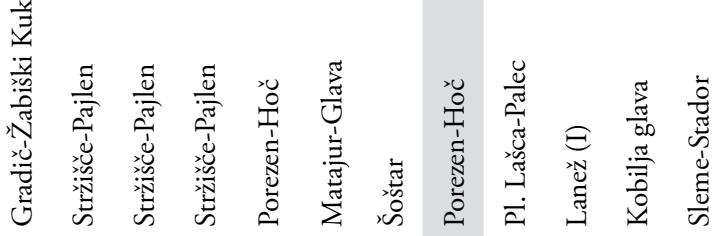

JA JA JA JA JA JA JA JA JA JA JA JA

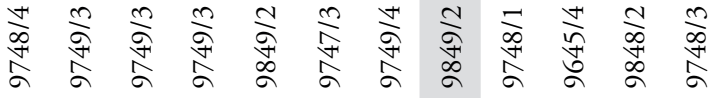

\begin{tabular}{|c|c|c|c|c|c|c|c|c|c|}
\hline 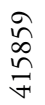 & 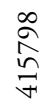 & $\begin{array}{l}\stackrel{*}{\hat{n}} \\
\hat{n} \\
\forall\end{array}$ & 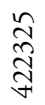 & $\underset{\substack{\infty \\
\sim}}{\stackrel{N}{N}}$ & 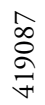 & 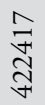 & 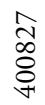 & $\begin{array}{l}\tilde{n} \\
\hat{\tilde{\sigma}} \\
\tilde{n}\end{array}$ & \\
\hline $\begin{array}{l}\text { S } \\
+ \\
\stackrel{+}{I}\end{array}$ & $\begin{array}{l}\stackrel{+}{0} \\
\stackrel{+}{\circ} \\
\text { I }\end{array}$ & 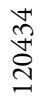 & 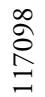 & $\begin{array}{l}\infty \\
\beth\end{array}$ & 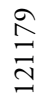 & $\begin{array}{l}\stackrel{2}{\circ} \\
\triangleq\end{array}$ & 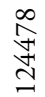 & $\begin{array}{l}8 \\
0 \\
\infty \\
0 \\
0\end{array}$ & \\
\hline
\end{tabular}


Diagnostic species of the association (Diagnostične vrste asociacije)

MuA Aconitum lycoctonum subsp. ranunculifolium

ML Paraleucobryum sauteri

VP Polystichum lonchitis

VP Clematis alpina

VP Luzula sylvatica

EP Pinus mugo

BA Sorbus chamaemespilus

CF Carex ferruginea

CA Laserpitium peucedanoides

EP Rhododendron hirsutum

PS Paederota lutea

BA Salix appendiculata

BA Salix appendiculata

CA Festuca calva

ES Aster bellidiastrum

VP Lonicera caerulea

VP Homogyne alpina

EP Rhodothamnus chamaecistus

\begin{tabular}{|c|c|c|c|c|c|c|c|c|c|c|c|c|c|c|}
\hline E1 & + & . & . & . & + & + & & 2 & . & + & + & & 32 & 65 \\
\hline E0 & + & + & . & + & . & . & + & . & + & . & + & . & 27 & 55 \\
\hline E1 & . & $\cdot$ & . & . & . & . & + & . & + & . & . & $r$ & 26 & 53 \\
\hline E2 & . & . & . & . & . & . & + & . & 1 & + & + & . & 24 & 49 \\
\hline E1 & + & . & . & . & + & . & + & + & + & + & . & . & 23 & 47 \\
\hline E2 & . & . & . & . & . & . & . & & . & . & . & . & 19 & 39 \\
\hline E2 & . & . & . & . & . & . & . & . & . & . & . & . & 16 & 33 \\
\hline E1 & . & . & · & . & . & . & . & . & . & . & . & . & 14 & 29 \\
\hline E1 & . & . & . & . & . & . & . & & . & . & $\cdot$ & . & 14 & 29 \\
\hline E2 & . & . & - & . & . & . & . & & . & . & $\cdot$ & . & 13 & 27 \\
\hline E1 & . & . & . & . & . & + & . & • & + & . & + & . & 10 & 20 \\
\hline E3 & . & . & . & . & . & . & . & & . & + & $\cdot$ & . & 1 & 2 \\
\hline E2 & . & . & . & . & . & $\mathrm{r}$ & . & & . & + & $\cdot$ & . & 8 & 16 \\
\hline E1 & . & . & . & . & . & . & + & & + & . & + & . & 8 & 16 \\
\hline E1 & . & . & 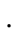 & . & . & . & . & & . & . & $\cdot$ & . & 7 & 14 \\
\hline E2 & . & . & . & . & . & . & . & & . & . & $\cdot$ & . & 4 & 8 \\
\hline E1 & 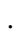 & . & . & . & . & . & . & & $\cdot$ & . & $\cdot$ & . & 2 & 4 \\
\hline E1 & . & . & . & . & . & . & . & $\theta^{\circ}$ & . & . & . & . & 3 & 6 \\
\hline
\end{tabular}

\section{Differential species of the subassociation (Razlikovalnice subasociacije)}

VP Calamagrostis arundinacea

VP Luzula luzuloides

FB Cirsium erisithales

EC Primula vulgaris

AF Aremonio-Fagion

Cardamine enneaphyllos

Cyclamen purpurascens

Cardamine trifolia

Anemone trifolia

Lamium orvala

Rhamnus fallax

Anemone x pittonii

Knautia drymeia

Aremonia agrimonoides

Hacquetia epipactis

Vicia oroboides

Omphalodes verna

EC Erythronio-Carpinion

Helleborus odorus

Ornithogalum pyrenaicum

$\begin{array}{ll}\text { E1 } & 1 \\ \text { E1 } & 1 \\ \text { E1 } & \text {. } \\ \text { E1 } & \text {. }\end{array}$

\section{Tilio-Acerion}

Adoxa moschatellina

Acer pseudoplatanus

Acer pseudoplatanus

Acer pseudoplatanus

Polystichum aculeatum

Aruncus dioicus

Geranium robertianum

Lunaria rediviva

Polystichum brauni

Chrysosplenium alternifolium

Cardamine flexuosa

Polystichum $x$ luerssenii

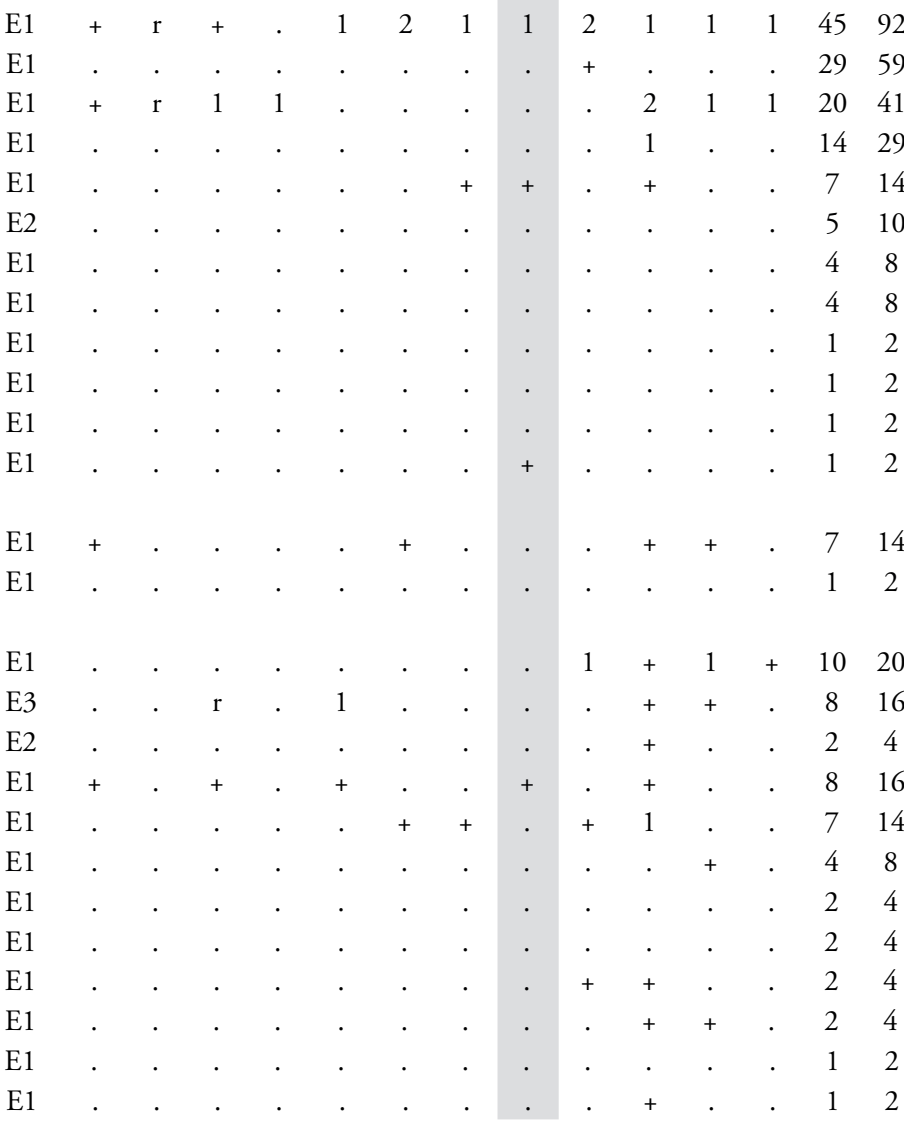




\begin{tabular}{|c|c|c|c|c|c|c|c|c|c|c|c|c|c|c|c|c|}
\hline & Number of relevé (Zaporedna številka popisa) & & 38 & 39 & 40 & 41 & 42 & 43 & 44 & 45 & 46 & 47 & 48 & 49 & Pr. & Fr. \\
\hline & Stellaria montana & E1 & . & $\cdot$ & . & . & . & . & . & . & . & . & 1 & . & 1 & 2 \\
\hline & Corydalis solida & E1 & . & 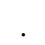 & . & . & . & . & . & ${ }^{\circ}$ & . & . & + & . & 1 & 2 \\
\hline \multirow[t]{44}{*}{ FS } & Fagetalia sylvaticae & & & & & & & & & & & & & & & \\
\hline & Fagus sylvatica & E3 & 5 & 5 & 5 & 5 & 5 & 5 & 5 & 5 & 5 & 5 & 5 & 5 & 49 & 100 \\
\hline & Fagus sylvatica & E2 & + & $\mathrm{r}$ & + & . & . & . & . & + & + & 1 & + & 1 & 31 & 63 \\
\hline & Fagus sylvatica & E1 & . & . & . & . & . & + & + & 1 & . & . & + & . & 13 & 27 \\
\hline & Lilium martagon & E1 & + & $\mathrm{r}$ & + & + & + & 1 & + & + & . & . & 1 & + & 41 & 84 \\
\hline & Galium laevigatum & E1 & + & + & . & . & + & + & + & + & . & . & + & 1 & 40 & 82 \\
\hline & Lonicera alpigena & E2 & . & $\mathrm{r}$ & . & + & + & + & . & . & + & + & . & . & 39 & 80 \\
\hline & Mercurialis perennis & E1 & . & . & . & . & 1 & . & + & . & + & + & . & . & 36 & 73 \\
\hline & Daphne mezereum & E2 & + & + & . & . & . & . & + & + & + & . & . & . & 35 & 71 \\
\hline & Galeobdolon flavidum & E1 & . & . & . & + & + & + & 1 & 1 & + & . & + & . & 33 & 67 \\
\hline & Symphytum tuberosum & E1 & . & + & + & + & + & . & + & 1 & . & + & 1 & 1 & 32 & 65 \\
\hline & Dryopteris filix-mas & $\mathrm{E} 1$ & . & $\mathrm{r}$ & + & $\mathrm{r}$ & 1 & 1 & + & 1 & 1 & 1 & 1 & 1 & 30 & 61 \\
\hline & Paris quadrifolia & E1 & . & . & $\mathrm{r}$ & . & 1 & + & . & + & 1 & . & + & . & 27 & 55 \\
\hline & Melica nutans & E1 & . & . & . & . & . & . & + & . & + & . & . & . & 25 & 51 \\
\hline & Actaea spicata & E1 & + & . & . & . & 1 & . & + & . & 1 & 1 & + & + & 22 & 45 \\
\hline & Prenanthes purpurea & E1 & 1 & 1 & + & 1 & . & . & + & . & . & . & . & . & 18 & 37 \\
\hline & Euphorbia amygdaloides & E1 & . & . & + & . & . & . & . & . & . & . & . & . & 15 & 31 \\
\hline & Epilobium montanum & E1 & . & . & . & . & + & . & 2 & + & + & + & 1 & + & 14 & 29 \\
\hline & Lathyrus vernus & E1 & + & + & . & . & . & + & + & . & 1 & + & + & . & 14 & 29 \\
\hline & Scrophularia nodosa & E1 & . & . & . & . & 1 & . & . & + & . & + & + & + & 9 & 18 \\
\hline & Mycelis muralis & E1 & . & . & . & . & + & . & 1 & . & . & . & . & . & 8 & 16 \\
\hline & Lathyrus vernus subsp. flaccidus & E1 & + & . & . & . & . & . & . & . & . & + & + & + & 7 & 14 \\
\hline & Luzula nivea & E1 & . & . & . & . & . & . & . & . & . & + & . & . & 7 & 14 \\
\hline & Campanula trachelium & E1 & . & . & . & . & . & . & . & . & . & . & . & . & 6 & 12 \\
\hline & Corydalis cava & E1 & . & . & . & . & 1 & . & . & + & . & 1 & . & . & 6 & 12 \\
\hline & Pulmonaria officinalis & E1 & . & . & . & . & . & . & + & . & . & . & . & . & 5 & 10 \\
\hline & Myosotis sylvatica agg. & E1 & . & . & . & . & . & . & . & . & . & + & + & . & 4 & 8 \\
\hline & Festuca altissima & E1 & . & . & . & . & . & . & . & . & . & . & . & . & 4 & 8 \\
\hline & Laburnum alpinum & E3 & . & . & . & . & . & . & . & . & . & . & . & $\mathrm{r}$ & 3 & 6 \\
\hline & Laburnum alpinum & E2 & . & . & . & . & . & . & + & . & . & . & . & . & 4 & 8 \\
\hline & Laburnum alpinum & E1 & . & . & . & . & . & . & . & . & . & . & . & + & 2 & 4 \\
\hline & Ranunculus lanuginosus & E1 & . & . & . & . & . & . & . & . & . & . & + & + & 3 & 6 \\
\hline & Galium odoratum & E1 & . & . & . & . & 1 & + & . & 1 & . & . & . & . & 3 & 6 \\
\hline & Petasites albus & E1 & . & . & . & . & . & . & . & . & . & . & . & . & 2 & 4 \\
\hline & Viola reichenbachiana & E1 & . & . & . & . & . & . & . & . & . & . & . & . & 2 & 4 \\
\hline & Asarum europaeum subsp. caucasicum & E1 & . & . & . & . & . & . & . & . & . & $\mathrm{r}$ & . & . & 2 & 4 \\
\hline & Neottia nidus-avis & E1 & + & . & . & . & . & . & . & . & . & . & . & . & 2 & 4 \\
\hline & Cardamine bulbifera & $\mathrm{E} 1$ & . & . & . & . & + & . & . & + & . & . & . & . & 2 & 4 \\
\hline & Salvia glutinosa & E1 & . & . & . & . & . & . & . & . & . & . & . & . & 1 & 2 \\
\hline & Asarum europaeum subsp. europaeum & E1 & . & . & . & . & . & . & . & . & . & . & . & . & 1 & 2 \\
\hline & Polygonatum multiflorum & $\mathrm{E} 1$ & . & . & . & . & . & . & . & . & . & . & . & . & 1 & 2 \\
\hline & Carex sylvatica & E1 & . & . & . & . & . & . & . & . & . & . & . & . & 1 & 2 \\
\hline & Cardamine impatiens & E1 & . & . & . & . & . & . & . & . & . & . & . & . & 1 & 2 \\
\hline & Leucojum vernum & E1 & . & . & . & . & + & . & . & . & . & . & . & . & 1 & 2 \\
\hline \multirow[t]{7}{*}{ QP } & Quercetalia pubescenti-petraeae & & & & & & & & & & & & & & & \\
\hline & Sorbus arias (Aria edulis) & E3 & . & . & . & . & . & . & . & + & . & . & . & . & 2 & 4 \\
\hline & Sorbus arias (Aria edulis) & E2 & . & . & . & . & . & . & . & . & + & + & $\mathrm{r}$ & . & 18 & 37 \\
\hline & Sorbus arias (Aria edulis) & E1 & . & . & . & . & . & . & . & . & . & . & . & . & 1 & 2 \\
\hline & Melittis melissophyllum & E1 & . & . & . & . & . & . & . & . & . & . & . & . & 7 & 14 \\
\hline & Convallaria majalis & E1 & . & . & . & . & . & . & . & . & . & . & . & . & 6 & 12 \\
\hline & Carex flacca & E1 & . & . & . & . & . & . & . & . & . & . & . & . & 1 & 2 \\
\hline
\end{tabular}


QF Querco-Fagetea

Anemone nemorosa

Hepatica nobilis

Poa nemoralis

Carex digitata

Festuca heterophylla

Carex pilosa

Dactylorhiza fuchsii

Stellaria holostea

Ranunculus auricomus agg. (R. braun-blanquetii)

Spiraea chamaedryfolia

Aegopodium podagraria

Scilla bifolia

Dactylis glomerata subsp. lobata (D. polygama)

Lonicera xylosteum

VP Vaccinio-Piceetea

Aposeris foetida

Gentiana asclepiadea

Maianthemum bifolium

Veronica urticifolia

Oxalis acetosella

Picea abies

Picea abies

Picea abies

Rosa pendulina

Solidago virgaurea

Hieracium murorum

Vaccinium myrtillus

Dryopteris dilatata

Huperzia selago

Saxifraga cuneifolia

Homogyne sylvestris

Gymnocarpium dryopteris

Lonicera nigra

Vaccinium vitis-idaea

Phegopteris connectilis

Larix decidua

Dryopteris expansa

Calamagrostis villosa

Lycopodium annotinum

Abies alba

Luzula luzulina

Avenella flexuosa

Monotropa hypopitys

Thelypteris limbosperma

Blechnum spicant

EP Erico-Pinetea

Rubus saxatilis

Calamagrostis varia

Aquilegia nigricans

Erica carnea

Carex alba

Carex ornithopoda
$\begin{array}{lllllllllllll}\mathrm{E} 1 & 1 & 1 & 1 & 1 & 2 & 2 & 1 & 1 & 1 & . & 1 & +\end{array}$

E1 . . . . . . . . 1224

E1 . . . . . . . . . . + 1020

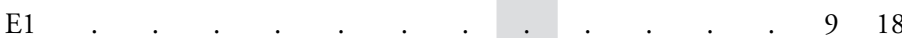

$\mathrm{E} 1+\ldots+\ldots+612$

$\mathrm{E} 1 . \mathrm{r}+\mathrm{H}$. . . . . . 510

$\mathrm{E} 1 . \quad . \quad . \quad . \quad . \quad . \quad . \quad+36$

$\mathrm{E} 1 .+. \quad . \quad . \quad 2.56$

$\mathrm{E} 1$. $. \quad . \quad . \quad . \quad . \quad . \quad . \quad . \quad . \quad . \quad 12$

E2 . . . . . . . . . . . 12

$\mathrm{E} 1 \ldots .+. \cdot 12$

$\mathrm{E} 1 .+. \quad . \quad . \quad . \quad . \quad . \quad . \quad 122$

$\mathrm{E} 2 . \quad . \quad . \quad . \quad . \quad . \quad . \quad . \quad 122$

$\mathrm{E} 1+1+1+.+\ldots++4286$

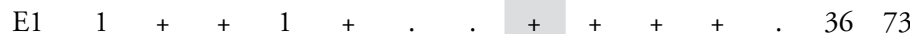

$\mathrm{E} 1+1+.+1+.+3061$

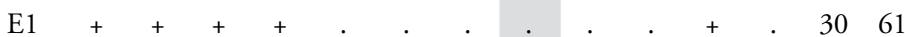

$\mathrm{E} 11+1 .+1 .+1+112959$

$\mathrm{E} 3 \mathrm{r}+\mathrm{r} . \mathrm{r}+. .2857$

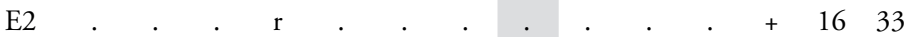

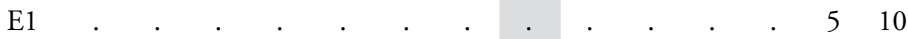

$\mathrm{E} 2 . \mathrm{r} . \quad . \quad . \quad . \quad . \quad . \quad 2857$

$\mathrm{E} 1++\mathrm{t} \cdot \mathrm{r}+.+2857$

$\mathrm{E} 1++\ldots+.+.+250.251$

$\mathrm{E} 1+.+.+.+. .2449$

$\mathrm{E} 11.1++{ }_{+}+t_{+}+2245$

$\mathrm{E} 1+. \quad . \quad . \quad . \quad . \quad . \quad . \quad . \quad 1531$

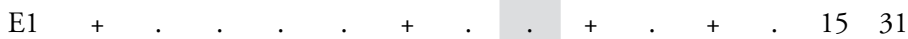

$\mathrm{E} 1+. \quad . \quad . \quad . \quad . \quad . \quad . \quad . \quad 1020$

$\mathrm{E} 1+$. . . . . ...$++ \quad 816$

E2 . $\quad . \quad . \quad 714$

E1 . . . . . . . . . . 612

$\mathrm{E} 1++{ }_{+}+6 \quad 612$

E3. . . . . . . . . . . 36

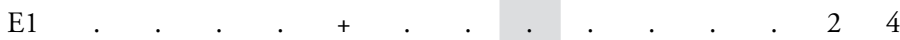

$\mathrm{E} 1 . \quad . \quad . \quad . \quad . \quad . \quad . \quad . \quad . \quad . \quad 2 \quad 24$

$\mathrm{E} 1 . .5 . \quad . \quad . \quad . \quad . \quad . \quad . \quad . \quad 2 \quad 44$

$\mathrm{E} 2 . \quad . \quad . \quad . \quad . \quad . \quad . \quad . \quad . \quad . \quad . \quad . \quad . \quad 244$

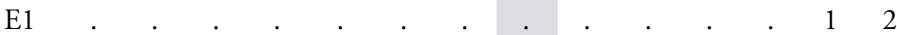

E1 $1 . \begin{array}{lll}1 & 1\end{array}$

$\mathrm{E} 1 .+. . \quad . \quad . \quad . \quad . \quad . \quad 122$

$\mathrm{E} 1 . \quad \mathrm{r} \quad . \quad . \quad . \quad . \quad . \quad . \quad . \quad 122$

$\mathrm{E} 1 .+.+. \quad . \quad . \quad . \quad . \quad 2347$

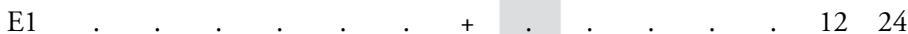

E1 . . . . . . . . . . . . 1122

E1 . . . . . . . . . . . . . 1020

$\mathrm{E} 1 . \quad . \quad . \quad . \quad . \quad . \quad . \quad . \quad . \quad 8016$

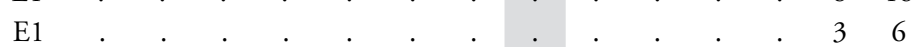


Genista radiata

Peucedanum austriacum subsp. rablense

E2

Chamaecytisus hirsutus

Polygala chamaebuxus

Peucedanum austriacum subsp. austriacum

SSC Sambuco-Salicion capreae, Rhamno-Prunetea

Sorbus aucuparia

Sorbus aucuparia

Sorbus aucuparia

Rosa sp.

Sambucus racemosa

BA Betulo-Alnetea

Alnus viridis

Ribes alpinum

Sorbus austriaca

Salix glabra

Juniperus sibirica

MuA Mulgedio-Aconitetea

Polygonatum verticillatum

Veratrum album

Athyrium filix-femina

Phyteuma ovatum

Senecio ovatus

Saxifraga rotundifolia

Ranunculus platanifolius

Tanacetum corymbosum subsp. clusii

Thalictrum aquilegiifolium

Aconitum degenii subsp. paniculatum

Chaerophyllum villarsii

Allium victorialis

Viola biflora

Geranium sylvaticum

Hypericum maculatum

Centaurea montana

Aconitum angustifolium

Pleurospermum austriacum

Senecio cacaliaster

Doronicum austriacum

Chaerophyllum hirsutum

Primula elatior

Rumex arifolius

Lathyrus occidentalis var. montanus

Geum rivale

Agropyron caninum

Myrrhis odorata

Milium effusum

Stellaria nemorum

Chaerophyllum aureum

Carduus personata

Poa hybrida

Streptopus amplexifolius

Silene dioica

Adenostyles alliariae

$$
\begin{aligned}
& 24 \\
& \begin{array}{ll}
1 & 2
\end{array} \\
& 12 \\
& 12 \\
& 12 \\
& 12 \quad 24 \\
& 10 \quad 20 \\
& \text { - } 16 \quad 33 \\
& \text { - } 12 \\
& 1 \\
& 510 \\
& 48 \\
& \text { - } 36 \\
& \text { - } 12 \\
& 12 \\
& 46 \quad 94 \\
& 4184 \\
& \begin{array}{ll}
34 & 69
\end{array} \\
& \begin{array}{ll}
33 & 67
\end{array} \\
& 27 \quad 55 \\
& \begin{array}{ll}
23 & 47
\end{array} \\
& 18 \quad 37 \\
& \begin{array}{ll}
18 & 37
\end{array} \\
& \begin{array}{ll}
18 & 37
\end{array} \\
& 14 \quad 29 \\
& 14 \quad 29 \\
& 13 \quad 27 \\
& 13 \quad 27 \\
& 12 \quad 24 \\
& 12 \quad 24 \\
& 11 \quad 22 \\
& 10 \quad 20 \\
& \begin{array}{ll}
9 & 18
\end{array} \\
& 714 \\
& 6 \quad 12 \\
& \text { 5 } 10 \\
& \begin{array}{ll}
4 & 8
\end{array} \\
& 36 \\
& 36 \\
& 36 \\
& 24 \\
& 24 \\
& \text { - } 24 \\
& 24 \\
& 12 \\
& 12 \\
& \text { 1 } 2 \\
& 12 \\
& 12
\end{aligned}
$$




\begin{tabular}{|c|c|c|c|c|c|c|c|c|c|c|c|c|c|c|c|c|}
\hline & Number of relevé (Zaporedna številka popisa) & & 38 & 39 & 40 & 41 & 42 & 43 & 44 & 45 & 46 & 47 & 48 & 49 & Pr. & Fr. \\
\hline & Cicerbita alpina & E1 & . & . & . & . & . & . & . & + & . & . & . & . & 1 & 2 \\
\hline \multirow[t]{2}{*}{$\mathrm{CF}$} & Caricion ferrugineae & & & & & & & & & & & & & & & \\
\hline & Cerastium subtriflorum & E1 & . & . & . & . & . & . & . & . & . & + & . & . & 2 & 4 \\
\hline \multirow[t]{12}{*}{ ES } & Elyno-Seslerietea & & & & & & & & & & & & & & & \\
\hline & Betonica alopecuros & E1 & . & . & . & . & . & . & + & . & . & . & . & . & 27 & 55 \\
\hline & Sesleria caerulea & E1 & . & . & . & . & . & . & + & . & + & . & . & + & 23 & 47 \\
\hline & Campanula witasekiana & E1 & . & . & . & . & . & + & . & . & . & . & . & . & 9 & 18 \\
\hline & Astrantia bavarica & E1 & . & . & . & . & . & . & . & . & + & . & . & . & 4 & 8 \\
\hline & Pimpinella alpina & E1 & . & . & . & . & . & . & . & . & + & . & . & . & 4 & 8 \\
\hline & Carex sempervirens & E1 & . & . & . & . & . & . & . & . & . & . & . & . & 3 & 6 \\
\hline & Phyteuma orbiculare & E1 & . & . & . & . & . & . & + & . & . & . & . & . & 3 & 6 \\
\hline & Ranunculus montanus & $\mathrm{E} 1$ & . & . & . & . & . & . & . & . & . & . & . & . & 1 & 2 \\
\hline & Achillea clavenae & E1 & . & . & . & . & . & . & . & . & . & . & . & . & 1 & 2 \\
\hline & Carduus crassifolius & $\mathrm{E} 1$ & . & . & . & . & . & . & . & . & . & . & . & . & 1 & 2 \\
\hline & Phleum hirsutum & E1 & . & . & . & . & . & . & + & . & . & . & . & . & 1 & 2 \\
\hline \multirow[t]{5}{*}{ NS } & Nardion strictae, Juncetea trifidi & & & & & & & & & & & & & & & \\
\hline & Campanula scheuchzeri & E1 & + & . & . & . & . & . & . & . & + & + & . & . & 7 & 14 \\
\hline & Festuca ovina agg. & E1 & . & . & . & . & . & . & . & . & . & . & . & . & 2 & 4 \\
\hline & Coeloglossum viride & E1 & . & . & . & . & . & . & . & . & . & . & . & . & 1 & 2 \\
\hline & Festuca nigrescens & E1 & . & . & . & . & . & . & . & + & . & . & . & . & 1 & 2 \\
\hline \multirow[t]{2}{*}{$\mathrm{CD}$} & Caricetalia davallianae & & & & & & & & & & & & & & & \\
\hline & Parnassia palustris & E1 & . & . & . & . & . & . & . & . & . & . & . & . & 1 & 2 \\
\hline \multirow[t]{8}{*}{ FB } & Festuco-Brometea & & & & & & & & & & & & & & & \\
\hline & Buphthalmum salicifolium & E1 & . & . & . & . & . & . & . & . & . & . & . & . & 3 & 6 \\
\hline & Carex humilis & $\mathrm{E} 1$ & . & . & . & . & . & . & . & . & . & . & . & . & 3 & 6 \\
\hline & Carlina acaulis & E1 & . & . & . & . & . & . & . & . & . & . & . & . & 2 & 4 \\
\hline & Bromopsis transsilvanica & E1 & . & . & . & . & . & . & . & . & . & . & . & . & 1 & 2 \\
\hline & Linum viscosum & E1 & . & . & . & . & . & . & . & . & . & . & . & . & 1 & 2 \\
\hline & Centaurea triumfettii & E1 & . & . & . & . & . & . & . & . & . & . & . & . & 1 & 2 \\
\hline & Koeleria pyramidata & E1 & . & . & . & . & . & . & . & . & . & . & . & . & 1 & 2 \\
\hline \multirow[t]{15}{*}{ TG } & Trifolio-Geranietea & & & & & & & & & & & & & & & \\
\hline & Digitalis grandiflora & E1 & . & . & . & . & . & . & + & + & . & . & . & . & 4 & 8 \\
\hline & Laserpitium latifolium & E1 & . & . & . & . & . & . & . & . & . & . & . & . & 3 & 6 \\
\hline & Arabis turrita & E1 & . & . & . & . & . & . & . & . & . & . & . & . & 3 & 6 \\
\hline & Calamintha sylvatica & E1 & . & . & . & . & . & . & . & . & . & . & . & . & 2 & 4 \\
\hline & Lilium carniolicum & E1 & . & . & . & . & . & . & . & . & . & . & . & . & 2 & 4 \\
\hline & Vicia sylvatica & E1 & . & . & . & . & . & . & + & . & . & . & . & . & 2 & 4 \\
\hline & Clinopodium vulgare & $\mathrm{E} 1$ & . & . & . & . & . & . & . & . & . & . & . & . & 2 & 4 \\
\hline & Iris graminea & E1 & . & . & . & . & . & . & . & . & . & . & . & . & 1 & 2 \\
\hline & Arabis pauciflora & $\mathrm{E} 1$ & . & . & . & . & . & . & . & . & . & . & . & . & 1 & 2 \\
\hline & Laserpitium siler & E1 & . & . & . & . & . & . & . & . & . & . & . & . & 1 & 2 \\
\hline & Polygonatum odoratum & E1 & . & . & . & . & . & . & . & . & . & . & . & . & 1 & 2 \\
\hline & Thalictrum minus & $\mathrm{E} 1$ & . & . & . & . & . & . & . & . & . & . & . & . & 1 & 2 \\
\hline & Verbascum lanatum & E1 & . & . & . & . & . & . & . & . & . & . & . & . & 1 & 2 \\
\hline & Grafia golaka & E1 & . & . & . & . & . & . & . & . & . & $\mathrm{r}$ & . & . & 1 & 2 \\
\hline \multirow[t]{5}{*}{$\mathrm{PaT}$} & Poo alpinae-Trisetetalia & & & & & & & & & & & & & & & \\
\hline & Trollius europaeus & E1 & . & . & . & . & . & . & . & . & . & . & . & . & 4 & 8 \\
\hline & Astrantia major & $\mathrm{E} 1$ & . & . & . & . & . & . & . & . & . & . & . & . & 1 & 2 \\
\hline & Ranunculus nemorosus & E1 & . & . & . & . & . & . & . & . & . & . & . & . & 1 & 2 \\
\hline & Poa alpina & E1 & . & . & . & . & . & $\mathrm{r}$ & . & . & . & . & . & . & 1 & 2 \\
\hline \multirow[t]{3}{*}{ MA } & Molinio-Arrhenatheretea & & & & & & & & & & & & & & & \\
\hline & Dactylis glomerata & E1 & . & . & . & . & . & . & . & . & . & . & . & . & 6 & 12 \\
\hline & Angelica sylvestris & E1 & . & . & . & . & . & . & + & . & + & . & . & 1 & 6 & 12 \\
\hline
\end{tabular}


Galium album

E1

Crepis paludosa

Vicia cracca

Deschampsia cespitosa

Taraxacum sect. Ruderalia

EA Epilobietea angustifolii, Galio-Urticetea Rubus idaeus Galeopsis speciosa

GU Urtica dioica

Hypericum hirsutum

Fragaria vesca

GU Alliaria petiolata

Stachys alpina

TR Thlaspietea rotundifolii

Adenostyles glabra

Heracleum pollinianum

Astrantia carniolica

Gymnocarpium robertianum

Arabis alpina

Dryopteris villarii

Hieracium bifidum

Ligusticum seguieri

Geranium macrorrhizum

Trisetum argenteum

Cy Cystopteridion fragilis

Asplenium viride

Valeriana tripteris

Cystopteris fragilis

Moehringia muscosa

Carex brachystachys

Cystopteris regia

Sedum hispanicum

PS Pvysoplexido comosae-Saxifragion petraeae

Campanula carnica

Campanula cespitosa

Primula auricula var. tolminensis

Saxifraga crustata

PC Potentilletalia caulescentis

Campanula cochleariffolia

Festuca stenantha

Primula auricula

AT Asplenietea trichomanis

Asplenium trichomanes

Asplenium ruta-muraria

Polypodium vulgare

O Other species (Druge vrste)

Hieracium sp.

ML Mosses and lichens (Mahovi in lišaji)

Ctenidium molluscum

Tortella tortuosa

Polytrichum formosum

Fissidens dubius

Schistidium apocarpum
E1

E1

E1

E2

E1

E1

E1

E1

E1

E1

E1

E1

E1

E1

E1

E1

E1

E1

E1

E1

E1

E1

E1

E1

E1

E1

E1

E1

E1

E1

E1

E1

E1

E1

E1

E1

E0

E0

E0

E0

E0
6

4

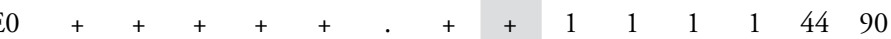

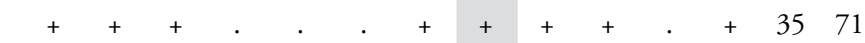

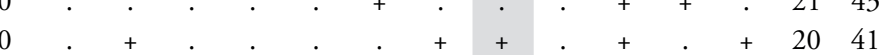




\begin{tabular}{|c|c|c|c|c|c|c|c|c|c|c|c|c|c|c|c|}
\hline Number of relevé (Zaporedna številka popisa) & & 38 & 39 & 40 & 41 & 42 & 43 & 44 & 45 & 46 & 47 & 48 & 49 & Pr. & Fr. \\
\hline Isothecium alopecuroides & E0 & . & . & . & . & + & + & + & + & 1 & + & + & + & 16 & 33 \\
\hline Peltigera canina & E0 & + & . & . & . & + & . & . & + & + & + & + & . & 12 & 24 \\
\hline Pseudoleskeella catenulata & E0 & . & . & . & . & + & . & + & . & 1 & + & + & . & 11 & 22 \\
\hline Mnium thomsonii & E0 & . & . & . & . & . & 1 & . & . & 1 & + & . & . & 8 & 16 \\
\hline Plagiochila porelloides & E0 & . & . & . & . & . & . & . & . & . & 1 & + & . & 8 & 16 \\
\hline Dicranum scoparium & E0 & . & . & . & . & . & + & . & . & . & . & . & . & 7 & 14 \\
\hline Bryum capillare & E0 & + & . & . & . & . & + & . & . & + & . & . & . & 5 & 10 \\
\hline Cladonia sp. & E0 & + & . & . & . & . & . & . & . & . & . & + & . & 5 & 10 \\
\hline Collema cristatum & E0 & . & . & . & . & . & . & . & . & . & . & . & . & 5 & 10 \\
\hline Homalothecium lutescens & E0 & . & . & . & . & . & . & . & + & . & . & . & 1 & 5 & 10 \\
\hline Conocephalum conicum & E0 & . & . & . & . & . & . & . & . & + & + & + & . & 4 & 8 \\
\hline Plagiothecium undulatum & E0 & + & . & . & . & . & . & . & . & . & . & . & . & 3 & 6 \\
\hline Brachythecium sp. & E0 & . & . & . & . & . & . & . & . & . & . & . & . & 3 & 6 \\
\hline Homalothecium philippeanum & E0 & . & . & . & . & . & . & + & . & . & . & . & . & 3 & 6 \\
\hline Encalypta streptocarpa & E0 & . & . & . & . & . & . & . & . & . & . & . & . & 3 & 6 \\
\hline Mnium marginatum & E0 & . & . & . & . & . & . & + & . & . & . & . & . & 3 & 6 \\
\hline Cladonia pyxidata & E0 & . & . & . & . & . & . & . & . & . & . & . & . & 2 & 4 \\
\hline Marchantia polymorpha & E0 & . & . & . & . & . & . & . & . & . & . & . & . & 2 & 4 \\
\hline Thuidium tamariscinum & E0 & . & . & . & . & . & . & . & . & + & . & . & . & 2 & 4 \\
\hline Hypnum cupressiforme & E0 & . & . & . & . & . & . & . & . & . & . & . & . & 2 & 4 \\
\hline Neckera crispa & E0 & . & . & . & . & . & . & . & . & . & + & . & . & 2 & 4 \\
\hline Plagiomnium undulatum & E0 & . & . & . & . & . & . & . & . & . & + & . & . & 2 & 4 \\
\hline Atrichum undulatum & E0 & . & . & . & . & . & . & . & + & . & . & . & . & 2 & 4 \\
\hline Dermatocarpon miniatum & E0 & . & . & . & . & . & . & . & . & . & . & . & . & 2 & 4 \\
\hline Rhizomnium punctatum & E0 & . & . & . & . & . & . & . & . & . & + & . & . & 2 & 4 \\
\hline Cladonia fimbriata & E0 & . & . & . & . & . & . & . & . & . & . & . & . & 2 & 4 \\
\hline Mnium sp. & E0 & + & . & . & . & . & . & . & . & 1 & . & . & . & 2 & 4 \\
\hline Peltigera leucophlebia & E0 & + & . & . & . & . & . & . & . & + & . & . & . & 2 & 4 \\
\hline Eurhynchium angustirete & E0 & . & . & . & . & + & . & . & . & 1 & . & . & . & 2 & 4 \\
\hline Anomodon longifolius & E0 & . & . & . & . & . & . & . & . & . & . & . & . & 1 & 2 \\
\hline Homalothecium sericeum & E0 & . & . & . & . & . & . & . & . & . & . & . & . & 1 & 2 \\
\hline Plagiomnium cuspidatum & E0 & . & . & . & . & . & . & . & . & . & . & . & . & 1 & 2 \\
\hline Entodon sp. & E0 & . & . & . & . & . & . & . & . & . & . & . & . & 1 & 2 \\
\hline Rhytidiadelphus triquetrus & E0 & . & . & . & . & . & . & . & . & . & . & . & . & 1 & 2 \\
\hline Plagiothecium denticulatum & E0 & + & . & . & . & . & . & . & . & . & . & . & . & 1 & 2 \\
\hline Plagiothecium sp. & E0 & + & . & . & . & . & . & . & . & . & . & . & . & 1 & 2 \\
\hline Hylocomium splendens & E0 & . & . & . & . & . & . & . & . & + & . & . & . & 1 & 2 \\
\hline
\end{tabular}

\section{Legend - Legenda}

ID Igor Dakskobler

A Limestone - apnenec

B Breccia - breča

D Dolomite - dolomit

G Claystone - glinavec

Gr Gravel - grušč

L Marlstone - laporovec

R Chert - roženec

Eu Eutryc brown soil - evtrična rjava tla

$\mathrm{Rj}$ Calcareous brown soil - rjava pokarbonatna tla

Re Rendzina - rendzina

JA Julian Alps - Julijske Alpe

I Italy (Italia, Italija)

Pr. Presence (number of relevés in which the species is presented) - število popisov, $\mathrm{v}$ katerih se pojavlja vrsta

Fr. Frequency in $\%$ - frekvenca $v \%$
Columns 1-34: Polysticho lonchitis-Fagetum luzuletosum luzuloidis Relevé No. 12, holotypus

Columns 35-49: Ranunculo platanifolii-Fagetum luzuletosum luzuloidis

Relevé No. 45, holotypus 
Table 4: Polysticho lonchitis-Fagetum calamagrostietosum variae - cluster 22. Relevé numbers 1-37.

\begin{tabular}{|c|c|c|c|c|c|c|c|c|c|c|c|c|c|c|c|}
\hline & \multicolumn{2}{|l|}{ Number of relevé (Zaporedna številka popisa) } & 1 & 2 & 3 & 4 & 5 & 6 & 7 & 8 & 9 & 10 & 11 & 12 & 13 \\
\hline & \multicolumn{2}{|l|}{$\begin{array}{l}\text { Database number of relevé } \\
\text { (Delovna številka popisa) }\end{array}$} & 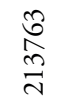 & $\begin{array}{l}\text { ত̃ } \\
\stackrel{n}{\sim} \\
\sim\end{array}$ & $\begin{array}{l}\hat{\sigma} \\
\hat{n} \\
\stackrel{\sim}{1}\end{array}$ & $\begin{array}{l}\stackrel{0}{\curvearrowleft} \\
\underset{\sim}{\sim}\end{array}$ & 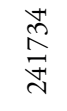 & $\begin{array}{l}\widehat{\sigma} \\
\infty \\
\infty \\
\curvearrowright \\
\curvearrowright\end{array}$ & $\begin{array}{l}\vec{n} \\
\infty \\
\infty \\
\sim\end{array}$ & $\begin{array}{l}\stackrel{0}{ } \\
\infty \\
\infty \\
\stackrel{\sim}{N}\end{array}$ & $\begin{array}{l}\underset{N}{\infty} \\
\infty \\
\sim \\
\sim\end{array}$ & $\begin{array}{l}\stackrel{N}{\infty} \\
\infty \\
\infty \\
\sim\end{array}$ & $\begin{array}{l}\text { f } \\
\infty \\
\infty \\
\curvearrowright \\
\curvearrowright\end{array}$ & $\begin{array}{l}\stackrel{0}{1} \\
\infty \\
\infty \\
\sim \\
\sim\end{array}$ & 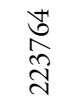 \\
\hline & \multicolumn{2}{|l|}{ Author of the relevé (Avtor popisa) } & ID & ID & ID & ID & ID & ID & ID & ID & ID & ID & ID & ID & ID \\
\hline & \multicolumn{2}{|l|}{ Elevation in $\mathrm{m}$ (Nadmorska višina $\mathrm{v} \mathrm{m})$} & $\begin{array}{l}\infty \\
\stackrel{\infty}{n}\end{array}$ & $\begin{array}{l}\stackrel{0}{0} \\
\stackrel{n}{n}\end{array}$ & $\begin{array}{l}\stackrel{\circ}{\infty} \\
\stackrel{1}{n}\end{array}$ & $\stackrel{\stackrel{̊}{n}}{\underset{\sharp}{\sharp}}$ & $\stackrel{n}{n}$ & 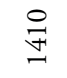 & 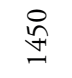 & 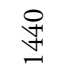 & $\stackrel{8}{\circ}$ & $\stackrel{尺}{\stackrel{Ð}{ \pm}}$ & \begin{tabular}{l}
$\stackrel{8}{0}$ \\
\multirow{+}{+}{}
\end{tabular} & 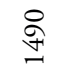 & $\stackrel{\stackrel{̊}{n}}{\underset{\sharp}{\sharp}}$ \\
\hline & \multicolumn{2}{|l|}{ Aspect (Lega) } & SW & SW & W & W & $\mathrm{E}$ & $\mathrm{E}$ & $\mathrm{NE}$ & $\mathrm{E}$ & SWW & SE & $\mathrm{E}$ & $\mathrm{NE}$ & SE \\
\hline & \multicolumn{2}{|l|}{ Slope in degrees (Nagib v stopinjah) } & 35 & 10 & 30 & 30 & 35 & 35 & 35 & 35 & 30 & 30 & 30 & 35 & 35 \\
\hline & \multicolumn{2}{|l|}{ Parent material (Matična podlaga) } & DA & A & DA & A & A & DA & DA & DA & DA & DA & DA & $\mathrm{DA}$ & DA \\
\hline & \multicolumn{2}{|l|}{ Soil (Tla) } & $\operatorname{Re}$ & $\operatorname{Re}$ & $\operatorname{Re}$ & $\operatorname{Re}$ & $\operatorname{Re}$ & $\operatorname{Re}$ & $\operatorname{Re}$ & $\operatorname{Re}$ & $\operatorname{Re}$ & $\operatorname{Re}$ & $\operatorname{Re}$ & $\operatorname{Re}$ & $\operatorname{Re}$ \\
\hline & \multicolumn{2}{|l|}{ Stoniness in \% (Kamnitost v \%) } & 5 & 20 & 10 & 30 & 30 & 30 & 20 & 30 & 20 & 30 & 20 & 20 & 30 \\
\hline & \multicolumn{15}{|l|}{ Cover in \% (Zastiranje v \%): } \\
\hline & Upper tree layer (Zgornja drevesna plast) & E3b & 70 & 60 & 60 & 40 & 80 & 80 & 90 & 70 & 70 & 80 & 90 & 80 & 80 \\
\hline & Lower tree layer (Spodnja drevesna plasti) & E3a & . & 30 & 20 & 60 & 5 & 10 & . & 20 & 10 & 10 & . & . & . \\
\hline & Shrub layer (Grmovna plast) & E2 & 40 & 20 & 30 & 10 & 10 & 10 & 5 & 10 & 20 & 10 & 10 & 30 & 20 \\
\hline & Herb layer (Zeliščna plast) & E1 & 70 & 60 & 50 & 60 & 70 & 60 & 40 & 60 & 50 & 70 & 50 & 50 & 50 \\
\hline & Moss layer (Mahovna plast) & E0 & 5 & 10 & 10 & 10 & 5 & 10 & 10 & 10 & 10 & 10 & 5 & 5 & 5 \\
\hline & Maximum tree diameter (Maks. premer dreves) & $\mathrm{cm}$ & 30 & 60 & 50 & 50 & 60 & 60 & 30 & 50 & 50 & 45 & 30 & 35 & 25 \\
\hline & Maximum tree height (Maksimalna višina dreves) & $\mathrm{m}$ & 14 & 15 & 12 & 15 & 22 & 17 & 18 & 16 & 16 & 14 & 12 & 14 & 8 \\
\hline & Number of species (Število vrst) & & 46 & 68 & 44 & 71 & 56 & 76 & 70 & 69 & 66 & 72 & 70 & 69 & 82 \\
\hline & Relevé area (Velikost popisne ploskve) & $\mathrm{m}^{2}$ & 200 & 200 & 200 & 400 & 400 & 400 & 400 & 400 & 400 & 400 & 400 & 400 & 400 \\
\hline & \multicolumn{2}{|l|}{ Date of taking relevé (Datum popisa) } & 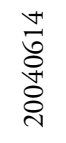 & 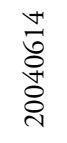 & 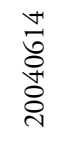 & 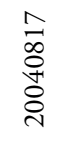 & 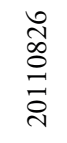 & 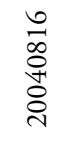 & 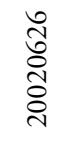 & $\begin{array}{l}0 \\
\stackrel{0}{0} \\
\circ \\
\stackrel{+}{0} \\
\stackrel{2}{0}\end{array}$ & $\begin{array}{l}0 \\
0 \\
0 \\
\stackrel{+}{0} \\
\stackrel{0}{0}\end{array}$ & $\begin{array}{l}0 \\
\stackrel{0}{1} \\
\stackrel{0}{\circ} \\
\stackrel{+}{\circ} \\
\stackrel{0}{1}\end{array}$ & 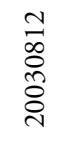 & 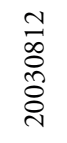 & 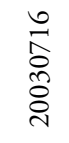 \\
\hline & \multicolumn{2}{|l|}{ Day (Dan) } & 14 & 14 & 14 & 17 & 26 & 16 & 26 & 16 & 16 & 16 & 12 & 12 & 16 \\
\hline & \multicolumn{2}{|l|}{ Month (Mesec) } & 6 & 6 & 6 & 8 & 8 & 8 & 6 & 8 & 8 & 8 & 8 & 8 & 7 \\
\hline & \multicolumn{2}{|l|}{ Year (Leto) } & 苍 & 苍 & 苍 & 苍 & $\overrightarrow{\vec{D}}$ & 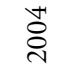 & ठิ & ஓे & ஓे & 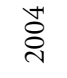 & ڤి & ڤి & ڤ̊ి \\
\hline \multicolumn{3}{|c|}{ Locality (Nahajališče) } & 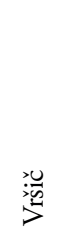 & 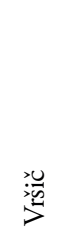 & 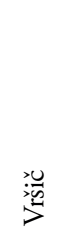 & 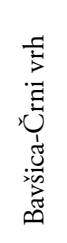 & 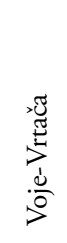 & $\begin{array}{l}\stackrel{\Xi}{\Xi} \\
\tilde{5} \\
\dot{\Xi}\end{array}$ & 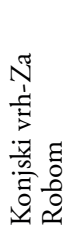 & $\begin{array}{l}\frac{\pi}{\vec{J}} \\
\tilde{D} \\
\vec{a}\end{array}$ & $\begin{array}{l}\frac{\pi}{F} \\
\vec{B} \\
\vec{\Xi}\end{array}$ & $\begin{array}{l}\frac{\tilde{Z}}{5} \\
\tilde{B} \\
\vec{a}\end{array}$ & $\begin{array}{l}\stackrel{\tilde{E}}{\tilde{O}} \\
\stackrel{\overrightarrow{0}}{0} \\
\dot{a}\end{array}$ & 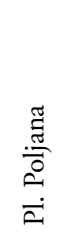 & 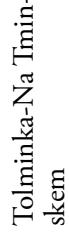 \\
\hline \multicolumn{3}{|c|}{ Mountain range (Pogorje) } & JA & JA & JA & JA & JA & JA & JA & JA & JA & JA & JA & JA & JA \\
\hline \multicolumn{3}{|c|}{ Quadrant (Kvadrant) } & $\begin{array}{l}\stackrel{+}{\infty} \\
\stackrel{+}{+} \\
\end{array}$ & $\begin{array}{l}\stackrel{+}{\infty} \\
\stackrel{\infty}{+} \\
\stackrel{+}{a}\end{array}$ & $\begin{array}{l}\stackrel{+}{\infty} \\
\stackrel{\infty}{+} \\
\stackrel{+}{a}\end{array}$ & 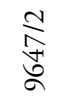 & 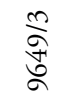 & 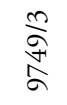 & 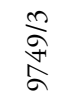 & $\underset{\substack{\stackrel{+}{a} \\
a}}{\stackrel{m}{a}}$ & $\underset{\substack{\stackrel{N}{a}\\
}}{\stackrel{n}{a}}$ & 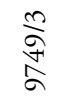 & $\underset{\substack{+\stackrel{+}{a}}}{\stackrel{m}{a}}$ & 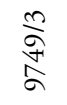 & 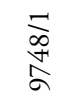 \\
\hline & Coordinate (Koordinate) GK Y (D-48) & $\mathrm{m}$ & 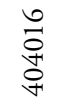 & 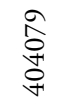 & $\begin{array}{l}\stackrel{+}{\hat{a}} \\
\text { ָे } \\
\stackrel{+}{+}\end{array}$ & 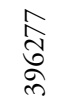 & $\begin{array}{l}\stackrel{ }{\vec{\sigma}} \\
\underset{\forall}{F}\end{array}$ & 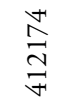 & 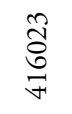 & 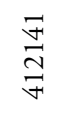 & $\begin{array}{l}\stackrel{n}{\infty} \\
\stackrel{\infty}{\exists} \\
\underset{\forall}{*}\end{array}$ & $\begin{array}{l}\stackrel{n}{+} \\
\infty \\
\exists \\
F\end{array}$ & $\underset{\stackrel{m}{n}}{\stackrel{m}{F}}$ & 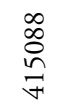 & $\begin{array}{l}\overrightarrow{2} \\
\text { ¿ิ } \\
\text { ठํ. }\end{array}$ \\
\hline & Coordinate (Koordinate) GK X (D-48) & $\mathrm{m}$ & 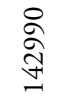 & 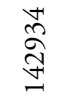 & $\begin{array}{l}\vec{尺} \\
\stackrel{\infty}{+}\end{array}$ & $\begin{array}{l}\text { : } \\
\stackrel{0}{0} \\
\stackrel{2}{2}\end{array}$ & 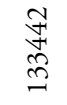 & 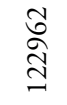 & \begin{tabular}{l}
0 \\
\multirow{2}{*}{} \\
$\stackrel{1}{U}$
\end{tabular} & 令 & \begin{tabular}{l} 
Oे \\
\multirow{2}{*}{} \\
I \\
I
\end{tabular} & $\begin{array}{l}\stackrel{+}{\circ} \\
\stackrel{\Xi}{\Xi}\end{array}$ & $\begin{array}{l}\text { ה్ } \\
\text { I }\end{array}$ & 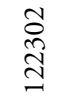 & $\begin{array}{l}8 \\
\infty \\
\stackrel{\infty}{1}\end{array}$ \\
\hline & \multicolumn{15}{|c|}{ Diagnostic species of the association (Diagnostične vrste asociacije) } \\
\hline VP & Clematis alpina & E2 & 1 & + & . & + & . & + & + & . & + & 2 & 1 & + & 1 \\
\hline MuA & Aconitum lycoctonum subsp. ranunculifolium & E1 & . & . & . & + & 1 & + & . & + & + & + & . & + & + \\
\hline CA & Laserpitium peucedanoides & E1 & 1 & + & . & + & + & + & + & . & + & + & + & + & . \\
\hline VP & Polystichum lonchitis & E1 & $\cdot$ & + & + & + & 1 & + & + & + & + & . & 1 & 1 & + \\
\hline
\end{tabular}




\begin{tabular}{|c|c|c|c|c|c|c|c|c|c|c|c|c|c|c|c|c|c|c|c|c|c|c|c|}
\hline & & & & & & & & & & & & & & & & & & & & 34 & & & \\
\hline $\begin{array}{l}\infty \\
\infty \\
\infty \\
\stackrel{\infty}{\sim}\end{array}$ & 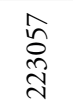 & $\begin{array}{l}\text { ñ } \\
\text { oे } \\
\text { }\end{array}$ & & 7 & $\begin{array}{l}\infty \\
\infty \\
\sim \\
\sim\end{array}$ & 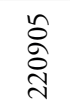 & ร & & ๙ेे & ఓิे & & & & & & & त & & & & & & \\
\hline & & & II & 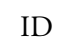 & ID & ID & & & & & ID & ID & ID & & & 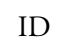 & ID & D & O & D & & & \\
\hline 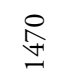 & 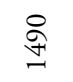 & $\begin{array}{l}\infty \\
\stackrel{\infty}{n}\end{array}$ & $\Xi$ & $\stackrel{\curvearrowleft}{\sim}$ & 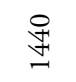 & $\stackrel{\ominus}{\underset{\exists}{\rightleftarrows}}$ & 吕 & $\stackrel{0}{\curvearrowleft}$ & $\simeq$ & $\simeq$ & 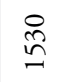 & $\underset{\mathbb{I}}{\mathbb{J}}$ & 呑 & 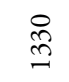 & $\stackrel{n}{n}$ & $\stackrel{\sim}{\sim}$ & $\stackrel{n}{n}$ & $\cong$ & $\begin{array}{l}n \\
\stackrel{n}{n} \\
n\end{array}$ & $\begin{array}{l}8 \\
\stackrel{+}{+}\end{array}$ & \pm & & \\
\hline SE & W & SE & & SE & & SE & S. & & SE & SI & SW & & SW & SSE & & & NW & SE & & & SSW & & \\
\hline 3 & 35 & & & & 40 & 35 & & & & & & 35 & 30 & 40 & & 30 & & & & & & & \\
\hline A & A & DA & 工 & & A & DA & A & & $N$ & 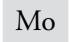 & & & $\mathrm{A}$ & & & & & & & & & & \\
\hline $\operatorname{Re}$ & $\mathrm{Re}$ & $\mathrm{R}$ & & $\mathrm{R}_{\mathrm{c}}$ & Re & $\mathrm{Re}$ & $\mathrm{Re}$ & & & & & Re & e & & & & & & & & & & \\
\hline 30 & 50 & & & & & & & & & & & & & & & & & & & & & & \\
\hline & & & & & & 80 & & & & & & & & & & & & & & & & & \\
\hline 10 & & & & & & 2 & & & & & & & & & & & & & & & & & \\
\hline 5 & 10 & & 10 & 5 & 10 & 2 & 5 & & & 20 & & & 10 & & & & & 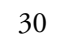 & & & & & \\
\hline & 50 & & & 5 & 6 & 60 & 20 & & & & 50 & & & & & & & & & & & & \\
\hline & 10 & & & & & & & & & & & & & & & & & & & & & & \\
\hline 60 & 45 & & 30 & 25 & 35 & 60 & 35 & & 40 & 25 & 40 & 35 & 30 & 40 & 25 & 40 & 25 & 30 & 50 & 35 & & & \\
\hline & & & & & & & 12 & & & & & & & & & & & & & & & & \\
\hline & & & & & & & & & & & & & & & & & & & & & & & \\
\hline & & & 400 & & & & & 400 & 400 & 400 & 400 & 400 & 00 & 400 & 200 & 400 & 200 & 400 & 400 & 400 & 400 & 200 & \\
\hline 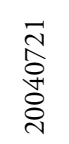 & $\begin{array}{l}\hat{\circ} \\
\text { बे }\end{array}$ & 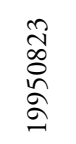 & $\stackrel{\hat{\circ}}{\curvearrowleft}$ & to & t & $\hat{a}$ & $\begin{array}{l}\overline{\hat{े}} \\
\text { ᄋे } \\
\text { ᄋ्र }\end{array}$ & $\begin{array}{l}\infty \\
\stackrel{\infty}{2} \\
\stackrel{\sim}{2}\end{array}$ & 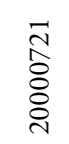 & 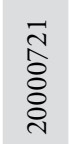 & $\widehat{\hat{D}}$ & $\begin{array}{l}\hat{\circ} \\
\text { } \\
\stackrel{\sim}{े}\end{array}$ & ○ & 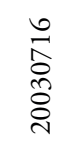 & $\begin{array}{l}\text { مे } \\
\text { ळे } \\
\text { ठें }\end{array}$ & $\begin{array}{l}\circ \\
\overrightarrow{0} \\
\stackrel{\circ}{\circ}\end{array}$ & वे & $\stackrel{\hat{\sigma}}{\vec{i}}$ & 兽 & $\frac{6}{0}$ & e & $\frac{b}{b}$ & \\
\hline & 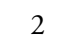 & & & & & & & & & & & & & & & & & 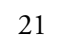 & & & & & \\
\hline & 7 & & & & & 7 & & & & & & & & & & & & & & & & & \\
\hline 容 & ฉे & 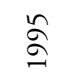 & $\tilde{\alpha}$ & 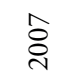 & $=$ & 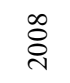 & ๖ & 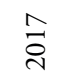 & & & & $\sqrt{ }$ & $\stackrel{\infty}{2}$ & 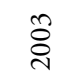 & 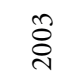 & ঃ্ণ & त) & $\overrightarrow{\vec{i}}$ & $\overrightarrow{\vec{i}}$ & $\vec{i}$ & & & \\
\hline 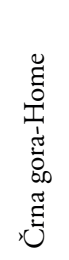 & 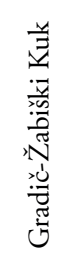 & 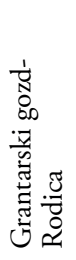 & 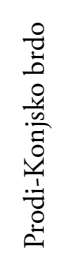 & 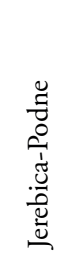 & 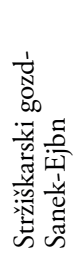 & 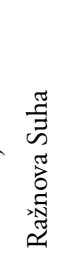 & 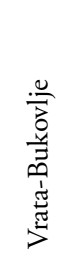 & 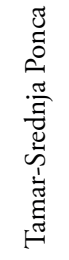 & 尊 & 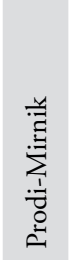 & 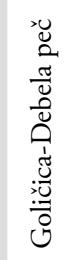 & 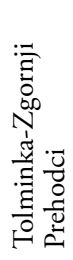 & 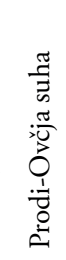 & 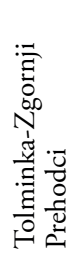 & $\pi$ & & & 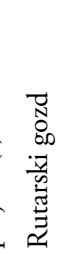 & 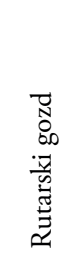 & 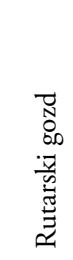 & 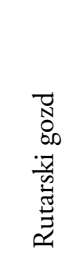 & $\frac{\vec{y}}{6}$ & \\
\hline 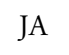 & $\mathrm{J} A$ & JA & $\mathrm{I}$ & 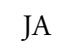 & JA & JA & & & $\mathrm{I}$ & JA & IA & $\mathrm{I}$ & A & $\mathrm{JA}$ & JA & IA & JA & JA & JA & JA & A & IA & \\
\hline$\frac{7}{9}$ & 冓 & $\stackrel{\text { s}}{3}$ & & $\frac{5}{1}$ & 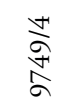 & $\delta$ & $\begin{array}{l}\underset{\infty}{\infty} \\
\underset{\delta}{+} \\
\sigma\end{array}$ & $\frac{5}{\partial}$ & 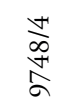 & & & 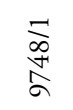 & 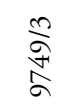 & $\bar{a}$ & & & $\frac{\pi}{n}$ & & $\frac{m}{\sigma}$ & $\stackrel{m}{a}$ & & & \\
\hline $\begin{array}{l}\hat{\infty} \\
\stackrel{\infty}{\infty}\end{array}$ & $\hat{n}$ & 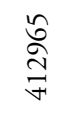 & ळ & $\stackrel{n}{\Sigma}$ & $\exists$ & $\underset{f}{F}$ & $\stackrel{\curvearrowright}{\approx}$ & शे & $\hat{\sigma}$ & $\begin{array}{l}\infty \\
\infty \\
\varnothing\end{array}$ & $\stackrel{\infty}{\stackrel{\vec{n}}{n}}$ & $\tilde{n}$ & $\begin{array}{l}\infty \\
\stackrel{2}{\circ}\end{array}$ & $\vec{\infty}$ & $\stackrel{\hat{\sigma}}{\curvearrowleft}$ & e & : & 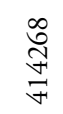 & $\hat{\text { సิ }}$ & $\hat{\tilde{c}}$ & $\infty$ & & \\
\hline $\begin{array}{l}\vec{\sim} \\
\infty\end{array}$ & 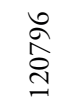 & $=$ & $\tilde{\approx}$ & $\begin{array}{l}\stackrel{\varpi}{\Xi} \\
\Xi\end{array}$ & $\stackrel{\infty}{a}$ & $\stackrel{\circ}{n}$ & $\tilde{\tilde{b}}$ & $\infty$ & $\stackrel{+}{\infty}$ & - & $\stackrel{n}{n}$ & d & 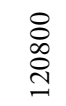 & $\stackrel{\infty}{\aleph}$ & $\vec{N}$ & $\begin{array}{l}\stackrel{8}{N} \\
\stackrel{N}{=}\end{array}$ & $\vec{n}$ & - & 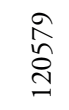 & $\begin{array}{l}\infty \\
\infty \\
\stackrel{\infty}{*} \\
\stackrel{N}{1}\end{array}$ & $\begin{array}{l}\text { ते } \\
\text { こิ } \\
\text { Iิ }\end{array}$ & 令 & \\
\hline
\end{tabular}




\begin{tabular}{|c|c|c|c|c|c|c|c|c|c|c|c|c|c|c|c|}
\hline & Number of relevé (Zaporedna številka popisa) & & 1 & 2 & 3 & 4 & 5 & 6 & 7 & 8 & 9 & 10 & 11 & 12 & 13 \\
\hline EP & Pinus mugo & E2 & + & + & + & . & . & . & . & . & + & . & $\mathrm{r}$ & + & + \\
\hline EP & Pinus mugo & E1 & . & . & . & . & . & . & . & . & . & . & . & . & . \\
\hline EP & Rhododendron hirsutum & E2 & . & + & . & . & . & . & + & . & 2 & + & 1 & 1 & ${ }^{\circ}$ \\
\hline $\mathrm{BA}$ & Sorbus chamaemespilus & E2 & + & + & + & . & . & . & . & . & + & + & + & 1 & + \\
\hline $\mathrm{CF}$ & Carex ferruginea & E1 & + & + & 1 & . & . & . & $\mathrm{r}$ & $\cdot$ & . & . & + & + & $\cdot$ \\
\hline ML & Paraleucobryum sauteri & E0 & . & . & . & . & . & + & + & . & + & + & + & . & + \\
\hline PS & Paederota lutea & E1 & . & + & $\cdot$ & . & . & + & + & . & . & + & . & + & + \\
\hline VP & Luzula sylvatica & E1 & + & 1 & 1 & + & . & . & . & . & . & . & . & . & . \\
\hline ES & Aster bellidiastrum & E1 & . & $\cdot$ & • & $\cdot$ & . & + & . & . & + & . & + & + & . \\
\hline VP & Lonicera caerulea & E2 & . & . & • & . & . & $\cdot$ & . & $\mathrm{r}$ & . & . & . & . & . \\
\hline CA & Festuca calva & E1 & . & . & . & + & $\mathrm{r}$ & . & . & • & . & . & . & . & + \\
\hline VP & Homogyne alpina & E1 & . & . & + & . & - & + & . & • & . & . & . & . & $\cdot$ \\
\hline $\mathrm{BA}$ & Salix appendiculata & E2 & . & $\cdot$ & . & . & . & . & . & . & + & + & . & . & . \\
\hline $\mathrm{EP}$ & Rhodothamnus chamaecistus & E1 & . & . & . & . & . & . & . & . & . & . & . & . & \\
\hline
\end{tabular}

Differential species of the subassociation and variant (Razlikovalnice subasociacije in variante)

EP Calamagrostis varia

AF Cyclamen purpurascens

AF Rhamnusfallax

EP Genista radiata

AF Aremonio-Fagion

Cardamine enneaphyllos

Anemone trifolia

Knautia drymeia

Helleborus niger

Cardamine trifolia

Lamium orvala

Anemone x pittonii

$\begin{array}{llllllllllllll}\text { E1 } & 2 & 1 & 1 & 2 & 1 & 2 & + & 1 & 1 & 2 & 1 & 1 & + \\ \text { E1 } & + & + & + & . & + & 1 & + & + & 1 & 1 & + & 1 & 1 \\ \text { E2 } & . & . & . & \text { r } & + & + & . & + & + & + & . & + & + \\ \text { E2 } & . & . & . & . & . & . & . & . & . & . & . & . & .\end{array}$

EC Erythonio-Carpinion

Primula vulgaris

$\begin{array}{lll}\text { E1 } & 1 & 1 \\ \text { E1 } & 1 & 1 \\ \text { E1 } & + & . \\ \text { E1 } & 1 & 2 \\ \text { E1 } & . & + \\ \text { E1 } & . & . \\ \text { E1 } & . & .\end{array}$

Helleborus odorus

TA Tilio-Acerion

Acer pseudoplatanus

Acer pseudoplatanus

Acer pseudoplatanus

Aruncus dioicus

Polystichum aculeatum

Adoxa moschatellina

Stellaria montana

Geranium robertianum

Hesperis candida

\section{FS Fagetalia sylvaticae}

Fagus sylvatica

Fagus sylvatica

Fagus sylvatica

Lonicera alpigena

Mercurialis perennis

Daphne mezereum

Galium laevigatum

Galeobdolon flavidum

Melica nutans

Lilium martagon

Prenanthes purpurea

Dryopteris filix-mas

$\begin{array}{llllllllllllll}\text { E3 } & 4 & 4 & 4 & 3 & 4 & 5 & 5 & 4 & 4 & 5 & 5 & 5 & 4 \\ \text { E2 } & 1 & + & 1 & 1 & . & 1 & + & 1 & 1 & + & + & 1 & . \\ \text { E3 } & . & + & 1 & + & + & 1 & . & . & + & + & + & . & . \\ \text { E2 } & + & + & + & + & + & + & + & + & 1 & 1 & 1 & 1 & 1 \\ \text { E1 } & . & \cdot & \cdot & 1 & . & 1 & 1 & 1 & . & 1 & + & 1 & 1 \\ \text { E2 } & + & + & + & . & + & + & + & + & + & + & + & 1 & 1 \\ \text { E1 } & . & . & . & + & 1 & 1 & 1 & 1 & + & 1 & 1 & 1 & 1 \\ \text { E1 } & . & . & . & + & + & + & + & 1 & + & + & + & + & 1 \\ \text { E1 } & + & + & + & 1 & + & + & + & + & 1 & + & . & + & + \\ \text { E1 } & + & + & 1 & + & . & + & + & + & + & . & + & + & + \\ \text { E1 } & + & . & . & . & . & + & + & . & . & . & + & . & . \\ \text { E1 } & . & . & . & + & + & + & + & 1 & + & . & . & + & +\end{array}$


- Hacquetia $20 / 2 \cdot 2021,373-564$

Igor Dakskobler \& Andre Rozman

Vegetation analysis of the subalpine beech forest on the upper forest line in the

Julian Alps (NW Slovenia and NW Italy) and in the northern Dinaric Alps

$\begin{array}{llllllllllll}14 & 15 & 16 & 17 & 18 & 19 & 20 & 21 & 22 & 23 & 24 & 25\end{array}$

$\begin{array}{llll}26 & 27 \quad 28 \quad 29\end{array}$

$\begin{array}{llllllll}30 & 31 & 32 & 33 & 34 & 35 & 36 & 37\end{array}$

$\begin{array}{llllllllll}+ & \cdot & \cdot & \cdot & \cdot & \cdot & \cdot & \cdot & + & + \\ + & \cdot & \cdot & \cdot & \cdot & \cdot & \cdot & \cdot & \cdot & \cdot \\ + & & & & & & & & + \\ +\end{array}$

r +

$1+$

$\begin{array}{ccc}35 & 36 & 37 \\ + & 1 & 1 \\ . & . & .\end{array}$

+
+
+
+
+

$\begin{array}{rrrrrrrrrrrr}. & + & . & + & 1 & 1 & 1 & + & 1 & 1 & 1 & 1 \\ 1 & 1 & 1 & 1 & 1 & 1 & 1 & + & 1 & 1 & 1 & 1 \\ + & + & . & . & . & + & . & \mathrm{r} & . & + & + & . \\ . & . & . & . & . & . & . & . & . & . & \mathrm{r} & .\end{array}$

$\begin{array}{ll}1 & 2 \\ 1 & 1\end{array}$

+
+
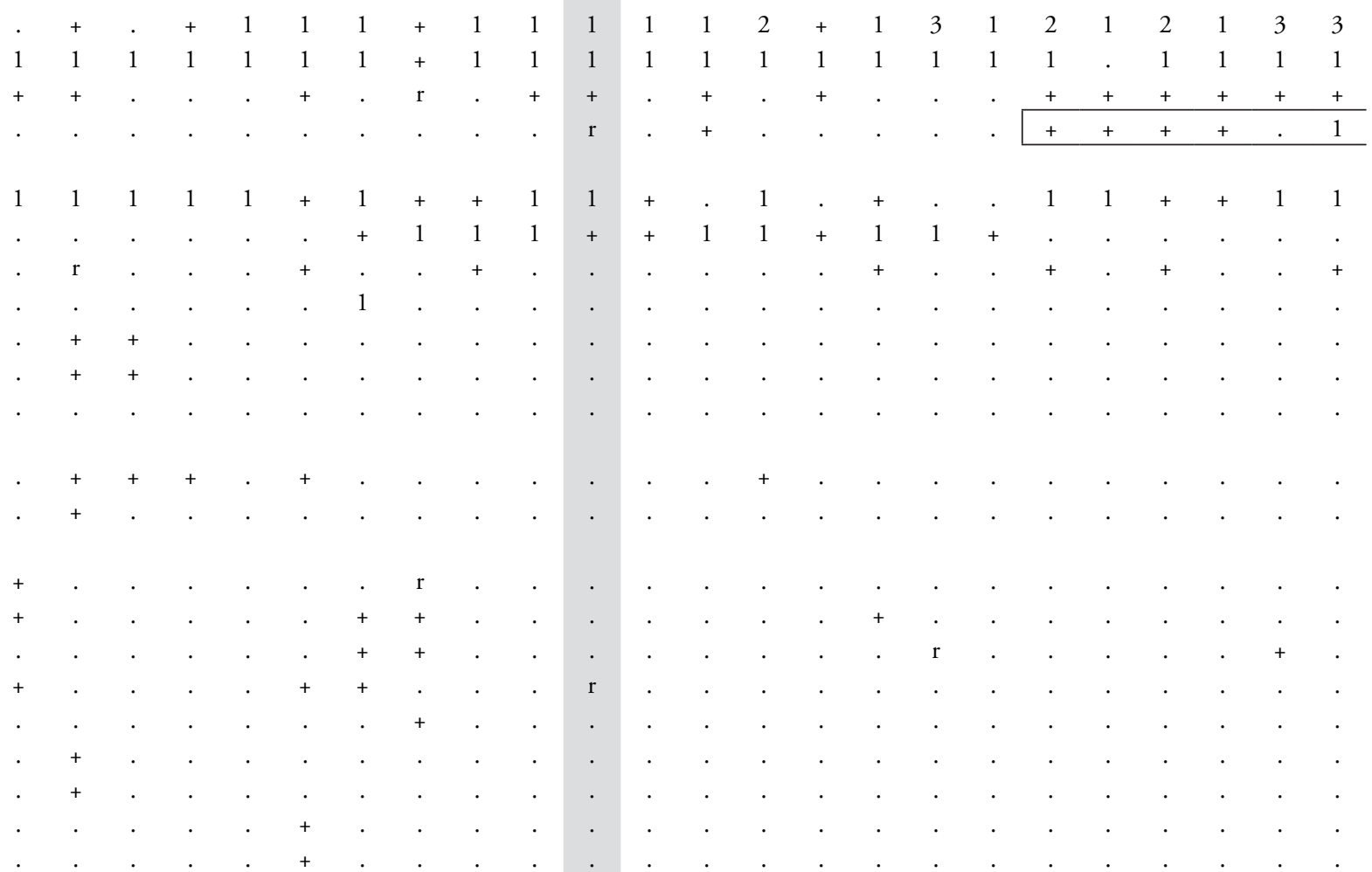

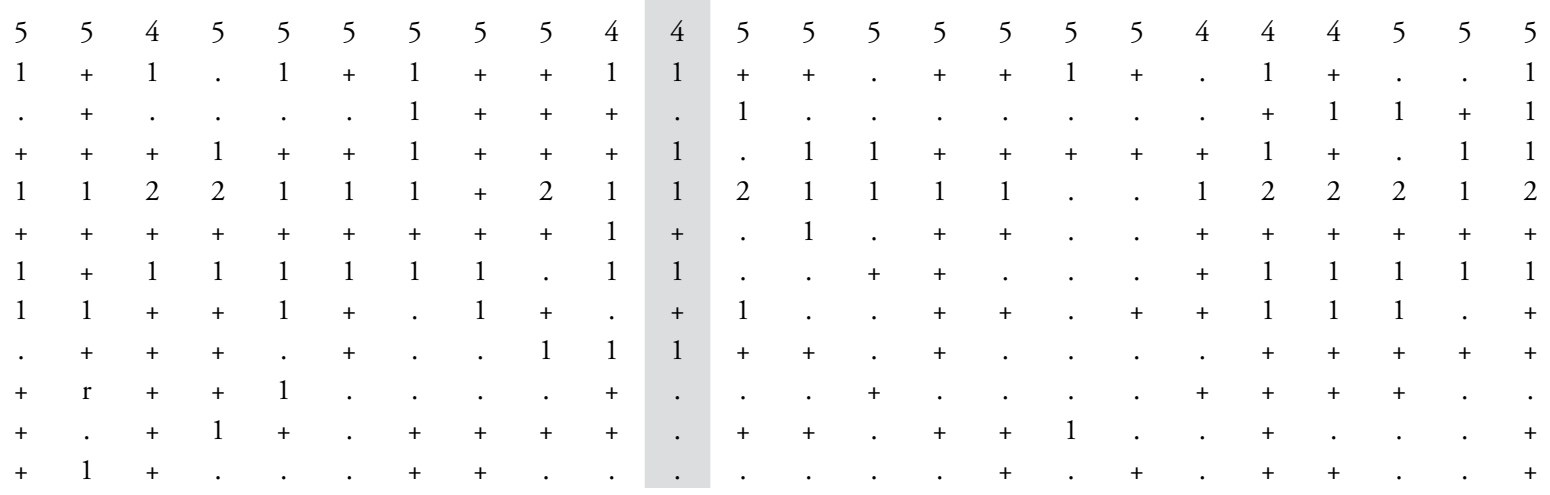

475 
Paris quadrifolia

Symphytum tuberosum

Actaea spicata

Euphorbia amygdaloides

Luzula nivea

Neottia nidus-avis

Laburnum alpinum

Laburnum alpinum

Laburnum alpinum

Mycelis muralis

Lathyrus vernus

Campanula trachelium

Polygonatum multiflorum

Epipactis helleborine

Epilobium montanum

Scrophularia nodosa

Festuca altissima

Myosotis sylvatica agg.

Petasites albus

Cardamine bulbifera

Cardamine impatiens

Carex sylvatica

Galium odoratum

Ranunculus lanuginosus

QP

Quercetalia pubescenti-petraeae

Sorbus aria (Aria edulis)

Sorbus aria (Aria edulis)

Sorbus aria (Aria edulis)

Melittis melissophyllum

Convallaria majalis

Carex flacca

QF Querco-Fagetea

Anemone nemorosa

Carex digitata

Hepatica nobilis

Poa nemoralis

Viola riviniana

Festuca heterophylla

Corylus avellana

Platanthera bifolia

VP

\section{Vaccinio-Piceetea}

Aposeris foetida

Rosa pendulina

Solidago virgaurea

Picea abies

Picea abies

Picea abies

Veronica urticifolia

Hieracium murorum

Calamagrostis arundinacea

Gentiana asclepiadea

Vaccinium myrtillus

Maianthemum bifolium
E1

E1

E1

E1

\begin{tabular}{|c|c|c|c|c|c|c|c|c|c|c|c|c|}
\hline E1 & 1 & 1 & 1 & . & 1 & . & + & . & 1 & 1 & + & 1 \\
\hline E2 & + & + & + & + & + & + & + & + & . & . & + & + \\
\hline E1 & + & . & + & + & . & 1 & $T$ & + & 1 & 1 & + & + \\
\hline E3 & + & 1 & 1 & 1 & + & . & . & . & . & . & . & 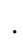 \\
\hline E2 & 1 & 1 & 1 & + & + & + & . & + & + & + & + & • \\
\hline E1 & . & + & . & . & . & . & + & . & . & . & . & + \\
\hline E1 & . & . & . & + & . & 1 & 1 & 1 & $\cdot$ & 1 & 1 & + \\
\hline E1 & + & + & + & + & $\mathrm{r}$ & . & • & . & . & + & + & + \\
\hline E1 & . & . & . & . & + & . & + & . & + & 1 & 1 & . \\
\hline E1 & . & + & + & . & . & + & + & + & 1 & 1 & 1 & + \\
\hline E1 & 1 & + & + & . & . & . & • & . & 1 & + & 1 & 1 \\
\hline E1 & + & 1 & . & + & + & + & + & . & + & + & + & + \\
\hline
\end{tabular}


- Hacquetia 20/2 • 2021,373-564

Igor Dakskobler \& Andre Rozman

Vegetation analysis of the subalpine beech forest on the upper forest line in the

Julian Alps (NW Slovenia and NW Italy) and in the northern Dineric Alps

$\begin{array}{lllllllllllllllllllllllll}14 & 15 & 16 & 17 & 18 & 19 & 20 & 21 & 22 & 23 & 24 & 25 & 26 & 27 & 28 & 29 & 30 & 31 & 32 & 33 & 34 & 35 & 36 & 37\end{array}$ $+$
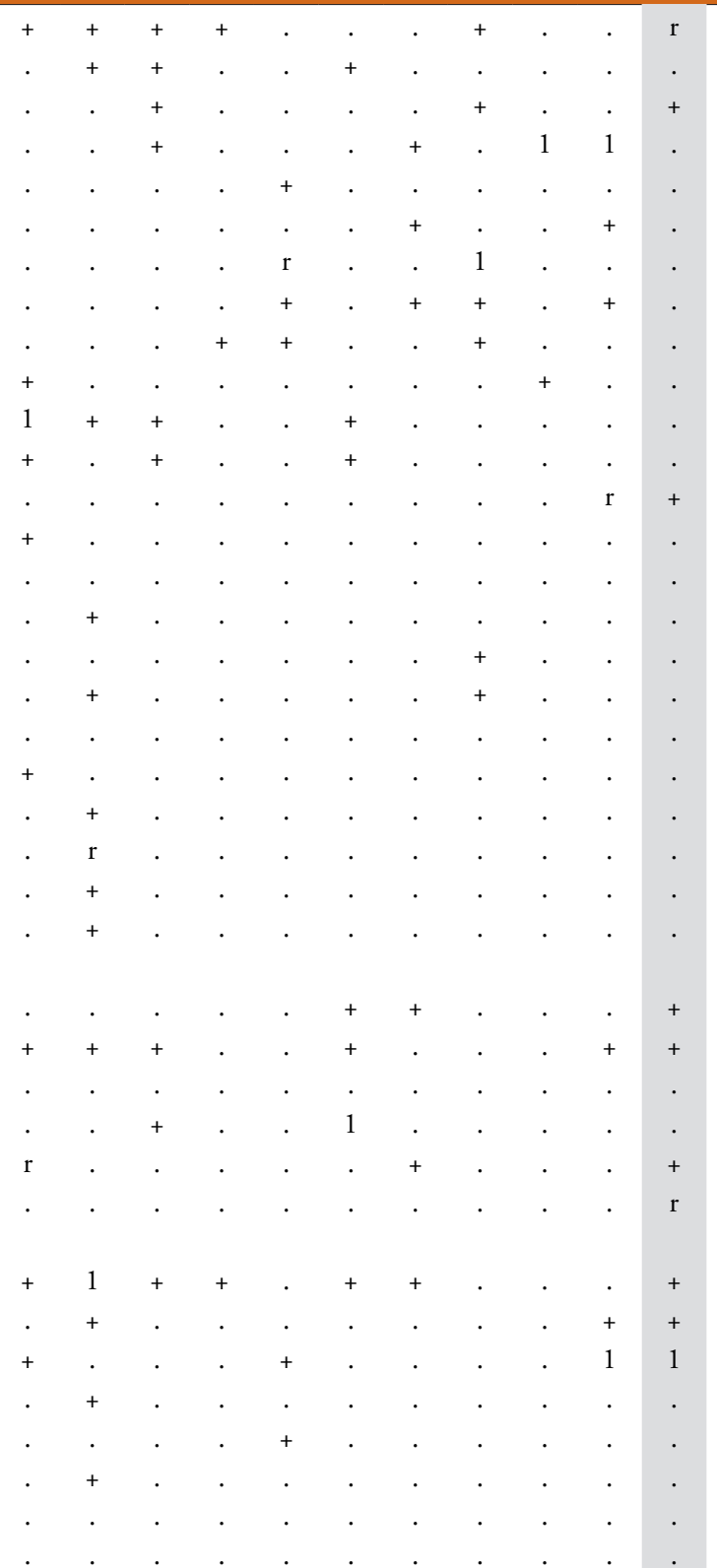

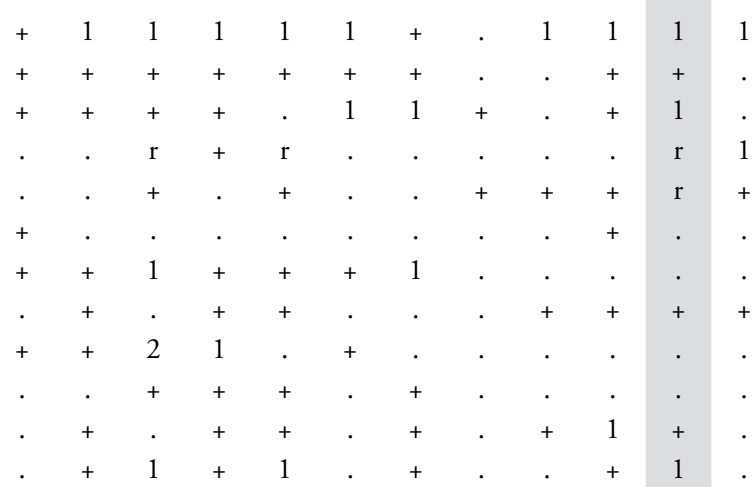

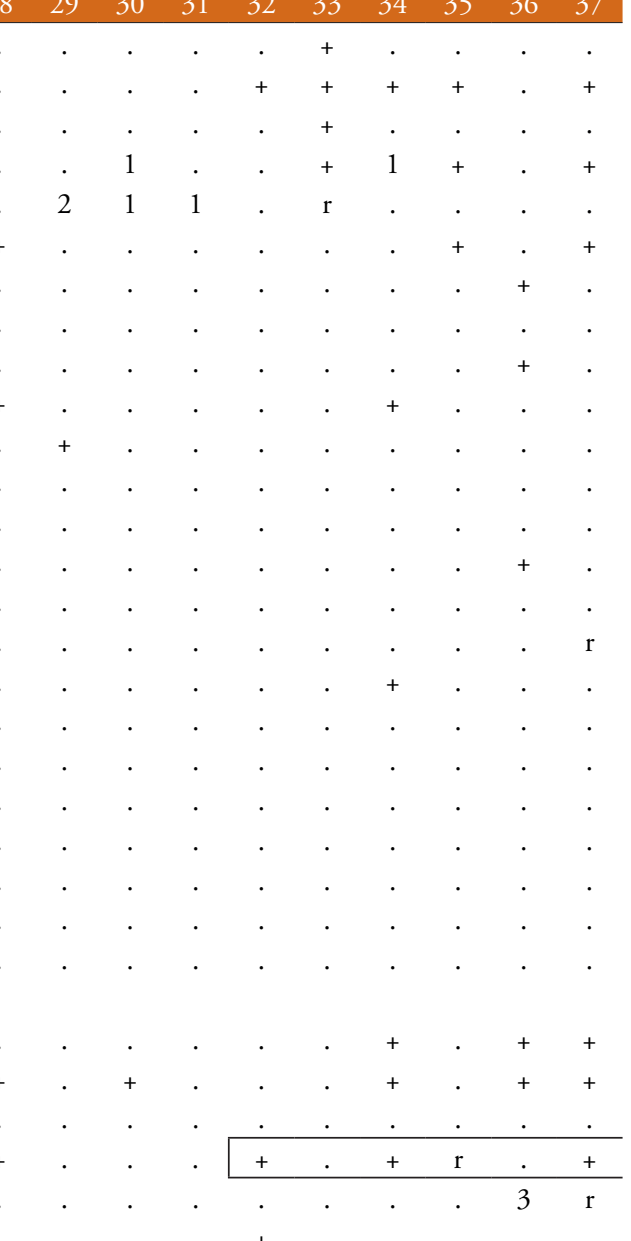

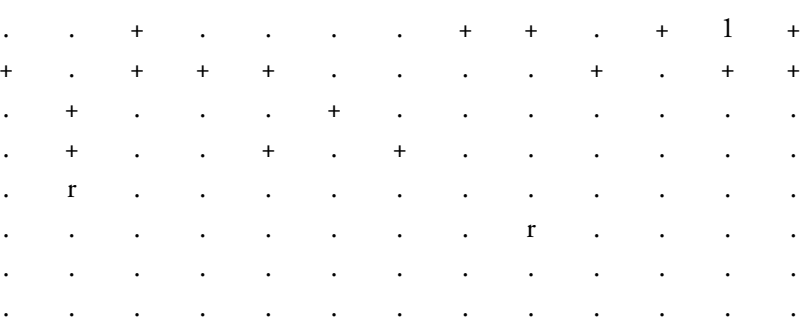

477 
Luzula luzuloides

Gymnocarpium dryopteris

Huperzia selago

Oxalis acetosella

Larix decidua

Larix decidua

Lonicera nigra

Homogyne sylvestris

Saxifraga cuneifolia

Dryopteris dilatata

Phegopteris connectilis

Abies alba

Abies alba

Abies alba

Vaccinium vitis-idaea

Calamagrostis villosa

Corallorhiza trifida

Luzula luzulina

EP Erico-Pinetea

Rubus saxatilis

Erica carnea

Carex alba

Aquilegia nigricans

Peucedanum rablense

Carex ornithopoda

Polygala chamaebuxus

Molinia arundinacea

Chamaecytisus hirsutus

Cotoneaster tomentosus

SSC Sambuco-Salicion capreae, Rhamno-Prunetea

Sorbus aucuparia

Sorbus aucuparia

Sorbus aucuparia

BA Betulo-Alnetea

Ribes alpinum

Sorbus austriaca

MuA Mulgedio-Aconitetea

Polygonatum verticillatum

Veratrum album

Athyrium filix-femina

Phyteuma ovatum

Senecio ovatus

Aconitum degenii subsp. paniculatum

Ranunculus platanifolius

Geranium sylvaticum

Thalictrum aquilegiifolium

Viola biflora

Chaerophyllum villarsii

Aconitum angustifolium

Centaurea montana

Myrrhis odorata

Saxifraga rotundifolia

Senecio cacaliaster
E1 


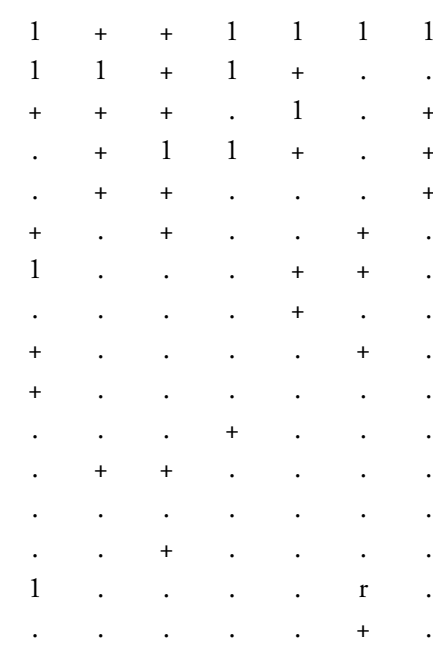


Pleurospermum austriacum

Chaerophyllum hirsutum

Hypericum maculatum

Lathyrus occidentalis var. montanus

Tanacetum corymbosum subsp. clusii

Agropyron caninum

Chaerophyllum aureum

Primula elatior

Adenostyles alliariae

Doronicum austriacum

Crepis pyrenaica

CA Caricion austroalpinae

Gentiana lutea subsp. symphyandra

Koeleria eriostachya

Centaurea haynaldii subsp. julica

Arabis vochinensis

Heracleum austriacum subsp. siifolium

CF Caricion ferrugineae

Campanula thyrsoides

Knautia longifolia

Cfir Caricion firmae

Carex firma

ES Elyno-Seslerietea

Betonica alopecuros

Sesleria caerulea

Campanula witasekiana

Carduus crassifolius

Carex sempervirens

Phyteuma orbiculare

Senecio abrotanifolius

Pimpinella alpina

Scabiosa lucida subsp. stricta

Leucanthemum heterophyllum

Galium anisophyllum

Astrantia bavarica

Ranunculus carinthiacus

Lotus alpinus

Thymus praecox subsp. polytrichus

Alchemilla alpigena

Arabis ciliata

Thesium alpinum

Anthyllis vulneraria subsp. alpestris

Bartsia alpina

NS Nardion strictae, Juncetea trifidi

Campanula scheuchzeri

Coeloglossum viride

Potentilla erecta

FB Festuco-Brometea

Cirsium erisithales

Buphthalmum salicifolium

Carex humilis

Carlina acaulis

Bromopsis transsilvanica
E1 $\begin{array}{lllllllllllll}1 & 2 & 3 & 4 & 5 & 6 & 7 & 8 & 9 & 10 & 11 & 12 & 13\end{array}$

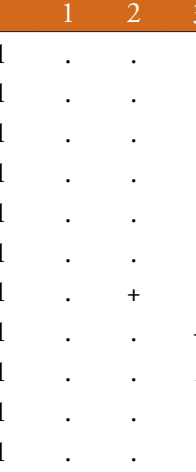

\section{El}

Elons

El

1

1

1

(1)

1

.


- Hacquetia 20/2 • 2021,373-564

Igor Dakskobler \& Andre Rozman

Vegetation analysis of the subalpine beech forest on the upper forest line in the Julian Alps (NW Slovenia and NW Italy) and in the northern Dinaric Alps

$\begin{array}{lllllllllllllllllllllllll}4 & 15 & 16 & 17 & 18 & 19 & 20 & 21 & 22 & 23 & 24 & 25 & 26 & 27 & 28 & 29 & 30 & 31 & 32 & 33 & 34 & 35 & 36 & 37\end{array}$ $+$

$+$
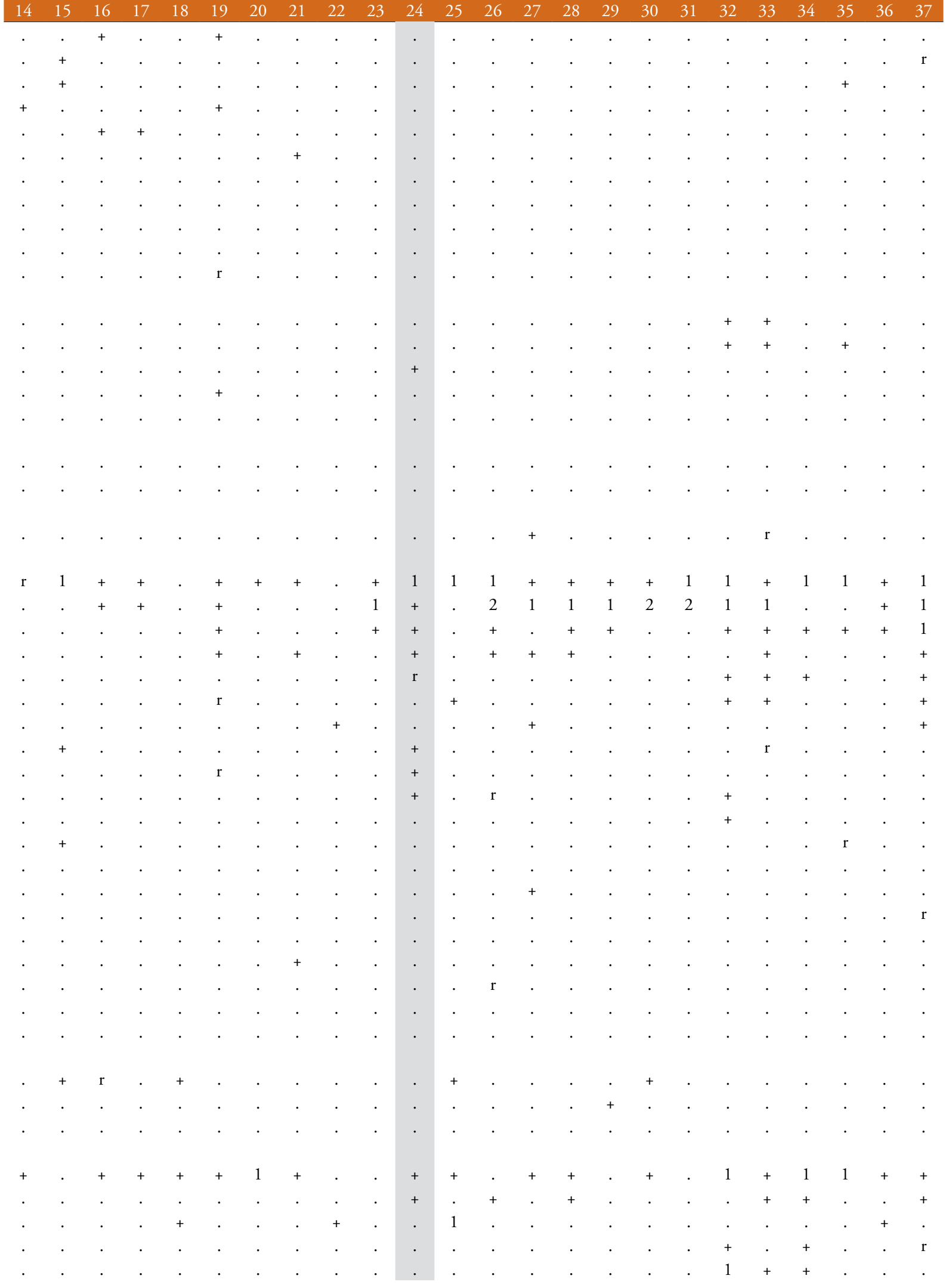

481 
Koeleria pyramidata

Dactylorhiza sambucina

Arabis hirsuta

Prunella grandiflora

Hippocrepis comosa

TG Trifolio-Geranietea

Laserpitium latifolium

Lilium carniolicum

Clinopodium vulgare

Verbascum lanatum

Thalictrum minus

Digitalis grandiflora

Arabis pauciflora

Laserpitium siler

Arabis turrita

Iris graminea

Viola hirta

PaT Poo alpinae-Trisetetalia

Poa alpina

Ranunculus nemorosus

Trollius europaeus

MA Molinio-Arrhenatheretea

Dactylis glomerata

Crepis paludosa

Deschampsia cespitosa

Angelica sylvestris

Festuca rubra agg.

Taraxacum sect. Ruderalia

EA Epilobietea angustifolii, Galio-Urticetea

Fragaria vesca

Rubus idaeus

Hypericum hirsutum

$\mathrm{GU}$ Urtica dioica

Galeopsis speciosa

TR Thlaspietea rotundifolii

Adenostyles glabra

Gymnocarpium robertianum

Heracleum pollinianum

Astrantia carniolica

Trisetum argenteum

Valeriana montana

Hieracium bifidum

Ligusticum seguieri

Aquilegia iulia

Biscutella laevigata

Molopospermum peloponnesiacum subsp. bauhinii Aquilegia einseleana

Petasites paradoxus

Hieracium glaucum

Festuca laxa

Hieracium austriacum

Cy Cystopteridion fragilis

Valeriana tripteris
E1

E1

E

E1

E

E1

E1

$\begin{array}{lll} & 1 & 2 \\ 1 & . \\ 1 & . \\ 1 & \cdot \\ 1 & .\end{array}$

1

1

1

.

.

E1

.


Number of relevé (Zaporedna števillka popisa)

Asplenium viride

Moehringia muscosa

Cystopteris fragilis

Carex brachystachys

Cystopteris regia

PS Physoplexido comosae-Saxifragion petraeae

Campanula carnica

Campanula cespitosa

Paederota bonarota

Campanula zoysii

PC Potentilletalia caulescentis

Festuca stenantha

Primula auricula

Campanula cochleariifolia

Potentilla caulescens

\section{E1

AT Asplenietea trichomanis

Asplenium ruta-muraria

Asplenium trichomanes

Polypodium vulgare

Kernera saxatilis

Sedum maximum

O Other species (Druge vrste)

Festuca sp.

ML Mosses and lichens (Mahovi in lišaji)

Ctenidium molluscum

Tortella tortuosa

Schistidium apocarpum

Fissidens dubius

Pseudoleskeella catenulata

Polytrichum formosum

Bryum capillare

Peltigera canina

Isothecium alopecuroides

Encalypta streptocarpa

Homalothecium lutescens

Cladonia sp.

Collema cristatum

Conocephalum conicum

Mnium thomsonii

Dermatocarpon miniatum

Dicranum scoparium

Homalothecium philippeanum

Mnium sp.

Cladonia pyxidata

Plagiochila porelloides

Hypnum cupressiforme

Peltigera leucophlebia

Lobaria pulmonaria

Rhizomnium punctatum

Plagiothecium sp.

Marchantia polymorpha

Bartramia pomiformis

Cetraria islandica

$\begin{array}{lllllllllllll}1 & 2 & 3 & 4 & 5 & 6 & 7 & 8 & 9 & 10 & 11 & 12 & 13\end{array}$

\section{(2)}

1

\section{1}

1

(1)

(1)


- Hacquetia $20 / 2 \cdot 2021,373-564$

Igor Dakskobler \& Andre Rozman

Vegetation analysis of the subalpine beech forest on the upper forest line in the

Julian Alps (NW Slovenia and NW Italy) and in the northern Dinaric Alps

$\begin{array}{llllllllllllllllllllllllll}14 & 15 & 16 & 17 & 18 & 19 & 20 & 21 & 22 & 23 & 24 & 25 & 26 & 27 & 28 & 29 & 30 & 31 & 32 & 33 & 34 & 35 & 36 & 37\end{array}$

$\begin{array}{llllll}14 & 15 & 16 & 17 & 18 & 19 \\ + & . & + & + & +\end{array}$

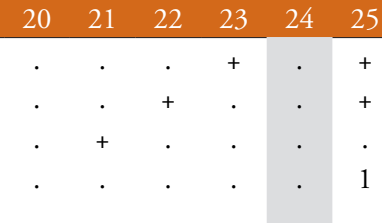

$+\quad+\quad+$
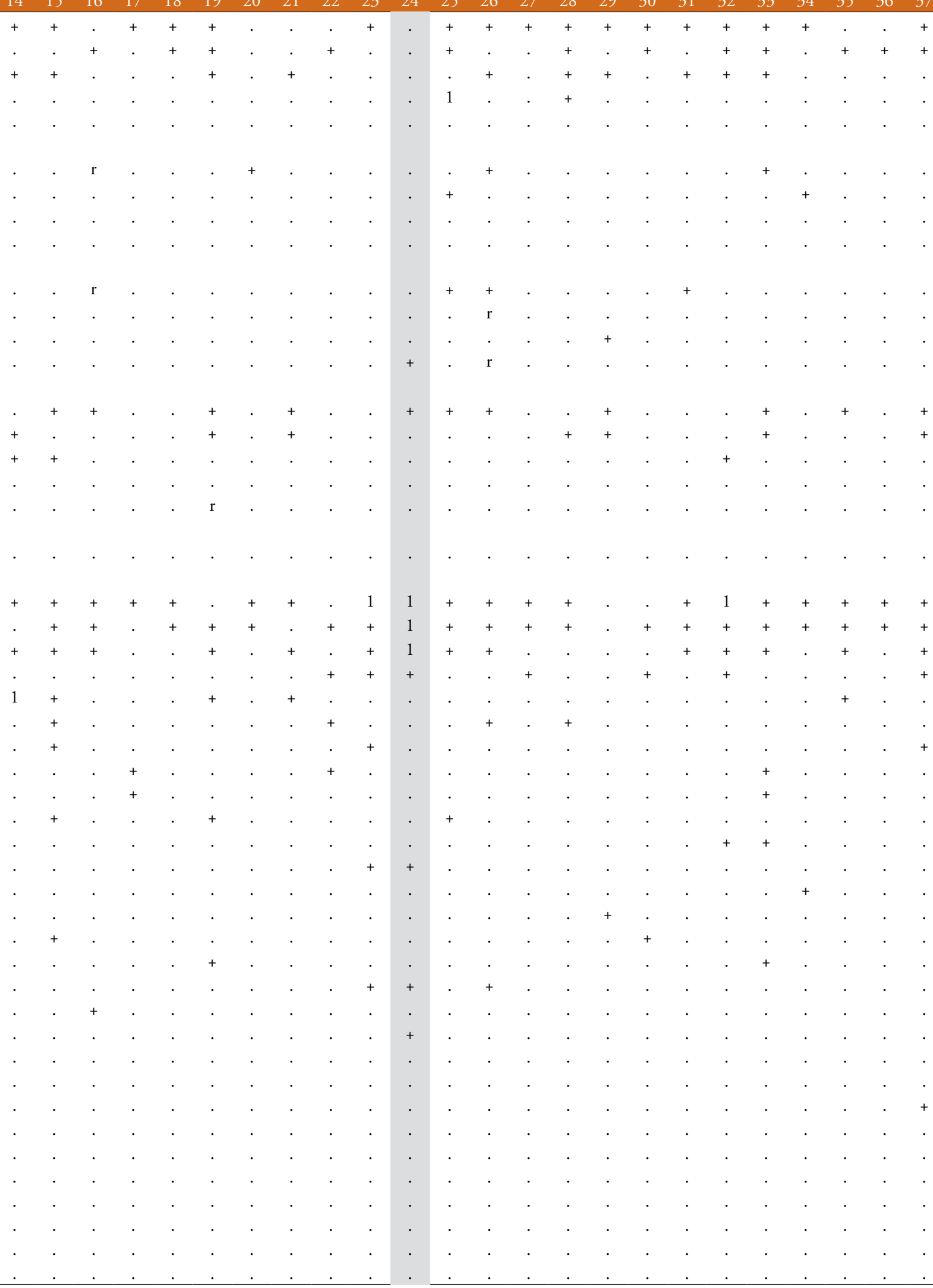

485


Table 4: Polysticho lonchitis-Fagetum calamagrostietosum variae - cluster 22. Relevé numbers 38-48.

Tabela 4: Polysticho lonchitis-Fagetum calamagrostietosum variae - skupina 22. Zaporedne številke popisov 38-48.

\begin{tabular}{|c|c|c|c|c|c|c|c|c|c|c|c|c|}
\hline Number of relevé (Zaporedna številka popisa) & & 38 & 39 & 40 & 41 & 42 & 43 & 44 & 45 & 46 & 47 & 48 \\
\hline $\begin{array}{l}\text { Database number of relevé } \\
\text { (Delovna številka popisa) }\end{array}$ & & 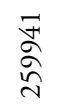 & 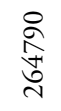 & $\begin{array}{l}\stackrel{8}{\stackrel{7}{\sharp}} \\
\underset{\sim}{\sim}\end{array}$ & $\begin{array}{l}\stackrel{2}{~} \\
\check{\curvearrowright} \\
\check{\gamma}\end{array}$ & 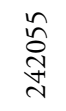 & 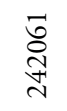 & $\underset{\stackrel{\sim}{\sim}}{\stackrel{\sim}{\sim}}$ & 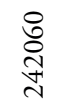 & 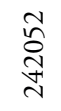 & 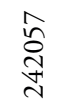 & $\stackrel{\text { \ }}{\sim}$ \\
\hline Author of the relevé (Avtor popisa) & & ID & ID & ID & ID & ID & ID & ID & ID & ID & ID & ID \\
\hline Elevation in $\mathrm{m}$ (Nadmorska višina $\mathrm{v} \mathrm{m})$ & & $\underset{Ð}{\stackrel{R}{\leftrightarrows}}$ & $\underset{\square}{\stackrel{\&}{ \pm}}$ & $\begin{array}{l}\stackrel{\infty}{\infty} \\
\stackrel{+}{\square}\end{array}$ & $\underset{\underset{J}{ \pm}}{\stackrel{f}{7}}$ & $\stackrel{ }{\underset{\varpi}{\ddagger}}$ & 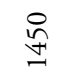 & 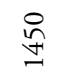 & 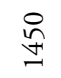 & $\begin{array}{l}8 \\
\stackrel{+}{ \pm}\end{array}$ & 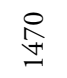 & $\stackrel{\overbrace{}}{+}$ \\
\hline Aspect (Lega) & & SW & $S$ & $S$ & SW & SSW & SW & $S$ & $S$ & SW & SW & SSW \\
\hline Slope in degrees (Nagib v stopinjah) & & 35 & 35 & 30 & 30 & 35 & 30 & 40 & 40 & 35 & 35 & 35 \\
\hline Parent material (Matična podlaga) & & DA & DA & Gr & DA & DA & DA & DA & DA & DA & DA & \\
\hline Soil (Tla) & & $\operatorname{Re}$ & $\operatorname{Re}$ & $\operatorname{Re}$ & $\operatorname{Re}$ & $\operatorname{Re}$ & $\operatorname{Re}$ & $\operatorname{Re}$ & $\operatorname{Re}$ & $\operatorname{Re}$ & $\operatorname{Re}$ & $\operatorname{Re}$ \\
\hline Stoniness in \% (Kamnitost v \%) & & 10 & 20 & 40 & 20 & 40 & 60 & 10 & 30 & 50 & 30 & 40 \\
\hline \multicolumn{13}{|l|}{ Cover in \% (Zastiranje v \%): } \\
\hline Upper tree layer (Zgornja drevesna plast) & $\mathrm{E} 3 \mathrm{~b}$ & 70 & 80 & 70 & 80 & 80 & 70 & 75 & 70 & 80 & 80 & 80 \\
\hline Lower tree layer (Spodnja drevesna plasti) & $\mathrm{E} 3 \mathrm{a}$ & . & . & 10 & . & 5 & 5 & . & 10 & 10 & 10 & 5 \\
\hline Shrub layer (Grmovna plast) & E2 & 20 & 1 & 40 & 10 & 20 & 30 & 10 & 20 & 10 & 10 & 5 \\
\hline Herb layer (Zeliščna plast) & E1 & 80 & 60 & 70 & 80 & 70 & 40 & 75 & 75 & 60 & 70 & 60 \\
\hline Moss layer (Mahovna plast) & E0 & 5 & 5 & 5 & 5 & 10 & 10 & 5 & 10 & 10 & 10 & 10 \\
\hline Maximum tree diameter (Maks. premer dreves) & $\mathrm{cm}$ & 40 & 35 & 40 & 30 & 50 & 45 & 50 & 35 & 40 & 60 & 40 \\
\hline Maximum tree height (Maksimalna višina dreves) & $\mathrm{m}$ & 10 & 10 & 14 & 10 & 14 & 8 & 10 & 12 & 13 & 15 & 14 \\
\hline Number of species (Število vrst) & & 50 & 51 & 61 & 63 & 60 & 75 & 57 & 67 & 75 & 62 & 59 \\
\hline Relevé area (Velikost popisne ploskve) & $\mathrm{m}^{2}$ & 400 & 400 & 400 & 400 & 400 & 400 & 400 & 400 & 400 & 400 & 400 \\
\hline Date of taking relevé (Datum popisa) & & 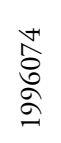 & 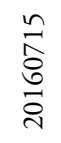 & 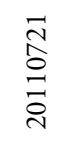 & 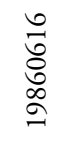 & $\begin{array}{l}\hat{\hat{O}} \\
\vec{ٍ} \\
\text { ㄱ. }\end{array}$ & $\begin{array}{l}\hat{} \\
\text { O } \\
\bar{\sigma} \\
\stackrel{2}{1}\end{array}$ & $\begin{array}{l}\hat{N} \\
\stackrel{\circ}{\Xi} \\
\stackrel{\sim}{\circ}\end{array}$ & $\begin{array}{l}\hat{N} \\
\stackrel{\circ}{\Xi} \\
\vec{ٍ}\end{array}$ & 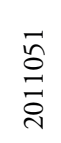 & 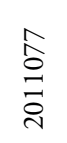 & 离 \\
\hline Day (Dan) & & 4 & 15 & 21 & 16 & 7 & 7 & 7 & 7 & 1 & 7 & 7 \\
\hline Month (Mesec) & & 7 & 7 & 7 & 6 & 7 & 7 & 7 & 7 & 5 & 7 & 7 \\
\hline Year (Leto) & & $\stackrel{2}{\check{\beth}}$ & $\begin{array}{l}\stackrel{0}{i} \\
\stackrel{\sim}{i}\end{array}$ & $\overrightarrow{\vec{i}}$ & $\stackrel{Ð}{\stackrel{2}{\beth}}$ & $\overrightarrow{\vec{i}}$ & $\vec{\sim}$ & $\vec{\sim}$ & ב্ & $\vec{\Xi}$ & ప্ & $\overrightarrow{\vec{D}}$ \\
\hline Locality (Nahajališče) & & 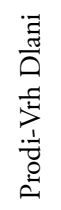 & 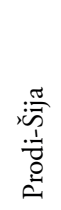 & 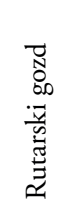 & 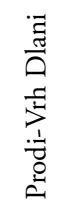 & 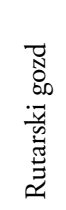 & 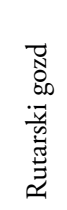 & 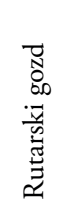 & 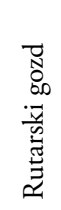 & 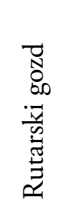 & 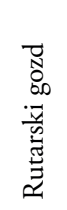 & 要 \\
\hline Mountain range (Pogorje) & & JA & JA & JA & JA & JA & JA & JA & JA & JA & $\mathrm{JA}$ & JA \\
\hline Quadrant (Kvadrant) & & $\underset{\sigma}{\stackrel{n}{a}}$ & $\underset{\stackrel{n}{a}}{\stackrel{n}{a}}$ & $\underset{\substack{+\\
}}{\stackrel{n}{a}}$ & 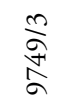 & 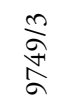 & 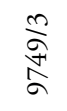 & 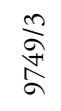 & $\frac{n}{\stackrel{+}{a}}$ & $\frac{n}{\stackrel{+}{a}}$ & 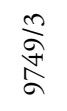 & $\stackrel{\frac{1}{9}}{\frac{1}{2}}$ \\
\hline Coordinate (Koordinate) GK Y (D-48) & $\mathrm{m}$ & 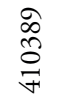 & 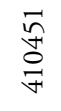 & $\underset{\stackrel{D}{+}}{\stackrel{+}{+}}$ & $\begin{array}{l}\vec{\infty} \\
\stackrel{0}{\circ} \\
\vec{F}\end{array}$ & 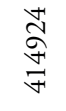 & $\begin{array}{l}\stackrel{\Re}{\approx} \\
\underset{\forall}{F}\end{array}$ & $\begin{array}{l}\stackrel{0}{ } \\
\infty \\
\infty \\
\stackrel{\forall}{\forall}\end{array}$ & 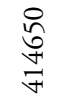 & 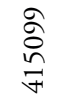 & 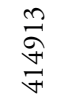 & \\
\hline Coordinate (Koordinate) GK X (D-48) & $\mathrm{m}$ & $\begin{array}{l}\widehat{ત} \\
\stackrel{\Xi}{\beth}\end{array}$ & $\begin{array}{l}\stackrel{0}{ } \\
\infty \\
\stackrel{\Xi}{\Xi}\end{array}$ & 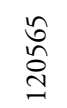 & 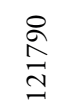 & $\begin{array}{l}\overrightarrow{\vec{n}} \\
\stackrel{\text { ㄱ }}{0}\end{array}$ & 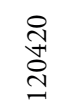 & 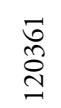 & $\begin{array}{l}\infty \\
\stackrel{0}{+} \\
\stackrel{ }{(}\end{array}$ & 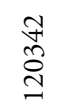 & $\begin{array}{l}\stackrel{+}{~} \\
\stackrel{\text { }}{\sigma}\end{array}$ & ֶֻ) \\
\hline
\end{tabular}

Diagnostic species of the association (Diagnostične vrste asociacije)

Pr. Fr.

MuA Aconitum lycoctonum subsp. ranunculifolium

CA Laserpitium peucedanoides

VP Polystichum lonchitis 


\begin{tabular}{|c|c|c|c|c|c|c|c|c|c|c|c|c|c|c|}
\hline & Number of relevé (Zaporedna številka popisa) & & 38 & 39 & 40 & 41 & 42 & 43 & 44 & 45 & 46 & 47 & 48 & Pr. \\
\hline $\mathrm{EP}$ & Pinus mugo & E2 & 1 & . & 1 & + & 1 & 1 & + & 1 & + & + & . & 27 \\
\hline EP & Pinus mugo & E1 & . & . & + & . & . & . & . & . & . & . & . & 1 \\
\hline EP & Rhododendron hirsutum & E2 & + & . & . & + & . & + & $\mathrm{r}$ & . & $\mathrm{r}$ & $\mathrm{r}$ & . & 19 \\
\hline BA & Sorbus chamaemespilus & E2 & . & . & + & . & . & . & . & + & . & . & . & 19 \\
\hline $\mathrm{CF}$ & Carex ferruginea & E1 & . & + & + & + & . & . & . & . & + & + & . & 18 \\
\hline ML & Paraleucobryum sauteri & E0 & . & . & . & + & . & + & . & . & + & + & . & 16 \\
\hline PS & Paederota lutea & E1 & . & . & . & . & . & + & . & . & . & . & . & 11 \\
\hline VP & Luzula sylvatica & E1 & . & $\cdot$ & . & . & . & . & . & . & . & . & . & 9 \\
\hline ES & Aster bellidiastrum & E1 & . & . & . & + & . & . & . & . & . & . & . & 9 \\
\hline VP & Lonicera caerulea & E2 & . & . & + & . & . & + & . & . & . & . & . & 8 \\
\hline $\mathrm{CA}$ & Festuca calva & E1 & . & . & . & . & . & . & . & . & . & . & . & 6 \\
\hline VP & Homogyne alpina & E1 & . & . & . & . & . & . & . & . & . & . & . & 4 \\
\hline BA & Salix appendiculata & E2 & . & . & . & . & . & + & . & . & . & . & . & 4 \\
\hline $\mathrm{EP}$ & Rhodothamnus chamaecistus & E1 & . & $\cdot$ & . & + & . & . & . & . & . & . & . & 1 \\
\hline
\end{tabular}

Differential species of the subassociation and variant (Razlikovalnice subasociacije in variante)

EP Calamagrostis varia

AF Cyclamen purpurascens

AF Rhamnus fallax

EP Genista radiata

AF Aremonio-Fagion

Cardamine enneaphyllos

Anemone trifolia

Knautia drymeia

Helleborus niger

Cardamine trifolia

Lamium orvala

Anemone x pittonii

EC Erythonio-Carpinion

Primula vulgaris

Helleborus odorus

TA Tilio-Acerion

Acer pseudoplatanus

Acer pseudoplatanus

Acer pseudoplatanus

Aruncus dioicus

Polystichum aculeatum

Adoxa moschatellina

Stellaria montana

Geranium robertianum

Hesperis candida

FS Fagetalia sylvaticae

Fagus sylvatica

Fagus sylvatica

Fagus sylvatica

Lonicera alpigena

Mercurialis perennis

Daphne mezereum

Galium laevigatum

Galeobdolon flavidum

Melica nutans

\begin{tabular}{llllllllllllll} 
E1 & 2 & 1 & 3 & 3 & 2 & 2 & 2 & 3 & 2 & 2 & 2 & 46 & 96 \\
E1 & 1 & 1 & 1 & 1 & 1 & 1 & 1 & 1 & 1 & 1 & 1 & 46 & 96 \\
E2 &. &. & + &. & + & 1 & + & + & + & + & + & 30 & 62 \\
\cline { 2 - 10 } & + & + & + & + &. & $\mathrm{r}$ & + &. & $\mathrm{r}$ &. &. & 14 & 29
\end{tabular}

$\mathrm{E} 11+\begin{array}{llllllllllll}1 & 1 & 1 & 1 & 1 & 1 & 1 & 1 & 1 & 43 & 90\end{array}$

E1 . . . . . . . . . . . . . . 25252

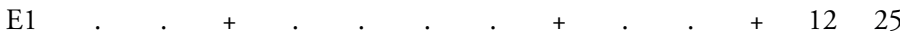

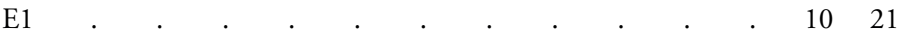

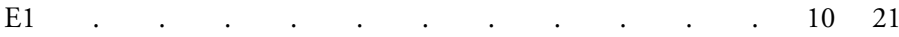

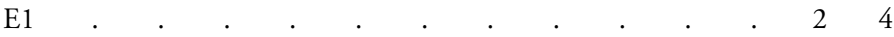

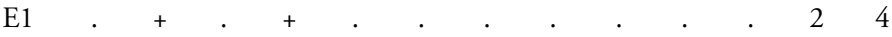

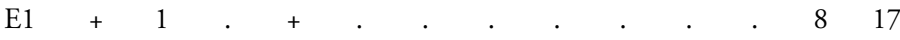

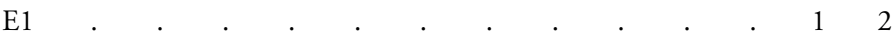

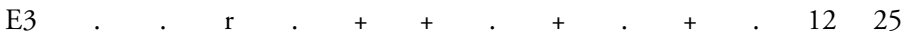

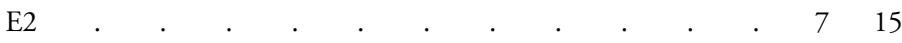

$\mathrm{E} 1$. . . . $.+.+29+1429$

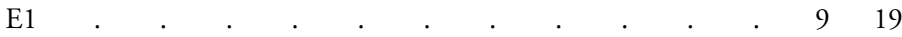

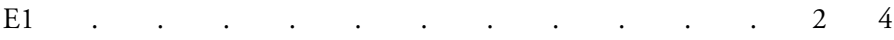

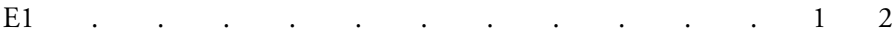

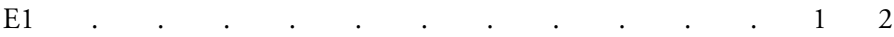

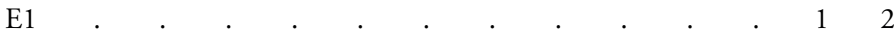

$\mathrm{E} 1$. $\quad . \quad . \quad . \quad . \quad . \quad . \quad . \quad . \quad . \quad . \quad 112$

$\begin{array}{llllllllllllll}\text { E3 } & 5 & 5 & 4 & 4 & 5 & 4 & 4 & 4 & 5 & 4 & 5 & 48 & 100 \\ \text { E2 } & + & \cdot & \cdot & + & + & \cdot & \cdot & + & + & + & + & 37 & 77 \\ \text { E3 } & \cdot & \cdot & + & \cdot & + & \cdot & + & + & + & + & + & 26 & 54 \\ \text { E2 } & \cdot & \cdot & \cdot & \cdot & + & + & 1 & . & \mathrm{r} & + & + & 41 & 85 \\ \text { E1 } & 2 & 1 & 1 & 1 & 1 & 1 & 2 & 2 & 1 & 2 & 1 & 41 & 85 \\ \text { E2 } & \cdot & \cdot & + & \cdot & + & + & + & + & 1 & + & + & 40 & 83 \\ \text { E1 } & + & + & + & + & + & + & + & 1 & 1 & + & 1 & 39 & 81 \\ \text { E1 } & \cdot & \cdot & 1 & \cdot & + & 1 & + & 1 & 1 & 1 & 1 & 36 & 75 \\ \text { E1 } & + & \cdot & + & + & + & + & . & + & + & + & . & 35 & 73\end{array}$


Number of relevé (Zaporedna številka popisa)

Lilium martagon

Prenanthes purpurea

Dryopteris filix-mas

Paris quadrifolia

Symphytum tuberosum

Actaea spicata

Euphorbia amygdaloides

Luzula nivea

Neottia nidus-avis

Laburnum alpinum

Laburnum alpinum

Laburnum alpinum

Mycelis muralis

Lathyrus vernus

Campanula trachelium

Polygonatum multiflorum

Epipactis helleborine

Epilobium montanum

Scrophularia nodosa

Festuca altissima

Myosotis sylvatica agg.

Petasites albus

Cardamine bulbifera

Cardamine impatiens

Carex sylvatica

Galium odoratum

Ranunculus lanuginosus

QP Quercetalia pubescenti-petraeae

Sorbus aria (Aria edulis)

Sorbus aria (Aria edulis)

Sorbus aria (Aria edulis)

Melittis melissophyllum

Convallaria majalis

Carex flacca

QF Querco-Fagetea

Anemone nemorosa

Carex digitata

Hepatica nobilis

Poa nemoralis

Viola riviniana

Festuca heterophylla

Corylus avellana

Platanthera bifolia

VP Vaccinio-Piceetea

Aposeris foetida

Rosa pendulina

Solidago virgaurea

Picea abies

Picea abies

Picea abies

\section{$\begin{array}{lllllllllllll}38 & 39 & 40 & 41 & 42 & 43 & 44 & 45 & 46 & 47 & 48 & \text { Pr. } & \text { Fr. }\end{array}$}

E1

E1

E1

E1

E1

E1

E1

E1

E3

E3

E2

E1

E1

E1

E1

E1

E1

E1

E1

E1

E1

E1

E1

E1

E1

E1

E1

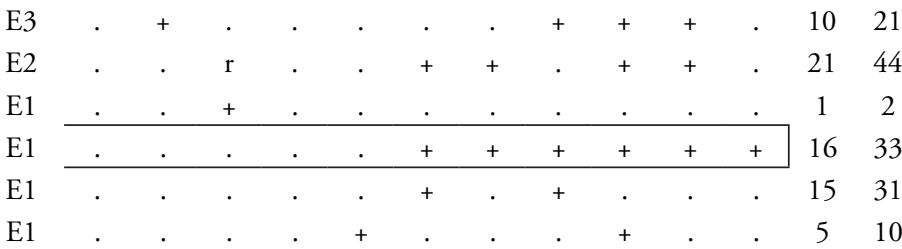

$\mathrm{E} 1+++1++++1++3165$

$\mathrm{E} 1+++++++++2756$

$\mathrm{E} 1 . .5 . \quad . \quad . \quad . \quad . \quad 1429$

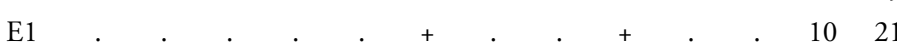

$\mathrm{E} 1 . .5 . \quad . \quad . \quad . \quad . \quad 24$

E1

E2

E1

$\begin{array}{llllllllllllll}\text { E1 } & 1 & + & 1 & 1 & . & . & + & 1 & 1 & 1 & 1 & 40 & 83 \\ \mathrm{E} 2 & + & + & . & + & + & + & + & + & + & + & + & 37 & 77 \\ \mathrm{E} 1 & . & + & . & . & + & + & . & + & + & + & + & 34 & 71 \\ \mathrm{E} 3 & + & . & + & + & . & + & + & + & . & 1 & + & 26 & 54 \\ \mathrm{E} 2 & + & . & . & + & + & + & + & + & + & . & + & 32 & 67 \\ \text { E1 } & . & . & . & . & . & . & . & . & + & + & . & 9 & 19\end{array}$ 
Number of relevé (Zaporedna števillka popisa)

Veronica urticifolia

Hieracium murorum

Calamagrostis arundinacea

Gentiana asclepiadea

Vaccinium myrtillus

Maianthemum bifolium

Luzula luzuloides

Gymnocarpium dryopteris

Huperzia selago

Oxalis acetosella

Larix decidua

Larix decidua

Lonicera nigra

Homogyne sylvestris

Saxifraga cuneifolia

Dryopteris dilatata

Phegopteris connectilis

Abies alba

Abies alba

Abies alba

Vaccinium vitis-idaea

Calamagrostis villosa

Corallorbiza trifida

Luzula luzulina

EP Erico-Pinetea

Rubus saxatilis

Erica carnea

Carex alba

Aquilegia nigricans

Peucedanum rablense

Carex ornithopoda

Polygala chamaebuxus

Molinia arundinacea

Chamaecytisus hirsutus

Cotoneaster tomentosus

SSC Sambuco-Salicion capreae, Rhamno-Prunetea

Sorbus aucuparia

Sorbus aucuparia

Sorbus aucuparia

BA Betulo-Alnetea

Ribes alpinum

Sorbus austriaca

MuA Mulgedio-Aconitetea

Polygonatum verticillatum

Veratrum album

Athyrium filix-femina

Phyteuma ovatum

Senecio ovatus

Aconitum degenii subsp. paniculatum

Ranunculus platanifolius

$\begin{array}{lllllllllllll}38 & 39 & 40 & 41 & 42 & 43 & 44 & 45 & 46 & 47 & 48 & \text { Pr. } & \text { Fr. }\end{array}$

E1

E1

E1

E1

E1

E1

E1

E1

E1

E1

E3

E1

E2

E1

E1

E1

E1

E3

E2

E1

E1

E1

E1

E1

$\mathrm{E} 11++1 .++\ldots+.43369$

$\mathrm{E} 1++++++++2654$

$\mathrm{E} 1+.+2 \quad+\quad 2 \quad 2 \quad 1 \quad 21+2246$

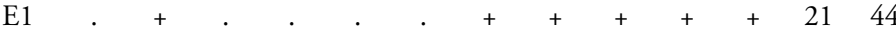

$\mathrm{E} 1$. . . $++++\ldots+1429$

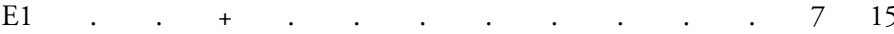

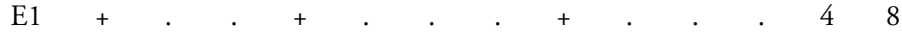

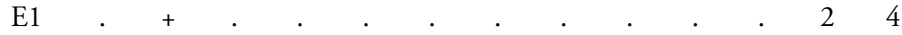

E1

E2

E3

E2

E1

E2

E2

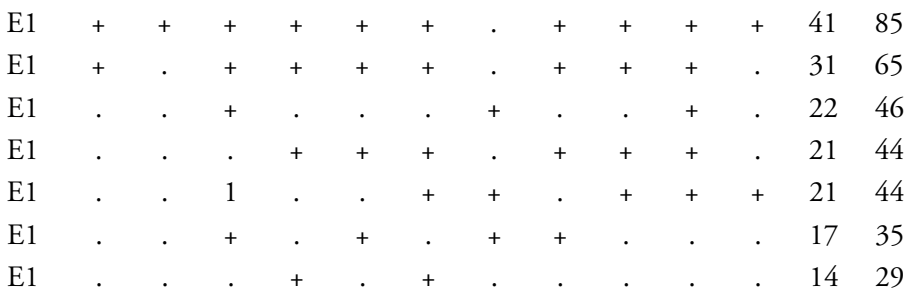


Number of relevé (Zaporedna številka popisa)

Geranium sylvaticum

Thalictrum aquilegiifolium

Viola biflora

Chaerophyllum villarsii

Aconitum angustifolium

Centaurea montana

Myrrhis odorata

Saxifraga rotundifolia

Senecio cacaliaster

Pleurospermum austriacum

Chaerophyllum hirsutum

Hypericum maculatum

Lathyrus occidentalis var. montanus

Tanacetum corymbosum subsp. clusii

Agropyron caninum

Chaerophyllum aureum

Primula elatior

Adenostyles alliariae

Doronicum austriacum

Crepis pyrenaica

CA Caricion austroalpinae

Gentiana lutea subsp. symphyandra

Koeleria eriostachya

Centaurea haynaldii subsp. julica

Arabis vochinensis

Heracleum austriacum subsp. siifolium

Caricion ferrugineae

Campanula thyrsoides

Knautia longifolia

Cfir

Caricion firmae

Carex firma

\section{ES Elyno-Seslerietea}

Betonica alopecuros

Sesleria caerulea

Campanula witasekiana

Carduus crassifolius

Carex sempervirens

Phyteuma orbiculare

Senecio abrotanifolius

Pimpinella alpina

Scabiosa lucida subsp. stricta

Leucanthemum heterophyllum

Galium anisophyllum

Astrantia bavarica

Ranunculus carinthiacus

Lotus alpinus

Thymus praecox subsp. polytrichus

Alchemilla alpigena

Arabis ciliata

Thesium alpinum
E1

E1

E1

E1

E1

E1

E1

E1

E1

E1

E1

E1

E1

E1

E1

E1

E1

E1

E1

E1

E1

E1

E1

E1

E1

E1

E1

E1

$\mathrm{E} 1+1 \quad 1 \quad 1 \quad 1+\quad+\quad 1 \quad 1 \quad 1 \quad 144696$

$\mathrm{E} 112+1++21 \quad 1 \quad 1 \quad 1 \quad 1 \quad 3267$

$\mathrm{E} 1+1++++++++2450$

$\mathrm{E} 1+++.+.+2+\ldots+1838$

$\mathrm{E} 1+.+2 .+1+\mathrm{r} \cdot+1225$

$\mathrm{E} 1++.+2 .+\mathrm{e}+\mathrm{r}+1225$

$\mathrm{E} 1++.+. \quad$. . . . . 1123

E1

E1

E1

E1

E1

E1

E1

E1

E1

E1

E1

\section{$\begin{array}{lllllllllllll}38 & 39 & 40 & 41 & 42 & 43 & 44 & 45 & 46 & 47 & 48 & \text { Pr. } & \text { Fr. }\end{array}$}

\section{7}

. . . . . . + r . . 1021

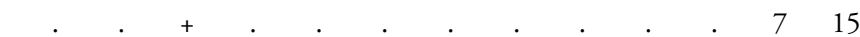

$\cdot+\cdot \cdot \cdot \cdot \cdot \cdot \cdot$

. . 48

$. .5 . .56$

. . . . . . . . . . 36

. . . . . . . . . . 24

24

2

2$$
2
$$

6 . 
Number of relevé (Zaporedna številka popisa)

Anthyllis vulneraria subsp. alpestris

Bartsia alpina

NS Nardion strictae, Juncetea trifidi

Campanula scheuchzeri

Coeloglossum viride

Potentilla erecta

FB Festuco-Brometea

Cirsium erisithales

Buphthalmum salicifolium

Carex humilis

Carlina acaulis

Bromopsis transsilvanica

Koeleria pyramidata

Dactylorhiza sambucina

Arabis hirsuta

Prunella grandiflora

Hippocrepis comosa

TG Trifolio-Geranietea

Laserpitium latifolium

Lilium carniolicum

Clinopodium vulgare

Verbascum lanatum

Thalictrum minus

Digitalis grandiflora

Arabis pauciflora

Laserpitium siler

Arabis turrita

Iris graminea

Viola hirta

PaT Poo alpinae-Trisetetalia

Poa alpina

Ranunculus nemorosus

Trollius europaeus

MA Molinio-Arrhenatheretea

Dactylis glomerata

Crepis paludosa

Deschampsia cespitosa

Angelica sylvestris

Festuca rubra agg.

Taraxacum sect. Ruderalia

EA Epilobietea angustifolii, Galio-Urticetea

Fragaria vesca

Rubus idaeus

Hypericum hirsutum

GU Urtica dioica

Galeopsis speciosa

TR Thlaspietea rotundifolii

Adenostyles glabra

Gymnocarpium robertianum

Heracleum pollinianum

\section{$\begin{array}{lllllllllllll}38 & 39 & 40 & 41 & 42 & 43 & 44 & 45 & 46 & 47 & 48 & \text { Pr. } & \text { Fr. }\end{array}$}

E1

E1

E1

E1

E1

$\mathrm{E} 1++++++++4879$

E1 . 1 . $\quad . \quad . \quad . \quad+\quad+\quad+\quad+\quad+1327$

E1 12 . . . . $+1+\ldots+1123$

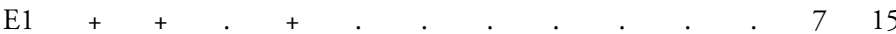

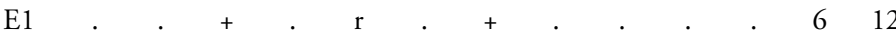

E1

E1

E1

E1

E1

E1

E1

E1

E1

E1

E1

E1

E1

E1

E1

E1

E1

E1

E1

E1

E1

E1

E1

E1

E2

E1

E1

E1

E1

E1
2

2

2

2

19

12

6

6

6

4

4

2

2

2

2

6

2

2

15

6

4

4

2

2

$14 \quad 29$

1021
2

24

24

12

\begin{tabular}{ll}
$37 \quad 77$ \\
\hline
\end{tabular}

- $18 \quad 38$

$\cdot \quad 18$
$+\quad 1838$ 
Number of relevé (Zaporedna števillka popisa)

Astrantia carniolica

Trisetum argenteum

Valeriana montana

Hieracium bifidum

Ligusticum seguieri

Aquilegia iulia

Biscutella laevigata

Molopospermum peloponnesiacum subsp. bauhinii

Aquilegia einseleana

Petasites paradoxus

Hieracium glaucum

Festuca laxa

Hieracium austriacum

Cy Cystopteridion fragilis

Valeriana tripteris

Asplenium viride

Moehringia muscosa

Cystopteris fragilis

Carex brachystachys

Cystopteris regia

PS Physoplexido comosae-Saxifragion petraeae

Campanula carnica

Campanula cespitosa

Paederota bonarota

Campanula zoysii

PC Potentilletalia caulescentis

Festuca stenantha

Primula auricula

Campanula cochleariifolia

Potentilla caulescen

AT Asplenietea trichomanis

Asplenium ruta-muraria

Asplenium trichomanes

Polypodium vulgare

Kernera saxatilis

Sedum maximum

O Other species (Druge vrste)

Festuca sp.

ML Mosses and lichens (Mahovi in lišaji)

Ctenidium molluscum

Tortella tortuosa

Schistidium apocarpum

Fissidens dubius

Pseudoleskeella catenulata

Polytrichum formosum

Bryum capillare

Peltigera canina

Isothecium alopecuroides

Encalypta streptocarpa

Homalothecium lutescens

\section{$\begin{array}{lllllllllllll}38 & 39 & 40 & 41 & 42 & 43 & 44 & 45 & 46 & 47 & 48 & \text { Pr. } & \text { Fr. }\end{array}$}

E1

E1

E1

E1

E1

E1

E1

E1

E1

E1

E1

E1

E1

E1

E1

E1

E1

E1

E1

E1

E1

E1

E1

E1

E1

E1

E1

E1

E1

E1

E1

E1

E1

$\mathrm{E} 0+++1+1+1+4288$

$\mathrm{E} 0+++++1++.+3879$

$\mathrm{E} 0+.+1+\ldots++\ldots+3165$

$\mathrm{E} 0++.+.+.+2 .+1940$

$\mathrm{E} 0 .++.+\ldots+.+1123$

$\mathrm{E} 0 .+.+. .+2 .+817$

$\mathrm{E} 0$. . . . . . . . +612

$\mathrm{E} 0$. . . . . . . . . . . 612

$\mathrm{E} 0$. . . . . . . . . . 612

$\mathrm{E} 0.5 .5 . .5 .510$ 
Number of relevé (Zaporedna številka popisa)

\begin{tabular}{|c|c|c|c|c|c|c|c|c|c|c|c|c|}
\hline 38 & 39 & 40 & 41 & 42 & 43 & 44 & 45 & 46 & 47 & 48 & Pr. & Fr. \\
\hline+ & . & . & . & . & . & . & . & . & . & . & 4 & 8 \\
\hline . & . & . & . & . & . & . & . & + & . & . & 3 & 6 \\
\hline . &. & . & . & . & . & . & . & . & . & . & 3 & 6 \\
\hline$\cdot$ & $\cdot$ & $\cdot$ & . & . & . & . & . & . & . & . & 3 & 6 \\
\hline . &. & . & . & . & + & . & . & . & . & . & 3 & 6 \\
\hline$\cdot$ & . & . & . & . & . & . & . & . & . & . & 3 & 6 \\
\hline . & . & . & . & . & . & . & . & . & . & . & 2 & 4 \\
\hline. &. & . & . & . & . & . & . & . & . & . & 2 & 4 \\
\hline . & . & . & . & + & . & . & . & . & . & . & 2 & 4 \\
\hline . & . & . & . & . & . & . & . & . & . & . & 2 & 4 \\
\hline . & . & . & . & . & + & . & . & . & . & . & 2 & 4 \\
\hline$\cdot$ & $\cdot$ & $\cdot$ & . & . & . & . & . & . & . & . & 1 & 2 \\
\hline$\cdot$ & $\cdot$ & $\cdot$ & $\cdot$ & . & . & . & $\cdot$ & $\cdot$ & $\cdot$ & . & 1 & 2 \\
\hline$\cdot$ & $\cdot$ & $\cdot$ & $\cdot$ & . & $\cdot$ & . & . & . & . & . & 1 & 2 \\
\hline . & $\cdot$ & $\cdot$ & . & . & . & . & . & . & . & . & 1 & 2 \\
\hline . & . & $\cdot$ & . & . & . & . & . & $\cdot$ & $\cdot$ & . & 1 & 2 \\
\hline . & $\cdot$ & $\cdot$ & + & . & . & . & . & . & . & . & 1 & 2 \\
\hline . & . & . & + & . & . & . & . & . & . & . & 1 & 2 \\
\hline
\end{tabular}

Cladonia sp.

Collema cristatum

Conocephalum conicum

Mnium thomsonii

Dermatocarpon miniatum

Dicranum scoparium

Homalothecium philippeanum

Mnium sp.

Cladonia pyxidata

Plagiochila porelloides

Hypnum cupressiforme

Peltigera leucophlebia

Lobaria pulmonaria

Rhizomnium punctatum

Plagiothecium sp.

Marchantia polymorpha

Bartramia pomiformis
E0

E0

E0

E0

E0

E0

E0

E0

E0

E0

E0

E0

E0

E0

E0

E0

E0

\begin{tabular}{llllllllllllll} 
& 38 & 39 & 40 & 41 & 42 & 43 & 44 & 45 & 46 & 47 & 48 & Pr. & Fr. \\
E0 & + &. &. &. &. &. &. &. &. &. &. & 4 & 8 \\
E0 &. &. &. &. &. &. &. &. & + &. &. & 3 & 6 \\
E0 &. &. &. &. &. &. &. &. &. &. &. & 3 & 6 \\
E0 &. &. &. &. &. &. &. &. &. &. &. & 3 & 6 \\
E0 &. &. &. &. &. & + &. &. &. &. &. & 3 & 6 \\
E0 &. &. &. &. &. &. &. &. &. &. &. & 3 & 6 \\
E0 &. &. &. &. &. &. &. &. &. &. &. & 2 & 4 \\
E0 &. &. &. &. &. &. &. &. &. &. &. & 2 & 4 \\
E0 &. &. &. &. & + &. &. &. &. &. &. & 2 & 4 \\
E0 &. &. &. &. &. &. &. &. &. &. &. & 2 & 4 \\
E0 &. &. &. &. &. & + &. &. &. &. &. & 2 & 4 \\
E0 &. &. &. &. &. &. &. &. &. &. &. & 1 & 2 \\
E0 &. &. &. &. &. &. &. &. &. &. &. & 1 & 2 \\
E0 &. &. &. &. &. &. &. &. &. &. &. & 1 & 2 \\
E0 &. &. &. &. &. &. &. &. &. &. &. & 1 & 2 \\
E0 &. &. &. &. &. &. &. &. &. &. &. & 1 & 2 \\
E0 &. &. &. & + &. &. &. &. &. &. &. & 1 & 2 \\
E0 &. &. &. & + &. &. &. &. &. &. &. & 1 & 2 \\
\hline
\end{tabular}

Cetraria islandica

\section{Legend - Legenda}

ID Igor Dakskobler

A Limestone - apnenec

D Dolomite - dolomit

Gr Gravel - grušč

M Moraine (Till) - morena (til)

Re Rendzina - rendzina

JA Julian Alps - Julijske Alpe

I (Italia, Italija)

Pr. Presence (number of relevés in which the species is presented) - število popisov, v katerih se pojavlja vrsta

Fr. Frequency in \% -- frekvenca $v \%$

Relevé No. 24, holotypus 
Table 5: Polysticho lonchitis-Fagetum luzuletosum niveae var. Festuca calva - cluster 28. Relevé numbers 1-38.

\begin{tabular}{|c|c|c|c|c|c|c|c|c|c|c|c|c|c|c|c|}
\hline Number of relevé (Zaporedna številka popisa) & & 1 & 2 & 3 & 4 & 5 & 6 & 7 & 8 & 9 & 10 & 11 & 12 & 13 & 14 \\
\hline $\begin{array}{l}\text { Database number of relevé } \\
\text { (Delovna številka popisa) }\end{array}$ & & $\begin{array}{l}\stackrel{0}{0} \\
\infty \\
\infty\end{array}$ & \begin{tabular}{l}
$\hat{n}$ \\
\multirow{2}{*}{} \\
$\stackrel{\sim}{n}$
\end{tabular} & 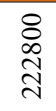 & 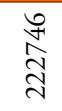 & 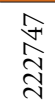 & $\begin{array}{l}\underset{\infty}{+} \\
\underset{\mathbb{N}}{\text { ते }}\end{array}$ & 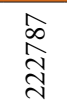 & $\begin{array}{l}\text { ลิ } \\
\text { तิ }\end{array}$ & 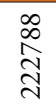 & 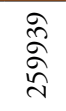 & $\begin{array}{l}\stackrel{ }{+} \\
\stackrel{\sigma}{\approx} \\
\text { }\end{array}$ & $\begin{array}{l}\stackrel{\infty}{\curvearrowright} \\
\underset{\text { तิ }}{\text { ते }}\end{array}$ & 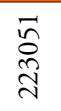 & 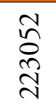 \\
\hline Author of the relevé (Avtor popisa) & & ID & ID & ID & ID & ID & ID & ID & ID & ID & ID & ID & ID & ID & ID \\
\hline Elevation in $\mathrm{m}$ (Nadmorska višina $\mathrm{v} \mathrm{m})$ & & $\stackrel{\stackrel{n}{n}}{\stackrel{n}{n}}$ & $\underset{\Im}{\stackrel{\Im}{\leftrightarrows}}$ & $\begin{array}{l}\stackrel{D}{\infty} \\
\stackrel{\sim}{n}\end{array}$ & $\begin{array}{l}\stackrel{n}{n} \\
\stackrel{n}{n}\end{array}$ & 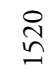 & $\begin{array}{l}\stackrel{\cap}{n} \\
n\end{array}$ & $\begin{array}{l}\stackrel{8}{0} \\
\end{array}$ & $\underset{-}{\stackrel{0}{0}}$ & $\begin{array}{l}\stackrel{i}{n} \\
n\end{array}$ & $\begin{array}{l}\stackrel{\wp}{\sharp} \\
\underset{\sim}{\sharp}\end{array}$ & $\underset{Ð}{\stackrel{尺}{\sharp}}$ & $\underset{Ð}{\stackrel{尺}{\unlhd}}$ & 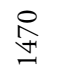 & $\begin{array}{l}\stackrel{\infty}{\infty} \\
\stackrel{+}{ \pm}\end{array}$ \\
\hline Aspect (Lega) & & SW & SW & SW & SE & SE & SE & SSW & SE & $\mathrm{E}$ & SSW & $S$ & $S$ & SE & SE \\
\hline Slope in degrees (Nagib v stopinjah) & & 30 & 35 & 25 & 25 & 25 & 25 & 25 & 30 & 30 & 35 & 35 & 10 & 30 & 25 \\
\hline Parent material (Matična podlaga) & & A & A & DL & $\mathrm{DA}$ & DA & DA & $\mathrm{DA}$ & DA & DA & $\mathrm{DA}$ & DA & DA & DA & DA \\
\hline Soil (Tla) & & $\mathrm{Re}$ & $\operatorname{Re}$ & $\mathrm{Re}$ & $\operatorname{Re}$ & $\operatorname{Re}$ & $\operatorname{Re}$ & $\operatorname{Re}$ & $\mathrm{Re}$ & $\mathrm{Re}$ & $\operatorname{Re}$ & $\operatorname{Re}$ & $\operatorname{Re}$ & $\operatorname{Re}$ & $\operatorname{Re}$ \\
\hline Stoniness in \% (Kamnitost v \%) & & 40 & 20 & 20 & 30 & 20 & 20 & 5 & 40 & 10 & 40 & 40 & 20 & 20 & 20 \\
\hline \multicolumn{16}{|l|}{ Cover in \% (Zastiranje v \%): } \\
\hline Upper tree layer (Zgornja drevesna plast) & $\mathrm{E} 3 \mathrm{~b}$ & 80 & 90 & 70 & 90 & 90 & 100 & 80 & 90 & 70 & 90 & 80 & 80 & 80 & 70 \\
\hline Lower tree layer (Spodnja drevesna plasti) & E3a & . & 10 & . & . & . & . & . & 10 & 10 & . & . & 10 & . & . \\
\hline Shrub layer (Grmovna plast) & E2 & 10 & 50 & 20 & 10 & 10 & 5 & 30 & 5 & 10 & 5 & 5 & 5 & 20 & 20 \\
\hline Herb layer (Zeliščna plast) & E1 & 40 & 10 & 40 & 20 & 30 & 20 & 50 & 60 & 60 & 30 & 40 & 60 & 50 & 60 \\
\hline Moss layer (Mahovna plast) & E0 & 10 & 5 & 10 & 10 & 10 & 5 & 10 & 10 & 10 & 5 & 5 & 5 & 5 & 5 \\
\hline Maximum tree diameter (Maks. premer dreves) & $\mathrm{cm}$ & 80 & 25 & 35 & 25 & 20 & 15 & 20 & 35 & 30 & 30 & 35 & 40 & 25 & 25 \\
\hline Maximum tree height (Maksimalna višina dreves) & $\mathrm{m}$ & 18 & 10 & 12 & 7 & 8 & 5 & 10 & 14 & 14 & 12 & 10 & 17 & 5 & 6 \\
\hline Number of species (Število vrst) & & 54 & 62 & 62 & 61 & 48 & 33 & 63 & 68 & 73 & 42 & 53 & 35 & 52 & 45 \\
\hline Relevé area (Velikost popisne ploskve) & $\mathrm{m}^{2}$ & 200 & 200 & 400 & 400 & 200 & 200 & 400 & 400 & 200 & 400 & 400 & 400 & 200 & 200 \\
\hline Date of taking relevé (Datum popisa) & & 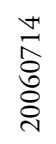 & 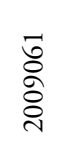 & 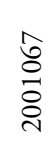 & 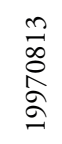 & $\begin{array}{l}\stackrel{n}{\infty} \\
\stackrel{\infty}{\rho} \\
\stackrel{2}{\sigma}\end{array}$ & 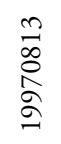 & 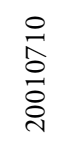 & $\begin{array}{l}\hat{\varnothing} \\
\stackrel{0}{0} \\
\stackrel{ }{0}\end{array}$ & $\begin{array}{l}\circ \\
\hat{\lambda} \\
0 \\
\stackrel{0}{\circ} \\
\stackrel{ }{N}\end{array}$ & 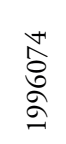 & 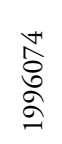 & $\begin{array}{l}\hat{ઠ} \\
\stackrel{0}{\circ} \\
\stackrel{ }{\circ}\end{array}$ & 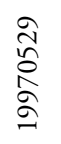 & 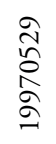 \\
\hline Day (Dan) & & 14 & 1 & 7 & 13 & 13 & 13 & 10 & 7 & 10 & 4 & 4 & 7 & 29 & 29 \\
\hline Month (Mesec) & & 7 & 6 & 6 & 8 & 8 & 8 & 7 & 6 & 7 & 7 & 7 & 6 & 5 & 5 \\
\hline Year (Leto) & & ঠ & 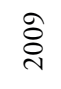 & ஓ্ণ & $\widehat{\widehat{\Omega}}$ & $\hat{\curvearrowright}$ & $\widehat{ૂ}$ & 홍 & ఠ্ & ळ্ & ڤ̆ & § & ஓे & $\hat{\check{\beth}}$ & $\widehat{\widehat{\partial}}$ \\
\hline Locality (Nahajališče) & & 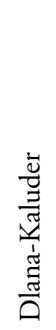 & 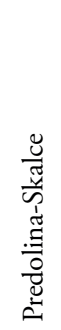 & 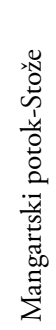 & 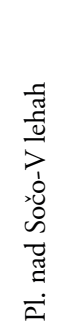 & 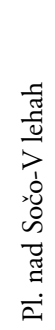 & 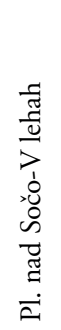 & 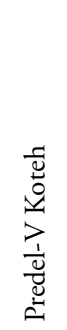 & 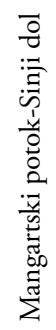 & 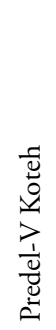 & 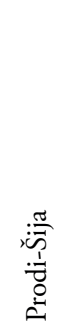 & 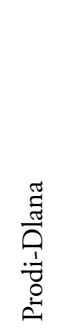 & 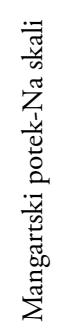 & 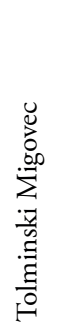 & 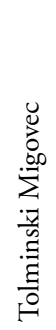 \\
\hline Mountain range (Pogorje) & & JA & JA & JA & JA & JA & JA & JA & JA & JA & JA & $\mathrm{JA}$ & JA & $\mathrm{JA}$ & JA \\
\hline Quadrant (Kvadrant) & & 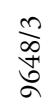 & 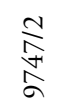 & 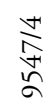 & 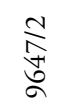 & $\underset{\curvearrowright}{\stackrel{N}{~}}$ & 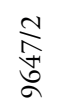 & 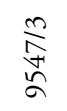 & $\underset{\stackrel{+}{*}}{\stackrel{+}{\sigma}}$ & 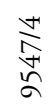 & $\underset{a}{\stackrel{n}{a}}$ & $\frac{n}{a}$ & $\begin{array}{l}\frac{\Downarrow}{\curvearrowright} \\
\stackrel{4}{n}\end{array}$ & $\begin{array}{l}\frac{+}{\infty} \\
\stackrel{\infty}{+} \\
\stackrel{+}{\alpha}\end{array}$ & $\begin{array}{l}\frac{+}{\infty} \\
\stackrel{\infty}{+} \\
\stackrel{\sigma}{\sigma}\end{array}$ \\
\hline Coordinate (Koordinate) GK Y (D-48) & $\mathrm{m}$ & $\begin{array}{l}\infty \\
\infty \\
\stackrel{\sigma}{\sigma}\end{array}$ & 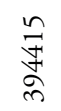 & $\frac{n}{\approx}$ & $\begin{array}{l}\text { त్రా } \\
\stackrel{\overbrace{}}{2}\end{array}$ & 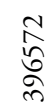 & 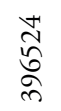 & $\begin{array}{l}\frac{+}{0} \\
\stackrel{2}{a} \\
\vec{n}\end{array}$ & $\begin{array}{l}\infty \\
\tilde{\approx} \\
\tilde{\approx} \\
\text { nิ }\end{array}$ & $\begin{array}{l}\Xi \\
\Xi \\
\bar{\sigma}\end{array}$ & 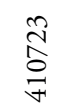 & 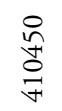 & 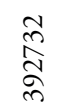 & 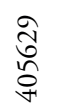 & 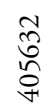 \\
\hline Coordinate (Koordinate) GK X (D-48) & $\mathrm{m}$ & $\begin{array}{l}\stackrel{0}{n} \\
\stackrel{8}{0} \\
\ddot{n}\end{array}$ & 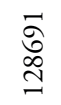 & $\begin{array}{l}\stackrel{+}{\infty} \\
\stackrel{+}{ \pm} \\
\pm \\
-\end{array}$ & $\frac{\pi}{\tilde{n}}$ & $\begin{array}{l}\infty \\
\infty \\
\infty \\
n \\
n\end{array}$ & $\begin{array}{l}\tilde{n} \\
\hat{n} \\
\tilde{n}\end{array}$ & 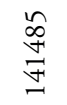 & $\underset{\stackrel{F}{\Psi}}{\stackrel{Ð}{ \pm}}$ & 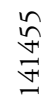 & 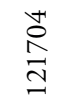 & $\begin{array}{l}\infty \\
\stackrel{\infty}{\infty} \\
\stackrel{\Xi}{\beth}\end{array}$ & 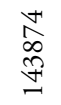 & 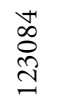 & $\frac{\sqrt[n]{n}}{\approx}$ \\
\hline
\end{tabular}

Diagnostic species of the association (Diagnostične vrste asociacije)
VP Polystichum lonchitis

VP Luzula sylvatica

VP Clematis alpina

MuA Aconitum lycoctonum subsp. ranunculifolium 
Tabela 5: Polysticho lonchitis-Fagetum luzuletosum niveae var. Festuca calva - skupina 28. Zaporedne številke popisov 1-38.
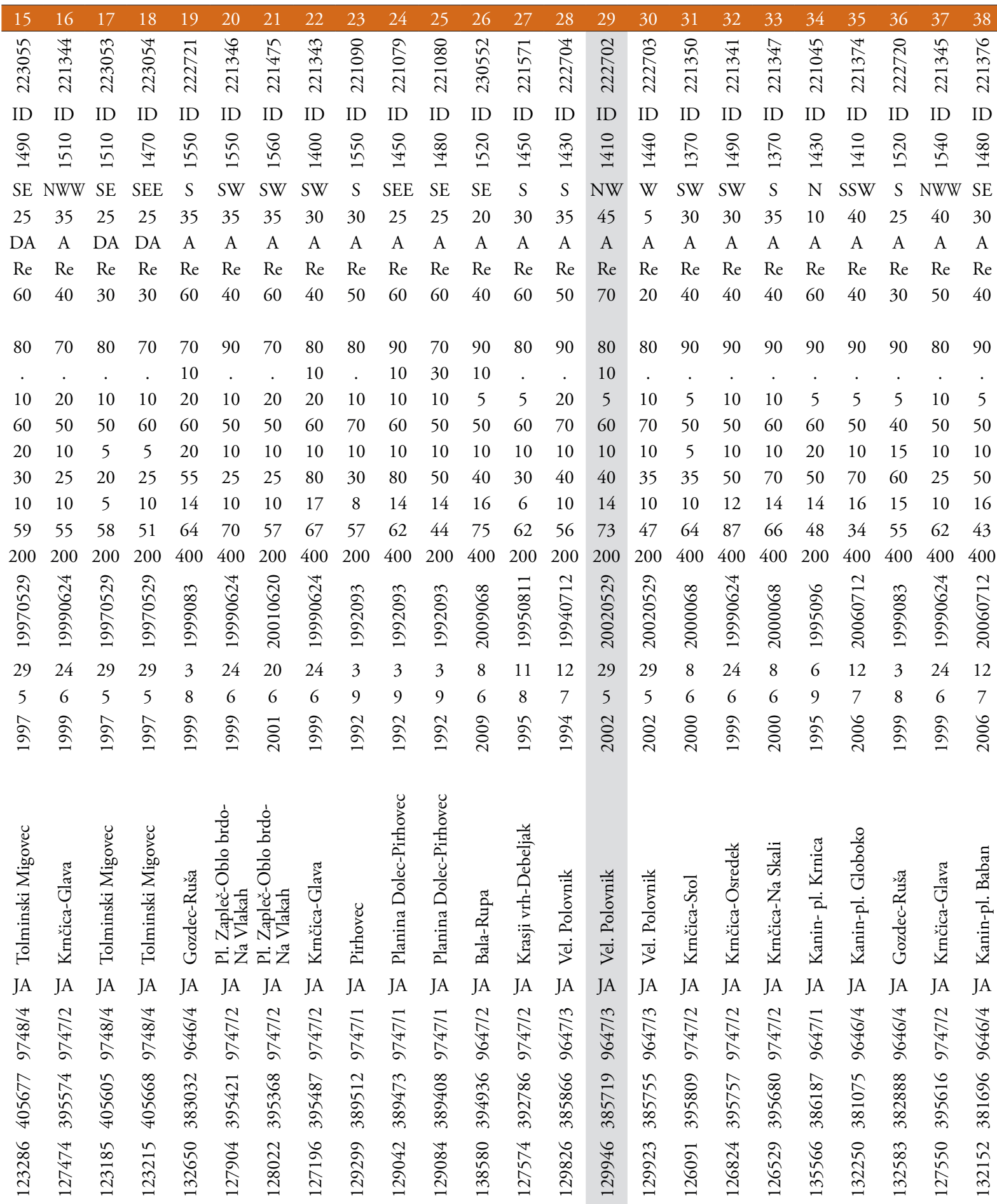


\begin{tabular}{|c|c|c|c|c|c|c|c|c|c|c|c|c|c|c|c|c|}
\hline & Number of relevé (Zaporedna številka popisa) & & 1 & 2 & 3 & 4 & 5 & 6 & 7 & 8 & 9 & 10 & 11 & 12 & 13 & 14 \\
\hline $\mathrm{CA}$ & Festuca calva & E1 & + & + & . & + & . & . & . & . & . & . & . & . & . & . \\
\hline EP & Rhododendron hirsutum & E2 & . & . & . & . & . & . & + & + & + & . & . & . & 1 & + \\
\hline $\mathrm{BA}$ & Sorbus chamaemespilus & E2 & . & . & + & + & + & . & + & + & + & . & . & . & + & . \\
\hline ML & Paraleucobryum sauteri & E0 & . & . & + & . & + & . & . & + & + & . & + & + & . & . \\
\hline CA & Laserpitium peucedanoides & E1 & . & . & . & . & . & $\mathrm{r}$ & . & + & . & + & + & . & . & + \\
\hline BA & Salix appendiculata & E2 & + & + & $\cdot$ & . & . & . & + & . & + & . & . & . & . & . \\
\hline $\mathrm{EP}$ & Pinus mugo & E2 & . & . & + & . & . & . & + & $\cdot$ & + & + & . & . & + & + \\
\hline $\mathrm{CF}$ & Carex ferruginea & E1 & . & . & + & . & . & . & $\mathrm{r}$ & + & . & + & + & . & . & $\mathrm{r}$ \\
\hline VP & Homogyne alpina & E1 & . & . & $\cdot$ & . & . & . & . & . & . & . & . & + & . & . \\
\hline PS & Paederota lutea & E1 & . & . & . & . & . & . & . & . & . & . & . & . & . & . \\
\hline VP & Lonicera caerulea & E2 & . & . & . & $\mathrm{r}$ & . & . & + & . & . & . & . & . & . & . \\
\hline ES & Aster bellidiastrum & E1 & . & . & . & . & . & . & . & . & . & + & + & . & . & . \\
\hline $\mathrm{EP}$ & Rhodothamnus chamaecistus & E1 & . & . & . & . & . & . & . & . & $\mathrm{r}$ & . & . & . & . & . \\
\hline
\end{tabular}

\section{Differential species of the subassociation (Razlikovalnice subasociacije)}

FS Luzula nivea

QF Poa nemoralis

TA Adoxa moschatellina

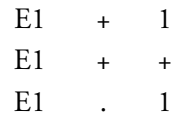

AF Aremonio-Fagion

Anemone trifolia

Cardamine enneaphyllos

Cyclamen purpurascens

Cardamine trifolia

Aremonia agrimonoides

Helleborus niger

Rhamnus fallax

Knautia drymeia

Lamium orvala

$\begin{array}{lllllllllllllll}\text { E1 } & 1 & 1 & 1 & 1 & 1 & 1 & 1 & 1 & 1 & 1 & . & 1 & 1\end{array}$.

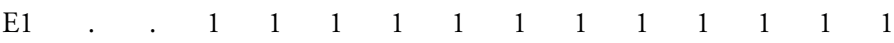

\section{Erythronio-Carpinion}

Helleborus odorus

Primula vulgaris

Galanthus nivalis

Ornithogalum pyrenaicum

TA Tilio-Acerion

Geranium robertianum

Acer pseudoplatanus

Acer pseudoplatanus

Acer pseudoplatanus

Polystichum aculeatum

Tilia platyphyllos

Aruncus dioicus

Euonymus latifolia

Chrysosplenium alternifolium

FS Fagetalia sylvaticae

Fagus sylvatica

Fagus sylvatica

Fagus sylvatica

Dryopteris filix-mas

Galeobdolon flavidum

Lilium martagon

Mercurialis perennis

Daphne mezereum

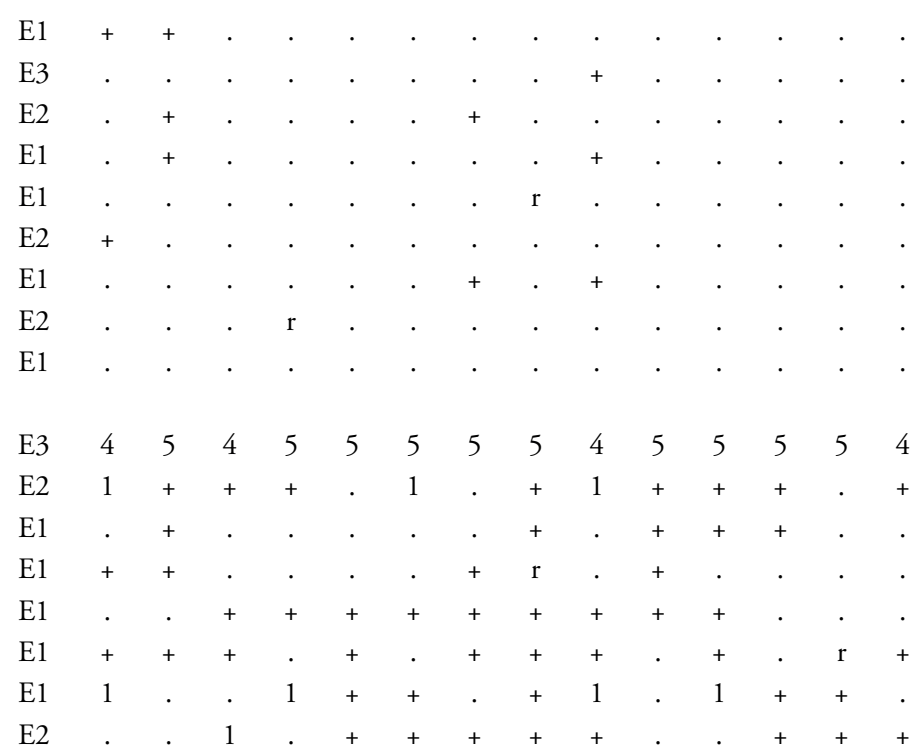


- Hacquetia 20/2 • 2021,373-564

Igor Dakskobler \& Andre Rozman

Vegetation analysis of the subalpine beech forest on the upper forest line in the

Julian Alps (NW Slovenia and NW Italy) and in the northern Dinaric Alps

$\begin{array}{llllllllllllllllllllllllll}15 & 16 & 17 & 18 & 19 & 20 & 21 & 22 & 23 & 24 & 25 & 26 & 27 & 28 & 29 & 30 & 31 & 32 & 33 & 34 & 35 & 36 & 37 & 38\end{array}$
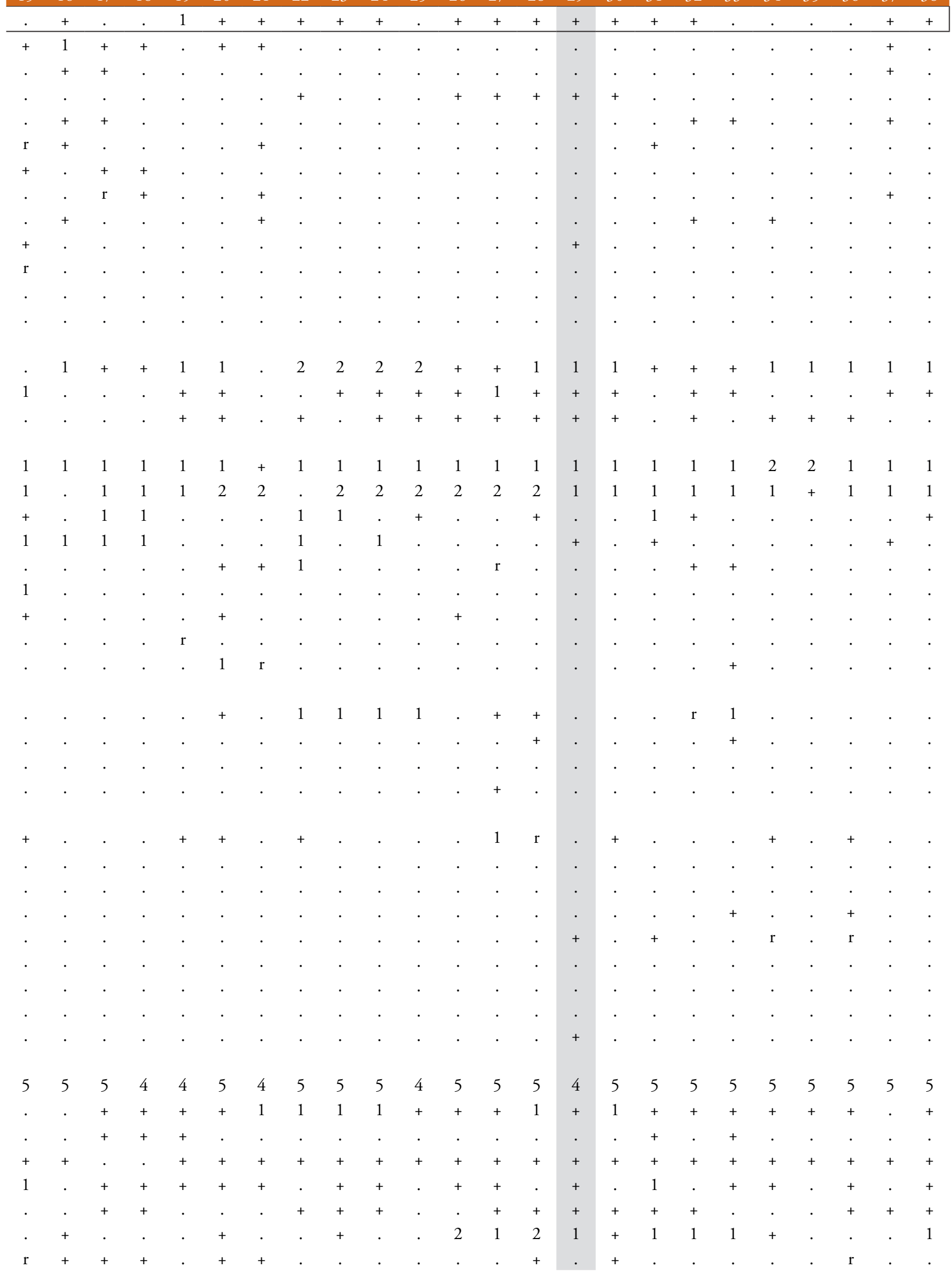

497 
Melica nutans

E1

Lathyrus vernus

Epilobium montanum

Lonicera alpigena

Paris quadrifolia

Actaea spicata

Galium laevigatum

Mycelis muralis

Euphorbia amygdaloides

Scrophularia nodosa

Prenanthes purpurea

Myosotis sylvatica agg.

Lathyrus vernus subsp. flaccidus

Symphytum tuberosum

Corydalis cava

Neottia nidus-avis

Polygonatum multiflorum

Ranunculus lanuginosus

Festuca altissima

Epipactis helleborine

Laburnum alpinum

Laburnum alpinum

Laburnum alpinum

Pulmonaria officinalis

Cardamine pentaphyllos

Petasites albus

Campanula trachelium

Cardamine impatiens

Galium odoratum

Cardamine bulbifera

Viola reichenbachiana

QP Quercetalia pubescenti-petraeae

Sorbus aria (Aria edulis)

Sorbus aria (Aria edulis)

Primula veris subsp. columnae

Convallaria majalis

Melittis melissophyllum

Hypericum montanum

Querco-Fagetea

Carex digitata

Anemone nemorosa

Hepatica nobilis

Festuca heterophylla

Aegopodium podagraria

Viola riviniana

Stellaria holostea

Moehringia trinervia

Ranunculus auricomus agg. ( $R$. braun-blanquetii)

Dactylis glomerata subsp. lobata (D. polygama)

Listera ovata

Scilla bifolia 


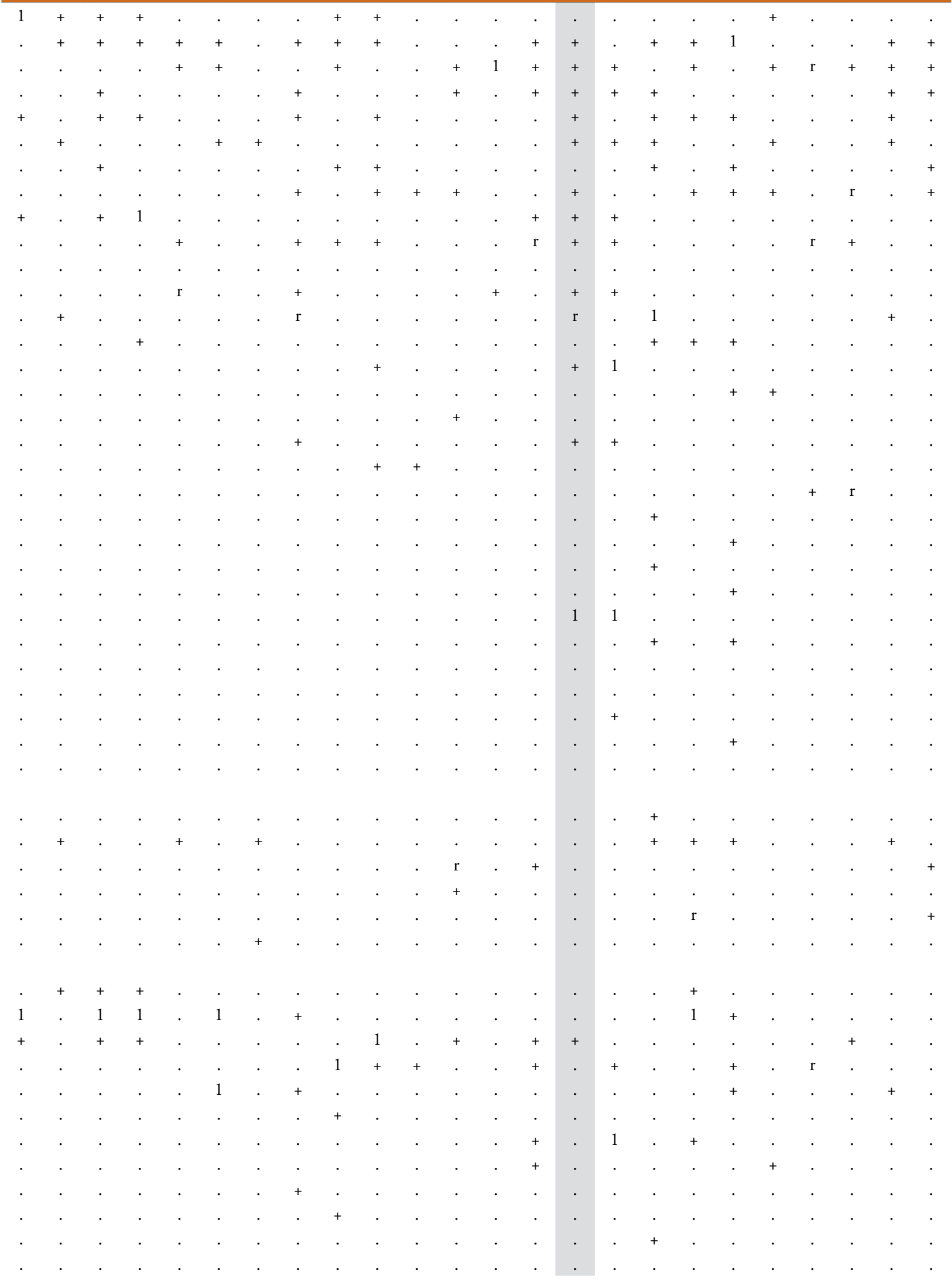


$\begin{array}{llllllllllllllll}\text { Number of relevé (Zaporedna številka popisa) } & 1 & 2 & 3 & 4 & 5 & 6 & 7 & 8 & 9 & 10 & 11 & 12 & 13 & 14\end{array}$

VP Vaccinio-Piceetea

Aposeris foetida

Vaccinium myrtillus

Calamagrostis arundinacea

Maianthemum bifolium

Hieracium murorum

Oxalis acetosella

Rosa pendulina

Picea abies

Picea abies

Picea abies

Solidago virgaurea

Gymnocarpium dryopteris

Veronica urticifolia

Dryopteris dilatata

Saxifraga cuneifolia

Corallorhiza trifida

Calamagrostis villosa

Huperzia selago

Luzula luzuloides

Vaccinium vitis-idaea

Gentiana asclepiadea

Dryopteris expansa

Phegopteris connectilis

Larix decidua

Luzula pilosa

Abies alba

Abies alba

Abies alba

Lonicera nigra

Lycopodium annotinum

Homogyne sylvestris

Ajuga pyramidalis

E1

E1

E1

Calamagrostis varia

Rubus saxatilis

Erica carnea

Carex ornithopoda

Peucedanum rablense

Carex alba

Polygala chamaebuxus

Genista radiata

Chamaecytisus hirsutus

Epipactis atrorubens

SSC Sambuco-Salicion capreae, Rhamno-Prunetea

Sorbus aucuparia

Sorbus aucuparia

Sorbus aucuparia

Salix caprea

Berberis vulgaris

Juniperus communis $+\quad \cdot \quad 1++\quad \cdot \begin{array}{lllllllll}1 & 1 & 1 & 1 & 1 & 1 & 1 & 1\end{array}$

. $++++++1++++$

. $1++++$. . ..+++

. $1 .+++\ldots+1+1$

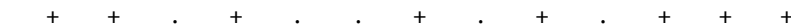

(1)

E3

$+$

E1

1

Elons

El

El

El

Elons

3

(1)

3

1

(1)

(1)

1 


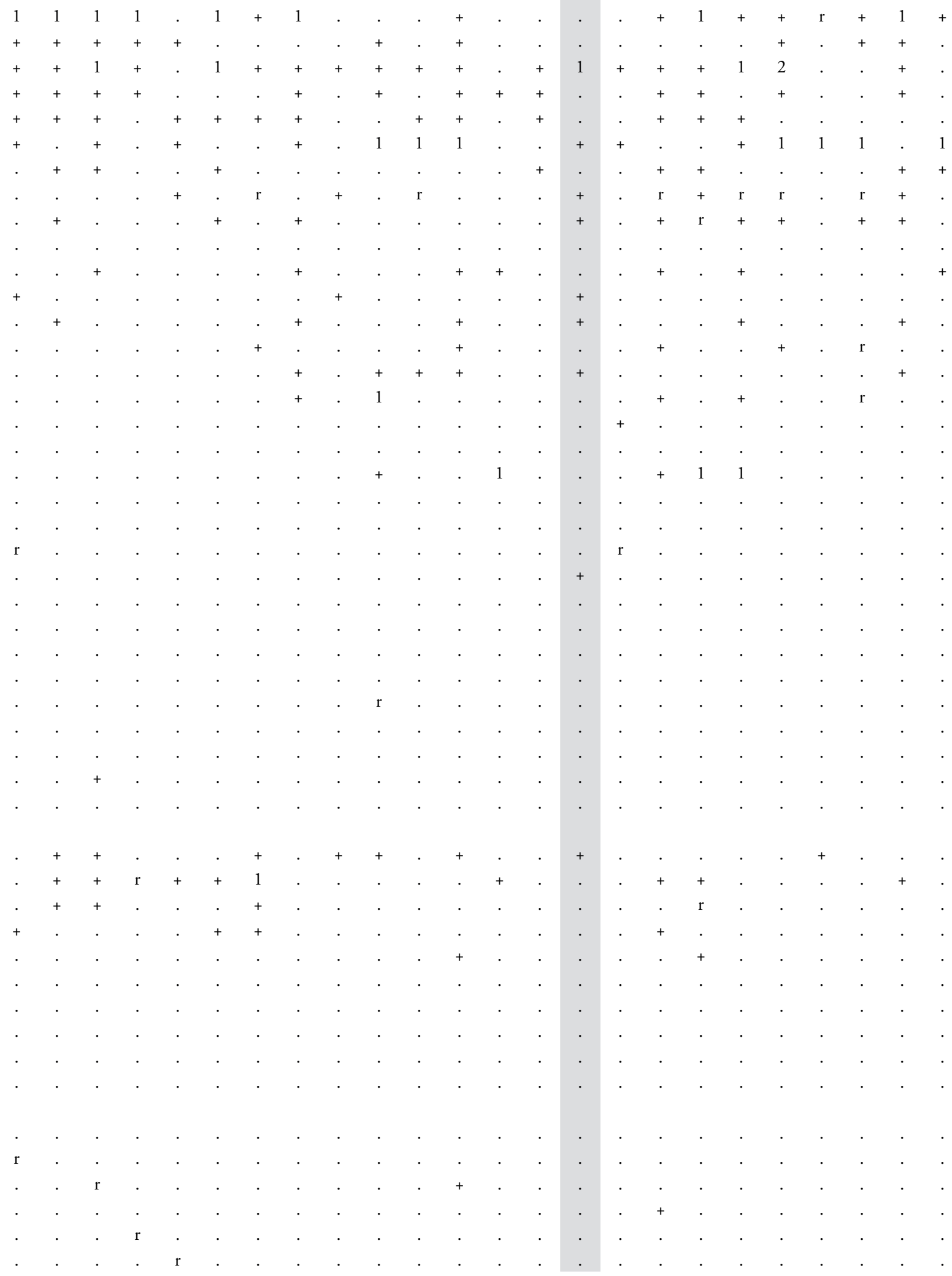


Sambucus racemosa

BA Betulo-Alnetea

Sorbus austriaca

Juniperus sibirica

Sorbus austriaca

Salix glabra

Alnus viridis

MA Mulgedio-Aconitetea

Polygonatum verticillatum

Veratrum album

Athyrium filix-femina

Saxifraga rotundifolia

Viola biflora

Senecio cacaliaster

Aconitum angustifolium

Geranium sylvaticum

Hypericum maculatum

Ranunculus platanifolius

Phyteuma ovatum

Chaerophyllum hirsutum

Aconitum degenii subsp. paniculatum

Chaerophyllum aureum

Thalictrum aquilegiifolium

Rumex arifolius

Senecio ovatus

Poa hybrida

Peucedanum ostruthium

Allium victorialis

Adenostyles alliariae

Tanacetum corymbosum subsp. clusii Epilobium alpestre

Chaerophyllum villarsii

Pleurospermum austriacum

CA Caricion austroalpinae

Arabis vochinensis

Gentiana lutea subsp. symphyandra

Pulsatilla alpina subsp. austroalpina

\section{Caricion ferrugineae}

Cerastium subtriflorum

Knautia longifolia

Elyno-Seslerietea

Betonica alopecuros

Sesleria caerulea

Campanula witasekiana

Pimpinella alpina

Carex sempervirens

Senecio abrotanifolius

Astrantia bavarica

Ranunculus carinthiacus

Galium anisophyllum

Carduus crassifolius
E2

E2

E2

E1

E2

E2

E1

E1

E1

E1

E1

E1

E1

E1

E1

E1

E1

E1

\section{$\begin{array}{llllllllllllll}1 & 2 & 3 & 4 & 5 & 6 & 7 & 8 & 9 & 10 & 11 & 12 & 13 & 14\end{array}$}

2

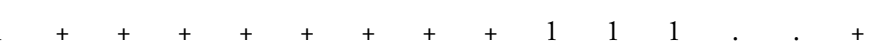

+ . . $+.+1+$. + . +1

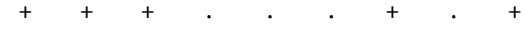

..+ ..++

$++$

$++$

(1)

$+$

(1)

$+$

(1)

(1)

(1)

(1)

(1)

(1)

1

E1

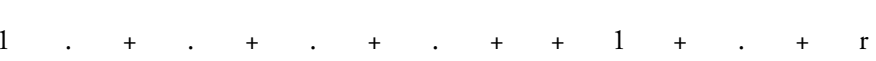

. $. \quad . \quad . \quad . \quad . \quad 1$. $+t_{+}+1$

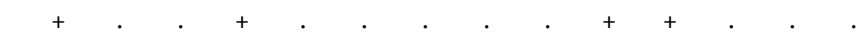

. + .

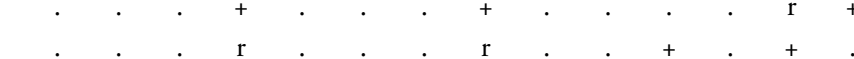

(1)

(5)


- Hacquetia 20/2 • 2021,373-564

Igor Dakskobler \& Andre Rozman

Vegetation analysis of the subalpine beech forest on the upper forest line in the

Julian Alps (NW Slovenia and NW Italy) and in the northern Dinaric Alps

$\begin{array}{llllllllllllllllllllllllll}15 & 16 & 17 & 18 & 19 & 20 & 21 & 22 & 23 & 24 & 25 & 26 & 27 & 28 & 29 & 30 & 31 & 32 & 33 & 34 & 35 & 36 & 37 & 38\end{array}$
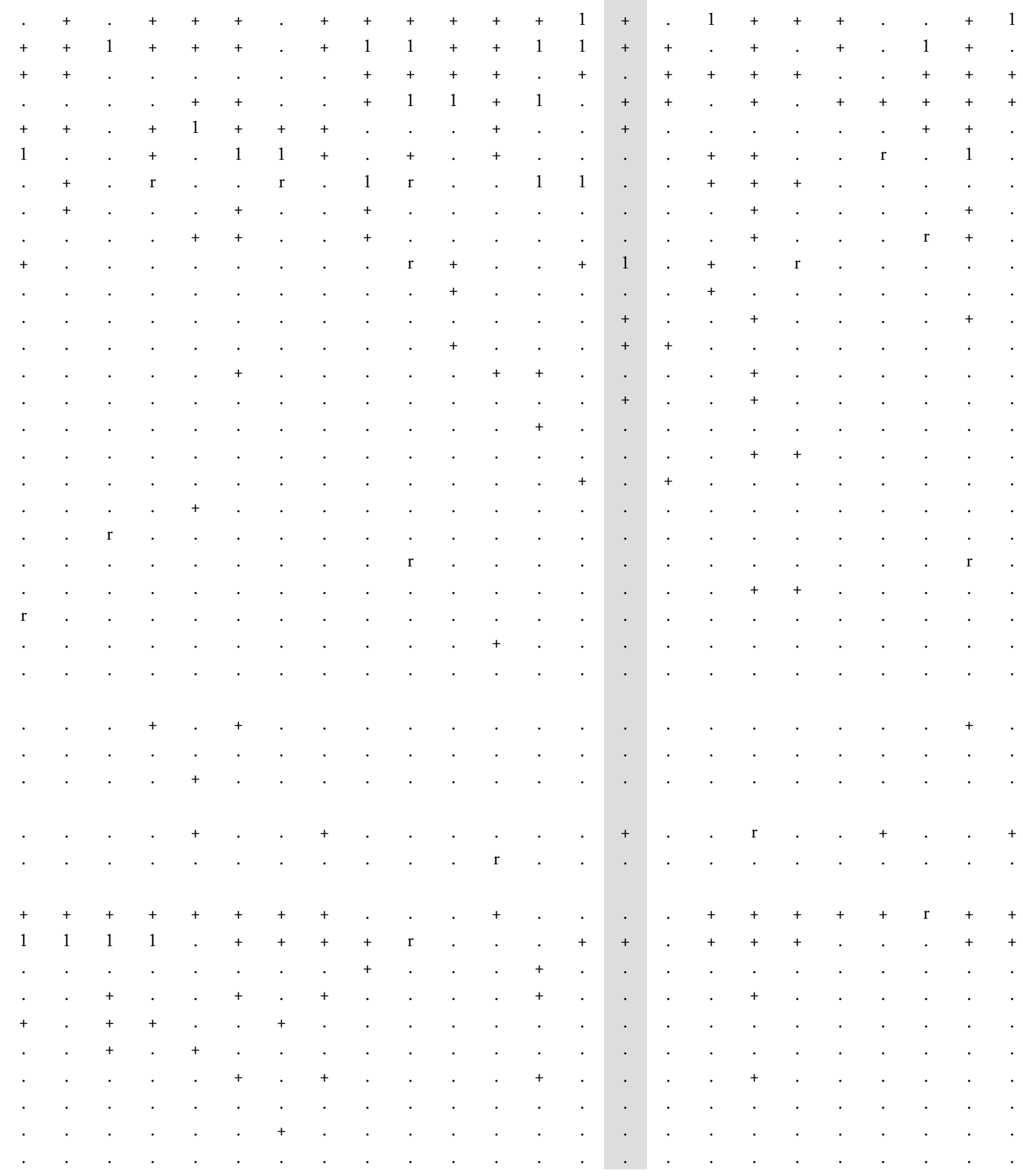

503 
Number of relevé (Zaporedna številka popisa)

$\begin{array}{llllllllllllll}1 & 2 & 3 & 4 & 5 & 6 & 7 & 8 & 9 & 10 & 11 & 12 & 13 & 14\end{array}$

Phyteuma orbiculare

E1

Alchemilla vulgaris agg.

E1

Alchemilla alpigena

Potentilla crantzii

Thymus praecox subsp. polytrichus

Scabiosa lucida subsp. stricta

Leontopodium alpinum

Lotus alpinus

Achillea clavenae

Leucanthemum heterophyllum

Erigeron glabratus

Ranunculus montanus

NS Nardion strictae, Juncetea trifidi

Campanula scheuchzeri

Phyteuma zahlbruckneri

Potentilla erecta

Coeloglossum viride

\section{E1}

E1

E1

E1

FB Festuco-Brometea

Cirsium erisithales

Buphthalmum salicifolium

Carlina acaulis

Koeleria pyramidata

Arabis hirsuta

Carex humilis

Hippocrepis comosa

Euphorbia cyparissias

ZG Trifolio-Geranietea

Verbascum lanatum

Clinopodium vulgare

Achillea distans

Laserpitium latifolium

Valeriana wallrothii

Laserpitium siler

Iris graminea

Vincetoxicum hirundinaria

E1

E1

E1

E1

E1

E1

E1

Vicia sylvatica

Lilium carniolicum

Hypericum perforatum

Libanotis sibirica subsp. montana

Origanum vulgare

$\mathrm{PaT}$ Poo alpinae-Trisetetalia

Poa alpina

E1

Crocus albiflorus

Trollius europaeus

Pimpinella major subsp. rubra

Cardaminopsis ovirensis

MA Molinio-Arrhenatheretea

Lathyrus pratensis

Deschampsia cespitosa

Dactylis glomerata

E1

E1

Galium album 
Crepis paludosa

Achillea millefolium

Festuca rubra agg.

Achillea roseoalba

Vicia sepium

Veronica chamaedrys

Angelica sylvestris

Leontodon hispidus

EA Epilobietea angustifolii

Fragaria vesca

Urtica dioica

Rubus idaeus

Galeopsis speciosa

Lamium maculatum

Tussilago farfara

Hypericum hirsutum

AC Arabidetalia caeruleae

Soldanella alpina

TR Thlaspietea rotundifolii

Adenostyles glabra

Gymnocarpium robertianum

Arabis alpina

Heracleum pollinianum

Dryopteris villarii

Astrantia carniolica

Geranium macrorrhizum

Molopospermum peloponnesiacum subsp. bauhinii Ligusticum seguieri

Rhodiola rosea

Cy Cystopteridion fragilis

Cystopteris fragilis

Asplenium viride

Valeriana tripteris

Moehringia muscosa

Cystopteris regia

Sedum hispanicum

Heliosperma pusillum

PS Physoplexido comosae-Saxifragion petraeae

Saxifraga petraea

Campanula carnica

Saxifraga crustata

Paederota bonarota

Saxifraga hostii

PC Potentilletalia caulescentis

Primula auricula

Campanula cochleariifolia

AT Asplenietea trichomanis

Asplenium trichomanes

Asplenium ruta-muraria

Polypodium vulgare

Sedum album
E1

E1

E1

E1

E1

E1

E1

E1

E1

E1

E1

E1

E1

E1

E1

E1

E1

E1

E1

E1

E1

E1

E1

E1

E1

E1

E1

E1

E1

E1

E1

E1

E1

E1

E1

E1

E1

E1

E1

E1

E1 
- Hacquetia 20/2 • 2021,373-564

Igor Dakskobler \& Andre Rozman

Vegetation analysis of the subalpine beech forest on the upper forest line in the

Julian Alps (NW Slovenia and NW Italy) and in the northern Dinaric Alps

15

$\begin{array}{lll}16 & 17 & 18\end{array}$

$19 \quad 20 \quad 21$

$\begin{array}{llllllll}22 & 23 & 24 & 25 & 26 & 27 & 28 & 29\end{array}$

$30 \quad 31$

$32 \quad 33$

$\begin{array}{lllll}34 & 35 & 36 & 37 & 38\end{array}$
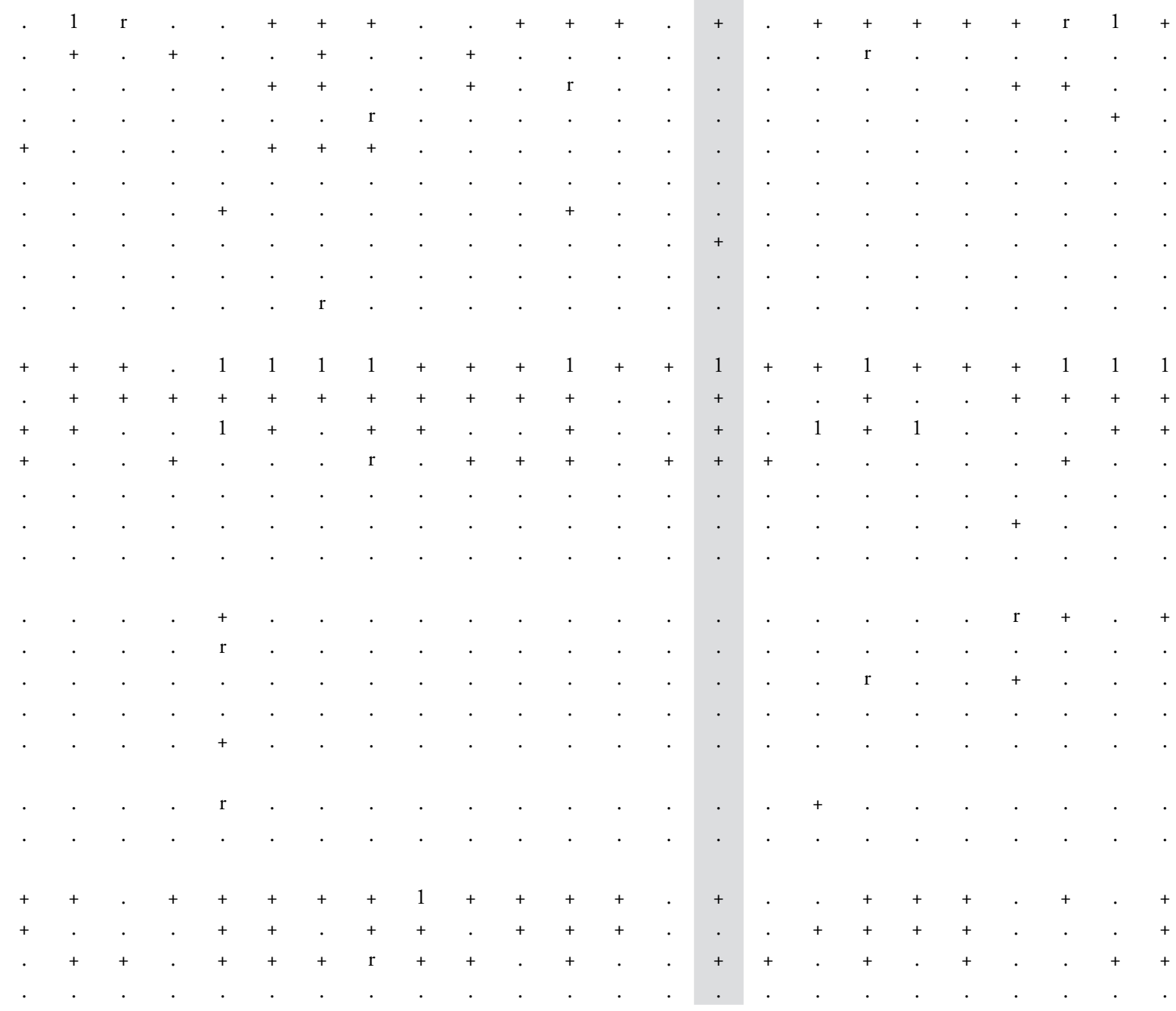

507 
Number of relevé (Zaporedna številka popisa)

$\begin{array}{llllllllllllll}1 & 2 & 3 & 4 & 5 & 6 & 7 & 8 & 9 & 10 & 11 & 12 & 13 & 14\end{array}$

$\mathrm{O}$ Other species (Druge vrste)

Hieracium sp.

E1

Viola sp.

Vicia sp.

Festuca sp.

ML Mosses and lichens (Mahovi in lišaji)

Ctenidium molluscum

Schistidium apocarpum

Tortella tortuosa

Homalothecium lutescens

Peltigera canina

Bryum capillare

Pseudoleskeella catenulata

Fissidens dubius

Polytrichum formosum

Mnium thomsonii

Isothecium alopecuroides

Dicranum scoparium

Homalothecium philippeanum

Cladonia sp.

Encalypta streptocarpa

Dermatocarpon miniatum

Brachythecium velutinum

Conocephalum conicum

Plagiochila porelloides

Lobaria pulmonaria

Cladonia pyxidata

Marchantia polymorpha

Anomodon attenuatus

Plagiomnium undulatum

Porella platyphylla

Bryum sp.

Atrichum undulatum

Peltigera sp.

Hypnum cupressiforme var. filiforme

Hypnum cupressiforme

Hypogymnia physodes

Porella arboris-vitae

Plagiomnium cuspidatum

Rhizomnium punctatum

Plagiothecium sp.

Neckera crispa

Peltigera leucophlebia

Plagiopus oederiana

Brachythecium rutabulum

Thuidium tamariscinum

Brachythecium sp. 


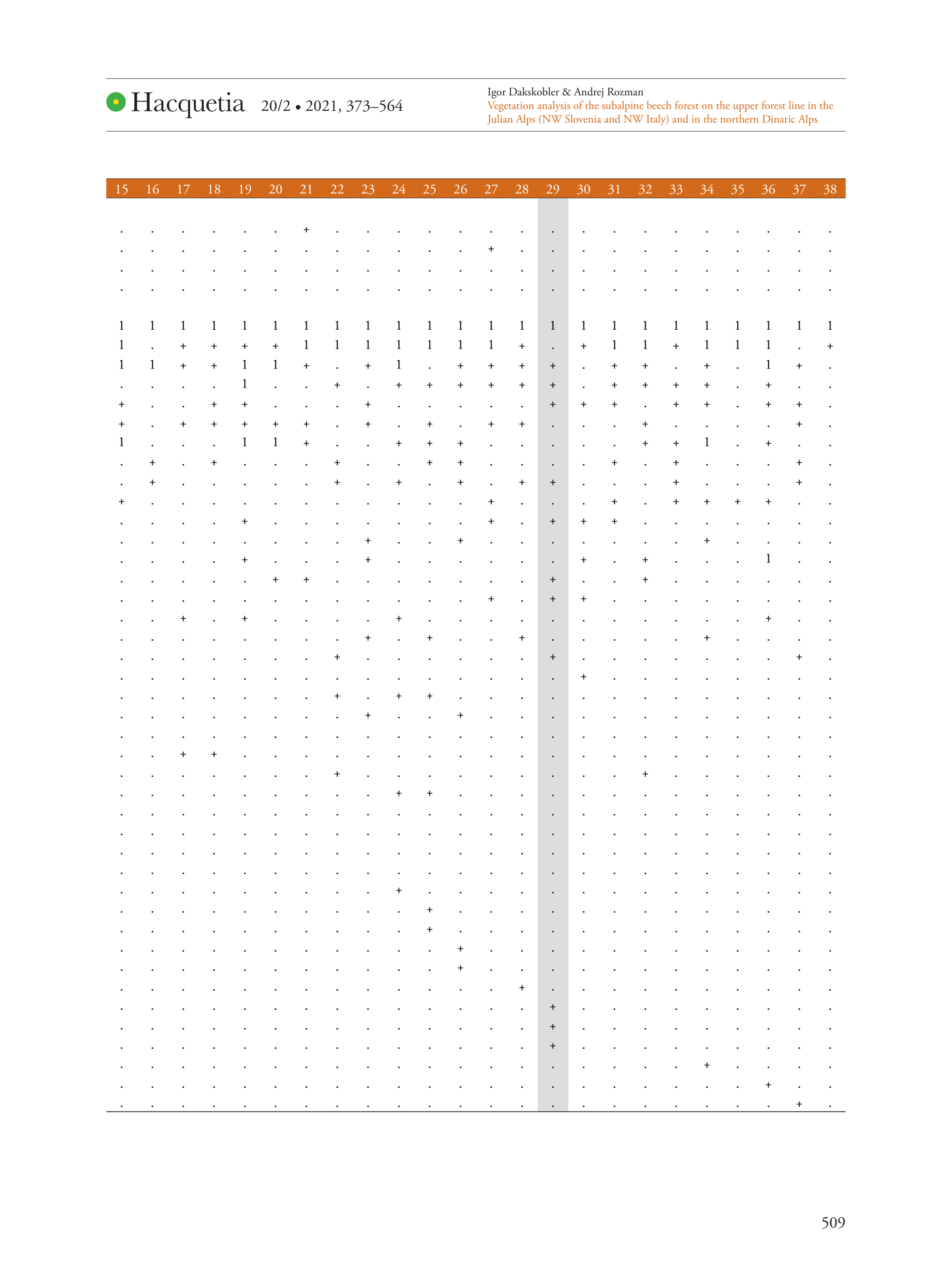


Table 5: Polysticho lonchitis-Fagetum luzuletosum niveae var. Festuca calva - cluster 28. Relevé numbers 39-50.

Tabela 5: Polysticho lonchitis-Fagetum luzuletosum niveae var. Festuca calva - skupina 28. Zaporedne številke popisov 39-50.

\begin{tabular}{|c|c|c|c|c|c|c|c|c|c|c|c|c|c|}
\hline Number of relevé (Zaporedna številka popisa) & & 39 & 40 & 41 & 42 & 43 & 44 & 45 & 46 & 47 & 48 & 49 & 50 \\
\hline $\begin{array}{l}\text { Database number of relevé } \\
\text { (Delovna številka popisa) }\end{array}$ & & 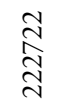 & $\begin{array}{l}\tilde{n} \\
\stackrel{\sim}{*} \\
\vec{\sim}\end{array}$ & 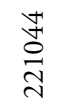 & 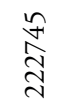 & 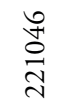 & 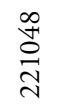 & $\begin{array}{l}\stackrel{\sim}{7} \\
\stackrel{\sim}{\sim}\end{array}$ & 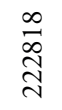 & $\begin{array}{l}\hat{\delta} \\
\text { ते } \\
\text { ते }\end{array}$ & 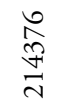 & $\underset{\sim}{\stackrel{\infty}{\sim}}$ & 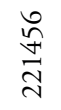 \\
\hline Author of the relevé (Avtor popisa) & & ID & ID & ID & ID & ID & ID & ID & ID & ID & ID & ID & ID \\
\hline Elevation in $\mathrm{m}$ (Nadmorska višina $\mathrm{v} \mathrm{m}$ ) & & 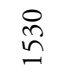 & 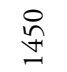 & $\stackrel{尺}{\stackrel{8}{ \pm}}$ & 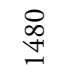 & 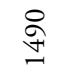 & $\underset{\Im}{\leftrightarrows}$ & O্ & $\stackrel{R}{\stackrel{R}{n}}$ & $\stackrel{\text { }}{\sim}$ & $\stackrel{2}{n}$ & $\underset{\Im}{\stackrel{\Im}{\unlhd}}$ & $\stackrel{R}{a}$ \\
\hline Aspect (Lega) & & SE & $\mathrm{NE}$ & S & SE & SE & $S$ & SW & $\mathrm{NE}$ & $\mathrm{NE}$ & $\mathrm{N}$ & $\mathrm{NE}$ & W \\
\hline Slope in degrees (Nagib v stopinjah) & & 25 & 30 & 35 & 25 & 30 & 30 & 35 & 35 & 20 & 25 & 15 & 10 \\
\hline Parent material (Matična podlaga) & & A & A & A & $\mathrm{DA}$ & A & A & A & A & A & $\mathrm{D}$ & A & Mo \\
\hline Soil (Tla) & & $\operatorname{Re}$ & $\operatorname{Re}$ & $\operatorname{Re}$ & $\operatorname{Re}$ & $\operatorname{Re}$ & $\operatorname{Re}$ & $\operatorname{Re}$ & $\operatorname{Re}$ & $\operatorname{Re}$ & $\operatorname{Re}$ & $\operatorname{Re}$ & $\operatorname{Re}$ \\
\hline Stoniness in \% (Kamnitost $\mathrm{v} \%)$ & & 30 & 30 & 30 & 30 & 60 & 35 & 40 & 20 & 40 & 20 & 50 & 60 \\
\hline \multicolumn{14}{|l|}{ Cover in \% (Zastiranje v \%): } \\
\hline Upper tree layer (Zgornja drevesna plast) & $\mathrm{E} 3 \mathrm{~b}$ & 90 & 90 & 90 & 100 & 90 & 90 & 80 & 70 & 80 & 90 & 90 & 90 \\
\hline Lower tree layer (Spodnja drevesna plasti) & E3a & . & . & . & . & . & . & . & . & 10 & 10 & 5 & 10 \\
\hline Shrub layer (Grmovna plast) & E2 & 5 & 5 & 5 & 10 & 5 & 5 & 5 & 40 & 5 & 5 & 10 & 5 \\
\hline Herb layer (Zeliščna plast) & E1 & 40 & 30 & 60 & 10 & 60 & 50 & 40 & 50 & 60 & 30 & 40 & 30 \\
\hline Moss layer (Mahovna plast) & E0 & 10 & 10 & 10 & 10 & 20 & 10 & 10 & 10 & 20 & 5 & 10 & 10 \\
\hline Maximum tree diameter (Maks. premer dreves) & $\mathrm{cm}$ & 80 & 30 & 30 & 25 & 35 & 35 & 25 & 30 & 35 & 60 & 50 & 40 \\
\hline Maximum tree height (Maksimalna višina dreves) & $\mathrm{m}$ & 15 & 15 & 10 & 8 & 12 & 12 & 12 & 8 & 18 & 18 & 17 & 18 \\
\hline Number of species (Število vrst) & & 64 & 58 & 65 & 35 & 70 & 39 & 63 & 67 & 54 & 42 & 60 & 53 \\
\hline Relevé area (Velikost popisne ploskve) & $\mathrm{m}^{2}$ & 400 & 200 & 200 & 200 & 400 & 200 & 400 & 200 & 400 & 400 & 400 & 400 \\
\hline Date of taking relevé (Datum popisa) & & 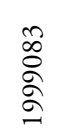 & 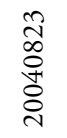 & 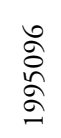 & $\begin{array}{l}\stackrel{2}{\infty} \\
\stackrel{\circ}{\circ} \\
\stackrel{2}{\Omega}\end{array}$ & 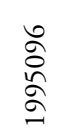 & 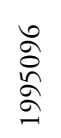 & 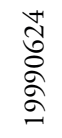 & 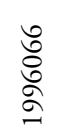 & 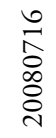 & 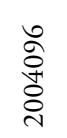 & 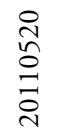 & 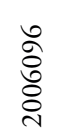 \\
\hline Day (Dan) & & 3 & 23 & 6 & 13 & 6 & 6 & 24 & 6 & 16 & 6 & 20 & 6 \\
\hline Month (Mesec) & & 8 & 8 & 9 & 8 & 9 & 9 & 6 & 6 & 7 & 9 & 5 & 9 \\
\hline Year (Leto) & & Әे & ๖̊ & 合 & $\widehat{\curvearrowright}$ & $\approx$ & $\cong$ & જે & $\stackrel{2}{\precsim}$ & $\stackrel{\infty}{\stackrel{\infty}{8}}$ & ๖̊ & $\overrightarrow{\stackrel{\sim}{~}}$ & ஓे \\
\hline Locality (Nahajališče) & & 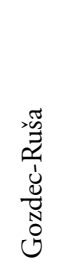 & 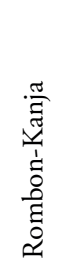 & 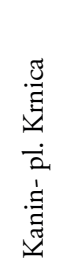 & 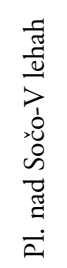 & 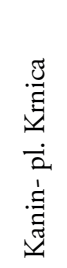 & 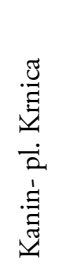 & 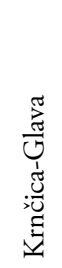 & 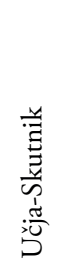 & 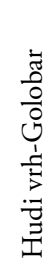 & 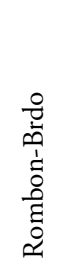 & 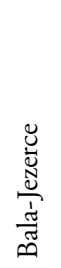 & 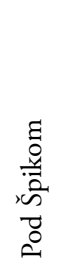 \\
\hline Mountain range (Pogorje) & & $\mathrm{JA}$ & JA & JA & JA & JA & JA & JA & JA & JA & JA & JA & JA \\
\hline Quadrant (Kvadrant) & & 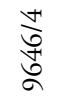 & 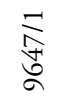 & 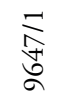 & 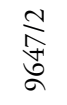 & 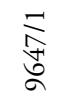 & 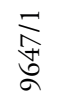 & 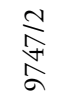 & 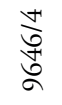 & 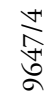 & $\underset{\text { }}{\stackrel{ }{\sigma}}$ & 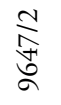 & 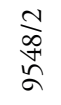 \\
\hline Coordinate (Koordinate) GK Y (D-48) & $\mathrm{m}$ & $\begin{array}{l}n \\
\hat{0} \\
\infty \\
\infty \\
\infty\end{array}$ & $\begin{array}{l}\stackrel{+}{ } \\
\text { तે } \\
\text { ळे }\end{array}$ & $\begin{array}{l}\infty \\
\approx \\
\infty \\
\sim \\
\infty \\
\infty\end{array}$ & 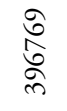 & $\begin{array}{l}\stackrel{2}{\overparen{\jmath}} \\
\infty \\
\infty\end{array}$ & 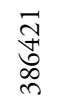 & $\underset{\hat{n}}{\hat{n}}$ & 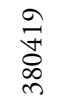 & $\begin{array}{l}\hat{n} \\
\stackrel{n}{+} \\
\stackrel{n}{n}\end{array}$ & 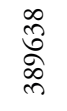 & 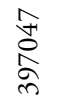 & 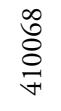 \\
\hline Coordinate (Koordinate) GK X (D-48) & $\mathrm{m}$ & 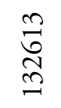 & $\underset{N}{\stackrel{n}{N}}$ & \begin{tabular}{l}
\multirow{6}{n}{} \\
$\tilde{n}$ \\
$\tilde{n}$
\end{tabular} & $\begin{array}{l}n \\
\infty \\
\tilde{n} \\
\sim\end{array}$ & 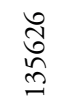 & 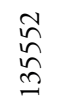 & $\underset{\overparen{N}}{\stackrel{\Xi}{\Xi}}$ & $\underset{\approx}{\approx}$ & $\begin{array}{l}\hat{n} \\
\hat{\sigma} \\
\end{array}$ & 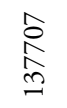 & $\begin{array}{l}\underset{\mathscr{H}}{\sigma} \\
\stackrel{\sim}{\sim}\end{array}$ & $\underset{⿱}{ \pm}$ \\
\hline
\end{tabular}

Diagnostic species of the association (Diagnostične vrste asociacije) 


\begin{tabular}{|c|c|c|c|c|c|c|c|c|c|c|c|c|c|c|c|c|}
\hline & Number of relevé (Zaporedna številka popisa) & & 39 & 40 & 41 & 42 & 43 & 44 & 45 & 46 & 47 & 48 & 49 & 50 & Pr. & Fr. \\
\hline $\mathrm{CA}$ & Festuca calva & E1 & + & + & + & . & . & + & + & + & . & . & + & . & 26 & 52 \\
\hline $\mathrm{EP}$ & Rhododendron hirsutum & E2 & . & . & . & . & . & . & $\mathrm{r}$ & + & . & + & $\mathrm{r}$ & + & 17 & 34 \\
\hline $\mathrm{BA}$ & Sorbus chamaemespilus & $\mathrm{E} 2$ & $\mathrm{r}$ & . & . & + & . & . & . & 1 & + & . & . & . & 14 & 28 \\
\hline ML & Paraleucobryum sauteri & E0 & . & + & . & . & + & . & . & . & . & . & . & . & 14 & 28 \\
\hline $\mathrm{CA}$ & Laserpitium peucedanoides & E1 & + & . & + & . & + & . & . & . & . & . & . & . & 13 & 26 \\
\hline BA & Salix appendiculata & E2 & $\mathrm{r}$ & . & . & . & . & . & . & + & . & . & + & . & 11 & 22 \\
\hline EP & Pinus mugo & E2 & $\mathrm{r}$ & . & . & . & . & . & . & . & . & . & . & . & 10 & 20 \\
\hline $\mathrm{CF}$ & Carex ferruginea & E1 & . & . & . & . & . & . & . & . & . & . & . & . & 10 & 20 \\
\hline VP & Homogyne alpina & E1 & . & . & . & . & . & . & + & + & . & . & + & . & 8 & 16 \\
\hline PS & Paederota lutea & E1 & $\mathrm{r}$ & + & . & . & . & . & . & . & + & + & + & + & 8 & 16 \\
\hline VP & Lonicera caerulea & E2 & . & . & . & . & . & . & . & + & . & . & . & . & 4 & 8 \\
\hline ES & Aster bellidiastrum & E1 & . & . & . & . & . & . & . & . & . & + & . & + & 4 & 8 \\
\hline EP & Rhodothamnus chamaecistus & E1 & . & . & . & . & . & . & . & . & . & . & . & . & 1 & 2 \\
\hline
\end{tabular}

Differential species of the subassociation (Razlikovalnice subasociacije)

\section{FS Luzula nivea \\ QF Poa nemoralis \\ TA Adoxa moschatellina \\ AF Aremonio-Fagion \\ Anemone trifolia \\ Cardamine enneaphyllos \\ Cyclamen purpurascens \\ Cardamine trifolia \\ Aremonia agrimonoides \\ Helleborus niger \\ Rhamnus fallax \\ Knautia drymeia \\ Lamium orvala}

$\begin{array}{llllllllllllll}\mathrm{E} 1 & 1 & 1 & 2 & 1 & 1 & 2 & 1 & 1 & + & 43 & 86\end{array}$

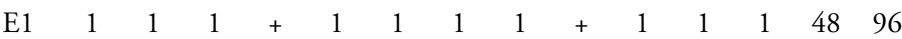

EC Erythronio-Carpinion

Helleborus odorus

Primula vulgaris

Galanthus nivalis

Ornithogalum pyrenaicum

E1

E1

E1

E1

E2

E1

E1

E1

E1

E1

E1

TA Tilio-Acerion

Geranium robertianum

Acer pseudoplatanus

Acer pseudoplatanus

Acer pseudoplatanus

Polystichum aculeatum

Tilia platyphyllos

Aruncus dioicus

Euonymus latifolia

Chrysosplenium alternifolium

FS Fagetalia sylvaticae

Fagus sylvatica

Fagus sylvatica

Fagus sylvatica

Dryopteris filix-mas

Galeobdolon flavidum

Lilium martagon

Mercurialis perennis

Daphne mezereum

\begin{tabular}{|c|c|c|c|c|c|c|c|c|c|c|c|c|c|}
\hline E1 & & 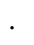 & $\cdot$ & $\cdot$ & $\cdot$ & . & ${ }^{\circ}$ & . & . & . & . & . & 11 \\
\hline E3 & . & . & . & . & . & . & . & & . & . & . & $\mathrm{r}$ & 2 \\
\hline E2 & . & . & . & . & . & . & . & & . & . & . & . & 2 \\
\hline E1 &. & . & . & . & . & . & . & • & . & $\mathrm{r}$ & . & 1 & 6 \\
\hline E1 & $\mathrm{r}$ & . & . & . & . & . & . & • & . & . & . & • & 6 \\
\hline E2 & $\mathrm{r}$ & . & . & . & . & . & . & • & . & . & . & & 2 \\
\hline E1 & . & . & . & . & . & . & . & $\cdot$ & . & . & . & & 2 \\
\hline E2 & . & . & . & . & . & . & . & & . & . & . & & 1 \\
\hline E1 & . & . & . & . & . & . & . & & . & & . & & 1 \\
\hline E3 & 5 & 5 & 5 & 5 & 5 & 5 & 5 & 4 & 5 & & 5 & 5 & 50 \\
\hline E2 & + & + & . & 1 & + & + & + & . & + & 1 & 1 & + & 42 \\
\hline E1 & . & . & . & . & . & . & . & . & + & 1 & 1 & 1 & 14 \\
\hline E1 &. & + & + & $\mathrm{r}$ & + & . & + & + & + & + & + & . & 36 \\
\hline E1 & + & 1 & + & + & + & + & . & . & . & . & . & + & 32 \\
\hline E1 & . & . & + & . & + & + & + & + & + & + & + & . & 32 \\
\hline E1 & . & 1 & 1 & + & 2 & 1 & 1 & . & . & . & + & . & 29 \\
\hline E2 & & + & + & & + & & & & & + & + & + & 24 \\
\hline
\end{tabular}


Melica nutans

Lathyrus vernus

Epilobium montanum

Lonicera alpigena

Paris quadrifolia

Actaea spicata

Galium laevigatum

Mycelis muralis

Euphorbia amygdaloides

Scrophularia nodosa

Prenanthes purpurea

Myosotis sylvatica agg.

Lathyrus vernus subsp. flaccidus

Symphytum tuberosum

Corydalis cava

Neottia nidus-avis

Polygonatum multiflorum

Ranunculus lanuginosus

Festuca altissima

Epipactis helleborine

Laburnum alpinum

Laburnum alpinum

Laburnum alpinum

Pulmonaria officinalis

Cardamine pentaphyllos

Petasites albus

Campanula trachelium

Cardamine impatiens

Galium odoratum

Cardamine bulbifera

Viola reichenbachiana

QP Quercetalia pubescenti-petraeae

Sorbus aria (Aria edulis)

E1

E1

E1

E2

E1

E1

E1

E1

E1

E1

E1

E1

E1

E1

E1

E1

E1

E1

E1

E1

E3

E2

E1

E1

E1

E1

E1

E1

E1

E1

E1

E3

E2

E1

Primula veris subsp. columnae

Convallaria majalis

Melittis melissophyllum

Hypericum montanum

QF

\section{Querco-Fagetea}

Carex digitata

Anemone nemorosa

Hepatica nobilis

Festuca heterophylla

Aegopodium podagraria

Viola riviniana

Stellaria holostea

Moehringia trinervia

Ranunculus auricomus agg. (R. braun-blanquetii)

Dactylis glomerata subsp. lobata (D. polygama)

Listera ovata

Scilla bifolia $+++.+++\ldots+2448$

+ . $. \mathrm{r} \cdot++.+2142$

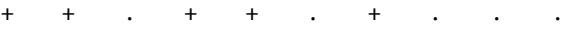

$\cdot \cdot+\cdot \cdot+{ }^{2} \cdot+1938$

$+\cdot \cdot \cdot \cdot \cdot \cdot \cdot \cdot \cdot 1530$

1326

$12 \quad 24$

$11 \quad 22$

$11 \quad 22$

$8 \quad 16$

$\begin{array}{ll}6 & 12\end{array}$

$5 \quad 10$

$\begin{array}{ll}5 & 10\end{array}$

48

48

36

36

36

36

12

12

24

24

24

24

$\begin{array}{ll}1 & 2\end{array}$

$\begin{array}{ll}1 & 2\end{array}$

12

12

2

12

$16 \quad 32$

$\begin{array}{ll}7 & 14\end{array}$

$\begin{array}{ll}5 & 10\end{array}$

$\begin{array}{lllllllllllllllll}\mathrm{E} 1 & . & . & . & . & \mathrm{r} & + & . & . & . & . & . & . & 5 & 10 \\ \mathrm{E} 1 & . & . & . & . & . & . & . & . & . & . & . & . & 1 & 2\end{array}$

$\mathrm{E} 1 .+.+.+.+.+1530$

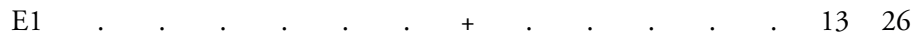

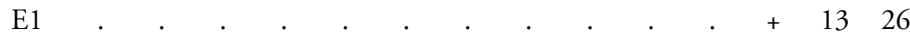

E1 . . . . . . . . . . . . . .

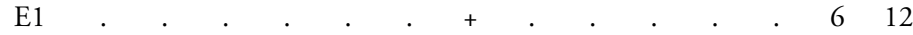

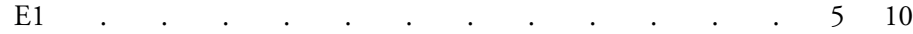

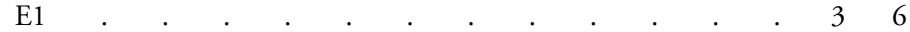

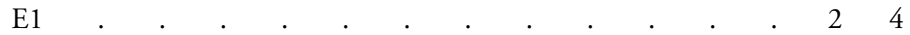

$\mathrm{E} 1$. $. \quad . \quad . \quad . \quad . \quad . \quad . \quad . \quad . \quad . \quad 12$

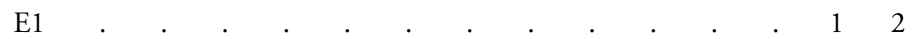

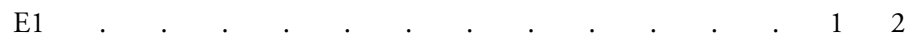

$\mathrm{E} 1 .+.+.+.+12$ 
VP Vaccinio-Piceetea

Aposeris foetida

Vaccinium myrtillus

Calamagrostis arundinacea

Maianthemum bifolium

Hieracium murorum

Oxalis acetosella

Rosa pendulina

Picea abies

Picea abies

Picea abies

Solidago virgaurea

Gymnocarpium dryopteris

Veronica urticifolia

Dryopteris dilatata

Saxifraga cuneifolia

Corallorhiza trifida

Calamagrostis villosa

Huperzia selago

Luzula luzuloides

Vaccinium vitis-idaea

Gentiana asclepiadea

Dryopteris expansa

Phegopteris connectilis

Larix decidua

Luzula pilosa

Abies alba

Abies alba

Abies alba

Lonicera nigra

Lycopodium annotinum

Homogyne sylvestris

Ajuga pyramidalis

E1

E1

E1

E1

E1

E1

E2

E3

E2

E1

E1

E1

E1

E1

E1

E1

E1

E1

E1

E1

E1

E1

E1

E3

E1

E3

E2

E1

E2

E1

E1

E1

E1

Calamagrostis varia

Rubus saxatilis

Erica carnea

Carex ornithopoda

Peucedanum rablense

Carex alba

Polygala chamaebuxus

Genista radiata

Chamaecytisus hirsutus

Epipactis atrorubens

SSC Sambuco-Salicion capreae, Rhamno-Prunetea

Sorbus aucuparia

Sorbus aucuparia

Sorbus aucuparia

Salix caprea

Berberis vulgaris

Juniperus communis

Sambucus racemosa

E2

E1

E1

E3

E2

E1

E3

E2

E2

E2 $\cdot+\cdot+1+1+++2+3774$

$+.+2+1+1 \quad 2 \quad 1 \quad 1 \quad 3162$

. . . 1 . ..+ .2958

$++\ldots+++4+2958$

$+\ldots+\ldots+2856$

$+1.5 .+1.11+2244$

$+.+\ldots+1+.+2244$

$\mathrm{r} \cdot .+.+.+. \quad 2142$

2142

36

$+\quad 1428$

1326

1224

$\begin{array}{ll}11 & 22\end{array}$

$\begin{array}{lll}11 & 22\end{array}$

$10 \quad 20$

$\begin{array}{ll}7 & 14\end{array}$

612

(5) 10

48

48

36

36

36

36

24

24

12

24

24

24

$26 \quad 52$

2652

1836

E1. . . . . . $\mathrm{r} . \quad . \quad 612$

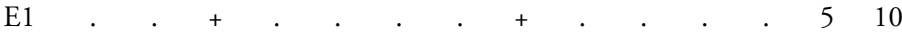

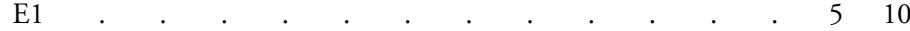

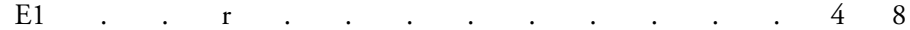


$\begin{array}{lllllllllllllllll}\text { Number of relevé (Zaporedna številka popisa) } & 39 & 40 & 41 & 42 & 43 & 44 & 45 & 46 & 47 & 48 & 49 & 50 & \text { Pr. } & \text { Fr. }\end{array}$

BA Betulo-Alnetea

Sorbus austriaca

Juniperus sibirica

Sorbus austriaca

Salix glabra

Alnus viridis

MA Mulgedio-Aconitetea

Polygonatum verticillatum

Veratrum album

Athyrium filix-femina

Saxifraga rotundifolia

Viola biflora

Senecio cacaliaster

Aconitum angustifolium

Geranium sylvaticum

Hypericum maculatum

Ranunculus platanifolius

Phyteuma ovatum

Chaerophyllum hirsutum

Aconitum degenii subsp. paniculatum

Chaerophyllum aureum

Thalictrum aquilegiifolium

Rumex arifolius

Senecio ovatus

Poa hybrida

Peucedanum ostruthium

Allium victorialis

Adenostyles alliariae

Tanacetum corymbosum subsp. clusii

Epilobium alpestre

Chaerophyllum villarsii

Pleurospermum austriacum

E2

E2

E1

E2

E2

E1

E1

E1

E1

E1

E1

E1

E1

E1

E1

E1

E1

E1

E1

E1

E1

E1

E1

E1

E1

E1

E1

E1

E1

E1

E1

E

E1

Pulsatilla alpina subsp. austroalpina

CF

Caricion ferrugineae

Cerastium subtriflorum

Knautia longifolia

ES Elyno-Seslerietea

Betonica alopecuros

Sesleria caerulea

Campanula witasekiana

Pimpinella alpina

Carex sempervirens

Senecio abrotanifolius

Astrantia bavarica

Ranunculus carinthiacus

Galium anisophyllum

Carduus crassifolius

Phyteuma orbiculare

Alchemilla vulgaris agg.

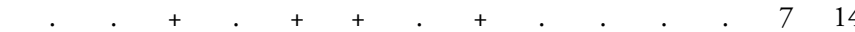

. . . . . . . . . . . 24

$\cdot \cdot \cdot \cdot \cdot \cdot \cdot \cdot \cdot \cdot \cdot \cdot \cdot$

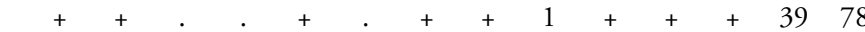

$++. \mathrm{r}+. \cdot 1+.+3672$

$++.+2+.+1632$

$\cdot+\cdot+\cdot \cdot \cdot \cdot \cdot 1530$

$\begin{array}{ll}11 & 22\end{array}$

$\begin{array}{ll}10 & 20\end{array}$

$10 \quad 20$

$8 \quad 16$

$\begin{array}{ll}6 & 12\end{array}$

$5 \quad 10$

48

48

36

36

36

36

24

24

24

$\begin{array}{ll}1 & 2\end{array}$

12

12

48

$\begin{array}{ll}1 & 2\end{array}$

12

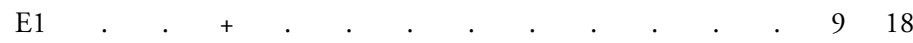

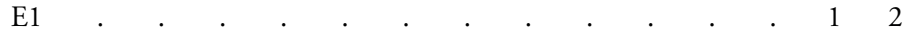

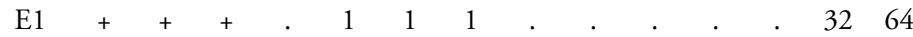

$\mathrm{E} 1$. $.+\ldots++\ldots++2958$

$\mathrm{E} 1 .++.+\ldots+.+.+1020$

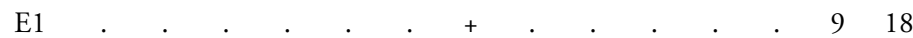

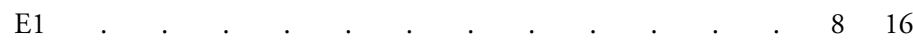

$\mathrm{E} 1.5 .6 .5612$

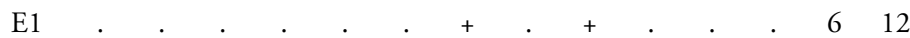

$\mathrm{E} 1.5 . \quad . \quad . \quad . \quad . \quad . \quad . \quad . \quad . \quad 510$

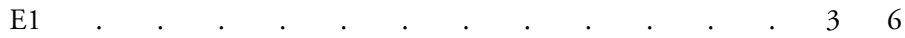

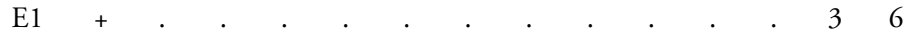

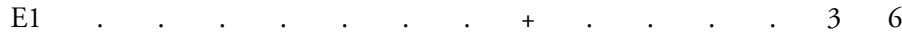

$\mathrm{E} 1$. $. \quad . \quad . \quad . \quad . \quad . \quad . \quad . \quad . \quad . \quad . \quad 24$ 
Alchemilla alpigena

E1

Potentilla crantzii

Thymus praecox subsp. polytrichus

Scabiosa lucida subsp. stricta

Leontopodium alpinum

Lotus alpinus

Achillea clavenae

Leucanthemum heterophyllum

Erigeron glabratus

Ranunculus montanus

NS Nardion strictae, Juncetea trifidi

Campanula scheuchzeri

Phyteuma zahlbruckneri

Potentilla erecta

Coeloglossum viride

FB Festuco-Brometea

Cirsium erisithales

Buphthalmum salicifolium

Carlina acaulis

Koeleria pyramidata

Arabis hirsuta

Carex humilis

Hippocrepis comosa

Euphorbia cyparissias

ZG Trifolio-Geranietea

Verbascum lanatum

Clinopodium vulgare

Achillea distans

Laserpitium latifolium

Valeriana wallrothii

Laserpitium siler

Iris graminea

Vincetoxicum hirundinaria

Vicia sylvatica

Lilium carniolicum

Hypericum perforatum

Libanotis sibirica subsp. montana

Origanum vulgare

PaT Poo alpinae-Trisetetalia

Poa alpina

Crocus albiflorus

Trollius europaeus

Pimpinella major subsp. rubra

Cardaminopsis ovirensis

MA Molinio-Arrhenatheretea

Lathyrus pratensis

Deschampsia cespitosa

Dactylis glomerata

Galium album

Crepis paludosa

Achillea millefolium

Festuca rubra agg.

$\begin{array}{ll} & 39 \\ & . \\ 1 & \text { r } \\ 1 & + \\ 1 & . \\ 1 & . \\ 1 & . \\ 1 & . \\ 1 & . \\ 1 & .\end{array}$

. . . . . . . . . . . 244

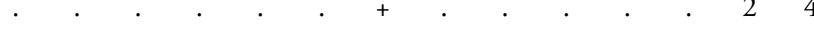

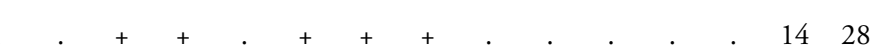

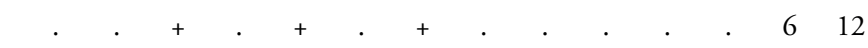

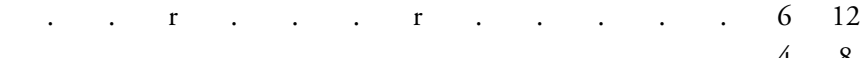

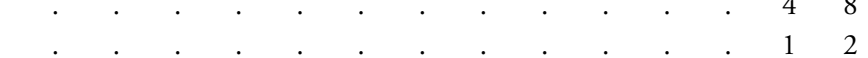

. $. \quad . \quad . \quad . \quad . \quad . \quad . \quad . \quad . \quad 112$

E1 . $1.0 \quad \mathrm{r}$.

. $+.+\ldots+. \quad$. . . . 1224

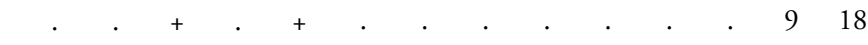

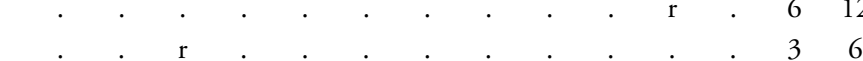

. . . . . . . . . . 36

24

24

12

12

$\begin{array}{ll}1 & 2\end{array}$

12 
Achillea roseoalba

Vicia sepium

Veronica chamaedrys

Angelica sylvestris

Leontodon hispidus

EA Epilobietea angustifolii

Fragaria vesca

Urtica dioica

Rubus idaeus

Galeopsis speciosa

Lamium maculatum

Tussilago farfara

Hypericum hirsutum

AC Arabidetalia caeruleae

Soldanella alpina

TR Thlaspietea rotundifolii

Adenostyles glabra

Gymnocarpium robertianum

Arabis alpina

Heracleum pollinianum

Dryopteris villarii

Astrantia carniolica

Geranium macrorrhizum

Molopospermum peloponnesiacum subsp. bauhinii

Ligusticum seguieri

Rhodiola rosea

Cy Cystopteridion fragilis

Cystopteris fragilis

Asplenium viride

Valeriana tripteris

Moehringia muscosa

Cystopteris regia

Sedum hispanicum

Heliosperma pusillum

PS Physoplexido comosae-Saxifragion petraeae

Saxifraga petraea

Campanula carnica

Saxifraga crustata

Paederota bonarota

Saxifraga hostii

PC Potentilletalia caulescentis

Primula auricula

Campanula cochleariifolia

AT Asplenietea trichomanis

Asplenium trichomanes

Asplenium ruta-muraria

Polypodium vulgare

Sedum album

O Other species (Druge vrste)

Hieracium sp.

Viola sp.
E1

E1

E1

E1

E1

E1

E1

E2

E1

E1

E1

E1

E1

E1

E1

E1

E1

E1

E1

E1

E1

E1

E1

E1

E1

E1

E1

E1

E1

E1

E1

E1

E1

E1

E1

E1

E1

E1

E1

E1

E1

E1

E1
. . . . . . . . . . . . . . 12

. . . . . . . . . . . . . . 112

. . . . . . . . . . . . 12

..$++++++22 .+24$

$12 \quad 24$

$10 \quad 20$

24

12

$\begin{array}{ll}1 & 2\end{array}$

12

48

3264

$10 \quad 20$

$\begin{array}{ll}9 & 18\end{array}$

$\begin{array}{ll}7 & 14\end{array}$

48

36

48

36

$\begin{array}{ll}1 & 2\end{array}$

12

$41 \quad 82$

3978

2754

$20 \quad 40$

36

12

12

$6 \quad 12$

36

24

12

12

36

1 . . . . . . . . . . . . . 412

$\mathrm{E} 1++++++++.+3162$

$++\mathrm{r}++++.+2346$

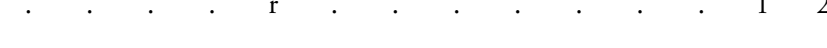

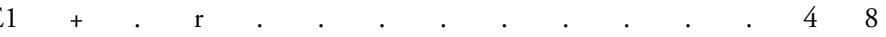

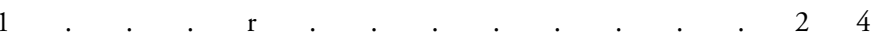




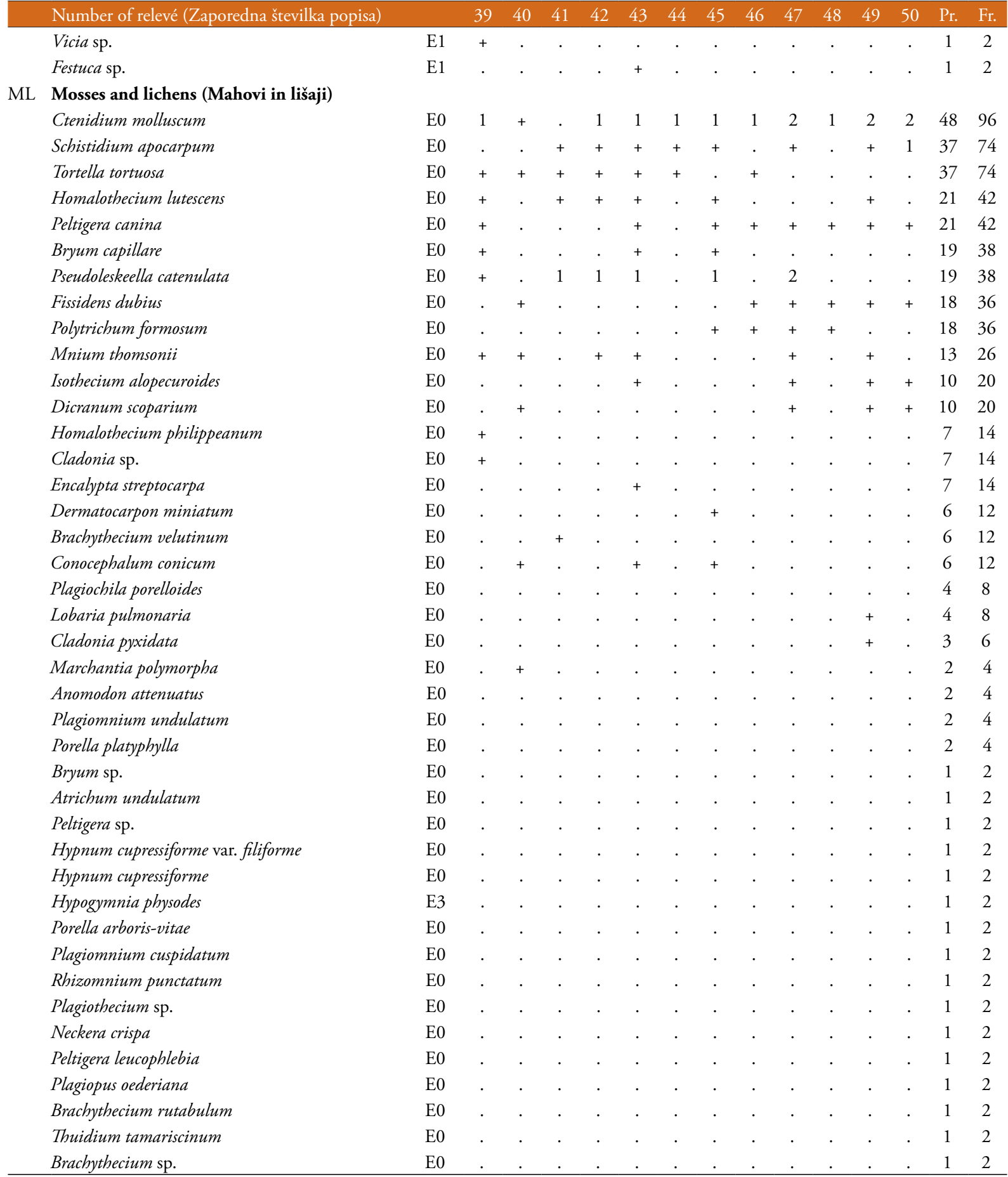

\section{Legend - Legenda}

ID Igor Dakskobler

A Limestone - apnenec

D Dolomite - dolomit

L Marlstone - laporovec

Mo Moraine (Till) - morena (Til)

Re Rendzina - rendzina
JA Julian Alps - Julijske Alpe

Pr. Presence (number of relevés in which the species is presented) - število popisov, v katerih se pojavlja vrsta

Fr. Frequency in $\%$ - frekvenca $v \%$

Relevé No. 29, holotypus 
Table 6: Polysticho lonchitis-Fagetum adoxetosum moschatelliane - cluster 24 and var. Helleborus niger - cluster 25. Table 6: Polysticho lonchitis-Fagetum adoxetosum moschatelliane - skupina 24 in var. Helleborus niger - skupina 25.

\begin{tabular}{|c|c|c|c|c|c|c|c|c|c|c|c|c|c|c|c|c|}
\hline Number of relevé (Zaporedna štev. popisa) & & 1 & 2 & 3 & 4 & 5 & 6 & 7 & 8 & 9 & 10 & 11 & 12 & 13 & 14 & 15 \\
\hline $\begin{array}{l}\text { Database number of relevé } \\
\text { (Delovna številka popisa) }\end{array}$ & & $\begin{array}{l}\stackrel{\infty}{\widetilde{\nearrow}} \\
\underset{\sim}{\sim}\end{array}$ & $\begin{array}{l}\widetilde{\approx} \\
\underset{\sim}{\sim}\end{array}$ & $\begin{array}{l}\stackrel{m}{\cong} \\
\stackrel{\sim}{\sim}\end{array}$ & $\begin{array}{l}\text { ते } \\
\stackrel{\pi}{\pi} \\
\text { స్ }\end{array}$ & $\begin{array}{l}\tilde{\tilde{n}} \\
\underset{\sim}{\vec{N}}\end{array}$ & 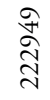 & 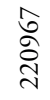 & $\begin{array}{l}\infty \\
\underset{\sim}{\pi} \\
\underset{\sim}{\pi}\end{array}$ & 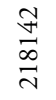 & $\frac{\stackrel{\cap}{n}}{\stackrel{\sim}{\sim}}$ & 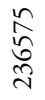 & $\begin{array}{l}\text { ț } \\
\stackrel{n}{\sim}\end{array}$ & 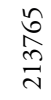 & 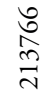 & \\
\hline Author of the relevé (Avtor popisa) & & ID & ID & ID & ID & ID & ID & ID & ID & ID & ID & ID & ID & ID & ID & \\
\hline Elevation in $\mathrm{m}$ (Nadmorska višina $\mathrm{v} \mathrm{m}$ ) & & $\stackrel{\stackrel{\overbrace{}}{\oplus}}{\leftrightarrows}$ & $\underset{Ð}{\stackrel{尺}{\unlhd}}$ & 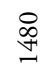 & $\underset{\overbrace{}}{\stackrel{Ð}{f}}$ & $\begin{array}{l}\text { \& } \\
\text { † }\end{array}$ & $\begin{array}{l}\stackrel{8}{\curvearrowleft} \\
\underset{f}{ \pm}\end{array}$ & $\begin{array}{l}\stackrel{\infty}{\infty} \\
\underset{+}{+}\end{array}$ & 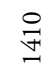 & $\underset{\Omega}{\stackrel{2}{\sigma}}$ & 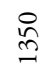 & 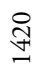 & : & $\begin{array}{l}\stackrel{8}{0} \\
\stackrel{2}{n}\end{array}$ & $\stackrel{尺}{\stackrel{2}{n}}$ & \\
\hline Aspect (Lega) & & 空 & 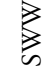 & 点 & 昰 & 学 & 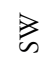 & $\stackrel{\sqrt[n]{n}}{\infty}$ & 8 & 乩 & 空 & 岂 & 乩 & 乩 & $n$ & \\
\hline Slope in degrees (Nagib v stopinjah) & & 35 & 35 & 35 & 35 & 35 & 30 & 35 & 30 & 35 & 30 & 35 & 30 & 25 & 25 & 35 \\
\hline Parent material (Matična podlaga) & & 孚 & $\varangle$ & $\varangle$ & $\ll$ & $\ddot{U}$ & $\varangle$ & $\varangle$ & $\varangle$ & $\varangle$ & $\varangle$ & $\overleftrightarrow{a}$ & $\overleftrightarrow{\Xi}$ & 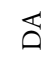 & $\overleftrightarrow{a}$ & $\ll$ \\
\hline Soil (Tla) & & $\mathrm{Rj}$ & $\operatorname{Re}$ & $\operatorname{Re}$ & $\operatorname{Re}$ & $\operatorname{Re}$ & $\operatorname{Re}$ & $\operatorname{Re}$ & $\operatorname{Re}$ & $\operatorname{Re}$ & $\operatorname{Re}$ & $\operatorname{Re}$ & $\operatorname{Re}$ & $\operatorname{Re}$ & $\operatorname{Re}$ & $\operatorname{Re}$ \\
\hline $\begin{array}{l}\text { Stoniness in \% (Kamnitost v \%) } \\
\text { Cover in \% (Zastiranje v \%): }\end{array}$ & & 60 & 20 & 30 & 30 & 50 & 70 & 30 & 30 & 40 & 40 & 20 & 10 & 10 & 20 & 0 \\
\hline Upper tree layer (Zgornja drevesna plast) & E3b & 80 & 80 & 90 & 80 & 70 & 80 & 80 & 90 & 80 & 90 & 80 & 90 & 90 & 90 & 70 \\
\hline Lower tree layer (Spodnja drevesna plasti) & E3a & 5 & . & . & & 20 & . & 10 & . & 10 & 5 & 10 & . & . & . & \\
\hline Shrub layer (Grmovna plast) & E2 & 5 & 10 & 5 & 5 & 10 & 10 & 5 & 5 & 5 & 5 & 2 & 20 & 10 & 10 & 20 \\
\hline Herb layer (Zeliščna plast) & E1 & 60 & 50 & 50 & 40 & 60 & 50 & 60 & 30 & 60 & 50 & 40 & 60 & 60 & 60 & 0 \\
\hline Moss layer (Mahovna plast) & E0 & 10 & 10 & 10 & 10 & 10 & 20 & 10 & 10 & 5 & 10 & 5 & 5 & 10 & 5 & 10 \\
\hline Max. tree diameter (Maks. premer dreves) & $\mathrm{cm}$ & 45 & 40 & 60 & 30 & 75 & 25 & 90 & 30 & 70 & 35 & 45 & 40 & 25 & 30 & 35 \\
\hline Maximum tree height (Maks. višina dreves) & $\mathrm{m}$ & 12 & 14 & 14 & 10 & 15 & 10 & 18 & 14 & 16 & 11 & 18 & 15 & 12 & 10 & 7 \\
\hline Number of species (Število vrst) & & 70 & 71 & 82 & 60 & 73 & 52 & 61 & 67 & 53 & 49 & 36 & 68 & 48 & 57 & 60 \\
\hline Relevé area (Velikost popisne ploskve) & $\mathrm{m}^{2}$ & ஓ̊ & ஓ & ஓి & 아 & ஓ & ஓ & ஓి & $\stackrel{+}{\circ}$ & \& & 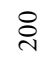 & \& & 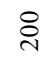 & 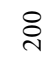 & 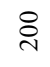 & $\stackrel{\text { ì }}{2}$ \\
\hline Date of taking relevé (Datum popisa) & & 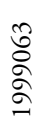 & 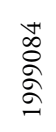 & 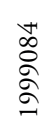 & 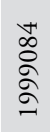 & 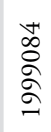 & 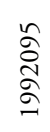 & $\begin{array}{l}\mathfrak{n} \\
\text { } \\
8 \\
8 \\
\stackrel{ᄋ}{1}\end{array}$ & 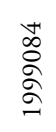 & 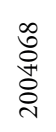 & 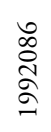 & 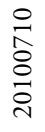 & 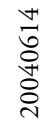 & 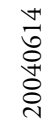 & 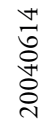 & $\begin{array}{l}\hat{\sigma} \\
\text { } \\
\text { }\end{array}$ \\
\hline Day (Dan) & & 3 & 4 & 4 & 4 & 4 & 5 & 5 & 4 & 8 & 6 & 10 & 14 & 14 & 14 & 3 \\
\hline Month (Mesec) & & 6 & 8 & 8 & 8 & 8 & 9 & 7 & 8 & 6 & 8 & 7 & 6 & 6 & 6 & 9 \\
\hline Year (Leto) & & $\stackrel{\text { }}{=}$ & $\stackrel{\curvearrowright}{\curvearrowright}$ & ळે & ळे & $\widehat{\sigma}$ & $\approx$ & ஓ্ণ & Әे & ষ্ঠ & $\widetilde{\Omega}$ & $\stackrel{\circ}{\stackrel{\overbrace{}}{\circ}}$ & 苍 & ষ্ণ & 苍 & ऽ \\
\hline Locality (Nahajališče) & & 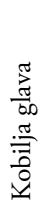 & 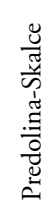 & 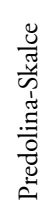 & 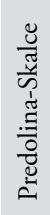 & 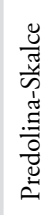 & $\begin{array}{l}\text { 营 } \\
\sum^{\pi / 2}\end{array}$ & 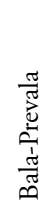 & 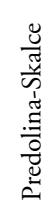 & 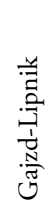 & 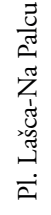 & 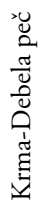 & 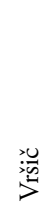 & 勇 & 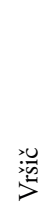 & 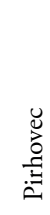 \\
\hline Mountain range (Pogorje) & & JA & JA & JA & JA & JA & JA & JA & JA & JA & JA & JA & JA & JA & JA & JA \\
\hline Quadrant (Kvadrant) & & $\begin{array}{l}\stackrel{N}{\infty} \\
\stackrel{+}{\infty} \\
\stackrel{\infty}{\circ}\end{array}$ & $\frac{\stackrel{N}{N}}{\stackrel{N}{a}}$ & 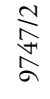 & 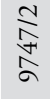 & 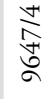 & 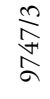 & 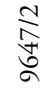 & $\underset{\stackrel{N}{N}}{\stackrel{N}{\sigma}}$ & 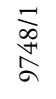 & $\begin{array}{l}\underset{\infty}{\infty} \\
\stackrel{+}{+} \\
\hat{\sigma}\end{array}$ & 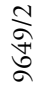 & 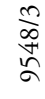 & $\begin{array}{l}\stackrel{n}{\infty} \\
\stackrel{+}{*} \\
\stackrel{+}{\alpha}\end{array}$ & 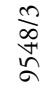 & $\Sigma$ \\
\hline Coordinate (Koordinate) GK Y (D-48) & $\mathrm{m}$ & 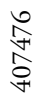 & 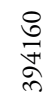 & 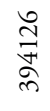 & 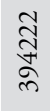 & $\begin{array}{l}n \\
\infty \\
\tilde{n} \\
\tilde{n}\end{array}$ & \begin{tabular}{l} 
ర్ర \\
\multirow{ర}{*}{} \\
$\infty$ \\
న
\end{tabular} & 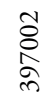 & $\begin{array}{l}m \\
\infty \\
\stackrel{\varpi}{\varpi}\end{array}$ & $\begin{array}{l}\infty \\
\stackrel{\infty}{\infty} \\
\stackrel{8}{8} \\
\stackrel{+}{1}\end{array}$ & 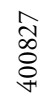 & $\begin{array}{l}\infty \\
\stackrel{\infty}{\circ} \\
\infty \\
\forall\end{array}$ & 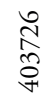 & 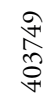 & $\begin{array}{c}\infty \\
\hat{\widehat{\gamma}} \\
\stackrel{+}{+}\end{array}$ & $\infty$ \\
\hline Coordinate (Koordinate) GK X (D-48) & $\mathrm{m}$ & $\underset{\sigma}{\sigma}$ & 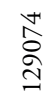 & $\frac{\widetilde{a}}{\stackrel{\Xi}{\beth}}$ & 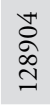 & 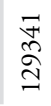 & 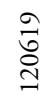 & 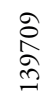 & 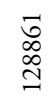 & 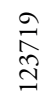 & 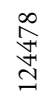 & 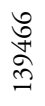 & $\begin{array}{l}\underset{f}{f} \\
\underset{f}{f}\end{array}$ & $\begin{array}{l}\stackrel{D}{\infty} \\
\stackrel{\infty}{\infty} \\
\stackrel{+}{+}\end{array}$ & $\begin{array}{l}\infty \\
\stackrel{\infty}{\infty} \\
\stackrel{\infty}{\Psi} \\
-1\end{array}$ & $\stackrel{\infty}{\widetilde{\alpha}}$ \\
\hline
\end{tabular}

Diagnostic species of the association (Diagnostične vrste asociacije)

Pr. Fr.1-10 Fr.11-15

VP Polystichum lonchitis

CA Festuca calva

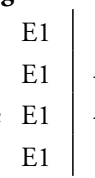

MuA Aconitum lycocto
VP Luzula sylvatica 


\begin{tabular}{|c|c|c|c|c|c|c|c|c|c|c|c|c|c|c|c|c|c|c|c|c|}
\hline \multicolumn{3}{|c|}{ Number of relevé (Zaporedna štev. popisa) } & \multirow{2}{*}{\multicolumn{2}{|c|}{$\begin{array}{ll}1 & 2 \\
. & .\end{array}$}} & \multirow{2}{*}{3} & \multirow{2}{*}{4} & \multirow{2}{*}{5} & \multirow{2}{*}{6} & \multirow{2}{*}{$\begin{array}{l}7 \\
+\end{array}$} & \multirow{2}{*}{8} & \multirow[t]{2}{*}{9} & \multirow[t]{2}{*}{10} & \multirow{2}{*}{11} & \multirow{2}{*}{$\begin{array}{l}12 \\
.\end{array}$} & \multirow{2}{*}{13} & 14 & 15 & Pr. & Fr.1-10 & Fr.11-15 \\
\hline $\mathrm{VP}$ & Clematis alpina & E2 & & & & & & & & & & & & & & & + & 3 & 10 & 40 \\
\hline ML & Paraleucobryum sauteri & E0 & + & . & . & . & . & . & + & . & . & . & . & . & . & . & . & 2 & 20 & . \\
\hline EP & Pinus mugo & E2 & $\cdot$ & . & . & . & . & . & . & . & . & . & . & + & . & + & . & 2 & . & 40 \\
\hline BA & Sorbus chamaemespilus & E2 & . & . & . & . & . & . & . & . & . & . & . & + & . & + & . & 2 & . & 40 \\
\hline ES & Aster bellidiastrum & E1 & . & . & . & . & . & . & . & . & . & . & + & + & . & . & . & 2 & . & 40 \\
\hline BA & Salix appendiculata & $\mathrm{E} 2$ & . & . & . & . & . & + & . & . & . & . & . & . & . & . & . & 1 & 10 & . \\
\hline $\mathrm{CF}$ & Carex ferruginea & E1 & . & . & . & . & . & . & . & . & . & . & + & . & . & . & . & 1 & . & 20 \\
\hline $\mathrm{CA}$ & Laserpitium peucedanoides & E1 & . & . & . & . & . & . & . & . & . & . & . & . & + & . & . & 1 & . & 20 \\
\hline PS & Paederota lutea & E1 & . & . & . & . & . & . & . & . & . & . & . & . & . & . & + & 1 & . & 20 \\
\hline & Differential species of the su & ad va & ant & $(\mathbf{R a}$ & like & aln & est & baso & iac & in & varia & nte) & & & & & & & & \\
\hline $\mathrm{TA}$ & Adoxa moschatellina & E1 & 1 & 1 & + & + & + & + & 1 & 1 & 1 & 1 & . & . & . & . & + & 11 & 100 & 20 \\
\hline TA & Geranium robertianum & E1 & . & . & + & + & + & 1 & 1 & + & + & + & . & . & . & . & . & 8 & 80 & . \\
\hline Сy & Sedum hispanicum & E1 & + & + & + & + & . & + & . & $\mathrm{r}$ & + & . & . & . & . & . & . & 7 & 70 & . \\
\hline $\mathrm{MuA}$ & Aconitum angustifolium & E1 & + & + & + & + & + & . & . & $\mathrm{r}$ & . & . & . & . & . & . & 1 & 7 & 60 & 20 \\
\hline $\mathrm{MuA}$ & Senecio cacaliaster & E1 & . & + & + & + & + & . & + & 1 & . & . & . & . & . & + & . & 7 & 60 & 20 \\
\hline QF & Aegopodium podagraria & E1 & + & 1 & 1 & 1 & 1 & . & . & + & . & . & . & . & . & . & . & 6 & 60 & . \\
\hline QF & Moehringia trinervia & E1 & + & . & + & + & . & . & . & + & + & . & . & . & . & . & . & 5 & 50 & . \\
\hline QF & Stellaria holostea & E1 & + & + & + & + & . & . & . & + & . & . & . & . & . & . & . & 5 & 50 & . \\
\hline $\mathrm{TA}$ & Stellaria montana & E1 & 1 & + & + & . & + & . & . & + & . & . & . & . & . & . & . & 5 & 50 & . \\
\hline $\mathrm{AF}$ & Lamium orvala & E1 & $\cdot$ & 1 & 1 & 1 & 1 & . & . & 1 & . & . & . & . & . & . & . & 5 & 50 & . \\
\hline TG & Verbascum lanatum & E1 & + & + & + & + & . & . & . & + & . & . & . & . & . & . & . & 5 & 50 & . \\
\hline TR & Heracleum pollinianum & E1 & . & + & . & . & + & . & + & . & . & . & . & . & + & . & . & 4 & 30 & 20 \\
\hline $\mathrm{AF}$ & Helleborus niger & E1 & . & . & . & . & . & . & . & . & . & . & 2 & 2 & 1 & 1 & . & 4 & . & 80 \\
\hline & Saxifraga cuneifolia & E1 & . & . & . & . & . & . & . & . & . & . & + & + & + & + & . & 4 & . & 80 \\
\hline & Hepatica nobilis & E1 & . & . & . & . & . & . & . & . & . & . & . & + & + & 1 & + & 4 & . & 80 \\
\hline $\mathrm{AF}$ & Aremonio-Fagion & & & & & & & & & & & & & & & & & & & \\
\hline & Cardamine enneaphyllos & E1 & + & 1 & + & + & 1 & 2 & 2 & 2 & 2 & 2 & 1 & 2 & 2 & 2 & 1 & 15 & 100 & 100 \\
\hline & Anemone trifolia & E1 & . & . & + & . & 1 & . & . & . & . & + & 1 & 1 & 1 & 1 & + & 8 & 30 & 100 \\
\hline $\mathrm{AF}$ & Cyclamen purpurascens & E1 & . & . & . & . & . & . & . & . & . & . & . & 1 & + & + & 1 & 4 & . & 80 \\
\hline & Aremonia agrimonoides & E1 & . & . & + & + & . & . & . & + & . & . & . & . & . & . & . & 3 & 30 & . \\
\hline & Cardamine trifolia & E1 & . & . & . & . & . & + & . & . & . & . & . & . & 1 & . & . & 2 & 10 & 20 \\
\hline EC & Erythronio-Carpinion & & & & & & & & & & & & & & & & & & & \\
\hline & Helleborus odorus & E1 & 1 & + & 1 & . & . & . & . & + & . & . & . & . & . & . & 1 & 5 & 40 & 20 \\
\hline & Galanthus nivalis & E1 & . & + & . & 1 & . & . & . & 1 & . & . & . & . & . & . & . & 3 & 30 & . \\
\hline TA & Tilio-Acerion & & & & & & & & & & & & & & & & & & & \\
\hline & Acer pseudoplatanus & E3 & + & . & . & . & . & . & . & . & + & . & . & + & . & . & . & 3 & 20 & 20 \\
\hline & Acer pseudoplatanus & E2 & . & . & . & . & . & . & + & . & + & . & . & . & . & . & . & 2 & 20 & . \\
\hline & Acer pseudoplatanus & E1 & + & 1 & + & . & $\mathrm{r}$ & . & . & + & + & . & . & 1 & . & . & . & 7 & 60 & 20 \\
\hline & Corydalis solida & E1 & + & + & + & . & . & + & . & + & . & . & . & . & . & . & . & 5 & 50 & . \\
\hline & Polystichum aculeatum & E1 & . & $\mathrm{r}$ & + & . & . & + & + & . & . & 1 & . & . & . & . & . & 5 & 50 & . \\
\hline & Chrysosplenium alternifolium & E1 & + & . & + & . & . & . & . & . & + & . & . & . & . & . & . & 3 & 30 & . \\
\hline & Lunaria rediviva & E1 & . & . & . & . & . & . & . & . & . & + & . & . & . & . & . & 1 & 10 & . \\
\hline FS & Fagetalia sylvaticae & & & & & & & & & & & & & & & & & & & \\
\hline & Fagus sylvatica & E3 & 5 & 5 & 5 & 5 & 4 & 5 & 5 & 5 & 5 & 5 & 5 & 4 & 5 & 5 & 4 & 15 & 100 & 100 \\
\hline & Fagus sylvatica & E2 & + & + & + & + & 1 & + & + & . & + & . & . & 1 & 1 & + & 1 & 12 & 80 & 80 \\
\hline & Fagus sylvatica & E1 & + & + & . & . & . & . & + & + & 1 & . & + & . & + & + & . & 8 & 50 & 60 \\
\hline & Dryopteris filix-mas & E1 & + & 1 & + & + & + & 1 & 1 & 1 & + & + & . & + & . & + & . & 12 & 100 & 40 \\
\hline & Galeobdolon flavidum & E1 & . & + & . & . & + & 1 & 1 & + & + & 1 & 1 & 1 & 1 & 1 & . & 11 & 70 & 80 \\
\hline & Epilobium montanum & E1 & 1 & 1 & 1 & 1 & 1 & 1 & 1 & + & + & . & . & . & . & . & + & 10 & 90 & 20 \\
\hline & Mercurialis perennis & E1 & . & . & + & 1 & 1 & . & 1 & 1 & 1 & 1 & 1 & 1 & . & . & + & 10 & 70 & 60 \\
\hline
\end{tabular}




\begin{tabular}{|c|c|c|c|c|c|c|c|c|c|c|c|c|c|c|c|c|c|c|c|c|}
\hline & Number of relevé (Zaporedna štev. popisa) & & 1 & 2 & 3 & 4 & 5 & 6 & 7 & 8 & 9 & 10 & 11 & 12 & 13 & 14 & 15 & & Fr.1-10 & r.11-15 \\
\hline & Actaea spicata & E1 & . & + & . & + & + & . & + & + & + & . & + & $\mathrm{r}$ & . & + & . & 9 & 60 & 60 \\
\hline & Luzula nivea & E1 & . & + & . & + & 1 & 1 & 1 & + & . & . & . & + & . & + & 1 & 9 & 60 & 60 \\
\hline & Daphne mezereum & E2 & + & . & . & . & . & . & . & + & + & + & . & + & + & + & + & 8 & 40 & 80 \\
\hline & Lilium martagon & E1 & + & + & + & . & . & . & + & + & . & . & . & 1 & . & + & + & 8 & 50 & 60 \\
\hline & Scrophularia nodosa & E1 & + & + & + & + & + & + & . & + & . & . & . & . & . & . & + & 8 & 70 & 20 \\
\hline & Paris quadrifolia & E1 & + & . & . & . & . & . & + & + & 1 & 1 & + & + & + & . & . & 8 & 50 & 60 \\
\hline & Lonicera alpigena & E2 & . & + & + & + & + & . & . & . & . & . & . & 1 & + & + & + & 8 & 40 & 80 \\
\hline & Lathyrus vernus & E1 & . & 1 & + & . & 1 & + & . & + & + & . & . & . & . & . & + & 7 & 60 & 20 \\
\hline & Galium laevigatum & E1 & . & . & . & . & + & + & + & . & + & . & + & . & . & + & + & 7 & 40 & 60 \\
\hline & Mycelis muralis & E1 & + & . & + & . & + & . & + & . & + & 1 & . & . & . & . & . & 6 & 60 & . \\
\hline & Myosotis sylvatica agg. & E1 & + & 1 & + & + & . & . & . & . & . & . & . & . & . & . & + & 5 & 40 & 20 \\
\hline & Ranunculus lanuginosus & E1 & + & 1 & + & . & . & + & . & + & . & . & . & . & . & . & . & 5 & 50 & . \\
\hline & Cardamine impatiens & E1 & + & + & + & . & + & . & . & + & . & . & . & . & . & . & . & 5 & 50 & . \\
\hline & Cardamine bulbifera & E1 & + & . & + & 1 & . & . & . & + & . & . & . & . & . & 1 & . & 5 & 40 & 20 \\
\hline & Campanula trachelium & E1 & & 1 & + & . & 1 & . & . & + & + & . & . & . & . & . & . & 5 & 50 & . \\
\hline & Symphytum tuberosum & E1 & + & . & . & . & . & + & . & . & . & . & . & + & + & + & . & 5 & 20 & 60 \\
\hline & Corydalis cava & E1 & . & . & . & . & . & + & . & . & 2 & . & . & 1 & 1 & 1 & . & 5 & 20 & 60 \\
\hline & Melica nutans & E1 & . & . & . & . & . & . & . & . & + & . & . & . & + & + & + & 4 & 10 & 60 \\
\hline & Epipactis helleborine & E1 & . & . & . & + & + & . & . & + & . & . & . & . & . & . & . & 3 & 30 & . \\
\hline & Laburnum alpinum & E3 & . & $\mathrm{r}$ & . & . & . & . & . & . & . & . & . & . & . & . & . & 1 & 10 & . \\
\hline & Laburnum alpinum & E2 & . & . & . & . & $\mathrm{r}$ & . & + & . & + & . & . & . & . & . & . & 3 & 30 & . \\
\hline & Laburnum alpinum & E1 & . & . & $\mathrm{r}$ & . & . & . & . & . & . & . & . & . & . & . & . & 1 & 10 & . \\
\hline & Lathyrus vernus subsp. flaccidus & E1 & + & . & . & . & . & . & . & . & . & . & . & . & . & . & . & 1 & 10 & . \\
\hline & Brachypodium sylvaticum & E1 & . & . & . & . & + & . & . & . & . & . & . & . & . & . & . & 1 & 10 & . \\
\hline & Dentaria pentaphyllos & E1 & . & . & . & . & . & + & . & . & . & . & . & . & . & . & . & 1 & 10 & . \\
\hline & Festuca altissima & E1 & . & . & . & . & . & . & 1 & . & . & . & . & . & . & . & . & 1 & 10 & . \\
\hline & Salvia glutinosa & E1 & . & . & . & . & . & . & . & . & + & . & . & . & . & . & . & 1 & 10 & . \\
\hline QP & Quercetalia pubescenti-petraeae & & & & & & & & & & & & & & & & & & & \\
\hline & Sorbus aria (Aria edulis) & E3 & . & . & . & . & . & . & . & . & $\mathrm{r}$ & . & . & . & . & . & . & 1 & 10 & . \\
\hline & Sorbus aria (Aria edulis) & E2 & . & . & . & + & + & . & $\mathrm{r}$ & . & . & . & . & . & + & . & . & 4 & 30 & 20 \\
\hline & Primula veris subsp. columnae & E1 & . & . & . & . & + & . & . & . & . & . & . & . & . & . & . & 1 & 10 & . \\
\hline QF & Querco-Fagetea & & & & & & & & & & & & & & & & & & & \\
\hline & Poa nemoralis & E1 & + & + & 1 & + & + & . & . & . & . & + & . & + & . & + & + & 9 & 60 & 60 \\
\hline & Anemone nemorosa & E1 & 1 & 1 & + & + & . & . & . & 1 & . & 1 & . & . & . & . & . & 6 & 60 & . \\
\hline & Festuca heterophylla & E1 & + & . & . & . & . & . & . & . & + & . & . & . & . & . & + & 3 & 20 & 20 \\
\hline & Carex digitata & E1 & . & . & . & . & . & + & . & . & . & . & + & + & . & . & . & 3 & 10 & 40 \\
\hline & Scilla bifolia & E1 & + & . & . & . & . & 1 & . & . & . & . & . & . & . & . & . & 2 & 20 & . \\
\hline & Corylus avellana & E2 & . & . & . & . & . & $\mathrm{r}$ & . & . & + & . & . & . & . & . & . & 2 & 20 & . \\
\hline & Listera ovata & E1 & . & . & . & . & . & . & $\mathrm{r}$ & . & . & . & . & + & . & . & . & 2 & 10 & 20 \\
\hline & Dactylorhiza fuchsii & E1 & . & . & . & . & + & . & . & . & . & . & . & . & . & . & . & 1 & 10 & . \\
\hline & Cruciata glabra & E1 & . & . & . & . & . & . & . & . & . & . & . & + & . & . & . & 1 & . & 20 \\
\hline & Viola riviniana & E1 & . & . & . & . & . & . & . & . & . & . & . & . & . & . & + & 1 & . & 20 \\
\hline VP & Vaccinio-Piceetea & & & & & & & & & & & & & & & & & & & \\
\hline & Oxalis acetosella & E1 & 1 & + & + & . & . & + & 1 & 1 & . & 1 & + & + & + & + & . & 11 & 70 & 80 \\
\hline & Aposeris foetida & E1 & . & + & + & + & + & . & . & + & + & . & 1 & 1 & + & 1 & . & 10 & 60 & 80 \\
\hline & Luzula luzuloides & E1 & 1 & + & 1 & + & + & . & . & + & + & 1 & . & . & . & . & . & 8 & 80 & . \\
\hline & Calamagrostis arundinacea & E1 & . & 1 & 1 & + & + & + & . & + & . & 1 & . & . & . & . & 1 & 8 & 70 & 20 \\
\hline & Gymnocarpium dryopteris & E1 & . & . & + & . & . & . & + & . & . & + & + & + & + & + & . & 7 & 30 & 80 \\
\hline & Maianthemum bifolium & E1 & . & . & . & . & + & . & + & . & . & + & + & 1 & 1 & + & . & 7 & 30 & 80 \\
\hline & Picea abies & E2 & . & $\mathrm{r}$ & . & . & + & . & . & + & . & . & . & + & . & + & + & 6 & 30 & 60 \\
\hline
\end{tabular}




\begin{tabular}{|c|c|c|c|c|c|c|c|c|c|c|c|c|c|c|c|c|c|c|c|c|}
\hline \multicolumn{3}{|c|}{ Number of relevé (Zaporedna štev. popisa) } & \multirow{2}{*}{1} & \multirow{2}{*}{$\begin{array}{l}2 \\
\mathrm{r}\end{array}$} & \multirow{2}{*}{3} & \multirow{2}{*}{4} & \multirow{2}{*}{$\begin{array}{l}5 \\
+\end{array}$} & \multirow{2}{*}{6} & \multirow{2}{*}{7} & \multirow{2}{*}{8} & \multirow{2}{*}{$\begin{array}{l}9 \\
+\end{array}$} & \multirow{2}{*}{$\begin{array}{l}10 \\
+\end{array}$} & \multicolumn{2}{|c|}{\begin{tabular}{|ll}
11 & 12 \\
\end{tabular}} & \multicolumn{3}{|c|}{$12 \quad 14 \quad 15$} & Pr. & Fr. $1-10$ & Fr.11-15 \\
\hline & Veronica urticifolia & E1 & & & & & & & & & & & + & . & . & . & . & 5 & 40 & 20 \\
\hline & Rosa pendulina & $\mathrm{E} 2$ & . & . & + & . & + & . & . & . & . & . & . & + & . & + & . & 4 & 20 & 40 \\
\hline & Solidago virgaurea & E1 & . & . & + & + & + & . & . & . & . & + & . & . & . & . & . & 4 & 40 & . \\
\hline & Picea abies & E3 & . & . & + & . & . & . & . & . & . & . & . & . & + & $\mathrm{r}$ & + & 4 & 10 & 60 \\
\hline & Hieracium murorum & E1 & + & . & + & . & + & . & . & . & . & . & . & . & . & . & . & 3 & 30 & . \\
\hline & Vaccinium myrtillus & E1 & . & . & . & . & . & . & . & . & . & . & . & . & + & + & + & 3 & . & 60 \\
\hline & Dryopteris dilatata & E1 & . & . & + & . & . & . & . & . & . & + & . & . & . & . & . & 2 & 20 & . \\
\hline & Larix decidua & E3 & . & . & . & . & . & . & . & . & . & . & + & 1 & . & . & . & 2 & . & 40 \\
\hline & Abies alba & E2 & . & $\mathrm{r}$ & . & . & . & . & . & . & . & . & . & . & . & . & . & 1 & 10 & . \\
\hline & Circaea alpina & E1 & . & . & . & . & . & + & . & . & . & . & . & . & . & . & . & 1 & 10 & . \\
\hline & Dryopteris expansa & E1 & . & . & . & . & . & . & . & . & . & + & . & . & . & . & . & 1 & 10 & . \\
\hline & Gentiana asclepiadea & E1 & . & . & . & . & . & . & . & . & . & . & + & . & . & . & . & 1 & . & 20 \\
\hline & Phegopteris connectilis & E1 & . & . & . & . & . & . & . & . & . & . & + & . & . & . & . & 1 & . & 20 \\
\hline & Larix decidua & E2 & . & . & . & . & . & . & . & . & . & . & . & + & . & . & . & 1 & . & 20 \\
\hline & Lonicera nigra & E2 & . & . & . & . & . & . & . & . & . & . & . & . & . & + & . & 1 & . & 20 \\
\hline EP & Erico-Pinetea & & & & & & & & & & & & & & & & & & & \\
\hline & Rubus saxatilis & E1 & . & . & + & . & . & . & . & . & . & . & . & + & + & . & . & 3 & 10 & 40 \\
\hline & Calamagrostis varia & E1 & . & . & . & . & . & . & + & . & . & . & . & . & . & + & . & 2 & 10 & 20 \\
\hline & Aquilegia nigricans & E1 & . & . & . & . & . & . & . & . & . & . & + & + & . & . & . & 2 & . & 40 \\
\hline & Erica carnea & E1 & . & . & . & . & . & . & . & . & . & . & . & + & . & . & + & 2 & . & 40 \\
\hline & Polygala chamaebuxus & E1 & . & . & . & . & . & . & . & . & . & . & . & + & . & . & . & 1 & . & 20 \\
\hline & Carex ornithopoda & E1 & . & . & . & . & . & . & . & . & . & . & . & . & + & . & . & 1 & . & 20 \\
\hline & Chamaecytisus hirsutus & E1 & . & . & . & . & . & . & . & . & . & . & . & . & . & . & + & 1 & . & 20 \\
\hline $\mathrm{AA}$ & Sambuco-Salicion capreae, Rhamn & tea & & & & & & & & & & & & & & & & & & \\
\hline & Sorbus aucuparia & E3 & . & . & . & . & . & + & . & . & . & . & . & . & + & + & . & 3 & 10 & 40 \\
\hline & Sorbus aucuparia & E2 & . & . & . & + & . & . & . & . & . & . & . & + & + & . & . & 3 & 10 & 40 \\
\hline & Sorbus aucuparia & E1 & . & . & . & . & . & . & . & . & . & . & . & . & . & + & . & 1 & . & 20 \\
\hline & Rosa sp. & E2 & . & . & . & $\mathrm{r}$ & . & . & . & . & . & . & . & . & . & . & . & 1 & 10 & . \\
\hline & Rubus fruticosus agg. & E2 & . & . & . & . & . & . & . & + & . & . & . & . & . & . & . & 1 & 10 & . \\
\hline $\mathrm{BA}$ & Betulo-Alnetea & & & & & & & & & & & & & & & & & & & \\
\hline & Ribes uva-crispa & E2 & . & . & $\mathrm{r}$ & + & . & . & . & $\mathrm{r}$ & . & . & . & . & . & . & . & 3 & 30 & . \\
\hline & Ribes alpinum & E2 & . & . & . & . & . & . & . & . & . & . & . & + & + & + & . & 3 & . & 60 \\
\hline & Juniperus sibirica & $\mathrm{E} 2$ & . & . & . & . & . & . & . & . & . & . & . & $\mathrm{r}$ & . & . & . & 1 & . & 20 \\
\hline & Mulgedio-Aconitetea & & & & & & & & & & & & & & & & & & & \\
\hline & Saxifraga rotundifolia & E1 & 1 & 2 & 1 & + & + & + & 1 & 1 & + & + & + & $\mathrm{r}$ & + & + & + & 15 & 100 & 100 \\
\hline & Polygonatum verticillatum & E1 & + & + & 1 & + & + & . & + & + & + & . & . & + & 1 & 1 & + & 12 & 80 & 80 \\
\hline & Veratrum album & E1 & 1 & + & + & . & + & . & + & + & + & + & 1 & + & + & + & . & 12 & 80 & 80 \\
\hline & Athyrium filix-femina & E1 & + & + & + & . & + & . & 1 & + & . & . & 1 & + & + & + & . & 10 & 60 & 80 \\
\hline & Primula elatior & E1 & . & + & + & + & . & . & . & + & . & . & . & + & . & + & . & 6 & 40 & 40 \\
\hline & Aconitum degenii subsp. paniculatum & E1 & + & + & . & . & . & + & + & . & + & . & . & . & . & . & . & 5 & 50 & . \\
\hline & Chaerophyllum hirsutum & E1 & + & + & . & . & . & + & . & . & + & . & . & . & . & . & . & 4 & 40 & . \\
\hline & Hypericum maculatum & E1 & . & + & + & + & + & . & . & . & . & . & . & . & . & . & . & 4 & 40 & . \\
\hline & Rumex arifolius & E1 & . & + & + & . & + & . & . & . & . & . & . & + & . & . & . & 4 & 30 & 20 \\
\hline & Senecio ovatus & E1 & . & . & . & . & . & 1 & . & . & 1 & 1 & + & . & . & . & . & 4 & 30 & 20 \\
\hline & Viola biflora & E1 & . & . & . & . & . & . & + & . & . & . & . & + & + & + & . & 4 & 10 & 60 \\
\hline & Thalictrum aquilegiifolium & E1 & . & . & . & . & . & . & + & . & . & . & . & + & . & + & . & 3 & 10 & 40 \\
\hline & Milium effusum & E1 & + & . & . & . & . & . & + & . & . & . & . & . & . & . & . & 2 & 20 & . \\
\hline & Phyteuma ovatum & E1 & . & . & . & . & + & . & . & . & + & . & . & . & . & . & . & 2 & 20 & . \\
\hline & Myrrhis odorata & E1 & . & . & . & . & . & . & . & . & + & . & . & $\mathrm{r}$ & . & . & . & 2 & 10 & 20 \\
\hline & Geranium sylvaticum & E1 & . & . & . & . & . & . & . & . & . & . & . & . & + & + & . & 2 & . & 40 \\
\hline
\end{tabular}




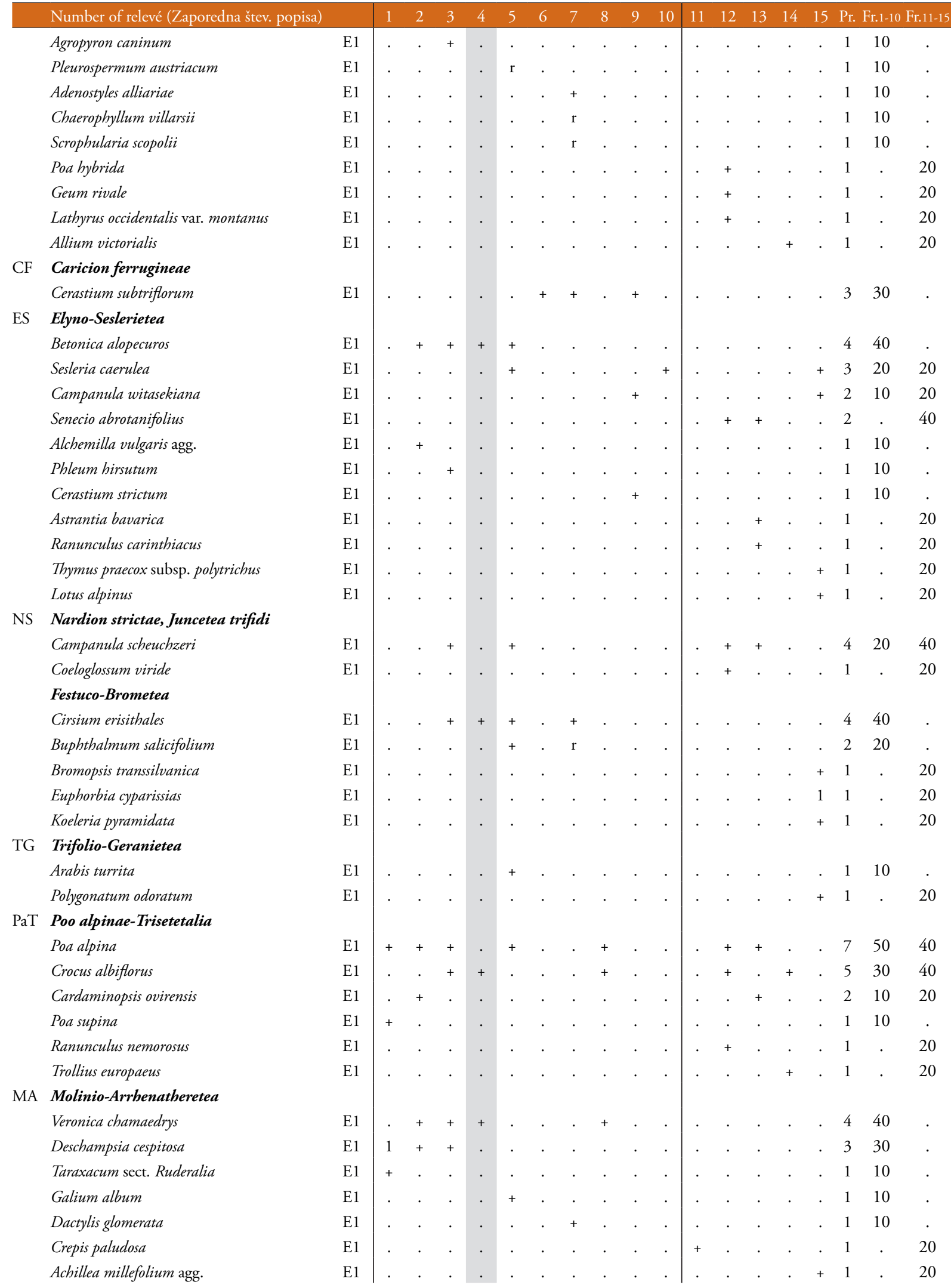




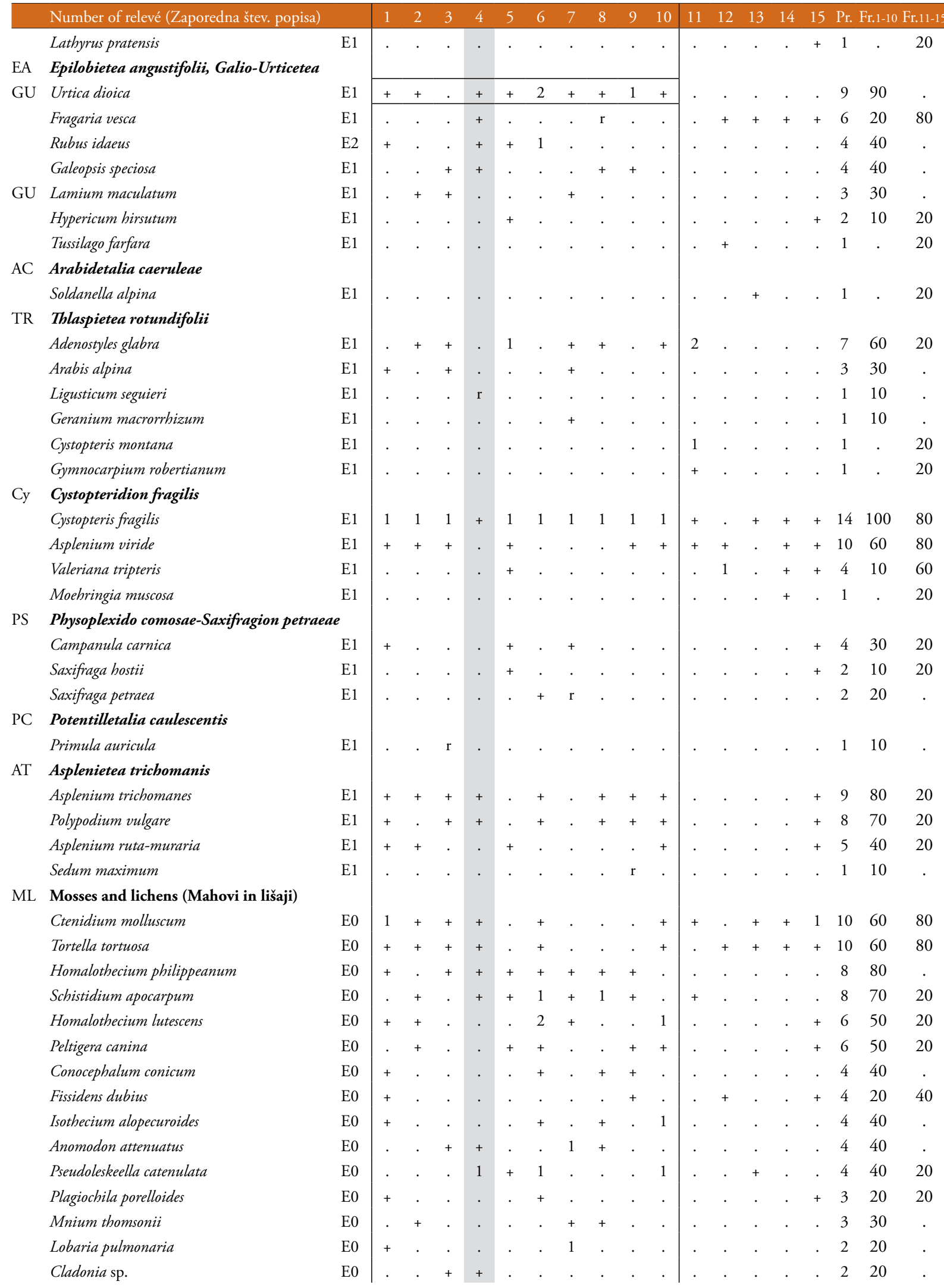


Dermatocarpon miniatum

Bryum capillare

Porella arboris-vitae

Hypogymnia physodes

Metzgeria furcata

Neckera crispa

Plagiothecium sp.

Porella platyphylla

Brachythecium sp.

Brachythecium rutabulum

Plagiomnium cuspidatum

Plagiothecium denticulatum

Collema cristatum

Marchantia polymorpha

Hypnum cupressiforme var. filiforme

Isothecium myosuroides

Mnium sp.

Plagiomnium undulatum

Polytrichum formosum

Cladonia pyxidata

\begin{tabular}{|c|c|c|c|c|c|c|c|c|c|c|c|c|c|c|c|c|c|c|}
\hline E0 & . & - & + & - & . & . & . & + & $\cdot$ & . & . & . & . & . & & 2 & 20 & . \\
\hline E0 & . & . & . & + & . & + & . & . & . & . & . & . & . & . & . & 2 & 20 & . \\
\hline E0 & . & . & . & $\cdot$ & . & . & 1 & . & . & + & $\cdot$ & . & . & . & . & 2 & 20 & . \\
\hline E3 & + & . & . & . & . & . & . & . & . & . & . & . & . & . & $\cdot$ & 1 & 10 & . \\
\hline E0 & + & . & . & . & . & . & . & . & . & . & . & . & . & . & . & 1 & 10 & . \\
\hline E0 & + & . & . & . & . & . & . & . & . & . & . & . & . & . & . & 1 & 10 & . \\
\hline E0 & + & . & . & . & . & . & . & . & $\cdot$ & . & $\cdot$ & . & . & . & . & 1 & 10 & . \\
\hline E0 & . & . & + & . & . & . & . & . & . & . & . & . & . & . & 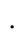 & 1 & 10 & . \\
\hline E0 & . & . & . & . & + & . & . & . & . & . & . & . & . & . & . & 1 & 10 & . \\
\hline E0 & . & . & . & . & . & + & . & . & . & . & . & . & . & . & . & 1 & 10 & . \\
\hline E0 & . & $\cdot$ & . & . & . & + & . & . & . & . & . & $\cdot$ & . & . & . & 1 & 10 & . \\
\hline E0 & . & . & . & . & . & + & . & . & $\cdot$ & . & . & . & . & . & . & 1 & 10 & . \\
\hline E0 & . & . & . & . & . & . & . & + & $\cdot$ & $\cdot$ & $\cdot$ & $\cdot$ & . & . & . & 1 & 10 & . \\
\hline E0 & . & · & . & $\cdot$ & . & . & . & . & + & $\cdot$ & . & . & . & . & . & 1 & 10 & . \\
\hline E0 & . & $\cdot$ & . & $\cdot$ & $\cdot$ & . & . & . & . & + & $\cdot$ & $\cdot$ & . & . & . & 1 & 10 & . \\
\hline E0 & . & . & . & . & . & . & . & . & . & + & . & $\cdot$ & . & . & $\cdot$ & 1 & 10 & . \\
\hline E0 & . & . & . & $\cdot$ & . & . & . & . & . & + & . & r & . & . & . & 1 & 10 & . \\
\hline E0 & . & . & . & . & . & . & . & . & . & + & . & . & . & . & . & 1 & 10 & . \\
\hline E0 & . & $\cdot$ & . & . & . & . & . & . & . & + & . & . & . & . & . & 1 & 10 & . \\
\hline E0 & . & . & . & . & . & . & . & . & . & . & . & . & . & . & + & 1 & . & 20 \\
\hline
\end{tabular}

\section{Legend - Legenda}

ID Igor Dakskobler

A Limestone - apnenec

D Dolomite - dolomit

Gr Gravel-grušč

L Marlstone - laporovec

$\mathrm{R}$ Chert - roženec

Re Rendzina - rendzina

JA Julian Alps - Julijske Alpe

Pr. Presence (number of relevés in which the species is presented) - število popisov, v katerih se pojavlja vrsta

Fr. Frequency in $\%-$ frekvenca $v \%$

Columns 1-10: Polysticho lonchitis-Fagetum adoxetosum moschatellinae

Relevé No. 4, holotypus

Columns 11-15: Polysticho lonchitis-Fagetum var. Helleborus niger prov. 
Table 7: Polysticho lonchitis-Fagetum allietosum victorialis - cluster 13.

Tabela 7: Polysticho lonchitis-Fagetum allietosum victorialis - skupina 13.

\begin{tabular}{|c|c|c|c|c|c|c|c|c|c|c|}
\hline Number of relevé (Zaporedna številka popisa) & & 1 & 2 & 3 & 4 & 5 & 6 & 7 & 8 & 9 \\
\hline Database number of relevé (Delovna številka popisa) & & 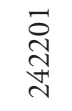 & 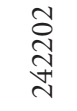 & 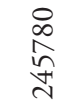 & \begin{tabular}{l}
$\curvearrowleft$ \\
$\infty$ \\
$\stackrel{\infty}{\sim}$ \\
\multirow{\sim}{*}{}
\end{tabular} & $\underset{\underset{\sim}{\sim}}{\stackrel{\infty}{\sim}}$ & 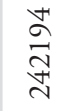 & $\underset{\stackrel{\infty}{\underset{T}{\sim}}}{\stackrel{\infty}{\sim}}$ & 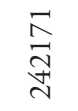 & 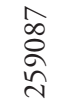 \\
\hline Author of the relevé (Avtor popisa) & & ID & ID & ID & ID & ID & ID & ID & ID & ID \\
\hline Elevation in $\mathrm{m}$ (Nadmorska višina $\mathrm{v} \mathrm{m})$ & & 1430 & 1430 & 1390 & 1390 & 1430 & 1440 & 1450 & 1450 & 14 \\
\hline Aspect (Lega) & & S & $\mathrm{NE}$ & NE & $\mathrm{NE}$ & SE & SW & SSE & SE & $S$ \\
\hline Slope in degrees (Nagib v stopinjah) & & 25 & 30 & 25 & 30 & 30 & 25 & 10 & 20 & 15 \\
\hline Parent material (Matična podlaga) & & A & A & A & A & A & A & A & A & $\mathrm{D}$ \\
\hline Soil (Tla) & & $\operatorname{Re}$ & $\operatorname{Re}$ & $\operatorname{Re}$ & $\operatorname{Re}$ & $\operatorname{Re}$ & $\operatorname{Re}$ & $\operatorname{Re}$ & $\operatorname{Re}$ & $\operatorname{Re}$ \\
\hline Stoniness in \% (Kamnitost v \%) & & 30 & 40 & 30 & 40 & 30 & 70 & 20 & 10 & P \\
\hline \multicolumn{11}{|l|}{ Cover in \% (Zastiranje v \%): } \\
\hline Upper tree layer (Zgornja drevesna plast) & $\mathrm{E} 3 \mathrm{~b}$ & 90 & 80 & 70 & 70 & 80 & 80 & 80 & 80 & 90 \\
\hline Lower tree layer (Spodnja drevesna plasti) & E3a & . & 5 & 5 & 10 & 5 & 10 & 10 & . & \\
\hline Shrub layer (Grmovna plast) & E2 & 5 & 15 & 20 & 10 & 20 & 10 & 10 & 20 & 10 \\
\hline Herb layer (Zeliščna plast) & E1 & 60 & 70 & 70 & 70 & 80 & 60 & 90 & 90 & 90 \\
\hline Moss layer (Mahovna plast) & E0 & 10 & 10 & 10 & 10 & 10 & 20 & 20 & 10 & 5 \\
\hline Maximum tree diameter (Maksimalni premer dreves) & $\mathrm{cm}$ & 35 & 50 & 40 & 50 & 45 & 40 & 45 & 40 & 30 \\
\hline Maximum tree height (Maksimalna višina dreves) & $\mathrm{m}$ & 10 & 10 & 14 & 12 & 12 & 10 & 12 & 12 & 12 \\
\hline Number of species (Število vrst) & & 71 & 55 & 55 & 65 & 66 & 57 & 47 & 53 & 53 \\
\hline Relevé area (Velikost popisne ploskve) & $\mathrm{m}^{2}$ & 400 & 400 & 400 & 400 & 400 & 400 & 200 & 400 & 40 \\
\hline Date of taking relevé (Datum popisa) & & 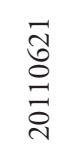 & 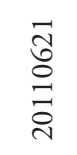 & 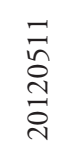 & 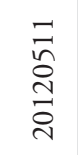 & 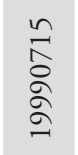 & 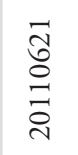 & 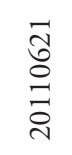 & 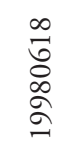 & \\
\hline Day (Dan) & & 21 & 21 & 11 & 11 & 15 & 21 & 21 & 18 & 2 \\
\hline Month (Mesec) & & 6 & 6 & 5 & 5 & 7 & 6 & 6 & 6 & 6 \\
\hline Year (Leto) & & 2011 & 2011 & 2012 & 2012 & 1999 & 2011 & 2011 & 1998 & \\
\hline
\end{tabular}

Locality (Nahajališče)

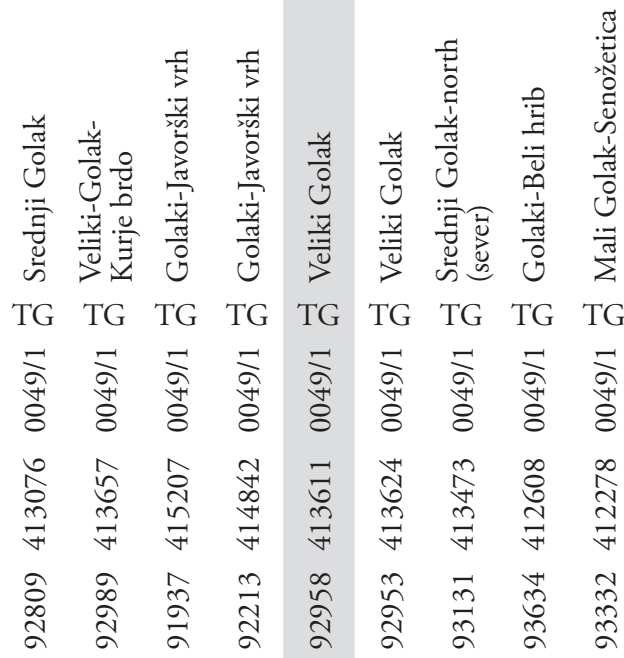

Pr. Fr.

Diagnostic species of the association (Diagnostične vrste asociacije)

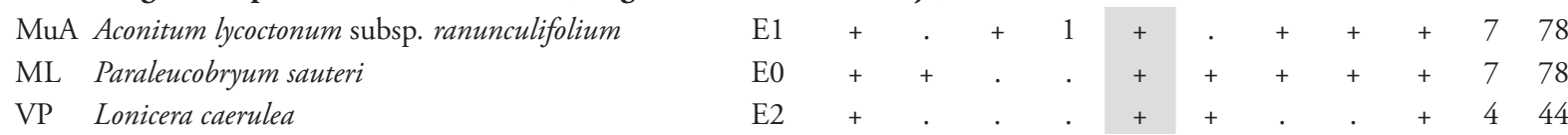




\begin{tabular}{|c|c|c|c|c|c|c|c|c|c|c|c|c|c|}
\hline & Number of relevé (Zaporedna številka popisa) & & 1 & 2 & 3 & 4 & 5 & 6 & 7 & 8 & 9 & Pr. & Fr. \\
\hline VP & Clematis alpina & E2 & . & + & . & . & + & + & . & + & . & 4 & 44 \\
\hline VP & Polystichum lonchitis & E1 & . & + & $\cdot$ & . & $\mathrm{r}$ & + & . & - & . & 3 & 33 \\
\hline BA & Salix appendiculata & E2 & . & + & . & + & + & $\cdot$ & . & $\sigma^{\circ}$ & . & 3 & 33 \\
\hline $\mathrm{EP}$ & Rhododendron hirsutum & E2 & . & + & . & . & . & + & . & . & . & 2 & 22 \\
\hline PS & Paederota lutea & E1 & . & . & . & + & . & . & . & . & . & 1 & 11 \\
\hline BA & Sorbus chamaemespilus & $\mathrm{E} 2$ & . & . & . & . & + & . & . & . & . & 1 & 11 \\
\hline VP & Luzula sylvatica & E1 & . & . & . & . & . & . & . & + & . & 1 & 11 \\
\hline
\end{tabular}

Differential species of the subassociation and variant (Razlikovalnice subasociacije in variante)

MuA Allium victorialis

VP Calamagrostis arundinacea

SSC Rubusidaeus

VP Lonicera nigra

TA Acerpseudoplatanus

TA Acerpseudoplatanus

TA Acerpseudoplatanus

TR Adenostyles glabra

TA Adoxa moschatellina

AF Aremonio-Fagion

Cardamine trifolia

Cardamine enneaphyllos

Cyclamen purpurascens

Omphalodes verna

Euphorbia carniolica

Rhamnus fallax

Knautia drymeia

TA Tilio-Acerion

Lunaria rediviva

Polystichum aculeatum

Aruncus dioicus

Polystichum braunii

Polystichum $x$ luerssenii

FS Fagetalia sylvaticae

Fagus sylvatica

Fagus sylvatica

Fagus sylvatica

Daphne mezereum

Dryopteris filix-mas

Lilium martagon

Lonicera alpigena

Actaea spicata

Heracleum sphondylium

Mercurialis perennis

Prenanthes purpurea

Cardamine bulbifera

Galeobdolon flavidum

Phyteuma spicatum subsp. coeruleum

Symphytum tuberosum

Corydalis cava

Epilobium montanum

Galium laevigatum

$\begin{array}{llllllllllll}\text { E1 } & 2 & 3 & 4 & 4 & 4 & 4 & 5 & 4 & 4 & 9 & 100 \\ \text { E1 } & 1 & 2 & + & + & 1 & 2 & + & 1 & 1 & 9 & 100 \\ \text { E2 } & 1 & + & 2 & 2 & 1 & 1 & 1 & 1 & 1 & 9 & 100 \\ \text { E2 } & 1 & 1 & + & + & 1 & 1 & . & + & 1 & 8 & 89 \\ \text { E3 } & 1 & . & + & + & + & + & 1 & + & 1 & 8 & 89 \\ \text { E2 } & . & + & + & . & + & . & . & + & + & 5 & 56 \\ \text { E1 } & + & . & + & . & . & + & . & + & + & 5 & 56 \\ \text { E1 } & 1 & + & + & 1 & . & . & . & + & . & 5 & 56 \\ \text { E1 } & + & + & + & + & . & . & . & . & . & 4 & 44\end{array}$

$\mathrm{E} 1+\begin{array}{llllllllll}1 & 1 & 1 & 1 & 1 & 1 & 1 & 1 & 9 & 100\end{array}$

$\begin{array}{llllllllllll}\mathrm{E} 1 & 2 & 1 & 1 & 1 & 1 & 1 & 1 & 1 & 1 & 9 & 100\end{array}$

$\mathrm{E} 1++.+1 .+67$

$\mathrm{E} 1++.+1+.+2+67$

$\mathrm{E} 1++.+.+.+444$

$\mathrm{E} 2 .+++\ldots+.+444$

E1

E1

E1

E1

E1

E1

E3

E2

E1

E2

E1

E1

E2

E1

E1

E1

E1

E1

E1

E1

E1

E1

E1

E1

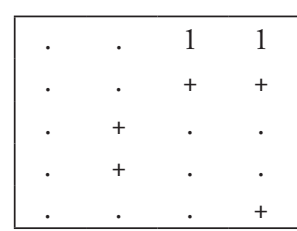

$\begin{array}{ccccccccccc}5 & 4 & 4 & 4 & 5 & 5 & 4 & 5 & 5 & 9 & 100 \\ + & . & 1 & 1 & 1 & 1 & 1 & 1 & 1 & 8 & 89\end{array}$

$+.1 .+1+1+667$

$++++1+++4100$

$1+11+1 \quad 1 \quad 1+9100$

$+++1++++4100$

$+++\quad 1 \quad 1+\ldots+89$

$+++1++.+78$

$+\quad+\quad 1++\quad+\quad+78$

$\begin{array}{lllllllllllll}1 & 1 & 1 & 1 & 1 & 1 & . & + & 7 & 78\end{array}$

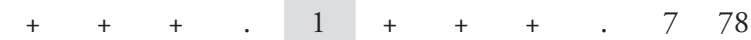

$+\quad 1 \quad 1 .+1+667$

$+11.11+. \quad 6 \quad 67$

+ . . +++++667

+ . . $+4++467$

$\begin{array}{lllllllll}1 & . & 1 & 1 & . & 1 & + & 5 & 56\end{array}$

$+++1 .+5656$ 


\begin{tabular}{|c|c|c|c|c|c|c|c|c|c|c|c|c|c|}
\hline & Number of relevé (Zaporedna številka popisa) & & 1 & 2 & 3 & 4 & 5 & 6 & 7 & 8 & 9 & Pr. & Fr. \\
\hline & Fraxinus excelsior & E3 & . & . & $\mathrm{r}$ & + & . & $\mathrm{r}$ & + & . & . & 4 & 44 \\
\hline & Mycelis muralis & E1 & + & . & . & + & . & + & . & . & . & 3 & 33 \\
\hline & Carex sylvatica & E1 & + & . & . & . & . & . & + & . & + & 3 & 33 \\
\hline & Galium odoratum & E1 & . & . & . & . & . & . & 1 & + & + & 3 & 33 \\
\hline & Campanula trachelium & E1 & + & . & . & + & . & . & . & . & . & 2 & 22 \\
\hline & Paris quadrifolia & E1 & . & . & + & + & . & . & . & . & . & 2 & 22 \\
\hline & Myosotis sylvatica agg. & E1 & . & . & + & . & . & . & . & . & + & 2 & 22 \\
\hline & Lathyrus vernus & E1 & . & . & . & . & . & . & . & + & + & 2 & 22 \\
\hline & Melica nutans & E1 & + & . & . & . & . & . & . & . & . & 1 & 11 \\
\hline & Allium ursinum & E1 & . & . & + & . & . & . & . & . & . & 1 & 11 \\
\hline & Scrophularia nodosa & E1 & . & . & + & . & . & . & . & . & . & 1 & 11 \\
\hline & Polygonatum multiflorum & E1 & . & . & . & + & . & . & . & . & . & 1 & 11 \\
\hline & Festuca altissima & E1 & . & . & . & . & . & . & . & + & . & 1 & 11 \\
\hline \multirow[t]{3}{*}{ QP } & Quercetalia pubescenti-petraeae & & & & & & & & & & & & \\
\hline & Sorbus aria (Aria edulis) & E3 & + & . & . & . & . & . & . & . & . & 1 & 11 \\
\hline & Sorbus aria (Aria edulis) & E2 & . & . & . & . & . & + & . & . & . & 1 & 11 \\
\hline \multirow[t]{8}{*}{ QF } & Querco-Fagetea & & & & & & & & & & & & \\
\hline & Anemone nemorosa & E1 & 1 & 1 & + & 1 & 1 & + & . & 1 & 1 & 8 & 89 \\
\hline & Anemone ranunculoides & E1 & . & . & . & . & + & + & . & . & . & 2 & 22 \\
\hline & Hepatica nobilis & E1 & + & . & . & . & . & . & . & . & . & 1 & 11 \\
\hline & Stellaria holostea & E1 & $\cdot$ & $\cdot$ & + & . & . & $\cdot$ & $\cdot$ & $\cdot$ & $\cdot$ & 1 & 11 \\
\hline & Lonicera xylosteum & E2 & $\cdot$ & $\cdot$ & . & + & . & $\cdot$ & $\cdot$ & $\cdot$ & $\cdot$ & 1 & 11 \\
\hline & Poa nemoralis & E1 & . & . & . & . & + & $\cdot$ & . & . & . & 1 & 11 \\
\hline & Viola riviniana & E1 & . & . & . & . & . & + & . & . & . & 1 & 11 \\
\hline \multirow[t]{19}{*}{ VP } & Vaccinio-Piceetea & & & & & & & & & & & & \\
\hline & Gentiana asclepiadea & E1 & + & 1 & + & + & + & + & . & + & + & 8 & 89 \\
\hline & Rosa pendulina & E2 & + & + & . & . & + & + & + & . & + & 6 & 67 \\
\hline & Oxalis acetosella & E1 & . & . & + & + & . & + & + & + & . & 5 & 56 \\
\hline & Aposeris foetida & E1 & + & . & . & . & + & + & . & + & . & 4 & 44 \\
\hline & Veronica urticifolia & E1 & + & . & + & . & + & + & . & . & . & 4 & 44 \\
\hline & Solidago virgaurea & E1 & . & . & . & + & + & + & . & + & . & 4 & 44 \\
\hline & Vaccinium myrtillus & E1 & . & . & . & . & + & + & . & + & + & 4 & 44 \\
\hline & Maianthemum bifolium & E1 & + & . & . & . & . & . & . & + & + & 3 & 33 \\
\hline & Abies alba & E2 & . & . & . & . & . & $\mathrm{r}$ & . & . & $\mathrm{r}$ & 2 & 22 \\
\hline & Abies alba & E1 & . & . & . & . & $\mathrm{r}$ & $\cdot$ & $\cdot$ & . & . & 1 & 11 \\
\hline & Dryopteris dilatata & E1 & . & + & . & + & . & . & . & . & . & 2 & 22 \\
\hline & Phegopteris connectilis & E1 & . & 1 & . & . & + & . & . & . & . & 2 & 22 \\
\hline & Gymnocarpium dryopteris & E1 & . & + & $\cdot$ & $\cdot$ & + & $\cdot$ & $\cdot$ & $\cdot$ & . & 2 & 22 \\
\hline & Picea abies & E3 & . & . & . & . & . & $\mathrm{r}$ & . & . & . & 1 & 11 \\
\hline & Picea abies & E2 & . & . & . & . & + & + & . & . & . & 2 & 22 \\
\hline & Luzula luzuloides & E1 & 1 & . & . & . & . & . & . & . & . & 1 & 11 \\
\hline & Homogyne sylvestris & E1 & $\cdot$ & + & . & $\cdot$ & . & $\cdot$ & $\cdot$ & $\cdot$ & $\cdot$ & 1 & 11 \\
\hline & Huperzia selago & E1 & . & + & . & . & . & $\cdot$ & . & . & . & 1 & 11 \\
\hline \multirow[t]{3}{*}{$\mathrm{EP}$} & Erico-Pinetea & & & & & & & & & & & & \\
\hline & Rubus saxatilis & E1 & . & + & $\cdot$ & $\cdot$ & + & $\cdot$ & $\cdot$ & + & $\cdot$ & 3 & 33 \\
\hline & Calamagrostis varia & E1 & . & . & + & + & . & . & . & . & . & 2 & 22 \\
\hline \multirow[t]{4}{*}{ SSC } & Sambuco-Salicion capreae, Rhamno-Prunetea & & & & & & & & & & & & \\
\hline & Sorbus aucuparia & E3 & + & + & $\mathrm{r}$ & + & . & $\cdot$ & . & $\mathrm{r}$ & + & 6 & 67 \\
\hline & Sorbus aucuparia & E2 & . & + & . & + & + & $\cdot$ & + & + & + & 6 & 67 \\
\hline & Sorbus aucuparia & E1 & + & . & + & . & . & + & . & . & + & 4 & 44 \\
\hline
\end{tabular}




\begin{tabular}{|c|c|c|c|c|c|c|c|c|c|c|c|c|c|}
\hline & Number of relevé (Zaporedna številka popisa) & & 1 & 2 & 3 & 4 & 5 & 6 & 7 & 8 & 9 & Pr. & Fr. \\
\hline \multicolumn{14}{|c|}{ MuA Mulgedio-Aconitetea } \\
\hline & Athyrium filix-femina & E1 & 1 & 1 & 1 & 1 & 1 & + & + & 1 & + & 9 & 100 \\
\hline & Veratrum album & E1 & 1 & 1 & 2 & 1 & 1 & + & 1 & 1 & 1 & 9 & 100 \\
\hline & Polygonatum verticillatum & E1 & + & 1 & . & + & + & + & + & + & + & 8 & 89 \\
\hline & Saxifraga rotundifolia & E1 & + & + & + & + & + & + & . & + & . & 7 & 78 \\
\hline & Ranunculus platanifolius & E1 & $\mathrm{r}$ & + & . & + & . & + & . & + & + & 6 & 67 \\
\hline & Senecio ovatus & E1 & + & . & + & . & . & + & + & . & + & 5 & 56 \\
\hline & Silene dioica & E1 & + & . & + & . & . & . & + & . & + & 4 & 44 \\
\hline & Cicerbita alpina & E1 & . & . & + & + & + & . & . & . & 1 & 4 & 44 \\
\hline & Doronicum austriacum & E1 & . & . & . & + & . & . & 1 & + & + & 4 & 44 \\
\hline & Aconitum degenii subsp. paniculatum & E1 & + & . & + & 1 & . & . & . & . & . & 3 & 33 \\
\hline & Pleurospermum austriacum & E1 & + & . & . & + & . & . & . & + & . & 3 & 33 \\
\hline & Poa hybrida & E1 & + & . & . & . & . & . & + & . & + & 3 & 33 \\
\hline & Thalictrum aquilegiifolium & E1 & . & . & . & + & + & . & + & . & . & 3 & 33 \\
\hline & Adenostyles alliariae & E1 & . & . & . & + & . & . & + & 1 & . & 3 & 33 \\
\hline & Stellaria nemorum & E1 & 2 & . & . & . & . & . & + & . & . & 2 & 22 \\
\hline & Myrrhis odorata & E1 & 2 & . & . & . & . & . & . & . & 1 & 2 & 22 \\
\hline & Senecio cacaliaster & E1 & + & . & . & . & . & . & + & . & . & 2 & 22 \\
\hline & Chaerophyllum hirsutum & E1 & + & . & . & . & . & . & . & + & . & 2 & 22 \\
\hline & Milium effusum & E1 & + & . & . & . & . & . & . & . & + & 2 & 22 \\
\hline & Centaurea montana & E1 & + & . & . & . & . & . & . & . & + & 2 & 22 \\
\hline & Chaerophyllum aureum & E1 & . & . & . & + & . & . & . & . & + & 2 & 22 \\
\hline & Rumex arifolius & E1 & . & . & . & + & . & . & . & . & + & 2 & 22 \\
\hline & Hypericum maculatum & $\mathrm{E} 1$ & . & . & . & + & . & . & . & . & . & 1 & 11 \\
\hline & Senecio nemorensis & E1 & . & . & . & . & . & $\cdot$ & . & + & . & 1 & 11 \\
\hline & Geranium sylvaticum & E1 & . & . & . & . & . & . & . & + & . & 1 & 11 \\
\hline & Geum rivale & E1 & . & . & . & . & . & . & . & . & + & 1 & 11 \\
\hline & Lathyrus occidentalis var. montanus & E1 & . & . & . & . & . & . & . & . & + & 1 & 11 \\
\hline \multirow[t]{4}{*}{$\mathrm{FB}$} & Festuco-Brometea & & & & & & & & & & & & \\
\hline & Cirsium erisithales & E1 & . & . & . & . & + & . & . & . & . & 1 & 11 \\
\hline & Trifolio-Geranietea & & & & & & & & & & & & \\
\hline & Laserpitium latifolium & E1 & . & . & . & . & + & + & . & . & . & 2 & 22 \\
\hline \multirow[t]{2}{*}{$\mathrm{PaT}$} & Poo alpinae-Trisetetalia & & & & & & & & & & & & \\
\hline & Poa supina & E1 & . & . & . & $\cdot$ & . & . & . & . & + & 1 & 11 \\
\hline \multirow[t]{4}{*}{ MA } & Molinio-Arrbenatheretea & & & & & & & & & & & & \\
\hline & Angelica sylvestris & E1 & + & + & + & . & . & . & + & . & + & 5 & 56 \\
\hline & Deschampsia cespitosa & E1 & . & . & . & + & . & . & . & . & . & 1 & 11 \\
\hline & Dactylis glomerata s.str. & E1 & . & . & . & . & . & . & + & . & . & 1 & 11 \\
\hline \multirow[t]{2}{*}{ EA } & Epilobietea angustifolii, Galio-Urticetea & & & & & & & & & & & & \\
\hline & Fragaria vesca & E1 & + & . & . & . & . & . & . & . & . & 1 & 11 \\
\hline \multirow[t]{2}{*}{ GU } & Urtica dioica & E1 & . & . & + & . & . & . & . & . & . & 1 & 11 \\
\hline & Galeopsis speciosa & E1 & . & . & . & + & . & . & . & . & . & 1 & 11 \\
\hline \multirow[t]{2}{*}{ TR } & Thlaspietea rotundifolii & & & & & & & & & & & & \\
\hline & Cystopteris montana & E1 & . & + & . & . & . & . & . & $\cdot$ & . & 1 & 11 \\
\hline \multirow[t]{6}{*}{ Cy } & Cystopteridion fragilis & & & & & & & & & & & & \\
\hline & Cystopteris fragilis & E1 & + & + & + & + & + & . & . & + & . & 6 & 67 \\
\hline & Valeriana tripteris & E1 & . & 1 & . & . & + & 1 & . & . & + & 4 & 44 \\
\hline & Moehringia muscosa & E1 & . & + & + & . & + & . & . & . & . & 3 & 33 \\
\hline & Sedum hispanicum & E1 & + & . & + & . & . & . & . & . & . & 2 & 22 \\
\hline & Asplenium viride & E1 & . & + & . & + & . & . & . & . & . & 2 & 22 \\
\hline
\end{tabular}




\begin{tabular}{|c|c|c|c|c|c|c|c|c|c|c|c|c|c|}
\hline & Number of relevé (Zaporedna številka popisa) & & 1 & 2 & 3 & 4 & 5 & 6 & 7 & 8 & 9 & Pr. & Fr. \\
\hline \multirow[t]{4}{*}{ AT } & Asplenietea trichomanis & & & & & & & & & & & & \\
\hline & Polypodium vulgare & E1 & + & . & . & $\cdot$ & + & + & . & . & . & 3 & 33 \\
\hline & Asplenium ruta-muraria & E1 & . & . & . & . & + & . & . & . & . & 1 & 11 \\
\hline & Asplenium trichomanes & E1 & . & . & . & . & $\mathrm{r}$ & . & . & . & . & 1 & 11 \\
\hline \multirow[t]{15}{*}{ ML } & Mosses and lichens (Mahovi in lišaji) & & & & & & & & & & & & \\
\hline & Ctenidium molluscum & E0 & 1 & . & 2 & 2 & 1 & 2 & 1 & + & . & 7 & 78 \\
\hline & Isothecium alopecuroides & E0 & 1 & 1 & + & 1 & + & + & + & . & . & 7 & 78 \\
\hline & Schistidium apocarpum & E0 & + & 1 & + & . & . & 1 & + & . & . & 5 & 56 \\
\hline & Tortella tortuosa & E0 & . & + & + & + & 1 & . & . & + & . & 5 & 56 \\
\hline & Peltigera canina & E0 & + & + & . & . & . & . & . & + & . & 3 & 33 \\
\hline & Homalothecium lutescens & E0 & + & . & . & 1 & . & . & . & . & . & 2 & 22 \\
\hline & Pseudoleskeella catenulata & E0 & . & . & 1 & 1 & . & . & . & . & . & 2 & 22 \\
\hline & Fissidens dubius & E0 & . & . & . & + & + & . & . & . & . & 2 & 22 \\
\hline & Cladonia sp. & E0 & . & . & . & . & + & . & . & + & . & 2 & 22 \\
\hline & Homalothecium philippeanum & E0 & . & . & . & . & . & . & + & + & . & 2 & 22 \\
\hline & Cladonia pyxidata & E0 & $\cdot$ & + & $\cdot$ & $\cdot$ & $\cdot$ & . & $\cdot$ & . & $\cdot$ & 1 & 11 \\
\hline & Mnium thomsonii & E0 & $\cdot$ & . & . & + & . & . & $\cdot$ & . & . & 1 & 11 \\
\hline & Bryum capillare & E0 & . & . & . & . & + & . & . & . & $\cdot$ & 1 & 11 \\
\hline & Mnium sp. & E0 & . & . & . & . & + & . & . & . & . & 1 & 11 \\
\hline
\end{tabular}

\section{Legend - Legenda}

ID Igor Dakskobler

A Limestone - apnenec

D Dolomite - dolomit

TG Trnovski Gozd Plateau - Trnovski gozd

Pr. Presence (number of relevés in which the species is presented) - število popisov, v katerih se pojavlja vrsta

Fr. Frequency in $\%-$ frekvenca $v \%$

Relevé No. 5, holotypus 
Table 8: Polysticho lonchitis-Fagetum stellarietosum nemorum and Ranunculo platanifolii-Fagetum stellarietosum nemorum - cluster 14.

\begin{tabular}{|c|c|c|c|c|c|c|c|c|c|c|c|c|c|c|}
\hline Number of relevé (Zaporedna številka popisa) & & 1 & 2 & 3 & 4 & 5 & 6 & 7 & 8 & 9 & 10 & 11 & 12 & 13 \\
\hline $\begin{array}{l}\text { Database number of relevé } \\
\text { (Delovna številka popisa) }\end{array}$ & & $\underset{\stackrel{\curvearrowright}{\sim}}{\stackrel{\sim}{\sim}}$ & 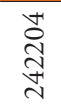 & 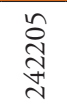 & 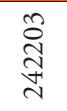 & 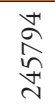 & 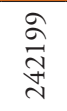 & $\begin{array}{l}\stackrel{\infty}{\curvearrowright} \\
\stackrel{n}{\sim}\end{array}$ & \begin{tabular}{l}
$\stackrel{\infty}{\infty}$ \\
$\stackrel{N}{\sim}$ \\
\multirow{\sim}{N}{}
\end{tabular} & \begin{tabular}{l}
$\infty$ \\
$\infty$ \\
$\stackrel{\infty}{n}$ \\
\multirow{\sim}{*}{}
\end{tabular} & $\begin{array}{l}\stackrel{+}{\infty} \\
\hat{n} \\
\stackrel{\sim}{\sim}\end{array}$ & 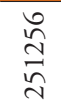 & 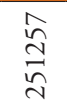 & $\begin{array}{l}\infty \\
\stackrel{\infty}{\sim} \\
\stackrel{\sim}{\sim}\end{array}$ \\
\hline Author of the relevé (Avtor popisa) & & ID & ID & ID & ID & ID & ID & ID & ID & ID & ID & ID & ID & ID \\
\hline Elevation in $\mathrm{m}$ (Nadmorska višina $\mathrm{v} \mathrm{m}$ ) & & 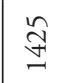 & 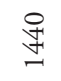 & 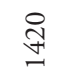 & $\stackrel{\Re}{\underset{I}{\leftrightarrows}}$ & 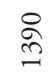 & 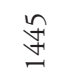 & ஓ & ஓ & $\stackrel{\circ}{\stackrel{m}{2}}$ & $\stackrel{n}{n}$ & $\stackrel{\circ}{\stackrel{n}{ \pm}}$ & $\begin{array}{l}\stackrel{0}{0} \\
\stackrel{+}{+}\end{array}$ & 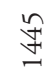 \\
\hline Aspect (Lega) & & SW & $\mathrm{NE}$ & NNE & $\mathrm{NE}$ & $\mathrm{NE}$ & $S$ & SW & NNE & SW & $\mathrm{NE}$ & $S$ & $S$ & SW \\
\hline Slope in degrees (Nagib v stopinjah) & & 15 & 30 & 40 & 30 & 15 & 25 & 30 & 25 & 15 & 30 & 20 & 15 & 15 \\
\hline Parent material (Matična podlaga) & & A & A & A & A & A & A & A & A & A & A & A & A & A \\
\hline Soil (Tla) & & $\operatorname{Re}$ & $\operatorname{Re}$ & $\operatorname{Re}$ & $\operatorname{Re}$ & $\mathrm{Re}$ & $\operatorname{Re}$ & $\operatorname{Re}$ & $\operatorname{Re}$ & $\operatorname{Re}$ & $\operatorname{Re}$ & $\operatorname{Re}$ & $\operatorname{Re}$ & $\operatorname{Re}$ \\
\hline Stoniness in \% (Kamnitost v \%) & & 30 & 40 & 40 & 35 & 10 & 40 & 40 & 40 & 30 & 50 & 70 & 30 & 40 \\
\hline \multicolumn{15}{|l|}{ Cover in \% (Zastiranje v \%): } \\
\hline Upper tree layer (Zgornja drevesna plast) & $\mathrm{E} 3 \mathrm{~b}$ & 80 & 80 & 70 & 80 & 70 & 80 & 80 & 80 & 80 & 70 & 80 & 80 & 90 \\
\hline Lower tree layer (Spodnja drevesna plasti) & E3a & 10 & 5 & 10 & 10 & 5 & 10 & 10 & 5 & 10 & 5 & 5 & . & . \\
\hline Shrub layer (Grmovna plast) & E2 & 20 & 20 & 10 & 20 & 20 & 30 & 10 & 10 & 10 & 20 & 20 & 10 & 10 \\
\hline Herb layer (Zeliščna plast) & E1 & 80 & 70 & 80 & 80 & 80 & 70 & 60 & 60 & 70 & 60 & 60 & 70 & 60 \\
\hline Moss layer (Mahovna plast) & E0 & 5 & 20 & 20 & 10 & 10 & 10 & 10 & 10 & 5 & 10 & 10 & 5 & 10 \\
\hline Maximum tree diameter (Maks. premer dreves) & $\mathrm{cm}$ & 60 & 45 & 45 & 60 & 50 & 50 & 40 & 45 & 50 & 60 & 40 & 20 & 40 \\
\hline Maximum tree height (Maks. višina dreves) & $\mathrm{m}$ & 15 & 10 & 15 & 16 & 12 & 16 & 12 & 12 & 15 & 14 & 12 & 7 & 12 \\
\hline Number of species (Število vrst) & & 58 & 78 & 74 & 65 & 48 & 59 & 62 & 69 & 60 & 60 & 62 & 82 & 55 \\
\hline Relevé area (Velikost popisne ploskve) & $\mathrm{m}^{2}$ & 400 & 400 & 400 & 400 & 400 & 400 & 400 & 400 & 400 & 400 & 200 & 200 & 200 \\
\hline Date of taking relevé (Datum popisa) & & $\begin{array}{l}\overrightarrow{\widetilde{J}} \\
\stackrel{0}{\sigma} \\
\vec{\sim}\end{array}$ & $\begin{array}{l}\overrightarrow{\widetilde{\sigma}} \\
\stackrel{0}{\circ} \\
\vec{ٍ}\end{array}$ & 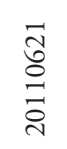 & 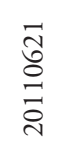 & 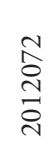 & 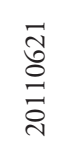 & 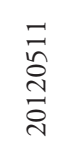 & 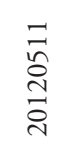 & 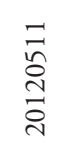 & 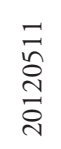 & 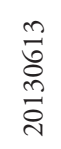 & 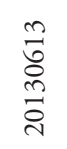 & 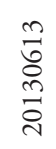 \\
\hline Day (Dan) & & 21 & 21 & 21 & 21 & 2 & 21 & 11 & 11 & 11 & 11 & 13 & 13 & 13 \\
\hline Month (Mesec) & & 6 & 6 & 6 & 6 & 7 & 6 & 5 & 5 & 5 & 5 & 6 & 6 & 6 \\
\hline Year (Leto) & & $\vec{\sim}$ & $\overrightarrow{\check{c}}$ & $\overrightarrow{\bar{c}}$ & ন্ & 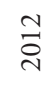 & $\overrightarrow{\bar{c}}$ & 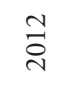 & $\stackrel{\sim}{\stackrel{4}{2}}$ & $\frac{\mathfrak{d}}{\stackrel{2}{2}}$ & $\stackrel{\sim}{\stackrel{i}{\sim}}$ & $\stackrel{n}{\stackrel{n}{i}}$ & $\stackrel{n}{i}$ & $\stackrel{n}{\text { i }}$ \\
\hline Locality (Nahajališče) & & 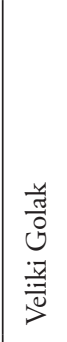 & 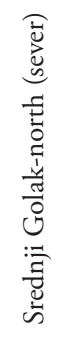 & 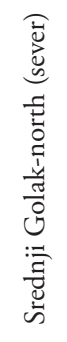 & 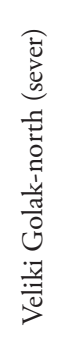 & 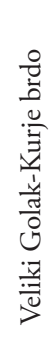 & 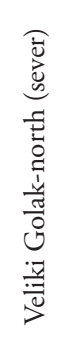 & 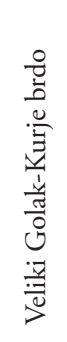 & 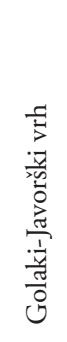 & 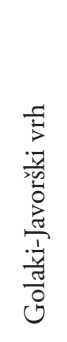 & 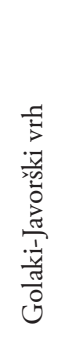 & 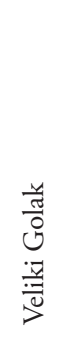 & $\begin{array}{l}\frac{y}{0} \\
0 \\
0 \\
\frac{\pi}{3} \\
\frac{\pi}{0}\end{array}$ & $\begin{array}{l}\frac{y}{\pi} \\
0 \\
0 \\
\bar{y} \\
\overline{0}\end{array}$ \\
\hline Mountain range (Pogorje) & & TG & TG & TG & TG & TG & TG & TG & TG & TG & TG & TG & TG & TG \\
\hline Quadrant (Kvadrant) & & $\begin{array}{l}\vec{a} \\
\stackrel{+}{8}\end{array}$ & $\begin{array}{l}\vec{a} \\
\stackrel{+}{8}\end{array}$ & 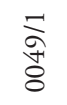 & $\underset{\grave{a}}{\stackrel{\vec{a}}{8}}$ & $\underset{\grave{a}}{\stackrel{े}{8}}$ & 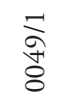 & 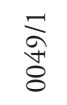 & $\begin{array}{l}\vec{a} \\
\stackrel{a}{8}\end{array}$ & $\underset{\grave{a}}{\stackrel{5}{8}}$ & $\underset{\grave{a}}{\stackrel{\vec{a}}{8}}$ & $\begin{array}{l}\vec{a} \\
\stackrel{a}{8}\end{array}$ & $\underset{\grave{a}}{\stackrel{5}{8}}$ & $\underset{\grave{a}}{\stackrel{\vec{a}}{8}}$ \\
\hline Coordinate (Koordinate) GK Y (D-48) & $\mathrm{m}$ & $\begin{array}{l}\frac{n}{n} \\
\stackrel{n}{F} \\
F\end{array}$ & $\begin{array}{l}\stackrel{+}{N} \\
\stackrel{\sim}{*}\end{array}$ & 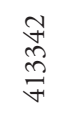 & $\begin{array}{l}\stackrel{\infty}{+} \\
\stackrel{\infty}{+} \\
\stackrel{\forall}{\forall}\end{array}$ & $\underset{\underset{F}{\sim}}{\stackrel{n}{7}}$ & 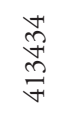 & $\underset{\underset{+}{\stackrel{+}{F}}}{\underset{F}{F}}$ & $\frac{n}{\stackrel{n}{n}}$ & $\begin{array}{l}\stackrel{n}{0} \\
\stackrel{n}{n} \\
\forall\end{array}$ & $\begin{array}{l}\stackrel{\curvearrowright}{\curvearrowright} \\
\stackrel{\infty}{+} \\
\vec{\forall}\end{array}$ & $\begin{array}{l}\stackrel{2}{~} \\
\stackrel{\approx}{F}\end{array}$ & $\begin{array}{l}\stackrel{\infty}{+} \\
\stackrel{+}{F} \\
\underset{\forall}{+}\end{array}$ & $\begin{array}{l}\stackrel{\infty}{n} \\
\stackrel{n}{\approx} \\
\stackrel{\forall}{*}\end{array}$ \\
\hline Coordinate (Koordinate) GK X (D-48) & $\mathrm{m}$ & $\begin{array}{l}\text { రె } \\
\text { } \\
\text { }\end{array}$ & 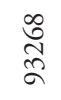 & $\begin{array}{l}\tilde{a} \\
\tilde{n}\end{array}$ & $\begin{array}{l}\stackrel{+}{+} \\
\text { n̆ }\end{array}$ & $\underset{\widetilde{\Omega}}{\stackrel{\overbrace{}}{\sim}}$ & $\begin{array}{l}\stackrel{0}{0} \\
\stackrel{1}{n}\end{array}$ & $\begin{array}{l}\stackrel{\infty}{n} \\
\stackrel{\leftrightarrow}{\sim} \\
\widetilde{\sigma}\end{array}$ & $\begin{array}{l}\stackrel{\wp}{\curvearrowright} \\
\stackrel{\sigma}{\sigma}\end{array}$ & 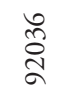 & $\begin{array}{l}\stackrel{\infty}{\sim} \\
\stackrel{\sim}{\sigma}\end{array}$ & 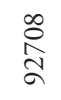 & 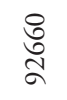 & $\begin{array}{l}\overrightarrow{\widetilde{S}} \\
\stackrel{\sigma}{ }\end{array}$ \\
\hline
\end{tabular}

Diagnostic species of the association (Diagnostične vrste asociacije)

ML Paraleucobryum sauteri

MuA Aconitum lycoctonum subsp. ranunculifolium

VP Luzula sylvatica

BA Salix appendiculata

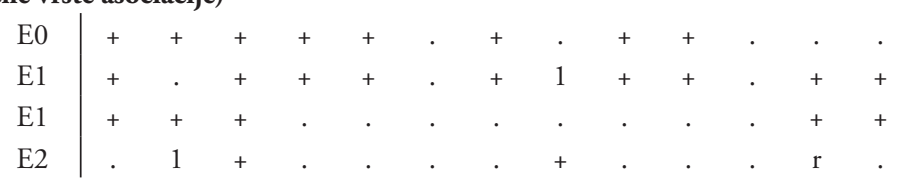




\begin{tabular}{|c|c|c|c|c|c|c|c|c|c|c|c|c|c|c|c|c|c|c|c|c|c|c|}
\hline & & & & & & & & & & & & 6 & & & & & 1 & 37 & 3 & & & \\
\hline 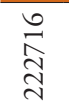 & 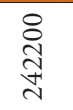 & 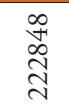 & $\begin{array}{l}\tilde{0} \\
\text { ปิ }\end{array}$ & 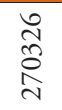 & तิ & $\stackrel{\infty}{\approx}$ & $\begin{array}{l}\infty \\
\infty \\
\sim\end{array}$ & $\begin{array}{l}\infty \\
\stackrel{\sim}{\sim}\end{array}$ & & $\begin{array}{l}\stackrel{\partial}{ } \\
\stackrel{\sim}{\sim}\end{array}$ & $\begin{array}{l}\text { के } \\
\text { }\end{array}$ & & & $\underset{\mathfrak{d}}{\vec{J}}$ & & $\underset{\widetilde{J}}{\widetilde{J}}$ & ָ̊ & & 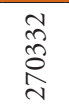 & & & \\
\hline & ID & & ID & ID & ID & & & ID & ID & & ID & D & & & D & $\mathrm{D}$ & ID & $\mathrm{D}$ & & $\mathrm{D}$ & D & \\
\hline$\stackrel{8}{ \pm}$ & 兽 & $\stackrel{\text { กิ }}{\sim}$ & 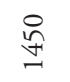 & $\begin{array}{l}\stackrel{\infty}{\infty} \\
\stackrel{+}{+}\end{array}$ & 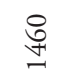 & $\stackrel{n}{n}$ & $\stackrel{\circ}{\curvearrowleft}$ & \pm & 离 & \begin{tabular}{l} 
S \\
\multirow{+}{*}{}
\end{tabular} & 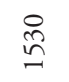 & $n$ & in & 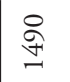 & $\begin{array}{l}\infty \\
\stackrel{\infty}{+} \\
+\end{array}$ & $\stackrel{\circ}{\stackrel{n}{n}}$ & 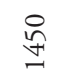 & 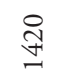 & 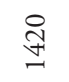 & $\simeq$ & 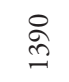 & \\
\hline IF & S & $\mathrm{NE}$ & W & SE & 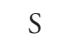 & E & SE & W & NW & SW & $\mathrm{NE}$ & $\mathrm{NE}$ & $\mathrm{NW}$ & & NW & & NW & $\mathrm{N}$ & NW & NW & I w & \\
\hline & 20 & & 20 & & 25 & 15 & & 3 & & 20 & & 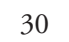 & & & & & & & & & & \\
\hline 4 & A & & $\mathrm{AI}$ & A & & A & AR & & & & & & & & & & & & & & & \\
\hline Re & $\mathrm{Re}$ & & $\mathrm{R}$ & $\mathrm{R}$ & $\mathrm{Re}$ & $\operatorname{Re}$ & & $\mathrm{R}$ & $\operatorname{Re}$ & & & & $\mathrm{R}$ & & & & & & & & & \\
\hline 30 & 30 & & 20 & 30 & 6 & & & & & & & & & & & & & & & & & \\
\hline 0 & & & 0 & & 90 & 90 & 1 & 80 & 90 & & 70 & 0 & & 0 & 0 & 0 & 75 & 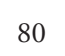 & & & & \\
\hline 10 & & & & & & & & & & & & & & & & & & & & & & \\
\hline 5 & 10 & 5 & 5 & 5 & 20 & 5 & & 1 & & & & & & & & & & & & & & \\
\hline 50 & 60 & & 70 & 60 & 5 & 60 & 60 & 8 & 80 & 60 & & & & & 70 & & & 30 & & & & \\
\hline 20 & & & & & & & & & & & & & & & & & & & & & & \\
\hline 45 & 35 & & 30 & & 3 & 25 & 25 & 5 & & & & 0 & & & 0 & & & & & & & \\
\hline 8 & & & 12 & & 1 & 7 & & & & & & & & & & & & & & & & \\
\hline & & & 54 & & 5 & 56 & & 46 & & & & 48 & & & & & & & & & & \\
\hline & & & 400 & & 400 & 400 & & 400 & 400 & 200 & 400 & 200 & 200 & J0 & 400 & 400 & 400 & 400 & 400 & 400 & 400 & \\
\hline ڤू & 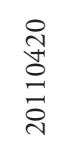 & $\begin{array}{l}\overparen{\hat{े}} \\
\hat{ٍ}\end{array}$ & $\begin{array}{l}\hat{\delta} \\
\stackrel{+}{+} \\
\stackrel{+}{0}\end{array}$ & $\begin{array}{l}\hat{o} \\
\stackrel{d}{+} \\
\stackrel{d}{\circ}\end{array}$ & 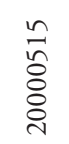 & $\begin{array}{l}\hat{o} \\
\text { Oे } \\
\stackrel{0}{0}\end{array}$ & $\begin{array}{l}\tilde{\delta} \\
\delta \\
\delta \\
\text { ¿े }\end{array}$ & $\begin{array}{l}\sigma \\
\vec{ठ} \\
\stackrel{\swarrow}{\sigma} \\
\sigma\end{array}$ & 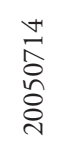 & $\begin{array}{l}\text { J゙ } \\
\stackrel{0}{\circ} \\
\stackrel{\sigma}{\sigma}\end{array}$ & 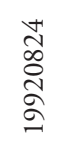 & \begin{tabular}{l}
\multirow{J}{*}{} \\
$\stackrel{\sim}{\Delta}$ \\
$\stackrel{\sigma}{\sigma}$
\end{tabular} & $\underset{\infty}{\mathbb{J}}$ & 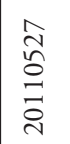 & $\begin{array}{l}\hat{\widehat{V}} \\
\hat{ٍ}\end{array}$ & $\hat{\tilde{N}}$ & 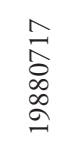 & 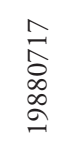 & $\begin{array}{l}\infty \\
\infty \\
\infty \\
\infty \\
\infty \\
ٍ\end{array}$ & $\begin{array}{l}\hat{O} \\
\infty \\
\infty \\
\approx\end{array}$ & $\begin{array}{l}\hat{\hat{O}} \\
\infty \\
\stackrel{0}{0}\end{array}$ & \\
\hline 6 & 20 & & & 7 & 15 & & 5 & 19 & 4 & 24 & 24 & 4 & 24 & & 77 & & 17 & 17 & & 17 & 17 & \\
\hline 9 & 4 & & & 6 & & & & & & & & & & & & & & & & & & \\
\hline & $\overline{\vec{d}}$ & $\bar{\circ}$ & 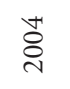 & ث゙ & 8 & ڤ్రి & గ & 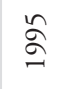 & ڤ̊ํे & Әे & Д̆ & $\tilde{\partial}$ & $g$ & $\overrightarrow{\widetilde{d}}$ & $\overrightarrow{\bar{\imath}}$ & $\bar{N}$ & $\begin{array}{l}\infty \\
\stackrel{\infty}{\beth}\end{array}$ & & $\begin{array}{l}\infty \\
\stackrel{\infty}{\Omega}\end{array}$ & $\begin{array}{l}\infty \\
\stackrel{\infty}{\beth}\end{array}$ & $\begin{array}{l}\infty \\
\stackrel{\infty}{\Omega}\end{array}$ & \\
\hline 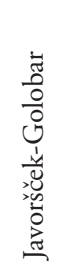 & 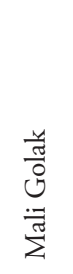 & 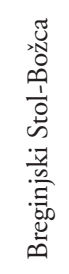 & 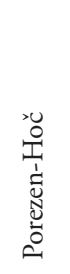 & 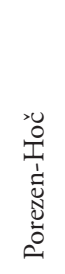 & 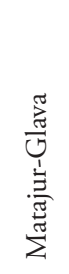 & ; & $;: \frac{\cup}{N}$ & 兰 & 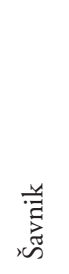 & 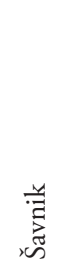 & 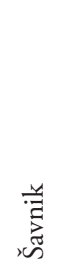 & $\frac{\breve{a}}{\vec{\Xi}}$ & 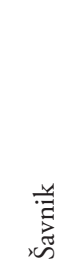 & 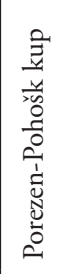 & 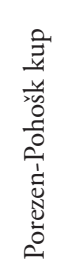 & 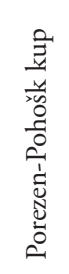 & 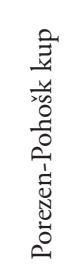 & 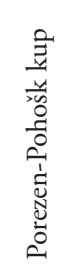 & 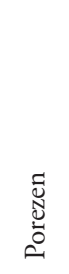 & 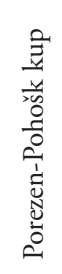 & 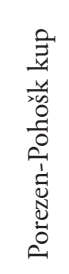 & \\
\hline 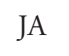 & $\mathrm{T}$ & $\mathrm{J} A$ & 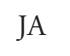 & & JA & & & JA & & JA & JA & & & & & & & & & A & 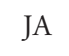 & \\
\hline$\stackrel{+H}{N}$ & $\begin{array}{l}\bar{a} \\
\text { वे }\end{array}$ & $\underset{S}{S}$ & $\frac{\mathfrak{d}}{\stackrel{T}{a}}$ & $\frac{a}{\sqrt[a]{2}}$ & $\stackrel{m}{\perp}$ & $\stackrel{m}{\triangleright}$ & $\overbrace{n}^{m}$ & $\stackrel{\Delta}{a}$ & $\frac{\Downarrow}{\stackrel{+}{a}}$ & $\frac{t}{a}$ & $\frac{+}{a}$ & $\frac{\vec{y}}{\stackrel{a}{a}}$ & $\stackrel{+}{9}$ & $\frac{2}{2}$ & बे & $\frac{a}{a}$ & $\approx$ & $\underset{\substack{+\sigma \\
\alpha}}{\frac{N}{\alpha}}$ & 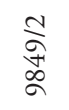 & જे & హे & \\
\hline ڤ్రి & 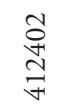 & $\underset{\Xi}{ت}$ & $\begin{array}{l}\hat{n} \\
\underset{\tilde{I}}{*}\end{array}$ & స్ & 육 & 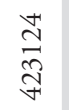 & $\tilde{c}$ & ळे & $\infty$ & $\widehat{\hat{m}}$ & $\stackrel{\unlhd}{J}$ & 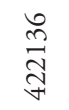 & ฉ & $\begin{array}{c}\infty \\
\infty \\
\infty\end{array}$ & $\stackrel{\infty}{\cdots}$ & $\hat{\infty}$ & $\approx$ & 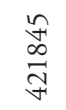 & $\vec{m}$ & $\underset{n}{n}$ & 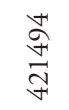 & \\
\hline Iิ & $\begin{array}{l}\infty \\
\tilde{N} \\
\tilde{\sigma}\end{array}$ & 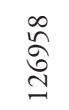 & $\hat{\sigma}_{\Xi}^{\infty}$ & $\tilde{g}$ & $\begin{array}{l}\stackrel{+}{ } \\
\stackrel{0}{\Xi} \\
\Xi\end{array}$ & 윰 & 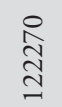 & $\stackrel{n}{n}$ & $\stackrel{\mathcal{F}}{\mathbb{T}}$ & $\underset{\widetilde{N}}{\stackrel{\Xi}{\Xi}}$ & 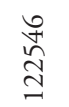 & $\hat{\circ}$ & $\begin{array}{l}\text { ్ㅠ } \\
\text { N } \\
\text { I }\end{array}$ & 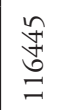 & $\overrightarrow{\tilde{n}}$ & $\begin{array}{l}\underset{\widetilde{ర}}{\Xi} \\
\underset{\Xi}{\sigma}\end{array}$ & $\begin{array}{l}\stackrel{n}{\hat{\sigma}} \\
\underline{=}\end{array}$ & $\begin{array}{l}\stackrel{0}{0} \\
\swarrow\end{array}$ & 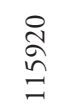 & $\begin{array}{l}\text { ్ㅠㅇ } \\
\text { the }\end{array}$ & 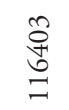 & \\
\hline
\end{tabular}




\section{Number of relevé (Zaporedna številka popisa)

$\begin{array}{lllllllllllll}1 & 2 & 3 & 4 & 5 & 6 & 7 & 8 & 9 & 10 & 11 & 12 & 13\end{array}$

VP Polystichum lonchitis

VP Clematis alpina

BA Sorbus chamaemespilus

VP Lonicera caerulea

PS Paederota lutea

CA Festuca calva

BA Salix appendiculata

CF Carex ferruginea

\begin{tabular}{l|lll} 
E1 & $\cdot$ & + & + \\
E2 & $\cdot$ & + & + \\
E2 & $\cdot$ & + & + \\
E2 & $\cdot$ & + & \\
E1 & $\cdot$ & + & + \\
E1 & $\cdot$ & $\cdot$ & \\
E1 & $\cdot$ & $\cdot$ & + \\
E1 & $\cdot$ & $\cdot$ & +
\end{tabular}

Differential species of the subassociation and variant (Razlikovalnice subasociacije in variante)

MuA Stellaria nemorum

FS Corydalis cava

$\mathrm{MuA}$ Adenostyles alliariae

MuA Myrrhis odorata

BA Ribes alpinum

MuA Chaerophyllum villarsii

BA Alnus viridis

AF Aremonio-Fagion

Cardamine enneaphyllos

Cardamine trifolia

Omphalodes verna

Rhamnus fallax

Vicia oroboides

Cyclamen purpurascens

Euphorbia carniolica

Anemone trifolia

Knautia drymeia

Lamium orvala

\begin{tabular}{l|ccccccccccccc} 
E1 &. & 1 & + & 2 & 1 & 1 &. & 1 &. &. &. &. & $\mathrm{r}$ \\
E1 & 1 &. &. &. & + & 1 & 1 & 1 & 1 & 1 & + & 1 &. \\
E1 & 1 & 2 & 2 & 2 & 3 &. & + & 2 & + &. &. &. &. \\
E1 & 1 & 1 & 1 &. &. &. & r &. & r &. & + & + & 1 \\
E2 &. & + & + & + &. &. &. & + &. & r & + & + &. \\
E1 &. &. &. &. &. &. &. &. &. &. &. &. &. \\
E2 &. &. &. &. &. &. &. &. &. &. &. &. &.
\end{tabular}

EC Erythronio-Carpinion

Primula vulgaris

Helleborus odorus

Ornithogalum pyrenaicum

Crocus vernus subsp. vernus (C. napolitanus)

TA Tilio-Acerion

Adoxa moschatellina

Acer pseudoplatanus

Acer pseudoplatanus

Acer pseudoplatanus

Polystichum aculeatum

Chrysosplenium alternifolium

Corydalis solida

Geranium robertianum

Stellaria montana

Aruncus dioicus

Lunaria rediviva

Polystichum braunii

Phyllitis scolopendrium

Hesperis candida

Polystichum $x$ illyricum

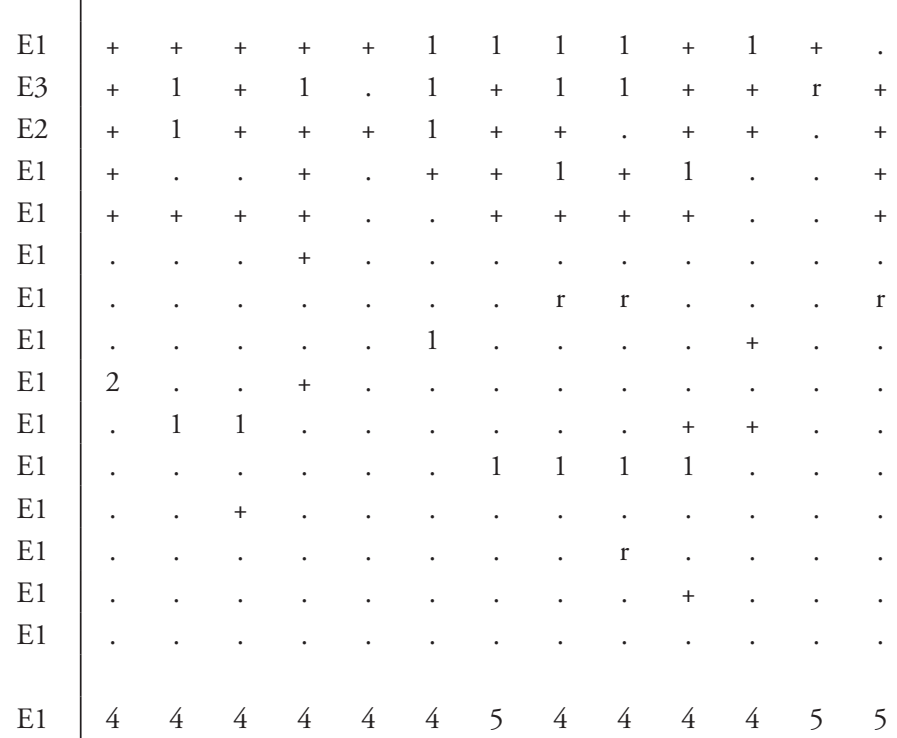

Fagus sylvatica 


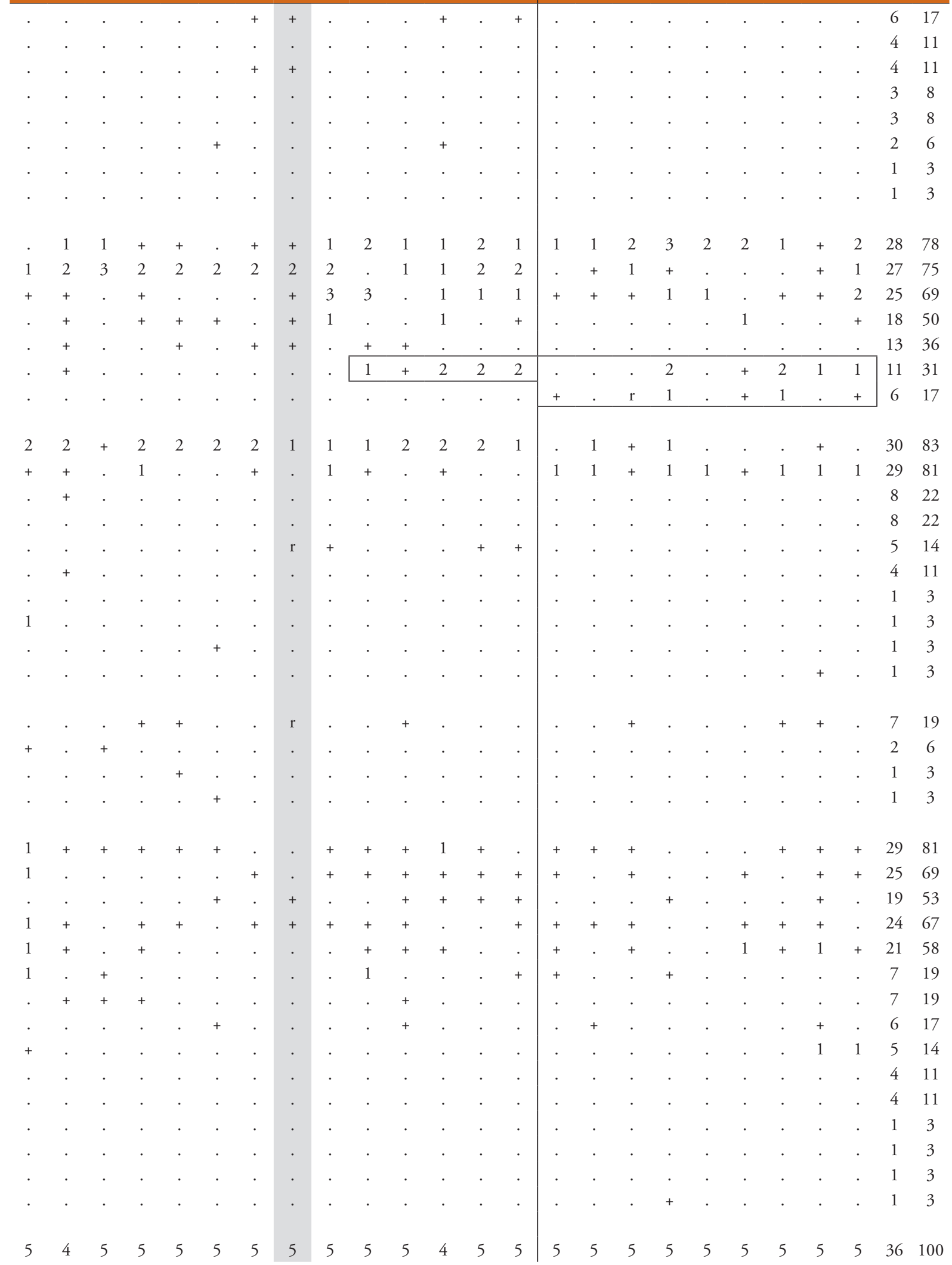


Fagus sylvatica

Fagus sylvatica

Dryopteris filix-mas

Galeobdolon flavidum

Lilium martagon

Paris quadrifolia

Actaea spicata

Symphytum tuberosum

Cardamine bulbifera

Daphne mezereum

Epilobium montanum

Lonicera alpigena

Galium laevigatum

Mercurialis perennis

Ranunculus lanuginosus

Heracleum sphondylium

Scrophularia nodosa

Myosotis sylvatica agg.

Mycelis muralis

Campanula trachelium

Carex sylvatica

Fraxinus excelsior

Galium odoratum

Prenanthes purpurea

Lathyrus vernus

Melica nutans

Phyteuma spicatum subsp. coeruleum

Festuca altissima

Leucojum vernum

Salvia glutinosa

Luzula nivea

Pulmonaria officinalis

Cardamine impatiens

Neottia nidus-avis

Polygonatum multiflorum

Lathyrus vernus subsp. flaccidus

Euphorbia amygdaloides

Prunus avium

Laburnum alpinum

QP Quercetalia pubescenti-petraeae

Sorbus aria (Aria edulis)

Sorbus aria (Aria edulis)

Melittis melissophyllum

Querco-Fagetea

Anemone nemorosa

Poa nemoralis

Anemone ranunculoides

Stellaria holostea

Gagea lutea

Ranunculus auricomus agg. (R. braun-blanquetii)

$$
\begin{aligned}
& \begin{array}{l|ccccccccccccc} 
& 1 & 2 & 3 & 4 & 5 & 6 & 7 & 8 & 9 & 10 & 11 & 12 & 13 \\
\text { E2 } & 1 & . & 1 & 1 & 1 & 2 & 1 & 1 & + & 1 & + & . & . \\
\text { E1 } & . & + & + & + & + & . & + & + & + & . & . & . & . \\
\text { E1 } & 2 & 2 & 2 & 2 & 2 & 2 & + & 1 & 1 & 1 & 1 & + & + \\
\text { E1 } & . & 1 & 1 & 1 & + & 1 & 1 & + & + & 1 & . & + & + \\
\text { E1 } & + & 1 & 1 & + & . & + & . & + & + & 1 & + & 1 & + \\
\text { E1 } & . & + & + & + & + & + & + & + & + & + & 1 & + & . \\
\text { E1 } & + & + & + & + & + & + & 1 & 1 & + & + & + & 1 & . \\
\text { E1 } & + & + & + & 1 & . & 1 & . & . & . & . & 1 & 1 & + \\
\text { E1 } & + & + & . & 1 & . & 1 & 2 & 1 & 2 & 1 & 1 & 1 & . \\
\text { E2 } & 1 & 1 & + & . & + & + & + & + & + & + & + & + & + \\
\text { E1 } & + & + & + & 1 & 1 & + & 1 & + & . & + & . & . & . \\
\text { E2 } & + & + & 1 & . & . & + & + & + & + & + & + & 1 & 1 \\
\text { E1 } & . & + & . & . & . & . & . & . & . & . & + & 1 & + \\
\text { E1 } & 1 & . & . & . & + & + & 1 & 1 & 2 & 2 & 1 & 1 & 1
\end{array} \\
& \text { E1 } \\
& \text { E1 }
\end{aligned}
$$




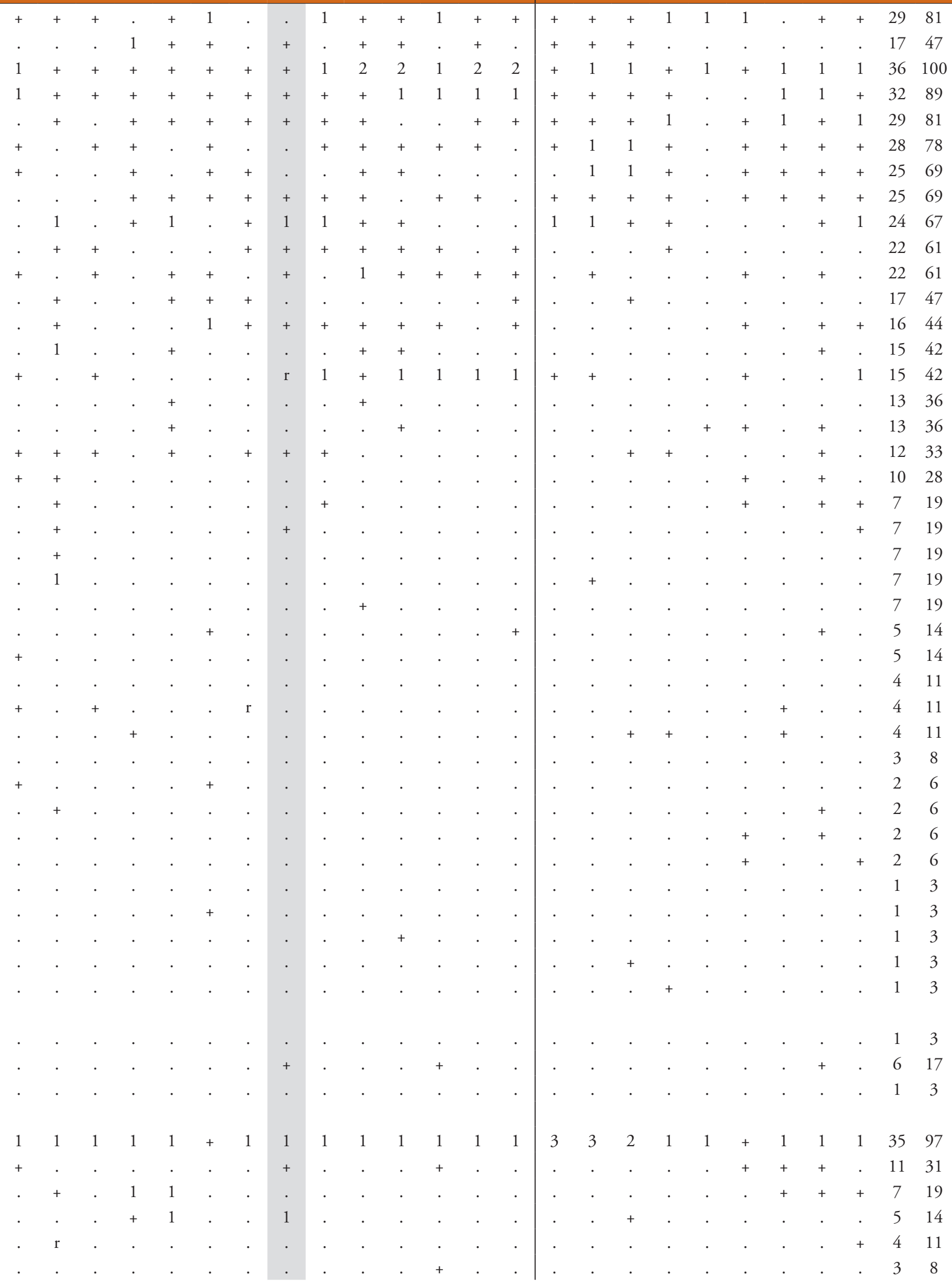


Lonicera xylosteum

Festuca heterophylla

Scilla bifolia

Dactylorhiza fuchsii

Viola riviniana

Carex pilosa

Hepatica nobilis

VP Vaccinio-Piceetea

Gentiana asclepiadea

Dryopteris dilatata

Aposeris foetida

Oxalis acetosella

Calamagrostis arundinacea

Luzula luzuloides

Lonicera nigra

Maianthemum bifolium

Veronica urticifolia

Vaccinium myrtillus

Picea abies

Picea abies

Picea abies

Gymnocarpium dryopteris

Solidago virgaurea

Rosa pendulina

Luzula luzulina

Dryopteris expansa

Phegopteris connectilis

Homogyne sylvestris

Huperzia selago

Circaea alpina

Abies alba

Larix decidua

Luzula pilosa

Thelypteris limbosperma

Avenella flexuosa

EP

Erico-Pinetea

Calamagrostis varia

E2

E1

E1

E1

E1

E1

E1

E1

E1

E1

E1

E1

E1

E2

E1

E1

E1

E3

E2

E1

E1

E1

E2

E1

E1

E1

E1

E1

E1

E3

E3

E1

E1

E1

Rubus saxatilis

SSC

Sambuco-Salicion capreae, Rhamno-Prunetea

Sorbus aucuparia

Sorbus aucuparia

Sorbus aucuparia

Sambucus racemosa

RP Berberis vulgaris

$\mathrm{RP}$ Cotoneaster integerrimus

Salix caprea

Betula pendula

Betulo-Alnetea

Ribes uva-crispa \\ $\begin{array}{lllllllllllll}1 & 2 & 3 & 4 & 5 & 6 & 7 & 8 & 9 & 10 & 11 & 12 & 13\end{array}$}

E1

(1)

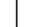

(1)

2

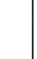

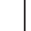

(1)

(1)

(1)

西

(1)




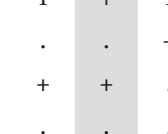

. 
Number of relevé (Zaporedna številka popisa)

MuA Mulgedio-Aconitetea

Veratrum album

Athyrium filix-femina

Polygonatum verticillatum

Saxifraga rotundifolia

Senecio ovatus

Doronicum austriacum

Milium effusum

Rumex arifolius

Ranunculus platanifolius

Viola biflora

Thalictrum aquilegiifolium

Senecio cacaliaster

Cicerbita alpina

Aconitum degenii subsp. paniculatum

Hypericum maculatum

Chaerophyllum hirsutum

Geum rivale

Allium victorialis

Poa hybrida

Phyteuma ovatum

Carduus personata

Centaurea montana

Silene dioica

Lathyrus occidentalis var. montanus

Anthriscus nitida

Pleurospermum austriacum

Chaerophyllum aureum

Athyrium distentifolium

Primula elatior

Geranium sylvaticum

Alchemilla xanthochlora

Agropyron caninum

Tanacetum corymbosum subsp. clusii

CA Caricion austroalpinae

Heracleum austriacum subsp. siifolium

ES Elyno-Seslerietea

Phyteuma orbiculare

Betonica alopecuros

NS Nardion strictae, Juncetea trifidi

Campanula scheuchzeri

Veronica officinalis

Festuco-Brometea

Cirsium erisithales

Trifolio-Geranietea

Laserpitium latifolium

Grafia golaka

Lilium carniolicum

Verbascum lanatum

Achillea distans
E1

E1

E1

E1

E1

E1

E1

E1

E1

E1

E1

E1

E1

E1

E1

E1

E1

E1

E1

E1

E1

E1

E1

E1

E1

E1

E1

E1

E1

E1

E1

E1

E1

E1

E1

E1

E1

E1

E1

E1

E1

E1

E1

E1 $\begin{array}{lllllllllllll}1 & 2 & 3 & 4 & 5 & 6 & 7 & 8 & 9 & 10 & 11 & 12 & 13\end{array}$

,

$\begin{array}{lllllllllllll}1 & 1 & 1 & 1 & 1 & 1 & 2 & 2 & 2 & 2 & 2 & 2 & 2\end{array}$

$\begin{array}{llllllllllll}1 & 1 & 2 & 2 & 1 & + & + & 1 & 1 & 1 & +\end{array}$

$1+1+++++++1+$

$+111++\ldots+++++$

1

$+1$

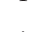

1

(1)

1

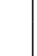

(1)

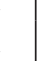




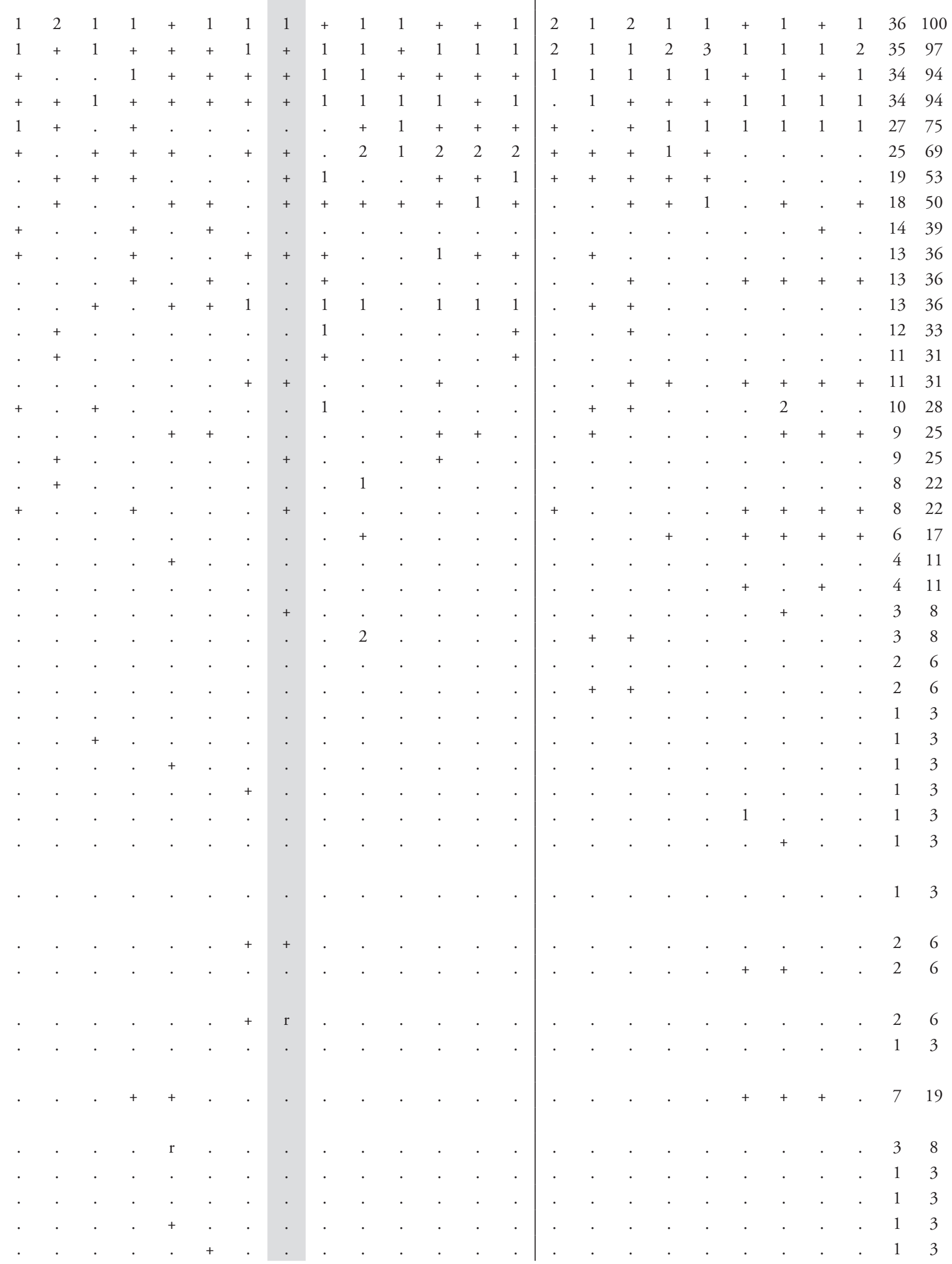




\begin{tabular}{|c|c|c|c|c|c|c|c|c|c|c|c|c|c|c|c|}
\hline & Number of relevé (Zaporedna številka popisa) & & 1 & 2 & 3 & 4 & 5 & 6 & 7 & 8 & 9 & 10 & 11 & 12 & 13 \\
\hline & Vicia sylvatica & E1 & . & . & . & . & . & . & . & . & . & . & . & . & . \\
\hline \multirow[t]{6}{*}{$\mathrm{PaT}$} & Poo alpinae-Trisetetalia & & & & & & & & & & & & & & \\
\hline & Poa alpina & $\mathrm{E} 1$ & . & . & . & . & . & . & . & . & . & . & . & + & . \\
\hline & Crocus albiflorus & $\mathrm{E} 1$ & . & . & . & . & . & . & . & . & . & . & . & . & . \\
\hline & Cardaminopsis ovirensis & $\mathrm{E} 1$ & . & . & . & . & . & . & . & . & . & . & . & . & . \\
\hline & Astrantia major & E1 & . & . & . & . & . & . & . & . & . & . & . & . & . \\
\hline & Trollius europaeus & $\mathrm{E} 1$ & . & . & . & . & . & . & . & . & . & . & . & . & . \\
\hline \multirow[t]{6}{*}{ MA } & Molinio-Arrbenatberetea & & & & & & & & & & & & & & \\
\hline & Angelica sylvestris & $\mathrm{E} 1$ & . & + & + & + & . & + & + & + & . & . & + & + & . \\
\hline & Deschampsia cespitosa & $\mathrm{E} 1$ & . & . & . & . & + & . & . & . & $\mathrm{r}$ & . & . & . & . \\
\hline & Dactylis glomerata s.str. & E1 & . & . & . & . & . & 1 & . & . & . & . & . & . & . \\
\hline & Crepis paludosa & $\mathrm{E} 1$ & . & . & . & . & . & . & . & . & . & . & . & . & . \\
\hline & Galium album & $\mathrm{E} 1$ & . & . & . & . & . & . & . & . & . & . & . & . & . \\
\hline \multirow[t]{10}{*}{ EA } & Epilobietea angustifolii, Galio-Urticetea & & & & & & & & & & & & & & \\
\hline & Rubus idaeus & E2 & 1 & 2 & 2 & 2 & 3 & 2 & 1 & 2 & 2 & 1 & + & + & + \\
\hline & Urtica dioica & $\mathrm{E} 1$ & . & . & . & + & . & + & + & + & + & . & + & . & . \\
\hline & Lamium maculatum & E1 & . & . & . & . & . & . & . & . & . & · & . & . & . \\
\hline & Fragaria vesca & E1 & + & + & . & . & . & . & . & . & . & · & . & . & . \\
\hline & Galeopsis speciosa & E1 & . & . & . & . & . & + & . & + & . & + & . & . & . \\
\hline & Solanum dulcamara & $\mathrm{E} 1$ & . & . & . & . & . & + & . & . & . & . & . & . & . \\
\hline & Hypericum hirsutum & E1 & . & . & . & . & . & . & . & . & . & + & . & . & . \\
\hline & Stachys alpina & E1 & . & . & . & . & . & . & . & . & . & . & . & . & . \\
\hline & Stellaria media & $\mathrm{E} 1$ & . & . & . & . & . & . & . & . & . & . & . & . & . \\
\hline \multirow[t]{2}{*}{$\mathrm{AC}$} & Arabidetalia caeruleae & & & & & & & & & & & & & & \\
\hline & Soldanella alpina & E1 & & & & & & & & & & & & & \\
\hline \multirow[t]{5}{*}{ TR } & Thlaspietea rotundifolii & & & & & & & & & & & & & & \\
\hline & Adenostyles glabra & $\mathrm{E} 1$ & + & + & 1 & + & . & 1 & . & + & + & + & . & . & . \\
\hline & Heracleum pollinianum & $\mathrm{E} 1$ & . & . & . & . & . & . & . & . & . & . & . & . & . \\
\hline & Arabis alpina & $\mathrm{E} 1$ & . & . & . & . & . & . & . & . & . & . & . & . & . \\
\hline & Molopospermum peloponnesiacum subsp. bauhinii & E1 & . & . & . & . & . & . & . & . & . & . & . & . & . \\
\hline \multirow[t]{7}{*}{ Cy } & Cystopteridion fragilis & & & & & & & & & & & & & & \\
\hline & Cystopteris fragilis & E1 & . & + & + & 1 & + & . & + & + & . & + & + & + & + \\
\hline & Asplenium viride & $\mathrm{E} 1$ & . & + & + & . & . & . & . & . & . & + & . & . & . \\
\hline & Valeriana tripteris & E1 & . & . & + & . & . & . & . & . & . & . & + & + & + \\
\hline & Sedum hispanicum & E1 & . & . & . & . & . & + & + & + & . & + & + & . & . \\
\hline & Moehringia muscosa & $\mathrm{E} 1$ & . & . & + & . & . & . & . & . & . & . & . & + & . \\
\hline & Cystopteris regia & E1 & . & . & . & . & . & . & . & . & . & . & . & . & . \\
\hline \multirow[t]{3}{*}{ AT } & Asplenietea trichomanis & & & & & & & & & & & & & & \\
\hline & Polypodium vulgare & $\mathrm{E} 1$ & . & + & . & + & . & . & + & . & + & . & . & . & . \\
\hline & Asplenium trichomanes & E1 & . & . & . & . & . & . & . & + & . & + & + & . & . \\
\hline \multirow[t]{10}{*}{ ML } & Mosses and lichens (Mahovi in lišaji) & & & & & & & & & & & & & & \\
\hline & Ctenidium molluscum & E0 & 1 & 1 & 2 & 1 & + & + & 1 & 2 & 2 & 2 & 1 & 1 & 1 \\
\hline & Isothecium alopecuroides & E0 & + & 1 & 1 & 1 & + & + & 1 & 1 & + & . & . & . & + \\
\hline & Pseudoleskeella catenulata & E0 & . & . & . & + & + & + & + & 1 & . & 1 & 1 & . & 1 \\
\hline & Schistidium apocarpum & E0 & 1 & + & 1 & + & . & 1 & + & . & 1 & . & + & . & . \\
\hline & Peltigera canina & E0 & + & + & . & + & + & + & + & + & . & + & . & . & . \\
\hline & Tortella tortuosa & E0 & . & + & 1 & . & . & + & + & . & . & + & . & + & . \\
\hline & Brachythecium rutabulum & E0 & . & . & . & 1 & . & . & . & . & . & . & . & . & . \\
\hline & Homalothecium lutescens & E0 & . & . & . & . & . & 1 & + & 1 & + & + & + & . & . \\
\hline & Mnium thomsonii & E0 & . & + & . & . & . & . & . & . & . & . & . & . & . \\
\hline
\end{tabular}




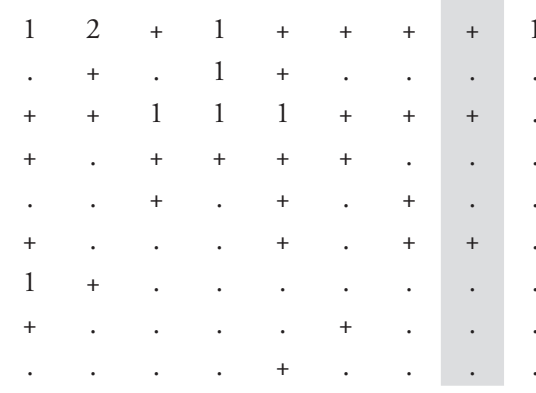


Number of relevé (Zaporedna številka popisa)

$\begin{array}{lllllllllllll}1 & 2 & 3 & 4 & 5 & 6 & 7 & 8 & 9 & 10 & 11 & 12 & 13\end{array}$

Plagiochila porelloides

Homalothecium philippeanum

Conocephalum conicum

Fissidens dubius

Polytrichum formosum

Cladonia pyxidata

Dicranum scoparium

Bryum capillare

Hypnum cupressiforme

Eurhynchium angustirete

Atrichum undulatum

Plagiomnium cuspidatum

Brachythecium velutinum

Plagiothecium denticulatum

Plagiomnium undulatum

Plagiothecium sp.

Plagiothecium nemorale

Mnium sp.

Lobaria pulmonaria

Hypogymnia physodes

Bryum sp.

Dicranella heteromalla

Cladonia fimbriata

Anomodon attenuatus

Mnium marginatum

Parmelia submontana

Collema cristatum

\begin{tabular}{|c|c|c|c|c|c|c|c|c|c|c|c|c|c|}
\hline E0 & . & . & . & . & . & . & . & . & . & . & . & . & . \\
\hline E0 & + & . & . & . & . & . & . & . & . & . & . & + & . \\
\hline E0 & . & . & + & . & . & . & . & . & . & . & . & . & . \\
\hline E0 & . & . & . & . & . & . & . & . & . & . & . & . & . \\
\hline E0 & + & . & . & . & . & . & . & . & . & . & . & . & . \\
\hline E0 & . & . & . & + & . & + & . & + & . & . & . & . & . \\
\hline E0 & . & . & . & . & . & . & . & + & . & . & . & . & . \\
\hline E0 & . & . & . & . & . & . & . & . & . & . & . & . & . \\
\hline E0 & . & . & . & . & . & . & . & . & . & . & . & . & . \\
\hline E0 & . & . & . & + & . & . & . & . & . & . & . & . & . \\
\hline E0 & . & . & . & . & + & . & . & . & . & . & . & . & . \\
\hline E0 & . & . & . & . & . & . & . & . & + & . & . & . & . \\
\hline E0 & . & . & . & . & . & . & . & . & . & . & . & . & . \\
\hline E0 & . & . & . & . & . & . & . & . & . & . & . & . & . \\
\hline E0 & . & . & . & . & . & . & . & . & . & . & . & . & . \\
\hline E0 & . & . & . & . & . & . & . & . & . & . & . & . & . \\
\hline E0 & . & . & . & . & . & . & . & . & . & . & . & . & . \\
\hline E0 & . & . & . & . & . & . & . & . & . & . & . & . & . \\
\hline E0 & . & . & . & . & . & . & . & . & . & . & . & . & . \\
\hline E1 & . & . & . & . & . & . & . & . & . & . & . & . & . \\
\hline E0 & . & . & . & . & . & . & . & . & . & . & . & . & . \\
\hline E0 & . & . & . & . & . & . & . & . & . & . & . & . & . \\
\hline E0 & . & . & . & . & . & . & . & . & . & . & . & . & . \\
\hline E0 & . & . & . & . & . & . & . & . & . & . & . & . & . \\
\hline E0 & . & . & . & . & . & . & . & . & . & . & . & . & . \\
\hline E0 & . & . & . & . & . & . & . & . & . & . & . & . & . \\
\hline E0 & . & . & . & . & . & . & . & . & . & . & . & . & \\
\hline
\end{tabular}

\section{Legend - Legenda}

ID Igor Dakskobler

A Limestone-apnenec

L Marlstone- laporovec

R Chert - roženec

Re Rendzina - rendzina

$\mathrm{Rj}$ Calcareous brown soil - rjava pokarbonatna tla

TG Trnovski Gozd Plateau - Trnovski gozd

JA Julian Alps - Julijske Alpe

Pr. Presence (number of relevés in which the species is presented) - število popisov, v katerih se pojavlja vrsta

Fr. Frequency in $\%$ - frekvenca $v \%$

Columns 1-27: Polysticho lonchitis-Fagetum stellarietosum nemorum

Relevé No. 21, holotypus

Columns 28-36: Ranunculo platanifolii-Fagetum stellarietosum nemorum 


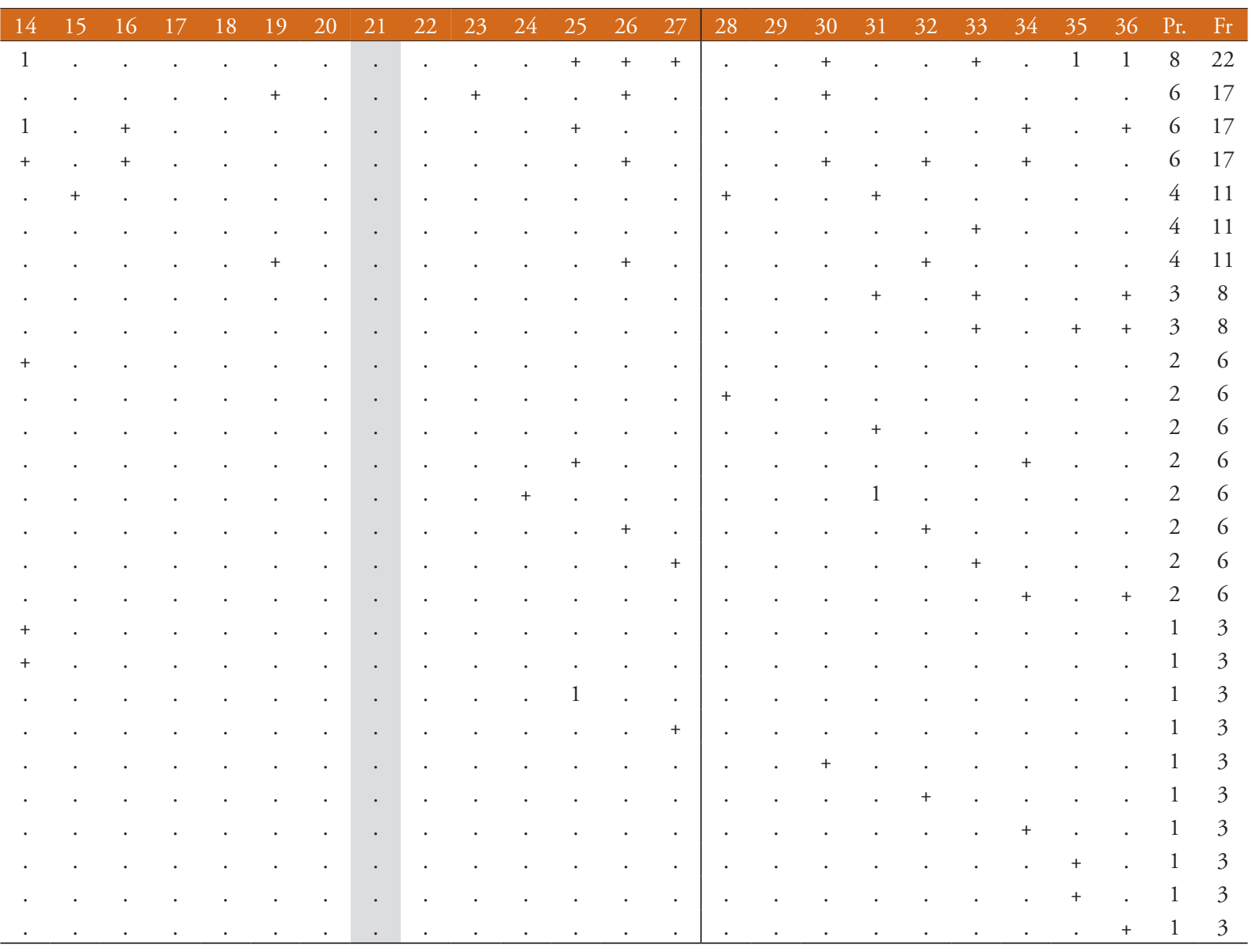


Table 9: Synoptic table of the altimontane and subalpine beech communities in the SE-Alps and NW-Dinaric Alps.

Tabela 9: Sintezna tabela altimontanskih in subalpinskih bukovih združb v Jugovzhodnih Alpah in severnem delu Dinarskega gorstva.

\begin{tabular}{|c|c|c|c|c|c|c|c|c|c|c|}
\hline Successive number (Zaporedna številka) & & 1 & 2 & 3 & 4 & 5 & 6 & 7 & 8 & 9 \\
\hline Sign for syntaxa (Oznaka sintaksonov) & & ApF & $\operatorname{SmF}$ & $\mathrm{CwF}$ & $\mathrm{MoF}$ & $\mathrm{AtF}$ & $\mathrm{SrF}$ & $\mathrm{RpF}$ & $\mathrm{PlF}$ & RhF \\
\hline Number of relevés (Število popisov) & & 8 & 16 & 7 & 2 & 231 & 73 & 236 & 614 & 232 \\
\hline \multicolumn{11}{|l|}{ Aremonio-Fagion } \\
\hline Cardamine enneaphyllos & E1 & 50 & 31 & 29 & 100 & 46 & 78 & 78 & 78 & 53 \\
\hline Anemone trifolia & E1 & 38 & . & . & . & 93 & 90 & 5 & 58 & 46 \\
\hline Cyclamen purpurascens & E1 & 25 & 19 & . & . & 66 & 36 & 39 & 53 & 84 \\
\hline Knautia drymeia & E1 & 12 & . & 57 & . & 10 & 12 & 1 & 17 & 17 \\
\hline Cardamine trifolia & E1 & 12 & 81 & 57 & 100 & 43 & 73 & 77 & 37 & 24 \\
\hline Lamium orvala & E1 & . & 56 & . & 100 & 8 & 8 & 16 & 4 & 2 \\
\hline Aremonia agrimonoides & $\mathrm{E} 1$ & . & 38 & . & . & 19 & 32 & 43 & 2 & 1 \\
\hline Vicia oroboides & E1 & . & 19 & . & 50 & 1 & 7 & 25 & 3 & . \\
\hline Cardamine waldsteinii & E1 & . & . & 86 & . & . & . & . & . & . \\
\hline Helleborus niger & $\mathrm{E} 1$ & . & . & . & . & 53 & 77 & 42 & 15 & 36 \\
\hline Rhamnus fallax & E2 & . & . & . & . & 3 & 5 & 4 & 16 & 35 \\
\hline Hacquetia epipactis & E1 & . & . & . & . & 2 & 12 & 18 & 1 & 4 \\
\hline Calamintha grandiflora & E1 & . & . & . & . & 2 & . & 24 & . & 2 \\
\hline Daphne laureola & E1 & . & . & . & . & 1 & . & . & . & . \\
\hline Omphalodes verna & E1 & . & . & . & . & . & . & 7 & 6 & 37 \\
\hline Euphorbia carniolica & E1 & . & . & . & . & . & . & 4 & 5 & 16 \\
\hline Laserpitium krapfii & E1 & . & . & . & . & . & . & 3 & 1 & 4 \\
\hline Anemone pittonii & E1 & . & . & . & . & . & . & . & 1 & 3 \\
\hline Hemerocallis lilioasphodelus & E1 & . & . & . & . & . & . & . & 1 & 4 \\
\hline Daphne laureola & $\mathrm{E} 2$ & . & . & . & . & . & . & . & . & 2 \\
\hline Potentilla carniolica & E1 & . & . & . & . & . & . & . & . & 2 \\
\hline Daphne blagayana & E2 & . & . & . & . & . & . & . & . & 1 \\
\hline Knautia drymea subsp. intermedia & E1 & . & . & . & . & . & . & . & . & 1 \\
\hline \multicolumn{11}{|l|}{ Erythronio-Carpinion } \\
\hline Primula vulgaris & E1 & . & . & . & . & 9 & . & 11 & 8 & 24 \\
\hline Isopyrum thalictroides & E1 & . & . & . & . & . & . & 11 & . & . \\
\hline Helleborus odorus & E1 & . & . & . & . & . & . & 8 & 6 & . \\
\hline Crocus vernus subsp. vernus & E1 & . & . & . & . & . & . & 9 & $\mathrm{r}$ & . \\
\hline Galanthus nivalis & E1 & . & . & . & . & . & . & 1 & 1 & . \\
\hline Ornithogalum pyrenaicum & E1 & . & . & . & . & . & . & . & 1 & . \\
\hline Epimedium alpinum & E1 & . & . & . & . & . & . & . & . & 1 \\
\hline \multicolumn{11}{|l|}{ Tilio-Acerion } \\
\hline Acer pseudoplatanus & E3 & 100 & 100 & 100 & 100 & 10 & 18 & 43 & 26 & 48 \\
\hline Acer pseudoplatanus & E2 & 88 & 75 & 29 & 50 & 12 & 5 & 45 & 25 & 44 \\
\hline Acer pseudoplatanus & E1 & . & 31 & 86 & . & 37 & 53 & 39 & 36 & 56 \\
\hline Adoxa moschatellina & E1 & 62 & 44 & 86 & 50 & . & 7 & 22 & 21 & . \\
\hline Impatiens noli-tangere & E1 & 50 & 6 & 43 & 50 & 1 & 4 & 2 & . & . \\
\hline Aruncus dioicus & E1 & 50 & 25 & . & 50 & 3 & 18 & 14 & 14 & 16 \\
\hline Geranium robertianum & E1 & 50 & 56 & . & 50 & 6 & 12 & 11 & 7 & 1 \\
\hline Polystichum aculeatum & E1 & 50 & 81 & . & 50 & 33 & 60 & 29 & 22 & 35 \\
\hline Chrysosplenium alternifolium & E1 & 38 & 50 & 14 & 50 & . & 1 & 9 & 4 & . \\
\hline
\end{tabular}




\begin{tabular}{|c|c|c|c|c|c|c|c|c|c|c|}
\hline \multicolumn{2}{|c|}{ Successive number (Zaporedna številka) } & \multirow{2}{*}{$\begin{array}{c}1 \\
25\end{array}$} & \multirow{2}{*}{$\begin{array}{c}2 \\
19\end{array}$} & \multirow{2}{*}{3} & \multirow{2}{*}{4} & \multirow{2}{*}{$\begin{array}{l}5 \\
1\end{array}$} & \multirow{2}{*}{$\begin{array}{l}6 \\
1\end{array}$} & \multirow{2}{*}{$\begin{array}{l}7 \\
2\end{array}$} & \multirow{2}{*}{$\begin{array}{l}8 \\
2\end{array}$} & \multirow{2}{*}{9} \\
\hline Lunaria rediviva & E1 & & & & & & & & & \\
\hline Phyllitis scolopendrium & E1 & 12 & 12 & . & . & . & . & 1 & . & 1 \\
\hline Stellaria montana & E1 & . & 81 & . & . & . & . & 8 & 3 & . \\
\hline Ulmus glabra & E3 & . & . & . & . & 1 & . & 1 & . & 3 \\
\hline Ulmus glabra & $\mathrm{E} 2$ & . & . & . & . & 2 & . & 1 & . & 1 \\
\hline Ulmus glabra & $\mathrm{E} 1$ & . & . & . & . & . & . & . & . & 1 \\
\hline Euonymus latifolius & E2 & . & . & . & . & 1 & . & . & . & 8 \\
\hline Tephroseris longifolia & E1 & . & . & . & . & 1 & . & . & . & 2 \\
\hline Dryopteris affinis & E1 & . & . & . & . & 1 & . & . & . & 1 \\
\hline Arum maculatum & E1 & . & . & . & . & . & . & 3 & . & . \\
\hline Corydalis solida & E1 & . & . & . & . & . & . & 1 & 3 & . \\
\hline Polystichum illyricum & E1 & . & . & . & . & . & . & . & 1 & 1 \\
\hline Polystichum braunii & E1 & . & . & . & . & . & . & . & 1 & . \\
\hline Polystichum $x$ luerssenii & E1 & . & . & . & . & . & . & . & 1 & . \\
\hline Acer platanoides & E3 & . & . & . & . & . & . & . & . & 3 \\
\hline Acer platanoides & E2 & . & . & . & . & . & . & . & . & 1 \\
\hline Acer platanoides & $\mathrm{E} 1$ & . & . & . & . & . & . & . & . & 2 \\
\hline Juglans regia & $\mathrm{E} 2$ & . & . & . & . & . & . & . & . & 2 \\
\hline Tephroseris pseudocrispa & E1 & . & . & . & . & . & . & . & . & 2 \\
\hline Tilia platyphyllos & E3 & . & . & . & . & . & . & . & . & 1 \\
\hline Tilia platyphyllos & $\mathrm{E} 2$ & . & . & . & . & . & . & . & . & 1 \\
\hline \multicolumn{11}{|l|}{ Fagetalia sylvaticae } \\
\hline Paris quadrifolia & E1 & 100 & 56 & 86 & 50 & 19 & 26 & 49 & 41 & 11 \\
\hline Dryopteris filix-mas & E1 & 100 & 100 & 100 & 50 & 48 & 48 & 65 & 59 & 29 \\
\hline Mercurialis perennis & E1 & 100 & 31 & 29 & 50 & 50 & 55 & 56 & 61 & 77 \\
\hline Fagus sylvatica & E3 & 100 & 100 & 100 & 100 & 100 & 99 & 97 & 100 & 100 \\
\hline Fagus sylvatica & E2 & 88 & 94 & 29 & 100 & 82 & 66 & 81 & 85 & 72 \\
\hline Fagus sylvatica & E1 & . & 12 & 71 & . & 51 & 27 & 33 & 45 & 38 \\
\hline Galeobdolon montanum & E3 & 100 & 88 & 100 & . & 3 & 1 & 14 & . & . \\
\hline Epilobium montanum & $\mathrm{E} 1$ & 88 & 75 & 57 & 100 & 15 & 23 & 20 & 30 & 3 \\
\hline Actaea spicata & $\mathrm{E} 1$ & 88 & 81 & 43 & . & 29 & 33 & 22 & 35 & 17 \\
\hline Myosotis sylvatica agg. & $\mathrm{E} 1$ & 75 & 56 & . & 50 & 2 & 12 & 11 & 10 & . \\
\hline Ranunculus lanuginosus & $\mathrm{E} 1$ & 75 & 69 & 57 & 100 & 4 & 18 & 35 & 10 & . \\
\hline Daphne mezereum & E2 & 75 & 44 & . & 50 & 57 & 56 & 59 & 70 & 80 \\
\hline Lonicera alpigena & E2 & 62 & 25 & . & . & 21 & 33 & 34 & 73 & 60 \\
\hline Lonicera alpigena & E1 & . & . & . & . & . & . & . & 2 & 1 \\
\hline Prenanthes purpurea & E1 & 62 & 31 & 100 & . & 62 & 67 & 52 & 47 & 61 \\
\hline Scrophularia nodosa & E1 & 50 & 88 & 43 & 50 & 5 & 4 & 22 & 11 & . \\
\hline Lilium martagon & E1 & 50 & 31 & 57 & 50 & 9 & 34 & 28 & 58 & 26 \\
\hline Melica nutans & E1 & 50 & . & . & . & 39 & 33 & 8 & 51 & 43 \\
\hline Galium aristatum & E1 & 38 & 6 & . & . & . & . & . & 1 & . \\
\hline Heracleum sphondylium & E1 & 25 & 31 & . & . & 1 & 1 & 19 & 6 & 2 \\
\hline Mycelis muralis & E1 & 25 & 81 & 14 & . & 58 & 56 & 48 & 19 & 22 \\
\hline Viola reichenbachiana & E1 & 12 & 19 & . & . & 42 & 12 & 12 & 2 & 20 \\
\hline Salvia glutinosa & $\mathrm{E} 1$ & 12 & 19 & . & 50 & 46 & 11 & 10 & 1 & 45 \\
\hline Galium odoratum & $\mathrm{E} 1$ & . & 81 & 100 & . & 3 & 3 & 39 & 4 & . \\
\hline Carex sylvatica & E1 & . & 56 & 29 & . & 8 & 14 & 31 & 2 & . \\
\hline
\end{tabular}




\begin{tabular}{|c|c|c|c|c|c|c|c|c|c|c|}
\hline Successive number (Zaporedna številka) & & 1 & 2 & 3 & 4 & 5 & 6 & 7 & 8 & 9 \\
\hline Cardamine bulbifera & E1 & . & 31 & . & 100 & 4 & 12 & 43 & 8 & . \\
\hline Lathyrus vernus & E1 & . & 31 & . & . & 7 & . & 20 & 20 & 11 \\
\hline Sanicula europaea & E1 & . & 31 & 43 & . & 22 & 16 & 18 & . & . \\
\hline Cardamine pentaphyllos & E1 & . & 25 & . & . & 6 & 7 & 1 & 1 & . \\
\hline Pulmonaria officinalis & E1 & . & 25 & . & 50 & 8 & 18 & 12 & 3 & 2 \\
\hline Corydalis cava & E1 & . & 25 & . & . & . & . & 9 & 11 & . \\
\hline Festuca altissima & E1 & . & 12 & 14 & . & 8 & 19 & 13 & 8 & . \\
\hline Petasites albus & E1 & . & 12 & 14 & 100 & 22 & 33 & 9 & 1 & . \\
\hline Phyteuma spicatum & E1 & . & 6 & 57 & . & 6 & . & 35 & 8 & 6 \\
\hline Symphytum tuberosum & E1 & . & 6 & 86 & 100 & 13 & 53 & 50 & 29 & 12 \\
\hline Leucojum vernum & E1 & . & 6 & 43 & . & . & . & 7 & 1 & . \\
\hline Galeobdolon flavidum & E1 & . & . & . & 100 & 44 & 60 & 56 & 56 & 16 \\
\hline Campanula trachelium & E1 & . & . & . & 50 & 13 & . & 17 & 6 & 5 \\
\hline Cardamine impatiens & E1 & . & . & . & 50 & . & . & 2 & 2 & . \\
\hline Euphorbia amygdaloides & E1 & . & . & . & . & 55 & 53 & 61 & 12 & 14 \\
\hline Neottia nidus-avis & E1 & . & . & . & . & 43 & 22 & 6 & 7 & 14 \\
\hline Epipactis helleborine & E1 & . & . & . & . & 22 & . & 5 & 4 & 35 \\
\hline Luzula nivea & E1 & . & . & . & . & 17 & . & 4 & 36 & 13 \\
\hline Galium laevigatum & E1 & . & . & . & . & 15 & 12 & 14 & 50 & 74 \\
\hline Cephalanthera damasonium & E1 & . & . & . & . & 12 & . & . & . & 1 \\
\hline Laburnum alpinum & E3 & . & . & . & . & 1 & . & 1 & 5 & 59 \\
\hline Laburnum alpinum & E2 & . & . & . & . & 7 & . & 4 & 9 & 54 \\
\hline Laburnum alpinum & E1 & . & . & . & . & 1 & . & . & 6 & 38 \\
\hline Brachypodium sylvaticum & E1 & . & . & . & . & 7 & . & . & . & . \\
\hline Polygonatum multiflorum & E1 & . & . & . & . & 6 & . & 13 & 2 & 6 \\
\hline Fraxinus excelsior & E3 & . & . & . & . & 1 & . & 3 & 2 & 3 \\
\hline Fraxinus excelsior & E2 & . & . & . & . & 1 & . & 1 & . & 1 \\
\hline Fraxinus excelsior & E1 & . & . & . & . & 4 & 1 & 1 & . & 1 \\
\hline Prunus avium & E2 & . & . & . & . & 3 & . & . & . & . \\
\hline Sambucus nigra & E1 & . & . & . & . & 2 & 1 & . & . & . \\
\hline Circaea lutetiana & E1 & . & . & . & . & 1 & 1 & 1 & . & . \\
\hline Sambucus nigra & E2 & . & . & . & . & 1 & 3 & 2 & . & 2 \\
\hline Euphorbia dulcis & E1 & . & . & . & . & 1 & . & 4 & . & 1 \\
\hline Asarum europaeum subsp. europaeum & E1 & . & . & . & . & 1 & . & . & . & 3 \\
\hline Asarum europaeum subsp. caucasicum & E1 & . & . & . & . & . & 3 & . & . & 13 \\
\hline Cypripedium calceolus & E1 & . & . & . & . & . & 1 & . & . & 1 \\
\hline Epipogium aphyllum & E1 & . & . & . & . & . & 1 & . & . & . \\
\hline Allium ursinum & E1 & . & . & . & . & . & . & 10 & . & . \\
\hline Hordelymus europaeus & E1 & . & . & . & . & . & . & 1 & . & . \\
\hline Tilia cordata & E3 & . & . & . & . & . & . & . & . & 2 \\
\hline Tilia cordata & E2 & . & . & . & . & . & . & . & . & 1 \\
\hline \multicolumn{11}{|l|}{ Quercetalia pubescenti-petraeae } \\
\hline Sorbus aria (Aria edulis) & E3 & . & . & . & . & 10 & 4 & 2 & 7 & 69 \\
\hline Sorbus aria (Aria edulis) & E2 & . & . & . & . & 16 & 7 & 8 & 30 & 69 \\
\hline Sorbus aria (Aria edulis) & E1 & . & . & . & . & 13 & 5 & . & 3 & 12 \\
\hline Carex flacca & E1 & . & . & . & . & 9 & 1 & 3 & 3 & 3 \\
\hline Melittis melissophyllum & E1 & . & . & . & . & 5 & 1 & 2 & 8 & 27 \\
\hline
\end{tabular}




\begin{tabular}{|c|c|c|c|c|c|c|c|c|c|c|}
\hline \multicolumn{2}{|c|}{ Successive number (Zaporedna številka) } & \multirow{2}{*}{$\begin{array}{l}1 \\
\text {. }\end{array}$} & \multirow{2}{*}{$\begin{array}{l}2 \\
.\end{array}$} & \multirow{2}{*}{$\begin{array}{l}3 \\
.\end{array}$} & \multirow{2}{*}{$\begin{array}{l}4 \\
.\end{array}$} & \multirow{2}{*}{$\frac{5}{4}$} & \multirow{2}{*}{$\begin{array}{l}6 \\
.\end{array}$} & \multirow{2}{*}{$\begin{array}{l}7 \\
1\end{array}$} & 8 & 9 \\
\hline Convallaria majalis & E1 & & & & & & & & 18 & 33 \\
\hline Fraxinus ornus & E3 & . & . & . & . & 1 & . & . & . & 40 \\
\hline Fraxinus ornus & E2 & . & . & . & . & 3 & 3 & . & . & 46 \\
\hline Fraxinus ornus & E1 & . & . & . & . & 3 & 3 & . & . & 22 \\
\hline Campanula persicifolia & E1 & . & . & . & . & 2 & . & 1 & 1 & . \\
\hline Hypericum montanum & E1 & . & . & . & . & 2 & . & 3 & 1 & 3 \\
\hline Ostrya carpinifolia & E3 & . & . & . & . & 1 & 3 & . & . & 58 \\
\hline Ostrya carpinifolia & $\mathrm{E} 2$ & . & . & . & . & 1 & . & . & $\mathrm{r}$ & 33 \\
\hline Ostrya carpinifolia & $\mathrm{E} 1$ & . & . & . & . & 1 & . & . & . & 3 \\
\hline Tanacetum corymbosum & E1 & . & . & . & . & . & . & 1 & 6 & 2 \\
\hline Primula veris subsp. columnae & E1 & . & . & . & . & . & . & . & 3 & . \\
\hline Euonymus verrucosus & E2 & . & . & . & . & . & . & . & . & 13 \\
\hline Euonymus verrucosus & E1 & . & . & . & . & . & . & . & . & 1 \\
\hline Cornus mas & E2 & . & . & . & . & . & . & . & . & 6 \\
\hline Sesleria autumnalis & E1 & . & . & . & . & . & . & . & . & 3 \\
\hline Mercurialis ovata & E1 & . & . & . & . & . & . & . & . & 2 \\
\hline Clematis recta & E2 & . & . & . & . & . & . & . & . & 1 \\
\hline \multicolumn{11}{|l|}{ Querco-Fagetea } \\
\hline Anemone nemorosa & E1 & 75 & 31 & 29 & 50 & 8 & 49 & 74 & 51 & 29 \\
\hline Galium schultesii & E1 & 50 & . & . & . & 2 & 7 & 3 & . & . \\
\hline Poa nemoralis & E1 & 25 & 6 & . & . & 2 & 11 & 4 & 32 & 1 \\
\hline Dactylorhiza fuchsii & $\mathrm{E} 1$ & 12 & 6 & 29 & 50 & 5 & 14 & 5 & 3 & 1 \\
\hline Lonicera xylosteum & $\mathrm{E} 2$ & 12 & 19 & . & . & 11 & 1 & 7 & 1 & 5 \\
\hline Lonicera xylosteum & E1 & . & . & . & . & 1 & . & . & . & 2 \\
\hline Carex digitata & $\mathrm{E} 1$ & 12 & 12 & . & . & 58 & 34 & 17 & 27 & 64 \\
\hline Hepatica nobilis & E1 & . & . & . & . & 39 & 22 & 21 & 37 & 51 \\
\hline Pteridium aquilinum & E1 & . & . & . & . & 15 & 1 & . & . & 21 \\
\hline Platanthera bifolia & E1 & . & . & . & . & 12 & . & 4 & . & 10 \\
\hline Melampyrum pratense & E1 & . & . & . & . & 10 & 3 & . & 1 & . \\
\hline Veronica officinalis & E1 & . & . & . & . & 8 & 5 & 1 & . & . \\
\hline Corylus avellana & E3 & . & . & . & . & 4 & . & . & . & . \\
\hline Corylus avellana & E2 & . & . & . & . & 7 & . & 4 & 2 & 18 \\
\hline Corylus avellana & E1 & . & . & . & . & 5 & . & . & . & 3 \\
\hline Cruciata glabra & E1 & . & . & . & . & 4 & 1 & 2 & . & 1 \\
\hline Clematis vitalba & $\mathrm{E} 1$ & . & . & . & . & 4 & 1 & . & . & 2 \\
\hline Cephalanthera longifolia & E1 & . & . & . & . & 3 & . & . & . & 1 \\
\hline Vinca minor & $\mathrm{E} 1$ & . & . & . & . & 3 & . & . & . & 1 \\
\hline Clematis vitalba & $\mathrm{E} 2$ & . & . & . & . & 3 & . & . & . & 5 \\
\hline Listera ovata & $\mathrm{E} 1$ & . & . & . & . & 2 & 1 & 2 & 1 & 2 \\
\hline Moehringia trinervia & $\mathrm{E} 1$ & . & . & . & . & 2 & 4 & 2 & 2 & . \\
\hline Quercus petraea & E3 & . & . & . & . & 1 & . & . & . & . \\
\hline Quercus petraea & E2 & . & . & . & . & 2 & . & . & . & . \\
\hline Quercus petraea & E1 & . & . & . & . & . & . & . & . & 1 \\
\hline Galium sylvaticum & E1 & . & . & . & . & 1 & . & 2 & 1 & . \\
\hline Aegopodium podagraria & E1 & . & . & . & . & 1 & 3 & 5 & 2 & 1 \\
\hline Viola riviniana & $\mathrm{E} 1$ & . & . & . & . & 1 & . & . & 9 & 5 \\
\hline Betonica officinalis & E1 & . & . & . & . & 1 & . & 2 & . & 2 \\
\hline
\end{tabular}




\begin{tabular}{|c|c|c|c|c|c|c|c|c|c|c|}
\hline \multicolumn{2}{|c|}{ Successive number (Zaporedna številka) } & \multirow{2}{*}{$\begin{array}{l}1 \\
.\end{array}$} & \multirow{2}{*}{$\begin{array}{l}2 \\
.\end{array}$} & \multirow{2}{*}{3} & \multirow{2}{*}{4} & \multirow{2}{*}{$\begin{array}{l}5 \\
1\end{array}$} & \multirow{2}{*}{$\begin{array}{l}6 \\
.\end{array}$} & \multirow{2}{*}{$\begin{array}{l}7 \\
.\end{array}$} & \multirow{2}{*}{$\begin{array}{l}8 \\
.\end{array}$} & \multirow{2}{*}{$\begin{array}{l}9 \\
3\end{array}$} \\
\hline Hedera helix & E1 & & & & & & & & & \\
\hline Hieracium lachenalii & $\mathrm{E} 1$ & . & . & . & . & 1 & 1 & . & . & . \\
\hline Alnus incana & $\mathrm{E} 2$ & . & . & . & . & 1 & . & . & . & . \\
\hline Malus sylvestris & E2 & . & . & . & . & 1 & . & . & . & . \\
\hline Viburnum opulus & $\mathrm{E} 2$ & . & . & . & . & . & . & . & . & 2 \\
\hline Viburnum opulus & E1 & . & . & . & . & 1 & . & . & . & . \\
\hline Hieracium laevigatum & E1 & . & . & . & . & . & 1 & . & . & . \\
\hline Quercus robur & E1 & . & . & . & . & . & 1 & . & . & . \\
\hline Veronica montana & E1 & . & . & . & . & . & . & 8 & . & . \\
\hline Carex pilosa & $\mathrm{E} 1$ & . & . & . & . & . & . & 5 & 1 & . \\
\hline Anemone ranunculoides & E1 & . & . & . & . & . & . & 4 & 1 & . \\
\hline Festuca heterophylla & E1 & . & . & . & . & . & . & 4 & 5 & 1 \\
\hline Ficaria verna & E1 & . & . & . & . & . & . & 4 & . & . \\
\hline Rubus hirtus & E2 & . & . & . & . & . & . & 3 & . & 9 \\
\hline Scilla bifolia & E1 & . & . & . & . & . & . & 2 & 1 & . \\
\hline Melica uniflora & E1 & . & . & . & . & . & . & 2 & . & . \\
\hline Gagea lutea & E1 & . & . & . & . & . & . & 1 & 1 & . \\
\hline Stellaria holostea & E1 & . & . & . & . & . & . & 1 & 5 & . \\
\hline Rosa arvensis & E2 & . & . & . & . & . & . & 1 & . & 1 \\
\hline Ranunculus auricomus agg. & E1 & . & . & . & . & . & . & . & 3 & . \\
\hline Veratrum nigrum & $\mathrm{E} 1$ & . & . & . & . & . & . & . & . & 12 \\
\hline Frangula alnus & E2 & . & . & . & . & . & . & . & . & 8 \\
\hline Ilex aquifolium & E3 & . & . & . & . & . & . & . & . & 2 \\
\hline Ilex aquifolium & E2 & . & . & . & . & . & . & . & . & 8 \\
\hline Spiraea chamaedryfolia & $\mathrm{E} 2$ & . & . & . & . & . & . & . & . & 8 \\
\hline Taxus baccata & E3 & . & . & . & . & . & . & . & . & 6 \\
\hline Taxus baccata & E2 & . & . & . & . & . & . & . & . & 3 \\
\hline Taxus baccata & E1 & . & . & . & . & . & . & . & . & 1 \\
\hline Viscum album & E3 & . & . & . & . & . & . & . & . & 4 \\
\hline Hedera helix & E3 & . & . & . & . & . & . & . & . & 2 \\
\hline Serratula tinctoria & E1 & . & . & . & . & . & . & . & . & 1 \\
\hline Spiraea chamaedryfolia & E1 & . & . & . & . & . & . & . & . & 1 \\
\hline \multicolumn{11}{|l|}{ Vaccinio-Piceetea } \\
\hline Gentiana asclepiadea & E1 & 88 & 62 & 86 & 100 & 25 & 45 & 48 & 55 & 45 \\
\hline Rosa pendulina & E2 & 75 & 19 & . & . & 22 & 26 & 26 & 70 & 70 \\
\hline Calamagrostis arundinacea & E1 & 62 & 12 & 43 & 50 & 12 & 30 & 8 & 57 & 29 \\
\hline Polystichum lonchitis & $\mathrm{E} 1$ & 62 & . & . & . & 24 & 47 & 19 & 78 & 12 \\
\hline Veronica urticifolia & $\mathrm{E} 1$ & 50 & 12 & . & 50 & 56 & 84 & 41 & 58 & 52 \\
\hline Picea abies & E3 & 50 & 38 & 100 & 100 & 85 & 82 & 45 & 40 & 42 \\
\hline Picea abies & E2 & 38 & 19 & 71 & . & 68 & 60 & 43 & 45 & 41 \\
\hline Picea abies & E1 & . & . & 43 & . & 34 & 30 & 7 & 18 & 20 \\
\hline Oxalis acetosella & E1 & 38 & 88 & 100 & 50 & 73 & 78 & 74 & 59 & 18 \\
\hline Lonicera nigra & E2 & 25 & 25 & 14 & . & 8 & 8 & 4 & 34 & 8 \\
\hline Lonicera nigra & E1 & . & . & . & . & 2 & 14 & . & . & . \\
\hline Dryopteris expansa & E1 & 25 & 50 & 71 & . & 8 & . & 8 & 9 & 3 \\
\hline Maianthemum bifolium & E1 & 25 & 44 & . & . & 36 & 18 & 27 & 55 & 22 \\
\hline Vaccinium myrtillus & E1 & 25 & . & 14 & . & 54 & 49 & 15 & 70 & 63 \\
\hline
\end{tabular}




\begin{tabular}{|c|c|c|c|c|c|c|c|c|c|c|}
\hline \multicolumn{2}{|c|}{ Successive number (Zaporedna številka) } & \multirow{2}{*}{$\begin{array}{c}1 \\
12\end{array}$} & \multirow{2}{*}{$\begin{array}{l}2 \\
6\end{array}$} & \multirow{2}{*}{$\begin{array}{c}3 \\
29\end{array}$} & \multirow{2}{*}{$\begin{array}{c}4 \\
50\end{array}$} & \multirow{2}{*}{$\frac{5}{15}$} & \multirow{2}{*}{$\begin{array}{l}6 \\
8\end{array}$} & \multirow{2}{*}{$\begin{array}{l}7 \\
2\end{array}$} & \multirow{2}{*}{$\begin{array}{c}8 \\
30\end{array}$} & \multirow{2}{*}{$\begin{array}{c}9 \\
22\end{array}$} \\
\hline Phegopteris connectilis & E1 & & & & & & & & & \\
\hline Luzula sylvatica & E1 & 12 & 19 & 86 & 50 & 18 & 53 & 32 & 61 & 5 \\
\hline Solidago virgaurea & E1 & 12 & . & 14 & . & 32 & 30 & 11 & 50 & 53 \\
\hline Luzula luzuloides & E1 & . & 25 & 29 & . & 11 & 25 & 25 & 25 & 5 \\
\hline Gymnocarpium dryopteris & E1 & . & 25 & 14 & . & 34 & 51 & 15 & 44 & 34 \\
\hline Abies alba & E3 & . & 19 & 71 & . & 51 & 68 & 18 & 11 & 25 \\
\hline Abies alba & $\mathrm{E} 2$ & . & 12 & 71 & . & 38 & 47 & 13 & 18 & 25 \\
\hline Abies alba & E1 & . & . & 43 & . & 33 & 29 & 9 & 4 & 15 \\
\hline Luzula pilosa & E1 & . & . & 57 & . & 17 & 3 & 5 & 3 & 1 \\
\hline Melampyrum sylvaticum & E1 & . & . & 43 & . & 22 & 26 & . & 8 & 3 \\
\hline Aposeris foetida & E1 & . & . & . & 100 & 40 & 77 & 54 & 71 & 18 \\
\hline Hieracium murorum & E1 & . & . & . & . & 66 & 59 & 14 & 40 & 49 \\
\hline Larix decidua & E3 & . & . & . & . & 39 & 56 & 9 & 22 & 10 \\
\hline Larix decidua & $\mathrm{E} 2$ & . & . & . & . & 2 & 4 & . & 8 & 2 \\
\hline Larix decidua & E1 & . & . & . & . & . & . & . & 2 & 1 \\
\hline Homogyne sylvestris & E1 & . & . & . & . & 34 & 55 & 17 & 27 & 70 \\
\hline Saxifraga cuneifolia & E1 & . & . & . & . & 20 & 37 & 10 & 31 & 9 \\
\hline Orthilia secunda & E1 & . & . & . & . & 19 & 1 & . & 1 & 6 \\
\hline Vaccinium vitis-idaea & $\mathrm{E} 1$ & . & . & . & . & 17 & 8 & . & 30 & 16 \\
\hline Clematis alpina & $\mathrm{E} 2$ & . & . & . & . & 13 & 27 & 13 & 67 & 43 \\
\hline Corallorrhiza trifida & E1 & . & . & . & . & 12 & 7 & . & 5 & . \\
\hline Ajuga pyramidalis & E1 & . & . & . & . & 10 & 3 & . & 3 & . \\
\hline Lycopodium annotinum & E1 & . & . & . & . & 9 & 4 & . & 14 & 7 \\
\hline Huperzia selago & E1 & . & . & . & . & 8 & 10 & 3 & 35 & 42 \\
\hline Homogyne alpina & E1 & . & . & . & . & 7 & 7 & 3 & 21 & . \\
\hline Luzula luzulina & E1 & . & . & . & . & 5 & 11 & . & 4 & . \\
\hline Calamagrostis villosa & E1 & . & . & . & . & 5 & 10 & . & 29 & 3 \\
\hline Dryopteris dilatata & E1 & . & . & . & . & 5 & 23 & 5 & 34 & 8 \\
\hline Pyrola chlorantha & E1 & . & . & . & . & 4 & . & . & . & . \\
\hline Monotropa hypopitys & E1 & . & . & . & . & 3 & . & . & 1 & . \\
\hline Pyrola rotundifolia & E1 & . & . & . & . & 2 & . & . & 1 & 2 \\
\hline Thelypteris limbosperma & E1 & . & . & . & . & 1 & 1 & . & 2 & . \\
\hline Pyrola minor & E1 & . & . & . & . & 1 & 3 & . & 2 & . \\
\hline Galium rotundifolium & E1 & . & . & . & . & 1 & . & . & . & . \\
\hline Moneses uniflora & E1 & . & . & . & . & 1 & . & . & . & . \\
\hline Blechnum spicant & E1 & . & . & . & . & . & 3 & . & . & . \\
\hline Lonicera caerulea & E2 & . & . & . & . & . & 1 & . & 22 & . \\
\hline Lonicera caerulea & E1 & . & . & . & . & . & . & . & 1 & . \\
\hline Circaea alpina & E1 & . & . & . & . & . & 1 & . & . & . \\
\hline Rhododendron ferrugineum & E1 & . & . & . & $\cdot$ & . & 1 & . & . & . \\
\hline Dryopteris carthusiana & E1 & . & . & . & . & . & . & 1 & . & 1 \\
\hline Hieracium murorum & $\mathrm{E} 2$ & . & . & . & . & . & . & 1 & . & . \\
\hline Listera cordata & E1 & . & . & . & . & . & . & . & . & 1 \\
\hline \multicolumn{11}{|l|}{ Erico-Pinetea } \\
\hline Calamagrostis varia & E1 & 38 & 38 & . & . & 63 & 55 & 35 & 51 & 81 \\
\hline Rubus saxatilis & E2 & 25 & 6 & . & . & 14 & . & 7 & 1 & . \\
\hline Carex alba & E1 & . & . & . & . & 42 & 11 & 11 & 13 & 65 \\
\hline
\end{tabular}




\begin{tabular}{|c|c|c|c|c|c|c|c|c|c|c|}
\hline \multicolumn{2}{|c|}{ Successive number (Zaporedna številka) } & \multirow{2}{*}{$\begin{array}{l}1 \\
.\end{array}$} & \multirow{2}{*}{$\begin{array}{l}2 \\
.\end{array}$} & \multirow{2}{*}{$\begin{array}{l}3 \\
.\end{array}$} & \multirow{2}{*}{$\begin{array}{l}4 \\
.\end{array}$} & \multirow{2}{*}{$\begin{array}{c}5 \\
20\end{array}$} & \multirow{2}{*}{$\begin{array}{l}6 \\
5\end{array}$} & \multirow{2}{*}{$\begin{array}{l}7 \\
1\end{array}$} & \multirow{2}{*}{$\begin{array}{l}8 \\
7\end{array}$} & \multirow{2}{*}{$\begin{array}{c}9 \\
35\end{array}$} \\
\hline Polygala chamaebuxus & E1 & & & & & & & & & \\
\hline Cephalanthera rubra & E1 & . & . & . & . & 14 & . & 1 & . & 1 \\
\hline Erica carnea & E1 & . & . & . & . & 12 & 18 & 3 & 40 & 77 \\
\hline Rubus saxatilis & E1 & . & . & . & . & 12 & 16 & 1 & 68 & 58 \\
\hline Pinus sylvestris & E3 & . & . & . & . & 11 & . & . & . & 2 \\
\hline Pinus sylvestris & $\mathrm{E} 2$ & . & . & . & . & 1 & . & . & . & 1 \\
\hline Rhododendron hirsutum & E2 & . & . & . & . & 5 & 10 & 2 & 52 & 91 \\
\hline Epipactis atrorubens & E1 & . & . & . & . & 5 & . & 1 & . & 2 \\
\hline Pinus mugo & E2 & . & . & . & . & 3 & 3 & . & 32 & 11 \\
\hline Cotoneaster tomentosus & E2 & . & . & . & . & 2 & 3 & . & 1 & 10 \\
\hline Rhodothamnus chamaecistus & E1 & . & . & . & . & 2 & 1 & . & 16 & 18 \\
\hline Molinia arundinacea & E1 & . & . & . & . & 1 & 1 & . & 3 & 21 \\
\hline Aquilegia nigricans & E1 & . & . & . & . & 1 & . & 4 & 17 & 13 \\
\hline Gymnadenia odoratissima & E1 & . & . & . & . & . & 3 & . & . & 1 \\
\hline Peucedanum rablense & E1 & . & . & . & . & . & . & . & 8 & 1 \\
\hline Carex ornithopoda & $\mathrm{E} 1$ & . & . & . & . & . & . & . & 8 & 10 \\
\hline Genista radiata & E2 & . & . & . & . & . & . & . & 6 & . \\
\hline Chamaecytisus hirsutus & E1 & . & . & . & . & . & . & . & 2 & 1 \\
\hline Amelanchier ovalis & $\mathrm{E} 2$ & . & . & . & . & . & . & . & 1 & 18 \\
\hline Amelanchier ovalis & $\mathrm{E} 1$ & . & . & . & . & . & . & . & . & 1 \\
\hline Peucedanum austriacum & E1 & . & . & . & . & . & . & . & . & 11 \\
\hline Pinus nigra & E3 & . & . & . & . & . & . & . & . & 6 \\
\hline Pinus nigra & E1 & . & . & . & . & . & . & . & . & 1 \\
\hline Allium ericetorum & E1 & . & . & . & . & . & . & . & . & 3 \\
\hline Pinus nigra & E2 & . & . & . & . & . & . & . & . & 2 \\
\hline Aquilegia atrata & E1 & . & . & . & . & . & . & . & . & 1 \\
\hline Chamaecytisus hirsutus & E2 & . & . & . & . & . & . & . & . & 1 \\
\hline \multicolumn{11}{|l|}{ Sambuco-Salicion capreae } \\
\hline Sambucus racemosa & E2 & 25 & 25 & 29 & . & 2 & 2 & . & 2 & 2 \\
\hline Sorbus aucuparia & E3 & . & 25 & 71 & . & 2 & 4 & 1 & 26 & 34 \\
\hline Sorbus aucuparia & $\mathrm{E} 2$ & 25 & 56 & 57 & . & 19 & 18 & 25 & 44 & 46 \\
\hline Sorbus aucuparia & $\mathrm{E} 1$ & . & . & 14 & . & 15 & 34 & 2 & 34 & 30 \\
\hline Salix caprea & E3 & . & . & . & . & 2 & . & . & 1 & . \\
\hline Salix caprea & E1 & . & . & . & . & . & . & . & . & 1 \\
\hline Betula pendula & E3 & . & . & . & . & . & . & 1 & . & 3 \\
\hline Betula pendula & E2 & . & . & . & . & . & 1 & . & . & 1 \\
\hline Betula pendula & $\mathrm{E} !$ & . & . & . & . & 2 & 1 & . & . & . \\
\hline Populus tremula & E3 & . & . & . & . & . & . & . & . & 1 \\
\hline \multicolumn{11}{|l|}{ Rhamno-Prunetea } \\
\hline Berberis vulgaris & E2 & . & . & . & . & 4 & . & . & . & 2 \\
\hline Rubus fruticosus agg. & E2 & . & . & . & . & 1 & . & . & . & . \\
\hline Rubus fruticosus & $\mathrm{E} 1$ & . & . & . & . & 2 & 1 & . & . & . \\
\hline Crataegus monogyna & $\mathrm{E} 2$ & . & . & . & . & 1 & . & . & . & 1 \\
\hline Viburnum lantana & $\mathrm{E} 2$ & . & . & . & . & 1 & . & . & . & 8 \\
\hline Rubus sp. & $\mathrm{E} 2$ & . & . & . & . & . & . & 4 & . & . \\
\hline Rhamnus cathartica & $\mathrm{E} 2$ & . & . & . & . & . & . & . & . & 6 \\
\hline Rhamnus cathartica & $\mathrm{E} 1$ & . & . & . & . & . & . & . & . & 1 \\
\hline
\end{tabular}




\begin{tabular}{|c|c|c|c|c|c|c|c|c|c|c|}
\hline \multicolumn{2}{|l|}{ Successive number (Zaporedna številka) } & 1 & 2 & 3 & 4 & 5 & 6 & 7 & 8 & 9 \\
\hline Juniperus communis & E2 & . & . & . & . & . & . & . & . & 6 \\
\hline Juniperus communis & E1 & . & . & . & . & . & . & . & . & 1 \\
\hline Cornus sanguinea & $\mathrm{E} 2$ & . & . & . & . & . & . & . & . & 2 \\
\hline Rosa canina & E1 & . & . & . & . & . & . & . & . & 1 \\
\hline \multicolumn{11}{|l|}{ Betulo-Alnetea } \\
\hline Salix appendiculata & E3 & . & . & . & . & . & . & . & 1 & 1 \\
\hline Salix appendiculata & $\mathrm{E} 2$ & 75 & . & . & . & 2 & 4 & 1 & 30 & 37 \\
\hline Salix appendiculata & E1 & . & . & . & . & . & . & . & 1 & 5 \\
\hline Sorbus chamaemespilus & E2 & 62 & . & . & . & 5 & 5 & . & 49 & 5 \\
\hline Sorbus aucuparia subsp. glabrata & E1 & 12 & . & . & . & . & . & . & . & . \\
\hline Sorbus aucuparia subsp. glabrata & E2 & 50 & . & . & . & . & . & . & . & . \\
\hline Ribes alpinum & E2 & 38 & 6 & . & . & . & . & 3 & 13 & . \\
\hline Ribes alpinum & E1 & . & . & . & . & . & 3 & . & . & . \\
\hline Cortusa matthioli & E1 & . & . & . & . & . & 1 & . & . & . \\
\hline Alnus viridis & E2 & . & . & . & . & . & . & . & 11 & . \\
\hline Juniperus sibirica & $\mathrm{E} 2$ & . & . & . & . & . & . & . & 6 & 1 \\
\hline Salix glabra & E2 & . & . & . & . & . & . & . & 6 & 22 \\
\hline Sorbus austriaca & $\mathrm{E} 2$ & . & . & . & . & . & . & . & 4 & 2 \\
\hline Salix waldsteiniana & E2 & . & . & . & . & . & . & . & 3 & . \\
\hline Betula pendula subsp. carpatica & E3 & . & . & . & . & . & . & . & 1 & . \\
\hline Ribes uva-crispa & E2 & . & . & . & . & . & . & . & 1 & . \\
\hline Sorbus mougeotii & E2 & . & . & . & . & . & . & . & 1 & . \\
\hline \multicolumn{11}{|l|}{ Mulgedio-Aconitetea } \\
\hline Saxifraga rotundifolia & E1 & 100 & 62 & 14 & 100 & 5 & 41 & 26 & 42 & . \\
\hline Aconitum degenii subsp. paniculatum & E1 & 100 & 6 & . & 100 & 2 & 1 & 7 & 14 & 5 \\
\hline Doronicum austriacum & E1 & 88 & 38 & 100 & . & 1 & 15 & 11 & 14 & . \\
\hline Thalictrum aquilegiifolium & E1 & 88 & 12 & 57 & 100 & 4 & 10 & 9 & 30 & 6 \\
\hline Chaerophyllum villarsii & E1 & 88 & 44 & 57 & . & . & 5 & 1 & 16 & . \\
\hline Geum rivale & E1 & 88 & 6 & . & 50 & . & 4 & 1 & 9 & . \\
\hline Cicerbita alpina & E1 & 75 & 31 & 71 & 50 & 1 & 14 & 11 & 5 & . \\
\hline Stellaria nemorum & E1 & 75 & 6 & 100 & 100 & 1 & 7 & 14 & 10 & . \\
\hline Myrrhis odorata & E1 & 75 & 25 & . & 100 & 1 & 12 & 3 & 9 & . \\
\hline Geranium sylvaticum & E1 & 75 & . & . & . & 4 & 5 & 1 & 32 & . \\
\hline Polygonatum verticillatum & E1 & 75 & 75 & 86 & 50 & 34 & 67 & 83 & 86 & 20 \\
\hline Athyrium filix-femina & E1 & 75 & 100 & 100 & 50 & 54 & 64 & 62 & 69 & 31 \\
\hline Rumex alpestris & E1 & 75 & 6 & 43 & . & . & . & 2 & 8 & . \\
\hline Silene dioica & E1 & 75 & 19 & 57 & . & . & 7 & 1 & 2 & . \\
\hline Ranunculus platanifolius & E1 & 62 & 31 & 57 & . & 2 & 12 & 50 & 33 & 2 \\
\hline Veratrum album & E1 & 62 & 56 & 57 & 50 & 11 & 67 & 73 & 72 & 20 \\
\hline Senecio ovatus & E1 & 62 & 88 & 100 & 100 & 32 & 48 & 71 & 28 & 30 \\
\hline Adenostyles alliariae & E1 & 62 & 44 & 57 & 50 & . & 15 & 22 & 15 & . \\
\hline Aconitum vulparia & E1 & 50 & 12 & . & . & 2 & 18 & 2 & 1 & . \\
\hline Viola biflora & E1 & 50 & . & 43 & 50 & 3 & 40 & 6 & 40 & 8 \\
\hline Senecio cacaliaster & E1 & 50 & . & . & . & . & 1 & . & 18 & . \\
\hline Milium effusum & E1 & 38 & 50 & 86 & . & 2 & 4 & 12 & 7 & . \\
\hline Aconitum lycoctonum subsp. ranunculifolium & E1 & 38 & 12 & . & . & 4 & . & 7 & 47 & 2 \\
\hline Phyteuma ovatum & E1 & 38 & . & . & 50 & 19 & 64 & 5 & 36 & 14 \\
\hline
\end{tabular}




\begin{tabular}{|c|c|c|c|c|c|c|c|c|c|c|}
\hline Successive number (Zaporedna številka & & 1 & 2 & 3 & 4 & 5 & 6 & 7 & 8 & 9 \\
\hline Hypericum maculatum & E1 & 25 & . & 29 & . & 1 & 14 & 2 & 18 & 2 \\
\hline Heracleum elegans & E1 & 25 & 6 & . & 50 & 2 & 12 & . & 1 & . \\
\hline Chaerophyllum hirsutum & E1 & . & . & . & 100 & 3 & 22 & 5 & 17 & . \\
\hline Agropyron caninum & E1 & . & . & . & 50 & . & 1 & . & 2 & . \\
\hline Cirsium carniolicum & E1 & . & . & . & . & . & 7 & . & 1 & . \\
\hline Primula elatior & E1 & . & . & . & . & . & 5 & 2 & 5 & . \\
\hline Knautia dipsacifolia & E1 & . & . & . & . & . & 5 & . & . & . \\
\hline Pedicularis recutita & E1 & . & . & . & . & . & 5 & . & . & . \\
\hline Senecio nemorensis & E1 & . & . & . & . & . & 4 & . & 1 & . \\
\hline Scrophularia scopolii & E1 & . & . & . & . & . & 4 & . & . & . \\
\hline Poa hybrida & E1 & . & . & . & . & . & 1 & . & 8 & . \\
\hline Lathyrus laevigatus & E1 & . & . & . & . & . & 1 & . & . & . \\
\hline Anthriscus nitidus & E1 & . & . & . & . & . & . & 10 & 2 & . \\
\hline Allium victorialis & E1 & . & . & . & . & . & . & 2 & 9 & . \\
\hline Pleurospermum austriacum & E1 & . & . & . & . & . & . & 1 & 7 & . \\
\hline Aconitum angustifolium & E1 & . & . & . & . & . & . & . & 10 & 7 \\
\hline Centaurea montana & E1 & . & . & . & . & . & . & . & 6 & 8 \\
\hline Lathyrus occidentalis & E1 & . & . & . & . & . & . & . & 4 & . \\
\hline Peucedanum ostruthium & E1 & . & . & . & . & . & . & . & 3 & . \\
\hline Carduus personata & E1 & . & . & . & . & . & . & . & 2 & . \\
\hline Chaerophyllum aureum & E1 & . & . & . & . & . & . & . & 2 & . \\
\hline Athyrium distentifolium & E1 & . & . & . & . & . & . & . & 1 & . \\
\hline Streptopus amplexifolius & E1 & . & . & . & . & . & . & . & 1 & . \\
\hline Tephroseris crispa & E1 & . & . & . & . & . & . & . & 1 & . \\
\hline Aconitum lycoctonum & E1 & . & . & . & . & . & . & . & . & 6 \\
\hline \multicolumn{11}{|l|}{ Caricion austroalpinae } \\
\hline Laserpitium peucedanoides & E1 & . & . & . & . & 5 & 7 & . & 31 & 26 \\
\hline Festuca calva & E1 & . & . & . & . & 1 & . & 2 & 21 & 1 \\
\hline Heracleum austriacum subsp. siifolium & E1 & . & . & . & . & . & 1 & . & 1 & . \\
\hline Pulsatilla alpina subsp. austroalpina & E1 & . & . & . & . & . & 1 & . & 3 & . \\
\hline Carduus crassifolius & E1 & . & . & . & . & . & . & . & 7 & 6 \\
\hline Gentiana lutea subsp. symphiandra & E1 & . & . & . & . & . & . & . & 2 & 2 \\
\hline Arabis vochinensis & E1 & . & . & . & . & . & . & . & 1 & . \\
\hline Koeleria eriostachya & E1 & . & . & . & . & . & . & . & 1 & . \\
\hline \multicolumn{11}{|l|}{ Caricion ferrugineae } \\
\hline Carex ferruginea & E1 & . & . & . & . & 2 & 26 & 1 & 23 & 33 \\
\hline Cerastium subtriflorum & E1 & . & . & . & . & . & . & 1 & 8 & 1 \\
\hline Knautia longifolia & E1 & . & . & . & . & . & . & . & 1 & . \\
\hline \multicolumn{11}{|l|}{ Elyno-Seslerietea } \\
\hline Betonica alopecuros & E1 & . & . & . & . & 12 & 5 & . & 43 & 38 \\
\hline Senecio abrotanifolius & E1 & . & . & . & . & 7 & . & 1 & 8 & 2 \\
\hline Sesleria caerulea & E1 & . & . & . & . & 4 & 1 & 2 & 41 & 30 \\
\hline Aster bellidiastrum & E1 & . & . & . & . & 3 & 4 & . & 27 & 30 \\
\hline Phleum hirsutum & E1 & . & . & . & . & 1 & . & . & 1 & . \\
\hline Phyteuma orbiculare & E1 & . & . & . & . & 1 & 3 & . & 8 & 6 \\
\hline Campanula witasekiana & E1 & . & . & . & . & 1 & . & 1 & 18 & 8 \\
\hline Astrantia bavarica & E1 & . & . & . & . & . & 5 & . & 12 & . \\
\hline
\end{tabular}




\begin{tabular}{|c|c|c|c|c|c|c|c|c|c|c|}
\hline \multicolumn{2}{|l|}{ Successive number (Zaporedna številka) } & \multirow{2}{*}{$\begin{array}{l}1 \\
.\end{array}$} & \multirow{2}{*}{2} & \multirow{2}{*}{3} & \multirow{2}{*}{4} & \multirow{2}{*}{5} & \multirow{2}{*}{$\begin{array}{l}6 \\
1\end{array}$} & \multirow{2}{*}{$\begin{array}{l}7 \\
.\end{array}$} & \multirow{2}{*}{$\begin{array}{l}8 \\
1\end{array}$} & \multirow{2}{*}{$\begin{array}{l}9 \\
.\end{array}$} \\
\hline Bartsia alpina & E1 & & & & & & & & & \\
\hline Galium anisophyllon & E1 & . & . & . & . & . & 1 & . & 2 & . \\
\hline Ranunculus carinthiacus & E1 & . & . & . & . & . & 1 & . & 3 & . \\
\hline Leucanthemum heterophyllum & $\mathrm{E} 1$ & . & . & . & . & . & 1 & . & 2 & . \\
\hline Polygonum persicaria (Persicaria vivipara) & E1 & . & . & . & . & . & 1 & . & 1 & . \\
\hline Euphrasia picta & E1 & . & . & . & . & . & 1 & . & . & . \\
\hline Hieracium scorzonerifolium & E1 & . & . & . & . & . & 1 & . & . & . \\
\hline Polygala amara & E1 & . & . & . & . & . & 1 & . & . & . \\
\hline Scabiosa lucida subsp. lucida & E1 & . & . & . & . & . & 1 & . & . & . \\
\hline Carex sempervirens & E1 & . & . & . & . & . & . & . & 7 & . \\
\hline Pimpinella alpina & E1 & . & . & . & . & . & . & . & 5 & 3 \\
\hline Scabiosa lucida subsp. stricta & E1 & . & . & . & . & . & . & . & 3 & 1 \\
\hline Ranunculus montanus & E1 & . & . & . & . & . & . & . & 3 & . \\
\hline Hieracium pilosum & E1 & . & . & . & . & . & . & . & 2 & . \\
\hline Lotus alpinus & E1 & . & . & . & . & . & . & . & 2 & . \\
\hline Alchemilla alpigena & $\mathrm{E} 1$ & . & . & . & . & . & . & . & 2 & . \\
\hline Carex firma & $\mathrm{E} 1$ & . & . & . & . & . & . & . & 1 & 2 \\
\hline Hieracium villosum & $\mathrm{E} 1$ & . & . & . & . & . & . & . & 1 & 3 \\
\hline Achillea clavennae & E1 & . & . & . & . & . & . & . & 1 & . \\
\hline Alchemilla vulgaris & E1 & . & . & . & . & . & . & . & 1 & . \\
\hline Cerastium strictum & E1 & . & . & . & . & . & . & . & 1 & . \\
\hline \multicolumn{2}{|c|}{ Helianthemum nummularium subsp. grandiflorum E1 } & . & . & . & . & . & . & . & 1 & . \\
\hline Selaginella selaginoides & E1 & . & . & . & . & . & . & . & 1 & . \\
\hline Thesium alpinum & E1 & . & . & . & . & . & . & . & 1 & . \\
\hline Carex mucronata & E1 & . & . & . & . & . & . & . & 1 & 5 \\
\hline Globularia cordifolia & E1 & . & . & . & . & . & . & . & $\mathrm{r}$ & 2 \\
\hline \multicolumn{11}{|l|}{ Nardion strictae, Juncete trifidi } \\
\hline Solidago minuta & E1 & 12 & . & 29 & . & . & . & . & . & . \\
\hline Campanula scheuchzeri & E1 & . & . & . & . & 5 & . & 1 & 37 & 2 \\
\hline Potentilla erecta & E1 & . & . & . & . & 2 & 1 & . & 5 & 3 \\
\hline Festuca nigrescens & E1 & . & . & . & . & . & 1 & 1 & . & . \\
\hline Phyteuma zablbruckneri & E1 & . & . & . & . & . & . & . & 2 & . \\
\hline Coeloglossum viride & E1 & . & . & . & . & . & . & . & 1 & . \\
\hline Gentiana pannonica & E1 & . & . & . & . & . & . & . & 1 & . \\
\hline Selaginella helvetica & E1 & . & . & . & . & . & . & . & . & 2 \\
\hline \multicolumn{11}{|l|}{ Caricetalia davallianae } \\
\hline Parnassia palustris & E1 & . & . & . & . & . & 3 & . & 3 & 3 \\
\hline Tofieldia calyculata & E1 & . & . & . & . & . & 1 & . & 1 & 2 \\
\hline Pinguicula alpina & E1 & . & . & . & . & . & . & . & 1 & 3 \\
\hline \multicolumn{11}{|l|}{ Montio-Cardaminetea } \\
\hline Cardamine amara & E1 & . & . & . & . & . & 1 & . & . & . \\
\hline \multicolumn{11}{|l|}{ Festuco-Brometea } \\
\hline Cirsium erisithales & E1 & 88 & 12 & . & . & 23 & 22 & 21 & 39 & 72 \\
\hline Buphthalmum salicifolium & E1 & 38 & . & . & . & 9 & 5 & 2 & 11 & 47 \\
\hline Fragaria viridis & E1 & 12 & 12 & . & . & . & . & . & . & . \\
\hline Euphorbia cyparissias & E1 & . & . & . & . & 4 & 1 & . & 1 & 1 \\
\hline Campanula rotundifolia & E1 & . & . & . & . & 3 & 3 & 2 & . & . \\
\hline
\end{tabular}




\begin{tabular}{|c|c|c|c|c|c|c|c|c|c|c|}
\hline \multicolumn{2}{|c|}{ Successive number (Zaporedna številka) } & \multirow{2}{*}{1} & \multirow{2}{*}{2} & \multirow{2}{*}{3} & \multirow{2}{*}{4} & \multirow{2}{*}{$\begin{array}{l}5 \\
2\end{array}$} & \multirow{2}{*}{6} & \multirow{2}{*}{$\begin{array}{l}7 \\
.\end{array}$} & \multirow{2}{*}{$\begin{array}{l}8 \\
5\end{array}$} & \multirow{2}{*}{$\begin{array}{c}9 \\
23\end{array}$} \\
\hline Carex humilis & E1 & & & & & & & & & \\
\hline Brachypodium rupestre & E1 & . & . & . & . & 2 & 1 & . & . & 8 \\
\hline Cirsium acaule & E1 & . & . & . & . & 2 & . & . & . & . \\
\hline Teucrium chamaedrys & E1 & . & . & . & . & 2 & . & . & . & . \\
\hline Pimpinella saxifraga & E1 & . & . & . & . & 2 & . & . & . & . \\
\hline Carlina acaulis & E1 & . & . & . & . & 1 & . & . & 4 & 2 \\
\hline Ajuga genevensis & E1 & . & . & . & . & 1 & 1 & . & . & 2 \\
\hline Stachys recta & E1 & . & . & . & . & 1 & . & . & . & . \\
\hline Galium lucidum & $\mathrm{E} 1$ & . & . & . & . & . & 1 & . & 1 & 4 \\
\hline Festuca pseudovina & $\mathrm{E} 1$ & . & . & . & . & . & 1 & . & . & . \\
\hline Koeleria pyramidata & E1 & . & . & . & . & . & . & . & 3 & . \\
\hline Thymus praecox & $\mathrm{E} 1$ & . & . & . & . & . & . & . & 2 & 1 \\
\hline Bromopsis transsilvanica & $\mathrm{E} 1$ & . & . & . & . & . & . & . & 2 & . \\
\hline Arabis hirsuta & E1 & . & . & . & . & . & . & . & 1 & . \\
\hline Silene vulgaris & E1 & . & . & . & . & . & . & . & 1 & . \\
\hline Salvia pratensis & $\mathrm{E} 1$ & . & . & . & . & $\cdot$ & . & . & . & 2 \\
\hline Festuca rupicola & E1 & . & . & . & . & $\cdot$ & . & $\cdot$ & . & 2 \\
\hline Bromus erectus & E1 & . & . & . & . & . & . & . & . & 1 \\
\hline Gentianella ciliata & E1 & . & . & . & . & . & . & . & . & 1 \\
\hline \multicolumn{11}{|l|}{ Trifolio-Geranietea } \\
\hline Laserpitium latifolium & E1 & 38 & . & . & . & 2 & . & . & 6 & 2 \\
\hline Digitalis grandiflora & E1 & . & . & . & . & 15 & 4 & 7 & 4 & 6 \\
\hline Vincetoxicum hirundinaria & E1 & . & . & . & . & 6 & . & . & 1 & 19 \\
\hline Clinopodium vulgare & E1 & . & . & . & . & 6 & . & . & 4 & 1 \\
\hline Origanum vulgare & E1 & . & . & . & . & 2 & . & . & 1 & 1 \\
\hline Vicia sylvatica & E1 & . & . & . & . & 1 & 3 & . & 1 & 1 \\
\hline Verbascum austriacum & E1 & . & . & . & . & 1 & . & . & . & . \\
\hline Viola collina & E1 & . & . & . & . & 1 & . & . & . & . \\
\hline Lilium carniolicum & E1 & . & . & . & . & . & 1 & 1 & 2 & 3 \\
\hline Laserpitium siler & E1 & . & . & . & . & . & . & 1 & 2 & 2 \\
\hline Hypericum perforatum & E1 & . & . & . & . & . & . & 1 & . & 1 \\
\hline Fragaria moschata & E1 & . & . & . & . & . & . & 1 & . & . \\
\hline Verbascum lanatum & E1 & . & . & . & . & . & . & . & 6 & 2 \\
\hline Valeriana wallrothii (V. collina) & E1 & . & . & . & . & . & . & . & 3 & 2 \\
\hline Hieracium bifidum & E1 & . & . & . & . & . & . & . & 3 & 8 \\
\hline Achillea distans & E1 & . & . & . & . & . & . & . & 2 & 1 \\
\hline Grafia golaka & E1 & . & . & . & . & . & . & . & 2 & 1 \\
\hline Iris graminea & E1 & . & . & . & . & . & . & . & 2 & 2 \\
\hline Thalictrum minus & E1 & . & . & . & . & . & . & . & 2 & 2 \\
\hline Arabis pauciflora & E1 & . & . & . & . & . & . & . & 1 & 1 \\
\hline Arabis turrita & E1 & . & . & . & . & . & . & . & 1 & 2 \\
\hline Polygonatum odoratum & E1 & . & . & . & . & . & . & . & 1 & 2 \\
\hline Viola hirta & E1 & . & . & . & . & . & . & $\cdot$ & 1 & 5 \\
\hline Silene nutans & E1 & . & . & . & . & . & . & . & 1 & . \\
\hline Campanula rapunculoides & E1 & . & . & . & . & . & . & . & . & 7 \\
\hline Libanotis montana & E1 & . & . & . & . & . & . & . & . & 3 \\
\hline Anthericum ramosum & E1 & . & . & . & . & . & . & . & . & 2 \\
\hline
\end{tabular}




\begin{tabular}{|c|c|c|c|c|c|c|c|c|c|c|}
\hline \multicolumn{2}{|l|}{ Successive number (Zaporedna številka) } & \multirow{2}{*}{$\begin{array}{l}1 \\
.\end{array}$} & \multirow{2}{*}{$\begin{array}{l}2 \\
.\end{array}$} & \multirow{2}{*}{$\begin{array}{l}3 \\
.\end{array}$} & \multirow{2}{*}{4} & \multirow{2}{*}{5} & \multirow{2}{*}{6} & \multirow{2}{*}{7} & \multirow{2}{*}{$\begin{array}{l}8 \\
.\end{array}$} & \multirow{2}{*}{$\begin{array}{l}9 \\
1\end{array}$} \\
\hline Thesium bavarum & E1 & & & & & & & & & \\
\hline Melampyrum velebiticum & E1 & . & . & . & . & . & . & . & . & 1 \\
\hline \multicolumn{11}{|l|}{ Poo alpinae-Trisetetalia } \\
\hline Ranunculus nemorosus & E1 & . & . & . & . & 1 & 5 & . & 4 & . \\
\hline Astrantia major s. lat. & E1 & . & . & . & . & 1 & 7 & 3 & 1 & . \\
\hline Trollius europaeus & E1 & . & . & . & . & . & 1 & 1 & 9 & . \\
\hline Poa alpina & E1 & . & . & . & . & . & 1 & 1 & 12 & . \\
\hline Crocus albiflorus & E1 & . & . & . & . & . & . & . & 4 & . \\
\hline Cardaminopsis halleri subsp. ovirensis & E1 & . & . & . & . & . & . & . & 1 & . \\
\hline \multicolumn{11}{|l|}{ Molinio-Arrbenatberetea } \\
\hline Crepis paludosa & E1 & 88 & 12 & 14 & 100 & 1 & 25 & 2 & 11 & . \\
\hline Deschampsia cespitosa & E1 & 12 & 19 & 71 & . & 1 & 3 & 4 & 8 & . \\
\hline Ajuga reptans & E1 & . & . & . & . & 18 & 5 & 5 & . & . \\
\hline Taraxacum sect. Ruderalia & E1 & . & . & . & . & 2 & 1 & . & 1 & 5 \\
\hline Lathyrus pratensis & E1 & . & . & . & . & 2 & . & . & 1 & . \\
\hline Veronica chamaedrys & E1 & . & . & . & . & 2 & 1 & 2 & 2 & . \\
\hline Galium album & E1 & . & . & . & . & 2 & 3 & . & 2 & . \\
\hline Lotus corniculatus & E1 & . & . & . & . & 2 & 1 & . & . & 2 \\
\hline Pimpinella major & E1 & . & . & . & . & 1 & . & . & 1 & . \\
\hline Centaurea jacea & E1 & . & . & . & . & 1 & . & . & . & 3 \\
\hline Galium mollugo & E1 & . & . & . & . & 1 & . & . & . & . \\
\hline Poa pratensis & E1 & . & . & . & . & . & 1 & . & . & . \\
\hline Symphytum officinale & $\mathrm{E} 1$ & . & . & . & . & . & 1 & . & . & . \\
\hline Dactylis glomerata & E1 & . & . & . & . & . & . & 1 & 7 & . \\
\hline Angelica sylvestris & E1 & . & . & . & . & . & . & . & 11 & 1 \\
\hline Festuca rubra & E1 & . & . & . & . & . & . & . & 2 & . \\
\hline Trifolium pratense & E1 & . & . & . & . & . & . & . & 1 & . \\
\hline Achillea millefolium & E1 & . & . & . & . & . & . & . & . & 2 \\
\hline \multicolumn{11}{|l|}{ Epilobietea angustifolii, Galio-Urticetea } \\
\hline Rubus idaeus & E2 & 75 & 81 & 86 & . & 4 & . & 35 & 34 & 21 \\
\hline Galeopsis speciosa & E1 & 62 & 31 & 29 & . & 1 & 3 & 4 & 4 & . \\
\hline Urtica dioica & E1 & 62 & 88 & 29 & . & 2 & 7 & 6 & 16 & 1 \\
\hline Fragaria vesca & E1 & 38 & 19 & 14 & . & 40 & 19 & 22 & 28 & 12 \\
\hline Lamium maculatum & E1 & 25 & 19 & 14 & . & . & 4 & . & 2 & . \\
\hline Rubus idaeus & E1 & . & . & . & . & 10 & 19 & . & . & 1 \\
\hline Eupatorium cannabinum & E1 & . & . & . & . & 8 & . & . & . & 10 \\
\hline Tussilago farfara & E1 & . & . & . & . & 3 & 3 & . & . & . \\
\hline Hypericum hirsutum & E1 & . & . & . & . & 2 & . & . & 3 & . \\
\hline Stachys sylvatica & E1 & . & . & . & . & 1 & . & 2 & . & . \\
\hline Galeopsis pubescens & E1 & . & . & . & . & 1 & . & 3 & . & . \\
\hline Solanum dulcamara & E1 & . & . & . & . & 1 & . & . & . & . \\
\hline Stachys alpina & E1 & . & . & . & . & 1 & . & . & . & . \\
\hline Bromus benekenii & E1 & . & . & . & . & . & 1 & . & . & . \\
\hline Bromus ramosus & E1 & . & . & . & . & . & . & 5 & . & . \\
\hline Poa annua & E1 & . & . & . & . & . & . & 1 & . & . \\
\hline Verbascum densiflorum & E1 & . & . & . & . & . & . & 1 & . & . \\
\hline Atropa bella-donna & E1 & . & . & . & . & . & . & . & . & 2 \\
\hline
\end{tabular}




\begin{tabular}{|c|c|c|c|c|c|c|c|c|c|c|}
\hline \multicolumn{2}{|l|}{ Successive number (Zaporedna številka) } & \multirow{2}{*}{1} & \multirow{2}{*}{$\begin{array}{l}2 \\
.\end{array}$} & \multirow{2}{*}{3} & \multirow{2}{*}{4} & \multirow{2}{*}{5} & \multirow{2}{*}{6} & \multirow{2}{*}{7} & \multirow{2}{*}{8} & \multirow{2}{*}{$\begin{array}{l}9 \\
1\end{array}$} \\
\hline Cirsium vulgare & E1 & & & & & & & & & \\
\hline \multicolumn{11}{|l|}{ Thlaspietea rotundifolii } \\
\hline Adenostyles glabra & E1 & 75 & 56 & 29 & 50 & 24 & 56 & 69 & 73 & 59 \\
\hline Gymnocarpium robertianum & E1 & . & . & . & . & 22 & 25 & 2 & 20 & 67 \\
\hline Aquilegia einseleana & E1 & . & . & . & . & 2 & . & . & 1 & 1 \\
\hline Valeriana montana & E1 & . & . & . & . & 2 & 3 & . & 3 & 1 \\
\hline Silene alpestris & E1 & . & . & . & . & 2 & 3 & . & 6 & . \\
\hline Astrantia carniolica & E1 & . & . & . & . & 2 & 3 & 1 & 14 & 20 \\
\hline Cystopteris montana & $\mathrm{E} 1$ & . & . & . & . & 1 & 10 & 1 & 1 & 1 \\
\hline Trisetum argenteum & E1 & . & . & . & . & 1 & 1 & . & 2 & . \\
\hline Senecio rupestris & E1 & . & . & . & . & 1 & 3 & . & . & . \\
\hline Soldanella alpina & E1 & . & . & . & . & 1 & . & . & 5 & . \\
\hline Petasites paradoxus & E1 & . & . & . & . & . & 3 & . & 1 & 5 \\
\hline Geranium macrorrhizum & E1 & . & . & . & . & . & . & 1 & 4 & . \\
\hline Heracleum pollinianum & E1 & . & . & . & . & . & . & . & 17 & . \\
\hline Dryopteris villarii & E1 & . & . & . & . & . & . & . & 8 & . \\
\hline Arabis alpina & E1 & . & . & . & . & . & . & . & 6 & . \\
\hline Molopospermum peloponesiacum & E1 & . & . & . & . & . & . & . & 5 & . \\
\hline Ligustricum sequieri & E1 & . & . & . & . & . & . & . & 2 & . \\
\hline Biscutella laevigata & E1 & . & . & . & . & . & . & . & 1 & 1 \\
\hline Festuca nitida & E1 & . & . & . & . & . & . & . & 1 & . \\
\hline Rhodiola rosea & E1 & . & . & . & . & . & . & . & 1 & . \\
\hline Hieracium porrifolium & E1 & . & . & . & . & . & . & . & . & 4 \\
\hline Aquilegia iulia & E1 & . & . & . & . & . & . & . & . & 2 \\
\hline Achnatherum calamagrostis & E1 & . & . & . & . & . & . & . & . & 1 \\
\hline Gypsophila repens & E1 & . & . & . & . & . & . & . & . & 1 \\
\hline Scrophularia juratensis & E1 & . & . & . & . & . & . & . & . & 1 \\
\hline \multicolumn{11}{|l|}{ Cystopteridion fragilis } \\
\hline Cystopteris fragilis & E1 & 75 & 44 & . & . & 4 & 12 & 17 & 56 & 10 \\
\hline Valeriana tripteris & E1 & 75 & 6 & . & . & 38 & 70 & 33 & 74 & 74 \\
\hline Asplenium viride & E1 & 50 & 19 & . & . & 32 & 30 & 18 & 75 & 67 \\
\hline Moehringia muscosa & E1 & . & . & . & . & 28 & 8 & 5 & 23 & 14 \\
\hline Carex brachystachys & E1 & . & . & . & . & 2 & 4 & . & 9 & 22 \\
\hline Sedum hispanicum & E1 & . & . & . & . & . & . & 1 & 4 & 1 \\
\hline Cystopteris regia (C. alpina) & E1 & . & . & . & . & . & . & . & 9 & 1 \\
\hline Heliosperma pusillum & E1 & . & . & . & . & . & . & . & 2 & . \\
\hline Primula carniolica & $\mathrm{E} 1$ & . & . & . & . & . & . & . & $\mathrm{r}$ & 12 \\
\hline \multicolumn{11}{|l|}{ Physoplexido-Saxifragion petraeae } \\
\hline Paederota lutea & E1 & . & . & . & . & 2 & 1 & 3 & 27 & 53 \\
\hline Campanula cespitosa & E1 & . & . & . & . & 1 & . & . & 4 & 26 \\
\hline Campanula carnica & E1 & . & . & . & . & . & . & 2 & 10 & 7 \\
\hline Saxifraga crustata & E1 & . & . & . & . & . & . & . & 3 & 6 \\
\hline Saxifraga petraea & E1 & . & . & . & . & . & . & . & 2 & . \\
\hline Phyteuma scheuchzeri subsp. columnae & E1 & . & . & . & . & . & . & . & 1 & 41 \\
\hline Campanula justiniana & E1 & . & . & . & . & . & . & . & 1 & . \\
\hline Saxifraga tenella & E1 & . & . & . & . & . & . & . & 1 & . \\
\hline Paederota bonarota & E1 & . & . & . & . & . & . & . & 1 & . \\
\hline
\end{tabular}




\begin{tabular}{|c|c|c|c|c|c|c|c|c|c|c|}
\hline \multicolumn{2}{|c|}{ Successive number (Zaporedna številka) } & \multirow{2}{*}{1} & \multirow{2}{*}{2} & \multirow{2}{*}{$\begin{array}{l}3 \\
.\end{array}$} & \multirow{2}{*}{4} & \multirow{2}{*}{5} & \multirow{2}{*}{6} & \multirow{2}{*}{$\begin{array}{l}7 \\
.\end{array}$} & \multirow{2}{*}{8} & \multirow{2}{*}{$\begin{array}{l}9 \\
2\end{array}$} \\
\hline Athamanta turbith & E1 & & & & & & & & & \\
\hline Micromeria thymifolia & E1 & . & . & . & . & . & . & . & . & 2 \\
\hline \multicolumn{11}{|l|}{ Potentilletalia caulescentis } \\
\hline Valeriana saxatilis & E1 & . & . & . & . & 1 & . & . & 6 & 34 \\
\hline Campanula cochleariifolia & E1 & . & . & . & . & 1 & 3 & . & 9 & 7 \\
\hline Galium pusillum & E1 & . & . & . & . & . & 1 & . & . & . \\
\hline Primula auricula & E1 & . & . & . & . & . & . & . & 6 & 12 \\
\hline Festuca stenantha & E1 & . & . & . & . & . & . & . & 4 & 4 \\
\hline Saxifraga hostii & $\mathrm{E} 1$ & . & . & . & . & . & . & . & 3 & . \\
\hline Potentilla caulescens & $\mathrm{E} 1$ & . & . & . & . & . & . & . & . & 9 \\
\hline \multicolumn{11}{|l|}{ Asplenietea trichomanis } \\
\hline Polypodium vulgare & E1 & . & 19 & . & . & 10 & . & 4 & 25 & 14 \\
\hline Asplenium trichomanes & E1 & . & . & . & . & 15 & 1 & 14 & 30 & 33 \\
\hline Asplenium ruta-muraria & E1 & . & . & . & . & 9 & . & 2 & 27 & 52 \\
\hline Kernera saxatilis & E1 & . & . & . & . & . & . & . & 1 & 8 \\
\hline Sedum album & E1 & . & . & . & . & . & . & . & 1 & . \\
\hline Sedum maximum & E1 & . & . & . & . & . & . & . & 1 & . \\
\hline \multicolumn{11}{|l|}{ Other species (Ostale vrste) } \\
\hline Aquilegia sp. & E1 & . & . & . & . & 3 & 15 & . & . & . \\
\hline Poa sp. & E1 & . & . & . & . & 1 & . & . & . & . \\
\hline Alchemilla sp. & E1 & . & . & . & . & . & 1 & . & . & . \\
\hline Corydalis sp. & E1 & . & . & . & . & . & 1 & . & . & . \\
\hline Galium sp. & E1 & . & . & . & . & . & 1 & . & . & . \\
\hline Knautia sp. & E1 & . & . & . & . & . & 1 & . & . & . \\
\hline Thymus sp. & E1 & . & . & . & . & . & 1 & . & . & . \\
\hline Hieracium sp. & E1 & . & . & . & . & . & . & . & 2 & 1 \\
\hline Festuca sp. & E1 & . & . & . & . & . & . & . & 2 & . \\
\hline \multicolumn{11}{|c|}{ Mosses and lichens (Mahovi in lišaji) } \\
\hline Ctenidium molluscum & E0 & 75 & 88 & . & . & 50 & 68 & 45 & 89 & 80 \\
\hline Anomodon attenuatus & E0 & 75 & 56 & . & . & . & . & . & 2 & . \\
\hline Fissidens taxifolius & E0 & 50 & 25 & . & . & 7 & 10 & 12 & . & . \\
\hline Tortella fragilis & E0 & 50 & 6 & . & . & . & . & . & . & . \\
\hline Mnium spinosum & E0 & 50 & 31 & . & . & . & . & . & . & . \\
\hline Peltigera leucophlebia & E0 & 38 & 31 & 14 & . & 1 & 1 & . & 10 & 3 \\
\hline Plagiochila asplenioides & E0 & 38 & 50 & 29 & . & 27 & 27 & 9 & 2 & 9 \\
\hline Grimmia pulvinata & E0 & 25 & 31 & . & . & 2 & . & 2 & . & . \\
\hline Camptothecium lutescens & E0 & 25 & 56 & . & . & 8 & 7 & 2 & . & 2 \\
\hline Tortella tortuosa & E0 & 25 & 38 & . & . & 28 & 56 & 15 & 78 & 74 \\
\hline Taxiphyllum depressum & E0 & 25 & 62 & 14 & . & . & . & . & . & . \\
\hline Plagiomnium elatum & E0 & 25 & 25 & 43 & . & . & . & . & . & . \\
\hline Pellia epiphylla & E0 & 25 & 12 & . & . & . & 1 & . & . & . \\
\hline Anomodon viticulosus & E0 & 25 & 12 & . & . & . & . & . & . & . \\
\hline Conocephalum conicum & E0 & 12 & 12 & . & 50 & 2 & 11 & 2 & 16 & 13 \\
\hline Rhizomnium punctatum & E0 & 12 & 19 & . & 100 & 2 & 8 & . & 8 & 3 \\
\hline Isothecium alopecuroides & E0 & 12 & 62 & . & . & 15 & 8 & 26 & 28 & 17 \\
\hline Orthodicranum montanum & E0 & 12 & 50 & 14 & . & . & . & . & . & . \\
\hline Cladonia pyxidata & E0 & 12 & 19 & . & . & . & . & 1 & 11 & . \\
\hline
\end{tabular}




\begin{tabular}{|c|c|c|c|c|c|c|c|c|c|c|}
\hline \multicolumn{2}{|c|}{ Successive number (Zaporedna številka) } & 1 & 2 & 3 & 4 & 5 & 6 & 7 & 8 & 9 \\
\hline Atrichum undulatum & E0 & . & 25 & . & 50 & 3 & 4 & 2 & 1 & 3 \\
\hline Eurinchium angustirete & E0 & . & 25 & . & . & . & . & . & 3 & . \\
\hline Platydictya subtilis & E0 & . & 25 & . & . & . & . & . & . & . \\
\hline Plagiomnium undulatum & E0 & . & 19 & . & . & 1 & . & 1 & 5 & 2 \\
\hline Polytrichum formosum & E0 & . & 19 & . & . & 10 & 10 & 4 & 32 & 70 \\
\hline Thamnobryum alopecurum & E0 & . & 19 & . & . & . & . & . & . & . \\
\hline Hypnum cupressiforme & E0 & . & 12 & 14 & . & 5 & 4 & 5 & 3 & 17 \\
\hline Mnium marginatum & E0 & . & . & . & 50 & . & 1 & . & 2 & 2 \\
\hline Brachythecium rutabulum & E0 & . & . & . & 50 & . & . & 2 & 3 & . \\
\hline Marchantia polymorpha & E0 & . & . & . & 50 & . & 1 & 1 & 5 & 4 \\
\hline Brachythecium reflexum & E0 & . & . & . & 50 & . & 1 & . & . & . \\
\hline Fissidens dubius & E0 & . & . & . & . & 15 & 30 & 3 & 45 & 59 \\
\hline Dicranum scoparium & E0 & . & . & . & . & 12 & 8 & 4 & 23 & 38 \\
\hline Eurhynchium striatum & E0 & . & . & . & . & 6 & 1 & . & . & 7 \\
\hline Encalypta streptocarpa & E0 & . & . & . & . & 5 & 4 & . & 4 & 11 \\
\hline Neckera crispa & E0 & . & . & . & . & 3 & 1 & 3 & 3 & 71 \\
\hline Hylocomium splendens & E0 & . & . & . & . & 3 & 1 & . & 3 & 9 \\
\hline Cladonia sp. & E0 & . & . & . & . & 3 & 3 & . & 11 & 18 \\
\hline Plagiothecium nemorale & E0 & . & . & . & . & 2 & . & . & 1 & . \\
\hline Metzgeria furcata & E0 & . & . & . & . & 2 & . & 4 & 3 & 4 \\
\hline Brachythecium velutinum & E0 & . & . & . & . & 2 & . & . & 3 & . \\
\hline Rhytidiadelphus triquetrus & E0 & . & . & . & . & 2 & 3 & 1 & 8 & 16 \\
\hline Mnium thomsonii & E0 & . & . & . & . & 2 & 4 & 3 & 22 & 6 \\
\hline Peltigera canina & E0 & . & . & . & . & 2 & . & 3 & 32 & 8 \\
\hline Schistidium apocarpum & E0 & . & . & . & . & 2 & 1 & 5 & 46 & 24 \\
\hline Paraleucobryum sauteri & E0 & . & . & . & . & 2 & . & 2 & 50 & 4 \\
\hline Peltigera sp. & E0 & . & . & . & . & 2 & . & . & . & . \\
\hline Hookeria lucens & E0 & . & . & . & . & 1 & 1 & . & 1 & 4 \\
\hline Dicranella heteromalla & E0 & . & . & . & . & 1 & . & . & 1 & 5 \\
\hline Cladonia fimbriata & E0 & . & . & . & . & 1 & . & . & 1 & . \\
\hline Bartramia pomiformis & E0 & . & . & . & . & 1 & . & 1 & 2 & 13 \\
\hline Cladonia rangiferina & E0 & . & . & . & . & 1 & . & . & 2 & . \\
\hline Dicranum polysetum & E0 & . & . & . & . & 1 & 1 & . & . & . \\
\hline Plagiothecium cavifolium & E0 & . & . & . & . & 1 & 5 & . & . & . \\
\hline Mnium stellare & E0 & . & . & . & . & 1 & 7 & . & . & . \\
\hline Cladonia coniocraea & E0 & . & . & . & . & 1 & . & . & . & . \\
\hline Eurhynchium hians & E0 & . & . & . & . & 1 & . & . & . & . \\
\hline Isopterygium seligeri & E0 & . & . & . & . & 1 & . & . & . & . \\
\hline Metzgeria conjugata & E0 & . & . & . & . & 1 & . & . & . & . \\
\hline Plagiothecium undulatum & E0 & . & . & . & . & . & 1 & . & 1 & 2 \\
\hline Solorina saccata & E0 & . & . & . & . & . & 1 & . & 1 & . \\
\hline Plagiothecium denticulatum & E0 & . & . & . & . & . & 1 & . & 4 & 6 \\
\hline Calypogeia azurea & E0 & . & . & . & . & . & 1 & . & . & . \\
\hline Calypogeia fissa & E0 & . & . & . & . & . & 1 & . & . & . \\
\hline Plagiothecium curvifolium & E0 & $\cdot$ & . & . & . & . & 1 & . & . & . \\
\hline Scapania aspera & E0 & . & . & . & . & . & 1 & . & . & . \\
\hline Homalothecium lutescens & E0 & . & . & . & . & . & . & 3 & 11 & 1 \\
\hline
\end{tabular}




\begin{tabular}{|c|c|c|c|c|c|c|c|c|c|c|}
\hline \multicolumn{2}{|c|}{ Successive number (Zaporedna številka) } & 1 & 2 & 3 & 4 & 5 & 6 & 7 & 8 & 9 \\
\hline Pseudoleskea catenulata & E0 & . & r & . & . & . & . & 2 & 20 & . \\
\hline Thuidium tamariscinum & E0 & . & . & . & . & . & . & 1 & 2 & 10 \\
\hline Eurhynchium sp. & E0 & . & . & . & . & . & . & 1 & 3 & . \\
\hline Lobaria pulmonaria & E0 & . & . & . & . & . & . & 1 & 3 & . \\
\hline Homalothecium philippeanum & E0 & . & . & . & . & . & . & 1 & 9 & 3 \\
\hline Plagiochila porelloides & E0 & . & . & . & . & . & . & . & 20 & 32 \\
\hline Bryum capillare & E0 & . & . & . & . & . & . & . & 13 & 4 \\
\hline Mnium sp. & E0 & . & . & . & . & . & . & . & 3 & 3 \\
\hline Collema cristatum & E0 & . & . & . & . & . & . & . & 3 & . \\
\hline Dermatocarpon miniatum & E0 & . & . & . & . & . & . & . & 3 & . \\
\hline Isothecium myosuroides & E0 & . & . & . & . & . & . & . & 2 & 6 \\
\hline Orthothecium rufescens & E0 & . & . & . & . & . & . & . & 2 & 16 \\
\hline Plagiomnium cuspidatum & E0 & . & . & . & . & . & . & . & 2 & . \\
\hline Porella arboris-vitae & E0 & . & . & . & . & . & . & . & 2 & . \\
\hline Porella platyphylla & E0 & . & . & . & . & . & . & . & 2 & . \\
\hline Bryum argenteum & E0 & . & . & . & . & . & . & . & 1 & 1 \\
\hline Bryum sp. & E0 & . & . & . & . & . & . & . & 1 & 1 \\
\hline Plagiothecium sp. & E0 & . & . & . & . & . & . & . & 1 & 2 \\
\hline Anomodon sp. & E0 & . & . & . & . & . & . & . & 1 & . \\
\hline Brachythecium sp. & E0 & . & . & . & . & . & . & . & 1 & . \\
\hline Distichium capillaceum & E0 & . & . & . & . & . & . & . & 1 & . \\
\hline Hypogymnia physodes & E3 & . & . & . & . & . & . & . & 1 & . \\
\hline Leucobryum glaucum & E0 & . & . & . & . & . & . & . & . & 32 \\
\hline Metzgeria sp. & E0 & . & . & & . & . & . & . & . & 9 \\
\hline Plagiopus oederi & E0 & . & . & . & . & . & . & . & . & 5 \\
\hline Dicranum sp. & E0 & . & . & . & . & . & . & . & . & 4 \\
\hline Scleropodium purum & E0 & . & . & . & . & . & . & . & . & 2 \\
\hline Bryhnia sp. & E0 & . & . & . & . & . & . & . & . & 1 \\
\hline Collema sp. & E0 & . & . & . & . & . & . & . & . & 1 \\
\hline Encalypta sp. & E0 & . & . & $\cdot$ & . & . & . & . & . & 1 \\
\hline Nardia scalaris & E0 & . & . & . & . & . & . & . & . & 1 \\
\hline Neckera complanata & E0 & . & . & & . & . & . & . & - & 1 \\
\hline Sphagnum sp. & E0 & . & . & & . & . & . & . & . & 1 \\
\hline
\end{tabular}

\section{Legend - Legenda}

ApF Aconito paniculati-Fagetum

SmF Stellario montanae-Fagetum

$\mathrm{CwF}$ Cardamino waldsteinii-Fagetum (Cardamino savensi-Fagetum var. Abies alba)

MoF Myrrhido-Fagetum

AtF Anemono trifoliae-Fagetum

SrF Saxifrago rotundifolii-Fagetum

$\mathrm{RpF}$ Ranunculo platanifolii-Fagetum

PIF Polysticho lonchitis-Fagetum

$\mathrm{RhF}$ Rhododendro hirsuti-Fagetum

r Frequency less than $1 \%$ (frekvenca manj kot $1 \%$ ) 
Table 10: Phytosociological groups in the syntaxa of the associations Polysticho lonchitis-Fagetum (columns 1-31), Ranunculo platanifolli-Fagetum (column 32) and Rhodothamno-Laricetum (column 34) in the Julian Alps and NW-Dinaric Alps (relative frequencies).

\begin{tabular}{|c|c|c|c|c|c|c|c|c|c|c|c|c|}
\hline Successive number (Zaporedna številka) & 1 & 2 & 3 & 4 & 5 & 6 & 7 & 8 & 9 & 10 & 11 & 12 \\
\hline Number of cluster (Številka skupine) & 6 & 5 & 2 & 32 & 31 & 1 & 8 & 9 & 10 & 12 & 11 & 30 \\
\hline Number of relevés (Število popisov) & 3 & 3 & 3 & 4 & 5 & 9 & 19 & 12 & 44 & 57 & 6 & 30 \\
\hline Aremonio-Fagion & 6,3 & 4,1 & 3,3 & 2,4 & 6,0 & 5,1 & 5,6 & 5,0 & 3,9 & 4,9 & 3,7 & 5,4 \\
\hline Erythronio-Carpinion & 0 & 0,4 & 0 & 0 & 0 & 0 & 0 & 0,1 & 0 & 0,0 & 0 & 0,1 \\
\hline Tilio-Acerion & 3,2 & 1,6 & 0,9 & 2,4 & 2,6 & 2,4 & 2,9 & 1,6 & 1,9 & 1,9 & 0,3 & 0,5 \\
\hline Fagetalia sylvaticae & 13,8 & 14,7 & 9,0 & 9,7 & 17,4 & 13,2 & 15,6 & 14,3 & 14,4 & 15,4 & 14,5 & 16,3 \\
\hline Quercetalia pubescenti-petraeae & 0,6 & 1,6 & 0 & 0,8 & 1,7 & 0,4 & 1,1 & 2,0 & 0,4 & 1,2 & 0,1 & 1,5 \\
\hline Querco-Fagetea & 2,5 & 2,8 & 4,3 & 1,6 & 2,3 & 2,6 & 3,8 & 2,4 & 2,0 & 1,9 & 2,2 & 3,2 \\
\hline Vaccinio-Piceetea & 32,1 & 22,4 & 32,1 & 36,0 & 18,5 & 31,0 & 31,7 & 20,4 & 29,1 & 28,1 & 25,6 & 21,8 \\
\hline Erico-Pinetea & 8,2 & 6,9 & 4,7 & 5,7 & 7,7 & 9,1 & 6,5 & 10,1 & 6,4 & 11,0 & 6,0 & 8,5 \\
\hline Sambuco-Salicion capreae, Rhamno-Prunetea & 3,2 & 2,8 & 0,9 & 2,0 & 1,1 & 1,4 & 1,6 & 1,9 & 2,5 & 1,7 & 1,5 & 1,1 \\
\hline Betulo-Alnetea & 5,7 & 2,4 & 0,9 & 4,5 & 0,6 & 1,6 & 1,8 & 3,5 & 2,7 & 2,5 & 2,2 & 1,4 \\
\hline Mulgedio-Aconitetea & 7,5 & 8,9 & 11,3 & 10,1 & 8,8 & 8,7 & 7,6 & 9,0 & 9,1 & 5,6 & 6,2 & 7,0 \\
\hline Caricion austroalpinae & 0 & 0,4 & 0,5 & 0,4 & 3,1 & 1,2 & 0,8 & 1,7 & 0,7 & 1,1 & 2,0 & 1,6 \\
\hline Caricion ferrugineae & 0 & 0,8 & 1,4 & 0,8 & 1,1 & 0,8 & 0,3 & 0,5 & 0,2 & 0,6 & 0,5 & 0,6 \\
\hline Caricion firmae & 0 & 1,2 & 0 & 0 & 0 & 0 & 0 & 0 & 0 & 0,1 & 0 & 0,1 \\
\hline Elyno-Seslerietea & 3,1 & 4,1 & 4,3 & 4,5 & 6,0 & 5,7 & 2,0 & 5,1 & 3,1 & 4,3 & 6,0 & 4,8 \\
\hline Nardion strictae, Juncetea trifidi & 0 & 0,8 & 0,9 & 2,4 & 0 & 0,1 & 0,5 & 1,3 & 1,1 & 0,7 & 1,7 & 1,4 \\
\hline Caricetalia davallianae & 0,6 & 0 & 0,9 & 0,8 & 0 & 0 & 0 & 0,1 & 0,0 & 0,1 & 0 & 0,1 \\
\hline Festuco-Brometea & 0,6 & 2,0 & 0 & 0,4 & 4,0 & 0,8 & 0,9 & 1,4 & 0,4 & 0,1 & 1,7 & 1,8 \\
\hline Trifolio-Geranietea & 0 & 1,2 & 0 & 0 & 3,1 & 0,2 & 0 & 1,5 & 0,0 & 0,1 & 0,1 & 1,4 \\
\hline Poo alpinae-Trisetetalia & 1,3 & 0 & 0,9 & 1,2 & 0,9 & 1,4 & 0,1 & 0,3 & 0,2 & 0,3 & 0,5 & 0,4 \\
\hline Molinio-Arrbenatberetea & 0,6 & 0 & 0,5 & 1,2 & 0,6 & 0,6 & 0,2 & 0,7 & 0,2 & 0,2 & 1,3 & 0,3 \\
\hline Epilobietea angustifolii, Galio-Urticetea & 1,9 & 2,0 & 0,5 & 0 & 0,3 & 1,2 & 0,1 & 0,5 & 1,0 & 0,7 & 2,0 & 1,0 \\
\hline Thlaspietea rotundifolii, Arabidetalia caeruleae & 1,2 & 3,2 & 5,2 & 3,2 & 6,0 & 2,2 & 2,8 & 3,0 & 2,9 & 3,2 & 2,5 & 3,0 \\
\hline Cystopteridion fragilis & 3,2 & 4,1 & 5,2 & 2,4 & 2,8 & 2,8 & 3,1 & 4,1 & 4,1 & 3,1 & 3,5 & 3,9 \\
\hline Physoplexido comosae-Saxifragion petraeae & 0 & 0,4 & 0,9 & 0,8 & 0,3 & 0,6 & 0,7 & 0,7 & 0,1 & 0,9 & 0,7 & 0,9 \\
\hline Potentilletalia caulescentis & 0 & 1,2 & 0,5 & 0 & 0,3 & 0,4 & 0,1 & 0 & 0,6 & 0,8 & 0,7 & 1,0 \\
\hline Asplenietea trichomanis & 0 & 1,6 & 0 & 0 & 1,1 & 0,4 & 0,4 & 0,7 & 1,2 & 0,6 & 2,2 & 1,5 \\
\hline Other species (Druge vrste) & 0 & 0 & 0 & 0 & 0 & 0 & 0 & 0,1 & 0 & 0 & 0,3 & 0,2 \\
\hline Mosses and lichens (Mahovi in lišaji) & 4,4 & 8,1 & 10,8 & 6,5 & 3,7 & 5,5 & 10,0 & 8,0 & 10,8 & 7,8 & 10,2 & 9,4 \\
\hline Total (Skupaj) & 100 & 100 & 100 & 100 & 100 & 100 & 100 & 100 & 100 & 100 & 100 & 100 \\
\hline
\end{tabular}


Tabela 10: Skupine diagnostičnih vrst v sintaksonih asociacij Polysticho lonchitis-Fagetum (stolpci 1-31), Ranunculo platanifolli-Fagetum (stolpec 32) in Rhodothamno-Laricetum (stolpec 34) v Julijskih Alpah in severozahodnje delu Dinarskega gorstva (relativne frekvence).

\begin{tabular}{|c|c|c|c|c|c|c|c|c|c|c|c|c|c|c|c|c|c|c|c|c|c|}
\hline 13 & 14 & 15 & 16 & 17 & 18 & 19 & 20 & 21 & 22 & 23 & 24 & 25 & 26 & 27 & 28 & 29 & 30 & 31 & 32 & 33 & 34 \\
\hline 7 & 20 & 18 & 19 & 22 & 23 & 26 & 28 & 27 & 29 & 24 & 25 & 13 & 14 & 21 & 17 & 4 & 15 & 16 & 3 & Total & L \\
\hline 40 & 41 & 25 & 49 & 48 & 8 & 25 & 50 & 17 & 13 & 10 & 5 & 9 & 36 & 5 & 4 & 3 & 5 & 4 & 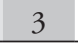 & 602 & 458 \\
\hline 4,8 & 4,8 & 41 & 4 & 60 & 1,8 & 4,9 & 53 & 4 & 31 & 3 & 7,4 & 7 & 4,0 & 3 & 6,0 & 4,2 & 2 & 3,7 & 6 & 4,7 & ,7 \\
\hline 0 & 0,0 & 0,1 & 0,9 & 0,3 & 0,2 & 0,3 & 0,5 & 0,6 & 0 & 1,1 & 0,4 & 0 & 0,5 & 0 & 0,3 & 0 & 0,5 & 0,3 & 1,0 & 0,3 & 0 \\
\hline 1,6 & 3,4 & 3,5 & 1,7 & 1,6 & 1,8 & 3,2 & 1,7 & 4,3 & 1,3 & 7,2 & 1,1 & 5,3 & 7,0 & L & 3,2 & 2,7 & 1,9 & 4,0 & 8,8 & 2,6 & 0,4 \\
\hline 13,7 & 16,0 & 17,4 & 20,1 & 18,3 & 20,6 & 17,2 & 18,2 & 20,3 & 17,6 & 22,9 & 20,6 & 27,4 & 24,1 & 20,6 & 18,9 & 17,2 & 21,1 & 19,3 & 31,0 & 17,5 & 6,8 \\
\hline 1,5 & 1,4 & 0,4 & 1,2 & 2,3 & 0,2 & 0,8 & 1,2 & 0,5 & 1,7 & 0,8 & 0,4 & 0,4 & 0,4 & 0,5 & 0,9 & 0,4 & 3,2 & 1,4 & 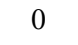 & 1,1 & 0,5 \\
\hline 2,7 & 2,4 & 3,9 & 3,4 & 3,0 & 3,5 & 2,8 & 3,2 & 3,2 & 2,0 & 5,7 & 4,6 & 2,7 & 3,4 & נו & 3,5 & 3,4 & 4,6 & 2,9 & 4,6 & 2,9 & 1,9 \\
\hline 26,0 & 23,9 & 21,4 & 19,6 & 16,6 & 17,2 & 20,2 & 16,5 & 14,3 & 13,6 & 9,3 & 17,7 & 14,8 & 12,0 & 19,5 & 19,2 & 17,2 & 15,4 & 13,8 & 6,7 & 21,1 & 24 \\
\hline 6,8 & 3,5 & 2,8 & 3,6 & 8,0 & 2,6 & 2,2 & 4,3 & 1,2 & 2,8 & 0,3 & 4,3 & 1,3 & 0,3 & 1,1 & 4,4 & 3,8 & 1,4 & 2,6 & 0 & 5,1 & 7,9 \\
\hline 2,0 & 2,2 & 2,0 & 1,3 & 1,2 & 1,1 & 1,8 & 0,8 & 1,2 & 1,0 & 0,6 & 1,8 & 2,9 & 2,2 & 2,7 & 1,3 & 0,8 & 0,5 & 0,9 & 2,0 & 1,7 & 0,8 \\
\hline 2,9 & 8 & 2,4 & ,3 & 0 & 1,5 & ,3 & 1,3 & 0,9 & 1,1 & 0 , & 2,1 & 0,7 & 1,4 & te & , & 2,3 & 1,4 & 0,9 & 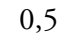 & 19 &, 2 \\
\hline 7,4 & 10,9 & 17,7 & 14,9 & 9,4 & 11,6 & 13,0 & 9,7 & 14,8 & 12,4 & 12,8 & 12,8 & 19,5 & 22,1 & 19,2 & 13,2 & 22,5 & 12,2 & 18,4 & 20,1 & 11,4 & 9 \\
\hline 0,8 & 0,5 & 0,4 & 0,8 & 1,6 & 0,9 & 0,9 & 1,5 & 1,5 & 2,1 & 1, & 1,1 & . & 0,1 & (נ) & , J & 0,7 & 1,4 & , & , & 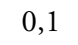 & 1,9 \\
\hline 0,4 & 0,2 & 0,4 & 05 & 0,7 & 0 & 0,6 & 0,7 & 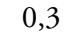 & , & 0 , & 0,4 & 0 & 0,0 & 0,3 & 0 & 0,8 & 0,5 & 0 & 0 & 0 & 0,8 \\
\hline 0,1 & 0 & 0 & U & 0,1 & 0 & 0 & 0 & 8 & 0 & 0 & 0 & 0 & 0 & c & 0 & 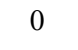 & 0 & 0 & 0 & 0,0 & 0,7 \\
\hline 2,9 & 2,4 & 2,2 & 2,8 & 4 & 2,4 & 1 & 4,6 & 7 & 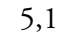 & 1, & 3,5 & 0 & 0,2 & 1,4 & 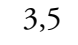 & 1,1 & 2,7 & 3 & 0 & 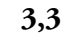 & 11 \\
\hline 1,1 & 0,4 & 0,5 & (5) & 列 & - & . & 1,3 & , & 0,9 & 0,3 & - & 0 & 0,1 & 0 & 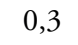 & 0,4 & 0,5 & 0,6 & & का & 2 \\
\hline 0,1 & 0,1 & 0,1 & 0,0 & 0 & 0 & 0,1 & 0 & 0,1 & 0 & 0 & 0 & 0 & 0 & 0 & 0 & 0,4 & 0 & 0 & 0 & 0,1 & 1 \\
\hline 0,9 & 0,9 & 0,3 & 1,3 & 2,7 & 0,9 & ? & 1,2 & 0,4 & 2,6 & 0,9 & 1,1 & 0,2 & 0,3 & 0 & 1,3 & 0 & 1,1 & 1,7 & 0 & 1 & 1,2 \\
\hline 0,2 & 0,4 & 0,4 & 0.8 & 1,0 & 09 & 04 & 15 & 08 & 22 & 0 & 0,4 & 0 & 0,4 & 03 & 0,6 & 1,1 & 1,4 & 2,3 & 0 & 0,7 & 0,3 \\
\hline 0,4 & 0,2 & 1,0 & 0,2 & 0 & 0 & 0,6 & 0,9 & 0 , & 1 & 1 , & 2,5 & 0 & 0,9 & 0,3 & 0 & 1,5 & 0,3 & 0,0 & 0 & 0,3 & 1 \\
\hline 0,2 & 0,6 & , & , & , & 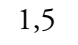 & 0,0 & 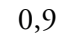 & , & , & 1,5 & 1, & , & 1,7 & , & 1,6 & 2, & $1, x$ & 1, & & (1) & 1,3 \\
\hline 0,8 & 1 & 1,6 & 0,8 & 0,1 & 2,0 & 1,8 & 1,7 & 3, & 17 & 3 , & 2,1 & 2 & 3,3 & 2,5 & 2,2 & 2,3 & 0,8 & 1,2 & 5,7 & 1,4 & 1 \\
\hline 3,3 & 2,0 & 2,6 & 2,7 & 3,8 & 4,4 & 2,7 & 2,7 & 2,3 & 3,7 & 2,1 & 1,8 & 1,1 & 1,4 & 1,4 & 1,3 & 3,1 & 2,2 & 3,2 & 1,0 & 2,8 & 5,7 \\
\hline 3,3 & 4,7 & 3,6 & 43 & 42 & 61 & 45 & 4,5 & 3,8 & 4 & 3,7 & 4,3 & 3, & 2,4 & 3,0 & 3,8 & 3,4 & 4,3 & 4,9 & 3,1 & 3,9 & 1 \\
\hline 1,2 & 1,1 & 0,2 & 0,6 & 0,7 & 2,4 & 1,3 & 0,7 & 0,9 & 16 & 0,9 & 1,1 & 0,2 & 0,1 & 0,8 & 0,9 & 0 & 1,4 & 0,9 & 0 & 0,8 & 1,6 \\
\hline 0,5 & 0,5 & 0,4 & 0,2 & 0,5 & 0 & 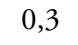 & 0,1 & $0, \ldots$ & , & 0,2 & 0 & 0 & 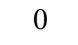 & $0, J$ & J & , 0 & 0,3 & 0 & 0 & נ, & 1,0 \\
\hline 0,1 & 1,1 & 0,9 & 1,3 & 1,3 & 3,3 & 2,0 & 2,5 & 3,0 & 2,7 & 3,1 & 1,1 & 0,9 & 0,5 & 2,2 & 1,6 & 0,4 & 2,2 & 2,3 & 1,0 & 1,4 & ,2 \\
\hline 0 & 0,2 & 0 & 0,1 & 0,0 & 0,4 & 0 & 0,3 & 0 & 0 & 0 & 0 & , & 0 & 0,3 & 0 & 0 & 0,3 & 0 & 0 & 0,1 & 0 \\
\hline 15,2 & 12,0 & 8,7 & 10,2 & 1,0 & 10,2 & 13,8 & 12,3 & 13,4 & 12,1 & 15,5 & 5,7 & 0,1 & 11,0 & 11,5 & 8,2 & 7,2 & 14,3 & 8,4 & 9,3 & 10,4 & 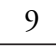 \\
\hline 100 & 100 & 100 & 100 & 100 & 100 & 100 & 100 & 100 & 100 & 100 & 100 & 100 & 100 & 100 & 100 & 100 & 100 & 100 & 100 & 100 & 10 \\
\hline
\end{tabular}


Table 11: Characteristic species combination in the stands of the association Polysticho lonchitis-Fagetum in the Southeastern Alps and Northwestern Dinaric Alps (species with constancy $40 \%$ and more).

Tabela 11: Značilna (stanovitna) kombinacija vrst v sestojih asociacije Poysticho lonchitis-Fagetum v Jugovzhodnih Alpah in severnem delu Dinarskega gorstva (vrste s stalnostjo 40 in več \%).

\begin{tabular}{|c|c|c|c|c|c|c|c|}
\hline FS & Fagus sylvatica & E3 & 100 & $\mathrm{VP}$ & Maianthemum bifolium & E1 & 55 \\
\hline $\mathrm{MuA}$ & Polygonatum verticillatum & E1 & 85 & $\mathrm{AF}$ & Cyclamen purpurascens & E1 & 54 \\
\hline VP & Picea abies & $\mathrm{E} 3,2,1$ & 81 & FS & Melica nutans & E1 & 52 \\
\hline $\mathrm{AF}$ & Cardamine enneaphyllos & E1 & 78 & $\mathrm{EP}$ & Calamagrostis varia & E1 & 52 \\
\hline VP & Polystichum lonchitis & E1 & 76 & $\mathrm{VP}$ & Gentiana asclepiadea & E1 & 51 \\
\hline $\mathrm{Cy}$ & Asplenium viride & E1 & 73 & EP & Rhododendron hirsutum & $\mathrm{E} 2$ & 51 \\
\hline $\mathrm{Cy}$ & Valeriana tripteris & E1 & 72 & FS & Galium laevigatum & E1 & 51 \\
\hline FS & Lonicera alpigena & E2 & 72 & $\mathrm{TA}$ & Acer pseudoplatanus & $\mathrm{E} 1, \mathrm{E} 2, \mathrm{E} 3$ & 49 \\
\hline TR & Adenostyles glabra & E1 & 71 & QF & Anemone nemorosa & E1 & 49 \\
\hline VP & Aposeris foetida & E1 & 71 & $\mathrm{BA}$ & Sorbus chamaemespilus & $\mathrm{E} 2$ & 48 \\
\hline $\mathrm{MuA}$ & Veratrum album & E1 & 70 & $\mathrm{MuA}$ & Aconitum lycoctonum subsp. ranun- & E1 & 48 \\
\hline $\mathrm{MuA}$ & Athyrium filix-femina & E1 & 70 & & culifolium (A. lupicida) & & \\
\hline FS & Daphne mezereum & E2 & 69 & $\mathrm{VP}$ & Solidago virgaurea & E1 & 48 \\
\hline $\mathrm{VP}$ & Vaccinium myrtillus & E1 & 69 & FS & Prenanthes purpurea & E1 & 47 \\
\hline VP & Rosa pendulina & E2 & 67 & ES & Betonica alopecuros & E1 & 44 \\
\hline EP & Rubus saxatilis & E1 & 67 & $\mathrm{VP}$ & Gymnocarpium dryopteris & E1 & 44 \\
\hline VP & Clematis alpina & $\mathrm{E} 2$ & 66 & SSC & Sorbus aucuparia & E2 & 42 \\
\hline $\mathrm{AF}$ & Anemone trifolia & E1 & 61 & ES & Sesleria caerulea & E1 & 42 \\
\hline FS & Mercurialis perennis & E1 & 61 & FS & Paris quadrifolia & E1 & 42 \\
\hline VP & Luzula sylvatica & E1 & 60 & $\mathrm{EP}$ & Erica carnea & E1 & 42 \\
\hline $\mathrm{VP}$ & Oxalis acetosella & E1 & 59 & $\mathrm{MuA}$ & Saxifraga rotundifolia & E1 & 41 \\
\hline FS & Lilium martagon & E1 & 59 & SSC & Sorbus aucuparia & $\mathrm{E} 3$;E2 & 41 \\
\hline FS & Dryopteris filix-mas & E1 & 58 & ML & Ctenidium molluscum & E0 & 88 \\
\hline VP & Calamagrostis arundinacea & E1 & 58 & ML & Tortella tortuosa & E0 & 76 \\
\hline VP & Veronica urticifolia & E1 & 57 & ML & Paraleucobryum sauteri & E0 & 50 \\
\hline FS & Galeobdolon flavidum & E1 & 56 & ML & Schistidium apocarpum & E0 & 47 \\
\hline Cy & Cystopteris fragilis & E1 & 55 & ML & Fissidens dubius & E0 & 44 \\
\hline
\end{tabular}


Table 12: Comparison of characteristic species combination in the stands of the associations Rhododthamno-Laricetum, Polysticho lonchitis-Fagetum and Rhododendro hirsuti-Fagetum.

Tabela 12: Primerjava značilne kombinacije vrst v sestojih asociacij Rhodothamno-Laricetum, Poysticho lonchitis-Fagetum in Rhododendro hirsuti-Fagetum.

\begin{tabular}{|c|c|c|c|c|c|}
\hline & & & $\begin{array}{l}\text { Rhodothamno- } \\
\text { Laricetum }\end{array}$ & $\begin{array}{c}\text { Polysticho lonchitis- } \\
\text { Fagetum }\end{array}$ & $\begin{array}{c}\text { Rhododendro hirsuti- } \\
\text { Fagetum }\end{array}$ \\
\hline & Number of relevés (Število popisov) & & 458 & 602 & 222 \\
\hline VP & Larix decidua & E3 & 100 & 25 & 10 \\
\hline EP & Rhododendron hirsutum & E2 & 90 & 51 & 91 \\
\hline VP & Vaccinium myrtillus & E1 & 85 & 69 & 63 \\
\hline AT & Asplenium viride & E1 & 81 & 73 & 67 \\
\hline VP & Vaccinium vitis-idaea & E1 & 81 & 30 & 16 \\
\hline EP & Pinus mugo & E2 & 80 & 33 & 11 \\
\hline VP & Luzula sylvatica & E1 & 79 & 60 & 5 \\
\hline VP & Picea abies & E3 & 78 & 81 & 73 \\
\hline VP & Polystichum lonchitis & E1 & 78 & 76 & 12 \\
\hline ES & Sesleria caerulea & E1 & 78 & 42 & 30 \\
\hline VP & Valeriana tripteris & E1 & 76 & 72 & 74 \\
\hline VP & Clematis alpina & E2 & 73 & 66 & 41 \\
\hline EP & Erica carnea & E1 & 72 & 42 & 77 \\
\hline EP & Calamagrostis varia & E1 & 71 & 52 & 81 \\
\hline PS & Paederota lutea & E1 & 71 & 27 & 53 \\
\hline $\mathrm{EP}$ & Rubus saxatilis & E1 & 70 & 67 & 58 \\
\hline $\mathrm{EP}$ & Rhodothamnus chamaecistus & E1 & 70 & 16 & 18 \\
\hline MuA & Viola biflora & E1 & 70 & 38 & 8 \\
\hline $\mathrm{CA}$ & Laserpitium peucedanoides & E1 & 69 & 33 & 5 \\
\hline FS & Daphne mezereum & E2 & 68 & 69 & 80 \\
\hline VP & Calamagrostis villosa & E1 & 65 & 29 & 3 \\
\hline VP & Aposeris foetida & E1 & 62 & 71 & 18 \\
\hline $\mathrm{BA}$ & Sorbus chamaemespilus & E2 & 62 & 48 & \\
\hline VP & Homogyne alpina & E1 & 61 & 22 & \\
\hline VP & Oxalis acetosella & E1 & 60 & 59 & 18 \\
\hline VP & Rosa pendulina & E2 & 60 & 67 & 66 \\
\hline $\mathrm{MuA}$ & Geranium sylvaticum & E1 & 59 & 31 & \\
\hline $\mathrm{BA}$ & Salix appendiculata & E2 & 58 & 29 & 5 \\
\hline VP & Hieracium murorum & E1 & 57 & 39 & 49 \\
\hline FS & Melica nutans & E1 & 57 & 52 & 42 \\
\hline ES & Aster bellidiastrum & E1 & 56 & 26 & 30 \\
\hline TR & Heliosperma alpestre & E1 & 52 & 6 & \\
\hline VP & Lycopodium annotinum & E1 & 50 & 15 & 7 \\
\hline VP & Dryopteris dilatata & E1 & 49 & 33 & 8 \\
\hline TR & Gymnocarpium robertianum & E1 & 49 & 21 & 67 \\
\hline VP & Homogyne sylvestris & E1 & 47 & 23 & 70 \\
\hline ES & Astrantia bavarica & E1 & 47 & 11 & \\
\hline SSC & Sorbus aucuparia subsp. aucuparia & E3 & 46 & 42 & 56 \\
\hline $\mathrm{MuA}$ & Veratrum album & E1 & 46 & 70 & 20 \\
\hline TR & Festuca nitida & E1 & 45 & 1 & \\
\hline VP & Solidago virgaurea & E1 & 45 & 48 & 53 \\
\hline MuA & Athyrium filix-femina & E1 & 44 & 70 & 31 \\
\hline $\mathrm{JT}$ & Campanula scheuchzeri & E1 & 44 & 36 & \\
\hline VP & Gymnocarpium dryopteris & E1 & 43 & 44 & 34 \\
\hline
\end{tabular}




\begin{tabular}{|c|c|c|c|c|c|}
\hline & & & $\begin{array}{l}\text { Rhodothamno- } \\
\text { Laricetum }\end{array}$ & $\begin{array}{c}\text { Polysticho lonchitis- } \\
\text { Fagetum }\end{array}$ & $\begin{array}{c}\text { Rhododendro hirsuti- } \\
\text { Fagetum }\end{array}$ \\
\hline $\mathrm{AF}$ & Anemone trifolia & E1 & 43 & 61 & 46 \\
\hline TR & Adenostyles glabra & E1 & 41 & 71 & 59 \\
\hline VP & Melampyrum sylvaticum & E1 & 40 & 8 & 3 \\
\hline $\mathrm{MuA}$ & Polygonatum verticillatum & E1 & 40 & 85 & 20 \\
\hline $\mathrm{AF}$ & Knautia drymeia & E1 & 40 & 18 & 17 \\
\hline $\mathrm{CF}$ & Carex ferruginea & E1 & 40 & 21 & 33 \\
\hline $\mathrm{MuA}$ & Saxifraga rotundifolia & E1 & 40 & 41 & \\
\hline $\mathrm{AF}$ & Cardamine enneaphyllos & E1 & 36 & 78 & 53 \\
\hline $\mathrm{AF}$ & Cyclamen purpurascens & E1 & 30 & 54 & 84 \\
\hline Сy & Cystopteris fragilis & E1 & 34 & 55 & 10 \\
\hline ES & Betonica alopecuros & E1 & 37 & 44 & 38 \\
\hline FS & Lonicera alpigena & E1 & 30 & 72 & 60 \\
\hline FS & Mercurialis perennis & E1 & 30 & 61 & 77 \\
\hline FS & Lilium martagon & E1 & 26 & 59 & 26 \\
\hline FS & Dryopteris filix-mas & E1 & 31 & 58 & 29 \\
\hline FS & Galium laevigatum & E1 & 18 & 51 & 74 \\
\hline FS & Galeobdolon flavidum & E1 & 29 & 56 & 16 \\
\hline FS & Prenanthes purpurea & E1 & 16 & 47 & 61 \\
\hline FS & Paris quadrifolia & E1 & 25 & 42 & 11 \\
\hline $\mathrm{MuA}$ & Aconitum lycoctonum s. lat. & E1 & 34 & 48 & 6 \\
\hline QF & Anemone nemorosa & E1 & 26 & 49 & 29 \\
\hline VP & Calamagrostis arundinacea & E1 & 17 & 58 & 29 \\
\hline VP & Veronica urticifolia & E1 & 23 & 57 & 52 \\
\hline VP & Mainathemum bifolium & E1 & 29 & 55 & 22 \\
\hline VP & Gentiana asclepiadea & E1 & 17 & 51 & 45 \\
\hline FS & Fagus sylvatica & E3 & 30 & 100 & 100 \\
\hline QP & Sorbus aria & $\mathrm{E} 3, \mathrm{E} 2, \mathrm{E} 1$ & 25 & 32 & 94 \\
\hline EP & Carex alba & E1 & 5 & 14 & 65 \\
\hline QF & Carex digitata & E1 & 21 & 26 & 64 \\
\hline FS & Laburnum alpinum & E3, E2, E1 & 10 & 16 & 86 \\
\hline QP & Ostrya carpinifolia & E3 & 8 & 0.1 & 68 \\
\hline AT & Asplenium ruta-muraria & E1 & 21 & 28 & 52 \\
\hline QF & Hepatica nobilis & E1 & 29 & 37 & 51 \\
\hline FB & Buphthalmum salicifolium & E1 & 13 & 11 & 47 \\
\hline QP & Fraxinus ornus & $\mathrm{E} 3, \mathrm{E} 2, \mathrm{E} 1$ & 3 & & 61 \\
\hline VP & Huperzia selago & E1 & 37 & 33 & 42 \\
\hline FS & Salvia glutinosa & E1 & 1 & 2 & 42 \\
\hline PS & Phyteuma scheuchzeri subsp. columnae & E1 & & 1 & 41 \\
\hline TA & Acer pseudoplatanus & E3 & 14 & 49 & 77 \\
\hline ML & Tortella tortuosa & E0 & 84 & 76 & 74 \\
\hline ML & Ctenidium molluscum & E0 & 72 & 88 & 80 \\
\hline ML & Rhytidiadelphus triquetrus & E0 & 66 & 8 & 18 \\
\hline ML & Dicranum scoparium & E0 & 54 & 21 & 38 \\
\hline ML & Hylocomium splendens & E0 & 48 & 2 & 9 \\
\hline ML & Paraleocobryum sauteri & E0 & 2 & 50 & 4 \\
\hline ML & Schistidium apocarpum & E0 & 26 & 47 & 24 \\
\hline ML & Fissidens dubius & E0 & 26 & 44 & 59 \\
\hline ML & Neckera crispa & E0 & 11 & 3 & 71 \\
\hline ML & Polytrichum formosum & E0 & 33 & 32 & 71 \\
\hline
\end{tabular}

